\title{
DOE/ER/40256-- T3
}

\section{PROGRESS IN RESEARCH}

APRIL 1, 1993 - MARCH 31, 1994

Prepared by

The Cyclotron Institute Staff

Texas A\&M University

College Station, TX 77843-3366

DISTRIBUTION OF THIS DOCUMENT IS UNLIMTEE 


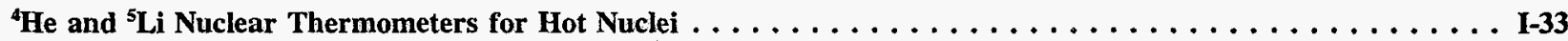

J. B. Natowitz, J. C. Hagel, R. Wada, X. Bin, J. Li, Y. Lou, and D. Utley

Time Scale of the Fission Process in the Reaction $50 \mathrm{AMeV}{ }^{20} \mathrm{Ne}+{ }^{165} \mathrm{Ho} \ldots \ldots \ldots \ldots \ldots \ldots \ldots$ N. Mdeiwayeh, R. Wada, G. Cibor, K. Hagel, J. Li, Y. Lou, Z. Majka, J. B. Natowitz, R. Tezkratt, D. Utley, and B. Xiao

Excitation Energy Dependence of the Fission Probability in ${ }^{200} \mathrm{~Pb}$ Compound Nuclei $\ldots \ldots \ldots \ldots \ldots \ldots$ D. Fabris, G. Viesti, E. Fioretto, M. Cinausero, N. Gelli, K. Hagel, F. Lucarelli, J. B. Natowitz, G. Nebbia, G. Prete, and R. Wada

Alpha Particle Emission as a Probe of the Level Density in Highly Excited A $\sim 200$ Nuclei $\ldots \ldots \ldots \ldots \ldots$ I-39 D. Fabris, E. Fioretto, G. Viesti, M. Cinausero, N. Gelli, K. Hagel, F. Lucarelli, J. B. Natowitz, G. Nebbia, G. Prete, and R. Wada

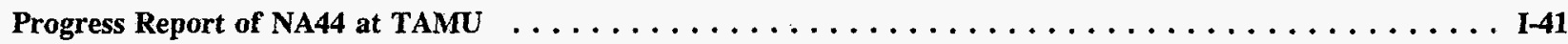
M. Murray

\section{NUCLEAR STRUCTURE AND FUNDAMENTAL INTERACTIONS}

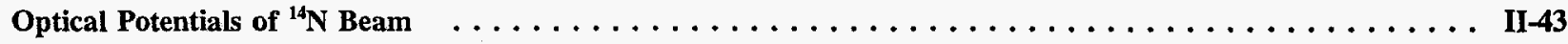

Y.-W. Lui, D. H. Youngblood, H. Clark, P. Oliver, G. F. Simler, and U. Garg

Search for Giant Resonances Above the GMR/GDR $\ldots \ldots \ldots \ldots \ldots \ldots \ldots \ldots \ldots \ldots \ldots \ldots$ II 4 46

D. H. Youngblood, Y.-W. Lui, P. Oliver, G. Simler, H. Clark, and U. Garg

The $\boldsymbol{\beta}$-Decay of ${ }^{57} \mathrm{Cu} \ldots \ldots \ldots \ldots \ldots \ldots \ldots \ldots \ldots \ldots \ldots \ldots \ldots \ldots \ldots \ldots \ldots \ldots$ II -48 D. R. Semon, M. Allen, H. D. Dejbakhsh, C. A. Gagliardi, S. Hale, L. M. Trache, R. E. Tribble, H. M. Xu, J. Jiang, S. J. Yennello, and X. G. Zhou

(d, $\left.{ }^{2} \mathrm{He}\right)$ Reactions with the Texas A\&M Proton Spectrometer $\ldots \ldots \ldots \ldots \ldots \ldots \ldots \ldots \ldots \ldots$ II-52

G. Ajupova, A. C. Betker, C. A. Gagliardi, B. Kokenge, H. M. Xu, and A. F. Zaruba

The $\bar{d}(x) / \bar{u}(x)$ Ratio in the Proton $\ldots \ldots \ldots \ldots \ldots \ldots \ldots \ldots \ldots \ldots \ldots \ldots \ldots \ldots$

C. A. Gagliardi, E. A. Hawker, and R. E. Tribble

The MEgA Experiment $\ldots \ldots \ldots \ldots \ldots \ldots \ldots \ldots \ldots \ldots \ldots \ldots \ldots \ldots \ldots \ldots \ldots \ldots$ II-59

C. A. Gagliardi, F. Liu, R. E. Tribble, X.-L. Tu, and L. Van Ausdeln

\section{NUCLEAR THEORY}

The Nuclear Spectral Function $\ldots \ldots \ldots \ldots \ldots \ldots \ldots \ldots \ldots \ldots \ldots \ldots \ldots \ldots \ldots \ldots \ldots$ III-62

G. M. Vagradov and S. Shlomo

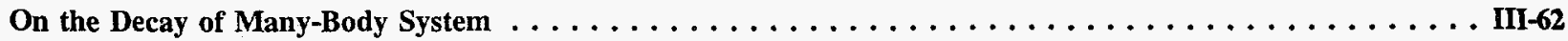

G. M. Vagradov and S. Shlomo

Stochastic Aspects of Nuclear Large Amplitude Motion $\ldots \ldots \ldots \ldots \ldots \ldots \ldots \ldots \ldots \ldots \ldots$ III-63 V. Kolomietz

Can Dileptons Reveal the In-Medium Properties of Vector Mesons? $\ldots \ldots \ldots \ldots \ldots \ldots \ldots \ldots \ldots$ III-65 G. Q. Li and C. M. Ko

Effective Mass and Width of Pions at $T \neq 0 \ldots \ldots \ldots \ldots \ldots \ldots \ldots \ldots \ldots \ldots \ldots \ldots \ldots \ldots \ldots$ III-67 Chungsik Song 
Phi Meson Width at Finite Temperatures $\ldots \ldots \ldots \ldots \ldots \ldots \ldots$ C. M. Ko and D. Seibert

Near-Threshold $\mathrm{K}^{+}$Production in Heavy-Ion Collisions Bao-An Li

Mean Free Path and Damping Rate of Kaons in Hot Hadronic Matter Chungsik Song

Effects of $\mathrm{N}^{*}(1440)$ Resonance on Subthreshold Kaon, Antikaon, and Antiproton Production III-75 B. A. Li, C. M. Ko, and G. Q. Li

Subthreshold Antikaon Production in Nucleus-Nucleus Collisions

G. Q. Li, C. M. Ko, and X. S. Fang

Antiproton Production in Heavy-Ion Collisions at Subthreshold Energies

G. Q. Li, C. M. Ko, X. S. Fang, and Y. M. Zheng

Fixed Exciton Number Level Density for a Finite Potential Well

S. Shlomo, Ye. A. Bogila, V. M. Kolomietz, and A. I. Sanzhur

Few-Body Problems in Nuclear Astrophysics. Asymptotic Behavior of Bound State Wave

Function in Two-Body Channels and Radiative Capture Reactions

L. D. Blokhintsev and A. M. Mukhamedzhanov

Coulomb Post Acceleration in Coulomb Break-Up Reactions in Asymptotic Three-Body Approach

E. O. Alt, B. F. Irgaziev, and A. M. Mukhamedzhanov

On the Possibility to Determine the Astrophysical S-Factor for the ${ }^{7} \mathrm{Be}(\mathrm{p}, \gamma)^{8} \mathrm{~B}$ Radiative

Capture Reaction from the Analysis of Proton Transfer Reactions

A. M. Mukhamedzhanov, N. K. Timofeyuk, and R. E. Tribble

The Overall Normalization of the Astrophysical S-Factor and the Asymptotic

Normalization Coefficient for ${ }^{7} \mathrm{Be}(\mathrm{p}, \gamma)^{8} \mathrm{~B}$ Reactions $\ldots \ldots \ldots \ldots \ldots \ldots \ldots \ldots$

H. M. Xu, C. A. Gagliardi, R. E. Tribble, A. M. Mukhamedzhanov, and N. K. Timofeyuk

A Comparison of BUU and QMD Calculations for ${ }^{40} \mathrm{Ca}+{ }^{40} \mathrm{Ca}$ Collisions Near the Fermi Energy . . . . . III-93

J. Cibor, J. Lukasik, Z. Majka, and H. M. Xu

Limiting Temperatures and Disappearance of Fusionlike Residues . . . . . . . . . . . . . . . . III-97 H. M. Xu

Fragmentation Barriers of Toroidal and Bubble Nuclei $\ldots \ldots \ldots \ldots \ldots \ldots$ H. M. Xu, C. A. Gagliardi, R. E. Tribble, and C. Y. Wong

Multifragmentation of Toroidal and Bubble Nuclei Within the BUU Approach . . . . . . . . . . . . III-101

H. M. Xu, C. A. Gagliardi, R. E. Tribble, and C. Y. Wong

Investigation of the Equation of State Properties using Maximum Linear Momentum Transfer . . . . . . . III-103

F. Haddad, K. Hagel, J. Li, N. Mdeiwayeh, J. B. Natowitz, R. Tezkratt, D. Utley, R. Wada, and B. Xiao

Role of the $a_{1}$ Meson in Dilepton Production from Hot Hadronic Matter . . . . . . . . . . . . . . III-105 Chungsik Song, C. M. Ko, and C. Gale

\section{ATOMIC MOLECULAR \& MATERIALS SCIENCE}

Electron Spectra from $\mathrm{Ag}$ and $\mathrm{NaCl}$ Targets Induced by Highly-Charged Ar Ions IV-107

C. Assad and R. E. Tribble 
Spectra of L X-Rays from Fast, Highly-Charged Xe Ions Traveling in Solid

V. Horvat, R. L. Watson, and R. Parameswaran

Determination of Charge Distributions for 6 and $8 \mathrm{MeV} / \mathrm{u}$ Xe Ions Traveling in

V. Horvat, R. L. Watson, and R. Parameswaran

Charge Distribution of $8 \mathrm{MeV} / \mathrm{u}$ Xe Ions Emerging from Carbon Foils $\ldots \ldots \ldots \ldots \ldots$

R. L. Watson, V. Horvat, R. Parameswaran, L. J. Cook, J. Knott, and A. Nash

Projectile and Target L X-Ray Spectra Produced in Fast Kr Ion Collisions

V. Horvat, J. M. Blackadar, R. L. Watson, and R. Parameswaran

Charge Distributions of Noble Gas Recoil-Ions Produced in Fast Heavy Ion-Atom Collisions

IV-123

R. Parameswaran, V. Horvat, and R. L. Watson

Lifetimes of Metastable Atomic Levels of Multicharged Ions

IV-125

D. A. Church

\section{SUPERCONDUCTING CYCLOTRON AND INSTRUMENTATION}

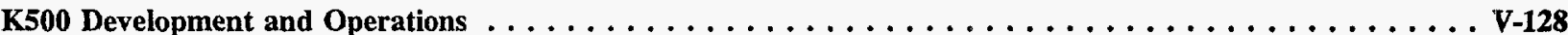
D. P. May, G. Mouchaty, G. J. Kim, and F. Abegglen

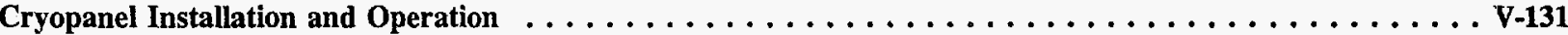
P. Smelser, G. J. Derrig, F. P. Abegglen, S. M. Bielamowicz, W. L. Dewees, D. P. May, W H. Peeler, R. D. Russell, and W. J. Walterscheid

Computing at the Cyclotron Institute $\ldots \ldots \ldots \ldots \ldots \ldots \ldots \ldots \ldots \ldots \ldots$

H. Dejbakhsh and K. Hagel

Single Event Effect Facility $\ldots \ldots \ldots \ldots \ldots \ldots \ldots \ldots \ldots \ldots \ldots \ldots \ldots \ldots \ldots \ldots \ldots \ldots$

R. C. Rogers, R. A. Gutierrez, D. P. May, D. L. Utley, and R. Wada

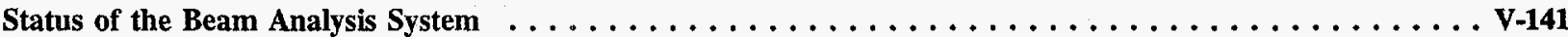

J. Bronson, D. H. Youngblood, Y.-W. Lui, G. Derrig, and F. Abegglen

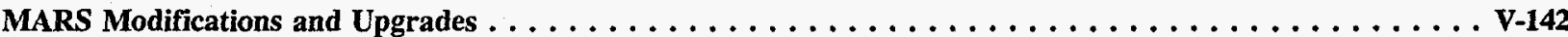

R. Tribble, H. Dejbakhsh, R. Eiserer, C. Gagliardi, S. Hale, R. Rogers, D. Semon, L. Trache, H. Xu, and X. Zhou

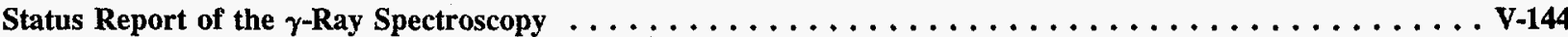

H. Dejbakhsh, C. A. Gagliardi, J. Jiang, D. R. Semon, L. Trache, R. E. Tribble, H. M. Xu, and X. G. Zhou

A High Resolution Large Dynamic Range Charged-Particle Detector for Nuclear Fragmentation Studies . . . . V-146

T. C. White, J. A. Winger, H. Johnston, and S. J. Yennello

Monte Carlo Code Simulations of the Neutron Ball Response $\ldots \ldots \ldots \ldots \ldots \ldots \ldots$

B. Hurst and R. P. Schmitt

Running with the Neutron Ball $4 \pi$ Detector in a Fast Mode $\ldots \ldots \ldots \ldots \ldots \ldots \ldots$

F. Haddad, K. Hagel, J. Li, N. Mdeiwayeh, J. B. Natowitz, R. Tezkratt, D. Utley, R. Wada, and B. Xiao

A New Method to Produce Plastic Scintillator Foils

V-153

B. Xiao, S. Lee, K. Hagel, F. Haddad, J. Li, R. Tezkratt, R. Wada, D. Utley, and J. B. Natowitz 
Status of the CsI Ball $\ldots \ldots \ldots \ldots \ldots \ldots \ldots \ldots \ldots \ldots \ldots \ldots \ldots$

B. Xiao, G. Derrig, K. Hagel, F. Haddad, S. Lee, J. Li, Y. Lou, N. Mdeiwayeh, R. Tezkratt, R. Wada, D. Utley, and

J. B. Natowitz

A Nearly Complete Detector System for Studying Heavy Ion Reactions . . . . . . . . . . . . . . . V-158

B. Xiao, G. Derrig, K. Hagel, F. Haddad, S. Lee, J. Li, Y. Lou, R. Tezkratt, R. Wada, D. Utley, and J. B. Natowitz

An Angle Calibration Technique for Large Aperture Spectra $\ldots \ldots \ldots \ldots \ldots \ldots$

H. L. Clark, Y.-W. Lui, and D. H. Youngblood

Design of a Position Sensitive PPAC

V-161

R. Parameswaran and R. Gutierrez

VI. PUBLICATIONS

Abstracts of Papers Published

April 1993 - March 1994

Abstracts of Papers Submitted

April 1993 - March 1994

VII. APPENDIX

Talks Presented .

Research Personnel and Engineering Staff

VII-180

Students Who Received Ph.D. Degrees

VII-181

Institute Colloquia and Seminars

VII-182 


\section{INTRODUCTION}

\section{April 1993 - March 1994}

The period 1 April 1993 - 31 March 1994 has seen a number significant developments of our research program as will be noted by the large increase in individual projects reviewed in this annual report. This increase reflects a number of convergent factors: The facility has moved to seven day a week operation of the accelerator; Many of the heavy ion experiments centered at the Neutron Ball have reached the stage of final analysis and publication; Experiments with the MARS and MDM Spectrometers are now well underway; An increase in the number of visiting scientists and collaborators has both increased the scientific manpower and stimulated some new activities.

Another, especially pleasing indicator of the activity level of the research program is the graduation of six of our Ph.D. students during this report period (see page VII 181).

Among the highlights of the K500 experimental program in Sections I, II, and IV are the investigations of excitation energy deposition and of fission dynamics employing both GDR and particle emission probes, measurements of isospin equilibration, studies of $\left(d,{ }^{2} \mathrm{He}\right)$ reactions with the proton spectrometer and of the $\beta$ decay of ${ }^{57} \mathrm{Cu}$ with MARS, and the precise studies of ionic charge state distributions using $\mathrm{x}$-ray measurements. Progress in theoretical studies of the nuclear spectral function and the decay of many body systems, on the properties of mesons in hot hadronic matter and on the determination of astrophysical S-factors from experimental studies of very peripheral reactions are presented in Section III.

The status of the LAMPF based MEGA experiment and of the CERN based NA66 experiment, both of which involve institute scientists, is also briefly presented in this report.

The shift to a seven day a week operation (thus avoiding the cycling on and off of many components) coupled with installation of cryopanels and more careful temperature control of the cooling water system have resulted in significant improvements in our operational efficiency and beam capabilities. The fractional use of the machine for higher energy beams $(E / Q \geq 110)$ increased from $10 \%$ last year to $49 \%$ this year. At the same time the hours of beam on target for experiments increased $14 \%$. Operating statistics are presented in Section V.
Also contained in Section V are descriptions of various development projects which will have a significant impact on our future program. The major upgrade of our computer capabilities continued in the past year with the installation of new VME based acquisition systems of the IUCF design. The beam analysis system is now half done and will be completed and operational by the end of this year. The new experimental station for Single Event Effect radiation damage measurements is installed and has been used by NASA scientists. Notable also are the preparations for $\gamma$ spectroscopy experiments using MARS with some Compton suppressed Ge detectors of the HERA array (on loan from LBL). This combination should prove to be particularly powerful for a number of structure studies.

The research described in this report is funded primarily by the Department of Energy under grants DE-FG03-93ER40773 (nuclear) and DE-FG0584ER13262 (atomic); by the National Science Foundation under grants PHY-8907986 and PHY9107008 (theory); and by The Robert A. Welch Foundation under grants A-330, A-355, A-558, A692, A-972, A-1082, A-1110, and A-1266. Operation of the facility is also supported by the University. The Texas A\&M MEGA collaboration is supported under grant DE-FG03-93ER40765. Research in the STAR collaboration is supported under grant DE-FG05-88ER40437. The LAMPF few nucleon studies have been funded under grant DEFG05-88ER40399. The MDM spectrometer and the analysis system are also funded by DOE under grant DE-FG03-93ER40773.

Some of the data and conclusions presented in this report are based upon preliminary analyses of the experiments. Until this research is published, it should not be cited without express consent of the investigators involved.

Finally, it is with great regret that I report the death of our longtime colleague and friend, Lee Northcliffe. Lee died June 2, 1994 as this report was in final preparation. We will miss him. This report is dedicated to his memory.
J. B. Natowitz July 1994 


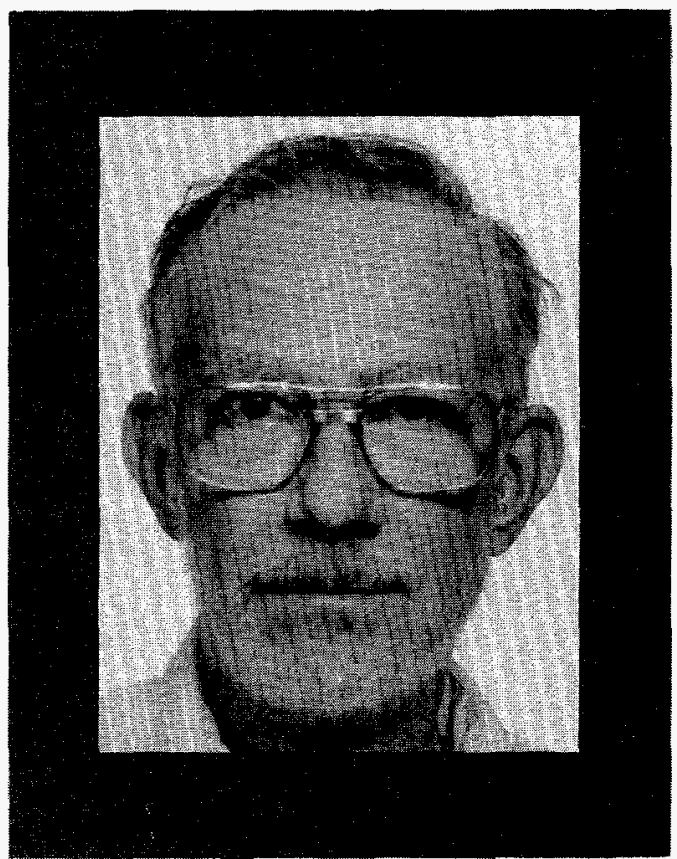

LEE C. NORTHCLIFFE

$1926-1994$ 


\section{heavy ION REACTIONS}




\section{Energy Deposition Systematics from Neutron Multiplicity Studies}

\section{J. Boger ${ }^{*}$, T. Botting, L. Cooke ${ }^{* *}$, B. Hurst, D. O'Kelly, R. P. Schmitt and W. Turmel ${ }^{* * *}$}

In recent years, considerable efforts have been directed at exploring the properties and decay modes of highly excited nuclear systems. Clearly, a knowledge of the excitation energy of the intermediate system is a fundamental ingredient needed in interpreting the results of these studies. At least for medium mass and heavy nuclei, one expects that a substantial fraction of the thermal excitation energy should be disposed of via neutron evaporation. Thus event-by-event neutron multiplicity measurements should offer an important handle on the initial excitation energy distributions. In order to explore this possibility, we have performed neutron multiplicity measurements for a variety of systems with expected excitation energies ranging from about $100 \mathrm{MeV}$ to more than $1000 \mathrm{MeV}$.

In the experiments beams of $30 \mathrm{MeV} / \mathrm{u}{ }^{14} \mathrm{~N},{ }^{20} \mathrm{Ne}$ and ${ }^{63} \mathrm{Cu}$ and $55 \mathrm{MeV} / \mathrm{u}{ }^{4} \mathrm{He}$ were used to irradiate targets ranging from ${ }^{12} \mathrm{C}$ to ${ }^{238} \mathrm{U}$. The associated neutron multiplicity distributions were measured with the neutron ball operated in a self-triggered mode. In this mode one can obtain a global view of the energy deposition in a reaction in a relatively short period of time (typically less than one hour per target + projectile system).

The multiplicity distributions were determined by scaling the number of delayed flashes from the ball during two successive $100 \mu$ s time intervals. The data from these gates were used to generate background corrected neutron multiplicity distributions for each reaction. These distributions were then corrected for losses due to the finite gate widths and pile-up effects. Additional details about the experimental methods and the analysis procedures can be found elsewhere. ${ }^{1.2}$

The neutron multiplicity distributions, $\mathbf{P}\left(\mathrm{M}_{\mathrm{n}}\right)$, obtained for the lightest systems show an approximately exponential decrease with increasing $M_{n}$. As seen in Fig. 1, the distributions medium mass and heavy systems show an additional Gaussian shaped component, which can be attributed to central collisions. In cases where this latter bump was visible, the multiplicity distributions were fit using a functional form consisting of an exponential plus a Gaussian. The efficiency corrected, most probable multiplicities (see below) are shown in Fig. 2 as a function of the target mass. Overall, the most probable multiplicity tends to increase with the target mass although there is considerable structure, particularly at the low mass end. The general rise in the multiplicity with mass can be attributed to the increasing center-of-mass energy and the decreasing probability of charged particle emission due to the larger barriers for the heavier systems. At the highest masses, the energy release from fission also contributes to the enhancement in the multiplicities. The structure seen at low masses is largely due to neutron binding energy effects. The multiplicity distributions for neutron deficient targets, such as ${ }^{112} \mathrm{Sn}$ and ${ }^{144} \mathrm{Sm}$, tend to peak at lower values than

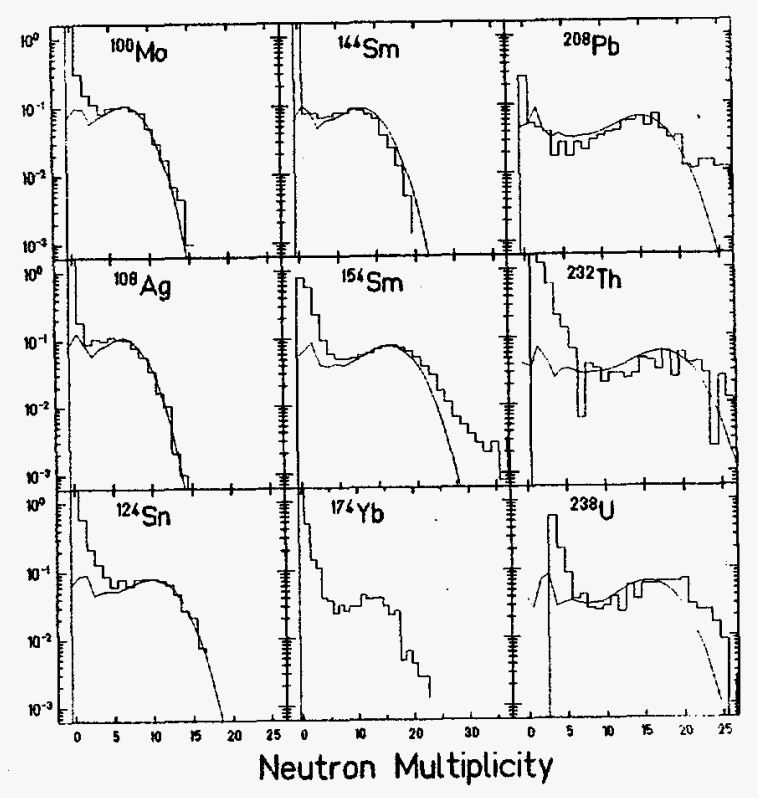

Figure 1. Measured (histograms) and calculated (curves) neutron multiplicity distributions for reactions of $30 \mathrm{MeV} / \mathrm{u}^{20 \mathrm{Ne}}$ and the indicated targets. 

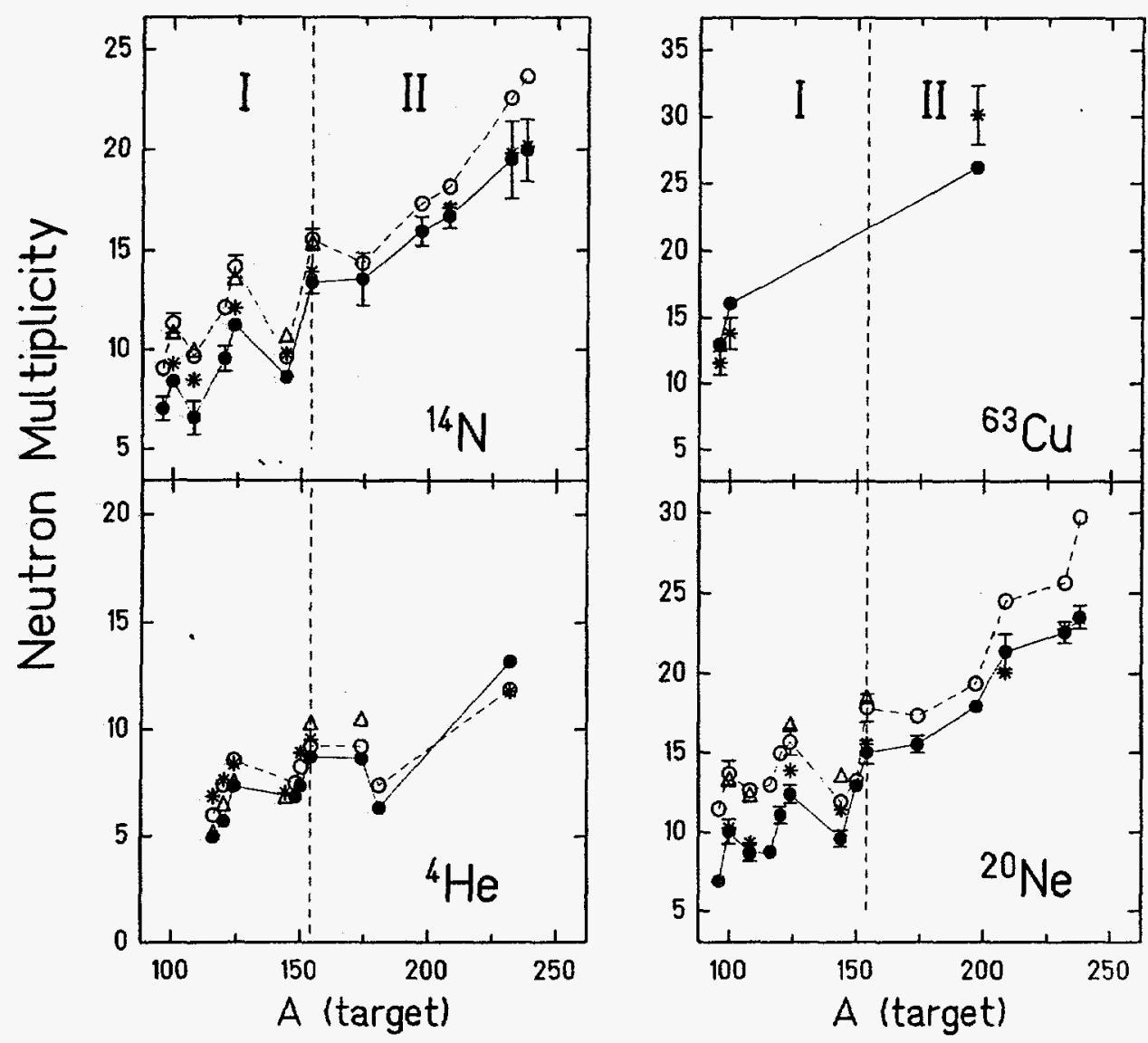

Figure 2. Most probable neutron multiplicity as a function of target mass for reactions induced with the indicated projectiles. The solid. points reflected the measured values. The circles, triangles and stars show the values predicted by CASCADE, GEMINI and EUGENE, respectively. Note that a suppressed zero has been employed on some plots.

those of their neutron rich counterparts, ${ }^{124} \mathrm{Sn}$ and ${ }^{154} \mathrm{Sm}$.

The experimental results have been compared with a variety of statistical model codes, such as CASCADE and GEMINI. In making the comparison, the initial conditions have been defined using what is probably the simplest of pictures, namely massive transfer. This approach assumes that a fraction of the projectile undergoes a complete fusion reaction with the target while the remaining spectator continues unscathed along the beam direction. The fractional mass (and energy) transfer has been determined with the aid of momentum transfer systematics. As an example, in the 30 $\mathrm{MeV} / \mathrm{u}{ }^{20} \mathrm{Ne}$ reactions where about $74 \%$ momentum transfer is expected, this translates into the complete fusion of ${ }^{15} \mathrm{~N}$ with the corresponding target nucleus.

Either the data or the statistical model calculations have to be corrected for the neutron detection efficiency in order to make a meaningful comparison. This is a complex problem since the multiplicity distributions reflect a wide variety of emission sources and temperatures. Lacking the detailed information required to fully untangle the web of competing processes, a simple picture has been adopted in which it was assumed that the neutrons were emitted from a single source moving along the beam axis (modifications were made when fission was estimated to occur with a greater than $10 \%$ probability). The velocity and associated temperature of this source were again estimated from momentum transfer systematics. These source 
parameters were fed into a highly modified version of the code DENIS, originally developed by Poitou and Signarbieux ${ }^{3}$ to determine the detection efficiency. The modified code incorporates the appropriate kinematics for a moving source and includes neutron cross section data up to $200 \mathrm{MeV}{ }^{4}$ Although there is significant spread in the estimated momentum transfers and corresponding emitter velocities and temperature, the calculated efficiency function for most of the systems is not particularly sensitive to these effects. With the possible exception of the ${ }^{63} \mathrm{Cu}$ induced reactions, the efficiencies are likely determined to within about $5 \%$.

The open circles in Fig. 2 depict the results of CASCADE calculations. Consistent with the data, the calculations reproduce the overall increase in $M_{n}$ with target mass. Moreover, the model calculations also tend to mirror the structure seen below about mass 150. However, close examination of Fig. 2 shows that the calculations invariably overestimate the multiplicities. The disparity is only around $10 \%$ for the heaviest systems but is closer to $30 \%$ for the lightest target-projectile combinations. Since CASCADE only treats the evaporation of light particles, one might speculate that the neglect of fragment emission could explain the discrepancy. However, calculations using GEMINI (triangles), which includes fragment emission, also overpredict the multiplicities.

One could attribute the mismatch between the data and the calculations to either the failure of the statistical model in correctly describing the competition between charged particle emission and neutron evaporation or some problem in the massive transfer assumption used in the model calculations. To further explore these possibilities, EUGENE calculations ${ }^{5}$ have been performed for a number of the systems. The results are indicated by stars in Fig. 2. The EUGENE code is more refined than the others in the sense that it makes some effort to model the entrance. channel dynamics and to include pre-equilibrium particle emission at least in a schematic way.
The agreement between the data and the EUGENE predictions is quite impressive. With few exceptions, the calculated multiplicitiesare very close to the measured values. As a further test of this model, the calculated multiplicity distributions have been compared with the experimental results. To correct for the detector response, the calculated distributions have been folded with the efficiency function determined by the Monte Carlo simulations. The results are shown by the curves in Fig. 1. Again, the agreement is very satisfactory. The central collision bump is well reproduced. Discrepancies are seen at the lowest multiplicities. However, this can be attributed largely to the neglect of deep inelastic processes and partially damped reactions in the model.

It is obviously important to trace out the reasons for the success of the EUGENE calculations. Sorting the event files generated by the program shows that the predicted momentum transfer is very much in line with that assumed in the other calculations. However, the predicted thermal energy deposition tends to fall some $20-30 \%$ below that given by the simplest massive transfer picture. This difference appears to result mainly from the pre-equilibrium emission. Although this is treated rather schematically in the model, it seems to account for most of the discrepancies seen with the other statistical model codes. It would be interesting to see how well this observation holds up for more highly excited systems. Such studies are planned for the near future.

\section{Present Address:}

Brookhaven National Laboratory, Upton, NY.

**Austin Community College, Austin, TX.

***Texas A\&M-Corpus Christi, Corpus Christi, TX.

\section{References}

1. R. P. Schmitt et al., New Nuclear Physics with Advanced Techniques (World Scientific, NY), p. 182. 
2. J. Boger et al., Progress in Research, 1992-1993, Cyclotron Institute, TAMU, p. 1.

3. J. Poitou and C. Signarbieux, Nucl. Instrum. Methods 114, 113 (1974).
4. B. Hurst and R. P. Schmitt, Progress in Research, 1993-1994, Cyclotron Institute, TAMU, p. 147.

5. D. Durand, Nucl. Phys. A541, 266 (1992).

\title{
GDR $\gamma$-Ray Emission in $144 \mathrm{MeV}{ }^{12} \mathrm{C}$-Induced Fission of ${ }^{197} \mathrm{Au}$
}

\author{
T. Botting, J. Boger, ${ }^{*}$ R. Erkert, B. Hurst, H. Jabs, J. Jackson, M. Murray, D. O'Kelly, B. \\ Sadhwani, P. Singh, R. P. Schmitt, and K. L. Wolf
}

Since their discovery, $\gamma$ rays emitted by giant dipole resonances (GDR) built on excited states ${ }^{1}$ have generated much interest because they can provide new information on shapes and other characteristics of hot nuclei. ${ }^{2}$ Recent studies involving fissioning systems $^{3}$ have shown that the GDR $\gamma$ rays can provide insights into the dynamics of the fission process similar to those obtained in fission-neutron coincidence experiments. ${ }^{4.5}$ Besides yielding the time scale of the fission process, GDR $\gamma$ ray emissions can also provide information on the shapes of rapidly rotating nuclei.

Previously, we have dealt with $120 \mathrm{MeV}{ }^{16} \mathrm{O}$ induced reactions, concentrating mainly on a ${ }^{208} \mathrm{~Pb}$ target and utilizing parallel plate avalanche counters (PPAC) in conjunction with NaI segments from the total energy crystal. ${ }^{6}$ However, low statistics and poor ability to separate neutrons from $\gamma$ rays led us to investigate other means. To that end, we have recently investigated $144 \mathrm{MeV}{ }^{12} \mathrm{C}$ on ${ }^{197} \mathrm{Au}$ utilizing the newly completed Barium Fluoride Arrays (BFA).

The experimental setup consisted of the ${ }^{197} \mathrm{Au}$ target at an angle of $75^{\circ}$ relative to the beam with two silicon detectors, placed at $90^{\circ}$ and $60^{\circ}$, respectively. The BFA's were configured into two "pods" of $19 \mathrm{BaF}_{2}$ detectors. The Pod A was placed at $90^{\circ}$ at a distance of $60 \mathrm{~cm}$, while the Pod B was positioned at $143^{\circ}$ at a distance of $77 \mathrm{~cm}$. To reduce the neutron background, a large block of polyethylene was placed directly in front of each pod. An array of $\mathrm{NaI}$ wedges from the Cyclotron's total energy counter was located behind Pod A to evaluate the leakage through the nineteen pack. Neutron counters were also placed at $20^{\circ}, 24^{\circ}, 29^{\circ}$, and $31^{\circ}$ with plastic scintillators in front as charged particle vetoes.

A preliminary analysis of this data suggests that pulse shape discrimination of the energy signals for neutrons and $\gamma$ rays is indeed possible from the BFA. This is evident in an event-by-event plot of the fast component versus the slow component for an individual crystal (Fig. 1). In this figure, the neutrons are represented by the band of points with the smaller slope and lower statistics. In addlition, we have a first look at the sum $\gamma$ ray energies from all the crystals for many runs (Fig. 2). The energy spectrum shown in Fig. 2 has gates on a common threshold of $3.5 \mathrm{MeV}$ on the fast component of each crystal, fission coincidence, and $\gamma$ rays as determined by fast versus slow plots, such as in Fig. 1 . Note the non-exponential behavior above $10 \mathrm{MeV}$ - the GDR $\gamma$ ray region.

In completing the analysis, the $\gamma$ ray analysis still needs to be optimized in a number of ways, including setting reliable timing gates, correlating two-fissionfragment events, evaluating the leakage through the BFA's, evaluating pileup in the BFA's, etc. If 


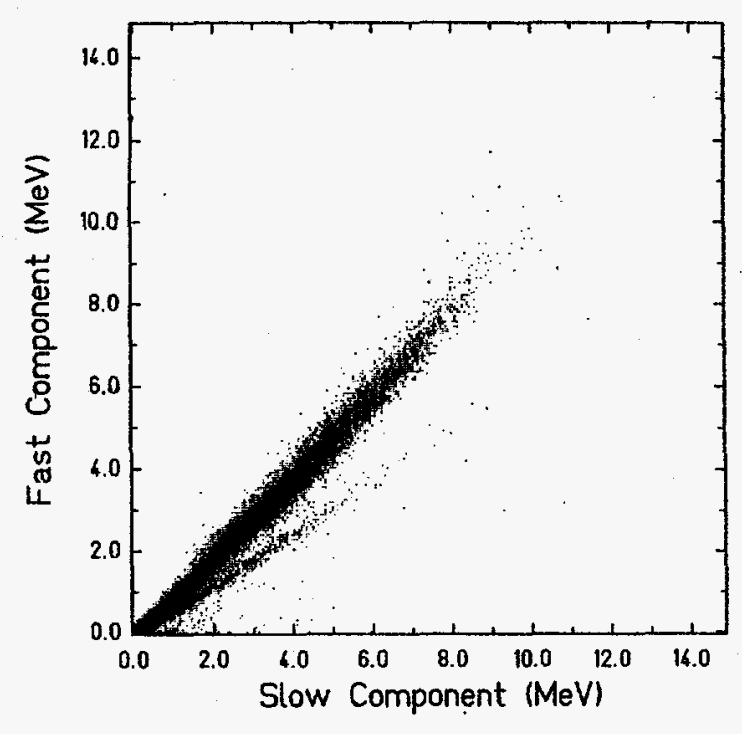

Figure 1. Cluster plot showing the separability of neutron energies from $\gamma$ ray energies using the pulse-shape discrimination capabilities of the BFA's.

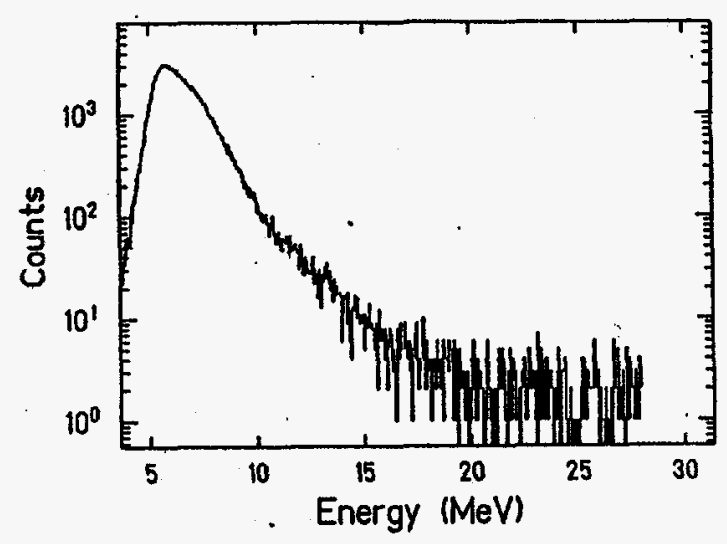

Figure 2. Sum spectrum of $\gamma$ ray energies detected in coincidence with fission fragments and gated on fast energy pulses $>3.5$ $\mathrm{MeV}$. statistics warrant, we also plan to investigate the effects of different mass asymmetries on the spectra in an effort to extract fission times and shape information from the data.

"Present address: Brookhaven National Lab, Upton, New York

\section{References}

1. J. O. Newton et al., Phys. Rev. Lett. 46, 1383 (1981).

2. K. A. Snover, Ann. Rev. Nucl. Part. Sci., 36 (1986).

3. M. Thoennessen et al., Phys. Rev. Lett. 59, 2860 (1987).

4. A. Gavron et al., Phys. Rev. C 35, 579 (1987), and references therein.

5. D. J. Hinde et al., Phys. Rev. C 45, 1229 (1992).

6. W. Turmel et al., Progress in Research, 19911992, Cyclotron Institute, Texas A\&M University, p. 17. 


\title{
Intermediate Mass Fragment Emission From the Reaction of ${ }^{40} \mathrm{Ar},{ }^{40} \mathrm{Ca}$ With ${ }^{58} \mathrm{Ni}$, ${ }^{58} \mathrm{Fe}$ at $\mathbf{E} / \mathbf{A}=35 \mathrm{MeV}$
}

\author{
H. Johnston, J. Winger, ${ }^{*}$ T. White, B. Hurst, D. O'Kelly, S. J. Yennello
}

Recently, there has been a great deal of interest in the isotopic composition of intermediate mass fragments emitted from intermediate energy light ion and heavy ion collisions. ${ }^{1-7}$ The isotopic composition of the reaction products contains important information about the reaction mechanism. If the composite system is equilibrated prior the emission of reaction products than the $N / Z$ ratio of those products should be independent of the $N / Z$ of the entrance channel. If the emission of the products occurs before equilibration than the products may resemble the $N / Z$ of the projectile and/or the target.

We recently performed an experiment to study the isotopic composition of nuclear reaction products produced in the reactions of $\mathrm{E} / \mathrm{A}=35 \mathrm{MeV}{ }^{40} \mathrm{Ar}+$ ${ }^{58} \mathrm{Fe},{ }^{58} \mathrm{Ni}$ and $\mathrm{E} / \mathrm{A}=35 \mathrm{MeV}{ }^{40} \mathrm{Ca}+{ }^{58} \mathrm{Fe},{ }^{58} \mathrm{Ni}$. These particular systems allow for the measurement of data over a range of $N / Z$ with a constant target mass and a constant projectile mass.

The reactions were carried out in the neutron ball. The intermediate mass fragment and light charged particles were measured by an array of six axial-field gas-ionization chamber telescopes covering an angular range of $10^{\circ}$ to $148^{\circ}\left(10^{\circ}, 44^{\circ}, 72^{\circ}\right.$, $\left.100^{\circ}, 128^{\circ}, 148^{\circ}\right)$. These detectors are described in detail elsewhere in this report. The coincident neutrons were detected by the neutron ball which will enable event characterization on an event by event basis.

Preliminary results (Fig. 1) shows the behavior of the isotopic ratios for the isotopes lithium through carbon at $44^{\circ}$. The data points represent the ratio of the number of counts of the more neutron rich isotope to that of the more proton rich isotope for the two most prominent isotopes of a given $\mathrm{Z}$.

$$
\left(\frac{N}{Z}\right)_{C S}=\frac{N_{\text {projectile }}+N_{\text {target }}}{Z_{\text {projectile }}+Z_{\text {target }}}
$$

A single line fits all of the data points for a given $\mathbf{Z}$. This indicates that the composite system may be equilibrated before the emission of reaction products. Further analysis of this data is currently in progress.

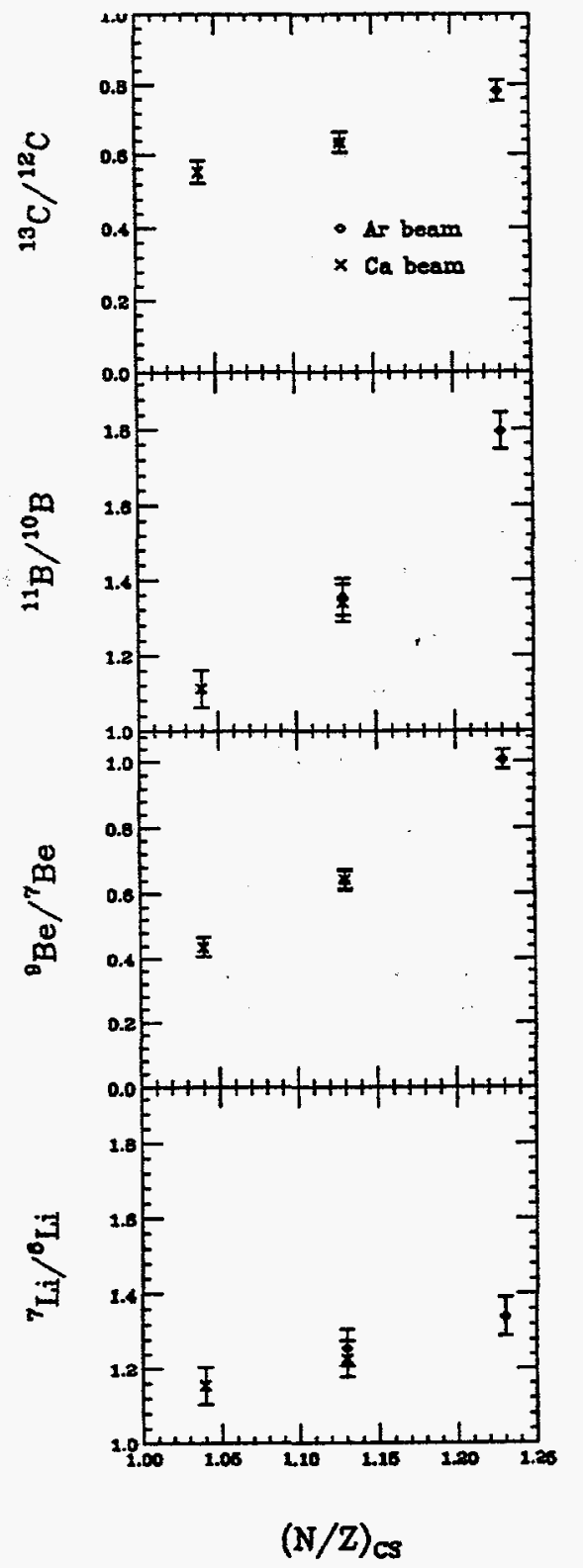

Figure 1. Isotopic ratios plotted as a function of $N / Z$ of the combined target and projectile system. 
"Present address: Mississippi State University, Mississippi State, Mississippi

\section{References}

1. S. J. Yennello et al., Phys. Lett. 321B, 15 (1994).

2. S. J. Yennello et. al., Proceedings from the 10th Winter Workshop on Nuclear Dynamics, Snowbird, UT, 1994, ed. W. Bauer.
3. J. Brzychczyk et. al., Phys. Rev. C 47, 1553 (1993).

4. E. Renshaw et. al., Phys. Rev. C 44, 2618 (1991).

5. R. Wada et. al., Phys. Rev. Lett. 58, 1829 (1987).

6. J. L. Wile et. al., Indiana University Nuclear Chemistry report \#INC-40007-77 (1990).

7. F. Deak et. al., Phys. Rev. C 43, 2432 (1991).

\section{Equilibration of the Isospin Degree of Freedom in the Reaction of $\mathrm{E} / \mathrm{A}=23 \mathrm{MeV}{ }^{40} \mathrm{Cl},{ }^{40} \mathrm{Ar}$ and}

${ }^{40} \mathrm{Ca}$ with ${ }^{58} \mathrm{Fe}$ and ${ }^{58} \mathrm{Ni}$

\section{S. J. Yennello, J. A. Winger ${ }^{*}$, H. Johnston, T. White, E. Gualtieri ${ }^{\ddagger}$, D. Craig ${ }^{\ddagger}$, S. Hannuschke ${ }^{\ddagger}$, J. Yee ${ }^{\ddagger}$, R. Pak ${ }^{\ddagger}$, A. VanderMolen ${ }^{\ddagger}$, W. Benenson ${ }^{\ddagger}$, G. D. Westfall ${ }^{\ddagger}$, T. Li $i^{\ddagger}$, W. J. Llope ${ }^{\ddagger}$, D. J. Morrissey $^{\ddagger}$, J. S. Winfield ${ }^{\ddagger}$ and M. Steiner ${ }^{\ddagger}$}

In a recent experiment the isospin degree of freedom was demonstrated not to be equilibrated on the timescale of emission of fragments from the reaction of $\mathrm{E} / \mathrm{A}=53 \mathrm{MeV}{ }^{40} \mathrm{Ar},{ }^{40} \mathrm{Ca},{ }^{40} \mathrm{Cl}$ beams with ${ }^{58} \mathrm{Fe}$ and ${ }^{58} \mathrm{Ni}$ targets. ${ }^{1}$ Previous work on similar systems at a much lower energy ${ }^{2}$ showed that the isospin degree of freedom was one of the fastest to equilibrate. In the higher energy data it appears that the timescale of interaction before fragmentation has been shortened to be on the order of that of equilibration of the isospin degree of freedom. If there is to be a consistent picture then a study similar to Ref. 1 must also see isospin equilibration at lower energies. There would then be a transition region where the onset of fragmentation before equilibration was observed. In order to search for the low energy equilibration we performed an experiment at Michigan State University/NSCL.

Primary beams of $\mathrm{E} / \mathrm{A}=25 \mathrm{MeV}{ }^{40} \mathrm{Ca}$ and ${ }^{40} \mathrm{Ar}$ were extracted from the K1200 cyclotron and focused on targets of ${ }^{58} \mathrm{Fe}$ and ${ }^{58} \mathrm{Ni}$ at the center of the $4 \pi$ array. The $\mathrm{A} 1200$ was used to separate $\mathrm{E} / \mathrm{A}=23$ $\mathrm{MeV}{ }^{40} \mathrm{Cl}$ fragments produced by an $\mathrm{E} / \mathrm{A}=80 \mathrm{MeV}$ ${ }^{40} \mathrm{Ar}$ beam incident on a $\mathrm{Be}$ production target. Additionally data from a secondary beam of $E / A=53$ $\mathrm{MeV}{ }^{40} \mathrm{Sc}$ was also measured to extend the previous study $^{1}$ to more extreme values of $\mathrm{N} / \mathrm{Z}$. These secondary beams of radioactive ions were focused on the same reaction targets at the center of the $4 \pi$. Isotopically separated intermediate-mass fragments and light charged particles were detected in an array of 13 silicon telescopes placed inside the $4 \pi$ array. These telescopes measured fragments at $14^{\circ}, 40^{\circ}$, and $140^{\circ}$. The experimental setup is similar to that in the previous experiment which is described in detail in Ref. 1.

Preliminary data showing the ratio of the two most abundant isotopes for a given $\mathrm{Z}$ plotted as a function of the neutron to proton ratio of the combined system are shown in Fig. 1. The nonequilibrium behavior as seen in the higher energy studies for the heaviest fragments does not appear to 
be present in this data. This would indicate that at $\mathrm{E} / \mathrm{A}=25 \mathrm{MeV}$ the system reaches a much greater degree of equilibration before disassembly.

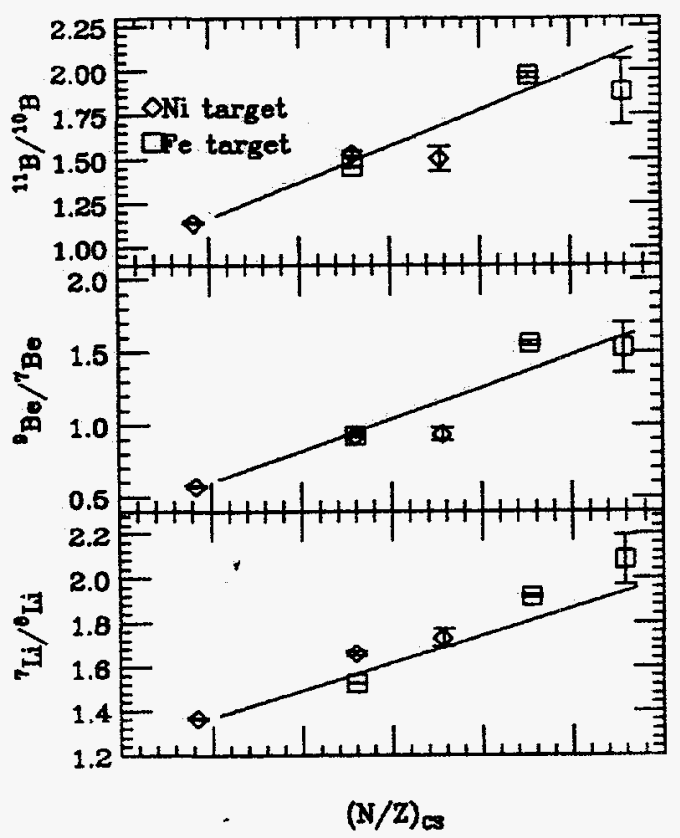

Figure 1. Isotopic ratios plotted as a function of the $N / Z$ of the combined target and projectile system. Lines are linear fits to the data.

The behavior demonstrated by the two most prominent isotopes is also reflected by the lower yield isotopes. Figure 2 shows the relative amount of the different carbon isotopes detected versus their mass number. Notice that the ${ }^{11} \mathrm{C}$ is similar to the ${ }^{12} \mathrm{C}$ in that the most proton rich system $(\mathrm{Ca}+\mathrm{Ni})$ produces the greatest percentage of these isotopes. The ${ }^{14} \mathrm{C}$ reflects the ${ }^{13} \mathrm{C}$ as the highest production of these isotopes is from the most neutron rich system ( $\mathrm{Ar}+$ $\mathrm{Fe})$. The same relative production of all carbon isotopes results from the two systems that differ only in entrance channel $(\mathrm{Ca}+\mathrm{Fe}$ and $\mathrm{Ar}+\mathrm{Ni})$. Further analysis is underway.
"Current address: Mississippi State University. ${ }^{\ddagger}$ Michigan State University National Superconducting Cyclotron Laboratory, East Lansing, MI

\section{References}

1. S. J. Yennello et. al., Phys. Lett. 321B, 15 (1994).

2. B. Gatty et. al., Z. Phys. A273, 65 (1975).

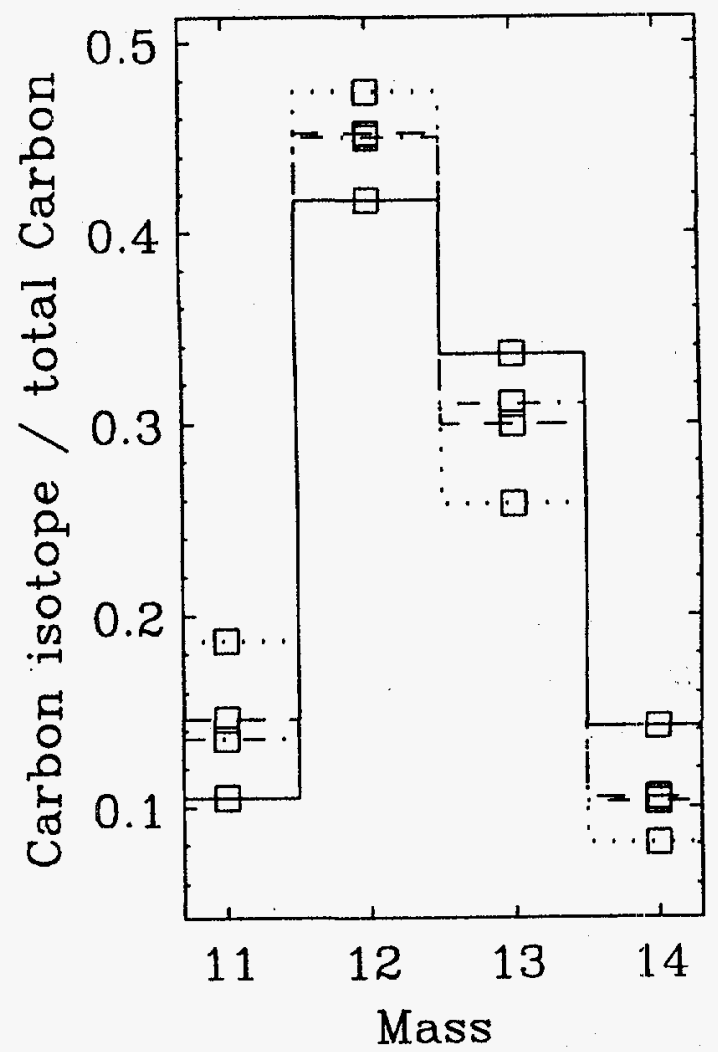

Figure 2. Ratio of carbon isotope yield to total carbon yield versus the mass number of the isotope for various target projectile combinations. Dotted line is ${ }^{40} \mathrm{Ca}+{ }^{58} \mathrm{Ni}$; dashed line is ${ }^{40} \mathrm{Ca}+$ ${ }^{58} \mathrm{Fe}$; dot dashed line is ${ }^{40} \mathrm{Ar}+{ }^{58} \mathrm{Ni}$; and solid line is ${ }^{40} \mathrm{Ar}+{ }^{58} \mathrm{Fe}$. 


\section{Comparisons of QMD Calculations to Violent Collisions in the ${ }^{40} \mathrm{Ca}+{ }^{40} \mathrm{Ca}$ Reaction at $35 \mathrm{MeV} / \mathrm{u}$}

K. Hagel, M. Gonin ${ }^{*}$, R. Wada, J. B. Natowitz, F. Haddad, Y. Lou, ${ }^{\ddagger}$ M. Gui, D. Utley, B. Xiao, J. Li, G. Nebbia, ${ }^{\S}$ D. Fabris,${ }^{\S}$ G. Prete,${ }^{\S}$ J. Ruiz, ${ }^{\S}$ D. Drain, ${ }^{\dagger}$ B. Chambon,${ }^{\dagger}$ B. Cheynis, ${ }^{\dagger}$ D. Guinet, ${ }^{\dagger}$ X. C. Hu, ${ }^{\dagger}$ A. Demeyer, ${ }^{\dagger}$ C. Pastor, ${ }^{\dagger}$ A. Giorni, ${ }^{* *}$ A. Lleres, ${ }^{* *}$ P. Stassi, ${ }^{* *}$ J. B. Viano, ${ }^{* *}$ and P. Gonthier ${ }^{* * *}$

The development of several microscopic computer simulations ${ }^{\mathrm{I}-3}$ with the potential capability of following the entire nuclear reaction from the early stages of the reaction through the final de-excitation has opened the possibility of understanding nuclear reactions on a microscopic level. We have made calculations using the QMD model developed by Maruyama et al. and compared the predictions of this model with our experimental data on the $35 \mathrm{MeV} / \mathrm{u}$ ${ }^{40} \mathrm{Ca}+{ }^{40} \mathrm{Ca}$ reaction. The experimental observables that we have compared are described in previous reports on the analysis of this data. ${ }^{4,5}$ As before all calculations are filtered through the detector acceptance and then compared to the experimental observables.

The QMD calculation, while similar in some respects to the BUU and VUU one-body models, provides a more realistic treatment of the fluctuations of the mean field and of cluster formation. Various quantities such as mass, charge and density of the emitting system in addition to the number of particles emitted can be followed as a function of time. In Fig. 1 we present as a function of time several calculated variables for collisions which passed through our detector acceptance. We observe for example that the mass and charge of the heaviest fragment, after reaching maxima near. 80 and 40 , respectively, quickly lose mass and charge between 50 and $100 \mathrm{fm} / \mathrm{c}$. The corresponding excitation energy per nucleon starting from a maximum near 8 $\mathrm{MeV} / \mathrm{u}$ decreases dramatically between 50 and 100 $\mathrm{fm} / \mathrm{c}$. During this time the density goes from a maximum near $1.5 \rho_{0}$ to a minimum near $.7 \rho_{0}$.

The solid points in Fig. 2 show the experimental elemental yield distribution. The predictions of the QMD for several different times at which the

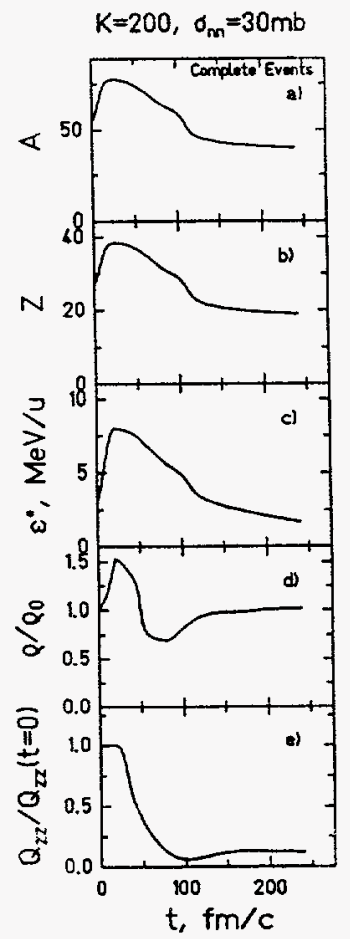

Figure 1. Time evolution of various quantities from the QMD calculation. a) Miss of heaviest fragment; b) Charge of heaviest fragment; c) Excitation energy per nucleon of the heaviest fragment; d) Density relative to density at point of contact; e) Quadrupole moment relative to quadrupole moment at point of contact.

afterburner is turned on are overlayed on the experimental data. The dotted line in Fig. 2 shows the QMD calculation in which GEMINI is used as an afterburner after $240 \mathrm{fm} / \mathrm{c}$. Turning on the GEMINI afterburner after $240 \mathrm{fm} / \mathrm{c}$ corresponds to allowing time for the system to compress, expand and then begin to return to normal nuclear density, during which time it loses a large amount of its excitation energy. We observe in Fig. 2, however, that the charge distribution generated in this way does not reproduce the data. This indicates that either 
GEMINI fails to treat the de-excitation correctly, or that the QMD is not adequately describing some aspect of the reaction before $240 \mathrm{fm} / \mathrm{c}$.

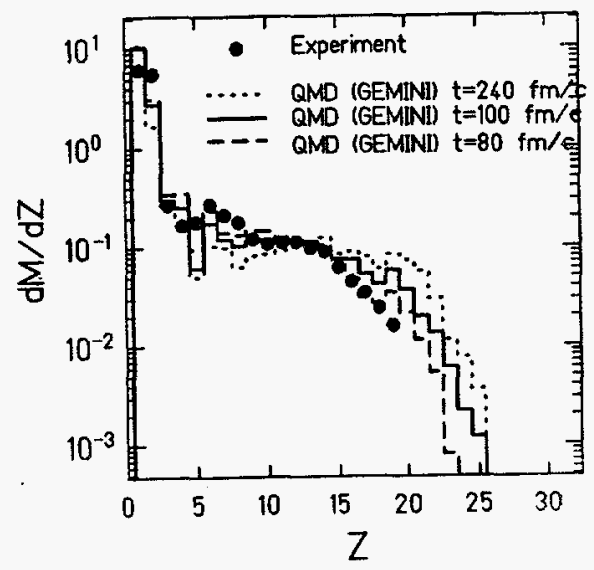

Figure 2. The elemental yield distribution. Solid points represent the experimental data, the dotted line represents the prediction of the QMD after the GEMINI afterburner is turned on after 240 $\mathrm{fm} / \mathrm{c}$, the dashed line represents the prediction of the QMD after the GEMINI afterburner us turned on after $80 \mathrm{fm} / \mathrm{c}$ and the solid line represents the prediction of the QMD after the GEMINI afterburner is turned on after $100 \mathrm{fm} / \mathrm{c}$.

We note a much better agreement with the experimental charge distribution if the afterburner is turned on after $80 \mathrm{fm} / \mathrm{c}$. This is represented by the dashed line in Fig. 2. However an examination of the quadrupole moment shown in Fig. 1(e) for events selected with our detector acceptance indicates that the system has not achieved complete equilibration until $100 \mathrm{fm} / \mathrm{c}$. Moreover, for those events which lead to two large fragments in the exit channel, a time of $100 \mathrm{fm} / \mathrm{c}$ is necessary for the two fragments to separate.

The solid line in Fig. 2 shows the charge distribution which is observed when the afterburner is used to model the de-excitation of the primary fragments after $100 \mathrm{fm} / \mathrm{c}$. This QMD (GEMINI) calculation reproduces the charge distribution up to atomic numbers of $\mathrm{Z}=15$ but exhibits a slight overprediction of the yield for the heaviest elements. Despite the poorer agreement (compared to turning on the afterburner after $80 \mathrm{fm} / \mathrm{c}$ ) we believe given the arguments in the preceding paragraph that $100 \mathrm{fm} / \mathrm{c}$ is a more appropriate time to turn on the afterburner.

As another means of exploring moments of the multiplicity distribution, we show in Fig. 3(a) the experimental event by event distribution of the logarithm of the atomic number of the largest fragment of an event versus the logarithm of the normalized second moment of the event $Z$ distribution, with the largest fragment excluded,

$$
S_{2}^{\prime}=\frac{\sum_{i: Z \neq Z_{\max }} Z_{i}^{2} M\left(Z_{i}\right)}{\sum_{i: Z \neq Z_{\max }} Z M\left(Z_{i}\right)}
$$

as described in our previous reports..$^{4,5}$ We note the two bumps in the experimental distribution, one at small $\ln S_{2}^{\prime}$ and the other at large $\ln S_{2}^{\prime}$. We recall from our previous reports ${ }^{5,6}$ that the lower $\ln S_{2}^{\prime}$ bump can be reproduced by a GEMINI calculation for a system with $A=70, Z=34$ and $E^{*}=420 \mathrm{MeV}$ while the large $\ln S_{2}^{\prime}$ bump can be explained by a multifragmentation calculation ${ }^{7}$ for the same system.

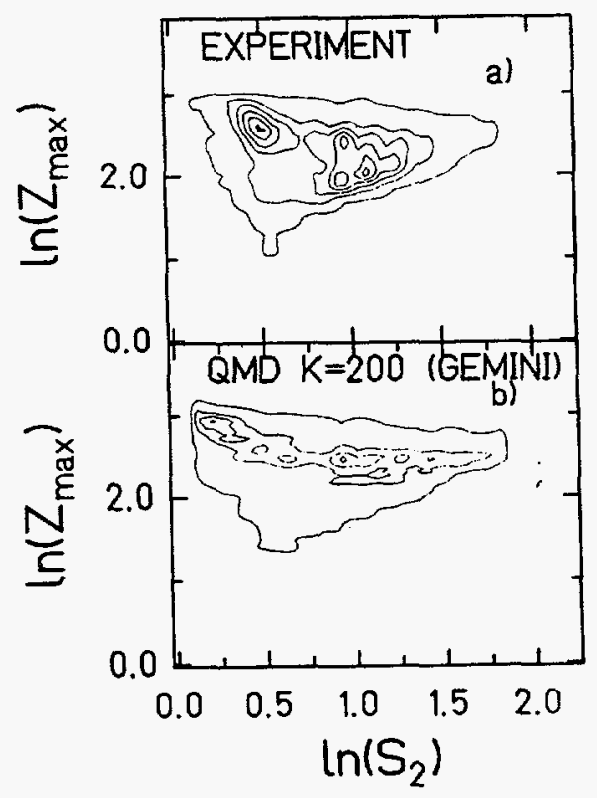

Figure 3. Log distribution of $Z_{\max }$ vs $S_{2}$ (see text). Each contour represents constant value in units of relative $\frac{d^{2} Y}{d \ln S_{1} d \ln Z_{\text {mex }}}$ where $Y$ is the yield. The outside contour is at a level of 10 , and eacla inner contour represents a progressive increase in yield of 150 . (a) Experiment, (b) QMD model with GEMINI afterburner. 


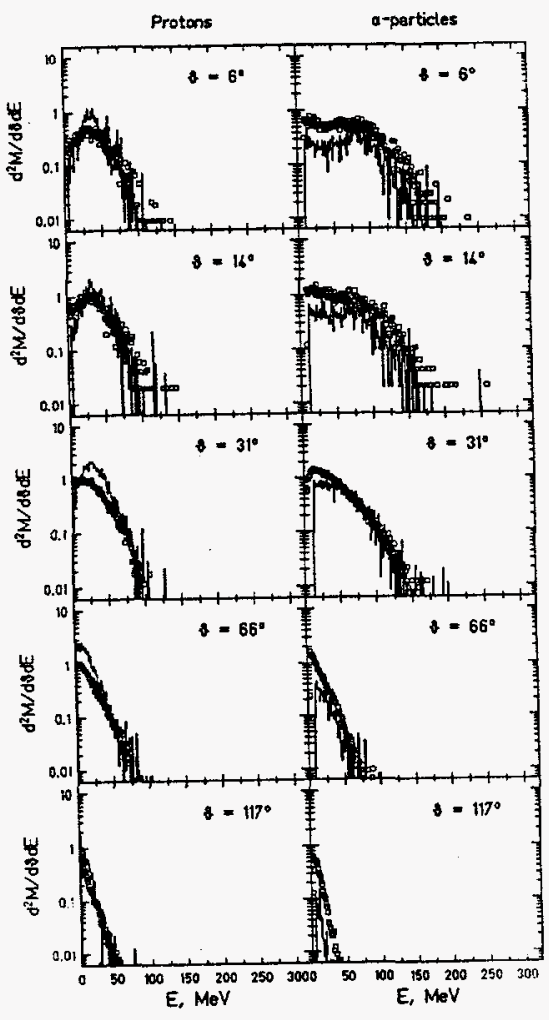

Figure 4. Left: Proton energy spectra of the QMD (GEMINI) calculation compared to experimental data for selected indicated AMPHORA angles. Right: Same for $\alpha$-particle energy spectra.

Figure 3(b) shows the distribution predicted by the QMD (GEMINI) calculation. We observe a distribution qualitatively similar to the data, that is, a spread of events over the entire range of $\ln S_{2}^{\prime}$ with enhancements at lower $\ln S_{2}^{\prime}$ and larger $\ln S_{2}^{\prime}$.

The energy spectra and angular distributions of emitted particles and fragments are also sensitive to the model assumptions and may provide some insights into the question of compression and expansion. The left panel of Fig. 4 shows the energy spectra of protons for the different angles in the AMPHORA detector compared to the results of the QMD (GEMINI) calculation. The QMD (GEMINI) calculation predicts proton spectral shapes in reasonable agreement with the experimental data, but suffers from an overprediction of the yield of protons as was already observed in Fig. 2.

The right panel of Fig. 4 shows energy spectra of $\alpha$-particles for the different angles in the
AMPHORA detector compared to the QMD (GEMINI) calculation. The shapes of the $\alpha$-particle energy spectra predicted by the QMD (GEMINI) calculation are also in reasonable agreement with the experimental spectra for angles forward of $45^{\circ}$ while there is some deficiency in yield for $\alpha$-particles at backward angles.

Figure 5 presents a comparison of the energy spectra of IMF's predicted by the QMD (GEMINI) calculation to the experimental data. Both the shapes of the energy spectra and the yield are qualitatively reproduced over the entire range of $Z$. For heavier fragments which are not shown in the figure, the angular distribution predicted by the QMD falls off slightly faster than the experiment.

Given the similarity of the QMD calculation to the data, we have explored the types of collisions which lead to the large and small $\ln S_{2}^{\prime}$ values. We show in Fig. 6 the impact parameter distributions which lead to small (Fig. 6(a)) and large (Fig. 6(b)) In $S_{2}^{\prime}$. In the QMD calculation the small In $S_{2}^{\prime}$ events result from the more central collisions and the large $\ln S_{2}^{\prime}$ events result from the more peripheral events indicating that the multifragment emission observed in the QMD results from events in which the binary nature of the collision is not forgotten or for which angular momentum plays a very important role.

We have examined all of the observables shown for a QMD (GEMINI) calculation using a hard equation of state, $K=378$. We find no significant changes of any of the observables presented with the increased value of $K$.

\section{Present address}

"Brookhaven National Laboratory, Upton, New York "Indiana University, Bloomington, Indiana ${ }^{\S}$ INFN - Legnaro, I-35020 Legnaro, Italy †IPN - Lyon 69622, Villeurbanne Cedex, France "*ISN - Grenoble 38026, Grenoble-Cedex, France ${ }^{* * *}$ Hope College, Holland, Michigan 


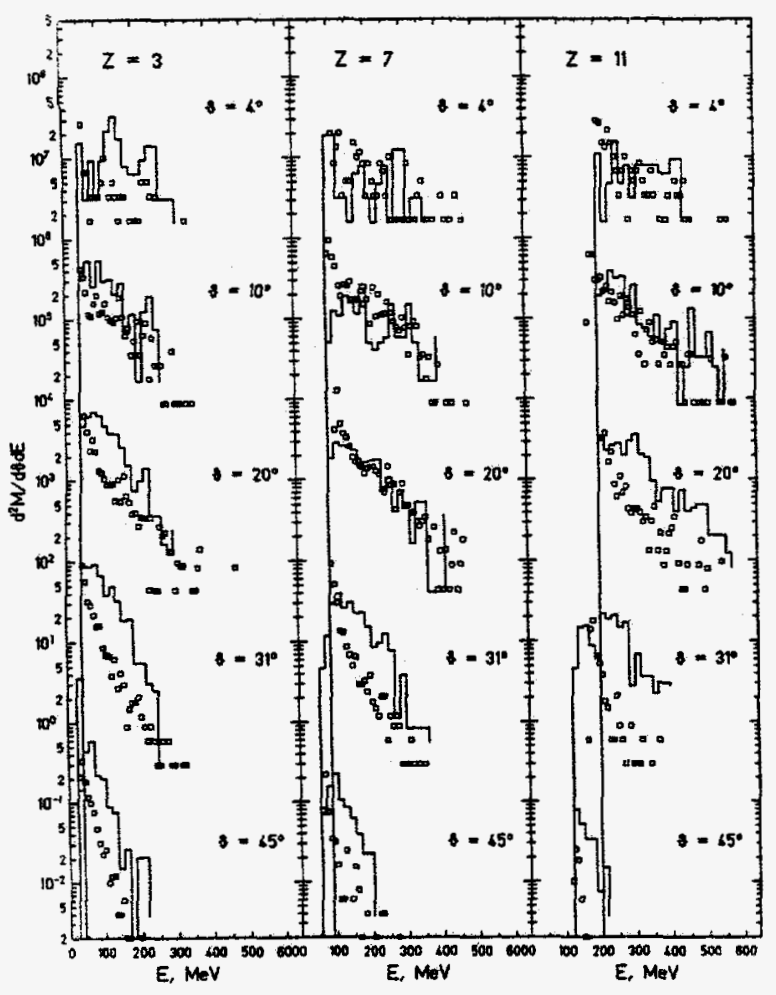

Figure 5. IMF energy spectra of the QMD (GEMINI) calcutation compare ti experimental data for selected indicated AMPIIOR: angles and selected charges.

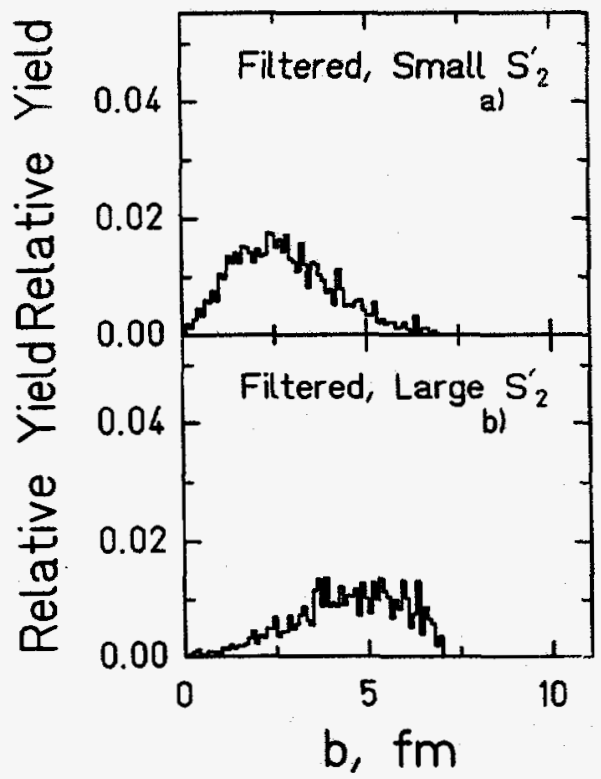

Figure 6. a) The QMD impact parameter, distribution allowed by the detector acceptance when small in $S_{2}$ events are selected; b) Same for large $\ln S_{2}$ events.

\section{References}

1. Jörg Aichelin, Phys. Rep. 202, 233 (1991).

2. T. Maruyama, A. Ono, A. Ohnishi, and $\mathrm{H}$. Horiuchi, Prog. Theo. Phys. 87, 1367 (1992).

3. J. Schnack, Diplomarbeit, Technische Hochschule Darmstadt, 1994.

4. K. Hagel, M. Gonin, R. Wada, J. B. Natowitz, B. H. Sa, Y. Lou, M. Gui, D. Utley, G. Nebbia, D. Fabris, G. Prete, J. Ruiz, D. Drain, B. Chambon, B. Cheynis, D. Guinet, X. C. Hu, A. Demeyer, C. Pastor, A. Giorni, A. Lleres, P. Stassi, J. B. Viano, and P. Gonthier, Phys. Rev. Lett. 68, 2141 (1992).

5. K. Hagel, M. Gonin, R. Wada, J. B. Natowitz, B. H. Sa, Y. Lou, M. Gui, D. Utley, G. Nebbia, D. Fabris, G. Prete, J. Ruiz, D. Drain, B. Chambon, B. Cheynis, D. Guinet, X. C. Hu, A. Demeyer, C. Pastor, A. Giorni, A. Lleres, P. Stassi, J. B. Viano, and P. Gonthier, Progress in Research, 1992-1993, Cyclotron Institute, TAMU, p. I-21.

6. K. Hagel, M. Gonin, R. Wada, J. B. Natowitz, F. Haddad, Y. Lou, M. Gui, D. Utley, B. Xiao, J. Li, G. Nebbia, D. Fabris, G. Prete, J. Ruiz, D. Drain, B. Chambon, B. Cheynis, D. Guinet, X. C. Hu, A. Demeyer, C. Pastor, A. Giomi, A. Lleres, P. Stassi, J. B. Viano, and P. Gonthier, in preparation, 1994.

7. D. H. E. Gross, Rep. Prog. Phys. 53, 605 (1990). 


\section{Light Particle Evaporation as a Function of Neutron Excess for Medium Mass Compound Nuclei with $\mathrm{E}_{\mathrm{x}} \approx 2 \mathrm{MeV} / \mathrm{u}^{*}$}

Y. Lou, ${ }^{* *}$ M. Gonin, ${ }^{* * *}$ R. Wada, K. Hagel, J. Li, X. Bin, M. Gui, D. Utley, R. Tezkratt, L. Cooke, T. Botting, B. Hurst, D. O'Kelly, G. Mouchaty, R. P. Schmitt, W. Turmel, J. B. Natowitz, B.

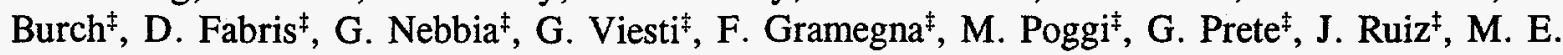
Brandan $^{\dagger}$, and A. Menchaca-Rocha ${ }^{\dagger}$

We have continued studies designed to characterize the decay of $\mathrm{A}=160$ compound nuclei by measurements of light particle emission from compound nuclei with very different neutron to proton ratios. In order to study this $\mathrm{N} / \mathrm{Z}$ dependence the reactions and the projectile energies used in this work were selected so that nuclei with $A \simeq 160$ and $\approx 280 \mathrm{MeV}$ excitation energy but very different neutron to proton ratios could be produced.

From measurements of fission fragment folding angles for the reactions $10 \mathrm{AMeV}{ }^{63} \mathrm{Cu}+{ }^{92,100} \mathrm{Mo}$ and $20 \mathrm{AMeV}{ }^{20} \mathrm{Ne}+{ }^{144,148,154} \mathrm{Sm}$, linear momentum transfers were determined and the initial excitation energies were inferred. The multiplicities of the light particles in coincidence with the fission fragments were obtained for neutrons, protons and $\alpha$ particles. Charged particle kinetic energies were measured directly while the neutron kinetic energies were derived from angular distributions measured in the median plane wedges of the Texas A\&M Neutron Ball. The experimental total multiplicities have been compared with results from a statistical calculation and the effect of delay time on the total multiplicities has been explored.

The Texas A\&M Neutron Ball was used to determine the total neutron multiplicities associated with fission decay. The dependence of these multiplicities on apparent linear momentum transfer (as determined from fission fragment folding angle measurements), and on the initial neutron to proton ratio of the excited composite system has been determined for nuclei with excitation energies near 2 MeV/nucleon and is shown in Figs. 1 and 2. The measured widths of the apparent LMT distributions are slightly wider than those expected from evaporation broadening of the folding angle distribution. In agreement with this, the variation of

$20 \mathrm{AMeV}{ }^{20} \mathrm{Ne}+\mathrm{Sm}$ or $10 \mathrm{AMeV}{ }^{63} \mathrm{Cu}+\mathrm{Mo}$

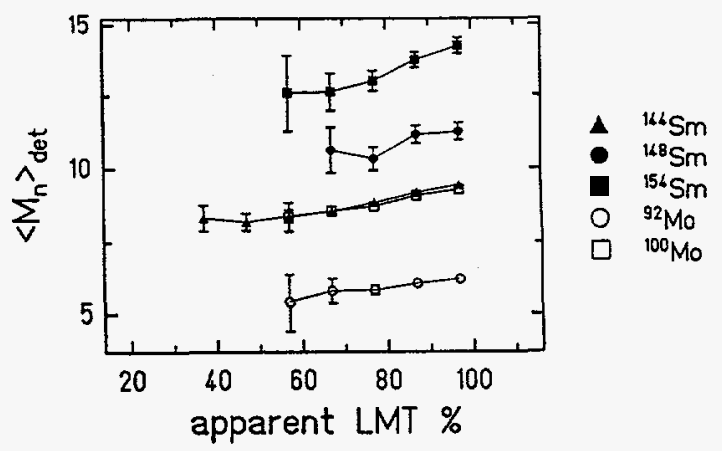

Figure 1. The detected total neutron multiplicities as a function of apparen $L M T$ for reactions of ${ }^{63} \mathrm{Cu}+{ }^{92,100} \mathrm{Mo}$ at $10 \mathrm{MeV} / \mathrm{t}_{\mathrm{i}}$ and ${ }^{20} \mathrm{Ne}, \cdots, 154 \mathrm{Sm}$ at $20 \mathrm{MeV} / \mathrm{u}$.

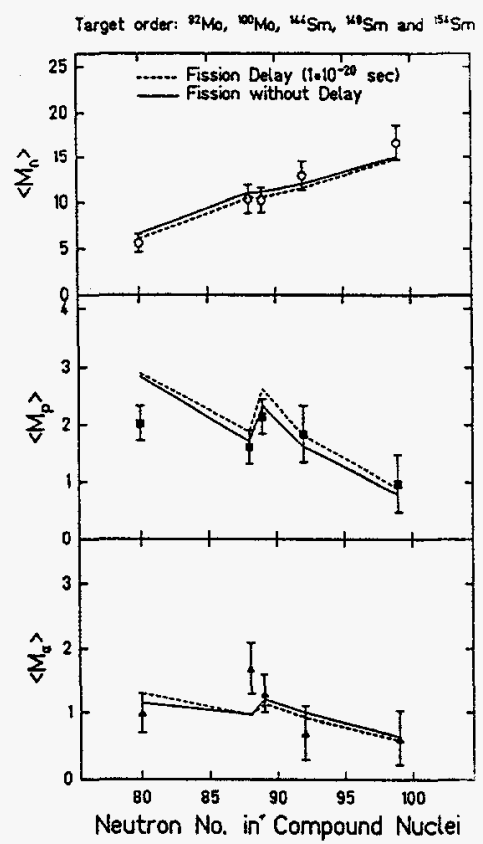

Figure 2. Experimental light particle multiplicities compared with the results of GEMINI calculations for excitation energy near 280 $\mathrm{MeV}$ : the squares and triangles present multiplicities of protons and $\alpha$ particles respectively in middle and bottom parts of the plot. In top part of the plot the open circles are evaporated neutron multiplicities. 
$<\mathrm{M}_{\mathrm{n}}>$ with apparent LMT indicates some spread in the primary LMT distribution and excitation energy.

The measured neutron multiplicities show a strong increase with increasing neutron to proton ratio of the composite system. These results are shown at the top of Fig. 2. For the most probable linear momentum transfers, the multiplicities (also presented in Fig. 2) and kinetic energies in the moving frame have also been measured for protons and $\alpha$ particles. Neutron angular distributions observed in the median plane wedges of the Neutron Ball have been analyzed to determine the average neutron kinetic energies in the frame of the moving hot nucleus.

Using the information on multiplicities and energies, a reconstruction of the total excitation energy has been made as seen in Fig. 3. The derived values compare favorably with the estimated excitation energies derived from the LMT data. IMF emission is negligible at these excitation energies.

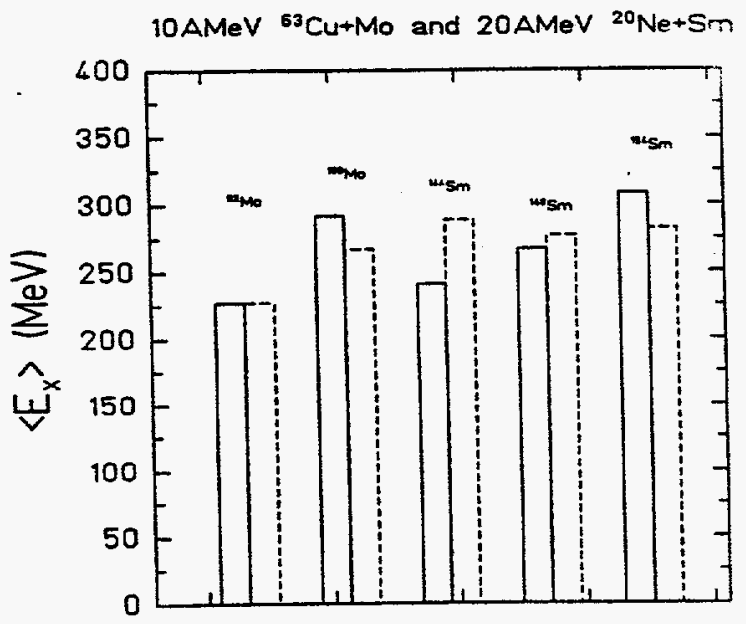

Figure 3. Comparison of the reconstructed excitation energy, $E_{x}^{(c)}$, (solid bar) and the results derived from the apparent LMT distributions, $E_{x}^{(a)}$, (dashed bar) for $10 \mathrm{MeV} / \mathrm{u}{ }^{63} \mathrm{Cu}+{ }^{92,100} \mathrm{Mo}$ and $20 \mathrm{MeV} / \mathrm{u}{ }^{20} \mathrm{Ne}+{ }^{144,148,154} \mathrm{Sm}$.
For the derived excitation energies, comparisons between statistical model calculations, made using the code GEMINI, and the experimental data, presented in Fig. 2, indicate that the evaporated neutron multiplicities are rather well reproduced at all excitation energies. While the calculated $\alpha$ multiplicities are also reasonably close to the measured values, the calculated proton multiplicities for the most neutron deficient nuclei tend to be significantly higher than those observed.

*Y. Lou, Ph.D. thesis, TAMU, 1993.

\section{Present Address}

\section{"Indiana University, Bloomington, Indiana \\ ***Brookhaven National Lab, Upton, New York \\ ${ }^{\ddagger}$ INFN and Universita di Padova, Padova, Italy and INFN, Lab. Naz. Legnaro, I-35020, Legnaro, Italy 'Instituto de Fisica, UNAM, Mexico}




\title{
The Fate of Compound Systems in Reactions of $40 \mathrm{MeV} / \mathrm{u}$ Ar with Selected Targets
}

\author{
D. Utley, X. Bin, M. Gui, F. Haddad, K. Hagel, J. Li, Y. Lou, J. B. Natowitz, R. Tezkratt, and \\ R. Wada
}

Beams from the K500 superconducting cyclotron have been used in experiments with the neutron ball to explore the limits to excitation energy in compound nuclei formed in the reactions ${ }^{40} \mathrm{Ar}+{ }^{232} \mathrm{Th},{ }^{197} \mathrm{Au}$, ${ }^{100} \mathrm{Mo},{ }^{159} \mathrm{~Tb}$ and $\mathrm{Ni}$ with projectile energies of 35 $\mathrm{MeV} / \mathrm{u}$ and $40 \mathrm{MeV} / \mathrm{u}^{1}$ Simulations based on the code EUGENE $^{2}$ and the statistical model code GEMINI $^{3}$ have been used to interpret product spectra resulting from these reactions. The experimental description may be found in earlier work." Highlights of the Th analysis have been published. ${ }^{4}$ The complete work may be found in the thesis of D. Utley. ${ }^{5}$

For the five targets, comparison of the relative yields and observed average neutron multiplicities for the experiment and the EUGENE simulations is made in Figs. 1 through 5. It is important to note that the calculated mass distributions are sensitive to the fission delay times selected for the simulations.

In Figs. 1 through 5 EUGENE simulations incorporating a fixed dynamic delay time for fission were used. In order to explore the yield of the various components in the spectra produced by the EUGENE code and facilitate the identification of fission fragments and residues, the fission delay time had previously been varied through a series of simulations. The simulation results for the neutron versus mass distributions for six delay time values are presented in Fig. 6 for ${ }^{40} \mathrm{Ar}+{ }^{232} \mathrm{Th}$. As the delay time for the onset of fission is increased the residue yield can be seen to increase in prominence. For short delay times intermediate impact parameters lead to fission events. For increasing delay times the excited nuclei produced in these collisions are more likely to survive fission. In this series the first figure represents effectively no fission delay and the last figure represents an infinite fission delay which allows only residues. A one-dimensional projection for three of these spectra is given in Fig.7. As the fission delay is increased the residue yield at higher mass values is seen to increase at the expense of the fission fragment yield. The fission delay represented in the simulations which led to reasonable agreement with the experiment used a value of $5 \times 10^{-20}$ seconds, a value in agreement with others in the literature. ${ }^{6,7}$ This should not be taken as a precise determination of the time scale for fission.

For the targets used in this work Fig. 8(a) shows a plot of the predicted average neutron multiplicities for mass windows $10 \mathrm{amu}$ wide, as determined from EUGENE simulations. This plot includes contributions of neutrons from all sources, i.e., preequilibrium, fission and evaporation. From this plot it is possible to arrive at the excitation energy associated with a given neutron multiplicity. As excitation energies increase the rate of increase of the neutron multiplicities diminish reflecting competition of charged particle evaporation.

The detected neutron multiplicities follow similar trends as seen in Fig. 8(b). A comparison between the two parts of Fig. 8 shows typical neutron detection efficiencies to be in the range of $55 \%$ for the hot moving sources resulting from central collisions for the heavier targets. As target mass decreases recoil velocities increase and the neutron detection efficiency decreases (to near $35 \%$ for the $\mathrm{Ni}$ target). These efficiencies markedly differ from efficiencies measured for ${ }^{252} \mathrm{Cf}$ which are in the range of $70 \%$ to $80 \%$. Care must be taken in arriving at neutron multiplicities (and related excitation energies) for hot systems.

Table 1 summarizes apparent excitation energies reached in the most central collisions for the systems studied here. The table includes: the average neutron multiplicity observed, the average mass of the product in the mass window the efficiency corrected average neutron multiplicity and the apparent excitation energy associated with the events having 
that average neutron multiplicity. The most central events are seen to have excitation energies on the order of $800 \mathrm{MeV}$ and $900 \mathrm{MeV}$ for the gold and thorium targets, respectively. These central collisions result in neutron multiplicitiesnear 40 . The apparent excitation energy for ${ }^{40} \mathrm{Ar}$ with the ${ }^{232} \mathrm{Th}$ target is 200 to $300 \mathrm{MeV}$ higher than the excitation energies reported by the GANIL HMI collaboration..$^{8-11}$ The results reported by that collaboration are derived from the most probable neutron multiplicity for the "central" collision neutron group and represent a wide range of products and impact parameters. That technique reflects a range of sources from differing excitation energies with the result being skewed by the relative cross-sections toward lower excitation energies.

A second determination of the excitation energy reached in the most central collisions may be made from the momentum transfer in the collision. The average velocities for the residues, identified by their neutron multiplicity and mass as coming from the most central collisions, was used to calculate the fraction of momentum transferred, the mass of the primary compound nucleus, the excitation energy of the compound nucleus and the excitation energy per nucleon for the compound nucleus. The results of these calculations are compared to a leading particle model calculation ${ }^{12}$ and the EUGENE simulations in Table 2. Results from the leading particle calculations are within the experimental uncertainties for the excitation energies from the neutron multiplicity measurements for all targets except the nickel. The EUGENE simulation results agree with the leading particle model and the experiment for the heavy target cases, but not so for the lighter targets.

*Indiana University, Bloomington, Indiana

\section{References}

1. D. Utley, X. Bin, M. Gui, K. Hagel, Y. Lou, J. Natowitz, and R. Wada, Progress in Research, 1992-1993, Cyclotron Institute, TAMU, p. 11.
2. D. Durand, Nucl. Phys. A541, 266 (1991).

3. R. J. Charity, M. A. McMahan, G. J. Wozniak, R. J. McDonald, L. G. Moretto, D. G. Sarantites, L. G. Sobotka, G. Guarino, A. Pantaleo, L. Fiore, A. Gobbi, and K. D. Hildenbrand, Nucl. Phys. A483, 371 (1988).

4. D. Utley, R. Wada, K. Hagel, J. Li, X. Bin, M. Gui, Y. Lou, R. Tezkratt, J. B. Natowitz, and M. Gonin, Phys. Rev. C 49, R1737 (1994).

5. D. Utley, Ph.D. thesis, TAMU, 1993.

6. D. Hilscher and H. Rossner, Ann. Phys. Fr. 17, 471 (1992).

7. E. M. Eckert, A. Kuhmichel, J. Pochodzalla, K. D. Hildenbrand, U. Lynen, W. F. J. Muller, H. J. Rabe, H. Sann, H. Stelzer, W. Trautmann, R. Trockel, R. Wada, C. Cerruti, P. Lhenore, R. Lucas, C. Mazur, C. Ngo, M. Ribrag, E. Tomasi, A. Demeyer, and D. Guinet, Fhys. Rev. Lett. 64, 2483 (1990).

8. B. Lott, J. L. Charvet, E. Crema, G. Duckene, H. Doubre, J. Frehault, J. Galin, B. Gatty, D. Guerreau, G. Ingold, D. Jacquet, U. Janke, D. X. Jiang, C. Magnago, M. Morjean, Y. Patin, E. Piasecki, J. Pouthas, Y. Pranal, F. SaintLaurent, E. Schwinn, A. Sokolov, J. L. Uzureau, and X. M. Wang, Z. Phys. A346, 201 (1993).

9. D. X. Jiang, H. Doubre, J. Galin, D. Guerreau, E. Piasecki, J. Pouthas, A. Sokolov, B. Cramer, G. Ingold, U. Janke, E. Schwin, J. L. Charvet, J. Frehaut, B. Lott, C. Magnago, M. Morjean, Y. Patin, Y. Pranal, J. L. Uzureau, B. Gatty, and D. Jacquet, Nucl. Phys. A503, 560 (1989).

10. E. Schwinn, U. Janke, J. L. Charvet, B. Cramer, H. Doubre, J. Frehaut, J. Galin, B. Gatty, D. Guerreau, G. Ingold, D. Jacquet, D. X. Jiang, B. Lott, M. Morjean, C. Magnago, Y. Patin, J. Pouthas, E. Piasecki, and A. Sokolov, to be published.

11. D. Guerreau, J. L. Charvet, H. Doubre, J. Frehaut, J. Galin, G. Ingold, D. Jacquet, U. Jahnke, D. X. Jiang, B. Lott, C. Magnaga, M. Morjean, Y. Patin, J. Pouthas, Y. Pranal, and J. 
L. Uzureau, Preprint GANIL P 87-23 (1987).

12. J. B. Natowitz, S. Leray, R. Lucas, C. Ngo, E. Tomasi, and C. Volant, Z. Phys. A325, 467 (1986).

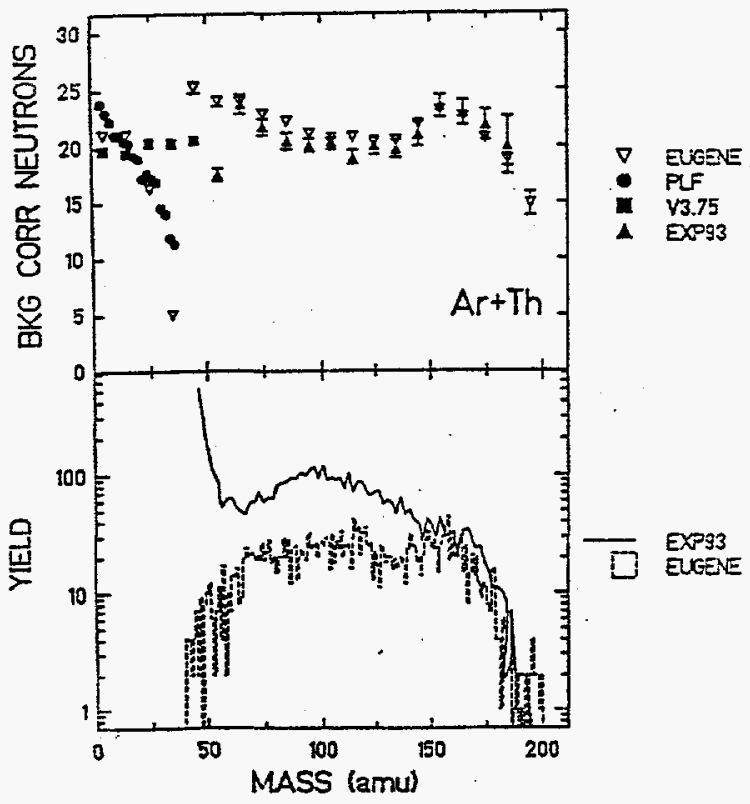

Figure 1. ${ }^{40} \mathrm{Ar}+{ }^{232} \mathrm{Th}$ : Comparisons hetween EUGENE simulation and the experiment: (Top) Background corrected average neutron multiplicities for different mass windows on coincident products and (Bottom) A comparison between experimental and simulated mass distributions.

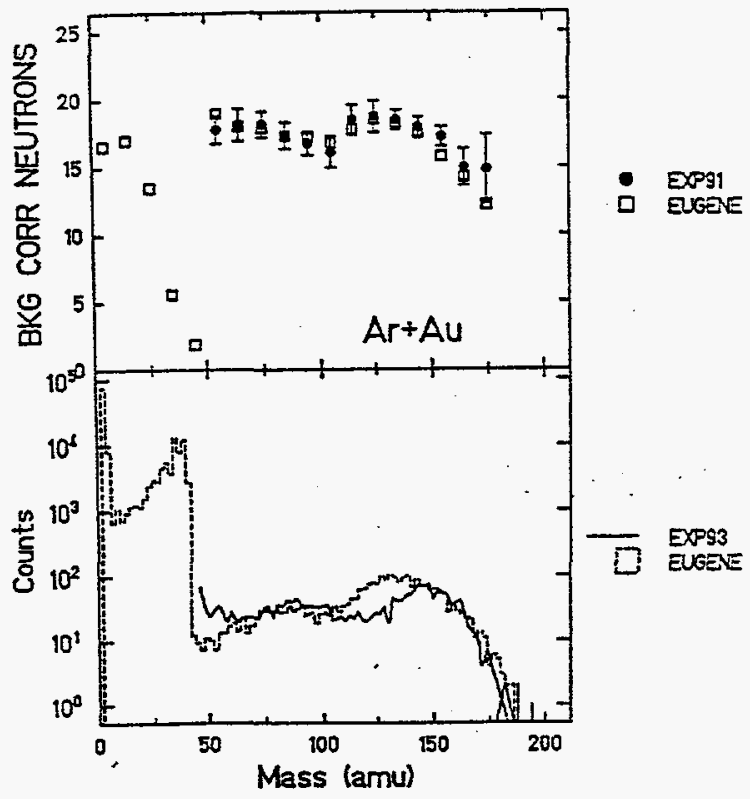

Figure 2. ${ }^{41} \mathrm{Ar}+{ }^{197} \mathrm{Au}$ : Comparisons between EUGENE simulation and the experiment: (Top) Background corrected average neutron multiplicities for different mass windows on coincident products and (Bottom) A comparison between experimental and simulated mass distributions.

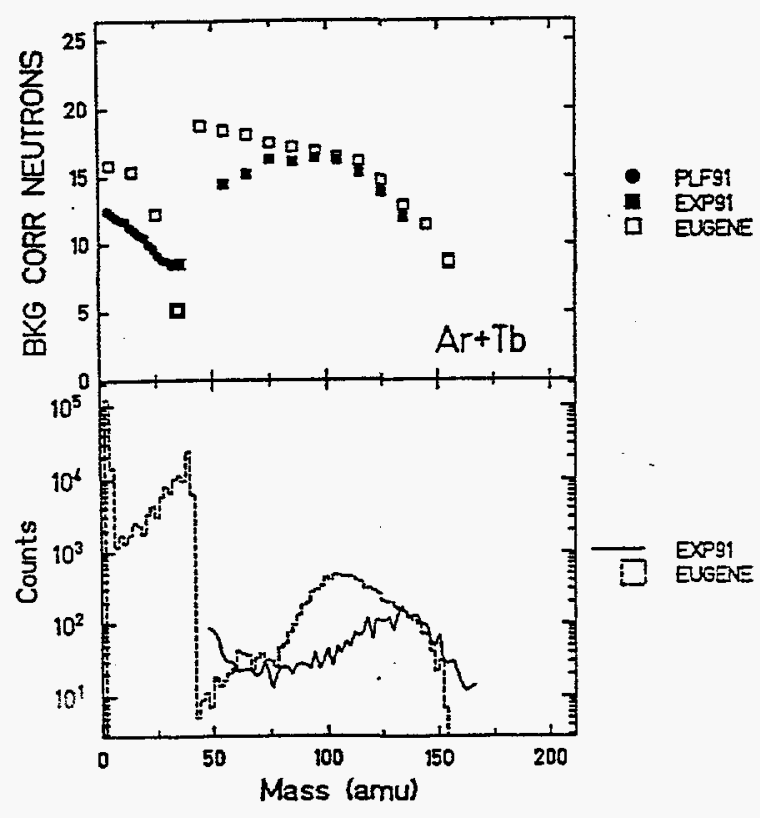

Tygi:is 3. ${ }^{41} \mathrm{Ar}+{ }^{159} \mathrm{~Tb}$ : Comparisons between EUGENE simulation and the experiment: (Top) Background corrected average neutron multiplicities for different mass windows on coincident products and (Bottom) A comparison betwe? experimental and simulated mass distributions.

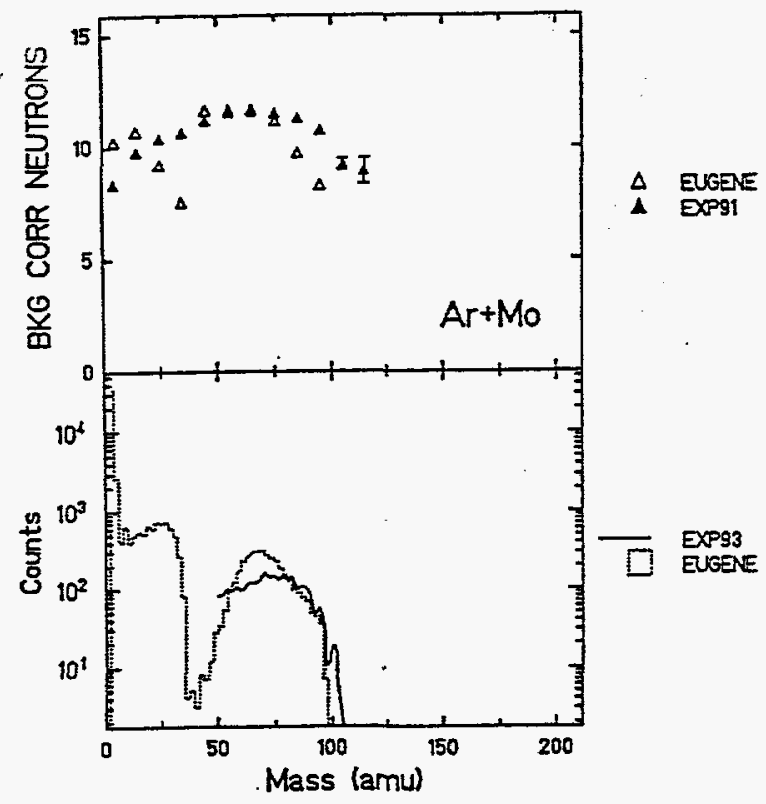

Figure 4. ${ }^{40} \mathrm{Ar}+{ }^{100} \mathrm{Mo}$ : Comparisons between EUGENE simulation and the experiment: (Top) Background corrected average neutron multiplicities for different mass windows on coincident products and (Bottom) A comparison between experimental and simulated mass distributions. 


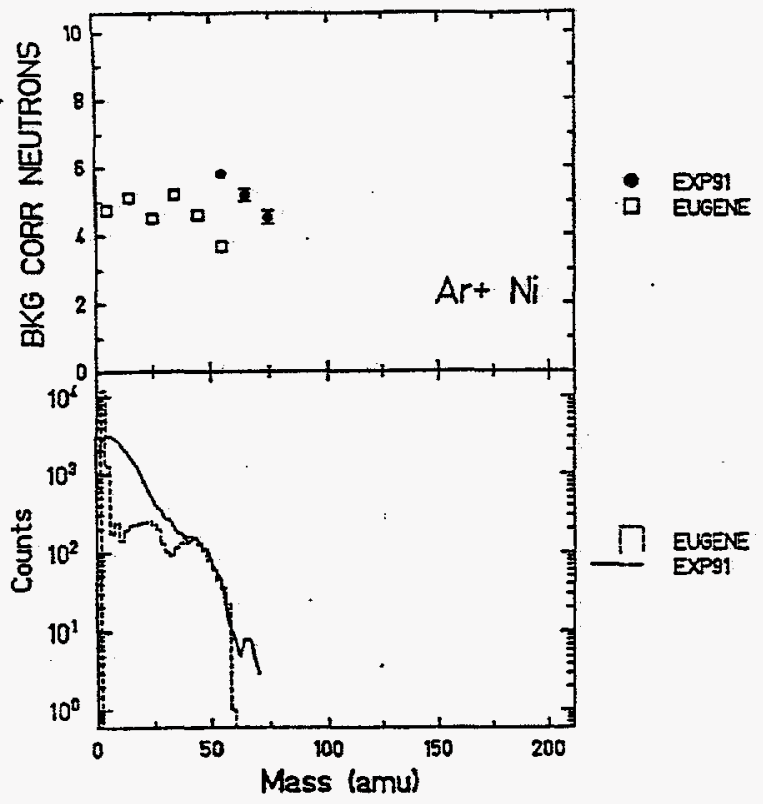

Figure 5. ${ }^{40} \mathrm{Ar}+\mathrm{Ni}$ : Comparisons between EUGENE simulation and the experiment: (Top) Background corrected average neutron multiplicities for different mass windows on coincident products and (Bottom) A comparison between experimental and simulated mass distributions.

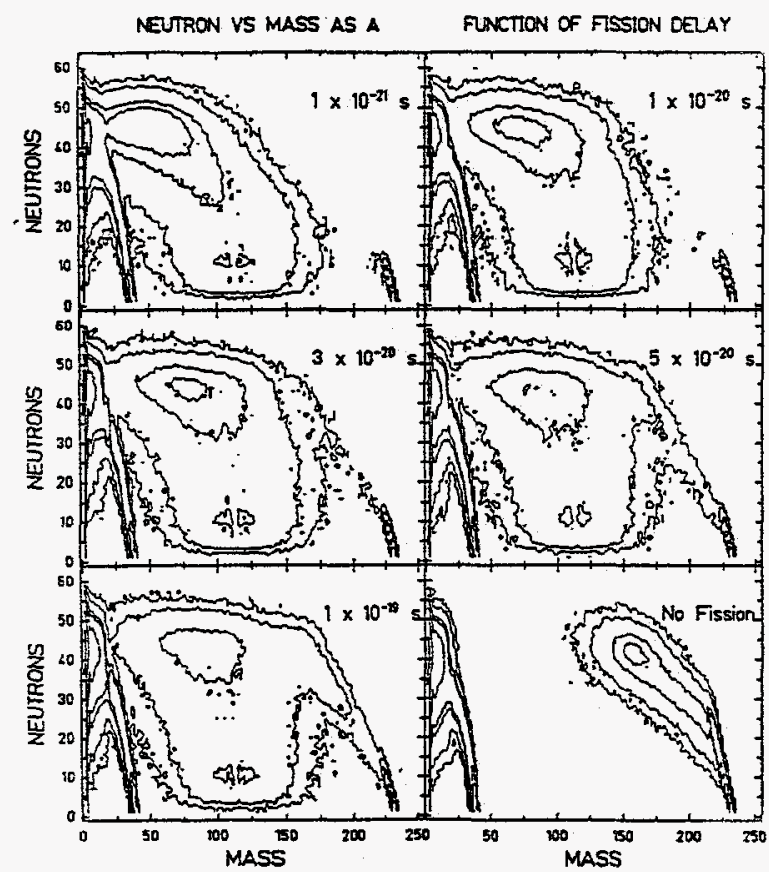

Figure $6 .{ }^{40} \mathrm{Ar}+{ }^{232} \mathrm{Th}$ : Neutron multiplicity versus product mass for six fission delay scenarios: delay times are shown on the plots. Zeros (0) mark regions with few products. Other contour levels are $1,5,10,100,1000$ etc.

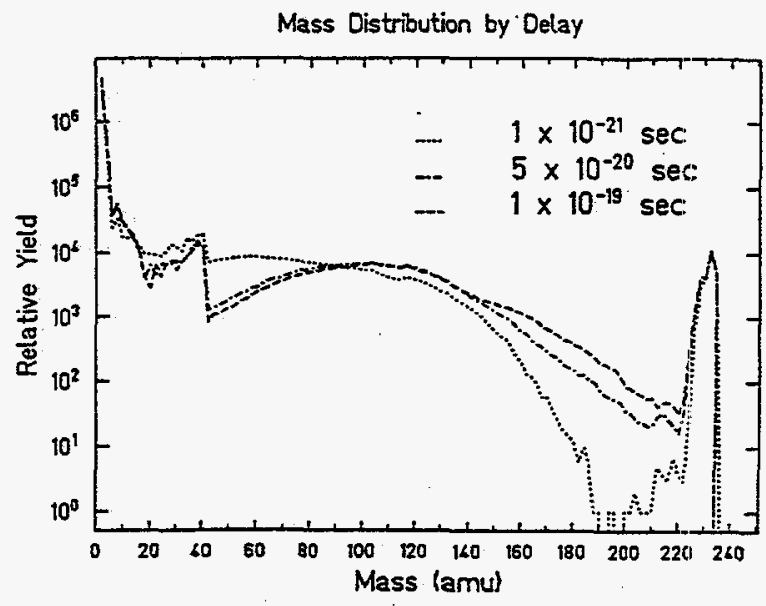

Figure $7 .{ }^{40} \mathrm{Ar}+{ }^{232} \mathrm{Th}$ : Simulated mass distributions for three delay times. Longer delays result in increasing product yields for heavier residues at the expense of lighter fission products. 


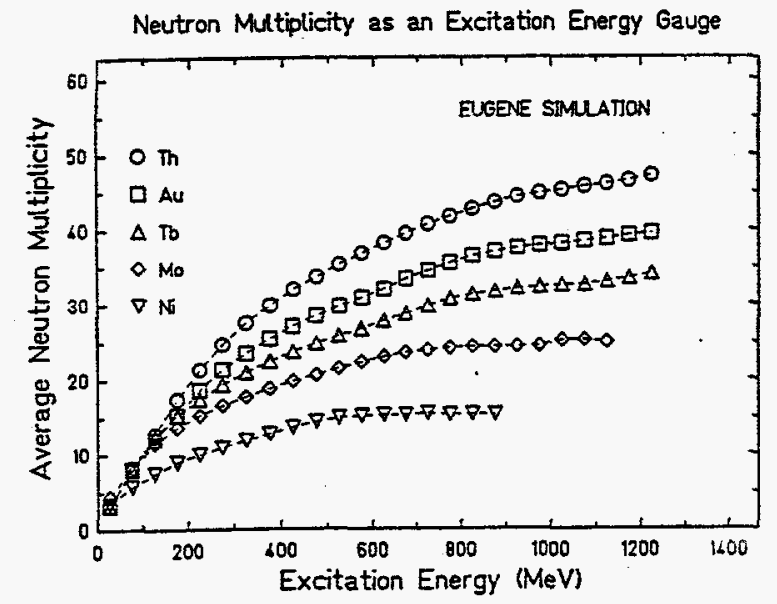

Figure 8(a). Calculated neutron multiplicities as a function of excitation energy in the reaction. Neutron multiplicities include neutrons from all sources.

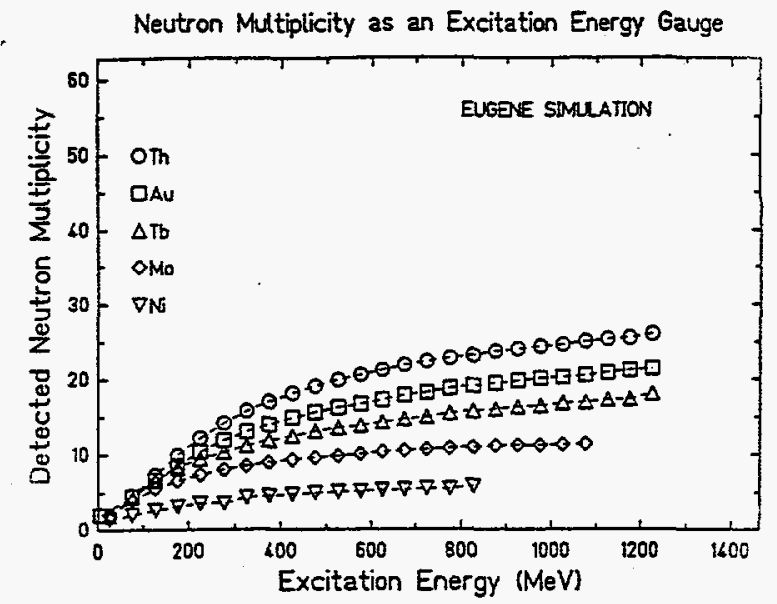

Figure 8(b). Calculated average detected neutron multiplicities as a function of excitation energy in the reaction. ${ }^{252} \mathrm{Cf}$ efficiency $69.8 \%$.

Table 1. Experimentally Derived Excitation Energies for Most Central Collisions of $40 \mathrm{AMeV}$ Ar with Selected Targets

\begin{tabular}{|c|c|c|c|c|c|c|c|c|}
\hline & \multicolumn{4}{|c|}{ Residues } & \multicolumn{4}{|c|}{ Fission Fragments } \\
\hline Target & $A_{\text {res }}$ & $<\operatorname{det}_{n}>\max$ & $\begin{array}{c}E_{x} \\
(\mathrm{MeV})\end{array}$ & $<\mathrm{M}_{\mathrm{n}}>^{(\mathrm{a}) \max }$ & $\mathrm{A}_{\mathrm{ff}}$ & $<\operatorname{det} M_{n}>\max$ & $\begin{array}{c}E_{x} \\
(\mathrm{MeV})\end{array}$ & $<M_{n}>^{(a) \max }$ \\
\hline Thorium-232 & 155 & $23.5 \pm 1.0^{(b)}$ & $870_{-110}^{+120}$ & $44 \pm 2$ & 65 & $23.7 \pm 0.9^{(b)}$ & $890_{-100}^{+110}$ & $44 \pm 2$ \\
\hline Gold-197 & 125 & $18.7 \pm 1.2^{(b)}$ & $770_{-120}^{+160}$ & $35 \pm 2$ & 70 & $18.2 \pm 1.2^{(b)}$ & $725_{-100}^{+150}$ & $34 \pm 2$ \\
\hline Terbium-159 & 95 & $18.04 \pm 0.2^{(\mathrm{c})}$ & $880_{-60}^{+70}$ & $32 \pm .4$ & & & & \\
\hline Molybdenum-100 & 65 & $11.6 \pm 0.1^{(c)}$ & $620_{-20}^{+20}$ & $23 \pm .2$ & & & & \\
\hline Nickel-58 & 55 & $5.8 \pm 0.1^{(\mathrm{c})}$ & $575_{-60}^{+100}$ & $15 \pm .3$ & & & & \\
\hline
\end{tabular}

${ }^{(a)}$ Efficiency corrected neutrons. Includes response functions of NBL.

(b) $\mathrm{Cf}-252$ neutron eff. $69.8 \%$.

${ }^{(c)} \mathrm{Cf}-252$ eff. $78.6 \%$. 
Table 2. Excitation Energies from Measured Momentum Transfer and Neutron Multiplicity Compared to Leading Particle Model Calculations ${ }^{(2)}$ and EUGENE Simulations

\begin{tabular}{|c|c|c|c|c|c|c|c|c|c|c|}
\hline & \multicolumn{2}{|c|}{ Leading Particle Model Calculations } & \multicolumn{2}{|c|}{ EUGENE } & \multicolumn{6}{|c|}{ Experiment } \\
\hline Target & $\mathrm{A}_{\mathrm{CN}}$ & $\mathrm{E}^{*}$ & $\mathrm{E}^{*} / \mathrm{A}$ & $\rho$ & $\mathrm{E}^{*}$ & $\rho_{\text {exp }}$ & $\mathrm{A}_{\mathrm{CN}}$ & $\mathrm{E}^{*}(\rho)$ & $\mathrm{E}^{*}\left(\mathrm{M}_{\mathrm{n}}\right)$ & $\mathrm{E}^{*}\left(\mathrm{M}_{\mathrm{n}}\right) / \mathrm{A}$ \\
\hline${ }^{232} \mathrm{Th}$ & 260 & 931 & 3.58 & .67 & 890 & .665 & 259 & 880 & $870_{-110}^{+120}$ & 3.36 \\
\hline${ }^{197} \mathrm{Au}$ & 224 & 890 & 3.96 & .62 & 820 & .604 & 221 & 804 & $780_{-120}^{+150}$ & 3.52 \\
\hline${ }^{159} \mathrm{~Tb}$ & 184 & 822 & 4.47 & .57 & 770 & .772 & 190 & 970 & $880_{-60}^{+70}$ & 4.63 \\
\hline${ }^{100} \mathrm{Mo}$ & 122 & 657 & 5.40 & .52 & 740 & .556 & 122 & 725 & $620_{-20}^{+20}$ & 5.08 \\
\hline${ }^{58} \mathrm{Ni}$ & 76 & 439 & 5.81 & .54 & 630 & .602 & 82 & 685 & $575_{-90}^{+100}$ & 7.01 \\
\hline
\end{tabular}

(a) Natowitz, et al., 1986.

\title{
Very Hot Nuclei Production in ${ }^{63} \mathrm{Cu}-$ Induced Reactions on $\mathrm{Au}, \mathrm{Tb}$, Mo at $35 \mathrm{AMeV}$
}

\author{
R. Tezkratt, K. Hagel, R. Wada, F. Haddad, J. B. Natowitz, Y. Lou, J. Li, D. Utley, B. Xiao, N. \\ Mdeiwayeh, G. Cibor, ${ }^{*}$ and Z. Majka*
}

In work done a few years ago on the system ${ }^{40} \mathrm{Ar}$ $+{ }^{232} \mathrm{Th}$, Jiang et al. ${ }^{1}$ reported that the thermal energy which can be deposited reaches a soft saturation around 600 to $700 \mathrm{MeV}$, when the incident energy increases from 27 to $77 \mathrm{MeV} / \mathrm{u}$. Recently, in work done on the same system, D. Utley et al. ${ }^{2}$ reported that about $900 \mathrm{MeV}$ excitation energy can be deposited. Is this value an upper limit or can one deposit more? This is what we are investigating in the present study of ${ }^{63} \mathrm{Cu}$-induced reactions on $\mathrm{Au}, \mathrm{Tb}$ and $\mathrm{Mo}$ targets. The ${ }^{63} \mathrm{Cu}$ beam at $35 \mathrm{MeV} /$ nucleon was delivered by the K500 Superconducting Cyclotron. The experiment was performed in the scattering chamber of the Texas A\&M Neutron Ball which allowed the measurement of the neutrons emitted from the reaction. A set of 35 telescopes made of gas ionization chambers and silicon detectors arranged in a $4 \pi$ configuration surrounded the target for the detection of the $\alpha$-particles and IMFs. A silicon detector $\left(900 \mathrm{~mm}^{2}\right)$ intended for energy and time of flight measurements of the heavy fragments emitted at forward angles $\left(6^{\circ}\right)$ was set at a distance of $1.7 \mathrm{~m}$ from the target. In order to detect projectile-like fragments a hodoscope made of 300 $\mu \mathrm{m}$ silicon backed by $5 \mathrm{~mm}$ CsI crystal scintillator was used. The gamma-flash signal delivered by the neutron ball was used as the start signal for time of flight measurements. Energy and time of flight calibration of the detectors was performed using radioactive sources $\left({ }^{252} \mathrm{Cf},{ }^{241} \mathrm{Am}\right)$ and by scattering ${ }^{129} \mathrm{Xe}$ and ${ }^{181} \mathrm{Ta}$ beams at $2 \mathrm{MeV} /$ nucleon off a thick Au-target.

Figure 1 displays mass vs velocity for products detected at $6^{\circ}$, for the system $\mathrm{Cu}+\mathrm{Au}$ at 35 $\mathrm{MeV} /$ nucleon. In this figure two main regions of event concentration can be distinguished, the first around mass 75-80 amu and the second around mass $150 \mathrm{amu}$. Their velocities are between $0.5 \mathrm{~cm} / \mathrm{ns}$ (which is determined by TOF-measurement threshold) and $2 \mathrm{~cm} / \mathrm{ns}$. In this reaction the velocity corresponding to full momentum transfer is about 2 $\mathrm{cm} / \mathrm{ns}$ and is indicated by an arrow in the figure. In Fig. 2 the corresponding neutron multiplicities for these two groups of events are given, they are 
corrected for background but not for efficiency of the neutron ball. The average background was 4 to 5 neutrons over the data acquisition period. Two results already reported in our previous work ${ }^{3}$ can be deduced from these data. The first is that both the fragments of mass around 75 and those around 150 mass units are associated with large neutron multiplicities indicating very dissipative collisions, and the second is that these neutron multiplicities increase with the velocity of the fragments. These observations are consistent with an Incomplete Fusion scenario, followed by either evaporation which gives rise to the observed products of mass 150 or by

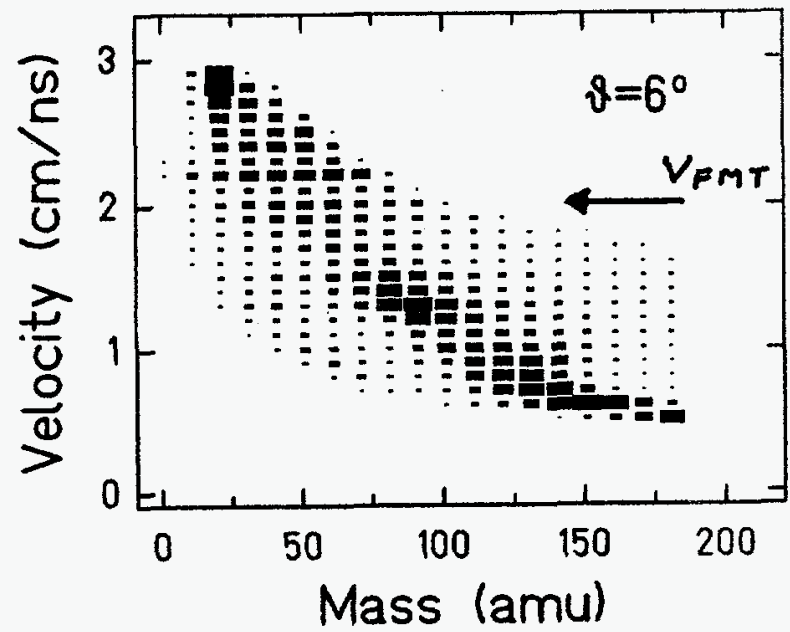

Figure 1: Density plot of mass-velocity distribution observed at lab angle $\theta=6^{\circ}$. The lower limit in velocity, $0.5 \mathrm{~cm} / \mathrm{ns}$ is the experimental TOF threshold. The arrow on the $y$-axis indicates the velocity corresponding to full momentum transfer reactions.

\section{$\mathrm{Cu}+\mathrm{Au}$ at $35 \mathrm{AMeV}$}

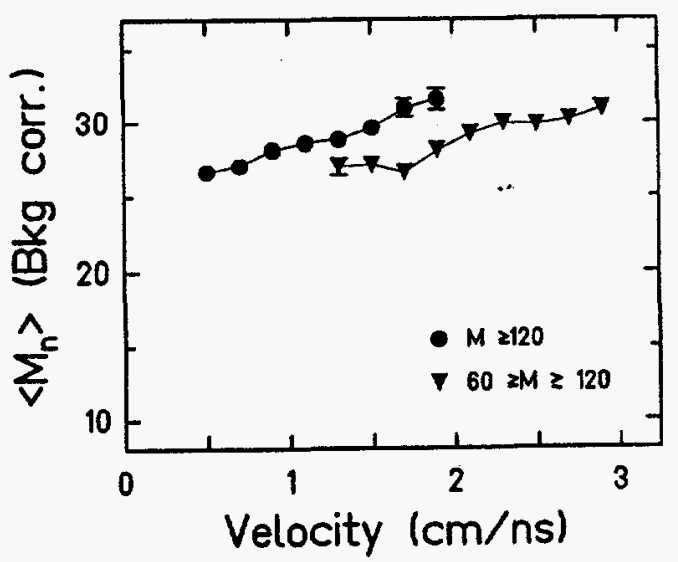

Figure 2: Average neutron multiplicities associated with the fragments observed at $\theta=6^{\circ}$ in the mass range of $A \geq 120$ (closed circles) and $60 \leq \mathrm{A} \leq 120$ (closed triangles). The data have been corrected for the background but not for efficiency.

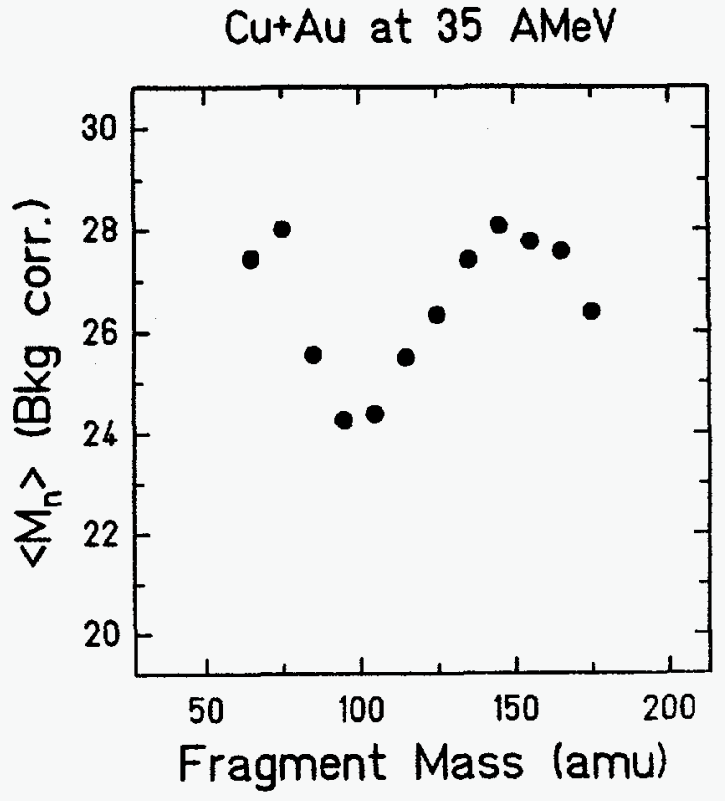

Figure 3: Background corrected neutron multiplicities as a function of fragment mass observed at $\theta=6^{\circ}$.

fission leading to the production of the fragments around mass 75 .

The former observation is confirmed by result displayed in Fig. 3, where the measured neutron multiplicities are represented as a function of the fragments mass. The largest fragment mass corresponds to a relatively low neutron multiplicity.

$\mathrm{Cu}+\mathrm{Au}$ at $35 \mathrm{AMeV}$ Eug-Gem

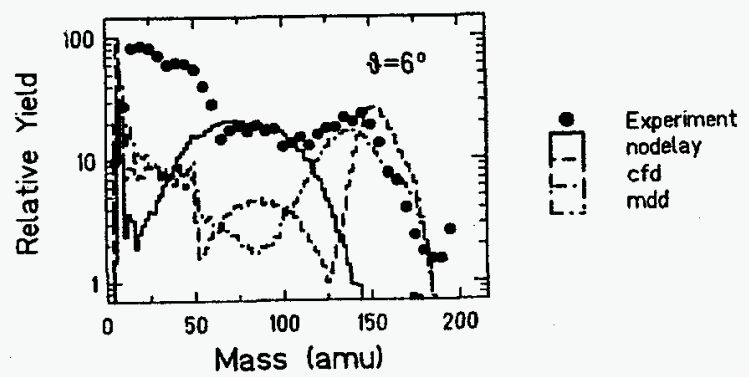

Figure 4: Differential mass distribution at $\theta=6^{\circ}$. Solid, dashed and dotted-dashed histograms correspond to no delay (ND), CFDdelay and MDD-delay respectively. The experimental distribution is represented by solid points.

When the fragment mass decreases the neutron number increases, which is consistent with the fact that the more mass the compound nucleus evaporates the lower is the residue mass and the larger the number of evaporated neutrons. The maximum 
neutron number is observed for mass around 145 . When approaching the fission fragments mass region the neutron multiplicities increases again reaching a maximum at the same value as for the evaporation residues. This observation suggests that these two groups of events originate from compound nuclei of similar excitation energies.

In order to determine the level of the primary excitation energy reached in the systems studied we performed simulations based on Incomplete Fusion processes. For the entrance channel the EUGENE ${ }^{4}$ code is used, in this code the whole energetics are based on massive transfer from the projectile to the target. To follow the decay of the generated compound nucleus in a more realistic way the code GEMINI $^{5}$ is used since Eugene does not treat the angular momentum in the deexcitation cascade. By comparison to the background corrected neutron multiplicity which gives a direct measure of the amount of thermalized energy in the compound nucleus, we extract the primary excitation energy deposited in the composite system. During these simulations the effect of the delay in the fission decay, a slow collective process which may be hindered at very high excitation energy, ${ }^{6}$ is explored by delaying the fission. Two dynamical delays have been investigated: constant fission delay (CFD) in which the emission of particles of mass higher than 30 is prohibited before $10^{-20} \mathrm{~s}$; and mass dependent delay (MDD) in which the delay is a function of the particle mass, ranging monotonically from $10^{-23} \mathrm{~s}$ for mass 10 to $10^{-20} \mathrm{~s}$ for mass 120 . The neutron multiplicities are quite insensitive to the dynamical delay. In order to reproduce the observed heavy fragment yields at lab angle $\theta=6^{\circ}$, a delay of the fission process is necessary as can be seen in Fig. 4 .

Figure 5 displays the evolution of the number of emitted neutrons from all sources (compound nucleus, projectile-like and preequilibrium) as a function of the primary excitation energy of the composite system. The primary neutron multiplicities are filtered by the experimental efficiency of the neutron ball. By comparison of the observed neutron multiplicities to the filtered ones predicted by the calculation, we extract the excitation energy of the composite system. The results are given in Table 1. Excitation energies

\section{Eugene-Gemini at $35 \mathrm{AMeV}$}

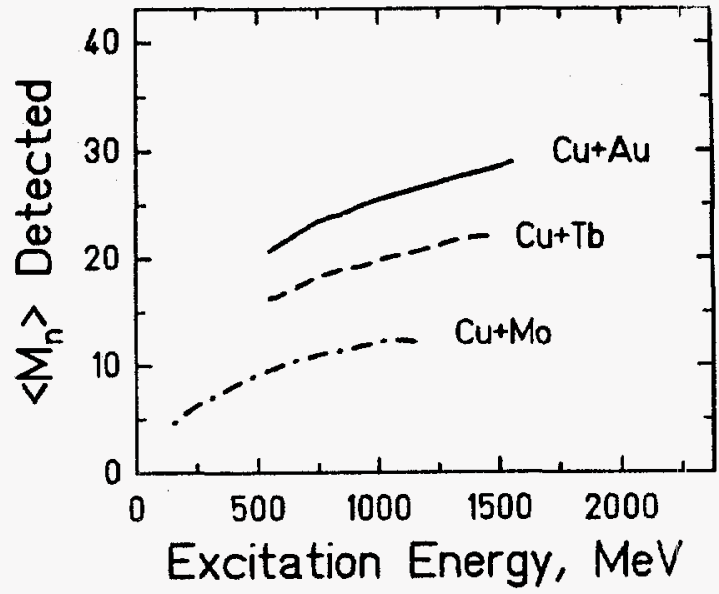

Figure 5: Detected neutron multiplicities as a function of the excitation energy deposited in the primary compound nucleus generated by Eugene-Gemini for ${ }^{63} \mathrm{Cu}+{ }^{197} \mathrm{Au},{ }^{159} \mathrm{~Tb}$ and ${ }^{92} \mathrm{Mo}$ reactions at $35 \mathrm{MeV} /$ nucleon.

of about $1250 \mathrm{MeV}$ are deposited in the systems ${ }^{63} \mathrm{Cu}$ $+{ }^{197} \mathrm{Au}$ and ${ }^{63} \mathrm{Cu}+{ }^{159} \mathrm{~Tb}$. For the system ${ }^{63} \mathrm{Cu}+$ ${ }^{92} \mathrm{Mo}$ an excitation energy of $1000 \mathrm{MeV}$ is deposited in the system. Separate work on this latter system using the linear momentum transfer derived from the folding angle distribution of the fission fragments led to a similar value of the excitation energy. ${ }^{7}$

Table 1. Excitation energy values extracted from EUGENE-GEMINI calculations for different targets ${ }^{197} \mathrm{Au},{ }^{159} \mathrm{~Tb},{ }^{92} \mathrm{Mo}$. The experimental neutron multiplicities are corrected for background but not efficiency.

\begin{tabular}{||c|c|c|}
\hline Target & $\begin{array}{c}\leq \mathrm{M}_{\mathrm{n}} \geq \text { det. } \\
\text { cent.coll }\end{array}$ & Excitation Energy \\
\hline${ }^{197} \mathrm{Au}$ & $26.90 \pm 0.03$ & $1250_{-50}^{+} 190 \mathrm{MeV}$ \\
\hline${ }^{159} \mathrm{~Tb}$ & $21.30 \pm 0.05$ & $1250_{-50}^{+} \mathrm{MeV}$ \\
\hline${ }^{92} \mathrm{Mo}$ & $12.02 \pm 0.03$ & $975_{-180 \mathrm{MeV}}^{+}$ \\
\hline
\end{tabular}

*Institute of Physics, Reymonta 4, PL-30053, Krakow, Poland 


\section{References}

1. D. X. Jiang et al., Nucl. Phys. A503, 560 (1989).

2. D. Utley, Ph.D. thesis (1993), Texas A\&M University.

3. R. Wada et al., Progress in Research, 19921993, Cyclotron Institute, TAMU, p. 6.
4. D. Durand, Nucl. Phys. A541, 266 (1992).

5. R. J. Charity et al., Nucl. Phys. A483, 371 (1988).

6. D. Hilsher et al., Phys. Rev. Lett. 62, 1099 (1989).

7. Y. Lou, Ph.D. thesis (1994), Texas A\&M University.

\title{
Formation and Decay of Hot Nuclei in the ${ }^{63} \mathrm{Cu}+{ }^{232} \mathrm{Th}$ Reaction at $35 \mathrm{MeV} /$ nucleon
}

\author{
Z. Majka*, J. Cibor*, K. Hagel, T. Kozik*, J. Li, Y. Lou, ${ }^{* *}$ J. B. Natowitz, Z. Sosin, R. Tezkratt, \\ D. Utley, R. Wada and B. Xiao
}

Intermediate energy nucleus-nucleus collisions provide opportunities for studying properties of nuclear matter at densities and temperatures far different than those encountered in nuclei near their ground states. This is related to the physics contained in the equation of state for infinite nuclear matter. ${ }^{1}$ Before serious attempts can be made to approach this fundamental problem, two crucial questions concerning the formation and decay of hot nuclei systems in nuclear collisions have to be solved, i.e. what is the maximum excitation energy which can be deposited into a nucleus before complete disintegration, and what are the dominant mechanisms responsible for the decay of the excited nuclear system.

In order to pursue this problem an exclusive experiment that imposed strong restrictive conditions was performed at the Texas A\&M K-500 superconducting cyclotron facility. The $35 \mathrm{AMeV}$ ${ }^{63} \mathrm{Cu}$ beam was incident on a $0.375 \mathrm{mg} / \mathrm{cm}^{2}{ }^{232} \mathrm{Th}$ target on a $0.030 \mathrm{mg} / \mathrm{cm}^{2} \mathrm{C}$ backing. The detectors for the charged fragments were placed inside the scattering chamber of the $4 \pi$ neutron detector $(\mathrm{NBL})^{2}$ containing about 1800 liters of Gadolinium loaded liquid scintillator. The scattering chamber has a cylindrical shape $(45 \mathrm{~cm}$ high and $40 \mathrm{~cm}$ in diameter) with a wedge-shaped forward extension covering \pm $20^{\circ}$ in the horizontal plane. A multi-detector arrangement (BALL) constructed at the Institute of Physics of the Jagellonian University was used mainly for the light charged particle, with $\mathrm{Z} \leq 2$ (LCP) and intermediate mass fragment (IMF) detection. The 35 ionization chamber - Si telescopes of this device were located between $50^{\circ}$ and $150^{\circ}$ in the laboratory, each covering a $42 \mathrm{msr}$ solid angle. A thin window (0.1 $\mathrm{mg} / \mathrm{cm}^{2}$ ) and low operation pressure (isobutane at 135 torr) applied to the ionization chambers of the telescopes allowed us to obtain low detection thresholds for the LCP and IMF. Four of these telescopes were tuned to detect the fission fragments (FF). The heavy residue (HR) Si detector (RES) was placed in a long extension tube $175 \mathrm{~cm}$ from the target at $6^{\circ}$. This detector was $900 \mathrm{~mm}^{2}$ in area and $200 \mu$ thick. Three large area Si detectors $300 \mu$ thick were used for fission fragment detection. Two of them labeled FF1 and FF2 were placed above and below the horizontal plane and covered the azimuthal angles from $38^{\circ}$ to $70^{\circ}$ and from $34^{\circ}$ to $72^{\circ}$ in laboratory, respectively. These detectors were $6 \mathrm{~cm}$ long and $4 \mathrm{~cm}$ wide and divided into 7 strips arranged horizontally. A third detector (FF3) was 5 $\mathrm{X} 5 \mathrm{~cm}^{2}$ and placed in the horizontal plane on the left 
side of the beam and covered the range from $9.5^{\circ}$ to $14.5^{\circ}$. The projectile like fragment (PLF) hodoscope (HODO) was located on the other side of the beam. The hodoscope consisting of one $5 \times 5 \mathrm{~cm}^{2}$ area and a $150 \mu \mathrm{m}$ thick Si detector, backed with a CsI crystal $6 \mathrm{~cm} \mathrm{X} 4 \mathrm{~cm}$ area and $0.5 \mathrm{~cm}$ thick covered a range from $5^{\circ}$ to $7^{\circ}$. Two $\mathrm{Si}$ detectors sited at $\pm 2^{\circ}$ were used as monitors.

The efficiency calibration of the NBL was done by using a ${ }^{252} \mathrm{Cf}$ source and computer simulations by the computer code DENIS. ${ }^{3,4}$ The energy calibrations of all charged particle detectors were performed by using $\alpha$ particles from ${ }^{148} \mathrm{Gd}$ and ${ }^{252} \mathrm{Cf}$ sources and observing the two fission maxima in the ${ }^{252} \mathrm{Cf}$ fission fragment energy spectrum. The total pulse height defects (TPHD) for the FF were obtained by putting the fission fragment detectors inside the gas, and adjusting the pressure in the chamber. The TPHD for the RES detector was determined using ${ }^{252} \mathrm{Cf}$ and scattered and degraded beams of $2 \mathrm{AMeV}{ }^{181} \mathrm{Ta}$ and ${ }^{136} \mathrm{Xe}$. Timing calibrations were made using delay lines in the timing circuits. Plasma delays were determined according to the measured times and known timing of the scattered reference beams.

To characterize the system which was formed after the pre-equilibrium phase the dynamical aspects of the collisions between projectile and target nuclei were simulated using the computer code CHIMERA ${ }^{5}$ which is based upon the molecular dynamics concept. The code is a compilation of two recently devised models, namely, the Quantum Molecular Dynamics (QMD) model of Aichelin and Stöcker ${ }^{6}$ and the Quasi-Particle Dynamics (QPD) model of Boal and Glosli. ${ }^{7}$ Recent calculations of the incomplete fusion reactions have shown ${ }^{8}$ that the time evolution of the longitudinal velocity of the fused system approaches a constant value at the end of the pre-equilibrium phase. If this observable was used to establish the time instant to calculate the characteristics of the equilibrated composite system formed in the nucleus-nucleus collision a time of $t=250 \mathrm{fm} / \mathrm{c}$ is suggested. The upper panel in Fig. 1 displays the scatter plot of a number of events for the fragment

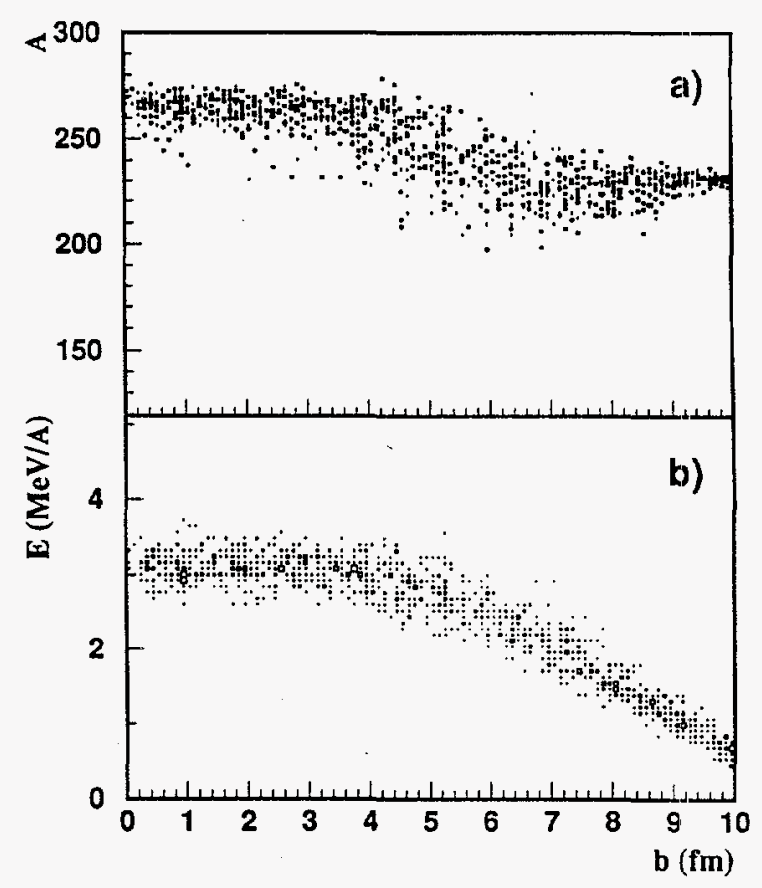

Figure 1. The scatter plots for a number of events for (a) the fragment mass versus the impact parameter and (b) the fragment excitation energy calculated at $t=250 \mathrm{fm} / \mathrm{c}$ for the ${ }^{63} \mathrm{Cu}+{ }^{232} \mathrm{Th}$ reaction at $35 \mathrm{MeV} /$ nucleon versus the impact parameter.

mass calculated for the ${ }^{63} \mathrm{Cu}+{ }^{232} \mathrm{Th}$ reaction at 35 $\mathrm{MeV} /$ nucleon versus the impact parameter. One can see that for the collisions below $4.5 \mathrm{fm}$ of the impact parameter the heavy composite system is predicted to be formed with mass in the range of $250 \mathrm{amu}$ to 280 amu. On the other hand, for collisions above $4.5 \mathrm{fm}$ the binary character of the reaction is observed. The lower panel in Fig. 1 shows the scatter plot of number of events for the heaviest fragment excitation energy versus the impact parameter. Here one can see that the model calculation predicts excitation energies of the fused system at $250 \mathrm{fm} / \mathrm{c}$ from 2.5 $\mathrm{MeV} /$ nucleon to $3.5 \mathrm{MeV} /$ nucleon. The number of events for the heaviest fragment masses versus excitation energy is shown in the upper panel in Fig. 2. The lower panel in Fig. 2 displays the number of events for the heaviest fragment velocity in the laboratory reference frame versus the excitation energy. Thus, the model calculation predicts that for the central collision about 70 per cent of the projectile linear momentum is transferred to the target 
nucleus and the heavy fused system is formed with the total excitation energy in the range from 625 $\mathrm{MeV}$ to $980 \mathrm{MeV}$.

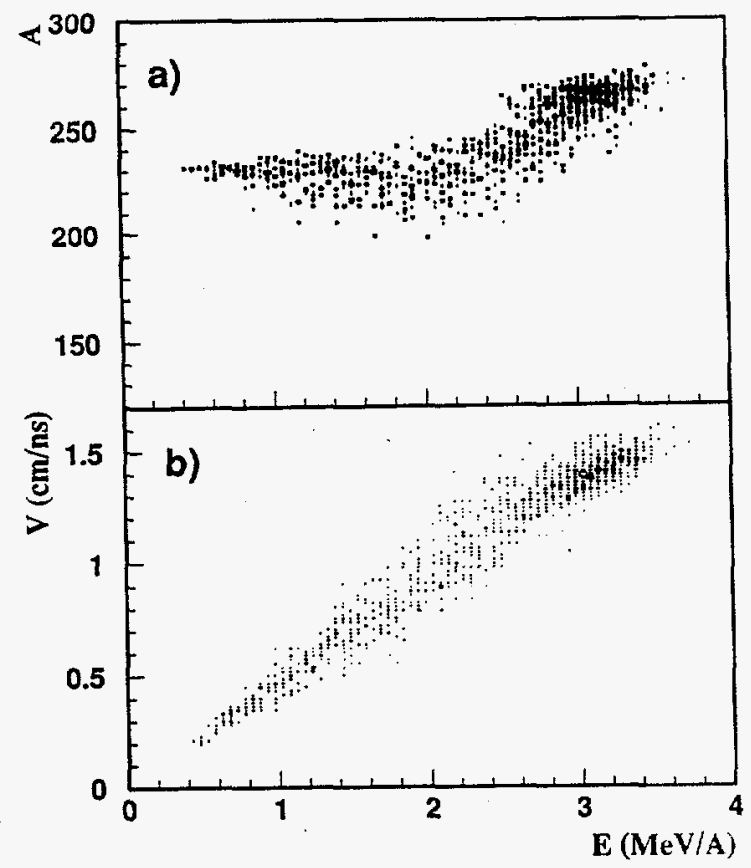

Figure 2. The scatter plots for a number of events for (a) the fragment mass versus the fragment excitation energy and (b) the fragment velocity calculated at $t=250 \mathrm{fm} / \mathrm{c}$ for the ${ }^{63} \mathrm{Cu}+{ }^{232} \mathrm{Th}$ reaction at $35 \mathrm{MeV} /$ nucleon versus the fragment excitation energy fragment.

From a preliminary analysis of the reported experiment the following observations can be made:

1. Fission is a dominant decay channel of the heavy composite system formed in the violent collisions. Measurements of two fission fragments in coincidence indicate that the velocity of the fissioning system is within the range predicted by the model calculation. The average background corrected neutron multiplicity associated with these fission fragments varies by less than 3 units in the source velocity range assigned for the violent collisions.

2. The experimental setup allowed collection of substantial statistics of triple coincidence events in which two fission fragments and an intermediate fragment were detected. Figure 3 displays the intermediate fragment multiplicities associated with two fission fragments. One can see that the intermediate fragment is more frequently associated with asymmetric fission than with symmetric fission. The average background corrected neutron multiplicity associated with these triple coincidence events is close to the value observed without the intermediate fragment detection.

3. The analysis of the events in which the heavy fragment was detected in the RES or HODO detectors suggests that the evaporation residues survive even for collisions with the highest momentum transfers. The intermediate fragment multiplicity for these events is higher than that seen in the fission decays. On the other hand the neutron multiplicities are similar.

The work was supported by the Scientific Research Committee under Grant No. 223929102. "Institute of Physics, Jagellonian University, PL-30-059 Krakow, Reymonta 4, Poland

**Indiana University, Bloomington, Indiana

\section{References}

1. H. Stöcker and W. Greiner, Phys. Rep. 202, 233 (1991).

2. R. P. Schmitt et al., Progress in Research, 1990-1991, Cyclotron Institute, TAMU, p. 47.

3. J. Poitou, Nucl. Instrum. Methods 114, 113 (1974).

4. B. J. Hurst, private communication.

5. J. Lukasik and Z. Majka, Acta Phys. Pol, 24B, 1959 (1993).

6. J. Aichelin et al., Phys. Rev. C 37, 2451 (1988); G. Peilert et al., Phys. Rev. C 39, 1042 (1989); J. Aichelin, Phys. Rep. 202, 233 (1991).

7. D. H. Boal and J. N. Glosli, Phys. Rev. C 38, 1870 (1988); D. H. Boal and J. N. Glosli, Phys. Rev. C 38, 2621 (1988).

8. J. Cibor, J. Lukasik and Z. Majka, Z. Phys. A in press. 


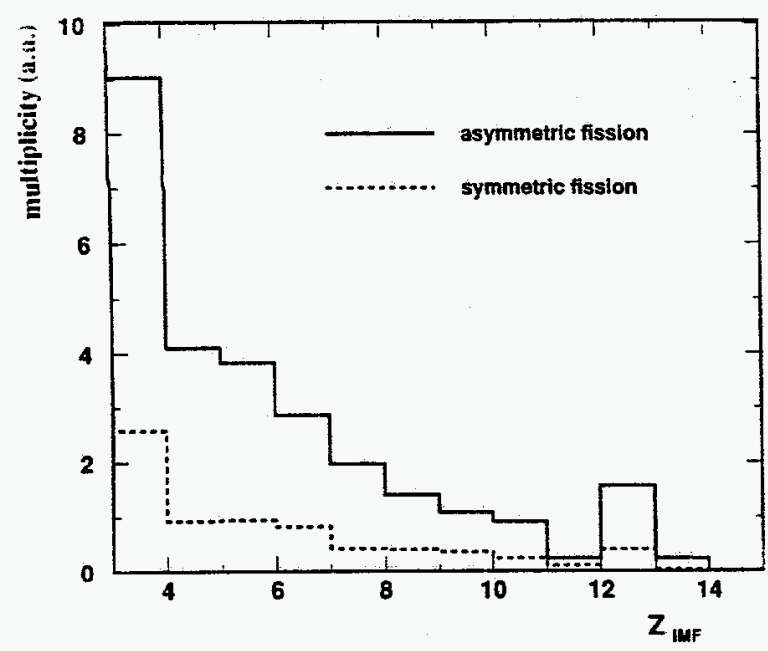

Figure 3. Multiplicities of the intermediate mass fragments detected in the coincidence of two fission fragments.

\section{A Study of the Reactions of ${ }^{63} \mathrm{Cu}+{ }^{63} \mathrm{Cu}$ at $35 \mathrm{MeV} / \mathrm{u}$}

J. Li, X. Bin, K. Hagel, F. Haddad, Y. Lou, ${ }^{*}$ N. Mdeiwayeh, R. Tezkratt, D. Utley, R. Wada, and J. B. Natowitz

To study the systematics of the reaction mechanisms and decay properties of hot nuclei, a 35 $\mathrm{AMeV}{ }^{63} \mathrm{Cu}$ beam from the Texas A\&M K500 superconducting cyclotron has been used to irradiate a $\mathrm{Cu}$ target. IMFs were detected by four $\mathrm{Si}+\mathrm{Si}+$ CsI $\left(\Delta \mathrm{E}_{1}+\Delta \mathrm{E}_{2}+\mathrm{E}\right)$ telescopes sitting at $15^{\circ}, 30^{\circ}$, $45^{\circ}, 60^{\circ}$ and two gas-ionization chamber $+\mathrm{Si}$ telescopes at $80^{\circ}$ and $120^{\circ}$. Residues and fragments were detected by a large $S i$ detector $(5 \mathrm{~cm} \times 5 \mathrm{~cm})$ at $\theta=6^{\circ}$ at a distance of $65 \mathrm{~cm}$ from the target. A hodoscope at $-6^{\circ}$ consisting of a 7 -strip Si detector (4 $\mathrm{cm} \times 6 \mathrm{~cm}$ ) backed by a $5 \mathrm{~mm}$ thick CsI crystal was used to detect projectile-like fragments (PLFs) and IMFs. Neutrons were detected by the Texas A\&M $4 \pi$ Neutron Ball. All the charged particles were detected in coincidence with the neutrons detected by the neutron ball.

Analysis is underway. At this point, energy and timing calibrations have been made for the hodoscope. The resultant energy spectra are shown in Fig. 1 for products of $5 \leq Z \leq 16$ which are identified in the hodoscope. A relatively high energy threshold is apparent. Since lower energy PLFs and IMFs are stopped in the $\mathrm{Si}$ detector, we have attempted to determine the masses of these stopped fragments, in order to complete the spectra. We do this by using the identified species to obtain a time calibration for the stopped fragments (assuming $\mathrm{A}=$ 2Z).

By comparing the time of flight calculated from the calibrated energy of ${ }^{12} \mathrm{C}$ with the experimental time of flight, we define the time calibration. Figure 2(a) shows the results of this timing calibration. In order to check if the derived time calibration is reasonable, we plot the calibrated time vs calculated time in Fig. 2(b) which shows that these two coincide well with each other.

Using the results of the energy and timing calibrations, we get the distribution of mass vs atomic number which is shown in Fig. 3. While the general trend of the distribution is reasonable, we see some clearly unphysical events. This suggests that the combination of relatively poor time resolution (from the neutron ball) and the derivation of the time 
calibration from ${ }^{12} \mathrm{C}$ alone lead to spurious masses.

We are presently continuing this analysis and refining the time calibration techniques.

"Indiana University, Bloomington, Indiana

\section{References}

1. B. Xiao et al., Progress in Research, 1992-1993, Cyclotron Institute, TAMU, p. I-19.

2. R. Wada et al., to be published.

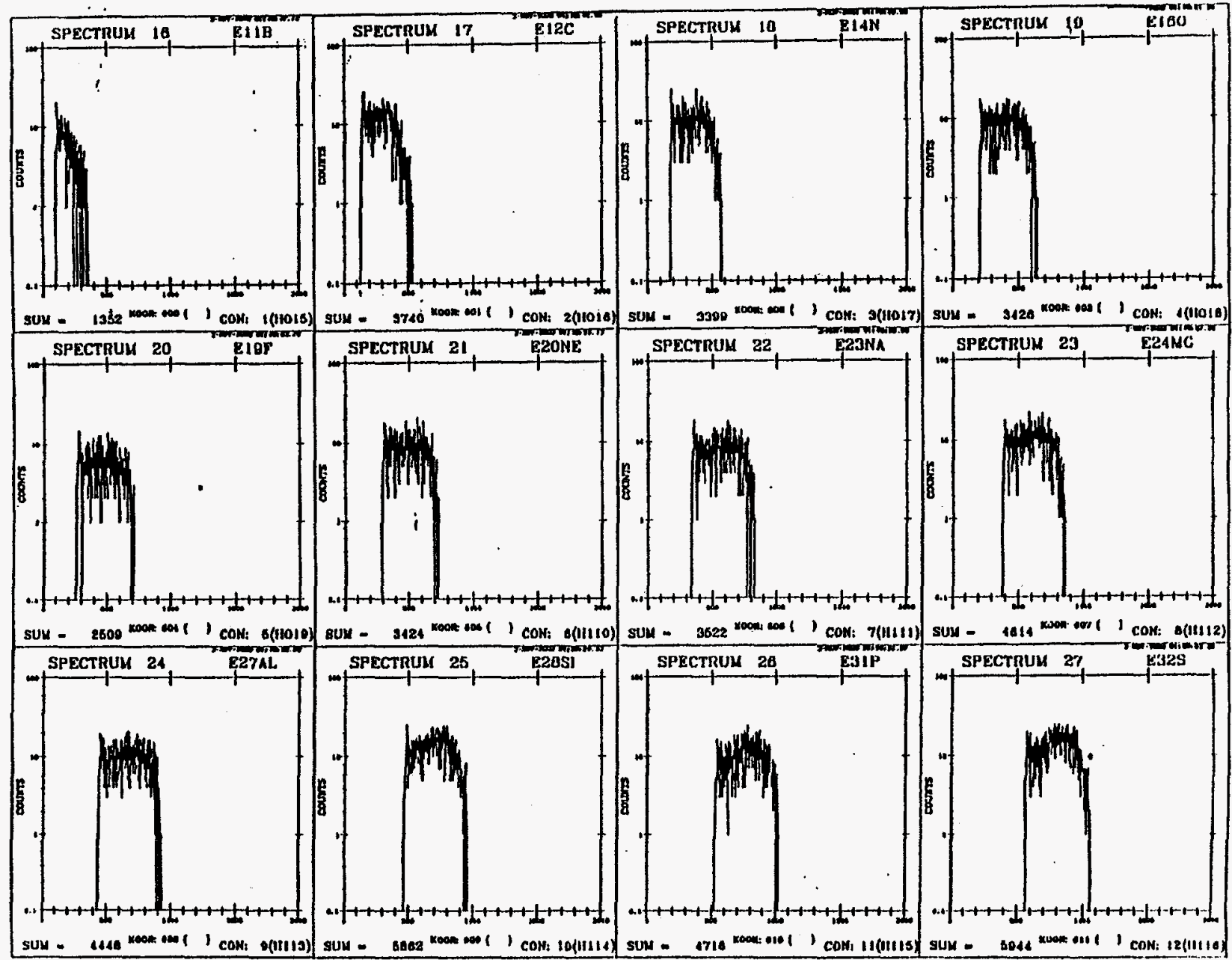

Figure 1. Energy spectra from the hodoscope. From left to right $Z=5$ to $Z=16$ products are shown. 


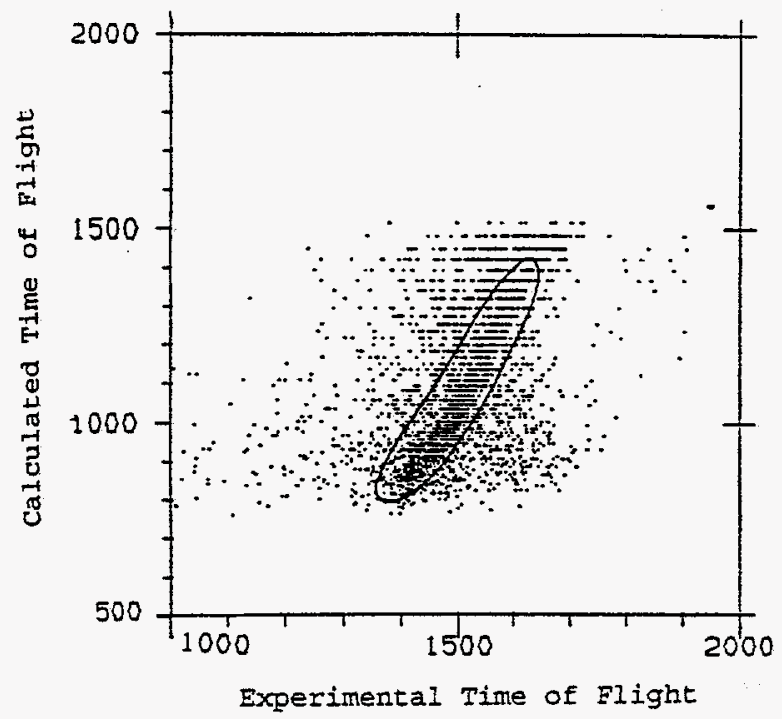

Figure 2(a). A scatter plot of the experimental flight time vs the flight time calculated from the energy for ${ }^{12} \mathrm{C}$.

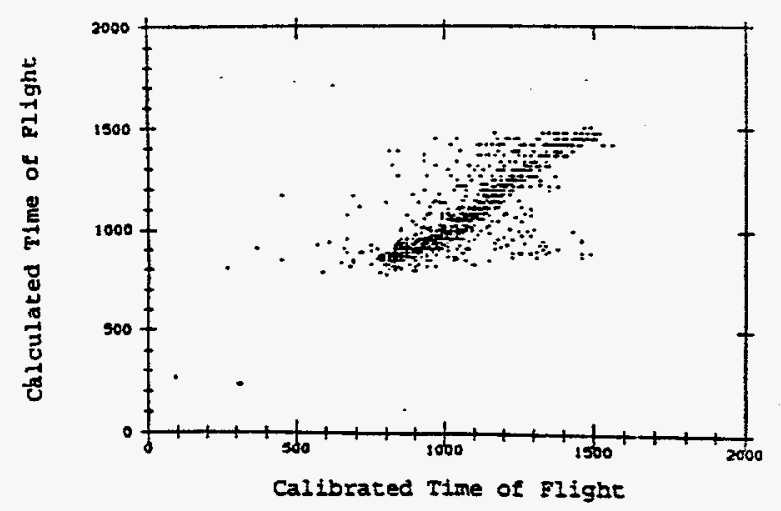

Figure 2(b). A comparison of the calibrated flight time to the same calculated flight time as in Fig. 2(a).

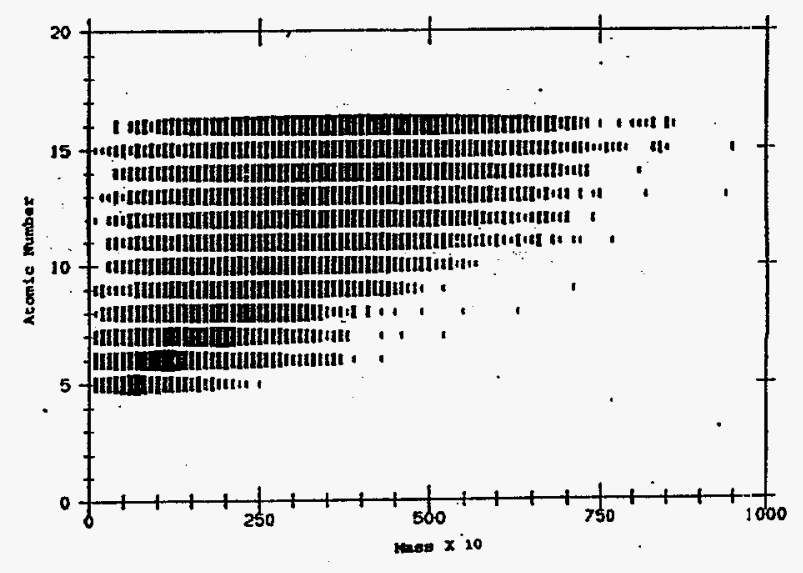

Figure 3. A distribution of mass vs. atomic number. 


\section{Hot Nuclei Produced in the Reaction ${ }^{64} \mathrm{Zn}+{ }^{n a t} \mathrm{Ti}$ at $35-79 \mathrm{AMeV}$}

R. Wada, J. C. Angélique ${ }^{*}$ G. Auger ${ }^{*}$, G. Bizard ${ }^{\ddagger}$, R. Brou ${ }^{\ddagger}$, C. Cabot ${ }^{\ddagger}$, Y. Cassgnou ${ }^{\dagger}$, E. Crema*, D. Cussol ${ }^{\ddagger}$, P. Eudes ${ }^{\ddagger}$, Y. El Masri**, M. Gonin, M. Gui, K. Hagel, A. Kerambrun ${ }^{\ddagger}$, R. Legrain ${ }^{\dagger}$, C. Lebrun $^{\ddagger}$, J. Li, Y. Lou, ${ }^{* * *}$ J. B. Natowitz, J. P. Patry ${ }^{\ddagger}$, A. Péghaire*, J. Péter ${ }^{\ddagger}$, R. Regimbart ${ }^{\ddagger}$, E. Rosato $^{\S}$, F. Saint-Laurent ${ }^{*}$, J. C. Steckmeyer ${ }^{\ddagger}$, B. Tamain ${ }^{\ddagger}$, D. Utley, E. Vient ${ }^{\ddagger}$, and B. Xiao

Reaction dynamics have been studied in the reaction of ${ }^{64} \mathrm{Zn}+{ }^{\text {nat }} \mathrm{Ti}$ at $35-79 \mathrm{AMeV}$. The experiment was performed at the GANIL facility in France, using a $4 \pi$ plastic scintillator array, MUR + TONNEAU, with seven additional Si-telescopes. The telescopes were set at forward angles between $4.3^{\circ}$ and $28.5^{\circ} .^{1}$

Events were sorted by the impact parameter which is evaluated from the $P \perp$ distribution of detected charged particles. For all impact parameters and all incident energies, velocity distributions of the particles indicate that most of them originate from a fast moving source which has about $60-70 \%$ of beam velocity. The source velocity is determined from the velocity distribution of fragments with $Z=6-9$ which shows a Gaussian distribution around the most probable source velocity. The velocity distributions of the lighter particles also show a peak around the source velocity but have a long tail on the slow velocity side, which is attributed to the contribution from preequilibrium particles. The angular distribution of light particles in the source rest frame shows an isotropic distribution up to $60^{\circ}$ for $Z=1$ and $90^{\circ}$ for $Z=2$. The angular distributions are enhanced at backward angles by the contribution from the preequilibrium particles in the reverse kinematics. The isotropic distribution of the light particles at forward angles suggests that the fast moving source is a thermally equilibrated compound system produced by a massive transfer process or incomplete fusion process. The mass and excitation energy of the source are extracted as a function of the impact parameter for each incident energy. In order to minimize the contribution from the preequilibrium particles, only the particles with a parallel velocity greater than the source velocity are selected in each event. The mass and excitation energy associated with these particles are determined and multiplied by a factor of two to get the source mass and the excitation energy, assuming an isotropic emission.
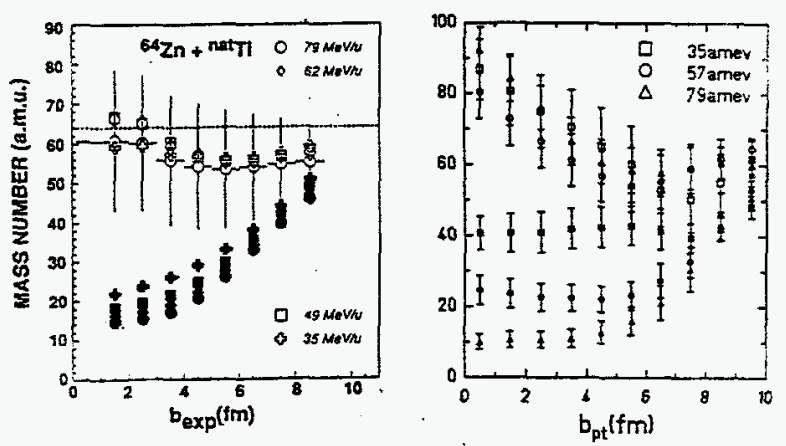

Figure 1. Reconstructed source mass and detected maximum mass as a function of the impact parameter. Experimental results and calculated ones are shown in the left and right side, respectively.

In the left side of Fig. 1 reconstructed source masses are plotted (open symbols) as a function of the experimentally derived impact parameter $b_{\text {exp }}$. The reconstructed source mass is rather constant around the projectile mass. The error bars indicate the width (FWHM) of the distribution. The solid symbols in the figure represent the average maximum mass detected by the telescopes. It depends strongly on the impact parameter. It is interesting that both of the masses show little dependence on the incident energy. The excitation energy of the source is extracted using the Q-value evaluated from the reconstructed source mass $M_{s}$ and sum of the observed charged particle mass $\Sigma M_{c p}=2 \Sigma Z_{c p}$, assuming the neutron multiplicity $M_{n}=M_{s}-\Sigma M_{c p}$. The evaluated excitation energy is plotted in the left side of Fig. 2. The excitation energy depends strongly on the impact parameter. It increases 
significantly between $35 \mathrm{AMeV}$ and $57 \mathrm{AMeV}$ whereas the increase is smaller between $57 \mathrm{AMeV}$ and $79 \mathrm{AMeV}$.
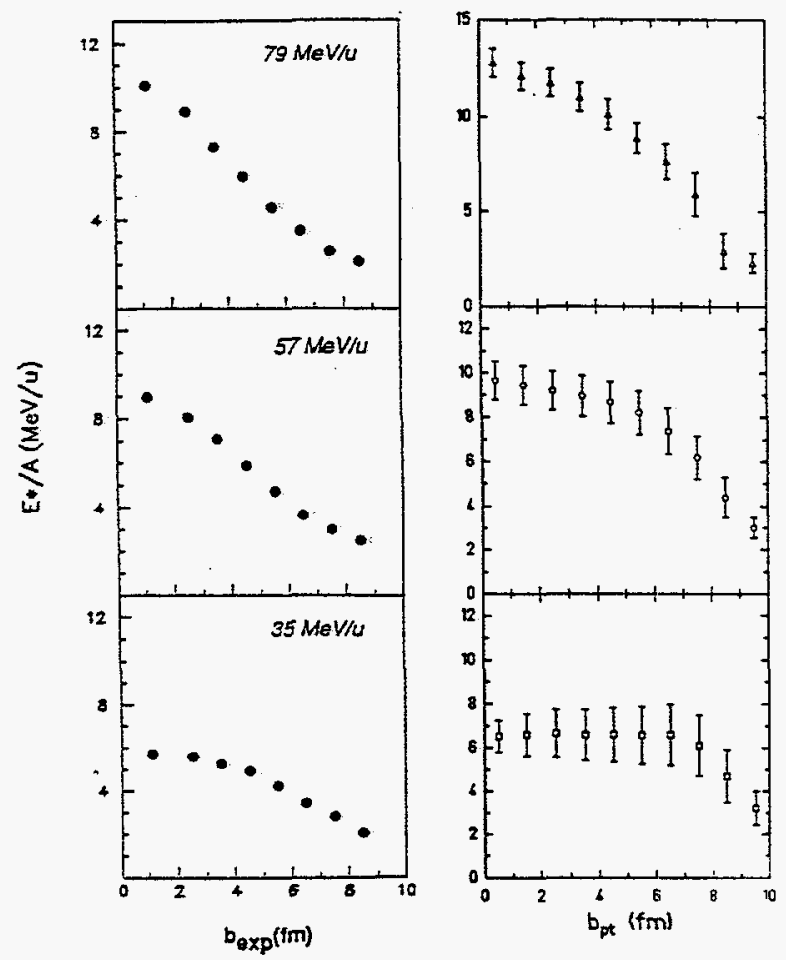

Figure 2. Excitation energy, $E_{x} / A$, is plotted as a function of the impact parameter. Experimental and calculated results are shown in the left and right side, respectively.

The experimental results are compared with QMD + GEMINI calculations. The QMD code was provided by $T$. Maruyama ${ }^{2}$ and the GEMINI code by R. Charity. ${ }^{3}$ QMD calculations are stopped at 250 $\mathrm{fm} / \mathrm{c}$ and switched to GEMINI. Here we show a preliminary results of the QMD calculations. In this QMD calculation a soft EOS $(\mathrm{K}=200 \mathrm{MeV})$ is used and the experimental nucleon-nucleon cross section is employed as the in medium cross section. In the GEMINI calculation fission delay is also employed. The delay is determined from the results of ${ }^{136} \mathrm{Xe}+$ ${ }^{48} \mathrm{Ti}$ at $18.5 \mathrm{AMeV} .{ }^{4}$

All calculated results are filtered by the experimental setup in order to make comparisons with the experimental results. In Fig. 3 the impact parameters, evaluated from the $\mathrm{P} \perp$ distribution calculated in the same manner as the experiment, are compared with those used as the input parameters in the QMD calculations. At higher incident energies one can see a good correlation but at $35 \mathrm{AMeV}$ the correlation becomes poor. This indicates that the results for central collisions at $35 \mathrm{AMeV}$, plotted in the left side of Figs. 1 and 2, may be contaminated significantly by events from more peripheral collisions. At the low incident energies one may have to find a better observable to select out events for the central collisions.

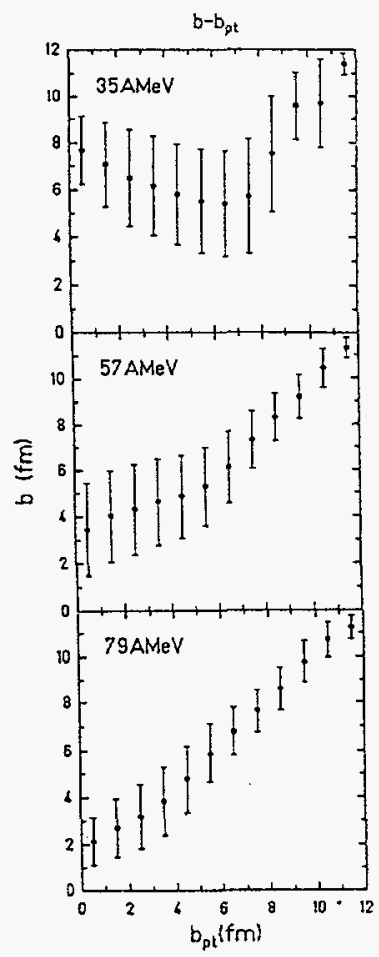

Figure 3. The calculated impact parameter $b_{p}$ in the same manner as the experiment is compared with the impact parameter $b$ used as an input parameter in the QMD calculation ( $Y$-axis) for different incident energies.

In ail QMD calculations a fast moving source is observed. The source mass and excitation energy have been extracted using the same procedure as in the experiment. The results for the reconstructed source mass and the detected maximum mass are shown in the right side of Fig. 1. The trends in the source mass are similar to the experimental results but the source mass for the central collisions is slightly higher for all incident energies. The agreement for the detected maximum mass is 
reasonable for the higher incident energies but poor at $35 \mathrm{AMeV}$. The calculated excitation energy is plotted in the right side of Fig. 2. The overall agreement is reasonable but the dependence of the calculated excitation energies on the impact parameter is much less than that derived from the experimental results. The absolute value at $79 \mathrm{AMeV}$ for central collisions is also much higher (note: change in y scale).

The simulations are still primitive. All parameters used in the calculations, such as the compressibility, nucleon-nucleon cross section in medium, fission delay and so on, have to be optimized to the experimental data. In order to extract the best parameter set further study is now underway.

*GANIL, BP5027, 14021 Caen, France.
${ }^{\ddagger}$ LPC Caen, ISMRA, IN2P3-CNRS, 14032 Caen, France.

*** Indiana University, Bloomington, Indiana.

${ }^{\dagger}$ CEN DphN/BE, Saclay 91191, Gif Sur Yvette, France.

${ }^{* *}$ Cyclotron Institute, Univ. Catholique de Louvain, Louvain La Neuve, Belgium.

${ }^{\S}$ Dipart. di Sienze Fisiche, Univ. di Napoli, Italy.

\section{References}

1. D. Cussol et al., LPC Caen preprint LPCC 93-02 (1993).

2. T. Maruyama et al., Phys. Rev. C 45, 2355 (1992).

3. R. Charity et al., Nucl. Phys. A483, 371 (1988).

4. M. Qui et al., Phys. Rev. C 48, 1791 (1993).

Isotopic Yield Ratio as a Probe of Reaction Dynamics in the Reaction ${ }^{64} \mathrm{Zn}+{ }^{58} \mathrm{Ni}$ at $35-79 \mathrm{AMeV}$

R. Wada, J. C. Angélique*, G. Auger*, G. Bizard ${ }^{\ddagger}$, R. Brou ${ }^{\ddagger}$, C. Cabot ${ }^{\ddagger}$, Y. Cassgnou ${ }^{\ddagger}$, E. Crema*, D. Cussol ${ }^{\ddagger}$, P. Eudes ${ }^{\ddagger}$, Y. El Masri ${ }^{* *}$, M. Gonin, M. Gui, K. Hagel, A. Kerambrun ${ }^{\ddagger}$, R. Legrain ${ }^{\dagger}$, C. Lebrun ${ }^{\ddagger}$, J. Li, Y. Lou, ${ }^{* * *}$ J. B. Natowitz, J. P. Patry ${ }^{\ddagger}$, A. Péghaire*, J. Péter ${ }^{\ddagger}$, R. Regimbart ${ }^{\ddagger}$, E. Rosato $^{\S}$, F. Saint-Laurent ${ }^{*}$ J. C. Steckmeyer ${ }^{\ddagger}$, B. Tamain ${ }^{\ddagger}$, D. Utley, E. Vient ${ }^{\ddagger}$, B. Xiao

Isotopic yields of Lithium to Nitrogen have been measured in the reaction of ${ }^{64} \mathrm{Zn}+{ }^{58} \mathrm{Ni}$ at $35-79$ AMeV. The experiment was performed at the GANIL facility in France. A $4 \pi$ plastic scintillator array, MUR + TONNEAU, was used for the detection of light particles. Seven additional Si-telescopes were used to measure fragments at forward angles between $4.3^{\circ}$ and $28.5^{\circ}$. Isotopes of $\mathrm{Z}=3$ to $\mathrm{Z}=7$ were clearly identified in most of the telescopes.

In Fig. 1 the isotopic yield distributions of Beryllium and Boron detected at $\theta=28.5^{\circ}$ are shown for different incident energies. The low energy threshold of the detector, determined by the thickness of the first detector of the telescope, is just below the expected Coulomb energies of the detected isotopes. In the figure the peaks corresponding to each isotope are clearly seen. The yield for $\mathrm{Be}$ isotopes shows a clear incident energy dependence. At $35 \mathrm{AMeV}$ yields of ${ }^{7} \mathrm{Be}$ and ${ }^{9} \mathrm{Be}$ are comparable and at $69 \mathrm{AMeV}{ }^{7} \mathrm{Be}$ yield is about twice that of ${ }^{9} \mathrm{Be}$. On the other hand, no significant incident energy dependence is observed for yields of Boron isotopes. Results of a more quantitative analysis are shown in Fig. 2. The energy integrated yield ratio of different isotopes at $\theta=28.5^{\circ}$ is plotted as a function of the 


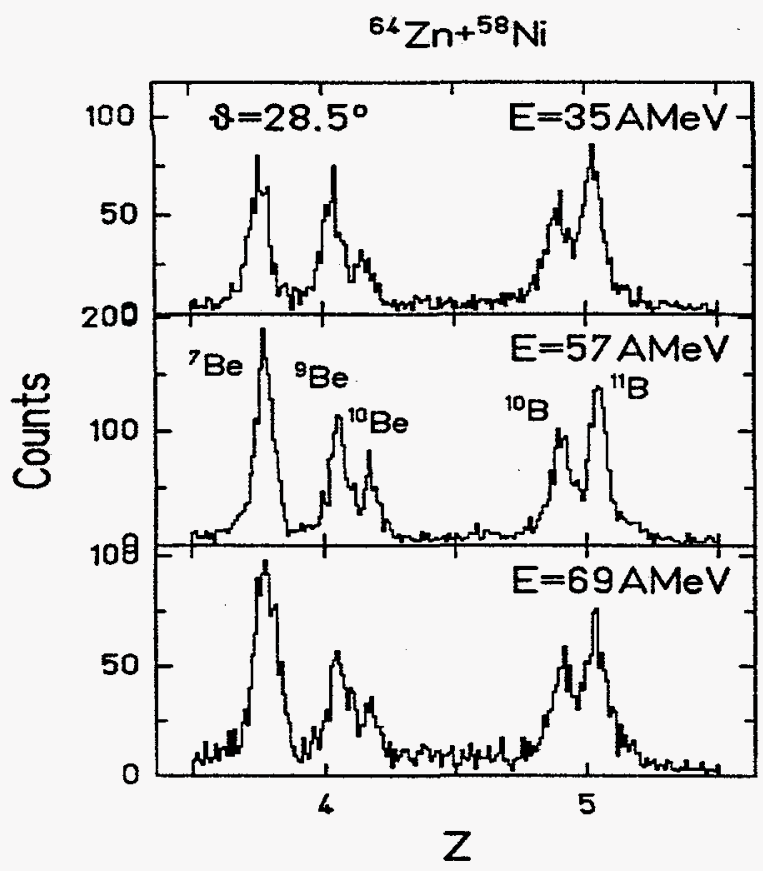

Figurs 1 rotopic yield distribution of Beryllium and Boron served at $\theta=28.5^{\circ}$ for different incident energies.

${ }^{64} \mathrm{Zn}+{ }^{58} \mathrm{Ni}$

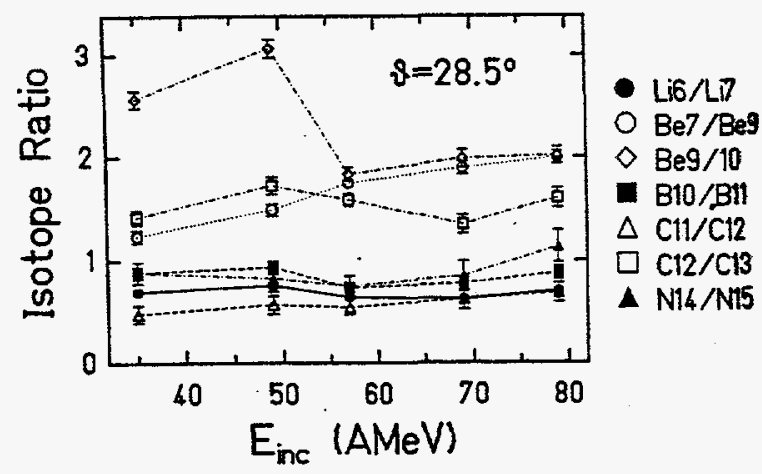

Figure 2. Isotopic yield ratio at $\theta=28.5^{\circ}$ as a function of the incident energy.

incident energy. Be isotopes show characteristic trends whereas all other isotopes show no significant dependence on the incident energy.
The yield of the isotopes results from a combination of two processes, one is the emission process at the early stage of the reaction, and the other is the sequential decay of the emitted isotopes at later stages. The yield in the emission process depends on temperature, density, mass, spin, N/Z of the source and binding energy of the isotope. The decay process is characterized by the excitation energy of the isotope when it is emitted and the $Q$ value for the particle decays. In the latter stages feeding from heavier fragments may also be important.

In order to interpret the results and extract physical properties of the emitting source from the isotope yield ratios, the above two processes have to be carefully taken into account. Detailed analysis is now underway, using a QMD code, provided by $T$. Maruyama et al., ${ }^{1}$ for the first stage and GEMINI, a statistical decay code provided by R. Charity et al., ${ }^{2}$ for the latter stages.

"GANIL, BP5027, 14021 Caen, France.

${ }^{\ddagger}$ LPC Caen, ISMRA, IN2P3-CNRS, 14032 Caen, France.

*** Indiana University, Bloomington, Indiana.

${ }^{\dagger}$ CEN DphN/BE, Saclay 91191, Gif Sur Yvette, France.

${ }^{* *}$ Cyclotron Institute, Univ. Catholique de Louvain, Louvain La Neuve, Belgium.

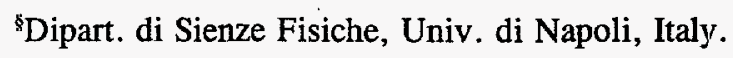

\section{References}

1. T. Maruyama et al., Phys. Rev. C 45, 2355 (1992).

2. R. Charity et al., Nucl. Phys. A483, 371 (1988). 


\title{
${ }^{4} \mathrm{He}$ and ${ }^{5} \mathrm{Li}$ Nuclear Thermometers for Hot Nuclei
}

\author{
J. B. Natowitz, J. C. Hagel, R. Wada, X. Bin, J. Li, Y. Lou, ${ }^{*}$ and D. Utley
}

For well-characterized compound nuclei with $\mathrm{A}=120$, produced in the collisions of $30 \mathrm{AMeV}{ }^{16} \mathrm{O}$ and ${ }^{32} \mathrm{~S}$ with $\mathrm{Ag}$, Wada et al. have reported the determinations of nuclear temperatures over a range of excitation energies from 2 to $4.4 \mathrm{MeV} /$ nucleon. $^{1}$ Over the same energy range, the $\alpha$ multiplicities have also been determined. The observed multiplicities have been used to correct the observed "apparent temperatures," which reflect the range of temperatures sampled during the deexcitation cascade, in order to obtain the initial temperatures at the start of the cascade. Above an excitation energy $\epsilon$, of 1 $\mathrm{MeV} /$ nucleon, the $\alpha$ multiplicities for this system increase linearly with excitation energy per nucleon, and can be simply parameterized as $M_{\alpha}=m \epsilon+b$, where $m=0.822$ and $b=-0.41$. At excitation energies in the 1-6 MeV/nucleon range, the linear increase of light particle multiplicity with $\epsilon$ appears to be generally observed. ${ }^{1,2}$ Assuming the Fermi-gas relationship $\epsilon=T^{2} / K$ with $K$ constant, we can write

$$
\begin{aligned}
& M_{\alpha}=\frac{m}{K} T^{2}+b, \\
& \frac{d M_{\alpha}}{d T}=2 \frac{m T}{K},
\end{aligned}
$$

above a lower limit $T_{L}$.

This leads to a simple expression for the weighted average apparent temperature for the spectrum which results from the deexcitation cascade:

$$
T_{\text {app }}=\frac{\int_{T_{L}}^{T_{U}}(2 m T / K) T d t}{\int_{T_{L}}^{T_{\nu}}(2 m T / K) d T}=\frac{2}{3} \frac{T_{U}^{3}-T_{L}^{3}}{T_{U}^{2}-T_{L}^{2}} .
$$

For purposes of evaluation of this equation, we use $T_{L}=3 \mathrm{MeV}$ corresponding to the temperature where $M_{\alpha}=0.5$. In Fig. 1, the solid line presents calculated values of $T_{a p p}$ vs $T_{U}$, the initial temperature of the deexciting nucleus. As a result of the cascade, the average or apparent temperature which is observed increasingly deviates from the initial temperature, $T_{U}$, as $T_{U}$ increases.

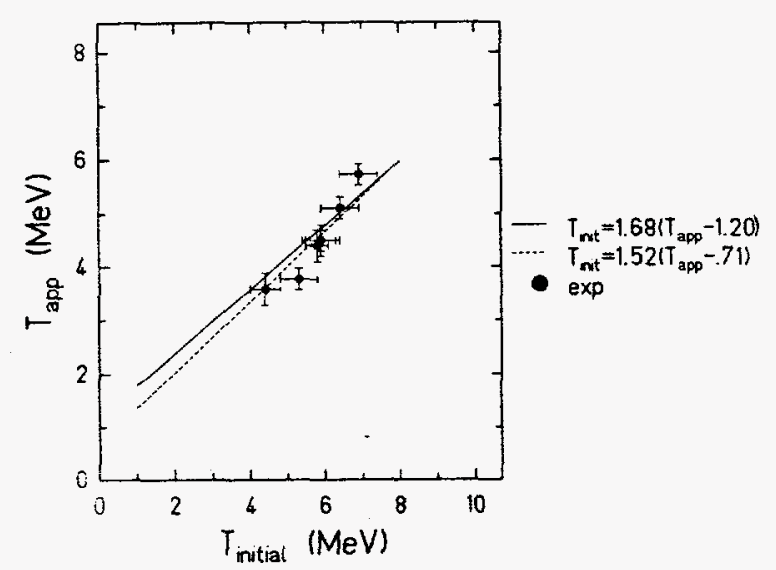

Figure 1: $T_{\text {iapp }}$ as a function of $T_{\text {inir }}$. The solid line represents the trend for spectral slope measurements for ${ }^{4} \mathrm{He}$. The dashed line represents the trend for state population ratio measurements for ${ }^{4} \mathrm{He}$. All calculations assume a constant level density parameter. The solid points with error bars are the values derived in Ref. 1 for $30 \mathrm{~A} \mathrm{MeV}{ }^{16} \mathrm{O},{ }^{32} \mathrm{~S}+\mathrm{Ag}$.

We also present in Fig. 1, as solid points, the apparent and initial temperatures derived from the spectral measurements of Ref. 1 . Note that in Table III of Ref. 1 only the final $T_{i n i t}$ values are shown with angular momentum corrections. In Fig. 1, we present angular momentum corrected values for both $T_{a p p}$ and $T_{i n i t}$. The data agree well with the simple calculation, but show a slightly different trend which reflects an apparent variation of the level density parameter with excitation energy. ${ }^{3}$

For a well-defined temperature, the use of state population ratios as thermometers is based on the assumption that an equilibrium Boltzmann distribution of states exists. The ratio of population of any states is then 


$$
R=\frac{2 J_{U}+1}{2 J_{L}+1} e^{-\Delta E / T}
$$

where $J_{V}$ and $J_{L}$ represent the angular momenta of the upper $(U)$ and lower states, $\Delta E$ is the energy difference between the states $\left(E_{U}-E_{L}\right)$, and $T$ is the temperature of the equilibrated system. Two systems well suited to such temperature measurements are the $0^{+}$ground state and $0^{-}$excited state at $20.21 \mathrm{MeV}$ in ${ }^{4} \mathrm{He}$ and the $3 / 2^{+}$"ground state," and the $3 / 2^{+} 16.66$ $\mathrm{MeV}$ state in ${ }^{5} \mathrm{Li}$. At temperatures below $6 \mathrm{MeV}$, modification of the population ratios by sequential feeding are expected to be negligible.

By analogy to the discussion of $T_{\text {app }}$ vs $T_{\text {init }}$ for the spectral slope measurements, it is clear that the observed state population ratios, even after correction for sequential feeding, can represent a weighted average of primary ratios over the range of temperatures sampled by the observed species during the deexcitation cascade. For ${ }^{4} \mathrm{He}$, in particular, our knowledge of the dependence of multiplicity on temperature allows us to write (assuming a constant inverse level density parameter, $K$ )

$$
\begin{gathered}
R_{a p p}=\frac{\int_{T_{L}}^{T^{v}}\left[\left(2 J_{U}+1\right) /\left(2 J_{L}+1\right)\right](2 m T / K) e^{-\Delta E / T_{d U}}}{\int_{T_{L}}^{T_{U}}(2 m T / K) d T} \\
=\frac{\int_{T_{L}}^{T^{U}} T e^{-\Delta E / T} d T}{T_{U}^{2}-T_{L}^{2}} .
\end{gathered}
$$

Once again using $T_{L}=3 \mathrm{MeV}$, this expression has been evaluated as a function of $T_{U}$, the initial temperature of a cascade.

In Figs. 2(a) and (b) the calculated values of $R_{\text {app }}$ are presented for both ${ }^{4} \mathrm{He}$ and ${ }^{5} \mathrm{Li}$ (in the latter we assume that the multiplicity of ${ }^{5} \mathrm{Li}$ also varies linearly over the same temperature range as ${ }^{4} \mathrm{He}$ ). In the same figures, we show the Boltzmann expression values corresponding to $T_{\text {init }}$. It is clear that the values averaged over the decay cascade are significantly lower than the initial values. Derivation of the initial temperatures from these values leads to temperatures corresponding to the cascade-weighted

\section{${ }^{4} \mathrm{He} 20.21 \mathrm{MeV}$ state}

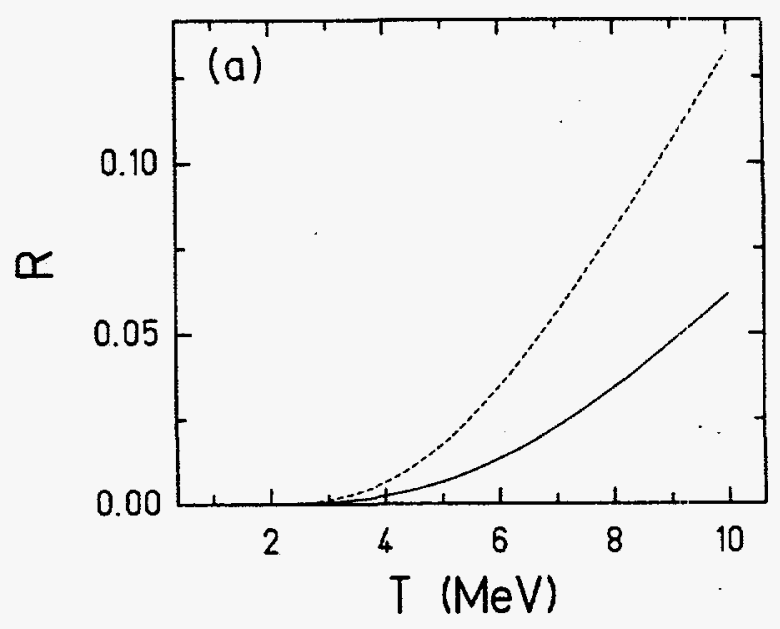

${ }^{5} \mathrm{Li} 16.7 \mathrm{MeV}$ state

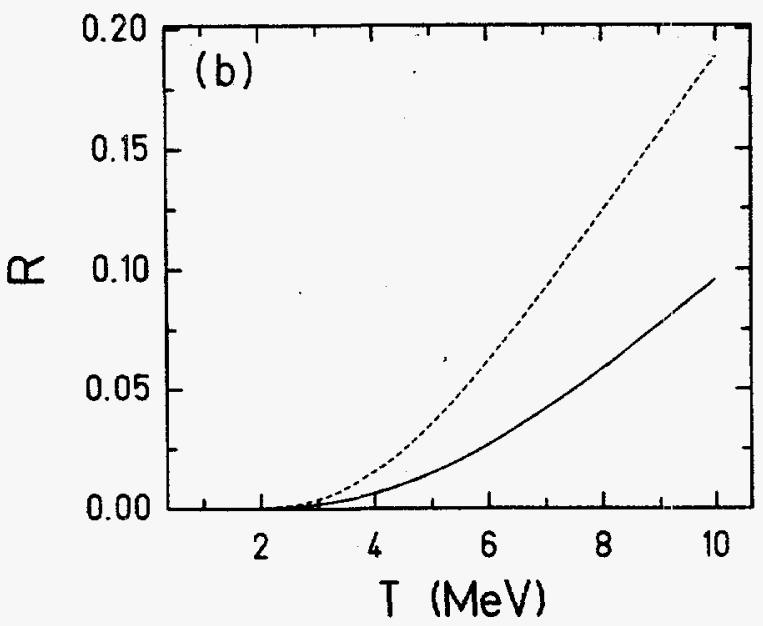

Figure 2: State population ratios as a function of temperature. The dashed lines represent the Boltzmann factor for states with identical angular momenta. The solid lines represent the decay cascade weighted values derived from Eq. 4. (a) ${ }^{4} \mathrm{He}$ ground state and $20.21 \mathrm{MeV}$ state. (b) ${ }^{5} \mathrm{Li}$ ground state and $16.66 \mathrm{MeV}$ state.

ratios are represented by the dashed lines in Fig. 1 . The variation of $T_{a p p}$ with $T_{i n i t}$ is slightly different 
than that derived for the spectral temperature determination. The similarities in the calculated variation of $T_{a p p}$ with $T_{\text {init }}$ shown in Fig. 1 indicate that, for a given initial temperature, similar values of $T_{\text {app }}$ (suitably corrected) should be observed using the different techniques.

We emphasize that the experimentally measured spectral slopes or yield ratios must be corrected for angular momentum effects and for recoil effects in the spectral shape analysis and, in the case of state ratios, for effects of decay of higher mass primary fragments before this calibration is used.

"Indiana University, Bloomington, Indiana

\section{References}

1. R. Wada, D. Fabris, K. Hagel, G. Nebbia, Y. Lou, M. Gonin, J. B. Natowitz, R. Billerey, B. Cheynis, A. Demeyer, D. Drain, D. Guinet, C. Pastor, L. Vagneron, K. Zaid, J. Alarja, A. Giorni, D. Heuer, C. Morand, B. Viano, C. Mazur, C. Ngô, S. Leray, R. Lucas, M. Ribrag, and E. Tomasi, Phys. Rev. C 39, 497 (1989).

2. G. Viesti, D. Fabris, G. Nebbia, G. Prete, K. Hagel, J. B. Natowitz, and R. Wada, (unpublished).

3. S. Shlomo and J. B. Natowitz, Phys. Lett. 252B, 187 (1990).

\title{
Time Scale of the Fission Process in the Reaction $50 \mathrm{AMeV}{ }^{20} \mathrm{Ne}+{ }^{165} \mathrm{Ho}$
}

\author{
N. Mdeiwayeh, R. Wada, G. Cibor, ${ }^{*}$ K. Hagel, J. Li, Y. Lou, ${ }^{* *}$ Z. Majka, ${ }^{*}$ J. B. Natowitz, \\ R. Tezkratt, D. Utley, and B. Xiao
}

In an attempt to increase our understanding of the nuclear viscosity, an experiment was performed on the K-500 cyclotron early in December 1993 in order to evaluate the fission time scale as a function of mass asymmetry. The reaction used was ${ }^{20} \mathrm{Ne}+$ ${ }^{165} \mathrm{Ho}$ at a projectile energy of $50 \mathrm{AMeV}$. In a previous study at the Cyclotron Institute a similar system, ${ }^{136} \mathrm{Xe}+{ }^{48} \mathrm{Ti}$, was studied for the same purpose. ${ }^{1}$ In this experiment it was found that the time scale decreases rapidly with increasing mass asymmetry. The advantage of using the reaction ${ }^{20} \mathrm{Ne}$ $+{ }^{165} \mathrm{Ho}$ was to minimize the contribution of those events resulting from deep inelastic collisions.

The reaction ${ }^{20} \mathrm{Ne}+{ }^{165} \mathrm{Ho}$ took place in the $4 \pi$ neutron ball scattering chamber. Inside the chamber, a $400 \mu \mathrm{g} / \mathrm{cm}^{2}{ }^{165} \mathrm{Ho}$ target was surrounded by 30 gas ionization chambers operating at a pressure of 50 torr of isobutane gas maintained by a gas flow system.
The ionization chambers were backed by Si detectors 1-2 $\mathrm{mm}$ thick and $2 \mathrm{~cm}$ in diameter in order to detect fission fragments. For detecting light-charged particles, a total of 7 additional telescopes were used. These telescopes consisted of $3 \mathrm{Si}$ detectors with active area of $50-200 \mathrm{~mm}^{2}$. The thickness of the first, second, and third set of the Si detectors was 20$50 \mu \mathrm{m}, 50-100 \mu \mathrm{m}$, and 300-2000 $\mu \mathrm{m}$ respectively. Behind the Si detectors were CsI crystals, $1.2 \times 1.2$ $x 2 \mathrm{~cm}^{3}$, optically coupled to photodiodes in order to detect high energy protons. This set-up provided a coverage of approximately $10 \%$ of $4 \pi$ for fission fragments and light-charged particles detection.

The energy, the atomic number, the angles $\theta$ and $\Phi$ of the detected fission fragments and of the lightcharged particles were measured. Singles and coincidence events for both symmetric and asymmetric cases were important to us. In the 
symmetric case, the atomic numbers of both fragments were identified. However, in the asymmetric case, the atomic number of the light fragment was identified, and the atomic number of the heavy one was determined from the relation $Z_{\mathrm{H}}=$ $Z_{\text {tot }}-Z_{L}$ where $Z_{\mathrm{H}}, Z_{L}$ are the atomic numbers of the heavy and light fragments, and $Z_{\text {tot }}$ is the average total atomic number obtained from the symmetric case. To find the masses of the fission fragments we employed the kinematic reconstruction method using the masses extracted from the atomic numbers of the detected fission fragments as trial masses in an iterative procedure. In that process results of the code EUGENE were used to estimate the most probable neutron/proton ratio of the fragments. ${ }^{2}$ Once the masses were obtained the mass asymmetry was defined by

$$
A S Y M=\frac{M_{H}-M_{L}}{M_{H}+M_{L}}
$$

where $M_{H}$ and $M_{L}$ are the masses of the heavy and light fragments. In the diffusion plot, Fig. 1., the total kinetic energy vs. atomic number is shown. Figure 2 shows the observed asymmetry as defined above. The energy spectra of the protons and alpha particles were also extracted. Steps that we will follow to evaluate the time scale of fission for this system will start with a moving source fit to the energy spectra of the light particles in order to determine the pre-scission and post-scission particle multiplicities. In this work three sources of light particles will be considered. These sources are the compound nucleus prior to scission, and the two fission fragments. Finally, to extract the fission time scale as a function of mass asymmetry we will determine the excitation energy as a function of mass asymmetry at scission.

"Institute of Physics, Reymonta 4, PL-30053 Krakow, Poland

"Indiana University, Bloomington, Indiana

\section{References}

1. M. Gui, Ph.D. Thesis, TAMU (1992) and references therein.

2. D. Durand, Nucl. Phys. A541, 266 (1992).

Total Kinetic Energy Vs. Charge

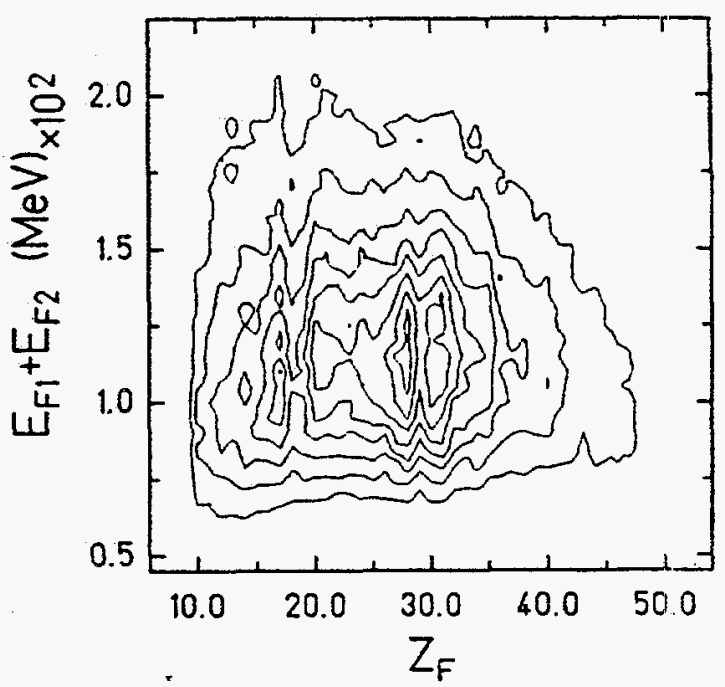

Figure 1. Total kinetic energy versus fragment atomic number.

\section{Asymmetry}

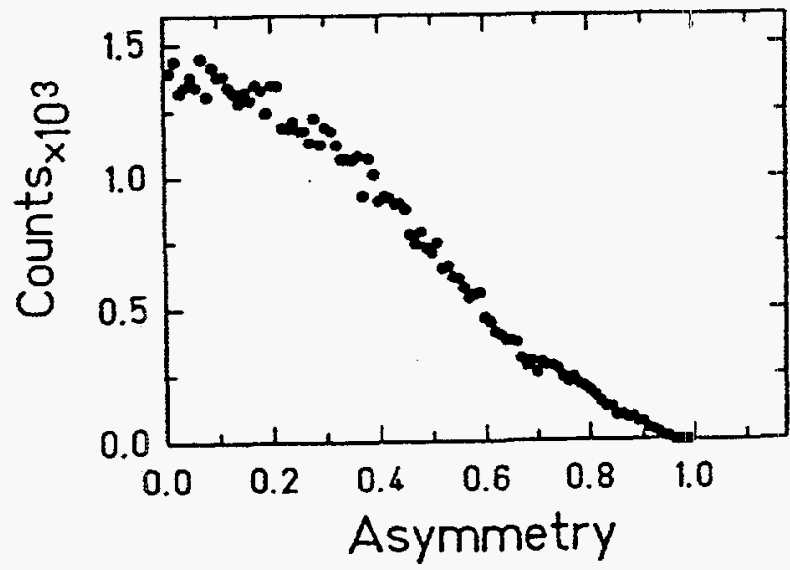

Figure 2. Distribution of detected fragments as a function of mass asymmetry. 


\title{
Excitation Energy Dependence of the Fission Probability in ${ }^{200} \mathrm{~Pb}$ Compound Nuclei
}

\author{
D. Fabris*, G. Viesti*, E. Fioretto ${ }^{\dagger}$, M. Cinausero ${ }^{\dagger}$, N. Gelli ${ }^{\dagger}$, K Hagel, F. Lucarelli ${ }^{\dagger}$, J. B. \\ Natowitz, G. Nebbia*, G. Prete ${ }^{\ddagger}$, and R. Wada
}

The excitation energy dependence of the fission probability in ${ }^{200} \mathrm{~Pb}$ has been determined from measurements of energetic $\gamma$-rays emitted in the deexcitation of the giant dipole resonance (GDR) in coincidence with both fission fragments and evaporation residues in the reactions of ${ }^{19} \mathrm{~F}$ with ${ }^{181} \mathrm{Ta}$.

The experiment was performed at the XTU Tandem facility of the Laboratori Nazionali di Legnaro. ${ }^{18} \mathrm{~F}$ beams at six energies between 90 and $140 \mathrm{MeV}$ were incident on a ${ }^{181} \mathrm{Ta}$ target of 100 $\mu \mathrm{g} / \mathrm{cm}^{2}$, evaporated onto a $20 \mu \mathrm{g} / \mathrm{cm}^{2}{ }^{12} \mathrm{C}$ backing.

The $\gamma$-rays were detected in a cluster of $19 \mathrm{BaF}_{2}$ scintillators ( $48 \mathrm{~cm}^{2}$ surface, $12 \mathrm{~cm}$ thick) placed at $12 \mathrm{~cm}$ from the target at $\theta_{\text {lab }}=90^{\circ}$. Each detector had an individual read-out and was connected to two different discriminators operated with low $\left(\mathrm{E}_{\mathrm{Th}}=200\right.$ $\mathrm{keV})$ and high $\left(\mathrm{E}_{\mathrm{Th}} \geq 3 \mathrm{MeV}\right)$ thresholds. The cluster was used to tag the reaction events (in the low threshold mode) providing the start signal for the time-of-flight (TOF) measurements for evaporation residues and fission fragments. Energetic $\gamma$-rays were tagged in the high threshold mode.

Fission Fragments, FF, were detected in a set of six 7-strip silicon detectors $300 \mu \mathrm{m}$ thick ( $4 \times 6 \mathrm{~cm}^{2}$ each) mounted in the backward hemisphere in a box geometry having near $2 \pi$ efficiency. The E-TOF correlation was used to discriminate FF from light particles.

An electrostatic separator was used to measure evaporation residues, $E R$, close to the beam axis, rejecting scattered projectiles. The separator, basically a plane capacitor, was $25 \mathrm{~cm}$ long and was operated at $10 \mathrm{KV} / \mathrm{cm}$ with an entrance collimator that allowed the selection of a slice of the kinematical cone around the beam axis from $\theta_{\text {lab }}=0^{\circ}$ to about $\theta_{\mathrm{lab}}=10^{\circ}$. The separator was followed by a set of three 7-strip silicon detectors $300 \mu \mathrm{m}$ thick (4 X 18 $\mathrm{cm}^{2}$ total area), placed on a movable arm at a distance of $177 \mathrm{~cm}$ from the target, to measure the energy and time-of-flight of ER.

Both ER and FF "singles" (i.e. ER- $\gamma$ and FF- $\gamma$ in low threshold mode events) and coincidences with energetic $\gamma$-rays (i.e. events having at least one of the $\mathrm{BaF}_{2}$ scintillators exceeding the high threshold) were measured at $E_{\text {Beam }}=90,100,120,130$ and 140 $\mathrm{MeV}$. The ${ }^{200} \mathrm{~Pb}$ compound nuclei were populated at average excitation energies $E_{\mathrm{x}}=58-103 \mathrm{MeV}$ with almost constant limiting angular momenta for fusionevaporation reactions, defined by the fission competition, at a value $J_{E R}^{\max } \sim 30 h$ for bombarding energies larger than $100 \mathrm{MeV}$.

The measured excitation function of energetic $\gamma$-rays $\left(E_{\gamma}>9 \mathrm{MeV}\right)$ in coincidence with ER is shown in Fig. 1. The solid line is the prediction from the statistical model code CASCADE $^{1}$ normalized to the data. GDR parameters and statistical model input data as determined for the same system at $E_{\text {Beam }}=105$ and $141 \mathrm{MeV}$ bombarding energies ${ }^{2}$ were used. With the normalization the calculated excitation function fits the experimental data well.

Results for the fission channel are also reported in Fig. 1. The comparison with computed quantities is more difficult in the FF case because both a prescission emission and a post-scission contribution have to be taken into account. In Fig. 1 it is shown that the experimental FF $\gamma$ multiplicity data at low bombarding energies are described by calculations considering the energetic $\gamma$-rays to be emitted only in the first step of the decay before fission, while the experimental point at $E_{\text {Beam }}=140 \mathrm{MeV}$ is bracketed by the two and three step calculations. This is a reflection of the expected onset of dynamical fission hindrance. To explore this hindrance, we have empirically reduced the fission width as a function of 
bombarding energy $\left(\Gamma_{f}=K\left(E_{x}\right) \times \Gamma_{\text {stat }}\right)$ for $E_{\text {Beam }}>$ $110 \mathrm{MeV}$. The reduction factors needed to fit the data are $\mathrm{K}=.10$ at $\mathrm{E}_{\text {Beam }}=120 \mathrm{MeV}$ and $\mathrm{K}=.01$ at $E_{\text {Beam }}=130,140 \mathrm{MeV}$.

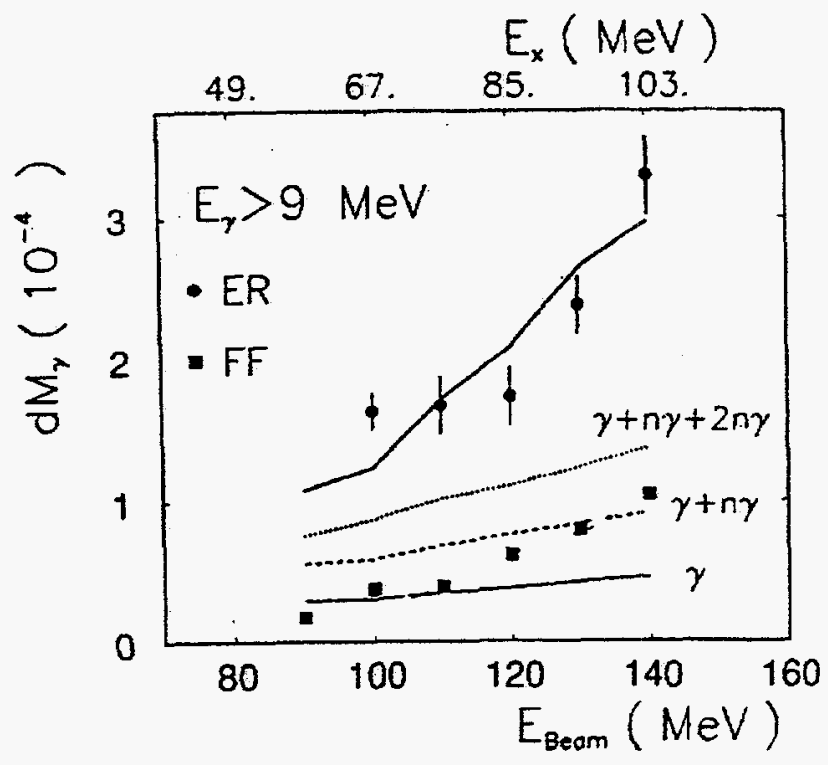

Figure 1. Excitation function for emission of energetic $\gamma$-rays $\left(E_{\gamma}\right.$ $\leq 9 \mathrm{MeV}$ ) in coincidence with evaporation residues (ER) and fission fragments (FF). Lines refer to statistical model calculations. For details see the text.

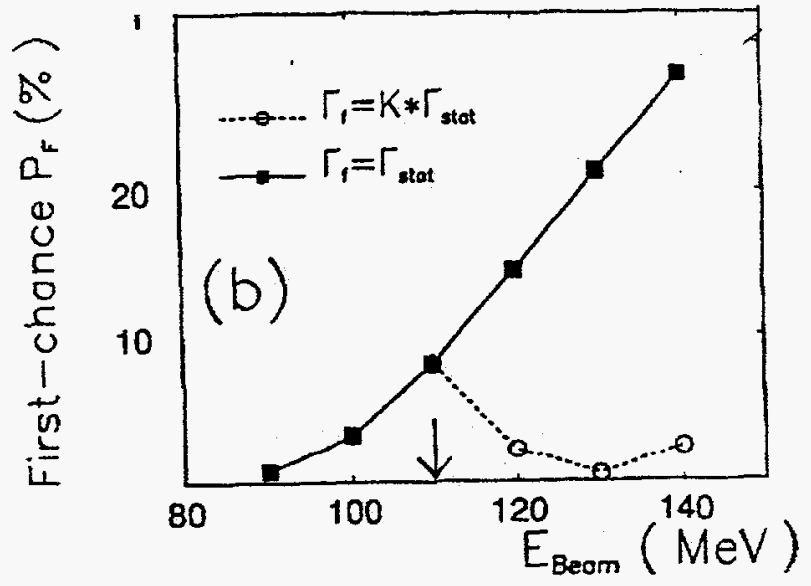

Figure 2. Calculated first-chance fission probability with and without hindrance of fission as derived in this work.
The distribution of $\mathrm{P}_{\mathrm{f}}\left(\mathrm{E}_{\mathrm{x}}\right)$ which is derived from our data is presented in Fig. 2. We note that the maximum energy for "statistical" pre-scission emission is consistent with results from the analysis of the neutron data presented in Ref. 3 as well as with the systematics of Thoennessen and Bertch. ${ }^{4}$ The sudden strong reduction of the fission partial width, $\Gamma_{f}$, due to dynamical effects is in very good agreement with the calculated results of T. Wada $e t$ $a l .{ }^{5}$ for this system and also agrees very well with the distributions derived in Ref. 6.

"INFN and Dipartmento di Fisica dell'Universita di Padova, I-35131 Padova, Italy

‡INFN Laboratori Nazionali di Legnaro, I-35020 Legnaro (Padova), Italy

'INFN and Dipartmento di Fisica dell'Universita di Firenze, I-50125 Firenze, Italy

\section{References}

1. N.B.L.-Milano extended version of the code CASCADE, see Puhlhofer et al., Nucl. Phys. A280, 267 (1977).

2. Butsch et al., Phys. Rev. C 41, 1530 (1990).

3. J. O. Newton et al., Nucl. Phys. A483, 126 (1988).

4. M. Thoennessen and G. Bertch, Phys. Rev. Lett. 71, 4303 (1993).

5. T. Wada, Y. Abe, and N. Carjan, Phys. Rev. Lett. 70, 3538 (1993).

6. J. B. Natowitz et al., Phys. Lett. 247B, 242 (1990). 


\title{
Alpha Particle Emission as a Probe of the Level Density in Highly Excited A $\sim 200$ Nuclei
}

\author{
D. Fabris*, E. Fioretto ${ }^{\S}$, G. Viesti*, M. Cinausero ${ }^{\ddagger}$, N. Gelli ${ }^{\ddagger}$, K. Hagel, F. Lucarelli ${ }^{\ddagger}$, \\ J. B. Natowitz, G. Nebbia*, G. Prete, and R. Wada
}

We present here first results obtained by measuring $\alpha$ particle spectra in coincidence with evaporation residues in the reaction ${ }^{19} \mathrm{~F}+{ }^{181} \mathrm{Ta}$ at 90 $140 \mathrm{MeV}$ bombarding energies. The experiment was performed using ${ }^{19} \mathrm{~F}$ beams from the XTU Tandem of the Laboratori Nazionali di Legnaro at six incident energies between 90 and $140 \mathrm{MeV}$. Targets consisted of $100 \mu \mathrm{g} / \mathrm{cm}^{2}{ }^{181}$ Ta evaporated onto a 20 $\mu \mathrm{g} / \mathrm{cm}^{2}{ }^{12} \mathrm{C}$ backing.

A wide angle electrostatic separator was used to measure evaporation residues close to the beam axis, rejecting both the primary beam and the elastic scattering events. The separator, basically a plane capacitor, was $25 \mathrm{~cm}$ long and was operated at 10 $\mathrm{KV} / \mathrm{cm}$. The entrance collimator allowed selection of a slice of the kinematical cone around the beam axis from $\theta_{l a b}=0^{\circ}$ to about $\theta_{l a b}=10^{\circ}$, thus covering basically the full evaporation residue angular distribution.

The evaporation residues (ER) were detected in a set of three 7-strip silicon detectors $300 \mu \mathrm{m}$ thick, with a total surface of $18 \times 4 \mathrm{~cm}^{2}$, placed on a movable arm $117 \mathrm{~cm}$ downstream from the target position, after the electrostatic separator.

Identification of the ER's was performed by measuring their energy and time-of-flight relative to prompt $\gamma$-rays detected in a cluster of $19 \mathrm{BaF}_{2}$ scintillator detectors placed $12 \mathrm{~cm}$ from the target. The total efficiency of the $\gamma$-ray cluster was $17 \%$ as measured with a ${ }^{60} \mathrm{Co}$ source.

$\alpha$ particles were detected in a set of six 7-strip silicon detectors mounted in the backward hemisphere with a box geometry having efficiency of $\approx 2 \pi$.

Evaporation residue singles (i.e. ER- $\gamma$ events) and $\alpha$-ER coincidences (i.e. ER- $\gamma-\alpha$ events) were measured. The average multiplicity $M_{\alpha}$ was extracted from the experimental data by integrating the measured experimental correlation function with the help of the Monte Carlo (MC) version of the CASCADE statistical model code. In the MC simulation the effective experimental geometry (electrostatic deflector, ER detector, silicon box) was properly introduced. $M_{\alpha}$ values are reported in Fig. 1. A strong experimental bombarding energy dependence of $M_{\alpha}$, in the presence of a saturated $\mathrm{J}_{\max }^{\mathrm{F}_{R}}$, indicates a sizable contribution of first chance emitted $\alpha$ particles.

Calculations were performed in which excitation energy dependent level density parameters $K=K\left(E_{x}\right)$ were employed. We found that the energy spectra of the particles are very sensitive to relatively small changes in the excitation energy dependence of the phase space open to the statistical decay induced by using $K=K\left(E_{x}\right)$.

A reasonable reproduction of the data was obtained using $K=8.3 \mathrm{MeV}^{-1}$ for excitation energies above the yrast line, $E_{x} \leq 20 \mathrm{MeV}$, and then linearly increasing $K$ up to the value $K=12 \mathrm{MeV}^{-1}$ at $E_{x}=$ $100 \mathrm{MeV}$, which corresponds to the $140 \mathrm{MeV}$ bombarding energy.

Results are compared with experimental data in Figs. 1 and 2. The high energy part of the $\alpha$ particle spectrum is very well described. The excess of calculated low energy $\alpha$ particles, emitted near the yrast line was eliminated by increasing the $\gamma$-ray competition as suggested in Ref. 1.

In the mass region $\mathrm{A} \sim 200$ the shapes of the $\alpha$ particle spectra can be reproduced either by very large changes in the average level density parameter for fixed spin distributions or by changes in the spin distribution leaving fixed the average level density parameters. However in both these cases an unrealistically constant excitation function is obtained for the $\alpha$ multiplicity. The only way we have found 
to reproduce simultaneously spectral shapes and multiplicities is the use of an excitation energy dependent level density parameter $K=K\left(E_{x}\right)$. The value $K \sim 12 \mathrm{MeV}^{-1}$ at $T \sim 2.5 \mathrm{MeV}$ obtained in this way compares well with the predictions of Ref. 2 .

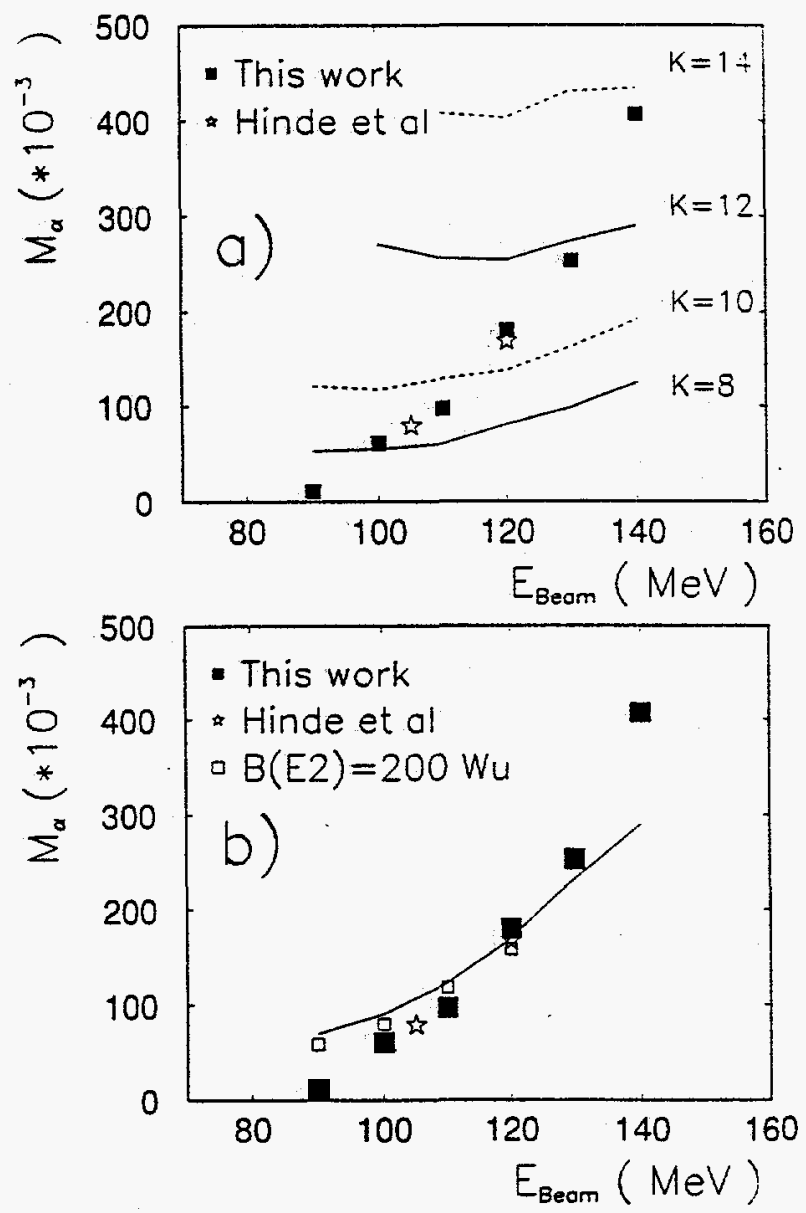

Figure 1. $\alpha$ particle multiplicity for the six bombarding energies compared with statistical model Monte Carlo calculations using inverse level density parameter $K=a / A$ (a) constant with excitation energy and (b) dependent on the excitation energy. For details see the text.

"INFN and Dipartmento di Fisica dell'Universitá di Padova, I-35131 Padova, Italy

${ }^{\ddagger}$ INFN and Dipartmento di Fisica dell'Universitá di Firenze, I-50125 Firenze, Italy

IINFN Laboratori Nazionali di Legnaro, I-35020 Legnaro (Padova), Italy

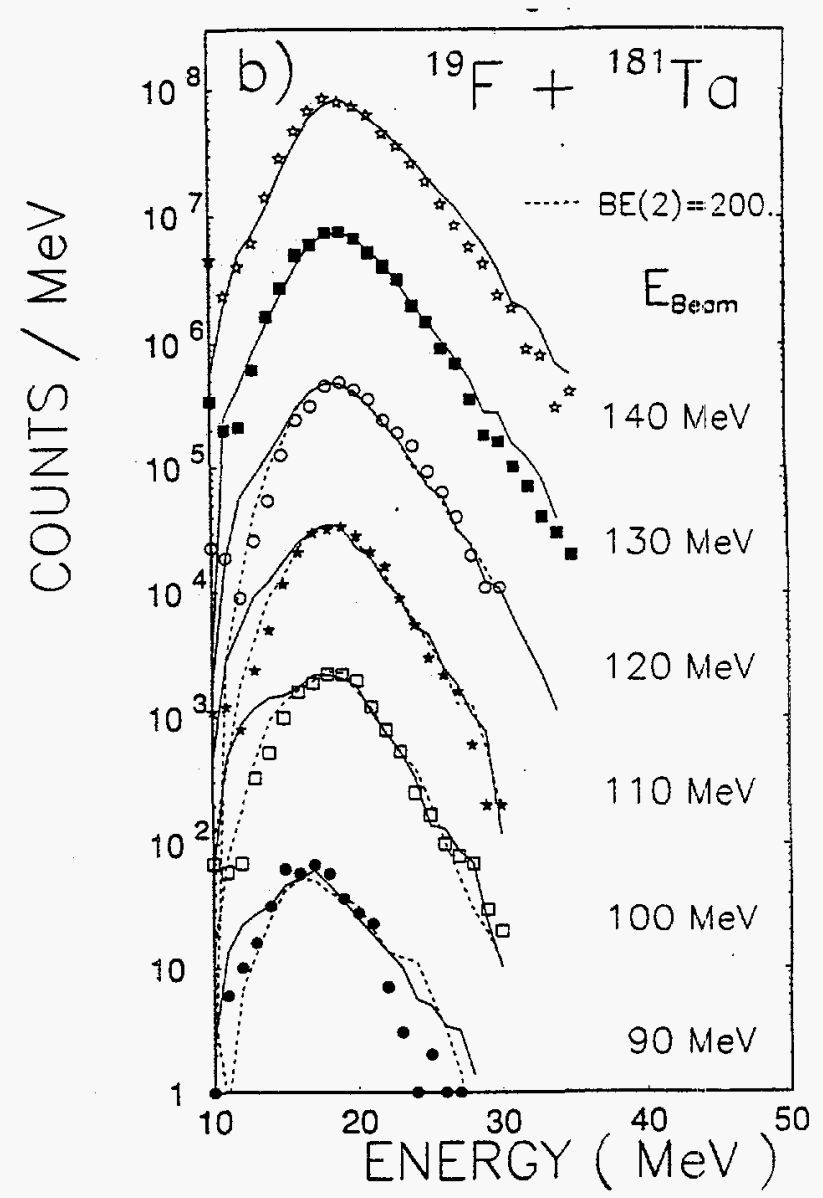

ligure 2. $\alpha$ particle energy spectra for the six bombarding thergies compared with statistical model calculations using an excitation energy dependent level density. See text.

\section{References}

1. B. Fornal et al., Phys. Rev. C 42, 1472 (1990).

2. S. Shlomo and J. B. Natowitz, Phys. Rev. C 44, 2878 (1991). 


\section{Progress Report for NA44 at TAMU}

\section{Murray}

The goal of experiment NA44 is to search for the breakdown of quark confinement under conditions of extreme density and temperature. A focusing spectrometer produces a magnified image of the target on 3 hodoscope planes. Two cerenkovs allow us to trigger on pairs or single rare particles. Thus we can make high statistics measurements over a large acceptance in a short amount of beam time. This year Michael Murray has worked on the analysis of the single particle spectra. The first task was to extend the transverse momentum, $P_{t}$, range of the spectra by combining data from the low and wide angle runs. The spectra now extend from a $P_{t}$ of zero up to $1.4 \mathrm{GeV}$. The data from backward rapidities have also been studied.

The antiproton to proton ratio is sensitive to annihilation effects and the difference in slopes may be due to a nuclear mean field. The pbar/p ratio at central rapidity has been measured for several systems. It decreases rapidly from $\mathrm{pBe}$ to $\mathrm{SPb}$ collisions and as one moves backward from rapidity 2.8 to 2, see Fig. 1. One can test the origin of the

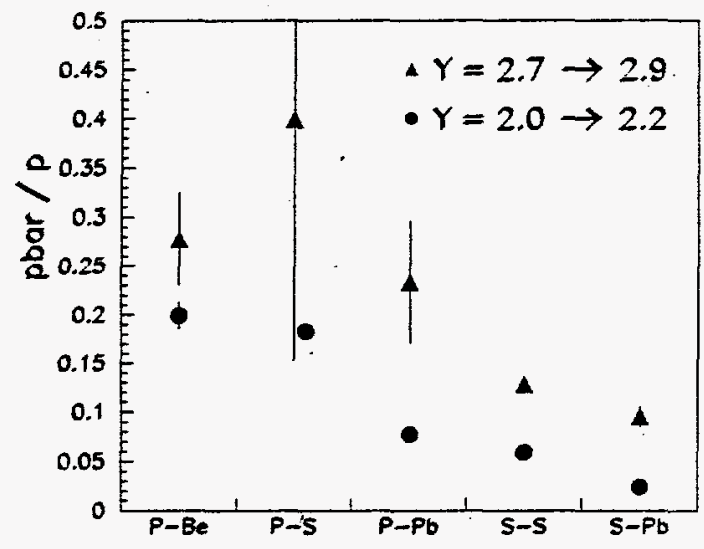

Figure 1. The ratios of antiprotons to protons versus system and rapidity.

drop in the $\mathrm{pbar} / \mathrm{p}$ ratio by normalizing the rapidity densities of protons and antiprotons to those of pions.
At mid rapidity protons become more abundant relative to pions as one goes to large systems. Since the pbar/p ratio is always low most of these protons were not produced at central rapidity but rather pushed forward by the projectile. In contrast antiprotons become relatively less abundant as one goes to heavier systems. This suggests that we are reaching a high enough baryon density to annihilate most antiprotons at central rapidity. These trends are qualitatively reproduced by RQMD, (Relativistic Quantum Molecular Dynamics) an event generator based on the "Dual Parton" model. Fritjof, an extension to AA systems of the "Lund String" model, fails to reproduce the data.

The single particle spectra for $\pi^{ \pm} \mathrm{K}^{ \pm}$and $\mathrm{p}^{ \pm}$ produced at central rapidity are well fit by simple exponentials in the transverse mass, $M_{t}=\operatorname{sqrt}\left(P_{t}^{2}+M_{0}\right)$. The inverse slopes of the $M_{T}$ spectra for $\mathrm{K}^{ \pm}$increase with the size of the target and projectile and are equal within their errors. For heavy ion collisions this effect has been suggested as a signal for quark gluon plasma formation. However the fact that the slopes are also equal for $\mathrm{pBe}$ and $\mathrm{pPb}$ collisions may simply imply that $\mathrm{K}^{ \pm}$have the same rescattering cross section in a region of low baryon density. The slopes of the $\pi^{+}$are roughly equal for all systems implying that the final stage of hadronization is independent of the initial conditions. The inverse slope of the protons almost doubles from $\mathrm{pBe}$ to $\mathrm{SPb}$ while that of the antiprotons increases by about $20 \%$. For $\mathrm{pBe}$ the slopes are equal within the errors. This effect may be due to the nuclear mean field boosting protons while retarding antiprotons as described by Koch et al. in Ref. 1. The fact that the proton and kaon slopes increase from $\mathrm{pBe}$ to $\mathrm{SPb}$ may be a hint of transverse flow. In June, Michael Murray presented these data at the Quark Matter '93 conference in Sweden. ${ }^{2}$

The ratio of protons to deuterons, at the same 
velocity, has been suggested as a way to measure the size of the proton source in relativistic heavy ion collisions. ${ }^{3}$ Assuming a gaussian source distribution, Fig. 2 shows the radius of the source versus Pt. The derived radius is about twice as large as lower energy measurements made at BNL suggesting that some of the additional beam energy is used to heat up the proton source or perhaps used to drive transverse flow. The correlation measurements of pions and kaons made by NA44 are also consistent: with transverse flow.

\section{References}

1. V. Koch, G. E. Brown, and C. M. Ko, Phys. Lett. 265B, 29 (1991).

2. M. Murray, Nucl. Phys. A566, 515 (1993).

3. M. Murray and S. Pratt, NA44 Note 171.

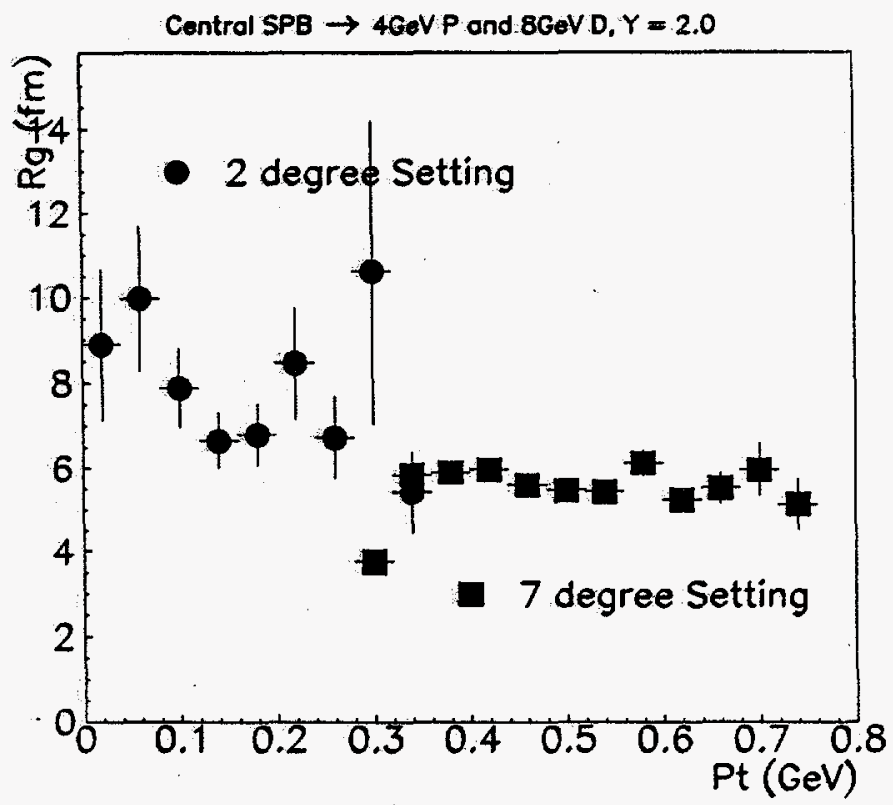

Figure 2. The radius of the proton source at rapidity 2.1 as a function of $\mathrm{Pt}$, for central $\mathrm{SPb}$ collisions at $200 \mathrm{GeV} /$ nucleon. The spectrometer took data at 2 and 7 degrees with respect to the beam. 


\section{NUCLEAR STRUCTURE AND FUNDAMENTAL INTERACTIONS}




\section{Optical Potentials of ${ }^{14} \mathrm{~N}$ Beam}

\section{Y. -W. Lui, D. H. Youngblood, H. Clark, P. Oliver, G. F. Simler and U. Garg*}

One of the important steps in studying Giant Resonances (GR) is to determine the multipolarity of the resonances. In the case of using inelastic scattering of alpha particles, the multipolarity of the GR was identified by comparing the cross-section calculated by Distorted-Wave Born Approximation (DWBA) to the experimental data. ${ }^{1}$ Each type of resonance has a distinct diffraction pattern at small angles $\left(0^{\circ}-10^{\circ}\right)$ in the DWBA calculations and these diffraction patterns are sensitive to the angular momentum ( $\mathrm{L}$ value) and are not very sensitive to the choice of optical potentials. These characteristics of inelastic alpha scattering provide a reliable tool to identify GR.

Recently, heavy ion projectiles, such as ${ }^{14} \mathrm{~N},{ }^{17} \mathrm{O}$, ${ }^{40} \mathrm{Ca}$ etc. ${ }^{2-4}$ have been used to study the properties of GR. The relatively low continuum in the inelastic scattering channel and a larger peak to background ratio in the GR region make heavy ions an attractive probe to study properties of the GR. Heavier projectiles carrying larger angular momentum may also provide a better chance to excite higher multipole resonances. However, these advantages of using heavy ion beams would not be very important, if the resonances cannot be identified uniquely. Unlike the alpha case, the calculated angular distributions for the inelastic scattering of heavy ions are sensitive to the choice of optical potentials. Therefore, it is important to obtain optical potentials that reproduce the experimental results in both the elastic and inelastic channels. ${ }^{14} \mathrm{~N}$ beams are useful for studying GR's because of the lack of pickup break-up reactions which could contribute peaks in the spectra. Thus we have studied elastic and inelastic scattering of ${ }^{14} \mathrm{~N}$ ions on ${ }^{92} \mathrm{Mo}$.

${ }^{14} \mathrm{~N}$ beams, of $490 \mathrm{MeV}$ and $700 \mathrm{MeV}$, were accelerated in the K500 cyclotron and used to bombard a ${ }^{92} \mathrm{Mo}$ target. Elastic and inelastic scattering of ${ }^{14} \mathrm{~N}$ particles were detected in two different magnetic spectrographs and detector systems. The elastic and inelastic scattering of 490 $\mathrm{MeV}{ }^{14} \mathrm{~N}$ particles were measured in the Enge split-pole spectrograph with a vertical drift chamber ${ }^{5}$ (VDC) located in the focal plane, while the scattering of $700 \mathrm{MeV}{ }^{14} \mathrm{~N}$ particles were measured in the recently installed Multipole-Dipole-Multipole(MDM) spectrograph. ${ }^{6}$ The detector system for the MDM includes two resistive wire proportional counters, and an ionization chamber backed by a BC400 plastic scintillator. ${ }^{7}$ Optical potentials obtained for ${ }^{12} \mathrm{C}$ and ${ }^{17} \mathrm{O}$ projectiles at similar energies and for similar targets were used to calculate the ${ }^{14} \mathrm{~N}$ elastic and inelastic scattering. However, they do not reproduce the shape of the angular distribution of the elastics or the low lying state as shown in Figs. 1 and 2, respectively.

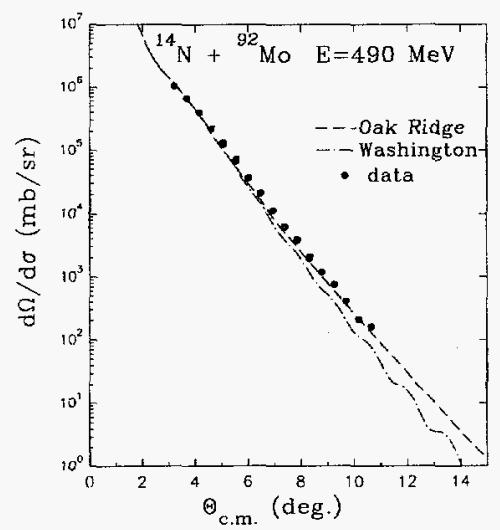

Figure 1. Elastic scattering calculations of ${ }^{14} \mathrm{~N}+{ }^{92} \mathrm{Mo}$ using optical potentials from Ref. 10 (dotted line) and from Ref. 11 (dash-dotted line).

Thus the elastic scattering data were analyzed by optical model analysis using the Coupled Channel Born Approximation (CCBA) code PTOLEMY. $^{8}$ The optical potential includes both the real and imaginary parts and has a Woods-Saxon form. Parameters of 


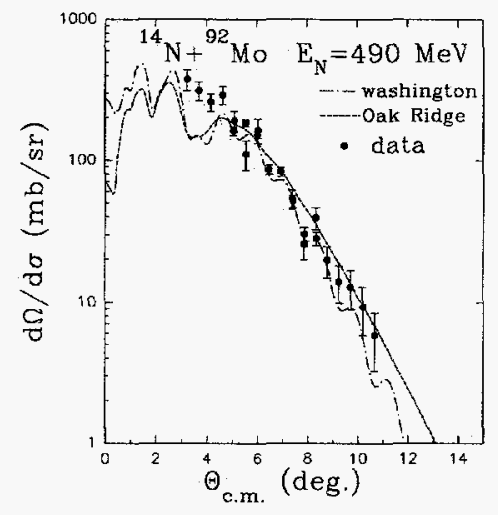

Figure 2. CCBA calculations of $E_{x}=1.509 \mathrm{MeV}$ in ${ }^{92} \mathrm{Mo}\left({ }^{14} \mathrm{~N}\right.$, ${ }^{14} \mathrm{~N}^{\prime}$ ) ${ }^{92} \mathrm{Mo}$ using optical potentials from Ref. 10 (dotted line) and from Ref. 11 (dash-dotted line).

the optical potential were adjusted systematically to find the best fit to the experimental data. In order to keep the optical parameters within physical values, some parameters were fixed and some parameters were varied together in the fitting process. The best fits to the experimental data were often obtained with a small diffuseness, such as 0.2 to 0.3 , in the optical potentials. This is different, however, from other reported heavy ion optical potentials ${ }^{9,10}$ which have diffuseness between 0.6 and 0.8 . In the analyses reported here, diffuseness values were kept close to 0.7 , and though $\chi^{2}$ was somewhat larger, quite good fits were obtained. The parameters of the optical potentials of these fits are listed in Table 1. The elastic scattering of $490 \mathrm{MeV}$ and $700 \mathrm{MeV}{ }^{14} \mathrm{~N}$ on ${ }^{92}$ Mo together with the optical model fits are shown in Fig. 3 and Fig. 4, respectively. As shown in Table 1, the chi-squares are quite different between these optical potentials. However, each of the 490 $\mathrm{MeV}$ potentials fits the elastic scattering data reasonably well. These optical potentials were then used to calculate the cross section for the $1.509 \mathrm{MeV}$ first excited state in ${ }^{92} \mathrm{Mo}$ with $490 \mathrm{MeV}{ }^{14} \mathrm{~N}$ ions. Different ratios of beta nuclear to beta Coulomb $\left(\beta_{\mathrm{N}} / \beta_{\mathrm{C}}\right),{ }^{12}$ were tried, as shown in Fig. 5 . The

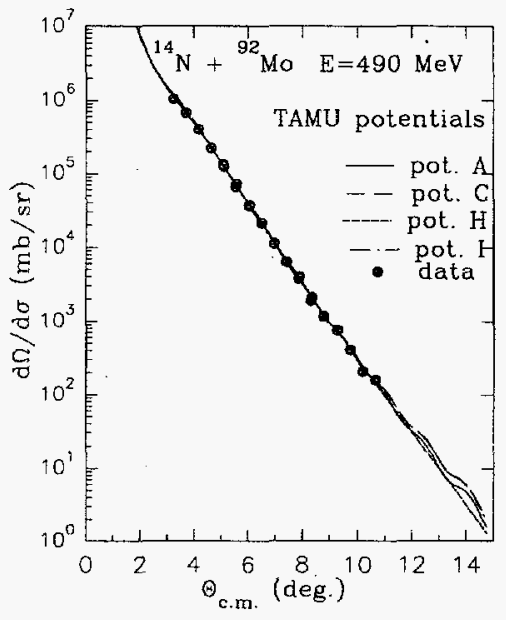

Figure 3. Angular distribution of elastic scattering of $490 \mathrm{MeV}$ ${ }^{14} \mathrm{~N}$ on ${ }^{92} \mathrm{Mo}$. Optical model fit with several optical potentials are also shown. The errors are smaller than the data points.

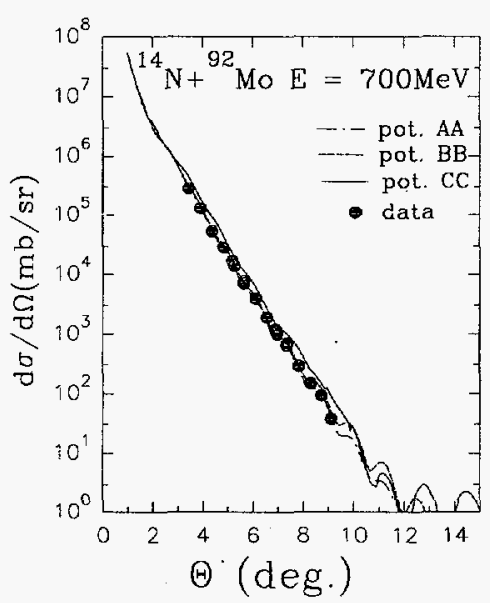

Figure 4. Same as Fig. 3 for $700 \mathrm{MeV}{ }^{14} \mathrm{~N}$ on ${ }^{92} \mathrm{Mo}$.

program PTOLEMY was used. Two different optical potentials were used, each with two different ratios of $\beta_{\mathrm{N}}$ to $\beta_{\mathrm{C}}$. The different potentials result in a difference in the magnitude of the cross-section and in the shape of the angular distribution, especially at smaller angles where nuclear and Coulomb interference are important. In general, potential C 
Table 1. Parameters of optical potentials obtained from fitting elastic scattering data of $490 \mathrm{MeV}{ }^{14} \mathrm{~N}$ and 700 $\mathrm{MeV}{ }^{14} \mathrm{~N}$ on ${ }^{92} \mathrm{Mo}$. An asterisk $\left({ }^{*}\right)$ indicates that the parameter was fixed in the fitting processes and an equal $\Leftrightarrow$ indicates that the parameters were varied together. The $\chi^{2}$ values are relative only. For these preliminary fits, a full error analysis has not been completed and uniform errors were assigned to all data points at each energy. Also, the errors assigned to the $490 \mathrm{MeV}$ data and the $700 \mathrm{MeV}$ data were different.

\begin{tabular}{|l|c|c|c|c|c|c|c|c|c|}
\hline $\begin{array}{c}\mathrm{E}_{\mathrm{lab}} \\
(\mathrm{MeV})\end{array}$ & $\begin{array}{c}\mathrm{V} \\
(\mathrm{MeV})\end{array}$ & $\begin{array}{c}\mathrm{r}_{\mathrm{v}} \\
(\mathrm{fm})\end{array}$ & $\begin{array}{c}\mathrm{a}_{\mathrm{v}} \\
(\mathrm{fm})\end{array}$ & $\begin{array}{c}\mathrm{W} \\
(\mathrm{MeV})\end{array}$ & $\begin{array}{c}\mathrm{r}_{\mathrm{w}} \\
(\mathrm{fm})\end{array}$ & $\begin{array}{c}\mathrm{a}_{\mathrm{w}} \\
(\mathrm{fm})\end{array}$ & $\begin{array}{c}\mathrm{r}_{\mathrm{c}} \\
(\mathrm{fm})\end{array}$ & $\begin{array}{c}\chi^{2} \\
(\mathrm{rel})\end{array}$ & Label \\
\hline 490.0 & 29.675 & $1.050^{=}$ & 0.419 & 22.692 & $1.050^{=}$ & 1.073 & 1.200 & 0.522 & $\mathrm{~A}$ \\
\hline 490.0 & $100.00^{*}$ & 0.933 & 0.737 & 310.050 & 0.852 & 0.744 & 1.300 & 2.298 & $\mathrm{C}$ \\
\hline 490.0 & 8.544 & $1.107^{=}$ & $0.761=$ & 28.331 & $1.107^{=}$ & $0.761=$ & 1.200 & 1.509 & $\mathrm{H}$ \\
\hline 490.0 & $50.000^{*}$ & $1.071=$ & 0.616 & $50.000^{*}$ & $1.071^{=}$ & 0.708 & 1.200 & 2.771 & $\mathrm{I}$ \\
\hline 700.0 & 42.857 & 1.043 & 0.557 & 25.255 & 1.067 & 0.822 & 1.200 & 0.375 & $\mathrm{AA}$ \\
\hline 700.0 & 32.440 & 1.035 & 0.497 & 301.400 & 0.672 & 0.914 & 1.200 & 0.285 & $\mathrm{BB}$ \\
\hline 700.0 & 29.297 & 0.919 & 0.546 & 17.877 & 0.872 & 0.903 & 1.200 & 0.584 & $\mathrm{CC}$ \\
\hline
\end{tabular}

reproduces the inelastic data much better than potential A, especially at smaller angles, and $=0.7$ gives the best description to the inelastic scattering

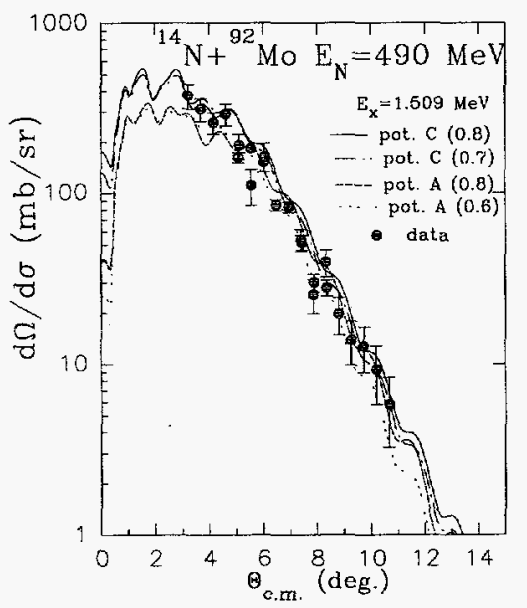

Figure 5. Angular distribution of differential cross-section for $E_{x}$ $=1.509 \mathrm{MeV}$ in ${ }^{92} \mathrm{Mo}$. CCBA calculations with two optical potentials and two $\beta_{\mathrm{N}} / \beta_{\mathrm{c}}$ ratios are also shown.

data. This ratio of $\beta_{\mathrm{N}} / \beta_{\mathrm{C}}$ is in reasonable agreement with Ref. 11.

It is well known that multiple sets of optical potentials can be found to fit the same sèt of elastic scattering data equally well in an optical model analysis. The measurements of inelastic scattering of the low lying states are very useful to further select the correct potentials. The analyses of the ${ }^{14} \mathrm{~N}$ on ${ }^{9} \mathrm{Mo}$ data show that potentials with relative small diffuseness and shallow potential depth are required to fit the experimental data. Whether this is due to lack of data at the larger angle region or the characteristics of ${ }^{14} \mathrm{~N}$ projectiles, remains uncertain. Measurements of the elastic scattering should include both a larger angular range and smaller angles. The larger angular range can better determine the shape of optical potential, and the smaller angles, especially at angles where only the Coulomb interaction is important, can determine the magnitude of the cross-section and reduce ambiguity due to normalization. The present analyses suggest that giant resonances can be identified in ${ }^{92} \mathrm{Mo}$ by the distinct diffraction patterns at angles greater than $4^{\circ}$, at least at $490 \mathrm{MeV}$.

"Present address: Department of Physics, University of Notre Dame, South Bend, IN 46556. 


\section{References}

1. D. H. Youngblood et al., Phys. Rev. C 13, 994 (1976).

2. Y. -W. Lui et al., Progress in Research, 19911992, Cyclotron Institute, TAMU, p. 37.

3. J. R. Beene et al., Phys. Rev. C 41, 920 (1990).

4. Nimet Frascaria, Institut de physique nucleaire Report, IPNO DRE 93-24 (1993).

5. K. Yates, M. Sc. thesis (1989), Texas A\&M University.

6. D. M. Pringle et al., Nucl. Instrum. Methods
A245, 230 (1986).

7. J. S, Winfield et al., Nucl. Instrum. Methods A251, 297 (1986).

8. M. H. Macfarlane and S. C. Peiper, Argonne National Laboratory Report No. ANL-76-11, Rev. 1, 1978 (unpublished).

9. D. J. Horen et al., Phys. Rev. C 44, 128 (1991).

10. C. -C. Sahm et al., Phys. Rev. C 34, 2165 (1986).

11. V. A. Madsen et al., Phys. Rev. Lett. 52, 176 (1984).

\section{Search for Giant Resonances Above the GMR/GDR}

\section{H. Youngblood, Y. -W. Lui, P. Oliver, G. Simler, H. Clark, and U. Garg*}

The region of excitation above the GMR/GDR has been studied in ${ }^{58} \mathrm{Ni},{ }^{90} \mathrm{Zr}$, ${ }^{92} \mathrm{Mo}$, and ${ }^{144} \mathrm{Sm}$ with $35 \mathrm{MeV} / \mathrm{n}{ }^{14} \mathrm{~N}$ ions, in ${ }^{92} \mathrm{Mo}$ with $50 \mathrm{MeV} / \mathrm{n}^{14} \mathrm{~N}$ ions, and in ${ }^{116} \mathrm{Sn}$ with $240 \mathrm{MeV}$ alpha particles. ${ }^{14} \mathrm{~N}$ ions were chosen because of the absence of pickup/breakup products that produce peaks in the spectra. $240 \mathrm{MeV}$ alpha particles were used because at this energy peaks from the break up of ${ }^{5} \mathrm{Li}-{ }^{5} \mathrm{He}$ reaction products are not in the region of interest. The $35 \mathrm{MeV} / \mathrm{n}$ data were taken in the Enge spectrometer using a large solid angle with a vertical drift chamber for ray tracing followed by an ionization chamber and a scintillator for particle identification. The $50 \mathrm{MeV} / \mathrm{n}$ and $240 \mathrm{MeV}$ alpha data were taken with the MDM spectrometer using a large solid angle and two resistive wires to determine position and angle followed by an ionization chamber and a scintillator for particle identification. Ray tracing was used to determine the scattering angle. Angle resolutions were generally about $0.3^{\circ}$. Considerable care was taken to minimize slit scattering contributions to spectra.

A spectrum obtained at $50 \mathrm{MeV} / \mathrm{n}$ is shown in
Fig. 1 and the GQR/GDR/GMR peak is indicated. After subtraction of a continuum, the spectra were fit with the known GQR and GMR peaks. Additional peaks at approximately 23 and $35 \mathrm{MeV}$ were required to fit the ${ }^{14} \mathrm{~N}$ spectra and a peak at about $23 \mathrm{MeV}$ was required to fit the alpha spectra. (The alpha spectra extend only to $E_{x}=40 \mathrm{MeV}$.) $A$ fit to the alpha data, after subtraction of the continuum, is shown in Fig. 2. Angular distributions obtained for the GQR, GMR, and one of the other peaks are shown in Figs. 3 and 4 along with Coupled Channel Elorn Approximation calculations for $100 \%$ of the respective sum rules. It can be seen that the peak at approximately $23 \mathrm{MeV}$ is consistent with an $\mathrm{L}=3$ transition exhausting all of the strength expected for the High Energy Octupole Resonance (HEOR). The energy and width are also consistent with published systematics for the HEOR. The angular distributions for the highest peak were inconsistent, and present evidence for its origin is inconclusive.

* Physics Department, University of Notre Dame, Notre Dame, Indiana 


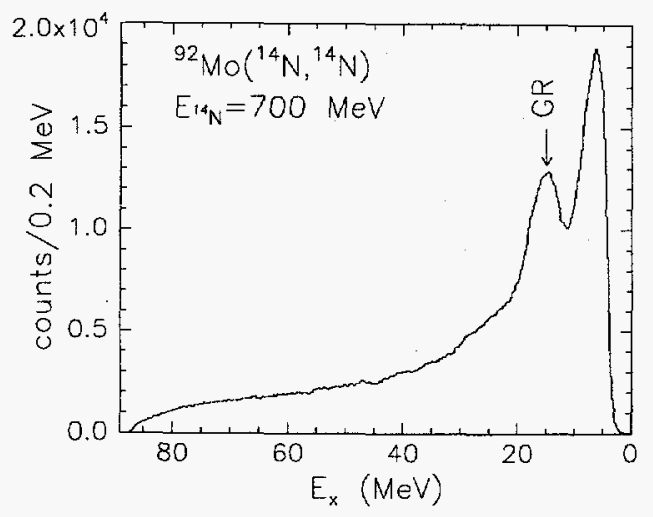

Figure 1. A spectrum obtained at $50 \mathrm{MeV} / \mathrm{n}$. The GR peak is indicated by the arrow.

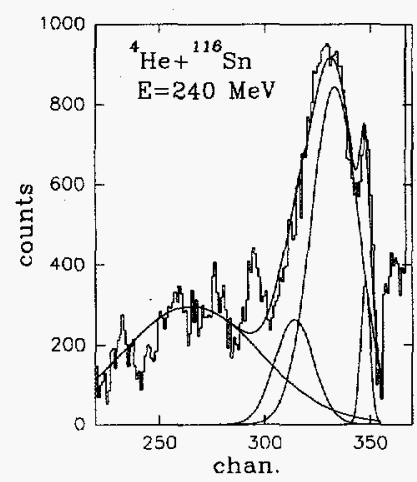

Figure 2. Alpha spectrum after subtraction of the continuum. A four peak fit, using known position and width for the GMR and GQR, is shown.
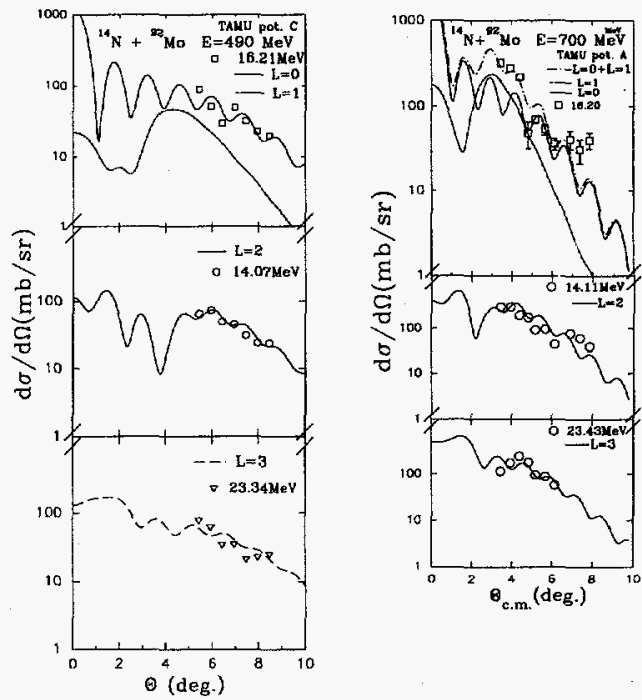

Figure 3. Angular distribution obtained with 35 and $50 \mathrm{MeV} / \mathrm{n}^{14} \mathrm{~N}$ ions for the indicated groups. CCBA calculations for $100 \%$ of the respective sum nules are shown by the lines.

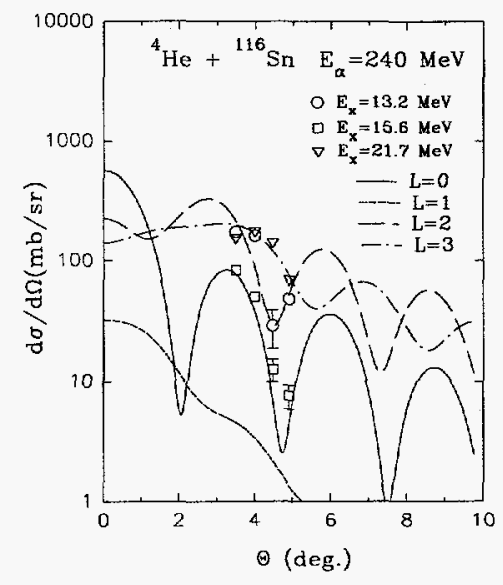

Figure 4. Angular distributions obtained with $240 \mathrm{MeV}$ alpha for the indicated groups. CCBA calculations for $100 \%$ energy weighted sum rules are shown by the lines. 


\title{
The $\beta$-Decay of ${ }^{57} \mathrm{Cu}$
}

\author{
D. R. Semon, M. Allen, A. Brown, ${ }^{*}$ H. D. Dejbakhsh, C. A. Gagliardi, S. Hale, L. M. Trache, R. \\ E. Tribble, H. M. Xu, J. Jiang, S. J. Yennello, and X. G. Zhou
}

\section{Introduction}

In the simplest shell model picture, ${ }^{57} \mathrm{Cu}$ is described by a single proton orbiting a closed ${ }^{56} \mathrm{Ni}$ core. While this picture adequately describes the essential features of the low-lying nuclear states, it neglects the extra-nucleonic effects arising from the detailed structure of the strong interaction. One possible way to investigate these effects is by comparing Gamow-Teller (GT) transitions between analog states. ${ }^{1}$ Single particle and single hole nuclei are the best candidates for this type of study since model calculations can be done more accurately near the closure of a shell than in the middle of a shell. The dependence of the matrix elements on the nuclear part of wavefunctions is further reduced if the transition is also $\ell$-forbidden. In this case, the nucleonic contribution to the matrix elements vanishes in first order. Towner and Khanna ${ }^{2}$ calculate that the most important non-nucleonic modifications to the transition rates come from $\Delta$-hole states and meson exchange currents and that second order configuration mixing (core polarization) is of approximately the same order of magnitude. While the experimental values obtained by Adelberger $e t a l{ }^{1}$ for the $1 d_{3 / 2} \rightarrow 2 s_{1 / 2} \ell$-forbidden, GT transition in $A=39$ are in reasonable agreement with these calculations, some discrepancies exist. To provide additional data for this problem, we have measured the branching ratio of ${ }^{57} \mathrm{Cu}$ decays to the ground state and first two excited states in ${ }^{57} \mathrm{Ni}$ and the lifetime of its $\beta$-decay (see Fig. 1). The results will be compared with fp shell model calculations. now being done at the Michigan State University National Superconducting Cyclotron Laboratory.

The level scheme for ${ }^{57} \mathrm{Cu} \beta$-decay is shown in Fig. 1. All three transitions are allowed by GT selection rules. The $2 \mathrm{p}_{3 / 2} \rightarrow 2 \mathrm{p}_{3 / 2}$ (gs $\rightarrow \mathrm{gs}$ ) transition is superallowed, while the $2 p_{3 / 2} \rightarrow 1 f_{5 / 2}$ transition is $\ell$ - forbidden. Standard $\beta$-decay theory predicts the branching ratio (BR) of the $2^{\text {nd }}$ forbidden $2 p_{3 / 2}-1 f_{5 / 2}$ transition to be approximately 1 part in $10^{4}$. This estimate, along with several preliminary runs and a background survey of the experimental area indicated that the overall BR could be determined more accurately by measuring the absolute $B R$ of the $2 p_{3 / 2} \rightarrow 2 p_{1 / 2}$ to the $2 p_{3 / 2} \rightarrow 2 p_{3 / 2}$ and the relative BR of the $2 \mathrm{p}_{3 / 2} \rightarrow 1 \mathrm{f}_{5 / 2}$ to the $2 \mathrm{p}_{3 / 2} \rightarrow 2 \mathrm{p}_{1 / 2}$ rather than a simultaneous measurement of all three branches. A third experiment was needed to determine the lifetime for the $\beta$-decay since there exists a large uncertainty in the only published value. ${ }^{3}$ Each of the experiments is described.

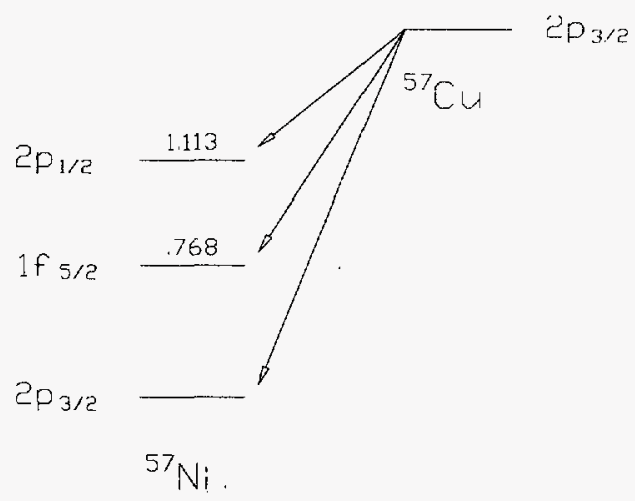

Figure 1. Level diagram for ${ }^{57} \mathrm{Cu} \beta$-decay

\section{Experiments}

In each of the experiments, we used ${ }^{58} \mathrm{Ni}$ beams between 29 and $31 \mathrm{MeV} / \mathrm{A}$ from the Texas A\&M K500 Cyclotron incident on a cryogenically cooled ${ }^{2} \mathrm{H}$ gas target (see Fig. 2) to produce samples of ${ }^{57} \mathrm{Cu}^{2}$ from the reaction $\mathrm{p}\left({ }^{58} \mathrm{Ni},{ }^{57} \mathrm{Cu}\right) 2 \mathrm{n}$. The use of inverse kinematics allows the spectrometer MARS to collect and transport the ${ }^{57} \mathrm{Cu}$ recoils to the focal plane with 
almost $100 \%$ efficiency (see progress report on MARS). Particles are identified from the Energy vs. Position spectra of a position sensitive, solid state detector (PSD) located at the MARS focal plane. Fully stripped ${ }^{57} \mathrm{Cu}$ is the least rigid $(\mathrm{M} / \mathrm{Q}=1.966)$, kinematically allowed recoil for this beam energy and appears in the highest channel numbers along the position axis. $\mathrm{N}=\mathrm{Z}$ recoils form three groups just below and well separated in position from the ${ }^{57} \mathrm{Cu}$ group. A set of adjustable slits located approximately $2 \mathrm{~cm}$ in front of the PSD is used to eliminate recoils other than ${ }^{57} \mathrm{Cu}$ from further transport. In all three experiments, the detectors used were close enough to the focal plane to require shielding from reactions occurring in the slits. A $10 \mathrm{~cm}$ thick $\mathrm{Pb}$ collimator located $7 \mathrm{~cm}$ behind the focal plane provided shielding from these backgrounds. Typical online Position vs. Energy spectra are shown in Fig. 3. The PSD is removed during actual data taking by means of a linear motion feedthrough. This enabled us to reinsert it periodically to check the stability of MARS without disturbing the rest of the system. The apparatus behind the focal plane differed for each experiment. ${ }^{152,154,155} \mathrm{Eu}$ and ${ }^{60} \mathrm{Co}$ sources were used for calibration of the HPGe detectors. The three isotopes in the Eu source provide a number of spectral lines with known strengths in an energy regime which is well suited to these measurements. While the ${ }^{60} \mathrm{Co}$ source does not provide enough information to serve as an independent calibration, it was useful as a consistency check.

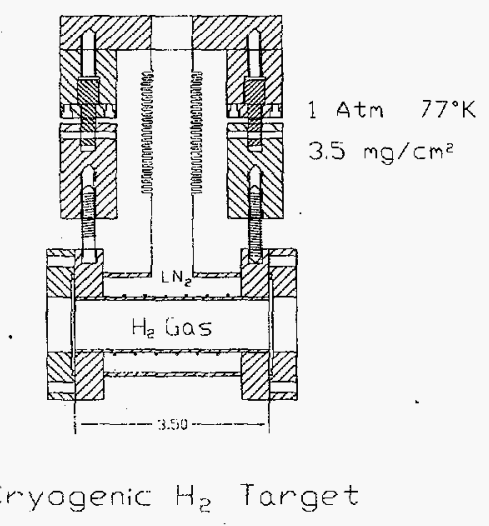

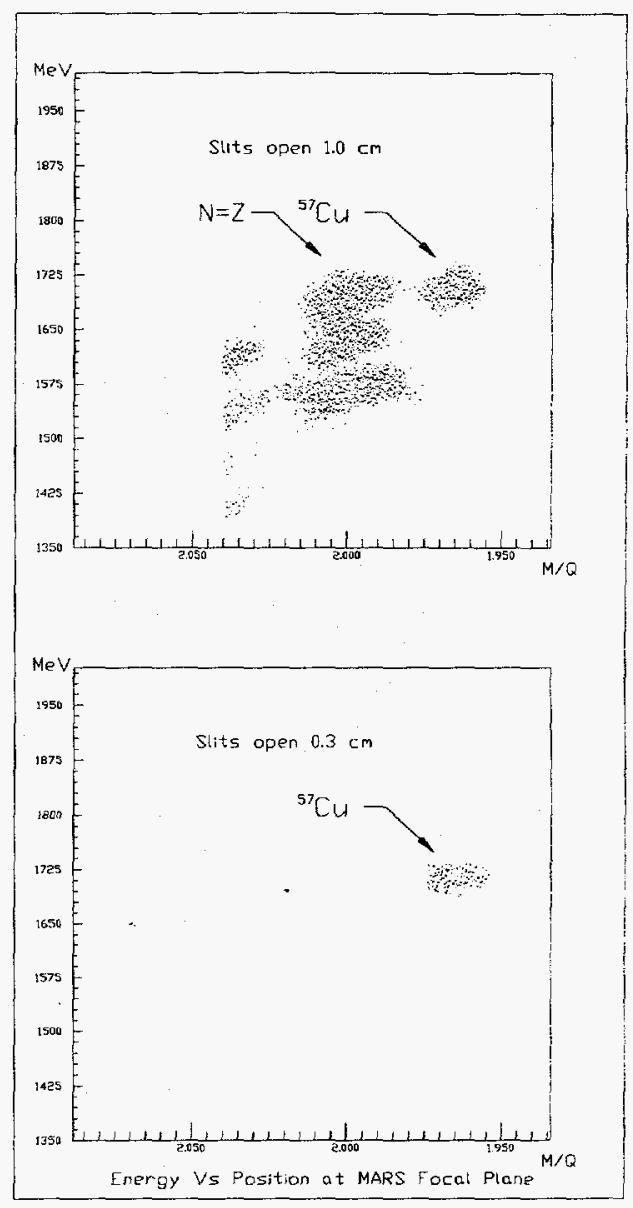

Figure 3. Typical on-line energy vs. position spectra.

\section{Relative BR}

We initially planned to perform this experiment by measuring the intensity of the $\gamma$ singles from ${ }^{57} \mathrm{Cu}$ decay by placing several Compton shielded High Purity Ge counters around an $\mathrm{Al}$ "catcher foil" that was our beam stop for the ${ }^{57} \mathrm{Cu}$. Unfortunately, the background from sources throughout the building produced a $\gamma$-ray at exactly the same energy as the $1^{\text {st }}$ excited state in ${ }^{57} \mathrm{Ni}$. Detection of the decay positron in coincidence with a ${ }^{57} \mathrm{Ni} \gamma$-ray eliminated the background. The detector geometry along with

Figure 2. Cryogenic gas target. 
typical on-line spectrum is shown in Fig. 4. Both the $1.113 \mathrm{MeV}$ and $0.768 \mathrm{MeV}$ states in ${ }^{57} \mathrm{Ni}$ are evident along with a third peak corresponding to the $4^{+} \rightarrow 2^{+}$ transition in ${ }^{54} \mathrm{Fe}$ from ${ }^{54 \mathrm{~m}} \mathrm{Co} \beta$-decay. Although closing the focal plane slits would have eliminated the ${ }^{54 \mathrm{~m}} \mathrm{Co}$, it was convenient to use it as a rough calibration. The introduction of the $\beta$ detectors added a potential source of background from Bremstrahlung. In order to examine this background, we took data in such a way that we can identify the $\beta$ and HPGe associated with each $\beta-\gamma$ coincidence. We can use this information to reject events where the coincidence occurs between one of the HPGe detectors and the $\beta$ counter directly in front of it. In order to eliminate Bremstrahlung in the HPGe detector itself, we stopped the positron in a lucite cone located in the front part of the Compton shield. Figure 4 does not have the Bremstrahlung background removed.

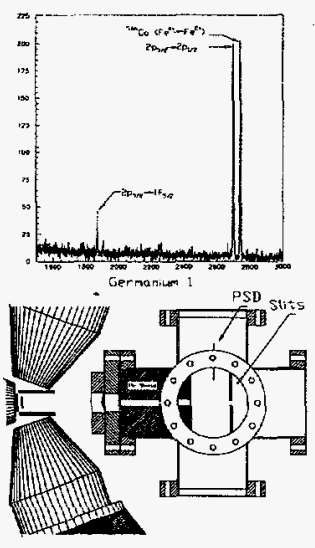

Figure 4. Detector geometry and $\gamma$-ray spectrum for relative BR measurement.

\section{Absolute BR}

For the measurement of absolute BR to the 1.113 $\mathrm{MeV}$ state in ${ }^{57} \mathrm{Ni}$, we used a single HPGe detector with no Compton shielding and a 500um thick $\mathrm{Si}$ detector as a "catcher foil" to count the total number of ${ }^{57} \mathrm{Cu}$ recoils arriving at the end of the beamline. The detector geometry and a typical on-line spectrum is shown in Fig. 5. The Compton shields are most useful when the region of interest is significantly obscured by the Compton edges of higher energy peaks. By carefully optimizing MARS for ${ }^{57} \mathrm{Cu}$, there were no strong peaks in the spectrum higher in energy than the $1.113 \mathrm{MeV} \gamma$ from ${ }^{57} \mathrm{Ni}$. The Compton shields in this case are possible sources of systematic error because good events can be rejected from real coincidences between the positron from ${ }^{57} \mathrm{Cu} \beta$-decay interacting in the Compton shield and photons in the HPGe. In order to shield the HPGe detector from room background, we surrounded it with $\mathrm{Pb}$ as shown in Fig. 5. By replacing the $\mathrm{Si}$ detector with a calibrated Eu source at the same location, we were able to obtain a calibration of the absolute efficiency of the HPGe detector. The source was moved across the region where the detector was located to determine what effect, if any, the finite size of the ${ }^{57} \mathrm{Cu}$ samples collected on the Si would have. We set limits on the ${ }^{57} \mathrm{Cu}$ sample size by layering Kapton degraders such that the successively larger apertures in the Kapton along the long axis of the detector corresponded to thicker films. The detector was then moved to various positions along its shorter axis to ensure that ${ }^{57} \mathrm{Cu}$ did not hit the edges of the counter.
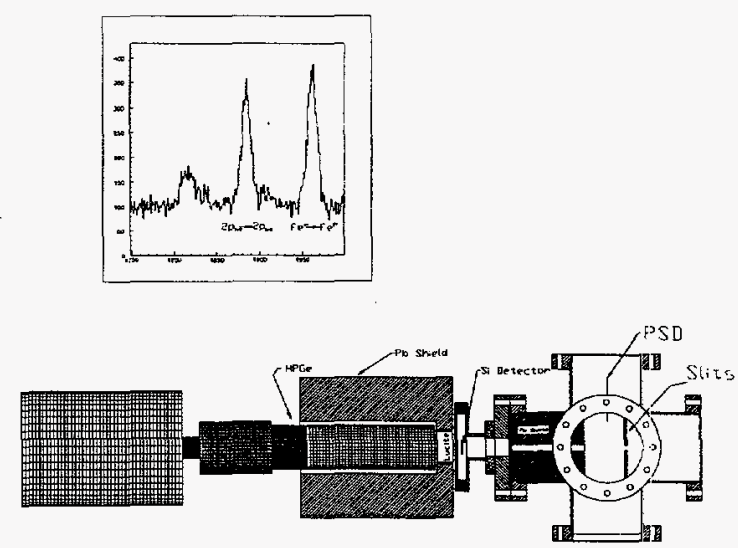

Figure 5. Detector geometry and $\gamma$-ray spectrum for absolute BR measurement. 


\section{Lifetime}

For the lifetime measurement, we used a three element $\beta$ telescope with a cosmic ray veto arranged as shown in Fig. 6. The data were taken in two different runs, each with a different trigger requirement. For the first run we required that there be a coincidence in two of the three elements of the telescope from a ${ }^{57} \mathrm{Cu}$ positron, without being vetoed by the cosmic ray shield. The trigger for the second data set required a coincidence in all three elements of the telescope, also without being vetoed by the cosmic ray shield. In order to eliminate any significant dead time, we took data with a PC set up as a multichannel scalar rather than the VAX based acquisition system. We reinserted the focal plane detector periodically to verify that the system had not drifted causing the ${ }^{57} \mathrm{Cu}$ to shift positions. Figure 6 also shows the $\beta$-decay spectrum from the triple coincidence run.
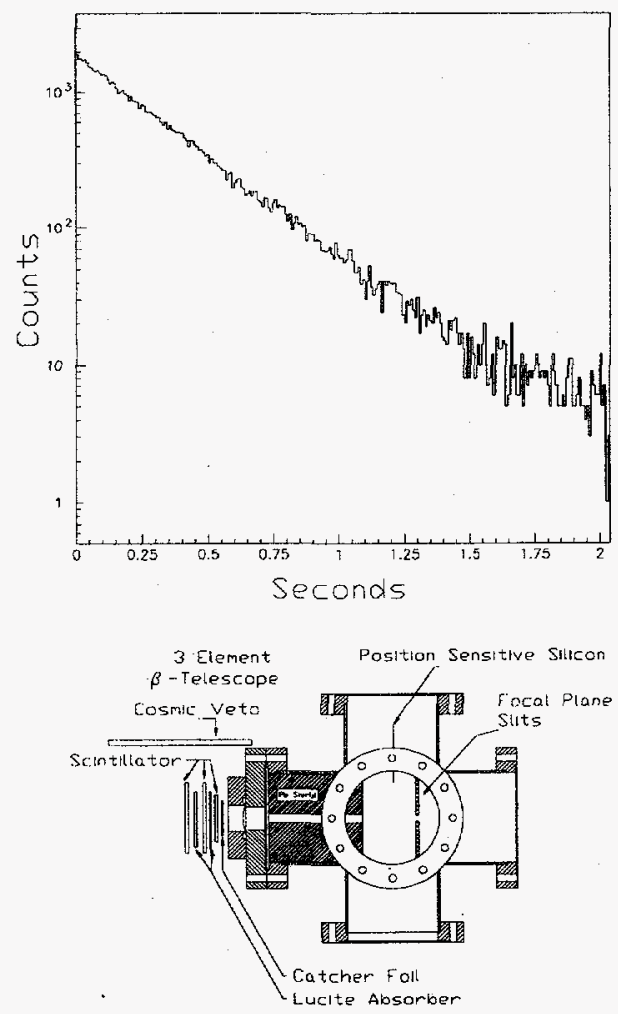

Figure 6. Detector geometry and $\beta$-decay spectrum for half-life measurement.

\section{Preliminary results}

Data analysis is almost complete. The branching ratio for the $2 \mathrm{p}_{3 / 2}-2 \mathrm{p}_{1 / 2}$ transition is about $8 \%$ of the total number of decays. This is larger than the result of Shinozuka et al. ${ }^{3}$ who obtained a value of $3.7 \% \pm$ $1.7 \%$. Their measurement technique involved a large systematic uncertainty in their total ${ }^{57} \mathrm{Cu}$ yield, which is absent with our procedure. While further analysis of the backgrounds are necessary, the uncertainty in the measurement at this point is small enough that further analysis will not change this result appreciably. The branching ratio for the $2 \mathrm{p}_{3 / 2}-1 \mathrm{f}_{5 / 2}$ transition is also much larger than expected. The number of decays to this state is about $1 \%$ of the total. Our value for the ${ }^{57} \mathrm{Cu}$ lifetime is $196 \pm 2 \mathrm{~ms}$ which disagrees with the only published value of $233 \pm 16 \mathrm{~ms}$ but does agree with a recent shell model prediction. ${ }^{4}$ Further analysis on the lifetime data is unlikely to change our value but probably will reduce our uncertainty.

"National Superconducting Cyclotron Laboratory, Michigan State University, East Lansing, MI 48824

\section{References}

1. Adelberger et al., Nucl. Phys. A417, 269 (1984).

2. I. S. Towner and F. C. Khanna, Nucl. Phys. A399, 334 (1983).

3. T. Shinozuka et al., Phys. Rev. C 30, 2111 (1984).

4. A. Brown, Private Communication. 


\section{(d, $\left.{ }^{2} \mathrm{He}\right)$ Reactions with the Texas A\&M Proton Spectrometer}

\section{G. Ajupova, A. C. Betker*, C. A. Gagliardi, B. Kokenge, H. M. Xu, and A. F. Zaruba}

During this past year, we have measured the $\left(\mathrm{d},{ }^{2} \mathrm{He}\right.$ ) reactions on ${ }^{\mathrm{t}} \mathrm{H},{ }^{11} \mathrm{~B},{ }^{12} \mathrm{C},{ }^{13} \mathrm{C},{ }^{26} \mathrm{Mg},{ }^{27} \mathrm{Al}$ targets over an angular range $0^{\circ}-17^{\circ}$, together with additional data on ${ }^{1} \mathrm{H}$ and ${ }^{12} \mathrm{C}$ targets covering $17^{\circ}-22^{\circ}$, at a deuteron beam energy of $127 \mathrm{MeV}$. As this is being written, we have just completed an additional run during which we added data on ${ }^{6} \mathrm{Li}$ and ${ }^{48} \mathrm{Ti}$ targets under similar experimental conditions. The goals of these runs are (1) to perform a self-consistent calibration, using the real data, for the proton spectrometer; (2) to measure the angular distributions of $\left(\mathrm{d},{ }^{2} \mathrm{He}\right)$ reactions on light targets to study the reaction mechanism in this energy regime; and (3) to measure the Gamow-Teller matrix elements for ${ }^{48} \mathrm{Ti}\left(\mathrm{d},{ }^{2} \mathrm{He}\right){ }^{48} \mathrm{Sc}$ reactions which are important to constrain calculations for the double beta decay of ${ }^{48} \mathrm{Ca}$.

In the present report, we update the current status of our data analysis for the first run. For a complete description of the Proton Spectrometer Facility, including its capacities, calibration procedures, and results of our earlier test runs, see Refs. 1-3. Consistent with previous measurements, ${ }^{1}$ we achieve a resolution of $\sigma \approx 0.20 \mathrm{~ns}$ in our scintillator triggers and a position resolution of $\approx 200 \mu \mathrm{m}$ FWHM for all DC layers. Using the distance vs time responses obtained from the SS data, the outgoing particle tracks are re-fitted with straight lines. The best fits from these lines, together with magnetic matrix elements obtained from RAYTRACE using results from our earlier field map, ${ }^{1}$ provide us information concerning the system acceptance, as well as physics information such as particle emission energies and angles.

We have devoted significant efforts, using the real data, to the self-calibration of the proton spectrometer. As noted in the previous report, ${ }^{1}$ a $1.3 \%$ discrepancy in the momentum scale between our field map and our observed particle trajectories was observed in our earlier analysis. Because of very limited SDNA data there and because of the complexity the issue involves, it was impossible to use the earlier data to do a complete self-consistent check. To do this, we employ several constraints imposed by physics as well as by the geometry of the proton spectrometer. First, we recall the broad acceptance of the proton spectrometer: it covers $\theta_{i n}=$ $\pm 7^{\circ}$ at each position. This allows us to use the cross over angles between ${ }^{1} \mathrm{H}\left(\mathrm{d},{ }^{2} \mathrm{He}\right) \mathrm{n}$ and ${ }^{26} \mathrm{Mg}\left(\mathrm{d},{ }^{2} \mathrm{He}\right){ }^{26} \mathrm{Na}$, as well as between ${ }^{1} \mathrm{H}\left(\mathrm{d},{ }^{2} \mathrm{He}\right) \mathrm{n}$ and ${ }^{12} \mathrm{C}\left(\mathrm{d},{ }^{2} \mathrm{He}\right){ }^{12} \mathrm{~B}$, to determine the incident beam angles. Furthernore, using data obtained at two different proton spectrometer angles provides a consistent check about the beam angles. Second, the image of entrance slits obtained in the SS mode provides a check on the incident angles. Third, the Q-value for the $\left({ }^{2}{ }^{2} \mathrm{He}\right)$ scattering on each target is independent of scattering angle, $\theta_{i n}$. Since the measured Q-values depend on the energy and angle of ${ }^{2} \mathrm{He}$, which, in turn, depend on the magnetic matrix elements and detector positions, the measured $\mathrm{Q}$ vs $\theta_{i n}$ relation provides a powerful constraint on all these parameters. Moreover, data from different targets, which have different $Q$ values, different masses, and therefore, different recoil effects, provide checks over a wide range of the acceptance of the proton spectrometer. If the magnetic matrix elements, the beam incident angle, as well as other parameters defining the geometry of our detecting system, were measured accurately, consistent results for $Q$ and $\theta_{\text {cross }}$ vallues should be obtained for all targets. This requirement puts a very strong constraint on our system and thus allow us to explore the dependence on the multi-dimensional space of input parameters. These parameters are allowed to be adjusted within limited ranges, constrained by the uncertainties in the field mapping or by the positioning accuracies of the drift chambers. In particular, these parameters include (1) 
those which define the magnetic field of the proton spectrometer, including the radii of effective boundary at both the entrance and exit, the distance the particles travel along the central ray inside the magnet, $A$, and therefore the central magnetic field, $B_{c}$, the angle between the central trajectory and the normal to the effective boundary at exit, $\beta$; (2) those defining the positions of our drift chambers. By adjusting these parameters, we find significant dependence on parameters, directly or indirectly related to the exit angles $\theta_{\text {our }}$, reflecting the point to parallel optics designed for this spectrometer. Using these trends we are able to iterate sequentially several key parameters, thus improving significantly the observed $Q$ vs $\theta_{i n}$ relation. As an example, Fig. 1 shows the measured $Q$ vs $\theta_{\text {in }}$ before (top panel) and
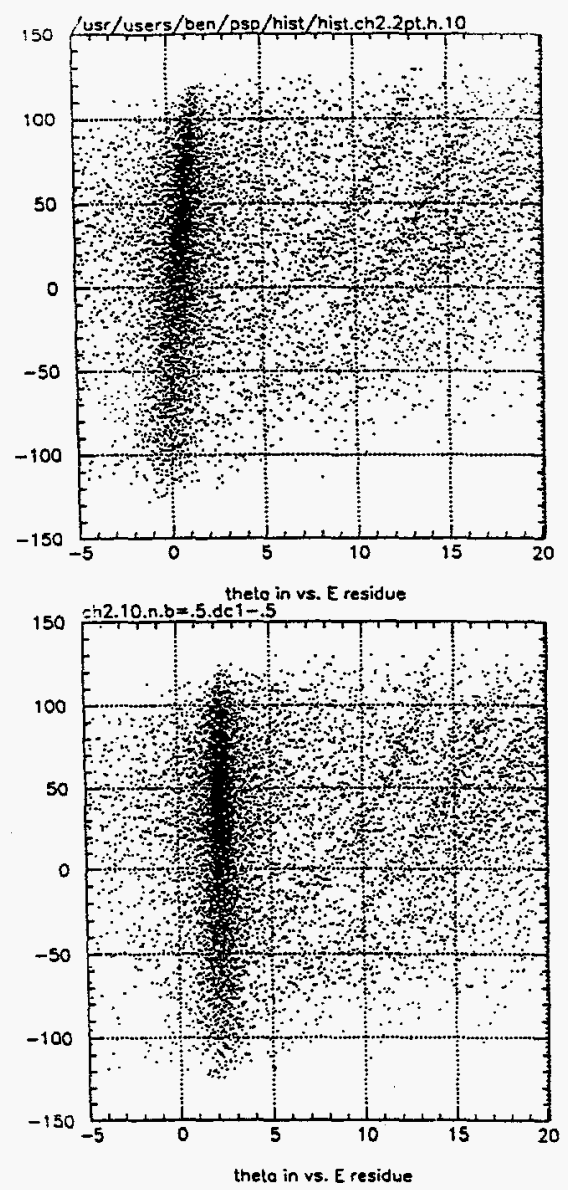

Figure 1. The $-Q$ (horizontal) vs $\theta_{\text {in }}$ (vertical) before (top panel) and after (bottom) the iterations discussed in the test ${ }^{\mathrm{fo}} \mathrm{H}{ }^{\mathrm{i}} \mathrm{H}\left(\mathrm{d},{ }_{2}{ }^{2} \mathrm{He}\right) \mathrm{n}$ reactions for proton spectrometer positioned at $\theta_{n u m}=10^{\circ}$. after (bottom) iterations for ${ }^{1} \mathrm{H}\left(\mathrm{d},{ }^{2} \mathrm{He}\right) \mathrm{n}$ scattering when the proton spectrometer is positioned at $\theta_{\text {nom }}=10^{\circ}$. This particular case is a severe test of our ability to reconstruct the ${ }^{2} \mathrm{He}$ scattering angle correctly due to the large, rapidly changing recoil energy of the unobserved neutron. Clearly, before the iterations, the $\mathrm{Q}$ vs $\theta_{\text {in }}$ is severely tilted with the $Q$ values ranging from $-Q \approx 0 \mathrm{MeV}$ to $-Q \approx 1.5$ $\mathrm{MeV}$ when the ${ }^{2} \mathrm{He}$ angle is changed from $\theta_{\text {in }} \approx-120$ $\mathrm{mr}$ to $120 \mathrm{mr}\left(\theta_{\text {in }} \approx-7^{\circ}\right.$ to $\left.7^{\circ}\right)$, indicating the problems with the system calibration. After the iterations, one sees a constant $Q$ at all angles with its value at $-Q \approx 2.3 \mathrm{MeV}$, close to the theoretical value of $-Q=2.225 \mathrm{MeV}$. Though we have investigated the dependence of $Q$ vs $\theta_{\text {in }}$ on many parameters, the final change, which converts $Q$ vs $\theta_{i n}$ from the top panel to the bottom one (Fig. 1), requires, in our analysis routines, only changing the nominal location of $\mathrm{DC} 1 \mathrm{X}$ by $-0.5 \mathrm{~mm}$, and pivoting the normal to the exit effective field boundary by $0.2^{\circ}$, while keeping its intersection with the central ray unchanged. To see how good these iterations reflect the true situation, we show, in Figs. 2 and 3, the measured $Q$ vs $\theta_{i n}$ plot before (top panels) and after (bottom) the iterations using the same iteration parameters as in Fig. 1, for ${ }^{12} \mathrm{C}\left(\mathrm{d},{ }^{2} \mathrm{He}\right){ }^{12} \mathrm{~B}$ and ${ }^{26} \mathrm{Mg}\left(\mathrm{d},{ }^{2} \mathrm{He}\right){ }^{26} \mathrm{Na}$, respectively. Clearly, for both ${ }^{12} \mathrm{C}$ and ${ }^{26} \mathrm{Mg}$ targets, the situations improve significantly: not only the slopes become more vertical, i.e., the measured $Q$ values become more nearly independent of scattering angle, but also, their values, $-Q \approx 15.0$ $\mathrm{MeV}$, for ${ }^{12} \mathrm{C}$, and $-Q \approx 11.0 \mathrm{MeV}$, for ${ }^{26} \mathrm{Mg}$, become much closer to the theoretical ones, $-Q=$ $14.8 \mathrm{MeV}$, for ${ }^{12} \mathrm{C}$, and $-Q \approx 10.8 \mathrm{MeV}$, for ${ }^{26} \mathrm{Mg}$.

Projecting onto the $\mathrm{x}$-axis, one obtains the excitation energy distributions. Figs. 4-7 show the excitation energy spectra at nominal scattering angles $0^{\circ}, 5^{\circ}, 10^{\circ}$, and $15^{\circ}$, for deuteron reactions on ${ }^{11} \mathrm{~B}$, ${ }^{12} \mathrm{C},{ }^{13} \mathrm{C}$, and ${ }^{26} \mathrm{Mg}$, respectively. These spectra are obtained with the angular and energy cuts: $|\delta \theta| \lesssim 3^{\circ},|\delta \phi| \lesssim 1^{\circ}$ about the central ray, and the relative energy between protons (internal energy of $\left.{ }^{2} \mathrm{He}\right) E_{r e l} \leq 1 \mathrm{MeV}$. With these cuts, the typical 

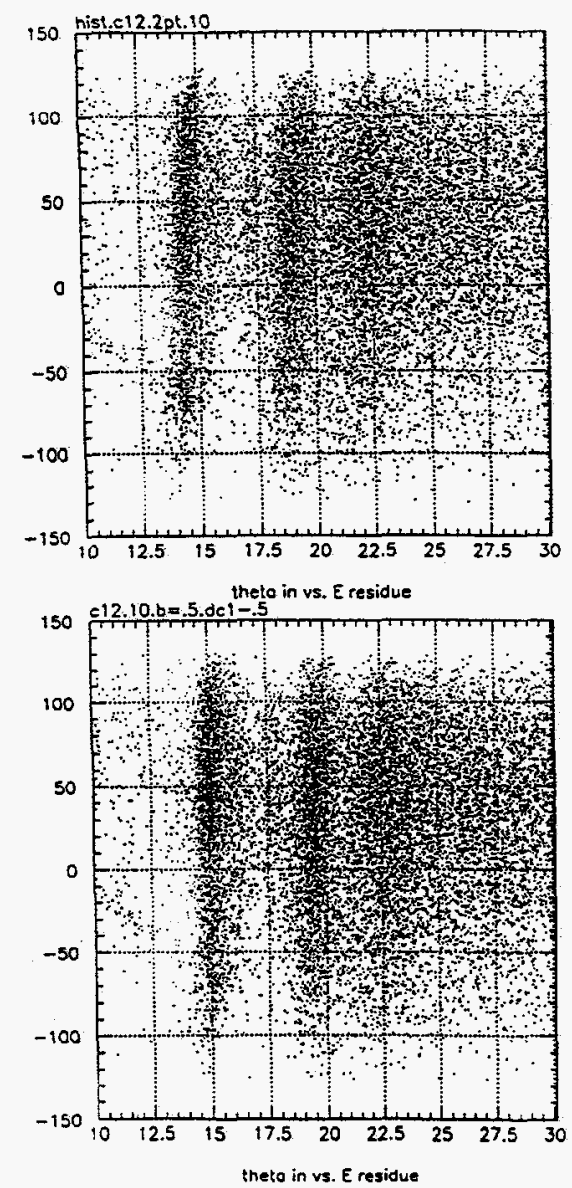

Figure 2. The $-Q$ (horizontal) vs $\theta_{i n}$ (vertical) before (top panel ? anu after (bottom) the iterations discussed in the text for ${ }^{12} \mathrm{C}\left(\mathrm{d},{ }^{2} \mathrm{He}\right){ }^{12} \mathrm{~B}$ reactions for the proton spectrometer positioned at $\theta_{\text {nom }}=10^{\circ}$.
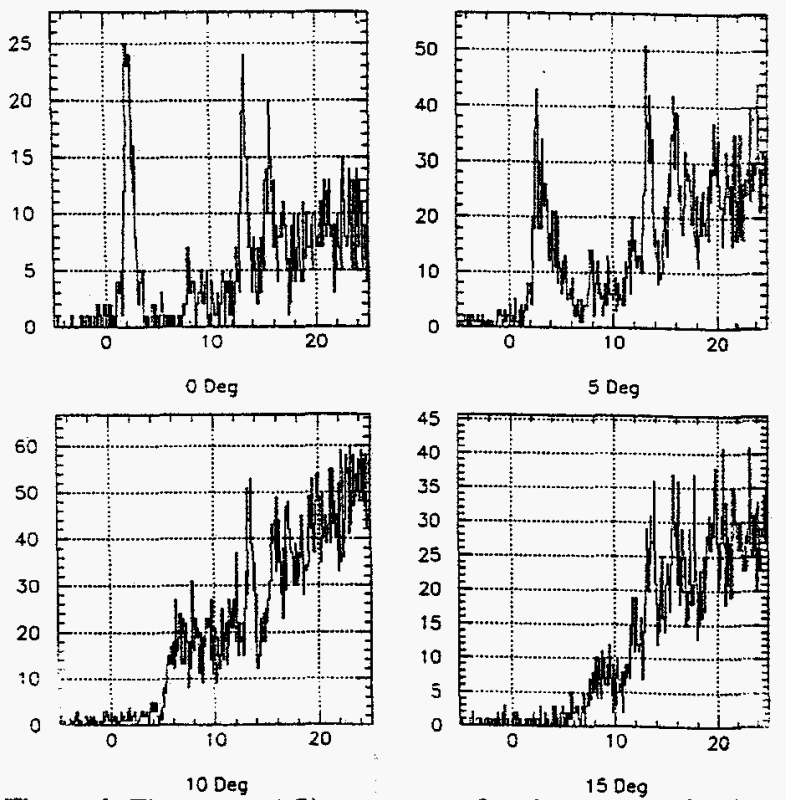

Figure 4. The energy (-Q) spectra as a function of angle for the proton spectrometer positioned at $0^{\circ}$ (upper left panel), $5^{\circ}$ (upper right) $10^{\circ}$ (lower left), and $15^{\circ}$ (lower right), respectively, for ${ }^{13} \mathrm{~B}\left(\mathrm{~d},{ }^{2} \mathrm{He}\right){ }^{11} \mathrm{Be}$ reactions. For details, see the text.
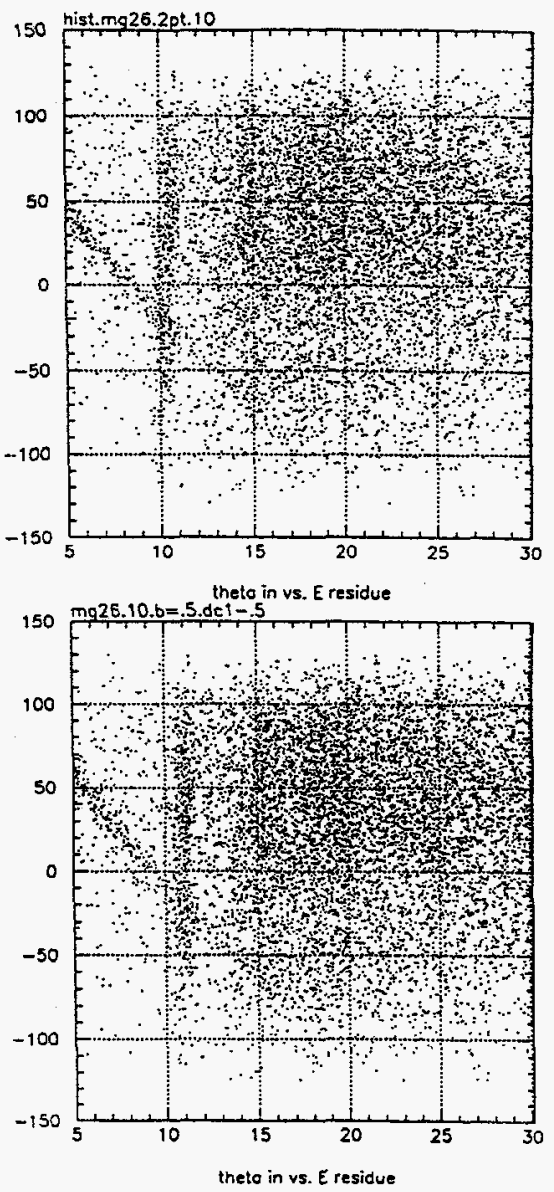

Figure 3. The $-\mathrm{Q}$ (horizontal) vs $\theta_{\text {in }}$ (vertical) before (top panel) and after (bottom) the iterations discussed in the text ${ }^{26} \mathrm{Mg}\left(\mathrm{d},{ }^{2} \mathrm{He}\right)^{26} \mathrm{Na}$ reactions for the proton spectrometer positioned at $\theta_{\text {nam }}=10^{\circ}$.
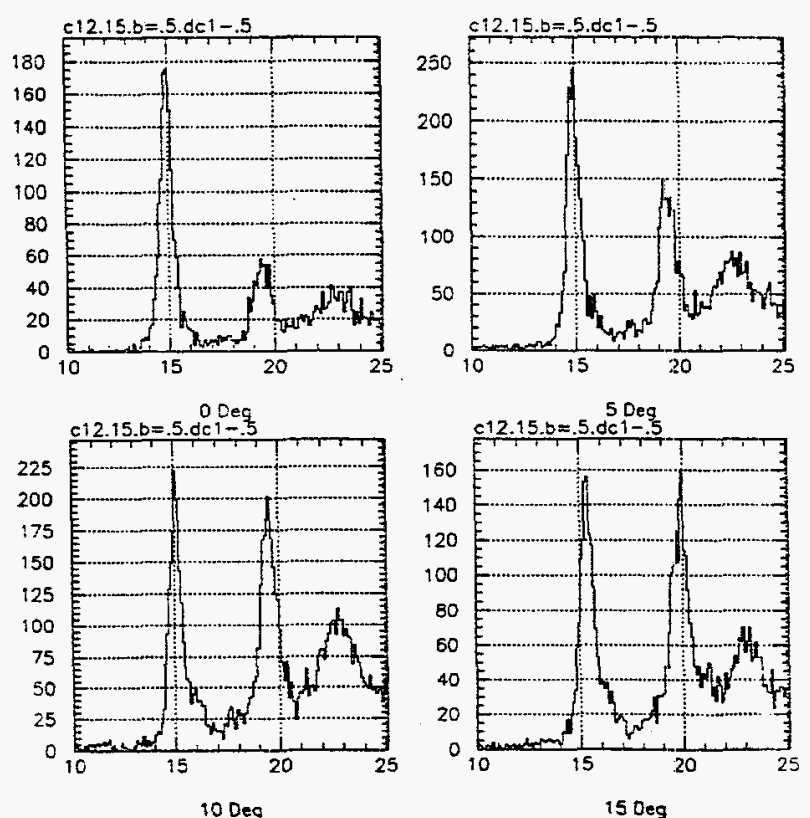

Figure 5. Same as Fig. 4 , but for ${ }^{12} \mathrm{C}\left(\mathrm{d},{ }^{2} \mathrm{He}\right)^{12} \mathrm{~B}$ reactions. 

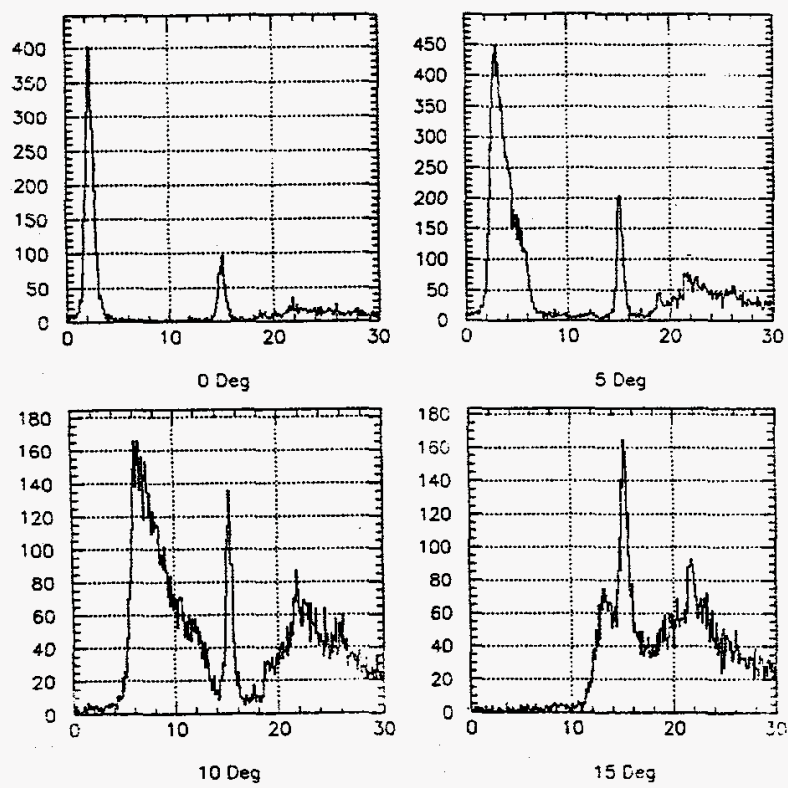

re 6. Same as Fig. 4 , but for ${ }^{13} \mathrm{C}\left(\mathrm{d},{ }^{2} \mathrm{He}\right){ }^{13} \mathrm{~B}$ reaction
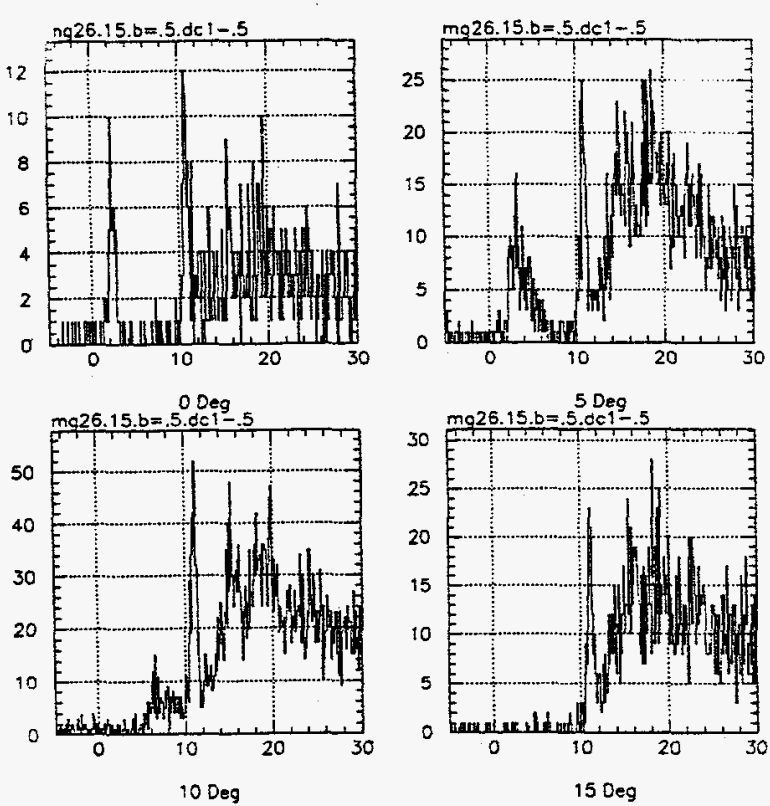

Figure 7. Same as Fig. 4, but for ${ }^{26} \mathrm{Mg}\left(\mathrm{d},{ }^{2} \mathrm{He}\right){ }^{26} \mathrm{Na}$ reactions.

energy resolution in these spectra is $650 \mathrm{keV}$ FWHM. Several features are clearly discernable: (1) As expected, we observe discrete $\delta \ell=0$ transitions for all targets. These transitions, peaked at $-Q \approx$ $13.3 \mathrm{MeV}$ for ${ }^{11} \mathrm{~B},-Q \approx 15.0 \mathrm{MeV}$ for ${ }^{12} \mathrm{C},-Q \approx$ $15.0 \mathrm{MeV}$ for ${ }^{13} \mathrm{C},-Q \approx 11.0 \mathrm{MeV}$ for ${ }^{26} \mathrm{Mg}$, correspond, respectively, to transitions to the first excited state in ${ }^{11} \mathrm{Be}$, ground state in ${ }^{12} \mathrm{~B}$, ground state in ${ }^{13} \mathrm{~B}$, and the first excited state in ${ }^{26} \mathrm{Na}$. All these transitions have well-known values of the GT matrix elements which will be used to calibrate our measurements. (2) Even without normalization, the fact that $\delta \ell=0$ transitions are peaked at zero degrees are clearly observed, in particular, in the scattering from the ${ }^{12} \mathrm{C}$ target. The second peak at $-Q \approx 20 \mathrm{MeV}$ (see Fig. 5), which corresponds to $\delta \ell=1$ transition, becomes increasingly larger at larger angles compared to the $\delta \ell=0$ transition at $-Q \approx 15$ $\mathrm{MeV}$, indicating the dominance of $\delta \ell=0$ transition at $\theta \approx 0^{\circ}$. This information is particularly important for our future study, for which we investigate the GT transitions in the high energy continuum where transitions from higher multipolarities are not separated from $\delta \ell=0$ transitions. (3) The ${ }^{1} \mathrm{H}\left(\mathrm{d},{ }^{2} \mathrm{He}\right) \mathrm{n}$ peaks, which are comparable or larger than the true peaks from targets such as ${ }^{11} \mathrm{~B},{ }^{13} \mathrm{C}$, or ${ }^{26} \mathrm{Mg}$ (see Figs. 4, 6, 7 respectively), not only become wider but also change their energy locations at large angles. This is caused by the strong angular dependence, as shown in Fig. 8, where the $\theta_{i n}$ vs $-Q$ is plotted. Because the recoil energies for all ${ }^{2} \mathrm{He}$ 's are corrected as if they were scattered from heavy residues, ${ }^{11} \mathrm{Be},{ }^{13} \mathrm{~B}$, or ${ }^{26} \mathrm{Na}$, respectively, the recoil energies for those that are scattered from ${ }^{1} \mathrm{H}$ target are underestimated, particularly at larger angles, leaving larger $-Q$ values at larger angles for those scattered from ${ }^{1} \mathrm{H}$ (see Fig. 8). In our final analysis, however, this background can be easily avoided by setting up gates away from the crossover regions (Fig. 8).

To summarize the present status, Fig. 9 shows the very preliminary angular distribution of $\delta \ell=0$ (open circles) and $\delta \ell=1$ (solid circles) transitions for ${ }^{12} \mathrm{C}\left(\mathrm{d},{ }^{2} \mathrm{He}\right){ }^{12} \mathrm{~B}$ reactions, using our best detector parameters to date. The $\delta \ell=0$ distributions are comparable to those from recently reported $\left({ }^{12} \mathrm{C},{ }^{12} \mathrm{~N}\right)$ reactions. ${ }^{4}$ Although we have improved significantly our detector parameters, more efforts are needed. For example, with our best detector parameters to date, the $Q$ value for ${ }^{12} \mathrm{C}\left(\mathrm{d},{ }^{2} \mathrm{He}\right){ }^{12} \mathrm{~B}$ is nearly 
independent of $\theta_{\text {in }}$ for each magnet position. However, this dependence becomes worse at larger angles and its mean values at different magnet positions, $-Q \approx 14.9 \mathrm{MeV}, 14.9 \mathrm{MeV}, 15.0 \mathrm{MeV}$, and $15.3 \mathrm{MeV}$, at $\theta_{\text {mag }}=0^{\circ}, 5^{\circ}, 10^{\circ}$, and $15^{\circ}$, respectively, still depend on the angle. The $-Q$ values at these four angles for ${ }^{1} \mathrm{H}\left(\mathrm{d},{ }^{2} \mathrm{He}\right) \mathrm{n}$ are 2.08 , $2.19,2.31,2.51 \mathrm{MeV}$, respectively. To resolve this problem, we have taken additional proton singles data in our most recent run using a newly-made narrow $\mathrm{x}$-slit, $\approx 4 \mathrm{~mm}$, positioned at the entrance of the proton spectrometer. Since proton emission angles are well defined, these new data will give us an additional tool to calibrate our detector parameters. Moreover, we have also taken new $\left(d,{ }^{2} \mathrm{He}\right)$ data for ${ }^{12} \mathrm{C}, \mathrm{CH}_{2}$ and ${ }^{6} \mathrm{Li}$ targets, with the proton spectrometer rotated in much smaller steps than our nominal step of about $5^{\circ}$. This will provide overlapping measurements of the reaction crossover angles to help pin down the residual problem associated with the calibration of the proton spectrometer.
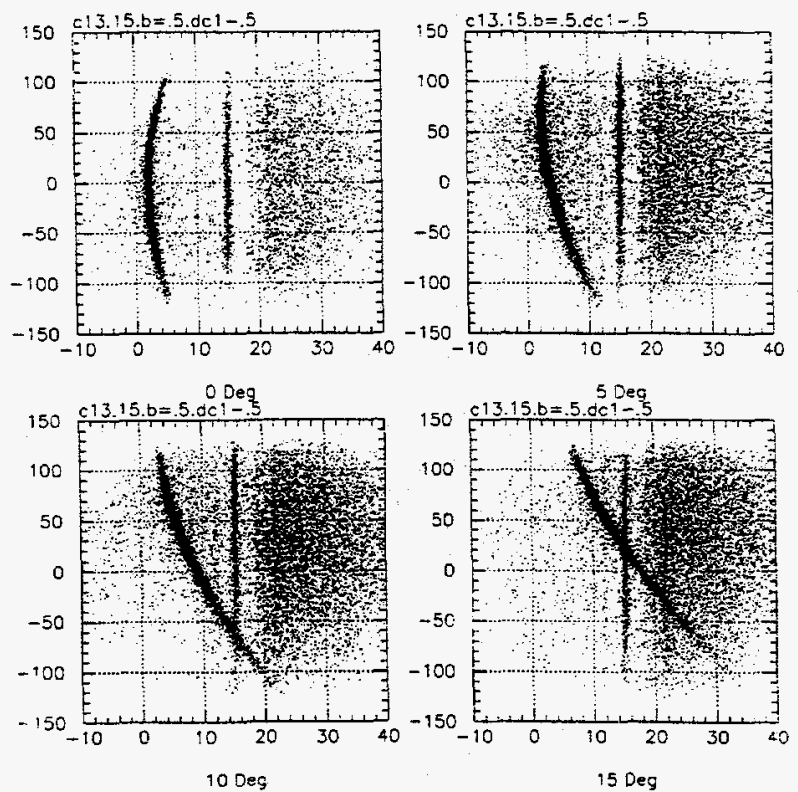

Figure 8. The $-Q$ (horizontal) vs $\theta_{\text {in }}$ (vertical) as a function of angle for the proton spectrometer positioned at $0^{\circ}$ (upper left panel), $5^{\circ}$ (upper right) $10^{\circ}$ (lower left), and $15^{\circ}$ (lower right), respectively, for ${ }^{26} \mathrm{Mg}\left(\mathrm{d},{ }^{2} \mathrm{He}\right)^{26} \mathrm{Na}$ reactions. See the text for details.

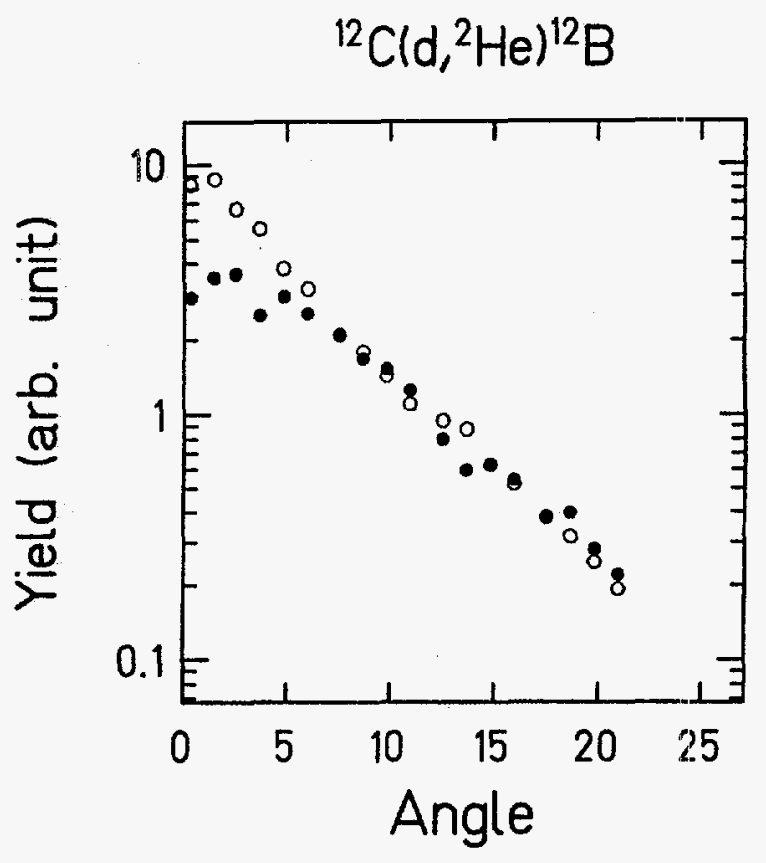

Figure 9. Angular distributions for both $\delta \ell=0$ (open circles) and $\delta \ell=1$ (closed circles) transitions, respectively, for ${ }^{12} \mathrm{C}\left(\mathrm{d},{ }^{2} \mathrm{He}\right){ }^{12} \mathrm{~B}$ reactions.

The analysis for the new data, as well as further analysis of the data from our earlier runs, is currently underway. At the same time, we are in the process of improving our Monte Carlo simulation of the proton spectrometer to improve our ability to extract absolute cross sections. We are also performing DWBA calculations for the various targets that we have investigated.

"Indiana University Cyclotron Facility, Bloomington, Indiana

\section{References}

1. A. C. Betker et al., Progress in Research, 1992-1993, Cyclotron Institute, TAMU, p. 131.

2. A. C. Betker, Ph.D. thesis (1993), Texas A\&M University.

3. A. C. Betker and C. A. Gagliardi, Nucl. Instrum. Methods A283, 76 (1989).

4. T. Ichihara et al., Phys. Lett. 323B, 278 (1994). 


\section{The $\bar{d}(x) / \bar{u}(x)$ Ratio in the Proton}

\section{A. Gagliardi, E. A. Hawker, and R. E. Tribble}

During the past year, we have continued our work on the hardware trigger system for Fermilab E866. This experiment will determine the ratio $\bar{d}_{p}(x) / \bar{u}_{p}(x)$ in the proton by comparing the Drell-Yan $\mu^{+} \mu^{-}$pair yields in $800 \mathrm{GeV} p p$ and $p d$ collisions. A general description of the experiment and its motivation, along with a discussion of our role in it, was given in last year's progress report. ${ }^{1}$ Since then, the scope of E866 has expanded, the time frame has shifted, and we have added several new collaborators. All of these developments have impacted on the design of the new trigger system that we are constructing for E866. Meanwhile, preliminary results from two new experiments, E665 at Fermilab and NA51 at CERN, have strengthened the physics motivation for E866.

E665 measured the deep inelastic muon scattering yields from hydrogen and deuterium targets, in a manner similar to the NMC experiment at CERN. ${ }^{2}$ The higher energy muon beam available at Fermilab permitted it to cover a broader range of quark momenta, reaching as low as $x$ of $10^{-5}$. In the $x$ region where E665 and NMC overlap, their results agree. But in the small $x$ region, E665 finds that $F_{2}^{n / F} z_{z}^{p}$ approaches 0.95 , rather than 1.0 as expected. The most likely explanation is that, even in the deuteron, shadowing sets in below some value of $x$, reducing the deep inelastic scattering yield relative to that for $\mu p+\mu n$. This implies that the NMC experiment conclusion that $\bar{d}_{p}>\bar{u}_{p}$ is model dependent. A direct measurement of $\bar{d}_{p}(x) / \bar{u}_{p}(x)$ is clearly needed. NA51 is the first such measurement. ${ }^{3}$ Like E866, it studied Drell-Yan pairs in $p p$ and $p d$ collisions, though with much lower statistical precision than E866 will achieve. Whereas E866 will concentrate on $\mu^{+} \mu^{-}$pairs with large positive rapidity, the acceptance in NA51 peaked near zero rapidity. This makes the interpretation of its results more sensitive to the assumed form of the valence quark distributions than we will be. But NA51 nonetheless finds that $\bar{u}_{p} / \bar{d}_{p}=0.56 \pm 0.04 \pm 0.05$ at $x=$ 0.18 . This asymmetry is much larger than predicted in recent theoretical calculations. E866 will be able to confirm this result, while increasing the statistical precision and decreasing the systematic effects. It will also extend measurements of this anti-quark asymmetry over the range $0.03<x<0.3$.

In the original E866 proposal, we only described plans to perform accurate measurements of the relative Drell-Yan cross sections in $p p$ and $p d$. Although we hoped to obtain absolute cross sections, we did not plan to devote extensive effort to them. Within the past year, several new collaborators have joined E866 from Argonne National Laboratory with their primary goal being to obtain accurate absolute cross sections to test QCD calculations of the Drell-Yan "K-factor". Although the trigger is not expected to be one of the primary systematic errors, this will clearly place an additional premium on our ability to determine and monitor its absolute efficiency.

We are now planning to study $\Upsilon$ 's produced in the beam dump at very large positive rapidities concurrently with our $\bar{d} / \bar{u}$ measurements. The physics motivation is to investigate the relative importance of gluon fusion and $q \bar{q}$ annihilation in $\Upsilon$ production. This implies that we will now need to have two essentially independent triggers running simultaneously, one for target events and the other for beam dump events.

There have been important developments at Fermilab that impact E866 during the past year. The start of the next fixed target beam cycle at Fermilab has been postponed until late 1995 , but it has also been extended to two consecutive 8 month long periods, together with a short break in between. When E866 was approved, it was to be one of three experiments to run in the Fermilab meson lab during 
the next fixed target period. The other two experiments were both SSC detector tests which have now been canceled. The current plan is to run the meson lab for E866 during the full first period, then to shut it down during the second period to save money. We believe that it will take as long as 3 months of beam time to complete the $\bar{d} / \bar{u}$ measurements as originally proposed. This means we will have several additional months at our disposal. We are considering various options to utilize this time effectively.

The additional effort associated with this expanded beam time makes our new collaborators particularly valuable. In addition to the Argonne group mentioned above, groups from Louisiana State, New Mexico State, and Valparaiso Universities have joined E866 during the past year. In particular, the Valparaiso group will likely be assisting us with trigger system, although their role has not been formalized at present.

Given the developments of the past year, we have chosen to refine our new trigger system in order to make it even more flexible and modular than planned at the time of last year's progress report. We now envision a three stage system consisting of "Track Finders," "Track Correlators," and a "Master Trigger OR."

The track finders will be similar in function to the "Trigger Matrices" that have been utilized by E605, E772, and E789. Their primary advantage compared to the old system will be that they will cover all possible scintillator hodoscope triple coincidences, making them far more flexible than the previous system. A single track finder will select events based on triple coincidences in either the $X$ or $\mathrm{Y}$ direction. In the $\mathrm{Y}$ (bend) direction, potential tracks originating at the target will be identified as Y1-Y2-Y4 coincidences. These will be separately tagged according to the particle charge and the quadrant in Y4 where the track terminates, a total of 8 different $Y$ track types. A second set of $8 Y$ track types will consist of tracks that originate at the beam dump. In the $\mathrm{X}$ (non-bend) direction, potential tracks will be identified as $\mathrm{X} 1-\mathrm{X} 3-\mathrm{X} 4$ triple coincidences that terminate in a specific octant in $\mathrm{X} 4$, once again leading to 8 target track types and 8 beam dump track types. The decision to separate tracks according to their location in Station 4 (the "Muon Hodoscopes") represents a change from the previous experiments. In the past, muon pairs have only been observed when one particle was on the left side of the system and the other was on the right. This implied that the scintillators involved in the two tracks were completely independent. We plan to accept $L-L$ and R-R muon pairs, in addition to L-R pairs, in E866. Mapping all of our candidate tracks into Station 4, which is substantially quieter than the other three, will minimize the chance that we might misidentify a single track as a pair due to random background hits.

The track correlators will be CAMAC modules that take an arbitrary set of 16 inputs and present them to a $64 \mathrm{~K} \times 4$ bit SRAM. These 16 inputs may, for example, be the 16 target track finder outputs mentioned above. The 4 SRAM output bits will represent 4 independent trigger combinations. Each one will be masked with a programmable prescaler, prior to generating a valid trigger output. During set up and debugging, we may choose to program the SRAM's to select various single track events, such as an $X$ triple, a $Y$ triple, or an $X-Y$ coincidence. Once we are satisfied with the performance of the spectrometer, we can then reprogram the SRAM's to trigger on more complex patterns, such as $X-X-Y$, $\mathrm{X}-\mathrm{Y}-\mathrm{Y}$, and $\mathrm{X}-\mathrm{Y}-\mathrm{X}-\mathrm{Y}$ coincidences. If computer dead time becomes an issue, we can trigger separately on the like-sign and unlike-sign muon pairs, with different prescale factors. The choice of CAMAC-programmable SRAM's and prescalers will make it easy to make such choices "on the fly," while keeping full documentation of the state of the trigger at any given time. We currently envision three parallel sets of track correlators -- one to process the target events, one to process the beam dump events, and a third diagnostic system which will receive OR's of the various hodoscope planes as inputs. The modularity of this system would make it easy to add 
further track correlators if we decide to add more independent triggers at a later time.

The master trigger OR will take the outputs of the various track correlators, together with additional triggers such as pulsers, apply the busy logic, and generate all of the gates that are required by the various parts of the data acquisition system.

We are also planning to use a different approach to test the trigger system than has been utilized in the previous experiments. In the past, the primary test of the trigger system was to read out the SRAM's in the trigger matrices between beam spills to confirm that they contain the same data that was originally downloaded into them. While important, this exercises only a small part of the total system. We plan to perform far more extensive diagnostics on the system between beam spills. The hodoscope phototube signals feed into LeCroy 4416 CAMAC discriminators. We will use these to generate test hit patterns for the trigger. We will then observe how the trigger system responds to these hit patterns by monitoring its intermediate stages. This will let us check the efficiency of all parts of the trigger except the scintillators and phototubes themselves. They can then be monitored with the redundant triggers described above.

As this is being written, we are in the process of deciding what technologies to adopt for the various stages in the trigger system. We will begin the actual circuit board design and construction this summer.

\section{References}

1. C. A. Gagliardi, E. A. Hawker, and R. E. Tribble, Progress in Research, 1992-1993, Cyclotron Institute, TAMU, p. II-49.

2. D. Geesaman, private communication, and E665 collaboration, to be published.

3. A. Baldil et al. (NA51 collaboration), Phys. Lett., to be published.

\title{
The MEGA Experiment
}

\author{
C. A. Gagliardi, F. Liu, R. E. Tribble, X.-L. Tu, L. Van Ausdeln
}

During the past year, we completed the installation of the full MEGA detector system and used it for an extended data run at LAMPF. The detector system, which has been described in detail in previous reports, was quite reliable over the course of the run. We were able to obtain sufficient data to improve our sensitivity for a possible $\mu \rightarrow$ er decay to a branching ratio of $3-4 \times 10^{-12}$, or about a factor of 15 below the present world limit, once the analysis is complete. In addition, we obtained new data on the Michel parameter $\rho$ using an electron spectrometer with much less electronics noise than we had during the ' 92 data run. Below we discuss the present status of the analysis for both the $\mu \rightarrow \mathrm{e} \gamma$ and $\rho$ data.

\section{$\mu \rightarrow$ e $\gamma$ Data Analysis}

Two working groups have been formed within the MEGA collaboration to attack the problem of data analysis. The two groups have been assigned to the photon and electron spectrometers. We at TAMU are coordinating the photon arm analysis effort.

There are several different types of analysis tasks that must be completed before the full data analysis can be carried out for the photon pair spectrometer. 
Calibration constants are needed for the delay lines and for the drift time versus radial position in the drift chambers. Also a correction is needed for the timing generated by the plastic scintillators due to the pulse height dependence of the discriminators. Different members of the analysis group are working on all of these calibration constants and expect to have final results by mid May. A major effort has gone into refining the pattern recognition algorithms that initially were developed at TAMU. By adding $z$ information, it now is possible to determine nearest neighbor drift chamber cells that belong to different loops and thus break large clusters of cells into smaller groups. A separate code has been put together to predict the location of the photon vertex based solely on the $\mathrm{z}$ information in the chambers. This code often helps reduce the ambiguity of multiple vertex candidates that are found in the end view pattern recognition code. The new on-line version of the code that was used in the DECstation farm in 1993 improved our efficiency for finding $52.8 \mathrm{MeV}$ photons by nearly $20 \%$ over the code that was used in 1992. In order to perform an end view circle fit and to extract the perpendicular momentum for the photon, it is necessary to pick out the drift chamber cells that correspond to the initial track through the chamber for both the electron and positron that are produced in the pair conversion of the photon. The procedure used for cell tagging is based on the work from Monte Carlo studies with refinements that were made following the ' 90 engineering test run. With the $\mathrm{z}$ information now available, some modifications have been made in cell tagging to make it more reliable.

Four other tasks are being carried out by members of the photon arm analysis group at Los Alamos. The first of these is to allow the existing code to look for solutions from more than one possible vertex. In the past, a "best guess" was used to pick a vertex when more than one candidate was found. By allowing for multiple vertex candidates, we should improve the efficiency of the detector system. The second task is a totally new approach to the problem of pattern recognition. One of the MEGA collaborators at LAMPF worked on pattern recognition algorithms for SSC detectors before joining MEGA and has carried over some of the ideas from that project to the pattern recognition problem for our pair spectrometers. Initial results from this new approach are encouraging and it could provide us ultimately with a second, independent, pattern recognition code. Another member of the analysis team at LAMPF has begun working on the identification of second loop tracks through the spectrometer. A full reconstruction of an event will require us to unravel all subsequent loops that both members of the electron-positron pair make as they traverse the spectrometer. Our initial effort: has centered on identifying the first loop since this is needed in order for us to extract the perpendicular momentum of the photon. The fourth task being undertaken at LAMPF is the implementation of a non-linear circle fitting algorithm that simultaneously fits both the electron and positron track. In a simultaneous circle fit, it is feasible to impose conditions on the track intersection region which can help constrain the possible solutions and hence help in the left-right ambiguity problem associated with choosing track locations through drift chambers. Our goal is to have solutions in place for each of these tasks by late May so that final filtering of the present data set can take place.

\section{Measurement of the Michel Parameter $\rho$}

In the fall of ' 92 , we collected the data for a measurement of the Michel parameter $\rho$. As discussed in last year's report, the objective of RHO was to test the Standard Model througl a measurement of $\rho$ to an accuracy of $\delta \rho / \rho=0.001$, which is about three times better than the current best value: $\rho=0.7518( \pm) 0.0026 .^{1}$ The positron spectrometer which is used for the measurement had several problems during the data acquisition including a tendency for many channels of the electronics on the spectrometer to trigger simultaneously on noise. This effect caused many events to be discarded since it was not possible to reconstruct them. While this 
could be viewed as a drop in efficiency, a more troublesome concern was that the events which were dropped might not represent a uniform sample of the Michel decays and thus they could produce a systematic change in the observed Michel spectrum. Modeling this problem in the Monte Carlo simulation would be very difficult if not impossible without understanding the source of the problem in detail. In order to circumvent this issue, new data for RHO was obtained during the ' 93 run when the chambers were working much better than they had been for the '92 period.

During six days of data taking, we were able to collect $4 * 10^{8}$ trigger events under various conditions. Three modes of data were taken with scintillator triggers as well as with the Snow White trigger. The three modes distinguish themselves by the type of beam used and magnetic field values. Two of them were taken with the surface muon beam and magnetic field at $15 \mathrm{kG}$ and $14.25 \mathrm{kG}$ respectively. For the third mode, the magnetic field was set at $15 \mathrm{kG}$ and the beam was switched to that of reversed polarization. The three data sets will help check and understand systematic errors.

As we noted in last year's report, many modifications of the positron chamber analysis programs had been made during the process of analyzing the '92 data. Further refinements have been made over the past year such that we are now able to reconstruct about $55 \%$ of the events that were sent to tape in '93. About $40 \%$ of the reconstructed events will be used in the final extraction of the $\rho$ parameter. The major analysis effort following the '93 run has centered on obtaining consistent efficiency determinations for both the positron wire chambers and plastic scintillators along with determining the beam profile and geometrical positions of the various detector elements. Also a new EGS4 based Monte Carlo code has been written by a collaborator (L. Piilonen of VPI) which allows for both more flexibility in the detector geometry and a non-uniform magnetic field. Tests with the new Monte Carlo indicate that the non-uniform field is important in the extraction of $\rho$ and all of the high statistics Monte Carlo runs will be done with this feature. The first pass analysis of the ' 93 data is now nearly complete. We expect to have a final result from the ' 93 data by late this fall.

\section{Reference}

1. J. Peoples, Columbia University, thesis, Nevis 147, 1966 (unpublished). 


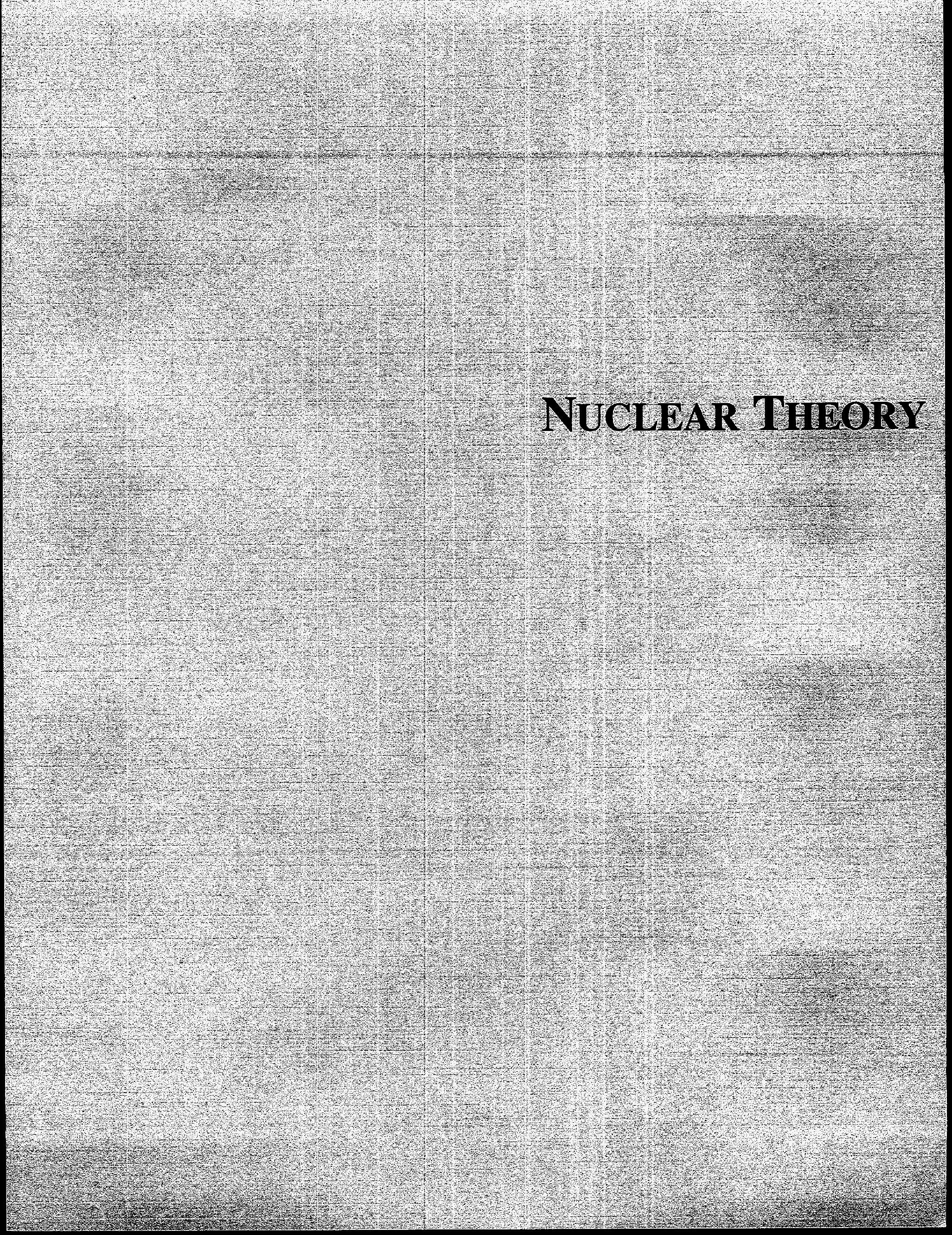




\title{
The Nuclear Spectral Function ${ }^{\star}$
}

\section{G. M. Vagradov** and S. Shlomo}

The nuclear spectral function $S(\bar{p}, \varepsilon)$, which has been $^{1}$ the subject of many experimental and theoretical investigations, is a basic single nucleon quantity determining the joint density distribution of energy $\varepsilon$ and momentum $\bar{p}$ of the nucleon in a nucleus. The spectral function $S(\bar{p}, \varepsilon)$ is given directly in terms of the imaginary part of the single nucleon Green's function which can be determined by solving the Dyson's equation. The aim of this work ${ }^{2}$ is to take a closer look at the microscopic theory of the nuclear spectral function and derive some properties. We also give the formulation of the theory of the spectral function in a field theoretical approach.

It is evident that the calculation of the spectral function is directly related to the solution of the many body problem. The aim of our approach is to obtain better physical insight on the complicated structure of the spectral function and uncover basic properties that should be maintained in simplified models.

We have demonstrated ${ }^{2}$ the usefulness of the energy dependent mass-operator introduced in this work. It allows the determination of the single particle spectrum as well as the spectral function. Experimental information on the energy dependent mass-operator can be extracted. In particular we have shown that the single particle spectrum has a lower level bound. We have also shown that an important consequence of the space translation invariance is the fact that the mass-operator $M(y, y)$ and the single particle propagator $G(y, y)$ depend only on the difference $y-y$. This leads to equations which parallel those for the case of an infinite system.

"Supported in part by the U. S. National Science Foundation under grant \#PHY9107008 and Fund for Fundamental Research of the Russian Government under grant \#92-02-143.

"* Permanent address: Institute for Nuclear Research, The Russian Academy of Science, 60th October Anniversary Prospect 7a, 117312 Moscow, Russia.

\section{References}

1. S. Frullani and J. Mougey, Adv. Nucl. Phys. 14, 1 (1984), and references therein.

2. G. M. Vagradov and S. Shlomo, submitted for publication.

\section{On the Decay of Many-Body System*}

\author{
G. M. Vagradov** and S. Shlomo
}

In this work we have concentrated mostly on the description of decaying states of a number of interacting particles and fields. We followed the approach of Ref. 1 and restricted ourselves to the consideration of a non-relativistic system with potential particle-particle interaction capable of forming bound states. In distinction to Ref. 1 we attempted here to make our exposition more transparent. At the same time, the present consideration is more general, since it is not based on the projection operator method in which the results are dictated by the choice of the unperturbed basis. 
We recall that in our approach ${ }^{1}$ we considered the outflow of a particle through a sphere around the system. Since the boundary conditions for the decay, namely the particle outflow through a sphere around the system, corresponds to a nonhermitian Hamiltonian, ${ }^{1}$ the relevant Schrödinger equation has two sets of eigenvectors and complex eigenvalues. These vectors (following Bohm ${ }^{2}$ we call them Gamow vectors) have some features other than the common ones. Nevertheless the decay state can be expanded on this two-set (bi-orthogonal) basis which leads to a mathematical description of the decaying states.

We have provided ${ }^{3}$ a description of particle decay and scattering using a bi-orthogonal set of eigenvectors. The arising of the bi-orthogonal sets of eigenvectors is governed by the decay and scattering boundary conditions. Some properties of such states can be observed in the decay and scattering, but each state occurs as an intermediate state. We also point out that our approach is non perterbative. Thus using the bi-orthogonal sets permits an extension of the common quantum mechanical approaches.

Our approach was formulated in the second quantization method. It has the advantage that it can be generalized to the case of systems of interacting fields. It can also be used for the case of relativistic dynamics. However, it is not clear how to formulate the covariant description of decay due to time isolation in this problem and due to difficulties of the relativistic covariant theory of a many-body system.

We have considered the effects related to the existence of particle flow. Clearly, it is applicable for finite systems of particles. For infinite systerns, the flow loses its meaning and the damping of excitations has a different physical origin.

*Supported in part by the U.S. National Science Foundation under grant PHY 9107008 and by Fund for Fundamental Research of the Russian Government under grant 92-02-1438

**Permanent address: Institute for Nuclear Research of the Russian Academy of Science, 60th October Anniversary, Prospect 7a, 117312 Moscow, Russia

\section{References}

1. J. Bang and G. M. Vagradov, Physica Scripta 31, 225 (1985).

2. A. Bohm, Quantum Mechanics: Foundations and Applications, 2nd Edn. Berlin, Heidelberg, New York: Springer (1986).

3. G. M. Vagradov and S. Shlomo, Submitted for publication.

\title{
Stochastic Aspects of Nuclear Large Amplitude Motion
}

\author{
V. M. Kolomietz*
}

The concept of macroscopic collective motion plays an important role in many phenomena in nuclear physics, such as large scale motion, fission etc. Usually these phenomena are treated in terms of only a few degrees of freedom, which are chosen to describe gross properties of the nucleus. Such an approach is acceptable for slow collective motion where the fast intrinsic degrees of freedom exert forces on the collective variables leading to a transport equation. The crucial point of this approach is the separation of total energy of the system into potential energy, collective kinetic energy and dissipative energy. This separation is non-trivial and gives rise to a Markovian transport equation without memory effects.

The study of macroscopic nuclear motion is 
simplified due to the use of an adiabatic moving basis. ${ }^{1}$ The motion along the energy terms of the moving basis produces virtual and real transitions. The virtual transitions lead to the collective mass while the real ones contribute to the dissipative energy. The difficulty appears in a region of the avoided crossings where an exact solution to the two-level problem has to be used. This can be done in the framework of the Landau-Zener approximation. ${ }^{2}$ However, the occurance of interlevel transitions change the occupation probability of excited terms. Thus, the picture of collective motion becomes much more complicated. Some simplification can be achieved by taking into account the statistical properties of multiples of avoided crossings of adiabatic terms. ${ }^{3}$ The subsequent spectral statistic smearing leads to coupled equations for the occupation probabilities and the collective variables. This aspect of nuclear large-scale dynamics is the main purpose of our investigation.

We have developed a consistent description of nuclear large amplitude dynamics, including the motion along a collective path and the internal excitation of the nucleus. Due to the use of the cranking model response function, averaged over the spectral statistics of avoided level crossings in the moving frame, it is possible to extract the smeared macroscopic transport parameters. In particular, the collective mass, friction and diffusion coefficients have been derived along the collective path. An essential advantage of such a smearing procedure is the elimination of the level quasi-crossing problem, which occurs for the quantum cranking model in a moving frame.

The averaged adiabatic mass coefficient is not sensitive to the choice of the spectral statistic smearing. The smeared mass coefficient is determined by the classical correlation function. The smooth part of this correlation function corresponds to the mass coefficient, which is related to the rotational hydrodynamic model. ${ }^{4}$ The additional energy dependent contribution to the mass coefficient appears from the fluctuating part of the correlation function.

The main contribution to the rate of dissipation energy is due to the Landau-Zener jump probabilities leading to a rate of dissipation which depends essentially on the total energy of the nucleus. The final result shows that a time irreversible energy exchange between the collective and internal degrees of freedom is possible when the level density increases with energy. The friction force which is proportional to the collective velocity appears here in the case of the GOE statistic for the motion close to the ground state. For other cases the velocity dependence of the friction force is much more complicated.

The transport coefficients for collective motion depend on the occupation probabilities on a moving basis. The short memory property of the response function calculated in a moving frame, allows us to reduce the master equation to a diffusion equation. The corresponding diffusion coefficient depends on the spectral statistics and determines the Landau-Zener evolution of the occupation probabilities over the terms of the moving basis. Numerical calculations show a sensitivity of the characteristics of the large scale motion, in particular, of the scission time in a nuclear fission to the spectral statistic.

This work was supported in part by the National Science Foundation under grant \#PHY9107008. The author would like to thank Prof. L. Wilets for stimulating discussions and comments. The author would like to thank Dr. S. Shlomo for numerous discussions and warm hospitality at the Cyclotron Institute, Texas A\&M University.

*Permanent address: Institute for Nuclear Research, Kiev, Ukraine

\section{References}

1. L. Wilets, Phys. Rev. 115, 372 (1959).

2. C. E. Zener, Proc. R. Soc. A137, 696 (1932); L. D. Landau, Phys. Z. Sow. 1, 88 (1932); 2, 46 (1932). 
3. M. Wilkinson, J. Phys. A21, 4021 (1988); A22, 2795 (1989).
4. V. M. Kolomietz and V. N. Kondratjev, Z. Phys. A344, 125 (1992).

\title{
Can Dileptons Reveal the In-Medium Properties of Vector Mesons?
}

\author{
G. Q. Li and C. M. Ko
}

Heavy-ion collisions at intermediate energies provide the possibility to form in the laboratory a piece of dense matter with density up to $2-3 \rho_{0}$ and thus make it possible to study experimentally the properties of hadrons in dense medium. Because of the partial restoration of chiral symmetry, hadronic properties are believed to be modified in nuclear medium. ' To probe their properties, dileptons have been considered a good observable due to their weak interaction with hadrons.

We have calculated the dilepton invariant-mass spectra from both pion-pion and kaon-antikaon annihilations to see whether one can learn about the in-medium properties of tho and phi mesons. We take as an example the $\mathrm{Ni}+\mathrm{Ni}$ collision at beam energy of $2.0 \mathrm{GeV} /$ nucleon and impact parameter of $0 \mathrm{fm}$. The dynamic evolution of this collision is described by the relativistic transport model ${ }^{2}$. Pions are produced from the decay of deltas which are excited in nucleon-nucleon inelastic interaction. Kaons and antikaons are produced in baryon-baryon interactions $s^{3}$; their scalar and vector potentials are obtained in the mean-field approximation to the chiral Lagrangain.

The in-medium properties of rho meson and phi meson enter our calculation through pion and kaon electromagnetic form factors in the vector dominance model. The effective masses of tho and phi mesons are taken from the QCD sum rules calculation, ${ }^{4}$ while their effective widths are calculated using corresponding effective masses. Because of reduced antikaon mass in the medium, we find that the phi-meson width increases with density although its mass decreases with density slightly.

For comparison we first carry out a calculation in which medium effects on kaon, antikaon, rho and phi mesons are neglected. The results are shown in. Fig. 1. For pion-pion annihilation we observe a broad but visible peak around the rho-meson mass. For kaon-antikaon annihilation we find a sharp peak around the phi-meson mass. This peak is about a factor of 4 above the background from the high-tail part of the pion-pion contribution.

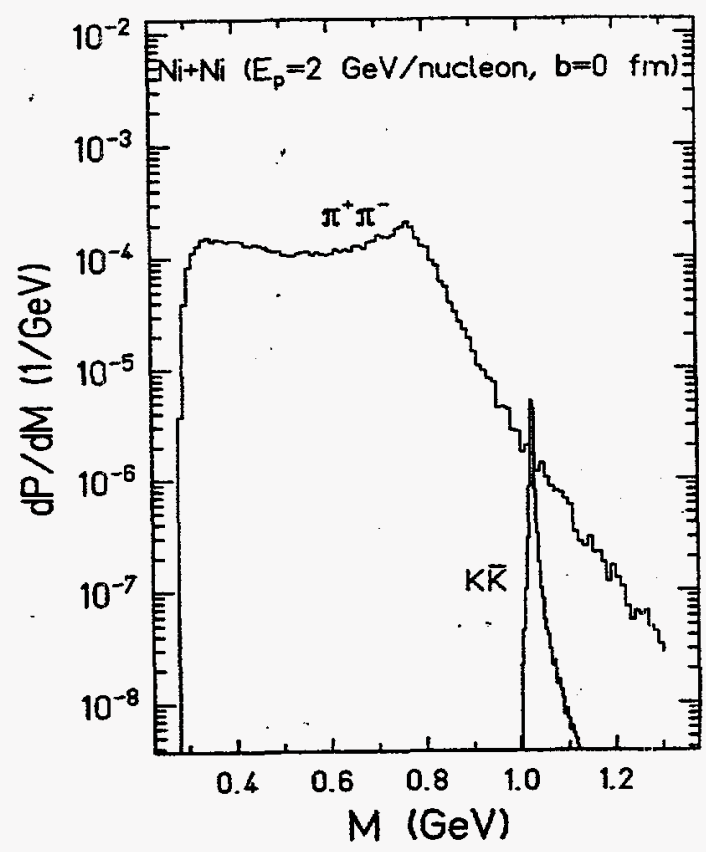

Figure 1. Dilepton invariant mass spectra from the pion-pion and kaon-antikaon annihilation without medium effects on kaon, antikaon, tho and phi mesons. 
In Fig. 2 we show our results when all medium effects discussed above are included in the calculation. The peak of the pion-pion contribution now shifts to lower invariant masses around 500 $\mathrm{MeV}$. This is due to reduced rho-meson mass in dense medium. The dilepton spectrum from the kaon-antikaon annihilation is also modified. The major peak now shifts to lower invariant masses around $950 \mathrm{MeV}$ due to the decrease of the phi-meson mass at high density. The height of this peak is about one order of magnitude above the pion-pion background. There is also a small peak around the free phi-meson mass which comes from kaon-antikaon annihilation at the surface of system.

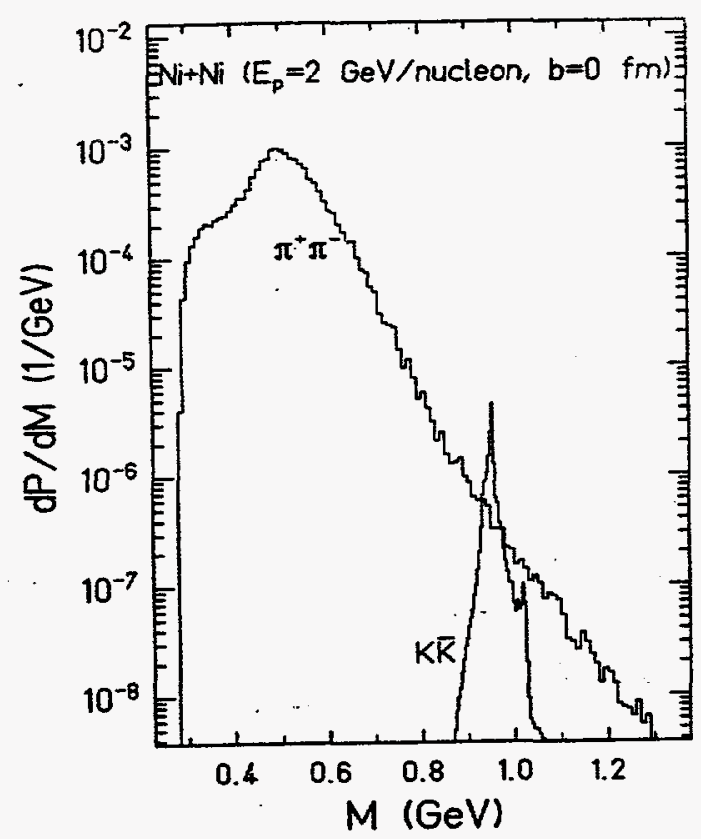

Figure 2. Same as Fig. 1, with medium effects.
Finally, we note that in order to study the in-medium properties of rho and phi mesons more quantitatively, we need to explicitly treat the formation, propagation and decay of these mesons. This is in progress.

\section{References}

1. G. E. Brown and M. Rho, Phys. Rev. Lett. 66, 2720 (1991).

2. C. M. Ko, Q. Li, and R. Wang, Phys. Rev. Lett. 59, 1084 (1987); C. M. Ko and Q. Li, Phys. Rev. C 37, 2270 (1988); Q. Li, J. Q. Wu, and C. M. Ko, Phys. Rev. C 39, 849 (1989).

3. X. S. Fang, C. M. Ko, G. Q. Li, and Y. M. Zheng, Phys. Rev. C 49, R609 (1994); G. Q. Li, C. M. Ko, and X. S. Fang, Phys. Lett. B, in press.

4. T. Hatsuda and S. H. Lee, Phys. Rev. C 46, R34 (1992). 


\section{Effective Mass and Width of Pions at $T \neq 0$}

\section{Chungsik Song}

The masses of mesons are studied in the connection to the symmetry properties of hot hadronic matter. ${ }^{1}$ However, the concept of the mass is not uniquely defined at finite temperature ${ }^{2}$ and various definitions are proposed in different context. It has been shown that there is discrepancy between these definitions of mass. ${ }^{3}$ This indicates that they represent different physical quantities and one should be very careful to relating whatever definition of the mass to physical ones. In this letter we calculate the effective mass and thermal width of the pions in hot hadronic matter based on an effective chiral lagrangian. The lagrangian includes the vector and axial-vector mesons explicitly, to describe the interaction of the pions in hot matter.

The properties of pions are modified in hot matter because of the interactions with particles in a heat bath. The modifications are included in the self-energy, $\Pi(\omega, k)$, and the propagation of the collective excitation is determined from the relation

$$
\omega^{2}=k^{2}+m_{\pi}^{2}+\Pi_{\pi}(\omega, k)
$$

where $\omega$ is the pion energy and $k=|k|$ is the pion momentum. The real part of the self-energy is related to the dispersion relation and the imaginary part determines the absorption of a particle in the heat bath.

The pole position of the propagator at $T \neq 0$ has been regarded as an effective mass of the collective mode in hot matter. This pole mass can be obtained from the Eq. (1) in the limit $k \rightarrow 0$,

$$
\omega^{2}-m_{\pi}^{2}-\operatorname{Re}\left[\Pi_{\pi}(\omega, k \rightarrow 0)\right]=0 .
$$

Eq. (2) can be solved self-consistently. From Eq. (2) the pole mass is obtained and given in Fig. 1 as a function of temperature. As temperature increases the pole mass of the pion decreases, but the change is very small, as expected from the Gell-Mann-Oakes-Renner relations $\left(\Delta m_{\pi}(T) \sim 10\right.$ $\mathrm{MeV}$ at $T=160 \mathrm{MeV}$ ). This behavior is opposite to the result of chiral perturbation theory, which shows an increase of the pion mass with temperature. The difference appears when $T>100 \mathrm{MeV}$.

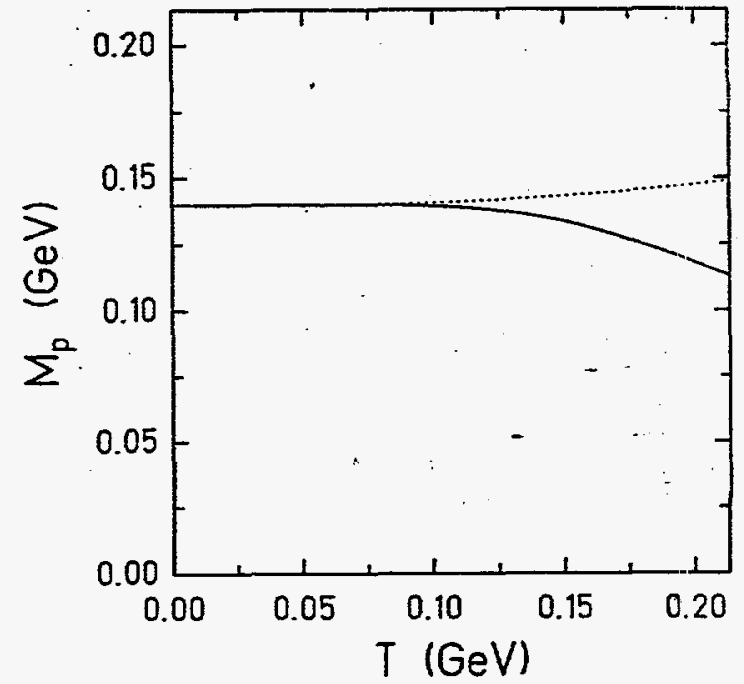

Figure 1. The effective mass (pole mass) of the pions in hot matter. The dotted line is the result of the chiral perturbation calculation at the lowest order.

At finite temperature, alternative definition of mass can be considered which in general is not equivalent to the one defined from the pole position of the propagator. One can define an effective mass by the inverse of the screening length which is obtained from the static infrared limit of the self-energy. This definition has been widely used in the lattice simulation and deduce the symmetry properties of hot hadronic matter. ${ }^{4}$ The screening mass is written as

$$
m_{s}=\sqrt{m_{\pi}^{2}+\Pi_{\pi}(\omega=0, k \rightarrow 0)} .
$$

The result is shown in Fig. 2. Even though there is a slight decrease, the screening mass is almost constant at temperatures we consider. When we compare the result with that obtained from the pole 
position, we get different values. However, both masses decrease with temperature and the screening mass decreases more slowly than the pole mass. This result reminds us to be careful of the definition of mass at finite temperature.

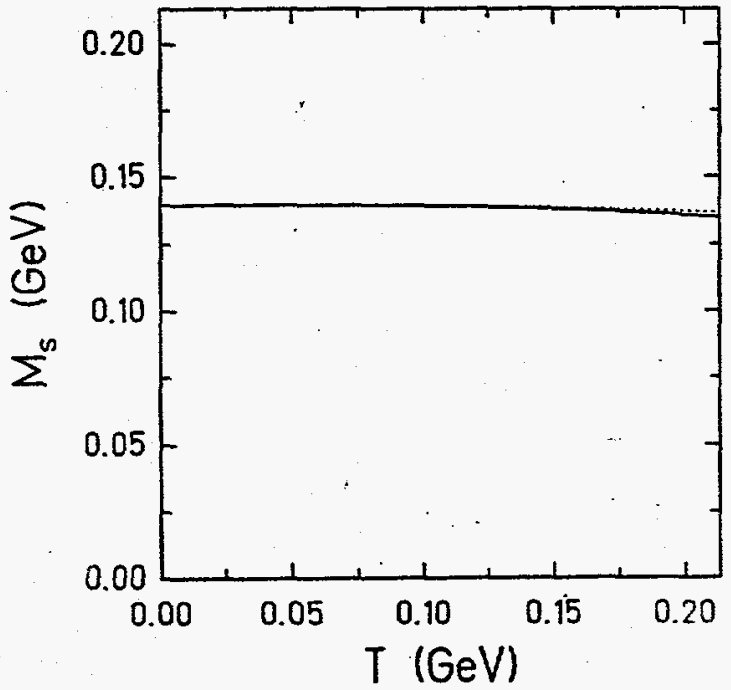

Figure 2. The effective mass (screening mass) of the pions in hot matter. The dotted line is the result of the chiral perturibation calculation at the lowest order.

In the chiral limit where $m_{\pi}=0$, we can see explicitly that the screening mass, defined from the static infrared limit of the real part for the self-energy, becomes zero in the chiral limit. One can also show that the pole mass goes to zero as the mass of the pion becomes zero. This means the pion mass is independent of temperature in the chiral limit. Since the pions are regarded as the massless Goldstone bosons corresponding to the spontaneously broken symmetry, the pion should remain massless at low temperature as long as the chiral symmetry remains broken. This is consistent with the result obtained from the chiral perturbation calculation: chiral symmetry protects all the masses from picking up a contribution of order $T^{2}{ }^{5}$

The thermal width $\gamma^{T}(k)$ of the pion can be obtained from the imaginary part of the self-energy;

$$
\gamma^{T}(k)=\frac{-\operatorname{Im} \Pi}{\omega} \text {. }
$$

We take the average value of the thermal width as

$$
\bar{\gamma}^{T}=\int d^{3} k \frac{\gamma^{T}(k)}{e^{\omega / T}-1} / \int d^{3} k \frac{1}{e^{\omega / T}-1}
$$

The results are shown in Fig. 3 and compared with current algebra result $\bar{\gamma}^{T} \approx T^{5} / 3 F_{\pi}^{4}$. The thermal width is almost negligible at low temperatures but increases rapidly with temperature when $T>100$ $\mathrm{MeV}$. The difference from the current algebra result shows up as temperature greater than $100 \mathrm{MeV}$.

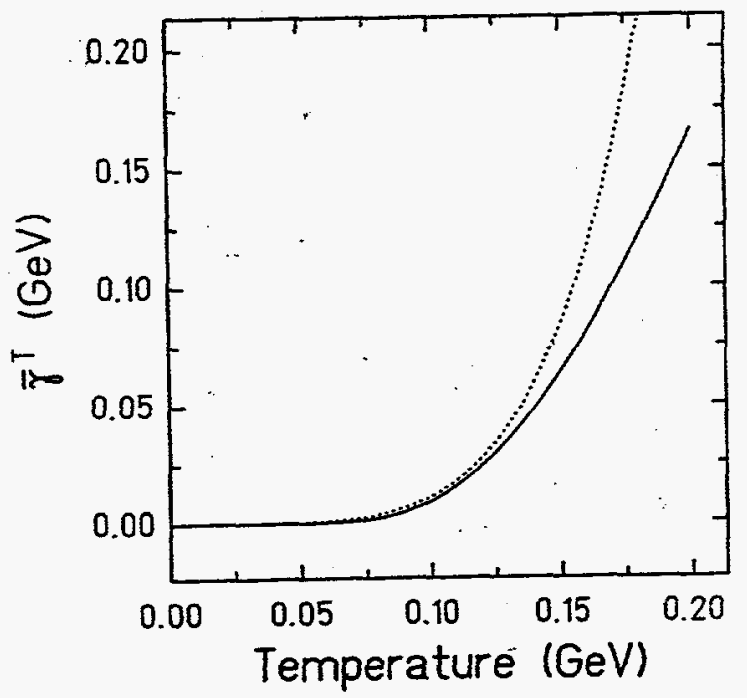

Figure 3. The thermal width of the pions at finite temperature. The dotted ine is the result of the current algebra.

We have obtained the effective mass and thermal width of the pions in hot hadronic matter based on the effective lagrangian. The effective mass cannot be uniquely defined. We have different values for the pion mass at finite temperature. However, both definitions give us a slight decrease of the mass when $T>100 \mathrm{MeV}$. When the temperature is lower than the pion mass the effective mass is almost constant in temperature. As temperature increases $(T>100$ $\mathrm{MeV}$ ), the thermal width of the pions rapidly increases. The increase in the thermal width will affect on the dilepton emissions from the $\pi-\pi$ annihilations in hot matter. Such an increase in the width implies the melting of the hadronic degrees of freedom in hot matter. 


\section{References}

1. T. Hatsuda, Y. Koike and Su H. Lee, Phys. Rev. D 47, 1225 (1993).

2. A. V. Smilga, Nucl. Phys. B335, 569 (1990).
3. Chungsik Song, Phys. Rev. D 48, 1375 (1.993).

4. C. DeTar and J. Kogut, Phys. Rev. D 36, 2828 (1987).

5. H. Leutwyler and A. V. Smilga, Nucl. Phys. B342, 302 (1990).

\title{
Phi Meson Width at Finite Temperatures
}

\author{
C. M. Ko and D. Seibert ${ }^{*}$
}

We have previously studied the mass of a phi meson in hot and dense matter. Using the QCD sum rules, we have found that the phi meson mass decreases in hot hadronic matter because of the appreciable number of strange particles. ${ }^{1}$ The phi meson mass drops below twice the free kaon mass when the temperature is above about $150 \mathrm{MeV}$. Since the kaon mass does not change much with temperature, the phi meson can only decay into a pion and a rho meson. Even including the decrease of the rho meson mass at finite temperatures, the decay width of a phi meson is substantially reduced from its width $(\sim 4 \mathrm{MeV})$ in free space.

This decrease of the phi meson mass has led to the proposal for a new signature for the quark-gluon plasma to hadronic matter transition in ultrarelativistic heavy-ion collisions. ${ }^{2}$ Specifically, it was shown that if there is a strong first-order phase transition between the quark-gluon plasma and the hadronic matter, then a double phi peak structure appears in the dilepton invariant mass spectrum. The low mass phi peak results from the decay of phi mesons with reduced in-medium mass in the mixed phase. Furthermore, it has been pointed out that due to the small transverse expansion of the matter during the phase transition, the transverse momentum distribution of these low mass phi mesons offers a viable means for determining the transition temperature.

In the above study, the change of the phi meson width in the medium is, however, not included. The interaction of a phi meson in hot baryon-free hadronic matter has recently been studied in Ref. 3 through the reactions $\phi \pi \rightarrow K K^{*}, \phi K \rightarrow \phi K$, $\phi \rho \rightarrow K \bar{K}$, and $\phi \phi \rightarrow K \bar{K}$. To take into account the complicated structure of the strong interaction vertices, we introduce at the vertex a monopole form factor with a cutoff parameter of $1.8 \mathrm{GeV}$. We also include the imaginary part of the kaon potential for the exchanged kaon. In Ref. 4, the kaon width $\Gamma_{K}$ in a medium has been evaluated and is shown to be about $10 \mathrm{MeV}$ at temperature $T=150 \mathrm{MeV}$, increasing to about $30 \mathrm{MeV}$ at $T=200 \mathrm{MeV}$.

In Fig. 1, we show the temperature dependence of the phi meson width, using $\Gamma_{K}=10$ and $30 \mathrm{MeV}$; the dependence on $\Gamma_{\mathrm{K}}$ is clearly unimportant for $T=$ $150-190 \mathrm{MeV}$. The total width at $T=190 \mathrm{MeV}$ is approximately $8 \mathrm{MeV}$. We note that the broadening due to $\phi \pi \rightarrow K K^{*}$ is larger than that from $\phi K \rightarrow \phi K, \phi \rho \rightarrow K \bar{K}$, and $\phi \phi \rightarrow K \bar{K}$.

The phi meson can also interact elastically with a pion through the rho meson exchange. The $\phi \rho \pi$ coupling constant is, however, an order of magnitude smaller than the $\phi K K$ coupling constant, as shown by the smaller branching ratio for phi decay into pion and tho meson. As a result, the phi-pion elastic 


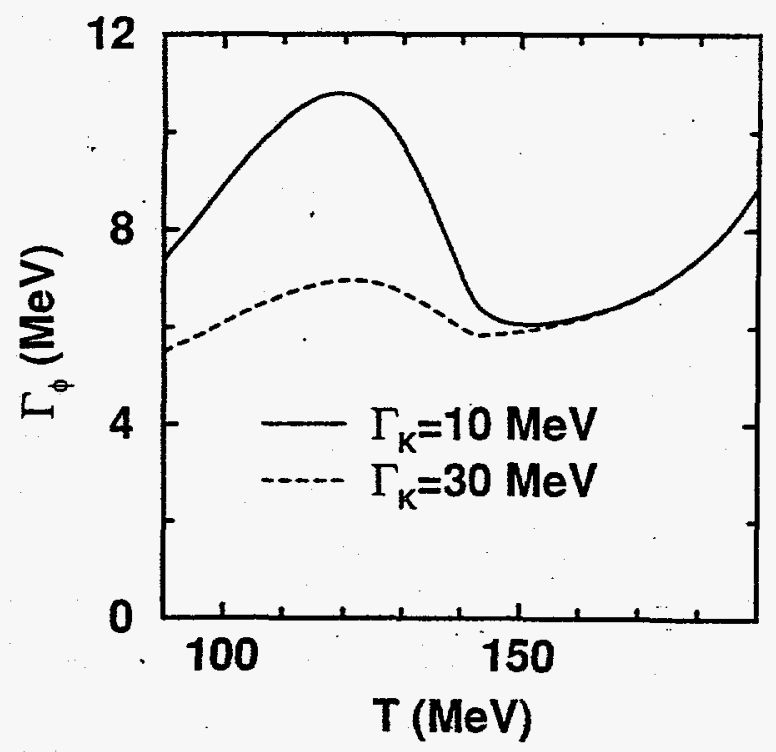

Figure 1. Phi meson width in hot hadronic matter.

scattering, which is proportional to the fourth power of the $\phi \rho \pi$ coupling, has a very small cross section. The phi collisional width due to this reaction turns out to be only a few $\mathrm{KeV}$. The reactions $\phi \rho \rightarrow \phi \rho, \phi \phi \rightarrow \pi \pi$, and $\phi \phi \rightarrow \rho \rho$ are also proportional to the fourth power of the $\phi \rho \pi$ coupling and are expected to be insignificant as well. The reactions $\phi \pi \rightarrow \rho \rho$ and $\phi K \rightarrow \phi K^{*}$ involve the square of the $\phi \rho \pi$ coupling, so their contributions to the phi collisional width are also negligible.

In a mixed-phase matter formed in ultrarelativisticheavy-ion collisions, the phi meson width is further enhanced by the presence of the quark-gluon plasma. However, the latter is seen to increase the phi meson width by only a few $\mathrm{MeV}$ above the width in a pure hadron matter. ${ }^{5}$

The narrow phi meson width justifies the assumption of Ref. 2 . If there is a strong first-order phase transition between the quark-gluon plasma and the hadronic matter in ultrarelativistic heavy-ion collisions, then a low mass secondary phi peak is expected to be observed in the dilepton spectrum. This second peak allows us to infer the transition temperature and the lifetime of the mixed phase in the case of a first-order transition, and also the range of temperatures over which the transition takes place in the case of a smooth but fast transition.

"Physics Department, Kent State University, Kent, OH 44242.

\section{References}

1. M. Asakawa and C. M. Ko, Nucl. Phys. A, in press.

2. C. M. Ko and M. Asakawa, Nucl. Phys. A566, 447c (1994); M. Asakawa and C. M. Ko, Phys. Lett. 322B, 33 (1994).

3. C. M. Ko and D. Seibert, Phys. Rev. C 49, 2198 (1994).

4. E. Shuryak and V. Thorsson, Nucl. Phys. A536, 739 (1992).

5. D. Seibert and C. M. Ko, submitted to Phys. Rev. C. 


\section{Near-Threshold $\mathrm{K}^{+}$Production in Heavy-Ion Collisions}

\section{Bao-An Li}

Kaons have long been proposed as one of the most promising messengers of the primary violent stage of relativistic heavy-ion collisions. ${ }^{1}$ Based on nuclear transport model calculations, it was further demonstrated that kaon production at subthreshold energies may be very useful in studying the nuclear equation of state $^{2}$ and in-medium properties of hadrons in the hot and dense zone formed in the reaction. ${ }^{3}$

The most recent data from the reaction of $\mathrm{Au}+\mathrm{Au}$ at a beam energy of $1.0 \mathrm{GeV} /$ nucleon taken by the KaoS collaboration ${ }^{4}$ at SIS/GSI has further stimulated much interest and theoretical work on kaon production. However, due to large discrepancies among the results of different model calculations, the interpretation of the experimental data has been difficult. To better understand the discrepancies among previous model calculations and most importantly to help interpret the experimental data more accurately, we have performed a study on several aspects of kaon production using a hadronic transport model for relativistic heavy-ion collisions detailed in Ref. 5.

Here we present some typical results of our study. More detailed discussions can be found in Ref. 6. Kaon spectra from seven collision channels $\left(N \Delta, N N, \Delta \Delta, N N^{*}, N^{*} N^{*}, \Delta N^{*}\right.$ and $\left.\pi N\right)$ calculated at $\theta_{\text {lab }}=44^{\circ}$ in the reaction of $\mathrm{Au}+\mathrm{Au}$ at $E_{\text {bean }} / A=$ $1.0 \mathrm{GeV}$ are displayed in Fig. 1. It is interesting to note that in the low momentum part $\left(P_{\text {lab }} \leq 0.5\right.$ $\mathrm{GeV} / \mathrm{c}) N \Delta$ and $N N$ collisions dominate, while in the high momentum part $\pi N$ and $N N^{*}$ collisions dominate. The fact that $\pi N$ collisions are the most important source for high momentum kaons is mainly due to the reaction kinematics.

A comparison between the model calculation and the experimental data is made in Fig. 2. It is seen that the experimental data can be well reproduced with the soft equation of state. We also found that
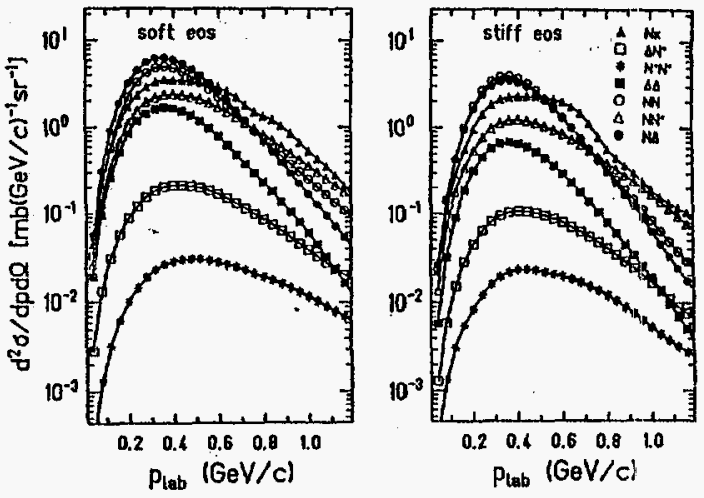

Kigure 1. Kaon spectra from 7 different collision channels in the reaction of $\mathrm{Au}+\mathrm{Au}$ at $E_{\text {beam }} / A=1.0 \mathrm{GeV}$.

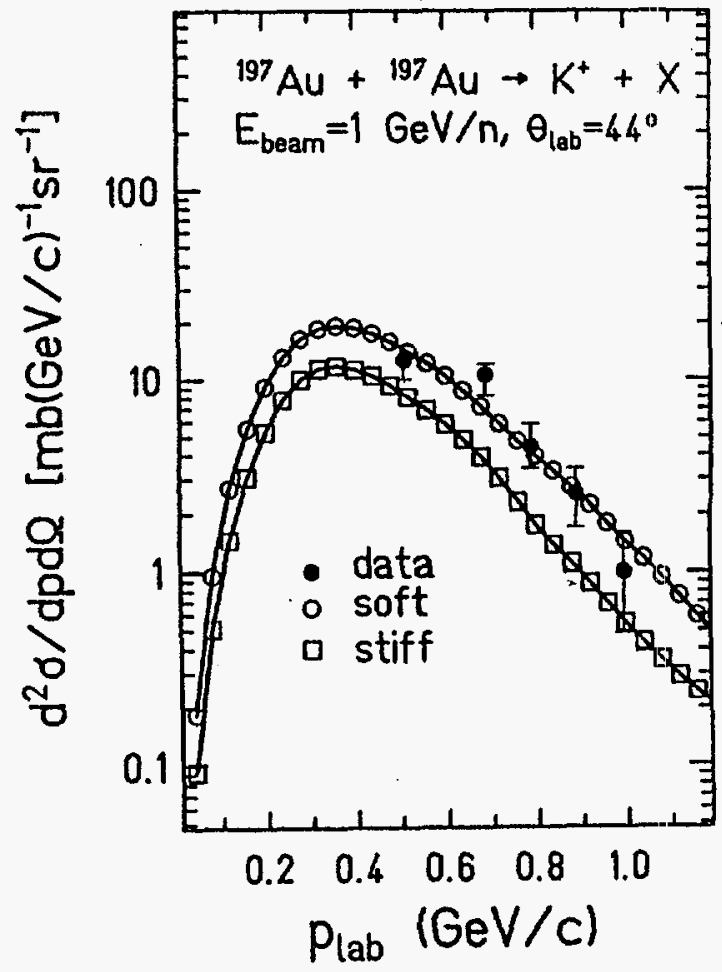

Figure 2. Comparison between the model calculations and the experimental data on kaon spectra from the reaction of $\mathrm{Au}+\mathrm{Au}$ at $E_{\text {beam }} / A=1.0 \mathrm{GeV}$.

with the soft equation of state $\pi^{-}$and $\pi^{+}$spectra from the same reaction can also be well described 
simultaneously as kaons. It indicates that the production of kaons is mainly determined by the $\pi$, $N, \Delta, N^{*}$ dynamics of the reaction and it has been well accounted for in the model. In addition, we found that the finite lifetime of baryon resonances affects significantly the shape of kaon spectra. The widely used frozen-resonance approximation in previous studies underestimates high energy parts of kaon spectra. $N^{*}(1440)$ resonances contribute only about $10 \%$ to the total kaon yield and have a minor effect on the kaon spectra. The initial Fermi momentum transformation has an effect on kaon yields and spectra as large as that of the nuclear equation of state.

\section{References}

1. J. Randrup and C. M. Ko, Nucl. Phys. A343, 529 (1980); A411, 537 (1983).

2. J. Aichelin and C. M. Ko, Phys. Rev. Lett. 55, 2661 (1985).

3. X. S. Fang, C. M. Ko, and Y. M. Zheng, Nucl. Phys. A556, 499 (1993).

4. E. Grosse, Prog. Part. Nucl. Phys. 30, 89 (1993).

5. B. A. Li and W. Bauer, Phys. Lett. 254B, 335 (1991); Phys. Rev. C 44, 450 (1991).

6. B. A. Li, TAMU preprint, submitted to Phys. Rev. C (1994).

\section{Mean Free Path and Damping Rate of Kaons in Hot Hadronic Matter}

\section{Chungsik Song}

Studying the properties of hadrons in hot matter can provide us with the precursor phenomena of the phase transition. The investigation of hot hadronic matter is also important to discriminate the signatures of QGP in nucleus-nucleus collisions. To describe hadronic matter below the phase transition, it is convenient to introduce an effective field theory in which mesons and baryons are regarded as elementary fields in an effective lagrangian. The effective lagrangian is constructed from symmetries and the anomaly structure of the fundamental theory and contains some parameters which can be inferred from experimental data. ${ }^{1}$

In this paper the properties of kaons in hot hadronic mater are investigated based on the effective lagrangian. We study the mean free path and damping rate of kaons in hot matter. The interaction of kaons in hadronic matter is described by an effective lagrangian which is invariant under the chiral $S U(3) \times S U(3)$ symmetry. To involve the effect of resonances the vector $\left(V_{\mu}\right)$ and axial-vector mesons $\left(A_{\mu}\right)$ are included as massive Yang-Mills fields of the chiral symmetry. ${ }^{2}$

We calculate the kaon self-energy in hot matter from the effective lagrangian using conventional finite temperature field theory. ${ }^{3}$ We approximate the self-energy with one loop diagrams shown in Fig. 1. It has been shown that hot hadronic matter is rather dilute even at $T \sim 150 \mathrm{MeV}$; the mean free path of pions in hot matter is $2-3 \mathrm{fm}$ at $T \sim 150 \mathrm{MeV} .{ }^{4}$ This fact indicates that the expansion in powers of density should be still meaningful at 100 $\mathrm{MeV}<T<T_{c}$. The leading contribution in this expansion is generated by one loop diagrams. There are diagrams with the same order, which include vector and axial-vector mesons only. Their 
contributions are almost negligible due to the large mass in the Boltzman factor ${ }^{4}$ and neglected in the present calculation.

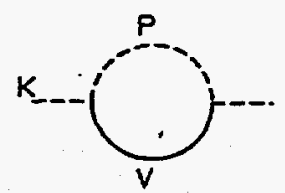

(a)

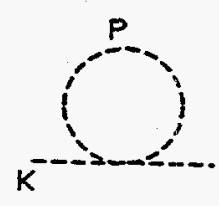

(b)
Figure 1. One-loop diagrams for the kaon self-energy. $P$ indicates pseudoscalar meson and $\boldsymbol{V}$ does vector meson. For (a) we consider $\left(\eta, K^{*}\right),\left(\pi, K^{*}\right),(K, \rho),(K, \omega),(K, \phi)$ meson loops and $(\pi, K, \eta)$ meson loops for (b).

The mean free path and damping rate of kaons in hot matter can be obtained from the imaginary part of the self-energy in the medium. Since the "tadpole" diagrams do not contain the imaginary part, we have contributions from pseudoscalar $(P)$ and vector $(V)$ meson loops. However, there are no contributions from the $\left(\eta, K^{*}\right),(K, \rho)$, and $(K, \omega)$ loops due to the kinematic reason. The imaginary part of the kaon self-energy is given by

$$
\operatorname{Im} \Pi_{K}=\frac{3}{8} \operatorname{Im} \Pi_{\pi K^{*}}+\frac{1}{4} \operatorname{Im} \Pi_{K \phi},
$$

where $\operatorname{Im} \Pi_{P V}$ is the contribution from pseudoscalar $(P)$ and vector $(V)$ meson loops. The mean free path of kaons in hot matter, $\lambda$, is obtained from the imaginary part of the self-energy as

$$
\lambda=-\frac{k}{\operatorname{Im}[\Pi(\omega, k)]} \text {. }
$$

The result is given in Fig. 2. We find that the mean free path depends on the momentum and continuously increases with momentum. The linear increase implies that the imaginary part of the self-energy is almost constant in momentum. The mean free path tends to zero as $k \rightarrow 0$, which is consistent with the fact the collision time for a massive particle at rest is finite. For kaons with high momentum, the mean free path is very large and might be bigger than the size of hot matter which is formed in the nucleus-nucleus collision. It may be possible that kaons with high momentum can escape hot matter without interaction and have no chance to be thermalized.

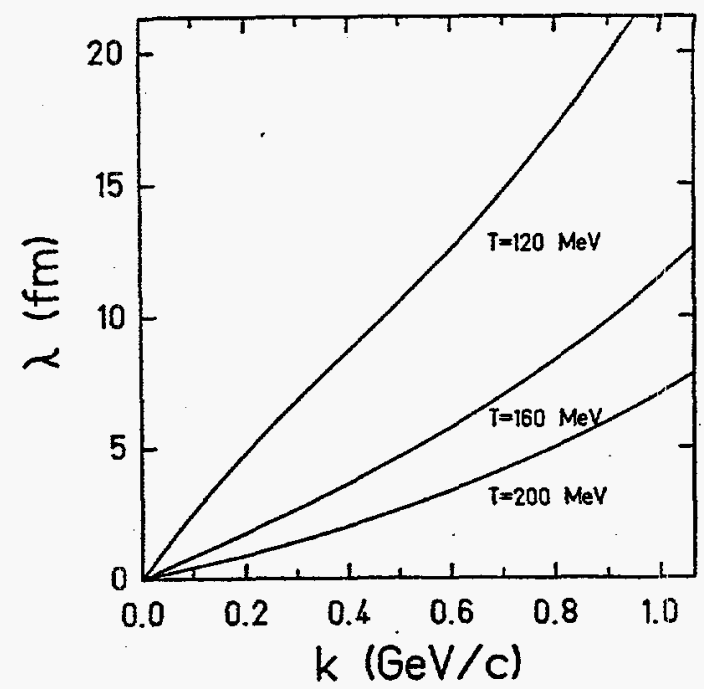

Figure 2. Mean free path of kaons in hot hadronic matter. The results are shown at $T=120,160$, and $200 \mathrm{MeV}$.

The damping rate is defined by

$$
\gamma^{T}(k)=\frac{-\operatorname{Im} \Pi(\omega, k)}{\omega}
$$

which can be related to the pure absorption of a particle in the medium. Since a particle can be absorbed and produced in a heat bath, the pure absorption of the particle is given by the difference of the absorption $\left(\gamma_{a b s}\right)$ and production rate $\left(\gamma_{p r o}\right)$;

$$
\gamma^{T}(k)=\gamma_{a b s}-\gamma_{p r o}
$$

We take the thermal average of the damping rate and the result is shown and compared with that of pions in hot matter in Fig. $3 .^{5}$ We find that the averaged 
damping rate almost negligible at low temperature but increases with temperature when $T>100 \mathrm{MeV}$. Such an increase of the damping rate might be related to the "melting" of hadronic degrees of freedom in hot matter as temperature increases. When we compare with the result for pion the damping rate increases from $T \sim 100 \mathrm{MeV}$ in both cases but the kaon damping rate slowly increases.

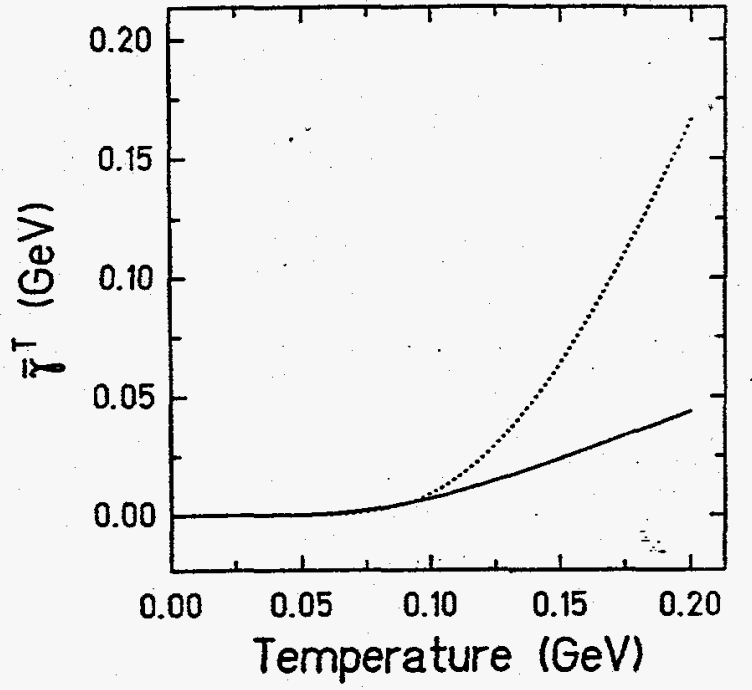

Figure 3. The thermal average of the damping rate of kaons at finite temperature. The dotted line is the result for pions.

In summary, the mean free path of kaon continuously increases with momentum and, for kaons with high momentum, may be larger than the size of the hot matter which is formed in nucleus-nucleus collisions. It is possible that the kaons with high momentum cannot be thermalized in hot matter. The non-equilibration of kaons might have signatures in kaon production as the same as in pion production. ${ }^{6}$ The thermal average of the damping rate increases with temperature as $T>100$ $\mathrm{MeV}$. This implies that the kaonic collective modes in hot matter have large damping rate and hardly propagates as temperature increases. This fact is related to the melting of the hadronic degrees of freedom as temperature gets close to the critical value. The damping rate increases slowly compared with the result for pions in hot matter. The modification in damping rate will affect the dilepton emission from hot hadronic matter. The investigation in this direction is now in progress.

\section{References}

1. S. Weinberg, Phys. Rev. Lett. 18, 188 (1967); Phys. Rev. 166, 1568 (1968); Physica 96A, 327 (1979).

2. S. Gasiorowicz and D. Geffen, Rev. Mod. Phys. 41, 531 (1969).

3. J. I. Kapusta, Finite Temperature Field Theory, (Cambridge University Press, Cambridge, 1989).

4. Chungsik Song, Phys. Rev. D 49, 1556 (1994).

5. Chungsik Song, to appear in Phys. Lett. B.

6. M. Kataja and P. Ruuskanen, Phys. Lett. 243B, 181 (1990). 


\section{Effects of $\mathrm{N}^{*}(1440)$ Resonance on Subthreshold Kaon, Antikaon, and Antiproton Production}

$$
\text { B. A. Li, C. M. Ko and G. Q. Li }
$$

To study the properties of hadrons in the hot and dense matter, which may be different from those in free space, ${ }^{1,2}$ has been one of the motivations for relativistic heavy-ion research. It has been shown recently via the relativistic transport model that the attractive scalar mean-field potential, which leads to a reduction of particle production thresholds in a medium, is required to account for the measured yields of kaons, ${ }^{3}$ antikaons, ${ }^{4}$ and antiprotons ${ }^{5}$ from heavy-ion collisions at subthreshold energies. In these studies, higher resonances other than the delta have been ignored. To extract information of the scalar mean-field potential for kaons, antikaons, and antiprotons from heavy-ion collisions, it is important to know the contribution of higher resonances to their production.

Based on the hadronic transport model for heavy-ion collisions, as described in details in Refs. 6 and 7, we have studied the effects of $N^{*}(1440)$ on subthreshold particle production. This model includes both $\Delta(1232)$ and $N^{*}(1440)$ resonances. The maximum number of $N .(1440)$ resonance increases from 7 to 17 as the beam energy increases from 1 to $2 \mathrm{GeV} /$ nucleon. For comparisons, it should be mentioned that the maximum number of $\Delta(1232)$ resonance increases from 52 to 89 in the same collision, and therefore the ratio of the abundance for the $N^{*}$ and the $\Delta$ resonance increases from $14 \%$ to $19 \%$ as the beam energy increases from 1 to 2 $\mathrm{GeV} /$ nucleon.

Our results on the production of kaons in $\mathrm{Au}+\mathrm{Au}$ collisions at beam energies between 1 and 2 $\mathrm{GeV} /$ nucleon and at an impact parameter $b=1 \mathrm{fm}$ are shown in Fig. 1. The total kaon production probability includes contributions from $N N, N \Delta, N N^{*}$, $\Delta \Delta, N^{*} N^{*}, \Delta N^{*}$ and $N \pi$ collisions. In the figure, the total probabilities are shown with the solid lines while the contributions from the $N^{*}$ involved collisions are shown with the dashed lines. It is seen that, the $N^{*}$ involved collisions contribute only about $11 \%$ in the whole energy range.

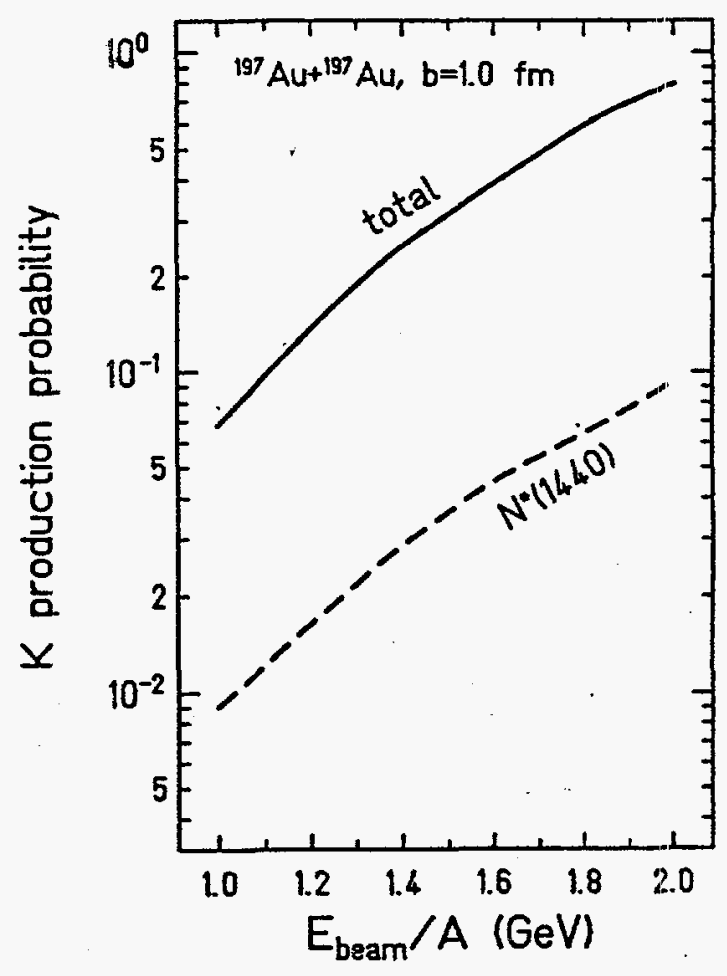

Figure 1. Total (solid) and $N(1440)$ induced (dashed) kaon production probability in the $\mathrm{Au}+\mathrm{Au}$ collisions for different beam energies.

Antikaon and antiproton production probabilities in the $\mathrm{Ni}+\mathrm{Ni}$ collisions at a beam energy of 1.85 $\mathrm{GeV} /$ nucleon and at an impact parameter $b=1 \mathrm{fm}$ are shown in Fig. 2 and Fig. 3, respectively. It is seen that at both beam energies the $N^{*}$ involved collisions contribute about $25 \%$ to the total antikaon yields. While for antiproton production the $N^{*}$ involved collisions contribute about $90 \%$ at 1.85 $\mathrm{GeV} /$ nucleon, this contribution decreases to about $70 \%$ at $2.1 \mathrm{GeV} /$ nucleon. This finding indicates that antiproton production at subthreshold energies may serve as a possible probe of $N^{*}(1440)$ resonance in the resonance matter formed in relativistic heavy-ion 
collisions.

In the above calculations, free-space properties of baryon resonances, i.e. their masses and widths, are used. In nuclei the decreasing mass and broadening width of $\Delta$ resonance are well known ${ }^{8}$ and have been

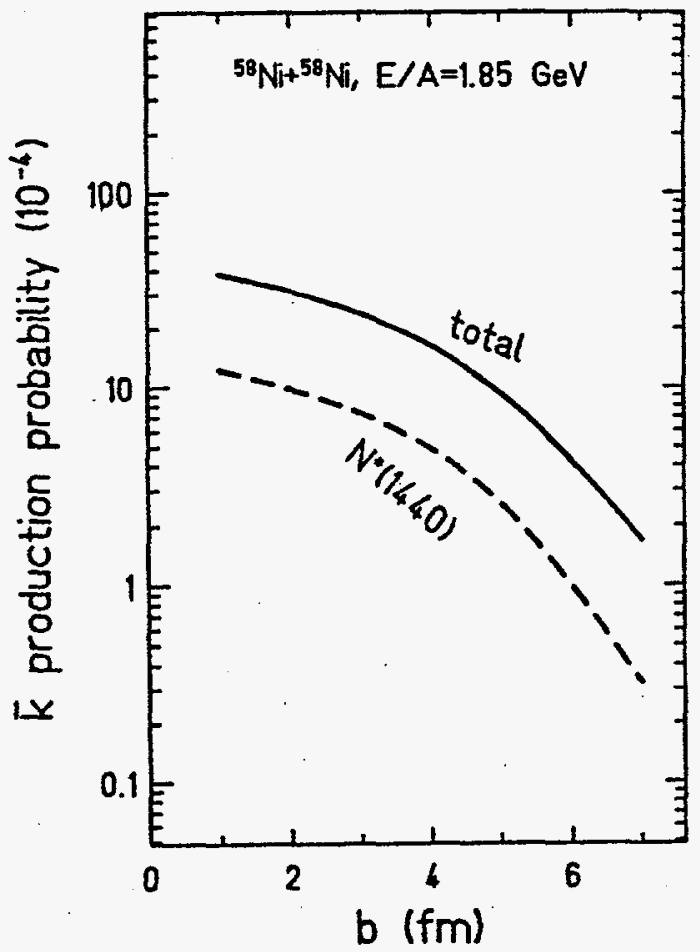

Figure 2. Total (solid) and $N^{*}(1440)$ induced (dashed) antikaon production probability in $\mathrm{Ni}+\mathrm{Ni}$ collisions at $1.85 \mathrm{GeV}$.

a subject of much interest. On the contrary, little is known about the in-medium behavior of higher resonances. The very recent analysis of photoabsorption cross sections on nuclei for photon energies between 500 and $1500 \mathrm{MeV}$ has shown that widths of higher resonances in medium are almost twice as large as those in free space, ${ }^{9}$ and thus there is a large overlapping between resonances. To see how the broadening of baryon resonances may change the effects of $N^{*}$ resonance discussed above, we have performed calculations by doubling the widths of $\Delta$ and $N^{*}$ resonance.

We have found that by doubling the widths of the resonances the total kaon production probability is slightly increased and the contribution from the $N^{*}$ involved collisions decrease to about $7 \%$. For antikaons the $N^{*}$ contribution decreases from $28 \%$ to
$20 \%$. For antiprotons, doubling the resonance widths results in a reduction of both the total and the $N^{*}$ involved production probability by about a factor of 3. Furthermore, the $N^{*}$ contribution is reduced from $70 \%$ to $65 \%$.

Another important in-medium effect is the changing masses of hadrons in a dense medium. This effect on the production of subthreshold kaons, antikaons, and antiprotons has been studied extensively. ${ }^{3.5}$ As the $N^{*}$ resonance is found

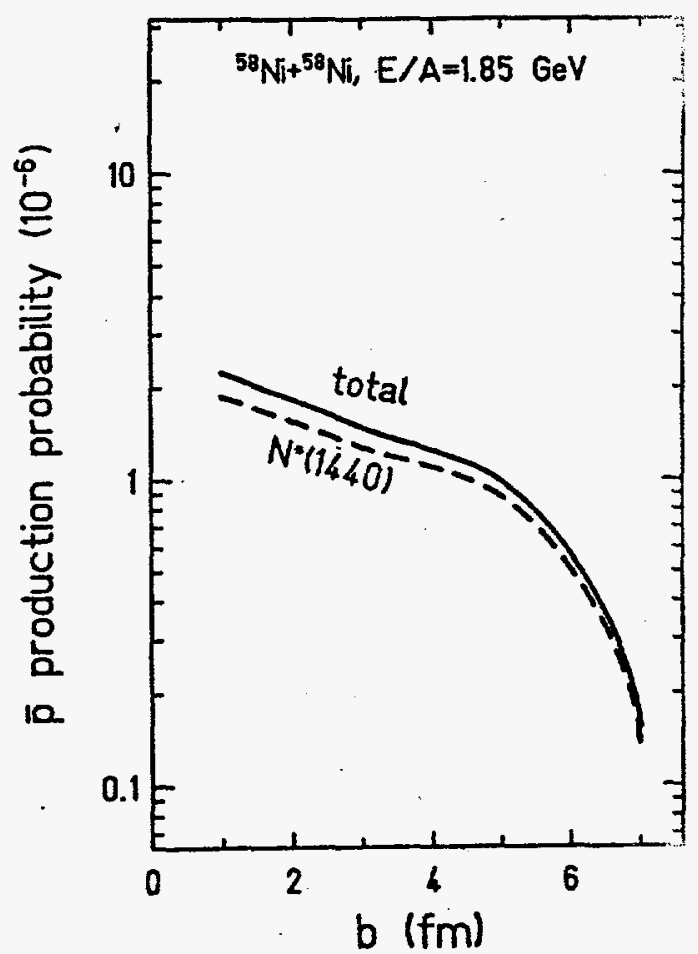

Figure 3. Total (solid) and $N^{*}(1440)$ induced (dashed) antiproton production probability in $\mathrm{Ni}+\mathrm{Ni}$ collisions at $1.85 \mathrm{GeV}$.

to have the most important effect on subthreshold antiproton production, we now study schematically how this effect may change if in-medium masses of hadrons are used. For this purpose, we use the empirical density-dependent nucleon effective mass in a medium as proposed in Refs. 1 and 2 for the two produced particles in the reaction $B B \rightarrow N N p \bar{p}$. Consequently, the density-dependent threshold for antiproton production in a nuclear medium can be written as

$$
T_{\bar{p}}=2 m_{n}\left[1+1 /\left(1+0.25 \rho / \rho_{0}\right)\right],
$$


here $m_{n}$ is the free nucleon mass and $\rho_{0}$ is the normal nuclear matter density. Using the reduced, density-dependent threshold we have found that the total and the $N^{*}$ involved antiproton yield increase by a factor of 35 and 27 , respectively, in the $\mathrm{Ni}+\mathrm{Ni}$ collision at a beam energy of $2.1 \mathrm{GeV} /$ nucleon and an impact parameter of $1.0 \mathrm{fm}$. The contribution of the $N^{*}$ involved collisions decreases to about $50 \%$ of the total probability.

Since the contribution of $N^{*}$ resonance to subthreshold kaon and antikaon production is small compared with that from the delta resonance. The results of Refs. 3 and 4 obtained without the $N^{*}$ resonance are therefore not much affected. However, the $N^{*}$ resonance does contribute appreciably to antiproton production from heavy-ion collisions at subthreshold energies. Since the medium effects due to the attractive scalar mean field are much larger than the effect from the $N^{*}$ resonance, the conclusion of Ref. 5 that the observed antiproton yield in subthreshold heavy-ion collisions is consistent with a reduced threshold as a result of the reduction in the in-medium antiproton mass remains valid. However, to study more quantitatively the in-medium effects on antiproton production, we need to include in the future the $N^{*}$ resonance. We therefore conclude that antiproton production from heavy-ion collisions at subthreshold energies not only provides information on the in-medium properties of the antiproton but also serve as a possible probe of the $N^{*}(1440)$ resonance in the resonance matter.

\section{References}

1. G. E. Brown, Nucl. Phys. A522, 397c (1991), and references therein.

2. M. Asakawa, C. M. Ko, P. Lévai and X. J. Qiu, Phys. Rev. C 46, R1159 (1992); M. Asakawa and C. M. Ko, Nucl. Phys. A560, 399 (1993).

3. X. S. Fang, C. M. Ko, and Y. M. Zheng, Nucl. Phys. A556, 499 (1993); X. S. Fang, C. M. Ko, G. Q. Li and Y. M. Zheng, Phys. Rev. C 49, R608 (1994).

4. G. Q. Li, C. M. Ko, X. S. Fang, and Y. M. Zheng, Phys. Rev. C 49, 1139 (1994); G. Q. Li and C. M. Ko, submitted to Phys. Rev. C.

5. G. Q. Li, C. M. Ko, and X. S. Fang, submitted to Phys. Lett. B.

6. B. A. Li and W. Bauer, Phys. Lett. 254B, 335 (1991); Phys. Rev. C 44, 450 (1991).

7. B. A. Li, W. Bauer and G. F. Bertsch, Phys. Rev. C 44, 2095 (1991).

8. E. Oset, H. Toki and W. Weise, Phys. Rep. 83, 282 (1982).

9. W. M. Alberico, G. Gervino and A. Lavagno, Phys. Lett. 321B, 177 (1994). 


\title{
Subthreshold Antikaon Production in Nucleus-Nucleus Collisions
}

\author{
G. Q. Li, C. M. Ko, and X. S. Fang
}

The possibility of $s$-wave kaon condensation in a dense nuclear matter was first suggested by Kaplan and Nelson ${ }^{1}$ and further studied by Brown et al. ${ }^{2}$ In the mean-field approximation to chiral Lagrangian and including both the scalar and vector interactions of a kaon with nucleons, we find that the kaon mass increases with density while the antikaon mass decreases with density, and the decrease of the antikaon mass is more significant than the increase of the kaon mass. The antikaon production threshold in the reaction $B B \rightarrow N N K \bar{K}$ is thus reduced in the medium, and this medium effect plays an important role in the antikaon production in nucleus-nucleus collisions at subthreshold energies. We have recently shown that the attractive scalar potential for kaon and antikaon is needed to explain the recent data from the SIS at GSI on antikaon production from heavy-ion collisions at subthreshold energies. ${ }^{3}$

Our studies are based on the relativistic transport model developed in Ref. 4. At energies considered in this work, the colliding system consists mainly of nucleons, deltas and pions. While pions are treated as free particles, nucleons and deltas are propagated in their mean-field potentials according to the classical equations of motion. The nucleon mean-field potentials are taken from the Dirac-Brueckner calculation with the Bonn nucleon-nucleon interaction. The elastic $(N N \rightarrow N N$, $N \Delta \rightarrow N \Delta$, and $\Delta \Delta \rightarrow \Delta \Delta)$ and inelastic ( $N N \leftrightarrow N \Delta, \Delta \leftrightarrow N \pi$ ) reactions among nucleons, deltas and pions are also included. The standard Cugnon parametrization and detailed-balance prescription are used for describing these reactions.

We use the parametrization of Ref. 5 for antikaon production cross section in the baryon-baryon interaction. Medium effects are taken into account by evaluating $p_{\max }$ of the produced antikaon using the in-medium masses. Antikaon production from baryon-baryon collisions is treated perturbatively as the production probability is very small. The propagation and interaction of produced antikaons in the medium are, however, included explicitly. The $K^{*} N$ elastic cross section $\sigma_{\text {ela }}$ and reaction cross sections are taken from Ref. 6.

We have carried out calculations for a ${ }^{58} \mathrm{Ni}+{ }^{59} \mathrm{Ni}$ collision at $1.85 \mathrm{GeV} /$ nucleon. The results are shown in Fig. 1. The dotted curve gives the results using the free kaon and antikaon masses. The results with the in-medium masses of kaon and antikaon are shown by the solid curve. Also shown in the figure by solid circles are recent experimental data from SIS at GSI. ${ }^{3}$ Using the free kaon and antikaon masses, our theoretical results are about a factor of 5-10 smaller than the experimental values. The calculation using the in-medium masses of kaon and antikaon leads to a factor of 4-5 enhancement of the antikaon yield. The theoretical results are now in reasonable agreement with the experimental data, except at $p_{\text {lab }} \bar{K}=1.0 \mathrm{GeV} / \mathrm{c}$ where our result still underestimates the experimental data by about a factor of two.

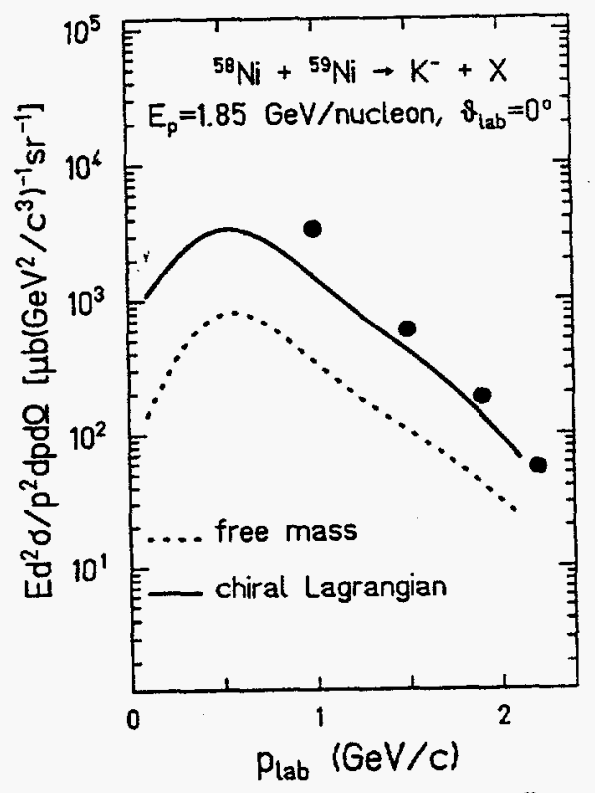

Figure 1. Antikaon production cross section in the ${ }^{58} \mathrm{Ni}+{ }^{59} \mathrm{Ni}$ collisions at $1.85 \mathrm{GeV} /$ nucleon. The solid curve gives the results using in-medium masses of kaon and antikaon, while the dotted curve gives the results using masses of kaon and antikaon in free space. Solid circles are the experimental data from Ref. 3. 
The effect of the attractive scalar potential of kaon and antikaon is expected to be more significant in antikaon production from nucleus-nucleus collisions at incident energies below 1.85 $\mathrm{GeV} /$ nucleon. To demonstrate this, we have carried out a similar calculation for the antikaon production cross section from the ${ }^{197} \mathrm{Au}+{ }^{197} \mathrm{Au}$ collision at 1 $\mathrm{GeV} /$ nucleon. The results are shown in Fig. 2.

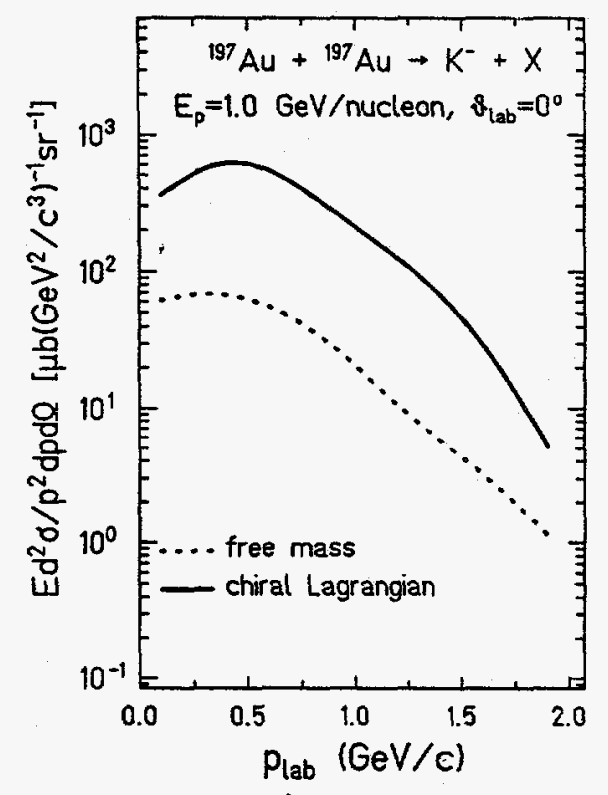

Figure 2. Same as Fig. 1 for ${ }^{197} \mathrm{Au}+{ }^{197} \mathrm{Au}$ collisions at 1 GeV/nucleon.
Indeed, the enhancement of the antikaon yield due to the attractive scalar potential of kaon and antikaon is more visible than the case at $1.85 \mathrm{GeV} /$ nucleon. Compared with the results using their masses in free space, the antikaon production cross section is increased by almost one order of magnitude. We therefore suggest that experimental measurements be carried out for this reaction.

\section{References}

1. D. B. Kaplan and A. E. Nelson, Phys. Lett. 175B, 57 (1986); A. E. Nelson and D. B. Kaplan, Phys. Lett. 192B, 193 (1987).

2. G. E. Brown, K. Kubodera, and M. Rho, Phys. Lett. 192B, 273 (1987); G. E. Brown, C. M. Ko, and K. Kubodera, Z. Phys. A341, 301 (1992).

3. A. Schröter et al., Nucl. Phys. A553, 775c (1993).

4. C. M. Ko, Q. Li, and R. Wang, Phys. Rev. Lett. 59, 1084 (1987); C. M. Ko and Q. Li, Phys. Rev. C 37, 2270 (1988).

5. W. Zwermann and B. Schürmann, Phys. Lett. 145B, 1 (1984).

6. C. B. Dover and G. E. Walker, Phys. Rep. 89, 1 (1982). 


\title{
Antiproton Production in Heavy-Ion Collisions at Subthreshold Energies
}

\author{
G. Q. Li, C. M. Ko, X. S. Fang, and Y. M. Zheng
}

The study of antiproton production in heavy-ion collisions at subthreshold energies has been a topic of great interests. Because of the attractive scalar field, both the nucleon and antinucleon masses are reduced in a medium. Assuming that the antiproton self-energies in a medium are given by the G-parity transformation of the nucleon self-energies, then the vector potential for the antiproton has an opposite sign from that for the nucleon. The vector potential therefore does not play any role in antiproton production as an antiproton is produced together with a nucleon. The reduction of nucleon and antinucleon masses in the medium then reduces the antiproton production threshold and enhances thus the primordial antiproton production in the dense matter formed in nucleus-nucleus collisions.

We have studied the antiproton production from nucleus-nucleus collisions at subthreshold energies in the relativistic Vlasov-Uehling-Uhlenbeck (RVUU) model. ${ }^{1}$ Extending the RVUU model to include the antiproton degree of freedom, we are able to include the medium effects on antiprotons and treat consistently their production, propagation, rescattering, and annihilation.

Antiprotons are mainly produced from $B_{1} B_{2} \rightarrow N N p \bar{p}$, where $B_{1}$ and $B_{2}$ are either a nucleon or a delta. The elementary antiproton production cross section in a medium is parametrized as

$$
\sigma_{B_{1}, B_{2}}^{\vec{p}}\left(\sqrt{s^{*}}\right)=0.012\left(\sqrt{s^{*}}-\sqrt{s_{0}^{*}}\right) .
$$

The total center-of-mass energy $\sqrt{s^{*}}$ of the colliding pair of baryons is $\sqrt{s^{*}}=\left(p_{1}^{*}+m_{1}^{*}\right)^{1 / 2}+\left(p_{2}^{*}+m_{2}^{*}\right)^{1 / 2}$, where $m_{i}^{*}$ and $p_{i}^{*}(i=1,2)$ are, respectively, the effective mass and kinetic momentum of the colliding baryons. The antiproton production threshold in the medium is $\sqrt{s_{0}^{*}}=4 m^{*}$.

The final-state interactions of primordial antiprotons with baryons are explicitly treated in our calculation. These include the propagation of antiprotons in the mean-field potential and their elastic scattering and annihilation by baryons. The mean-field potential is determined from the self-energies of the antiproton. For both elastic scattering and annihilation, the cross sections in free space as parametrized in Ref. 2 are used in the calculation.

The comparison of our theoretical results with the experimental data is given in Fig. 1 for $\mathrm{Ni}+\mathrm{Ni}$ collisions at $1.85 \mathrm{GeV} /$ nucleon. The dashed curve gives the results for the primordial antiprotons, while the solid curve is the final antiproton spectrum with all final-state interactions taken into account. The experimental data from SIS $^{3}$ are shown in the figure by solid circles. It is seen that the theoretical results are in reasonable agreement with the data. The calculated cross section at $p_{l a b}=1.0 \mathrm{GeV} / \mathrm{c}$ is somewhat below the experimental value which has, however, a large uncertainty.

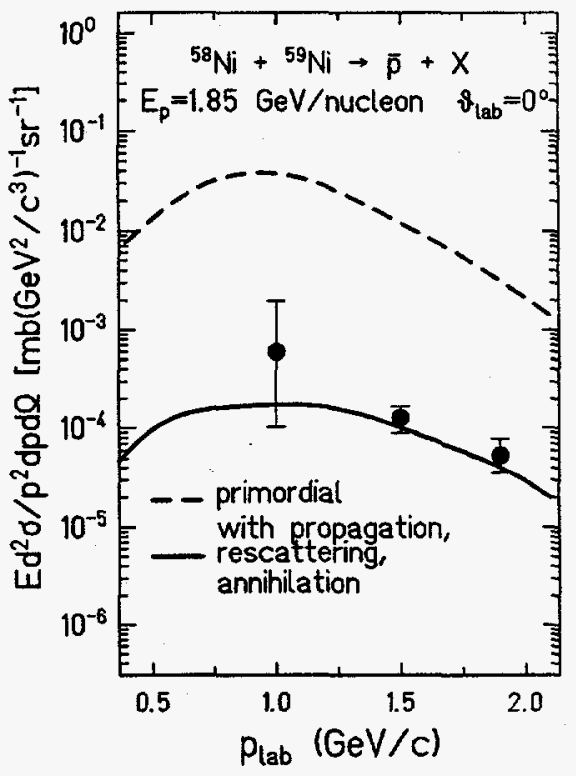

Figure 1. Antiproton momentum spectrum at $\theta_{k b b}=0^{\circ}$ in a $\mathrm{Ni}+\mathrm{Ni}$ collision at $1.85 \mathrm{GeV} /$ nucleon. The dashed curve is for the primordial antiprotons, while the solid curve is the final results obtained with antiproton propagation, elastic rescattering, and annihilation. The experimental data are taken from Ref. 3 . 
The reduction of in-medium nucleon and antiproton masses leads to an enhancement of primordial antiproton production as a result of the decreasing production threshold. To see this explicitly, we have carried out three calculations for the antiproton production cross section. The first calculation is done in the usual non-relativistic VUU model. In this case, baryon masses do not change with density as the mean-field potential is momentum independent. The result is shown in Fig. 2 by the dotted curve. The other two calculations are carried out in the RVUU model. In one calculation, the bare antiproton mass is used, i.e., only the nucleon mass decreases with density. The threshold in this case is thus $3 m^{*}+m$. The result of this calculation is shown in Fig. 2 by the dashed curve. The antiproton production cross section in this case is enhanced by about a factor of 12 over the result with the bare nucleon mass. In the final calculation, both the nucleon and the antiproton effective mass are used and the threshold is therefore $4 m^{*}$. The result is shown in Fig. 2 by the solid curve. It is seen that the antiproton production cross section is further enhanced by about a factor of 8 as compared to the second case. Overall, the antiproton production cross section is enhanced by about two orders of magnitude due to the reduction of baryon masses in a medium. Our study thus indicates that it is essential to include the attractive scalar potentials for both nucleon and antinucleon in accounting for the measured antiproton yield.

\section{References}

1. C. M. Ko, Q. Li, and R. Wang, Phys. Rev. Lett. 59, 1084 (1987); C. M. Ko and Q. Li, Phys. Rev. C 37, 2270 (1988).

2. J. Cugnon and J. Vandermeulen, Ann. Phys. 14, 49 (1989).

3. A. Schröter, E. Berdermann, H. Geissel, A. Gillitzer, J. Homolka, P. Kienle, W. Koenig, B. Povh, F. Schumacher, and H. Ströher, Nucl. Phys. A553, 775c (1993).

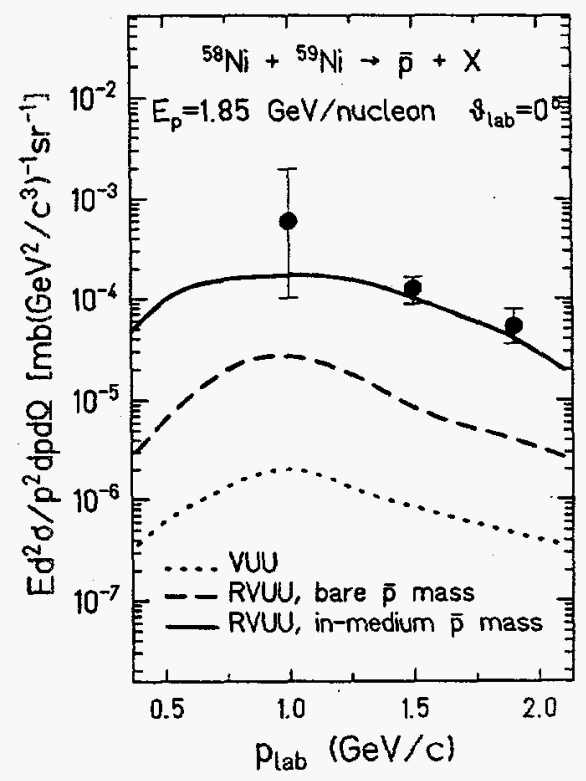

Figure 2. Same as Fig. 1. The dotted curve gives the results obtained from the non-relativistic VUU calculation. The results obtained from the RVUU calculation with the bare antiproton mass are given by the dashed curve. The solid curve gives the results of the RVUU calculation with both the nucleon and antiproton inmedium masses. 


\section{Fixed Exciton Number Level Density for a Finite Potential Well}

\section{S. Shlomo, Ye. A. Bogila**, V. M. Kolomietz ${ }^{* *}$, and A. I. Sanzhur"*}

An essential element of the exciton model of nuclear reactions is the level density $\omega_{n}(E)$ of the nucleus in a state with excitation energy $E$ and $n$ excited quasiparticles. The value of $\omega_{n}(E)$ depends crucially on the single particle level density $g(\epsilon)$. Usually the assumption of a constant single particle level density is made to obtain the density $\omega_{n}(E)$ in a convenient analytical form. ${ }^{1.5}$ Some generalization can be made by taking into account a slow $\epsilon$-dependence of $g(\epsilon)$ at the Fermi energy. ${ }^{6}$ These approaches provide a good approximation in the low energy region, where the influence of the finite depth of the potential well can be neglected. However, increasing the excitation energy $E$ leads to an increase of the partial contribution of high excited single particle states to $\omega_{n}(E)$. Therefore, a proper accounting of the continuum states, ${ }^{7}$ i.e. the decrease of $g(\epsilon)$ with $\epsilon$ and the effect of the diffuseness of the potential well should be taken into account in determining $\omega_{n}(E)$, especially for the case of a low-number of quasiparticle configurations. The aim of this work is to investigate the influence of the finite depth and the diffuseness of the single particle potential on the particle-hole excitation density.

The level density of a nucleus in a state with energy $E$ and $n$ excitons is given by,

$$
\omega_{n}(E)=\omega_{p h}(E)=\int_{0}^{E} d E^{\prime} \omega_{p}\left(E^{\prime}\right) \omega_{h}\left(E-E^{\prime}\right),
$$

where $\omega_{p(h)}(E)=\left.\omega_{p h}(E)\right|_{h=0(p=0)}$ and $p(h)$ is the number of particles (holes). Under the conditions of high excitation energy $E$ and low-number quasiparticle configurations the effect of the Pauli principle in $\omega_{p(h)}(E)$ is neglected and $\omega_{p(h)}(E)$ are proportional to the number of possible accommodations of $p$ particles and $h$ holes of the single-particle levels by the condition of energy conservation. Thus, the density $\omega_{p(h)}(E)$ is related to the single-particle level density $g(\epsilon)$ by the definition. ${ }^{8}$

$$
\begin{aligned}
& \omega_{p}(E)=\frac{1}{p !} \int d \tilde{\epsilon}_{1} g\left(\epsilon_{1}\right) \ldots \int d \bar{\epsilon}_{p} g\left(\epsilon_{p}\right) \delta\left(E-\sum_{i=1}^{p} \bar{\epsilon}_{i}\right),(2) \\
& \omega_{h}(E)=\frac{1}{h !} \int d \tilde{\epsilon}_{1} g\left(\epsilon_{1}\right) \ldots \int d \tilde{\epsilon}_{h} g\left(\epsilon_{h}\right) \delta\left(E-\sum_{i=1}^{n} \tilde{\epsilon}_{j}\right),(3)
\end{aligned}
$$

where $\tilde{\epsilon}_{i}=\epsilon_{i}-\epsilon_{F}\left(\tilde{\epsilon}_{j}=\epsilon_{F}-\epsilon_{j}\right)$ is the energy of $i$-th particle ( $j$-th hole) at the corresponding single-particle level and $\epsilon_{F}$ is the Fermi energy.

The simplest equidistant spacing approximation, when $g(\epsilon)=g\left(\epsilon_{F}\right) \equiv g_{F}$, gives from (1) - (3) the known Ericson-Strutinsky formula

$$
\omega_{p h}^{E S}(E)=\frac{g_{F}\left(g_{F} E\right)^{p+h-1}}{p ! h !(p+h-1) !}
$$

This result can be improved ${ }^{6}$ by considering a slow energy dependence of $g(\epsilon)$ at $\epsilon \approx \epsilon_{F}$.

$$
g(\epsilon) \approx g_{F}+g_{f}^{\prime}\left(\epsilon-\epsilon_{F}\right)+\frac{1}{2} g_{F}^{\prime \prime}\left(\epsilon-\epsilon_{F}\right)^{2}
$$

where $g_{F}{ }^{\prime}$ and $g^{\prime \prime}{ }_{F}$ are the first and second derivative of $g(\epsilon)$ at $\epsilon=\epsilon_{F}$, respectively. The final result obtained from (1)-(3) and (5) is the following,

$$
\begin{aligned}
& \omega_{p h}(E)=\omega_{p h}^{E S}(E)\left\{1+\Delta\left(\frac{g_{F}{ }^{\prime}}{g_{F}}\right) \frac{E}{n}+\left[\frac{\Delta^{2}-n}{2}\left(\frac{g_{F}{ }^{\prime}}{g_{F}}\right)^{2}\right.\right. \\
& \left.+n\left(\frac{g_{F}^{\prime \prime}}{g_{F}}\right)\right] \frac{E^{2}}{n(n+1)}+\left[\frac{\Delta^{3}-3 n \Delta+2 \Delta}{6}\left(\frac{g_{F}{ }^{\prime}}{g_{F}}\right)^{3}\right. \\
& \left.+\Delta(n-1)\left(\frac{g_{F}^{\prime} g^{\prime \prime}}{g_{F}^{2}}\right)\right] \frac{E^{3}}{n(n+1)(n+2)} \\
& +\left[\frac{\Delta^{4}-6 \Delta^{2} n+8 \Delta^{2}+3 n^{2}-6 n}{24}\left(\frac{g_{f}^{\prime \prime}}{g_{F}}\right)^{4}\right. \\
& +\frac{\Delta^{2} n-2 \Delta^{2}-n^{2}+2 n}{2}\left(\frac{g_{F}^{\prime}}{g_{F}}\right)^{2}\left(\frac{g_{F}^{\prime \prime}}{g_{F}}\right)^{2} \\
& \left.\left.+\frac{n^{2}-n}{2}\left(\frac{g_{F}^{\prime \prime}}{g_{F}}\right)^{2}\right] \frac{E^{4}}{n(n+1)(n+2)(n+3)}+\ldots\right\},
\end{aligned}
$$


where $n=p+h$ and $\Delta=p-h$.

We shall compare below the results of numerical calculations of the level density $\omega_{p h}(E)$ for these three cases using eqs. (1)-(3), (4) and (6). In the numerical calculations we shall consider two model potentials: (i) Infinite square-well (SQ) potential and (ii) Finite trapezoidal (TR) potential. The corresponding Thomas-Fermi expressions for the single particle level density and the parameters of these potentials are given in Ref. 7.

To show the influence of the finite depth of the potential well on the exciton level density in a transparent way we shall consider the energy behavior of the value

$$
\xi_{p h}(E)=\omega_{p h}(E) / \omega_{p h}^{E S}(E),
$$

where $\omega_{p h}^{E S}(E)$ is given by eq. (4).

The results for ${ }^{40} \mathrm{Ca}$ are presented in Fig. 1. The deviation of $\xi_{p h}(E)$ from 1 reflects the influence of the energy dependence of the single particle level density $g(\epsilon)$. The effect of diffuseness in the $T R$ potential is exhibited as a faster increase of $g(\epsilon)$ as compared to that of the $S Q$ potential well. Thus in the case of $T R$ potential the equidistant spacing approximation becomes more crude and the value of $\xi_{p h}^{T R}$ exceeds $\xi_{p h}^{S Q}$. This is clearly seen in Fig. 1 by considering the cases of the infinite $S Q$ and $T R$ potential wells. Similar results were obtained for ${ }^{208} \mathrm{~Pb}$. A detailed discussion can be found in Ref. 9 .

"Supported in part by the U.S. National Science Foundation under grant \#PHY9107008.

\section{References}

1. T. Ericson, Adv. in Phys. 9, 425 (1960).

2. F. C. Williams, Nucl. Phys. A166, 231 (1971).

3. C. Kalbach, Z. Phys. A332, 157 (1989).

4. E. Beták, J. Dobes, Z. Phys. A279, 319 (1976).

5. C. K. Cline, Nucl. Phys. A174, 73 (1971).

6. Ye. A. Bogila, V. M. Kolomietz and A. I. Sanzhur, Z. Phys. A341, 373 (1992).

7. S. Shlomo, Nucl. Phys. A539, 17 (1992).

8. E. Gadioli, E. Gadioli Erba and P. G. Sona, Nucl. A207, 589 (1973).

9. S. Shlomo, Ye. A. Bogila, V. M. Kolomietz and A. I. Sanzhur, Submitted for publication.
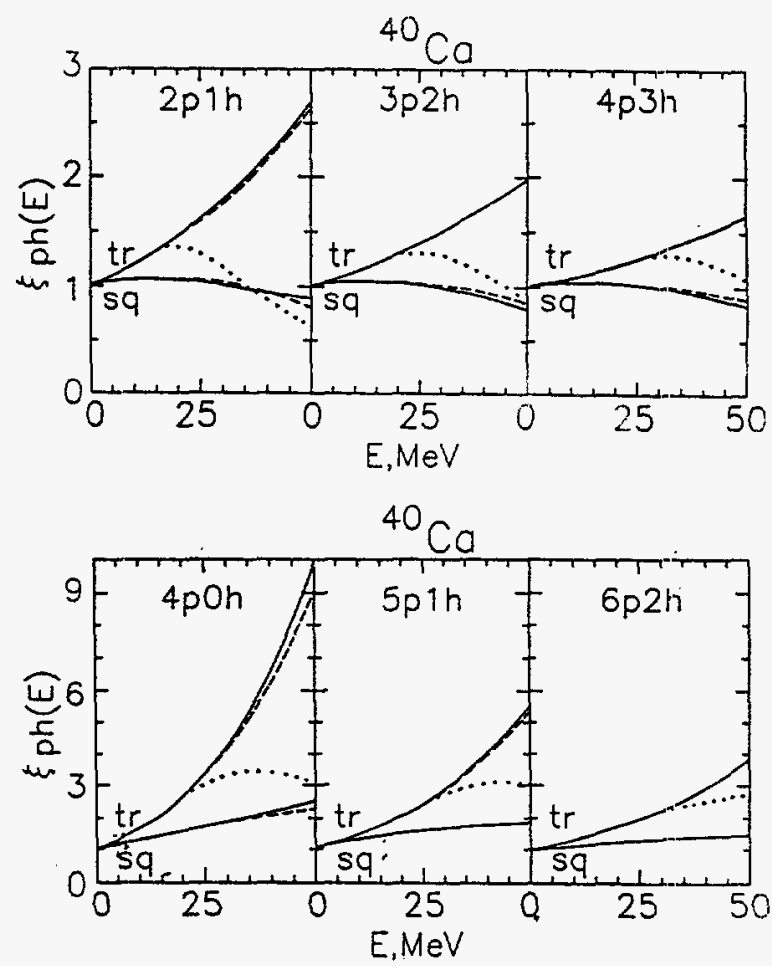

Figure 1. The function $\xi_{p h}(E)$, eq. (7), for the nucleus "Ca. Solid lines are numerical calculations according to the convolution formulas eqs. (1) - (3) for the infinite trapezoidal (tr) and square (sq) potentials, dotted lines are the same calculations for the finite depth trapezoidal potential well and dashed lines are calculations using eq. (6). 


\section{Few-Body Problems in Nuclear Astrophysics. Asymptotic Behavior of Bound State Wave Functions in Two-Body Channels and Radiative Capture Reactions}

\section{D. Blokhintsev* and A. M. Mukhamedzhanov}

Many nuclear reactions which are of interest for nuclear astrophysics are totally peripheral due to the strong Coulomb repulsion in the entrance channel. That is why when calculating the cross sections of such reactions it is important to know the tail of the many-particle bound state wave functions in two-body channels. Consider the direct radiative capture $b+$ $c \rightarrow a+\gamma$ at relative kinetic energy of colliding particles $E_{i} \rightarrow 0$. The reaction amplitude is

$$
M=<\varphi_{a}\left(\zeta_{b}, \zeta_{c} ; \vec{\rho}_{b c}\right)|O| \varphi_{b}\left(\zeta_{b}\right) \varphi_{c}\left(\zeta_{c}\right) \Psi_{i}^{(+)}\left(\vec{\rho}_{b c}\right)>,(\mathbf{1})
$$

where $\varphi_{\alpha}$ is the bound state wave function of particle $\alpha, \zeta_{\alpha}$ denotes the set of internal coordinates of particle $\alpha, \vec{\rho}_{b c}$ is the relative coordinate between the centers of mass of particles $b$ and $c, \hat{O}$ is the electromagnetic operator and $\psi_{i}^{(+)}\left(\vec{\rho}_{b c}\right)$ is the scattering wave function of particles $b$ and $c$ in the entrance channel. For simplicity all particles are assumed to be spinless. At $E_{i} \rightarrow 0$ and small binding energies of particles $b$ and $c, \epsilon_{b c}$ only large $\rho_{b c}$ values contribute to $M$, i.e. radiative capture at very low energies occurs at large interparticle distances. Hence, when calculating $M$ the wave function $\varphi_{a}$ can be approximated by its asymptotic at large $\rho_{b c}$. The usual approximation in that case is

$$
\begin{gathered}
\varphi_{a}\left(\zeta_{b}, \zeta_{c} ; \vec{\rho}_{b c}\right) \approx \varphi_{b}\left(\zeta_{b}\right) \varphi_{c}\left(\zeta_{c}\right) I_{a b c}\left(\rho_{b c}\right) \\
\approx \varphi_{b}\left(\zeta_{b}\right) \varphi_{c}\left(\zeta_{c}\right) C_{b c} \frac{e^{-\kappa_{b c} \rho_{b c}-\eta_{b c} \ln \left(2 \kappa_{b c} \rho_{b c}\right)}}{\rho_{b c}},
\end{gathered}
$$

where the overlap integral of the bound state wave function of $a$ and $b, c I_{a b c}\left(\rho_{b c}\right)=\left\langle\varphi_{b} \varphi_{c} \mid \varphi_{a}\right\rangle$ has been replaced by its asymptotic. Here $\eta_{b c}=z_{b} z_{c} \mu_{b c} / \mathbf{k}_{b c}, \kappa_{b c}=\sqrt{2 \mu_{b c} \epsilon_{b c}}, \mu_{b c}=m_{b} m_{c} / m_{b c}, m_{b c}=m_{b}+m_{c} m_{\alpha}$ and $z_{\alpha}$ is the mass and charge of particle $\alpha, C_{b c}$ is the asymptotic normalization coefficient (ANC) of the overlap integral $I_{a b c}\left(\rho_{b c}\right)$. Taking into account eq. 2 we derive for $M$

$$
M \approx C_{b c}\left\langle\frac{e^{-x_{b c} \rho_{b c}-\eta_{k} \ln \left(2 x_{k x} \rho_{k b}\right)}}{\rho_{b c}}\left|\hat{O}\left(\vec{\rho}_{b c}\right)\right| \psi_{i}^{(+)}\left(\vec{\rho}_{b c}\right)\right\rangle
$$

It is clear from eq. 3 that the only unknown quantity to calculate the peripheral astrophysical process is the corresponding ANC. That is why the calculation of this coefficient is one of the interesting and important application of few-body methods for nuclear reactions and nuclear astrophysics. Usually the microscopic resonating-group method (RGM) is made use of to calculate astrophysical cross sections. However it is important to stress that the value of $C_{b c}$ depends on the choice of the NN-potential, which figures in the RGM. Different potentials may reproduce the scattering phase shifts equally well and some of electromagnetic properties of nuclei but lead to different values of $C_{b c}$. In particular, when using Gauss-type potentials in the RGM to describe electromagnetic properties of nuclei the quick falloff of the wave function is compensated by overestimation of the ANC that can lead to an overestimation of the astrophysical cross section. An alternative microscopic method to calculate the ANC has been suggested. ${ }^{1}$ The NN-potential was fit to reproduce the reliable phenomenological values of the ANC. Using this potential without changing its parameters, the astrophysical factors at $E_{i}=O$ for reactions ${ }^{7} B e+p \rightarrow{ }^{8} B+\gamma$ and ${ }^{8} B+p \rightarrow{ }^{9} C+\gamma$ were calculated: $S_{17}(0)=0.0165 \mathrm{keV}^{2}$ and $S_{18}(0)=$ $0.053 \mathrm{keVb} .^{3}$ The first one defines the high energy solar neutrino flux and turns out to be about $30 \%$ smaller than the value $S_{I 7}(0)=0.024 \mathrm{keV}$ used by Bahcall in the standard solar model (SSM). With the new value of $S_{17}(0)$ the high energy neutrino flux in the SSM becomes 5.9 SNU instead of $7.9 \mathrm{SNU}$ 
calculated with the old value of $S_{17}(0)$. The recently derived ANC for the virtual decay ${ }^{6} L i \rightarrow \alpha+d C_{\alpha d}$ $=2.3 \mathrm{Fm}^{-1 / 2}$ using the extrapolation of the s-wave phase shifts of $\alpha d$-scattering ${ }^{4}$ and variational threebody calculations of ${ }^{6} \mathrm{Li}$ bound state wave function ${ }^{5}$, allows one to calculate the astrophysical factor $S_{24}(0)$ for the important astrophysical process $\alpha+d \rightarrow{ }^{6} \mathrm{Li}$ $+\gamma$.

"Present address: Institute of Nuclear Physics, Moscow State University, Moscow 119899, Russia.

\section{References}

1. A. M. Mukhamedzhanov and N. K. Timofeyuk, Sov. J. Nucl. Phys. 51, 431 (1990).

2. A. M. Mukhamedzhanov and N. K. Timofeyuk, JETP Lett. 51, 247 (1990).

3. N. K. Timofeyuk, Proceedings of the Intern. School-Seminar on Heavy Ion Physics, Dubna, Russia, 1993, v. 2, p. 335.

4. L. D. Blokhintsev et al., Phys. Rev. C 48, 2390 (1993).

5. A. M. Mukhamedzhanov et al., Phys. At. Nucl. (Russia) 56, 937 (1993)

\title{
Coulomb Post Acceleration in Coulomb Break-Up Reactions in Asymptotic Three-Body Approach
}

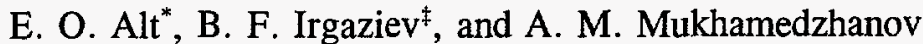

The disintegration of light projectiles in the Coulomb field of heavy targets is a tool to extract the cross sections of the astrophysically important radiative capture processes. ${ }^{1}$ A heavy target provides the domination of the Coulomb dissociation at small scattering angles. However the presence of a heavy target has a negative effect: three-body final state Coulomb interaction (Coulomb post acceleration (CPA)) becomes important and can distort the information extracted from the Coulomb break-up reactions. Accurate theoretical investigation of the CPA requires a genuine three-body approach. We present here a method to evaluate the CPA effect using the asymptotic behavior of a three-body scattering wave function in the configuration domain relevant for Coulomb break-up reactions at large impact parameters. ${ }^{2}$

Consider the break-up reaction (12) $+3 \rightarrow 1+$ $2+3$ in three-body model, where projectile (12) is the bound state of particles 1 and 2 , and 3 is a heavy target. The prior-form DWBA break-up amplitude is given by

$$
M=\left\langle\Psi_{f}^{(-)}\left|V_{13}+V_{23}-V_{i}\right| \Phi_{i}^{(+)}\right\rangle .
$$

Here $\Psi_{f}^{(-)}$is the solution of the Schrödinger equation with the full three-particle Hamiltonian describing the final state three-particle relative motion in the continuum, $\Phi_{i}^{(+)}=\phi_{12} \chi_{i}^{(+)}$is the entrance channel wave function, $\phi_{12}$ is the wave function of the bound state $12, \chi_{i}^{(+)}$is the wave function of the relative motion of the center of mass of (12) and particle 3 interacting via channel optical potential $V_{i}(\vec{\rho}), V_{\alpha \beta}\left(\vec{r}_{\alpha \beta}\right)$ is the interaction potential between particles $\alpha$ and $\beta, \vec{r}_{\alpha \beta}$ is the relative coordinate between particles $\alpha$ and $\beta$, and $\vec{\rho}$ is the relative coordinate between the center of mass of (12) and particle 3. Let us assume that the break-up reaction occurs at large $\rho\left(\rho>r_{12}\right)$. Then all the potentials in eq. 1 can be approximated by their Coulomb parts and $\Psi_{f}^{(-)}$by its asymptotics 
found in Ref. 2. We keep only the dipole term in the asymptotic expansion of $V_{13}^{C}+V_{23}^{C}-V_{i}^{C}$ in powers of $r_{12} / \rho:$

$$
\begin{gathered}
V_{13}^{c}\left(r_{13}\right)+V_{23}^{c}\left(r_{23}\right)-V_{i}^{c}(\rho) \approx \frac{4 \pi}{3} z_{3} \mu_{12}\left(\frac{z_{1}}{m_{1}}-\frac{z_{2}}{m_{2}}\right) \frac{r_{12}}{\rho^{2}} \\
\left.x \sum_{m} Y_{l m}^{*}(\hat{\vec{r}})\right\} Y_{l m}(\hat{\vec{p}}),
\end{gathered}
$$

where $V^{C}$ stands for the corresponding Coulomb potential, $m_{\alpha}$ and $z_{\alpha}$ are the mass and the charge of particle $\alpha$, and $\mu_{I 2}$ is the reduced mass of particles 1 and $2, \hat{\vec{r}}=\vec{r} / r$. The most important part of the asymptotics of $\Psi_{f}^{(-)}$is given by the wave function of the relative motion of particles 1 and 2 in the field of particle 3, $\Psi_{\vec{k}_{12}(\overrightarrow{\hat{p}})}^{(-)}\left(\vec{F}_{12}\right)$. The presence of the third particle results in changing the relative momentum of particles 1 and 2: instead of $\vec{k}_{12}$, this wave function depends on the local momentum $\vec{k}_{12}(\vec{\rho})$. The appearance of $\vec{k}_{12}(\vec{\rho})$ is a genuine three-body effect which is due to the infinite range of the Coulomb forces. Expanding this wave function in powers of $1 / \rho$ and keeping only the two first terms, we get

$$
\Psi_{\vec{k}_{12}(\bar{\rho})}^{(-)}\left(\vec{r}_{12}\right) \approx \Psi_{\vec{k}_{12}}^{(-)}\left(\vec{r}_{12}\right)+\frac{\vec{a}_{3}}{\rho} \nabla_{\vec{k}_{12}} \psi_{\vec{k}_{12}}^{(-)}\left(\vec{r}_{12}\right),
$$

where $\vec{a}_{3}$ is given in 2 . In approximation (3) the CPA effect is defined by the contribution of the second term of eq. 3. Taking into account eqs. 2 and 3 , we can derive for small $k_{12}$ the ratio of the breakup cross sections with and without $\left(\vec{a}_{3}=0\right) \mathrm{CPA}$ :

$$
X\left(E_{12}, E_{i}\right)=\left|1+i \frac{\mu_{12} \eta_{f} \eta_{12}}{z_{12} k_{12} \rho_{\min }}\right| \frac{z_{1}}{m_{1}}-\frac{z_{2}}{m_{2}}\left|\left[\frac{\pi}{2}-i \psi\left(1+i \eta_{12}\right)\right]\right|^{2} .(8)
$$

Here $E_{12}$ is the relative kinetic energy of particles 1 and 2 in the final state, $\eta_{i}=z_{12} z_{3} \mu_{f} / k_{f}, \eta_{12}=z_{1} z_{2} \mu_{12} / k_{12}, z_{12}=z_{1}+z_{2}, \psi\left(1+i \eta_{12}\right)$ is the special function psi, $k_{f}$ is the relative momentum of the center of mass of the system $1+2$ and particle 3 in the exit channel, and $\rho_{\min }$ is the cut-off radius in the radial integral of the matrix element. When deriving eq. 4 we assumed that $\rho_{\text {min }}>\kappa_{12}^{-1}, \kappa_{12}=\sqrt{2 \mu_{12} \epsilon_{12}}, \epsilon_{12}$ is the binding energy of particles 1 and 2 in the projectile and $x_{12}<k_{f}$. Eq. 4 defines the CPA effect at large impact parameters.

Figures 1-4 show the results of calculation of the ratio $X\left(E_{12}, E_{i}\right), E_{i}$ is the relative kinetic energy of the particles (12) and 3 in the entrance channel. In the approximation made, the CPA effect depends on $z_{1} / A_{1}$ - $z_{2} / A_{2}$, where $A_{\alpha}$ is the atomic number of particle $\alpha$. For reactions with $z_{1} / A_{1}-z_{2} / A_{2} \neq 0$, the CPA effect turns out to be significant for $E_{12}<40 \mathrm{keV}$. It decreases rapidly with increasing of $E_{12}$. For $z_{l} / A_{l}$ $z_{2} / A_{2}=0$ the CPA effect is much smaller. It is important to stress that our evaluations of $X$ have been made for large impact parameters and the value of $X$ can be much larger for realistic impact parameters close to the grasing one. For smaller impact parameters the quadrupole term in expansion (2) should be taken into account especially for the cases when $z_{1} / A_{1}-z_{2} / A_{2}=0$.

"Present address: Institut für Physik, Universität Mainz, D-55099 Mainz, Germany.

‡Present address: Department of Physics, Tashkent State University, Voozgorodok, Tashkent, Republic Uzbekistan.

\section{References}

1. G. Baur, C. A. Bertulani, and H. Rebel, Nucl. Phys. A458, 188 (1986).

2. E. O. Alt and A. M. Mukhamedzhanov, Phys. Rev. C 47, 2004 (1993). 
$200_{\mathrm{pb}} 1^{150}, \alpha^{12}{ }^{12} 1^{20 \theta_{\mathrm{pb}}}$

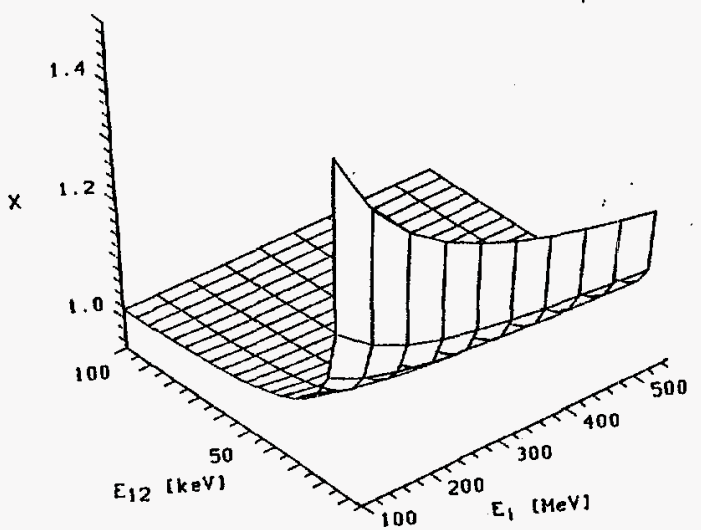

Figure 1: $X\left(E_{12}, E_{i}\right)$ for the reaction ${ }^{208} \mathrm{~Pb}\left({ }^{16} \mathrm{O}, \alpha{ }^{12} \mathrm{C}\right)^{208} \mathrm{~Pb}$.

${ }^{40} \mathrm{C} a,\left[\mathrm{E}_{\mathrm{L}, 1, \alpha, d}\right)^{40} \mathrm{Ca}$

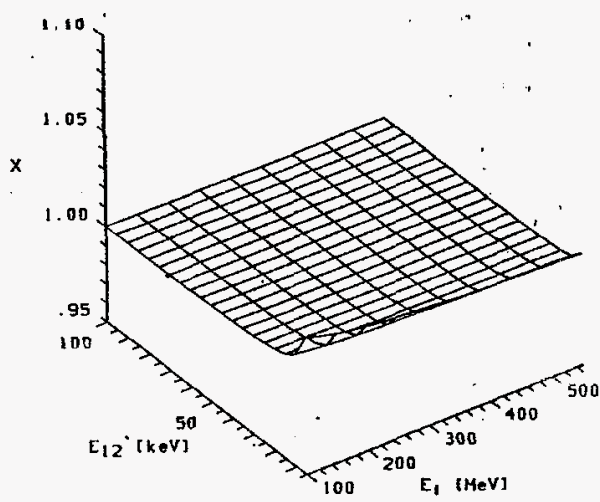

Figure 2: $X\left(E_{12}, E_{j}\right)$ for the reaction ${ }^{208} \mathrm{~Pb}\left({ }^{6} \mathrm{Li}, \alpha \mathrm{d}\right)^{200} \mathrm{~Pb}$.

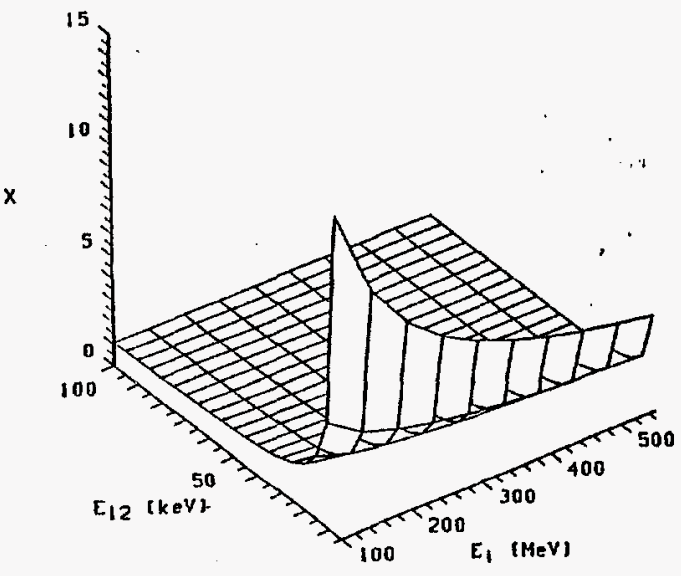

Figure 3: $X\left(E_{12}, E_{j}\right)$ for the reaction ${ }^{208} \mathrm{~Pb}\left({ }^{7} \mathrm{Be}, \alpha \mathrm{t}\right)^{208} \mathrm{~Pb}$.

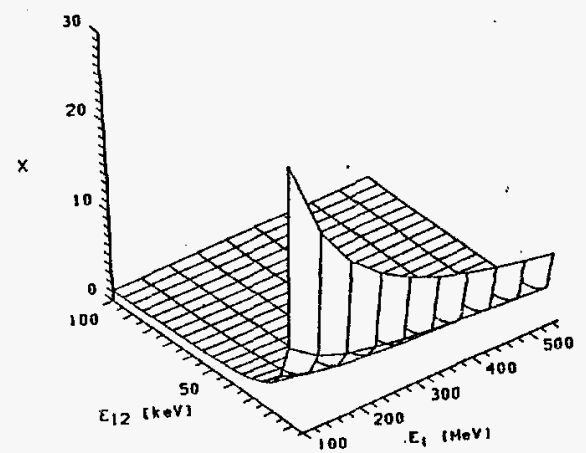

Figure 4: $\mathrm{X}\left(E_{12}, E_{i}\right)$ for the reaction ${ }^{208} \mathrm{~Pb}\left({ }^{13} \mathrm{~N},{ }^{12} \mathrm{Cp}\right){ }^{208} \mathrm{~Pb}$. 


\title{
On the Possibility to Determine the Astrophysical S-Factor for the ${ }^{7} \mathrm{Be}(p, \gamma)^{8} \mathrm{~B}$ Radiative Capture Reaction from the Analysis of Proton Transfer Reactions
}

\author{
A. M. Mukhamedzhanov, N. K. Timofeyuk ${ }^{*}$, and R. E. Tribble
}

The reaction ${ }^{7} \mathrm{Be}(p, \gamma)^{8} \mathrm{~B}$ is very important in the $p p$-chain defining the hydrogen burning of mainsequence stars. The reaction rate of this process at very small energies $(E<25 \mathrm{keV})$ is a quantity of fundamental importance to define both the branching ratios between different $p p$-chains and to calculate the high-energy solar neutrino flux which is of special interest for the solar neutrino puzzle.

However, at astrophysical energies the cross section of this reaction is so small that its measurement is very difficult in laboratory experiments. So, the experiments have been done at energies $\mathrm{E}>117 \mathrm{keV}$ and then the experimental cross sections are extrapolated to zero energy. ${ }^{1}$ Here we would like to draw attention to another possibility to get information about the S-factor, $S_{17}(E)$, of the reaction ${ }^{7} \mathrm{Be}(p, \gamma)^{8} \mathrm{~B}$ as $\mathrm{E} \rightarrow 0$ by measuring cross sections of proton transfer reactions induced by ${ }^{7} \mathrm{Be}$ nuclei.

The idea of our proposal is based on the fact that at astrophysical energies the radiative capture of protons by ${ }^{7} \mathrm{Be}$ nuclei occurs at very large distances, i.e. the overall normalization of the radiative capture cross section is defined by the so-called asymptotic normalization coefficient (ANC) of the overlap wave function of the bound state wave functions between ${ }^{8} \mathrm{~B}$ and ${ }^{7} \mathrm{Be}, I_{8_{B}{ }^{7} B e}=\left\langle\phi_{7_{B e}} \mid \phi_{8_{B}}\right\rangle$. This ANC can be extracted from analysis of surface proton transfer reactions. Consider, for example the reaction

$$
{ }^{7} \mathrm{Be}+{ }^{3} \mathrm{He} \rightarrow{ }^{8} \mathrm{~B}+d .
$$

We have calculated the cross section of this reaction within the framework of the DWBA to find the energy interval where this reaction is totally surface. Different initial and final state optical potentials used in the calculations are given in Table 1. The calculations have been made at incident energies of ${ }^{7} \mathrm{Be} \mathrm{E}=49,63,77$ and $105 \mathrm{MeV}$ which correspond to energies for ${ }^{3} \mathrm{He}$ of $\mathrm{E}=21,27,33$ and $45 \mathrm{MeV}$. The angular distributions corresponding to different choices of the optical potentials are shown in Fig. 1. One can inspect typical angular distributions for nucleon transfer reactions with the main stripping peak. To investigate the peripheral character of the reaction we introduced the cut-off radius $\mathbf{R}_{\text {cut }}$ in the radial integral defining the matrix element and investigated the contribution of the nuclear interior to the cross section by changing $\mathrm{R}_{c u \text {. }}$. One can see from Table 2 that at ${ }^{7} \mathrm{Be}$ energies of 49 and $63 \mathrm{MeV}$ and small scattering angles $\left(<15^{\circ}\right.$ in the $\left.\mathrm{CM}\right)$ there is no contribution from the nuclear interior, i.e. reaction (1) turns out to be surface. But for surface reaction (1), the differential cross section can be written as

$$
\frac{d \sigma}{d \Omega}=\lambda C_{0 \frac{1}{2}}^{2}\left(C_{1 \frac{1}{2}}^{2}+C_{1 \frac{3}{2}}^{2}\right) \tilde{\sigma}
$$

Here, $\lambda$ is the kinematical factor, $\tilde{\sigma}$ is the reduced cross section which does not contain any nuclear structure information; $C_{0 \frac{1}{2}}$ is the ANC for the virtual decay ${ }^{3} \mathrm{He} \rightarrow d+p, C_{1 \frac{1}{2}}$ and $C_{1 \frac{3}{2}}$ are the ANC for the virtual decay ${ }^{8} \mathrm{~B} \rightarrow{ }^{7} \mathrm{Be}+p$ for the total angular momentum of the proton of $1 / 2$ and $3 / 2$, respectively. It follows from Eq. 2 that for the surface reaction (1) $d \sigma / d \Omega$ is proportional to $C_{0 \frac{1}{2}}^{2}\left(C_{1 \frac{1}{2}}^{2}+C_{1 \frac{3}{2}}^{2}\right)$. This factor can be defined by normalizing the theoretical cross section (Eq. 2) to the experimental one in the main peak. The value of the ANC for the virtual decay ${ }^{3} \mathrm{He} \rightarrow d+p$ is well established: $C_{0 \frac{1}{2}}=2.07 \mathrm{Fm}^{-\frac{1}{2}} \cdot{ }^{2}$ That is why we can extract the factor $C_{1 \frac{1}{2}}^{2}+C_{1 \frac{3}{2}}^{2}$ which calibrates the absolute normalization of the radiative capture process ${ }^{7} \mathrm{Be}+$ $p \rightarrow{ }^{8} \mathrm{~B}+\gamma$ at astrophysical energies. ${ }^{3}$ However, we 
Table 1. Optical potentials for the ${ }^{3} \mathrm{He}+{ }^{7} \mathrm{Be}$ and $d+{ }^{8} \mathrm{~B}$ scattering. The Coulomb radius $r_{c}=1.3 \mathrm{Fm}$

\begin{tabular}{|l|l|l|l|l|l|l|l|l|l|l|c|}
\hline E,MeV & & $\mathrm{V}_{\mathrm{R}}$ & $\mathrm{r}_{\mathrm{R}}$ & $\mathrm{a}_{\mathrm{R}}$ & $\mathrm{W}_{\mathrm{v}}$ & $\mathrm{r}_{\mathrm{v}}$ & $\mathrm{a}_{\mathrm{v}}$ & $\mathrm{W}_{\mathrm{d}}$ & $\mathrm{r}_{\mathrm{d}}$ & $\mathrm{a}_{\mathrm{d}}$ & Ref. \\
\hline 21,27 & $\mathrm{H} 1$ & 62.7 & 1.432 & 0.746 & 21.8 & 1.525 & 1.055 & & & & 5 \\
\hline & $\mathrm{H} 2$ & 108.1 & 1.08 & 0.857 & 21.7 & 1.636 & 0.893 & & & & 5 \\
\hline 33 & $\mathrm{H} 3$ & 69.5 & 1.18 & 0.76 & & & & 20.3 & 1.18 & 0.76 & 5 \\
\hline & $\mathrm{H} 4$ & 176.6 & 1.11 & 0.707 & 51.7 & 0.96 & 0.801 & & & & 6 \\
\hline & $\mathrm{H} 5$ & 146.9 & 1.39 & 0.684 & 29.1 & 1.91 & 0.407 & & & & 6 \\
\hline & $\mathrm{H} 6$ & 79.8 & 1.2 & 0.7 & 8.24 & 2.15 & 1.07 & & & & 7 \\
\hline & $\mathrm{H} 7$ & 66.9 & 1.3 & 0.73 & 21.4 & 1.0 & 1.38 & & & & 7 \\
\hline 12 & $\mathrm{H} 8$ & 129.5 & 1.3 & 0.57 & 7.8 & 1.6 & 1.64 & & & & 7 \\
\hline 17 & $\mathrm{D} 1^{\mathrm{a}}$ & 78.0 & 0.967 & 1.04 & 30. & 1.07 & 0.81 & & & & 5 \\
\hline 22 & $\mathrm{D} 2$ & 65.0 & 1.25 & 0.79 & & & & 7.2 & 1.25 & 1.025 & 5 \\
\hline & $\mathrm{D} 3$ & 59.4 & 1.447 & 0.776 & 26.0 & 1.447 & 0.776 & & & & 5 \\
\hline 33 & $\mathrm{D} 4$ & 74.03 & 1.239 & 0.736 & & & & 11.63 & 1.239 & 0.736 & 5 \\
\hline
\end{tabular}

${ }^{\mathrm{W}}$ We use also spin-orbital potential for D1 parameter set with $\mathrm{V}_{\mathrm{so}}=6.05 \mathrm{MeV}, r_{\mathrm{so}}=0.967 \mathrm{Fm}$ and $a_{\mathrm{so}}=1.04 \mathrm{Fm}$.

Table 2. Ratio of the cross sections of the reaction ${ }^{7} \mathrm{Be}\left({ }^{3} \mathrm{He}, d\right)^{8} \mathrm{~B}$ calculated with the cut-off radii equal to $4 \mathrm{Fm}$ and to $0 \mathrm{Fm}$ at different energies with the optical parameters taken from Table 1.

\begin{tabular}{|c|c|c|c|c|c|}
\hline \multirow[t]{3}{*}{$\mathrm{E}, \mathrm{MeV}$} & \multirow{3}{*}{$\begin{array}{r}\text { Optical } \\
\text { potential }\end{array}$} & \multicolumn{4}{|c|}{$\theta^{\circ}$} \\
\hline & & 1 & & & 15 \\
\hline & & \multicolumn{4}{|c|}{$\sigma\left(\mathrm{R}_{\text {cut }}=4 \mathrm{Fm}\right) / \sigma\left(\mathrm{R}_{\text {cut }}=0\right)$} \\
\hline 21 & H1-D1 & 1.02 & 1.02 & 1.01 & 0.98 \\
\hline \multirow[t]{2}{*}{27} & H1-D2 & 0.97 & 1.01 & 1.00 & 1.00 \\
\hline & H2-D2 & 1.02 & 1.02 & 0.95 & 0.93 \\
\hline \multirow[t]{2}{*}{33} & H3-D3 & 0.93 & 0.93 & 0.88 & 0.80 \\
\hline & H5-D3 & 1.02 & 1.03 & 0.97 & \\
\hline \multirow[t]{3}{*}{45} & H8-D4 & 0.97 & 0.96 & 0.85 & 0.68 \\
\hline & H7-D4 & 0.87 & 0.86 & 0.79 & \\
\hline & H6-D5 & 0.74 & 0.73 & 0.66 & 0.60 \\
\hline
\end{tabular}




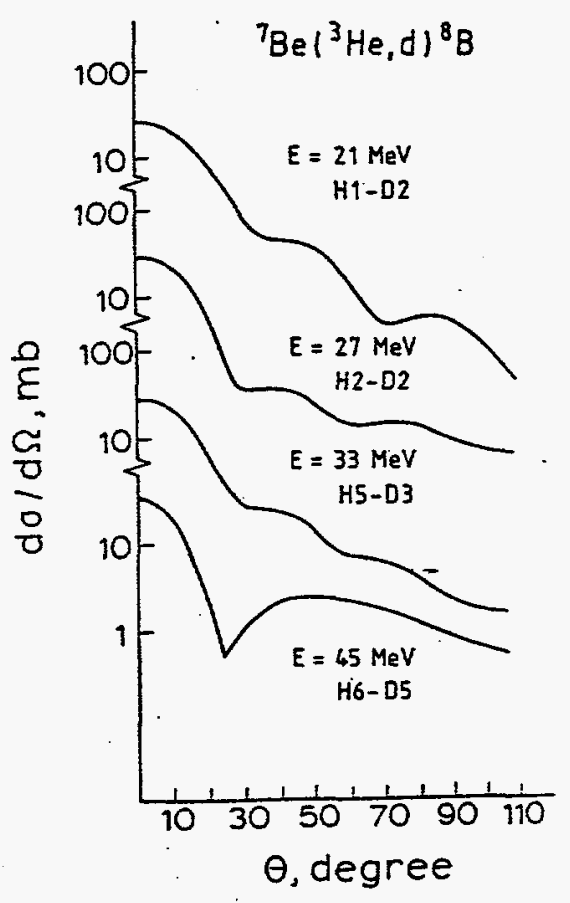

Figure 1. Angular distribution for the ${ }^{7} \mathrm{Be}\left({ }^{3} \mathrm{He}, \mathrm{d}\right)^{8} \mathrm{~B}$ reaction as a function of the $E_{3_{H e}}$.

would like to point our that it is quite difficult to measure the cross section of the reaction (1) at small scattering angles in the $\mathrm{CM}$ using ${ }^{7} \mathrm{Be}$ as a beam because the mass of the target (here ${ }^{3} \mathrm{He}$ ) is less than the mass of projectile. For this reason one needs to perform measurements of the angular distributions in the lab system at very small angles to cover the main stripping peak in the $\mathrm{CM}$ system. Also the $\mathrm{CM}$ angular range for the reaction is quite compact in the lab frame, where $1^{\circ}$ in the laboratory corresponds to about $5^{\circ}$ in the $\mathrm{CM}$. The divergence of the radioactive ${ }^{7} \mathrm{Be}$ beam would result in averaging over several degrees in the $\mathrm{CM}$ independent of the angular resolution in an experimental setup. That is why, from the technical point of view, the better nominees for targets would be ${ }^{10} \mathrm{~B}$ or ${ }^{14} \mathrm{~N}$. The ANCs corresponding to proton removal from these targets have been calculated in Ref. 4, but additional experiments to find these ANCs independently are highly desirable.

"Institute of Nuclear Physics, Uzbek Academy of Sciences, Tashkent, 702132, Ulugbek, Uzbekistan.

\section{References}

1. B. W. Fillippone, Ann. Rev. Nucl. Part. Sci. 36, 717 (1986).

2. I. Borbely and A. M. Mukhamedzhanov, Proceedings of the 34th Conference on Nuclear Spectroscopy and Structure, Alma-Ata, 1984, p. 413.

3. A. M. Mukhamedzhanov and N. K. Timofeyuk, JETP Lett. 51, 282 (1990).

4. A. M. Mukhamedzhanov and N. K. Timofeyuk, Sov. J. Nucl. Phys. 51, 431 (1990).

5. C. M. Perey and F. G. Perey, At. Data and Nucl. Data Tables 17, 1 (1976).

6. A. K. Basak, O. Karban, S. Roman et al., Nucl. Phys. A368, 74 (1981).

7. R. Goergen, F. Hinterberger, R. Jahn et al., Nucl. Phys. A320, 296 (1979). 


\title{
The Overall Normalization of the Astrophysieal S-factor and the Asymptotic Normalization Coefficient for ${ }^{7} \operatorname{Be}(p, \gamma)^{8} \mathrm{~B}$ Reactions
}

\author{
H. M. Xu, C. A. Gagliardi, R. E. Tribble, A. M. Mukhamedzhanov ${ }^{*}$ and N. K. Timofeyuk
}

Because of its unique role in the "8 $\mathrm{B}$ solar neutrino puzzle" the production rate of ${ }^{8} \mathrm{~B}$ at solar energies $\left(E_{c m} \lesssim\right.$ of $0-20 \mathrm{keV}, E_{c m}$ is the proton c.m. energy) through ${ }^{7} \mathrm{Be}(\mathrm{p}, \gamma)^{8} \mathrm{~B}$ reactions, or more precisely, its S-factor, has been studied extensively during the last three decades. ${ }^{1-12}$ Because of its vanishing cross sections at solar energies, the ${ }^{7} \mathrm{Be}(\mathrm{p}, \gamma)^{8} \mathrm{~B}$ S-factor was normally measured by direct radiative capture reactions at laboratory energies. The measurements were then extrapolated to solar energies using the theoretically derived energy dependence. ${ }^{6-9}$ At present, the energy dependence of the S-factor seems fairly well established. ${ }^{6-9}$ However, there are still large uncertainties, both experimentally and theoretically, concerning the absolute normalization of the S-factor.

In the present work, we address the issue of the normalization of the S-factor. For this purpose, we recall that the radiative capture reaction for $b+c \rightarrow$ $a+\gamma$ at $E_{c m} \rightarrow 0$ has an amplitude

$$
\begin{gathered}
M=<\Psi_{a}\left(\xi_{b}, \xi_{c}, \vec{r}\right)|\hat{O}(\vec{r})| \Psi_{b}\left(\xi_{b}\right) \Psi_{c}\left(\xi_{c}\right) \phi_{\vec{k}_{i}}^{(+)}(\vec{r})>(1) \\
=<I_{b c}^{a}(\vec{r})|\hat{O}(\vec{r})| \phi_{\overrightarrow{k_{i}}}^{(+)}(\vec{r})>
\end{gathered}
$$

where $\Psi_{i}$ is the bound state wave function of particle $i$, and $\xi_{b}, \xi_{c}$ are internal coordinates for bound states of nuclei $b$ and $c$, respectively; $\vec{r}$ represents the relative coordinate between $b$ and $c ; \hat{O}$ is the electromagnetic operator, and in the case of ${ }^{7} \mathrm{Be}(\mathrm{p}, \gamma)^{8} \mathrm{~B}$, the $\mathrm{E} 1$ operator; $\phi_{\vec{k}_{i}}^{(+)}(\vec{r})$ is the distorted wave in the initial channel, $b+c$; and $I_{b c}^{a}$ is the overlap wave function for $a \rightarrow b+c$, whose radial part, $I_{b c e s}^{a}(r)$, has the asymptotic behavior

$$
I_{b c \ell S}^{a}(r) \approx C_{\ell S} \frac{W_{-\eta, \ell+1 / 2}(2 K r)}{r}, \quad r>R_{N^{2}}
$$

where $R_{N}$ is the nuclear interaction radius between proton and ${ }^{7} \mathrm{Be} ; W_{-n, \ell+1 / 2}$ is the Whittaker function and $C_{B S}$ is the asymptotic normalization coefficient (ANC). In the standard potential model, ${ }^{6,7}$ however, $I_{b c e s}^{a}$ is approximated by the product of two factors, the spectroscopic factor $J_{R S}$ and the bound-state radial wave function $U_{e S}(r) / r$, as follows,

$$
I_{b c \ell S}^{a}(r) \approx J_{\ell S^{1 / 2}} \frac{U_{\ell S}(r)}{r} .
$$

Outside the core $\left(r>R_{N}\right), I_{b c e s}^{a}$ becomes

$$
I_{b c e s}^{a}(r) \approx J_{e S^{1 / 2} \beta_{e S}} \frac{W_{-\eta, \ell+1 / 2}(2 K r)}{r}
$$

here $\beta_{R S}$ is the normalization coefficient of the asymptotic part of the bound state wave function $U_{\mathrm{eS}}$. Thus $\beta_{\ell S}$ relates to the ANC, $C_{\ell S}$, by

$$
C_{\ell S}=J_{\ell S}{ }^{1 / 2} \beta_{\ell S} .
$$

As one can see from Eq. (2), the transition matrix element $M$ is completely determined by the knowledge of $I_{b c}^{a}(\vec{r}), \hat{O}(\vec{r})$, and $\phi_{\vec{k}_{i}}^{(+)}(\vec{r})$. Here, $O(\vec{r})$, the electromagnetic operator, is well known, and $\phi_{\overrightarrow{k_{i}}}^{(+)}(\vec{r})$, the distorted wave in the entrance channel, whose radial part, for capture at $E_{c m} \approx 0$ (the nuclear phase shifts $\delta_{e S}\left(E_{c m}\right) \approx 0$ ), is simply the regular Coulomb function, $F_{\ell}\left(\eta_{i}, r\right)$. Thus the value of $M$, and therefore, the $S_{17}(0)$-factor, is completely determined by the overlap wave function, $I_{b c}^{a}(\vec{r})$. If protons are captured both inside and outside of the core nucleus (e.g., ${ }^{7} \mathrm{Be}$ ), then, the whole knowledge of the overlap wave function, both $J_{e S}$ and $U_{e S}$ (see Eq. (4)), is required. If, on the other hand, the protons are captured well outside the core, then, only the knowledge of a single parameter, $C_{Q S}$, is required. As 
we will show later, it is precisely the value of $C_{R S}$, that completely determines the normalization of the $S_{17}(0)$-factor.

Because of the requirements that $\int U_{\ell=1}^{2} d r=1$ in the standard approaches, 6.7 wave functions that have small amplitudes at small radii, $r \lesssim 5 \mathrm{fm}$, have larger amplitudes at large radii, thus yielding higher values of $S_{17}(0)$. To see this more quantitatively, we have extracted the asymptotic values, $\left(\beta_{\ell=1}\right)_{i}=\left(U_{\ell=1}\right)_{i} / W_{-\eta, 3 / 2}$, where $i=1,2,3$, corresponds to solutions of different potential parameters used by Barker, ${ }^{7}$ and the results are listed in Table 1 . We find that all three wave functions become asymptotic at radii $r \geqslant 5 f m\left(\left(\beta_{1}\right)_{i}\right.$ becomes constant). From these $\beta$ values (Table $\mathrm{l}$ ), one can easily verify that $\left(\beta_{1}\right)_{i}^{2} / S_{17}(0)_{i} \approx 0.026$, a universal constant for all three wave functions. When the ANC of the overlap wave functions were normalized to specific values ${ }^{11}$ of ANC, $C_{\ell S}$, deduced from the values of nuclear vertex constants (NVC) predicted for the virtual decay of ${ }^{8} \mathrm{~B} \rightarrow{ }^{7} \mathrm{Be}+\mathrm{p}$ by Ref. 11 , a single value, $S_{17}(0)=17.6 \mathrm{eV} \cdot \mathrm{b}$, is obtained. Thus the values of $S_{t 7}(0)$ are entirely determined by the tails of the wave functions (at radius $r \gtrsim 5 \mathrm{fm}$ ). Further calculations ${ }^{13}$ indicate that most contributions to the capture come from protons well outside the core, with the maximum capture at $r \geq 40 \mathrm{fm}$ for energies $E_{c m} \approx 0-20$ $\mathrm{keV}$. In fact, we find that more than $99 \%$ of proton captures occur at distance $r \geqslant 5 \mathrm{fm}$ from the core at energies $E_{c m} \leqslant 20 \mathrm{keV}$. Thus it is the tail of the overlap wave function, or more precisely, the normalization constant of the tail, $C_{\ell S}$, that solely determines the value of $S_{17}(0)$ for ${ }^{7} \mathrm{Be}(\mathrm{p}, \gamma)^{8} \mathrm{~B}$ reactions.

In Fig. 1, we compare the calculated S-factor with data for ${ }^{7} \mathrm{Be}(\mathrm{p}, \gamma)^{8} \mathrm{~B}$. Overall, our calculations of the direct capture $S$-factor agree remarkably well with data points, both by Filippone et al. at low energies, and by Vaughn et al. at high energies, without any renormalization. However, the predicted value at solar energies, $S_{l,}(0) \approx 17.6 \mathrm{eV} \cdot \mathrm{b}$, appears to be slightly lower than those extrapolated from the data, $S_{i},(0) \approx 20.6 \pm 3.0 \mathrm{eV} \cdot \mathrm{b},{ }^{4}$ and $S_{i 7}(0) \approx 21.4 \pm 2.2$
$\mathrm{eV} \cdot \mathrm{b}^{3} \quad$ At present, we do not propose to set an independent value from our method, but rather to conclude that our value is consistent with lower values $^{3,4}$ instead of higher values from earlier measurements ${ }^{1,2}$ or from calculations based on the resonating group method. ${ }^{12}$

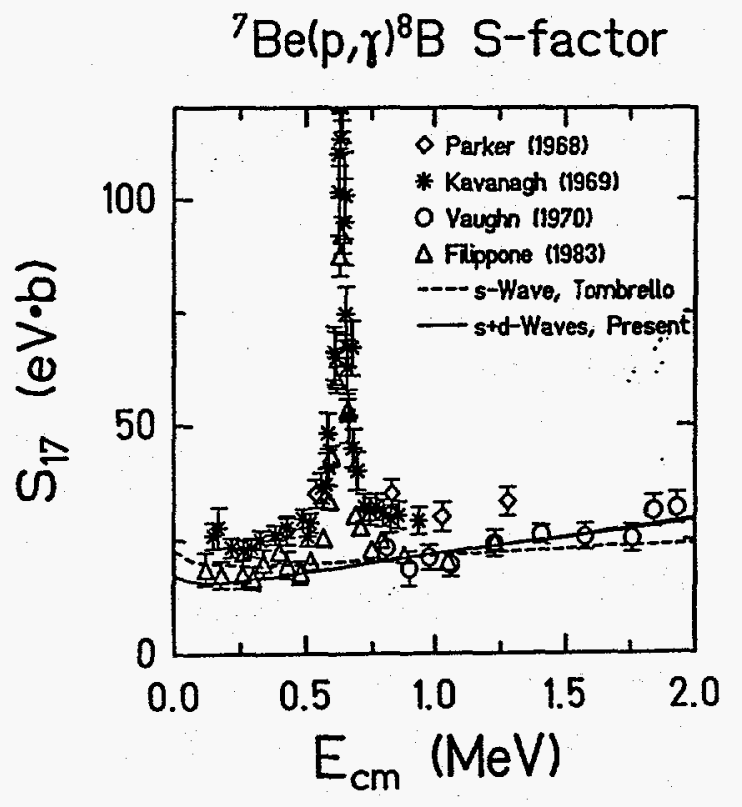

Figure 1. The $S_{17}$ factor for ${ }^{7} \mathrm{Be}(\mathrm{p}, \gamma)^{8} \mathrm{~B}$ as a function of proton energy $E_{\mathrm{cm}}$. The solid line indicates our calculations while the dashed line shows calculations of Tombrello when normalized to the low energy data points of Filippone et al.

In conclusion, we point out a simple relation between the nuclear vertex constant (NVC) and the overall normalization of the astrophysical S-factor. This unique relation, allows new possibilities, such as transfer reactions, to independently measure the astrophysical S-factors. ${ }^{13}$

"Institute for Nuclear Physics, Uzbek Academy of Science, Tashkent, 702132, Ulugbek, Uzbekistan.

\section{References}

1. P. D. Parker, Ap. J. 153, L85 (1968); Phys. Rev. 150, 851 (1966). 
2. R. W. Kavanagh et al., Bull. Am. Phys. Soc. 14, 1209 (1969).

3. F. J. Vaughn et al., Phys. Rev. C 2, 1657 (1970).

4. B. W. Filippone et al., Phys. Rev. Lett. 50, 412 (1983); Phys. Rev. C 28, 2222 (1983).

5. T. Motobayashi et al., Preprint Rikkyo RUP 94-2 (submitted to Phys. Rev. Lett.); T. Motobayashi, private communications.

6. T. A. Tombrello, Nucl. Phys. 71, 459 (1965).

7. F. C. Barker, Aust. J. Phys. 33, 177 (1980).
8. T. Kajino, Nucl. Phys. A460, 559 (1986).

9. C. W. Johnson et al., Ap. J. 392, 320 (1992).

10. A. M. Mukhamedzhanov and N. K. Timofeyuk, Pis'ma Zh. Eksp. Teor. Fiz. 51, 247 (1990).

11. A. M. Mukhamedzhanov and N. K. Timofeyuk, Yad. Fiz. 51, 679 (1990).

12. P. Descouvemont and D. Baye, Nucl. Phys. A567, 341 (1994); A487, 420 (1988).

13. H. M. Xu et al., (to be published).

Table I. The potential parameters used to calculate the bound state wave functions for ${ }^{8} \mathrm{~B} \rightarrow{ }^{7} \mathrm{Be}+\mathbf{p}$. The spectroscopic factors are the same as given by Barker, ${ }^{9}$ satisfying $J_{11}+J_{l 2} \approx 1.0$. Other parameters are discussed in the text.

\begin{tabular}{|c|c|c|c|c|c|r|}
\hline Set & $\mathrm{V}_{0}(\mathrm{MeV})$ & $r_{0}(\mathrm{fm})$ & $a(\mathrm{fm})$ & $\beta_{\ell}\left(\mathrm{fm}^{-1 / 2}\right)^{a}$ & $S_{J 7}(0)(\mathrm{eV} \cdot \mathrm{b})$ & $\beta_{\ell}^{2} / S_{17}(0)$ \\
\hline 1 & 46.56 & 1.25 & 0.65 & 0.764 & 22.5 & 0.0259 \\
\hline 2 & 151.76 & 0.53 & 0.65 & 0.609 & 14.4 & 0.0258 \\
\hline 3 & 47.91 & 1.25 & 0.27 & 0.592 & 13.5 & 0.0260 \\
\hline
\end{tabular}

${ }^{\text {a}} \mathrm{Here} \ell=1 ; \beta_{\ell}$ and $\mathrm{U}_{\ell}$ do not depend on channel spin $\mathrm{S}$ since the potentials do not include the spin-orbit term.

\section{A Comparison of BUU and QMD Calculations for ${ }^{40} \mathrm{Ca}+{ }^{40} \mathrm{Ca}$ Collisions Near the Fermi Energy}

\section{J. Cibor", J. Lukasik*, Z. Majka*, and H. M. Xu}

The reaction dynamics for ${ }^{40} \mathrm{Ca}+{ }^{40} \mathrm{Ca}$ collisions near the Fermi energy is investigated with a one-body BUU model and an n-body quantum molecular dynamics (QMD) model.' Numerical details concerning the $\mathrm{n}$-body QMD model and the one-body BUU mode used in the present study are described in Ref. 2 and Ref. 3, respectively. With both models, we have performed a comparative study for the following observables characterizing the reaction dynamics: (1) the pre-equilibrium emission rate, the thermalization time scale, and the compression-expansion dynamics; (2) the excitation energy for equilibrated residues; and (3) the residue cross sections.

To investigate the time scale for preequilibrium and thermalization, we display, in Fig. 1, the nucleon emission rates and the quadrupole moment of the momentum distributions for ${ }^{40} \mathrm{Ca}+{ }^{40} \mathrm{Ca}$ collisions at $\mathrm{E} / \mathrm{A}=35 \mathrm{MeV}$. The top panels show the emission rate of nucleons at selected impact parameters ranging between 0 and $6 \mathrm{fm}$. Clearly, both the BUU and QMD models predict similar emission rates, a large 
component at early times of the reaction and a small component at later times. These two components correspond to, respectively, significant early pre-equilibrium emission and small later evaporations characteristic of thermalized residues. Similar values in both the rates and time scales are obtained for both models. The middle panels in Fig. 1 show the time evolution of the $z$-th components of the quadrupole moment tensor in the momentum space given by $Q_{z}=\left[\int\left(3 p_{z}^{2}-p^{2}\right) g(\vec{p}) d^{3} p\right] / \int g(\vec{p}) d^{3} p$, where $g(\vec{p})$ is the one-body density in momentum space. This quantity is motivated by the fact that when equilibrium is reached, $Q_{z z}=0$. We note that, the time scale for equilibrium, ( $t \approx 100-120 \mathrm{fm} / \mathrm{c}$ ), as reflected by the slope change in the emission rates, is consistent with that obtained from the $Q_{z z}$, for which, both the BUU and QMD again predict similar values.
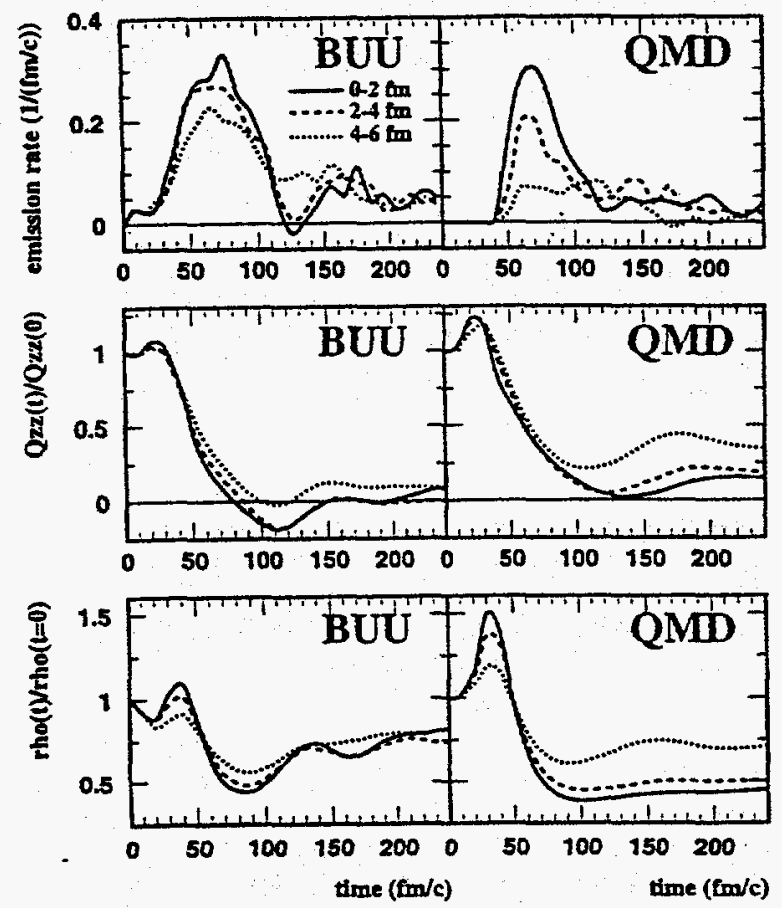

Figure 1. Time evolution of the emission rate (upper panels), quadrupole moment in momentum space (middle panels) and mean density (lower panels), calculated for ${ }^{40} \mathrm{Ca}+{ }^{40} \mathrm{Ca}$ collisions at $\mathrm{E} / \mathrm{A}$ $=35 \mathrm{MeV}$.

The bottom panels display the time evolution of the mean density of the whole system, defined as $\langle\rho\rangle=\int \rho^{2}(\vec{r}) d^{3} r / \int \rho(\vec{r}) d^{3} r$. Again, the overall features are qualitatively similar for both models, with the maximum compressions occurring at $\mathrm{t} \approx$ 30-40 fm/c and the maximum expansions (minimum densities) occurring at $t \approx 80-100 \mathrm{fm} / \mathrm{c}$. These low densities, $\rho \approx(0.3-0.4) \rho_{0}$, are consistent with the low densities assumed in the statistical models. ${ }^{4,5}$ In fact, the time scale for maximum expansion (bottom panels), $t \approx 80-100 \mathrm{fm} / \mathrm{c}$, as reflected by both models, are close to the time scale for the end of preequilibrium emission (top panels) and onset of thermalization (middle panels), $t \approx 100-120 \mathrm{fm} / \mathrm{c}$, indicating that reasonable assumptions are made in the statistical models (these models assume maximum expansion and thermalization occur at the same times).

In Fig. 2 we present the scatter plot of the number of events versus fragment charge and excitation energy per nucleon calculated by the QMD model for the ${ }^{40} \mathrm{Ca}+{ }^{40} \mathrm{Ca}$ reaction at $35 \mathrm{MeV} /$ nucleon beam energy when equilibrium is initially reached $(t \approx 120 \mathrm{fm} / \mathrm{c})$. For comparison, the diamonds depict the same quantities predicted by the BUU model. Because of lack of fluctuations and correlations, the BUU model only gives average values for both the charge and excitation energy. Both models predict a gradual decrease of average charge and excitation energy with impact parameter, indicating a gradual change from formation of hot residues in central collisions to formation of projectile and target like residues in peripheral collisions (binary processes).

In Fig. 3, we show the inclusive mass distributions (solid lines) for the ${ }^{40} \mathrm{Ca}+{ }^{40} \mathrm{Ca}$ reaction at $25 \mathrm{MeV} /$ nucleon obtained at $t=120 \mathrm{fm} / \mathrm{c}$ (upper panel) and $t=300 \mathrm{fm} / \mathrm{c}$ (lower panel), respectively. To select the fusion-like reactions we chose events in which there was only one fragment with $A \geq 20$. This gives us a single well-defined residue group, as indicated by the dashed line in the bottom panel of Fig. 3 (not shown for the top panel, since it is indistinguishable from the solid lines for mass $A \geq$ 50). Thus, evaluation of yields for residues at $t \approx 120$ $\mathrm{fm} / \mathrm{c}$, which is comparable to the time for which the thermalization is reached (see Fig. 1), provides an estimate for the cross sections of hot equilibrated 


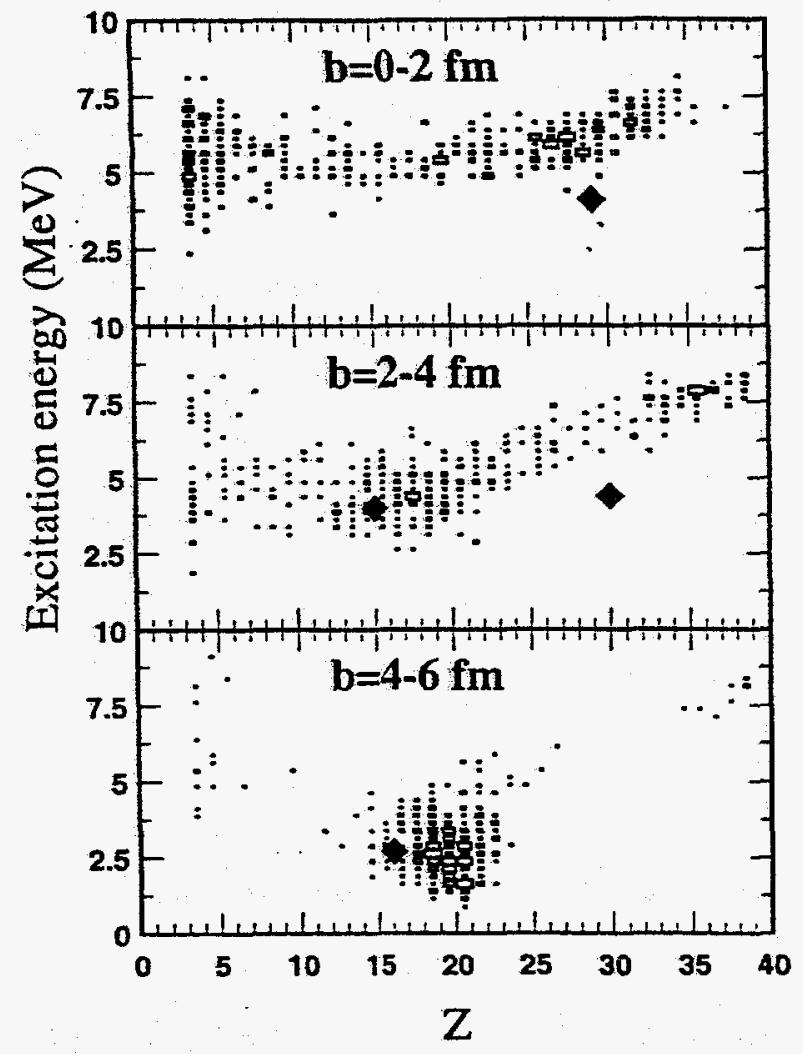

Figure 2. Excitation energy per nucleon for fragments obtained from QMD calculation at $\mathrm{t}=120 \mathrm{fm} / \mathrm{c}$. See the text for details.

fusionlike residues formed in collisions. On the other hand, evaluation of yields for residues at later time, $t$ $\approx 300 \mathrm{fm} / \mathrm{c}$, provides an estimate for cross sections of residues going through the evaporation decay channel. In Fig. 4, we show the probability for fusionlike residues formed as a function of impact parameter at 25 and $35 \mathrm{MeV} /$ nucleon calculated at $\mathrm{t}=120 \mathrm{fm} / \mathrm{c}$. For BUU models, one always sees well defined residues up to a certain critical impact parameter. However, for the QMD model, one sees a gradual decrease of fusion probability with impact parameter. Overall, similar results are obtained between the BUU and QMD models concerning the formation of fusionlike residues at an early stage of the reactions.

Integrating over all impact parameters, one obtains the fusion cross section. In Fig. 5, we present the incident energy dependence of the fusion cross section for the ${ }^{40} \mathrm{Ca}+{ }^{40} \mathrm{Ca}$ reaction. Both the BUU (circles) and QMD (stars) models predict that the composite system formation cross section decreases rapidly with energy. The QMD model provides further information concerning the later disintegration. As one can see from the Fig. 5, the cross sections of evaporation residues are much smaller than the cross

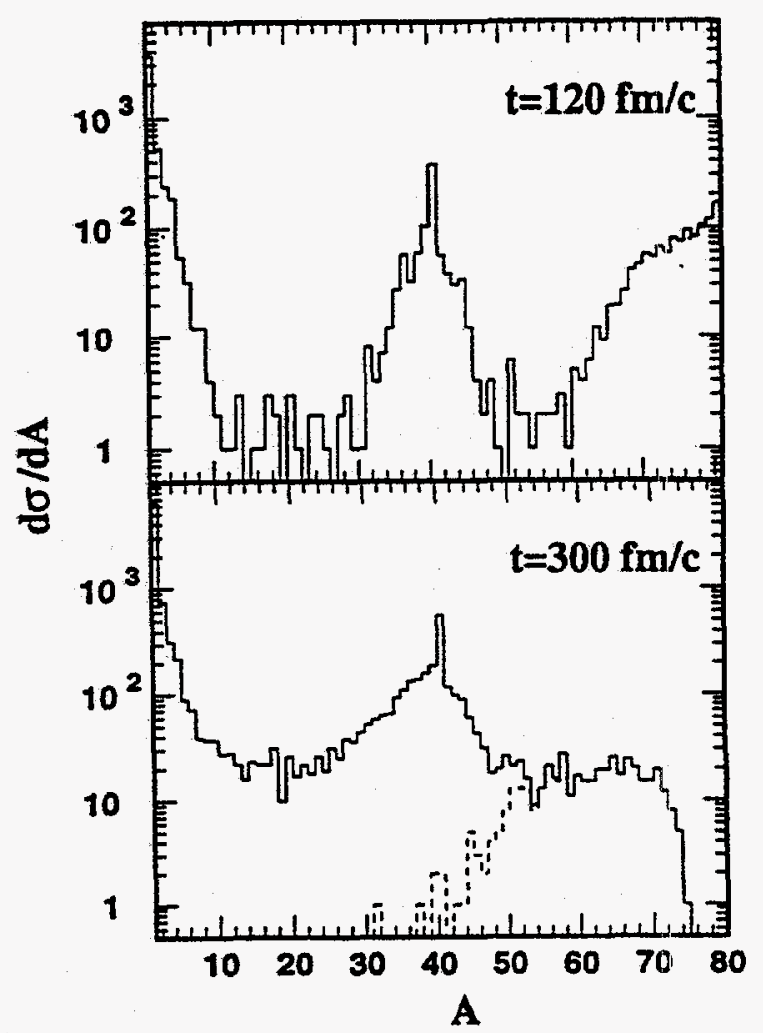

Figure 3. Mass distributions of fragments calculated by the QMD model. See the text for details.

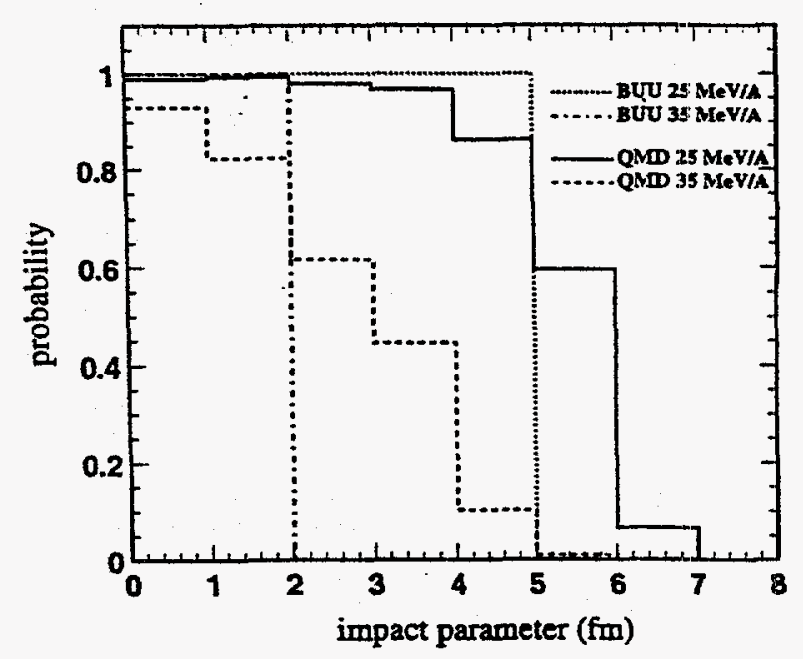

Figure 4. Probability distributions for fusionlike residues formed in ${ }^{40} \mathrm{Ca}+{ }^{40} \mathrm{Ca}$ collisions at 25 and $35 \mathrm{MeV} /$ nucleon beam energy. 
sections of fusionlike composite system formed. Moreover, the calculated cross sections for evaporation residues (squares) are similar to those taken from the systematics (triangles) ${ }^{6}$ and show a much slower decrease with the beam energy, suggesting that the evaporation residues could survive even higher incident energies.

In conclusion, both models give similar predictions for quantities characterizing the early stages of reactions, i.e., the time scales for pre-equilibrium and thermalization, excitation energies and cross sections for residues surviving the collisions. The QMD model further predicts that evaporation residues can be produced in central collisions at energies much higher than those indicated by the BUU calculations.

"Institute of Physics, Jagellonian University, PL-30-059 Kraków, Reymonta 4, Poland

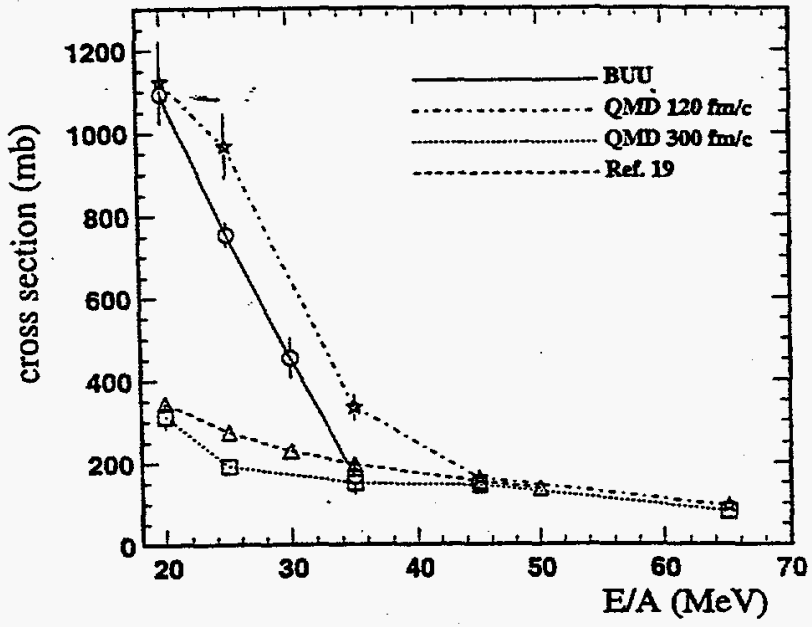

Figure 5. Beam energy dependence for cross sections of composite system formation and the evaporation residues in ${ }^{40} \mathrm{Ca}+{ }^{40} \mathrm{Ca}$ collisions.

\section{References}

1. J. Cibor, et al., Phys. Lett. B, (submitted).

2. J. Lukasik and Z. Majka, Acta Phys. Pol. B24, 1959 (1993); J. Lukasik et. al., Phys. Lett. 298B, 27 (1993).

3. H. M. Xu et. al., Phys. Rev. Lett. 65, 843 (1990); Phys. Lett. 261B, 240 (1991); Phys. Lett. 299B, 199 (1993).

4. D. H. E. Gross, Rep. Prog. Phys. 53, 605 (1990)

5. J. P. Bondorf et. al., Nucl. Phys. A443, 321 (1985).

6. W. W. Wilcke et. al., At. Data Nucl. Data Tables 25, 389 (1980). 


\section{Limiting Temperatures and Disappearance of Fusionlike Residues}

H. M. Xu

With an improved Boltzmann-Uehling-Uhlenbeck model, ${ }^{1}$ we have investigated the reaction dynamics which limits the formation of fusionlike residues in ${ }^{40} \mathrm{Ar}+{ }^{27} \mathrm{Al}$ collisions at energies near the Fermi energy. ${ }^{2}$ In particular, we have performed comparative studies using two different parameter sets: (1) $\sigma_{n i}=25 \mathrm{mb}$ and a stiff EOS and (2) $\sigma_{n n}=50 \mathrm{mb}$ and a soft EOS, which yield the same residue cross sections, as shown in Fig. 1. Using these two exclusive parameters, we have calculated the thermal excitation energy, the angular momentum, and the temperature for the fusionlike residues surviving the collisions. These calculations suggest that reaction dynamics, not the Coulomb or thermal instabilities, plays the most important role in limiting the production of fusionlike residues at energies less than the Fermi energy.

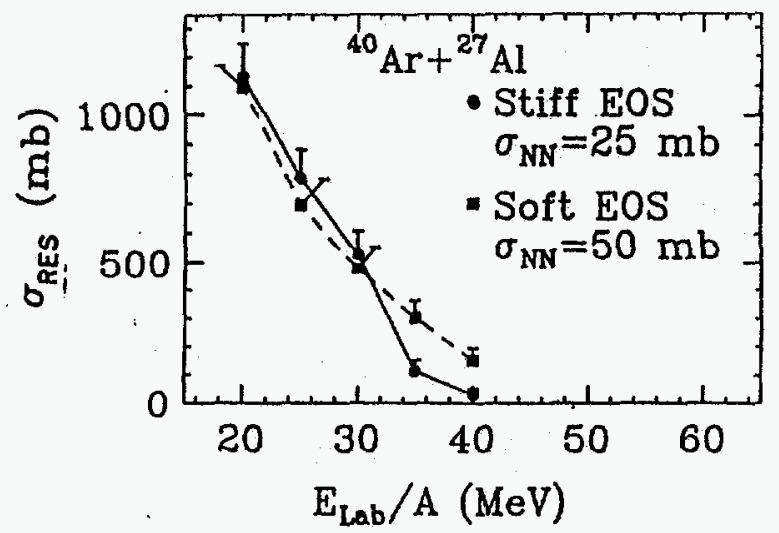

Figure 1. Residue cross sections for ${ }^{417} \mathrm{Ar}+{ }^{27} \mathrm{Al}$ collisions. The solid circles and solid squares describe calculations with the stiff and soft equations of state, respectively.

As an example, Fig. 2 shows the total angular momenta for residues (see Refs. 1 and 2 for further details), obtained for both the stiff equation of state (solid circles) and the soft equation of state (squares), as a function of impact parameter for ${ }^{40} \mathrm{Ar}+{ }^{27} \mathrm{Al}$ collisions at $\mathrm{E} / \mathrm{A}=30 \mathrm{MeV}$. The angular momentum increases linearly with impact parameter to a value of $J_{\max } \approx 58 \hbar$ at $\mathrm{b}=4.3 \mathrm{fm}$ for the stiff EOS ( $J_{\max } \approx 44 \hbar$ at $b=4 \mathrm{fm}$ for the soft EOS), comparable to the maximum orbital angular momentum predicted by the liquid-drop model for mass $A=56$ ( $A=52$ for soft EOS). This suggests that the formation of a residue at $\mathrm{E} / \mathrm{A}=30 \mathrm{MeV}$ may be partially limited by the maximum arigular momentum that a nucleus can sustain.

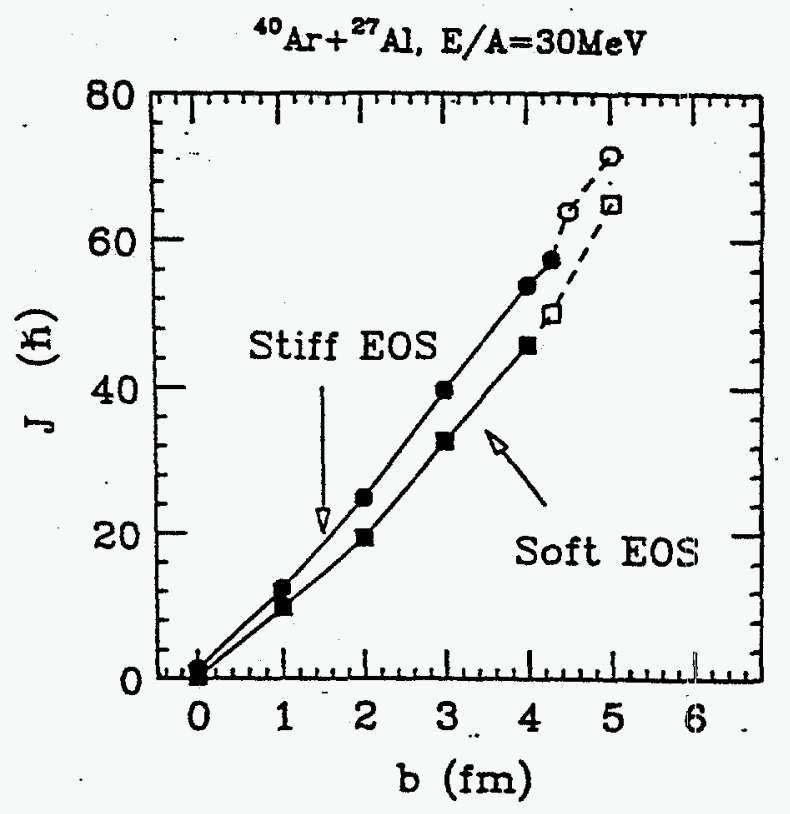

Figure 2. Angular momenta of residues as a function of impact parameter. The solid symbols correspond to calculations for which well defined residues are survived while the open symbols correspond to calculations for which distinct projectile and target like residues are observed in the final states.

Fig. 3 displays the temperatures for the fusionlike residues which have survived from ${ }^{40} \mathrm{Ar}+{ }^{27} \mathrm{~A} 1$ collisions at impact parameter $\mathrm{b}=2 \mathrm{fm}$. For both the stiff EOS (dashed line) and the soft EOS (solid line), the residue temperatures increase with incident energy. The stiff EOS predicts consistently larger values of temperature at all incident energies. Within the experimental uncertainties, the present calculations with both EOS's are comparable with experimental data. This result is significant, since it 
reproduces simultaneously the disappearance of fusionlike cross sections and the observed limiting temperatures, for the first time, within a dynamical model which includes the entrance channels that lead to the formation of the hot residues. This result also indicates that though the residue cross sections are primarily limited by dynamical processes (incomplete damping) at critical impact parameter collisions, the thermal limits may also be reached for the most

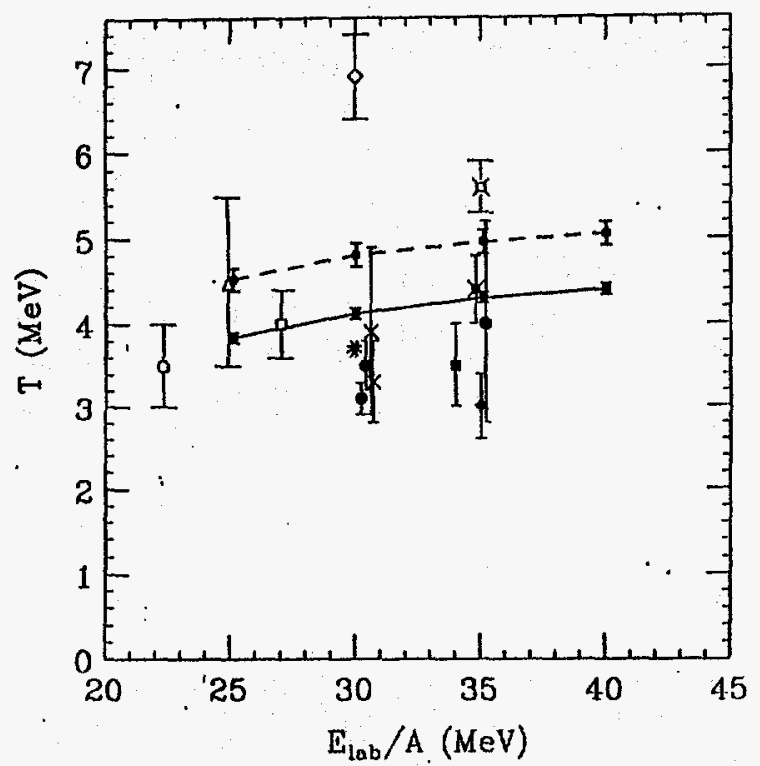

Figure 3. Dependence of the temperature on the incident energy for residues which have survived the ${ }^{40} \mathrm{Ar}+{ }^{27} \mathrm{Al}$ collisions. The data points are experimental data taken from literatures. For details see Ref. 1 . central collisions, where well defined fusionlike residues are observed in the present code. This thermal limit, or the thermal instability, could cause further reductions in the residue cross sections.

On the other hand, on a more quantitative level, the calculations also indicate that although the same residue cross sections can be obtained for both the stiff EOS and the soft EOS by adjusting separately the in-medium nucleon-nucleon cross sections, one would still see significant difference in the residue temperatures. At present, calculations with the soft EOS appear closer to the experimental data. However, due to the strong impact parameter dependencies of the calculated residue temperatures, which is not well addressed by many of the measurements, we can not make quantitative conclusions about the stiffness of the EOS from the present comparisons. More systematical experimental studies, including the impact parameter dependencies, are called for, in particular, to address the questions concerning the equation of state.

\section{References}

1. H. M. Xu, et al., Phys. Rev. Lett. 65, 843 (1990); Phys. Lett. 261B, 240 (1991); Phys. Lett. 299B, 199 (1993); H. M. Xu, W. G. Lynch, and P. Danielewicz, (to be published).

2. H. M. Xu, Nucl. Phys. A568, 365 (1994). 


\title{
Fragmentation Barriers of Toroidal and Bubble Nuclei
}

\author{
H. M. Xu, C. A. Gagliardi, R. E. Tribble, and C. Y. Wong*
}

Recently, we have observed the formation of toroidal and bubble nuclei in our BUU calculations. ${ }^{1-3}$ The purpose of this work ${ }^{4}$ is to compare the configurations of the system when the toroidal or bubble nuclei are formed, with the assumptions used in the standard statistical models. ${ }^{5-7}$ In particular, we investigate, from a dynamical (time-dependent) view, the following two key issues which are important in making connections to statistical models: (1) whether the barriers are reached at the times when the exotic shapes are formed; and (2) whether the low density assumption in the fragmentation models is valid for studying the decay of these exotic shapes. For this purpose, we have calculated, with an improved BUU model,,$^{1-3}$ the deformation energy surface following the dynamics of entrance channels leading to the formation of these exotic shapes.

As an example, we show in the top panel of Fig. 1 the evolution of the average density, defined as $\langle\rho\rangle=\int_{D} \rho^{2} d^{3} r /\left(\int_{D} \rho d^{3} r\right),{ }^{8}$ as a function of time. Here, $\mathrm{D}$ indicates the regions with $\rho \geq 0.1 \rho_{0}$, where $\rho_{0}$ is the nuclear saturation density. The corresponding residue masses are shown in the bottom panel. The residue mass curves exhibit two distinct tendencies: a rapid decrease, resembling the preequilibrium emission at earlier times, and a gradual decrease, resembling slow evaporation of particles, at later times. The compression-expansion dynamics is clearly illustrated in the top panel: for calculations with both the stiff EOS (solid line) and the soft EOS (dashed line), a maximum average density of about $\langle\rho\rangle_{\max } \approx 1.2\left\langle\rho_{0}\right\rangle$ is reached quite early at $t \approx 25-30 \mathrm{fm} / \mathrm{c}$. Following this modest compression, significant expansion is developed at later times with minimum densities $\langle\rho\rangle_{\min } \approx 0.2\langle\rho\rangle$, which are reached at $t \approx 100 \mathrm{fm} / \mathrm{c}$. The average densitics gradually increase at later times, $t \geq 100$ $\mathrm{fm} / \mathrm{c}$, reflecting the condensation into individual fragments. Though densities obtained for both equations of state at early times are about the same, calculations with the stiff EOS (solid lines) have slightly higher densities at later times than those calculated with the soft EOS. This is because the stiff EOS has a larger surface tension strength, and thus the tendency for the residues to expand or break up is less. We note in Fig. 1 that quite low densities are reached at the times $t \approx 100 \mathrm{fm} / \mathrm{c}$, which are close to the times when the toroidal and bubble geometries are formed. ${ }^{1-3}$ The occurrence of low densities with exotic shapes coincides with the low freeze-out density assumption used in the statistical fragmentation models. ${ }^{6,7}$ Nonetheless, such low densities have not been considered in estimating the multi-fission or multifragmentation barriers based on liquid drop models. ${ }^{9,10}$ While the effects of compression or expansion can be ignored in low energy processes, such effects clearly can not be ignored in processes which lead to the formation of the toroidal and bubble nuclei at high energies.

${ }^{92} \mathrm{Mo}+{ }^{92} \mathrm{Mo}, b=0$

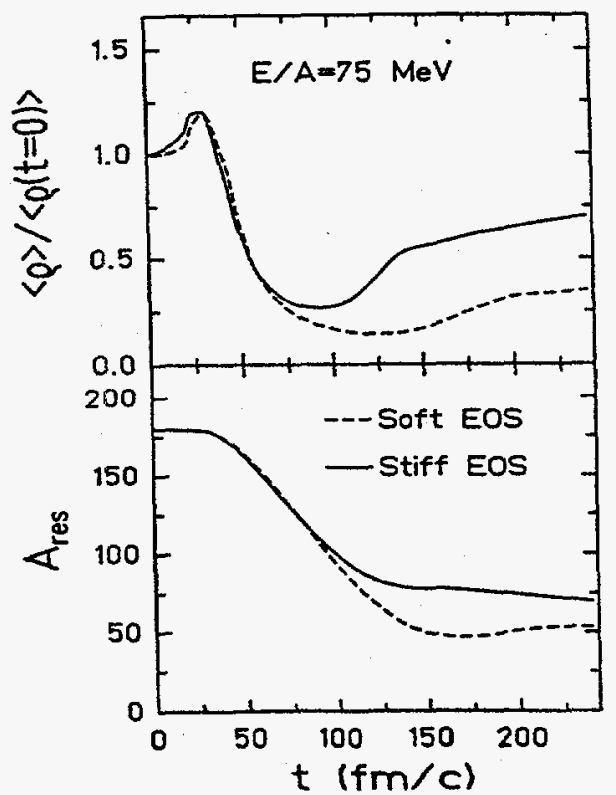

Figure 1. Top panel: the average density as a function of time for both the stiff EOS (solid line) and the soft EOS (dashed line); bottom panel: the corresponding residue mass as a function of time. 
To assess qualitatively the influence of compression and expansion on the values of the barrier height, we follow the dynamics of BUU calculations and estimate the deformation energy using an energy decomposition technique discussed in detail in Ref. 8. Figure 2 shows, respectively, the deformation energy (top panel), the transverse thermal energy (center), and the collective energy (bottom) as a function of time for residues with local density $\rho \geq 0.1 \rho_{0}$. As exhibited in this figure, both the collective energies, $E_{\text {coll }}^{*}$ and $E_{\text {coll }}^{*} / A_{\text {res }}$ (bottom panels), and the thermal energies, $E_{\text {the }}^{*}$ and $E_{\text {the }}^{*} / A_{\text {res }}$ (central panels), reach maximum values at early times $\mathrm{t} \approx 50 \mathrm{fm} / \mathrm{c}$. As a result, the systems expand and the deformation energies, both $E_{d e f}^{*}$ and $E_{\text {def }}^{*} / A_{\text {res }}$ (top panels), reach maximum values at slightly later times. It is clear from this figure that surprisingly large values of barrier heights, $E_{\text {def }}^{*} / A_{\text {res }}-4 \mathrm{MeV}$, for both equations of state), are obtained. They occur at times $t \approx 120-130 \mathrm{fm} / \mathrm{c}$ for the soft EOS and at $t \approx 80-100 \mathrm{fm} / \mathrm{c}$ for the stiff EOS (Fig. 2), which are close to the times when the toroidal or bubbles are already formed. ${ }^{4}$ At these times, the average densities of these exotic shapes are near their minimum values (see Fig. 1). Thus, because of the formation of these exotic shapes at very low densities, the barrier heights for fragmentation are significantly high: two to three times higher than those estimated from liquid-drop models. ${ }^{9,10}$ The fact that these barrier heights are reached at times comparable to the times for which the toroidal or bubble nuclei are well-developed indicates that the decay of such exotic shapes could readily be studied within the transition-state models, ${ }^{5}$ provided the fragmentation barrier heights are properly modified to include the effects of expansion. Since the toroidal and bubble nuclei are formed at very low densities, their decay can also be easily incorporated in the multi-particle phase space models ${ }^{6,7}$ which assume very low densities. In fact, at the times when these exotic shapes are formed $(t \approx 100 \mathrm{fm} / \mathrm{c}$ ), most of the remaining excitation energies are in the form of thermal energies (central panel, Fig. 2) with

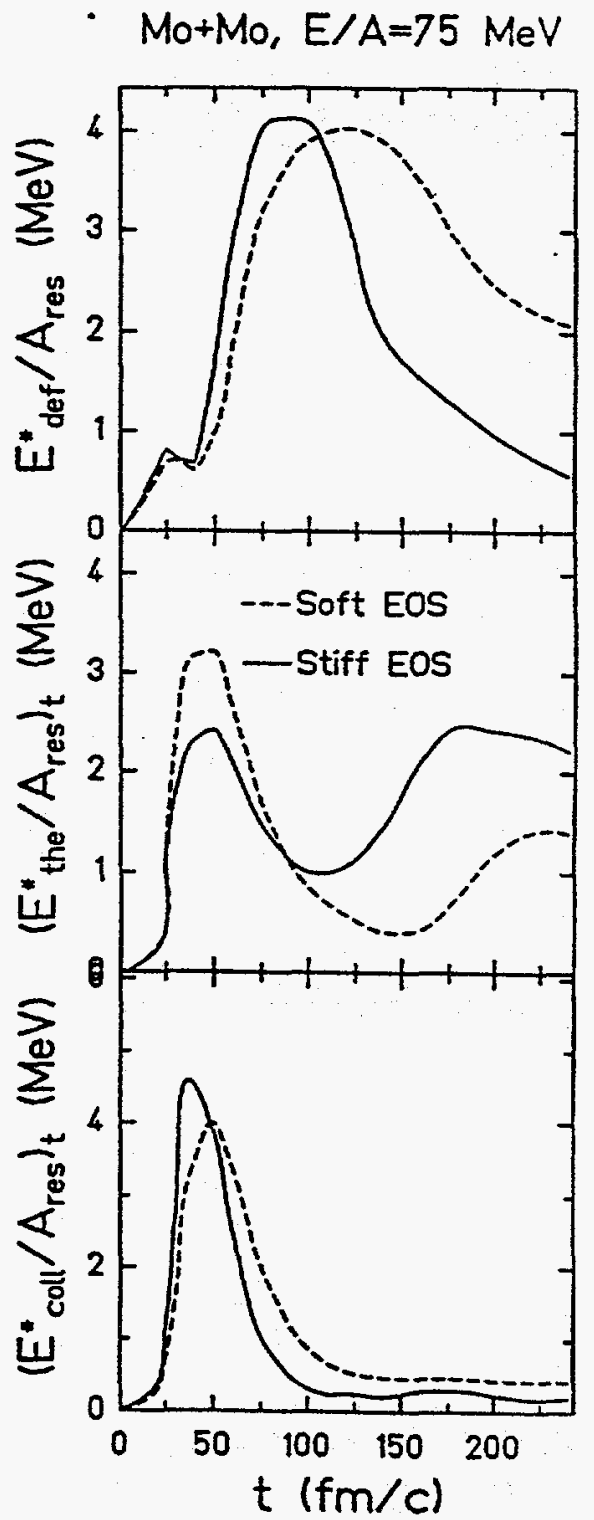

Figure 2. The components of the excitation energy per nucleon for ${ }^{2} \mathrm{Mo}+{ }^{22} \mathrm{Mo}$ collisions at $\mathrm{E} / \mathrm{A}=75 \mathrm{MeV}$ and $\mathrm{b}=0$ with both the stiff (sold lines) and the soft (dashed lines) EOS. Top panel: the deformation energy; Central panel: the thermal energy; Bottom panel: collective energy. For details, see the text.

negligible energies in the form the collective excitations (bottom panel), which are neglected in the statistical models. The gradual increase in the thermal excitation energy at later times reflects the 
breakup and condensation into individual clusters. Although the exotic nuclei are formed only at high energies, the thermal excitation energies at the barriers are very low; e.g., for stiff EOS, $\left(E_{\text {the }}^{*} / A_{\text {res }}\right)_{t} \approx 1 \mathrm{MeV}$ for these incident energies, because (1) significant amounts of mass and energy are taken away by the emission of particles before the system reaches the barriers; and (2) significant amounts of energy are stored in the form of potential energy $E_{\text {def }}^{*}$, which raises the barrier heights significantly. These low thermal excitations explain why the final clusters are nearly equal as observed in our BUU model.

In summary, with an improved BUU model which accurately conserves energy, we demonstrate that the potential maximum, or the fragmentation barrier, indeed occurs at times close to the times when these exotic shapes are formed. We find that these exotic shapes are formed when the systems expand to very low densities. However, due to the dynamics of large amplitude compression and expansion which are responsible for the formation of these exotic shapes, the fragmentation barriers are significantly higher than those estimated recently from liquid-drop models. ${ }^{9.10}$ Our results demonstrate that the decay of these exotic shapes can be readily incorporated into the standard statistical models such as the transition-state models ${ }^{5}$ or the multi-particle phase-space models. 6.7
"Physics Division, Oak Ridge National Laboratory, Oak Ridge, TN 37831

\section{References}

1. H. M. Xu, et al., Progress in Research, 19931994, Cyclotron Institute, TAMU, p. 101.

2. H. M. Xu, et al., Progress in Research, 1992-1993, Cyclotron Institute, TAMU, p. 68; Phys. Rev. C 48, 933 (1983).

3. H. M. Xu, et al., Nucl. Phys. A569, 575 (1994).

4. H. M. Xu, Phys. Rev. C 49, R1778 (1994).

5. L. G. Moretto, Phys. Lett. 40B, 185 (1972); Nucl. Phys. A247, 211 (1975).

6. D. H. E. Gross, Rep. Prog. Phys. 53, 605 (1990); B. H. Sa, Y. M. Zheng, and X. Z. Zhang, Int. J. Mod. Phys. A5, 843 (1990).

7. J. P. Bondorf, et al., Nucl. Phys. A443, 321 (1985); Nucl. Phys. A444, 460 (1985).

8. H. M. Xu, Phys. Rev. C 46, R2144 (1992).

9. F. Haddad and G. Royer, J. Phys. G 18, L153 (1992).

10. G. X. Dai, et al., Nucl. Phys. A568, 601 (1994).

\title{
Multifragmentation of Toroidal and Bubble Nuclei Within the BUU Approach
}

\author{
H. M. Xu, C. A. Gagliardi, R. E. Tribble, and C. Y. Wong*
}

In a previous report, ${ }^{1}$ we presented the initial results of our BUU study on the formation and decay of toroidal and bubble nuclei in central ${ }^{92} \mathrm{Mo}+{ }^{92} \mathrm{Mo}$ collisions. The dependencies on the equations of state were reported: With a stiff equation of state, simultaneous explosion into several nearly-equal fragments (NEFs) in a ring-like manner occurs due to the formation of metastable toroidal nuclei; in contrast, with a soft equation of state, simultaneous explosion into several NEFs in a volume-like manner occurs due to the formation of metastable bubble nuclei. Guided by our BUU model, two signatures 
were proposed: (1) because of the geometries of bubbles and toroids and because of the cold breakup at low temperatures, we predict enhanced cross sections for NEFs at small center-of-mass energies. (2) the coplanarity of these NEFs could carry important information concerning the geometry of the sources. This, in turn, could provide information about the stiffness of the equation of state.

In the present study, ${ }^{2}$ we investigate the details of the reaction dynamics that led to the formation of these exotic shapes. In particular, we study the dependence on various input parameters, including the dependence on impact parameters, the number of test particles, and the initial random seeds. As an example, Fig.1 shows the dependence on impact parameters calculated at impact parameters $b=1 \mathrm{fm}$ (left two columns, top and side views, respectively), $b=2 \mathrm{fm}$ (central two columns), and $b=3 \mathrm{fm}$ (right two columns) for the stiff equation of state. Instead of an emission plane perpendicular to the beam direction observed at $b=0,{ }^{1}$ the emission plane (oblate shape when calculated with the stiff EOS) is now rotated by an angle whose value depends on the impact parameter at impact parameters $b<3 \mathrm{fm}$. For the calculation with the soft EOS (not shown), an emission pattern corresponding to a prolate shape is found with its major axis rotated. ${ }^{2}$ For both cases, the transverse energies in the center of mass for the final fragments are surprisingly small and they are emitted at angles $\theta_{l a b} \leq 20^{\circ}$. For both equation of states at central collisions, $b \leqslant 2 \mathrm{fm}$, the fragments appear to be nearly-equal in size, consistent with what one would expect for the decay of toroidal and bubble nuclei at low temperatures.

At large impact parameters, $b \gtrsim 3 \mathrm{fm}$, whether the toroidal or bubble geometry is formed becomes questionable and one observes remnants of the projectile-like and target-like residues. Clearly, these peripheral collisions differ significantly from the central collisions, where the bubble or toroidal geometry is formed and one observes several fragments of nearly-equal sizes with very small C.M. energies. Thus even without the precise knowledge

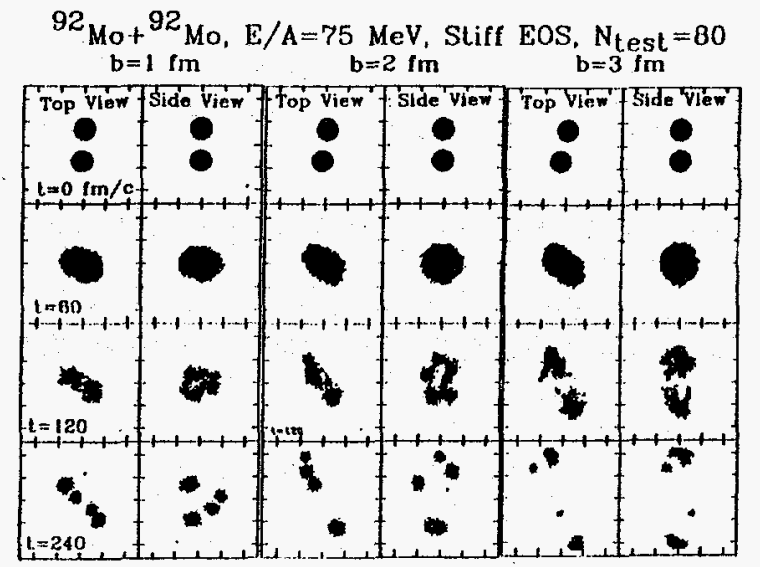

Figure 1. Dependence of density patterns on the impact parameters for ${ }^{92} \mathrm{Mo}+{ }^{92} \mathrm{Mo}$ collisions at $\mathrm{E} / \mathrm{A}=75 \mathrm{MeV}$. calculated with the stiff EOS.

of the impact parameters, one could still separate central events, where the bubbles or toroids are formed, from more peripheral events, if one analyzes the data in the specific kinematic regions discussed here.

Figure 2 shows the dependence on the number of test particles, $N_{\text {test }}=80$ (left two columns, top and front views, respectively) and $N_{\text {test }}=200$ (right two

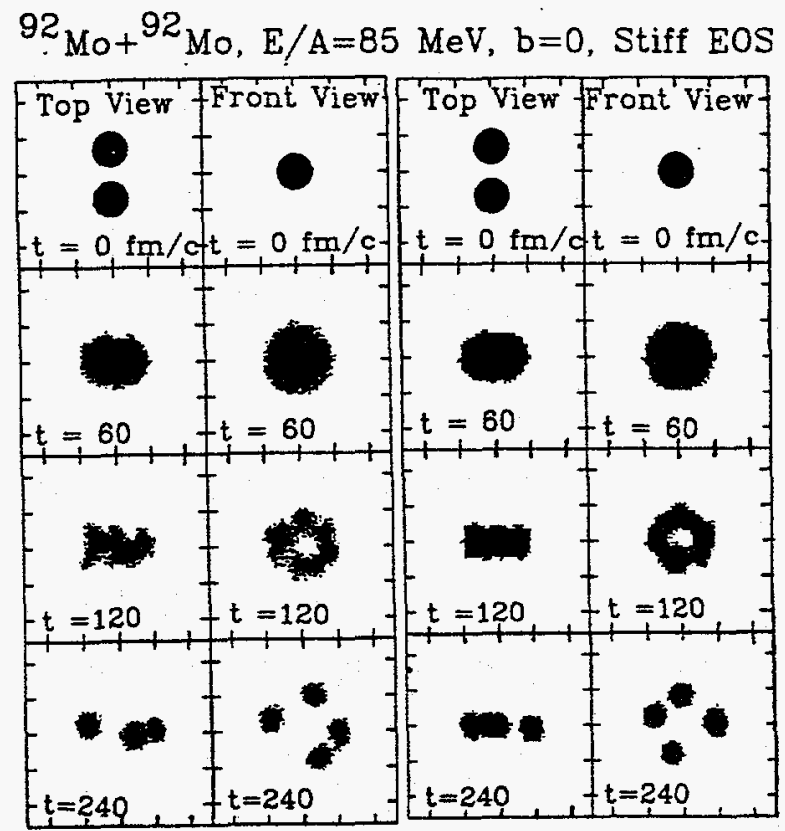

Figure 2. Dependence of density patterns on the number of test particles, $N_{\text {test }}$ for ${ }^{92} \mathrm{Mo}+{ }^{92} \mathrm{Mo}$ collisions at $\mathrm{E} / \mathrm{A}=85 \mathrm{MeV}$ calculated with the stiff EOS at $b=0$. 
columns) using the stiff EOS. From this figure, we observe clearly that the formation of toroids as well as the decay patterns are very similar for calculations with the two different values of $N_{\text {tess }}$. Further studies indicate that bubbles are formed for calculations with the soft EOS independent of the values of $N_{\text {test }}$. Figure 3 shows the dependence on the initial random seeds. In particular, results were shown for three different initializations calculated with the stiff EOS, $N_{\text {test }}=200$ and at $\mathrm{E} / \mathrm{A}=85 \mathrm{MeV}, b=0$. Here we see clearly that toroidal nuclei are consistently produced for all different initializations. These toroidal nuclei are unstable and eventually decay into three, or four clusters at this energy due to the minimum fluctuations associated with the BUU code.

In conclusion, this study confirms our earlier observation that these exotic nuclei are formed only at very central collisions. Moreover, our two major conclusions mentioned in the first paragraph, including the dependence of exotic shapes on equation of state, are not changed due to the change in initial input parameters of our BUU code.

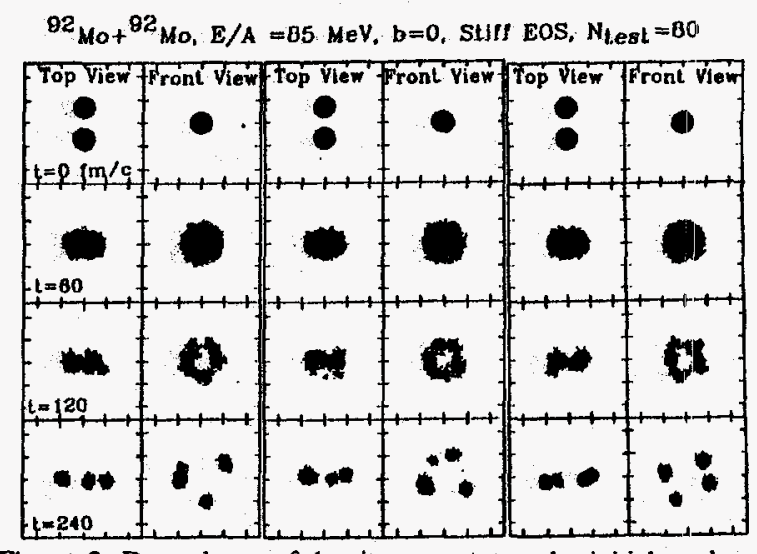

Figure 3. Dependence of density patterns on the initial random seeds with three different initializations (top and front views, respectively, for each calculation) for ${ }^{92} \mathrm{Mo}+{ }^{92} \mathrm{Mo}$ collisions at $\mathrm{E} / \mathrm{A}=85 \mathrm{MeV}$ calculated with the stiff $\mathrm{EOS}$ at $b=0$ and $N_{\text {ten }}=$ 200.

"Physics Division, Oak Ridge National Laboratory, Oak Ridge, TN 37831

\section{References}

1. H. M. Xu, et al., Progress in Research, 1992-1993, Cyclotron Institute, TAMU, p. 68; Phys. Rev. C 48, 933 (1983).

2. H. M. Xu, et al., Nucl. Phys. A569, 575 (1994).

\title{
Investigation of the Equation of State Properties using Maximum Linear Momentum Transfer
}

\author{
F. Haddad, K. Hagel, J. Li, N. Mdeiwayeh, J. B. Natowitz, R. Tezkratt, D. Utley, R. Wada, and
} B. Xiao

One of the main purposes of Heavy-ion experiments is to obtain some information about the Equation of State (E.O.S.) of nuclear matter. The incompressibility modulus, $K_{\infty}$, which governs the capacity of nuclear matter to be compressed, is of particular interest. This quantity is important for many other fields in physics, such as astrophysics or nuclear structure.

Several observables have been suggested to obtain this coefficient: nuclear flow measurements, giant isoscalar monopole resonance, etc. In this report, we explore another way which was suggested by Cibor J. et al. ${ }^{1}$ : The Maximum Linear Momentum Transfer (M.L.M.T.) induced in heavy-ion reaction.

This study has been performed using the well established Landau-Vlasov model. ${ }^{2}$ This model is based on the Landau-Vlasov equation which gives the time evolution of the one-body distribution function, $\mathrm{f}(\mathrm{r}, \mathrm{p})$, in phase space: 


$$
\frac{\partial f}{\partial t}+\{f, H\}=I_{\text {coll }}(f)
$$

where $\{$,$\} stands for the Poisson bracket, \mathrm{H}$ is the Hartree Fock Hamiltonian and $I_{\text {coll }}$ is the UehlingUhlenbeck collision term taking into account the twobody interaction. The local Zamick potential with an isospin dependence has been used in this study.

$$
U_{q}(\rho, \xi)=t_{0} \frac{\rho}{\rho_{0}}+t_{3}\left(\frac{\rho}{\rho_{0}}\right)^{1+v}+C \frac{\xi^{2}}{\rho_{0}^{2}}+4 C \frac{q \rho^{\xi}}{\rho^{2}}
$$

where $q=\frac{1}{2}$ for neutrons and $q=-\frac{1}{2}$ for protons. $\rho(r)$ is the local density:

$$
\begin{aligned}
& \rho(r)=\rho_{n}(r)+\rho_{p}(r) \\
& \xi(r)=\rho_{n}(r)-\rho_{p}(r)
\end{aligned}
$$

The parameters are $\rho_{0}$, the saturation density, and C, related to the volume symmetry coefficient of the mass formula. The parameters $t_{0}, t_{3}$, and $\nu$ are determined by the density and the energy at the saturation point and by choosing a value of the incompressibility modulus. In this study, two different sets of parameters are used. One with $K_{\infty}$ $=200 \mathrm{MeV}$ which corresponds to a so called soft E.O.S. and the other with $K_{\infty}=380 \mathrm{MeV}$ governing a stiff one. Table I contains all the coefficient values.

Table I. Zamick Interactions Parameters

\begin{tabular}{|c|c|c|c|c|}
\hline E.O.S. & $t_{0}$ & $t_{3}$ & $\nu$ & $\mathrm{C}$ \\
\hline soft & $-356 \mathrm{MeV}$ & $303 \mathrm{MeV}$ & $\frac{1}{6}$ & $20 \mathrm{MeV}$ \\
\hline stiff & $-123 \mathrm{MeV}$ & $70 \mathrm{MeV}$ & 1 & $20 \mathrm{MeV}$ \\
\hline
\end{tabular}

In the beginning of the 80's, a great amount of work was devoted to the determination of the linear momentum transfer in the actinide target region. A representative sample of those data is reported in Fig. 1. We have calculated, for four different systems, the M.L.M.T. To obtain the maximum value, only central collisions, $b=0 \mathrm{fm}$, have been investigated. The L.M.T. is determined at a freeze out time when a compound nucleus is formed or just before the splitting occurs in collisions where a mononucleus is not formed. In all cases, thermal equilibration is reached. Figure 1-a shows the results for the soft E.O.S. while Fig. 1-b shows those for the stiff E.O.S. For the soft E.O.S., the calculation is in very good agreement with the data. At low incident energy, the stiff E.O.S. calculations are compatible with the experimental results but give too much momentum transfer for the large incident energies.

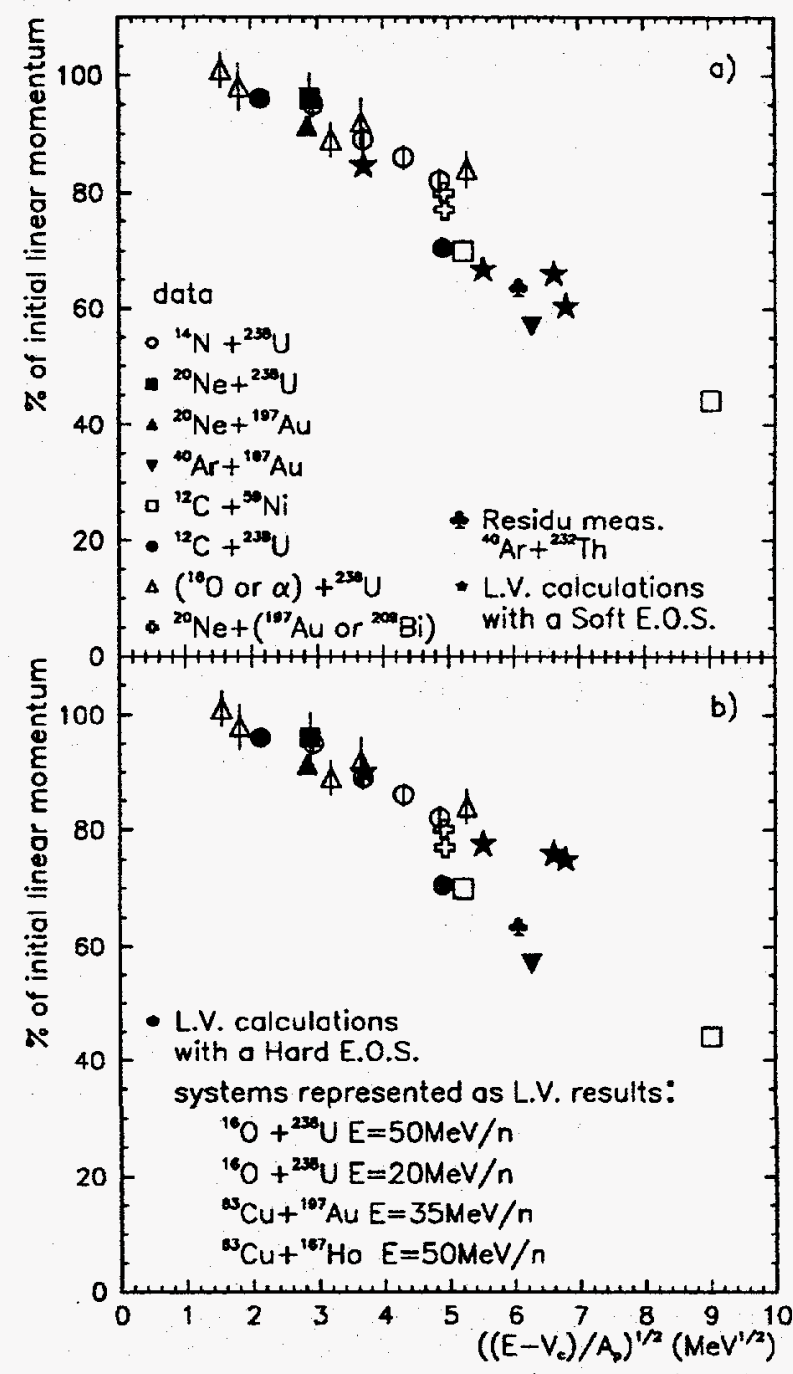

Figure 1. Maximum linear momentum transfers obtained in heavy ion reactions. Calculations correspond to an impact parameter $b$ $=0 \mathrm{fm}$. In a), the soft E.O.S. results are reported while those for a stiff E.O.S. are plotted in b). 
This fact must be related to the global feature of the reactions. Compound nucleus formation is always reached in this case, contrary to the case for the soft E.O.S.

The M.L.M.T. value seems to give some information on the E.O.S. A small value of $K_{\infty}$ allows reproduction of the data for a large range of systems as was the case in Ref. 1. Some complementary studies including the momentum dependence of the potential and the use of lighter systems are in progress. From an experimental point of view, it could be interesting to obtain precise data at high energies. A lack of information exists for high energy where the common measurement, based on the folding angle determination, fails. The use of our $4 \pi$ neutron detector, to select the most central collisions, associated with the measurement of the residue formed in those reactions could be an alternative way to obtain this information. A value derived from such a measurement, for ${ }^{40} \mathrm{Ar}+{ }^{232} \mathrm{Th},{ }^{3}$ is shown in Fig. 1.

\section{References}

1. J. Cibor, J. Lukasik, and Z. Majka, Z. Phys. (in press).

2. B. Remaud, C. Grégoire, F. Sébille, and P. Schuck, Nucl. Phys. A488, 423c (1988).

3. D. Utley et al., Phys. Rev. C 49, 1737 (1994).

\title{
Role of the $a_{1}$ Meson in Dilepton Production from Hot Hadronic Matter
}

\author{
Chungsik Song, C. M. Ko, and C. Gale*
}

In nucleus-nucleus collisions at ultrarelativistic energies, a hot and dense matter consisting of quarks and gluons is expected to be formed in the initial stage of the collision. This quark-gluon plasma eventually transforms into the hadronic matter as it expands and cools below some critical temperature. Since dileptons and photons produced from the quark-gluon plasma do not suffer final-state interactions, they carry information about their production and have been considered as possible signatures for the formation of the quark-gluon plasma. ${ }^{1}$ However, dileptons and photons can also be produced from the hot and dense hadronic matter. To use them as signatures for the quark-gluon plasma, one needs to distinguish them from those produced from the hadronic matter.

For dileptons with invariant masses larger than the $J / \Psi$ mass $(3 \mathrm{GeV})$, the dominant contributions are from the Drell-Yan process and the direct charm decay, while for invariant masses lower than the phi meson mass $(1 \mathrm{GeV})$, radiative and direct decays, together with the $\pi \pi$ annihilation, form the most important sources. To identify the quark-gluon plasma with these low invariant mass dileptons is thus difficult. Nevertheless, for dileptons of invariant masses that are between the phi and $J / \Psi$ masses, $m_{\Psi}<M<m_{J / \Psi}$, the contribution from the quark-gluon plasma may dominate over that from the hadronic matter, so these dileptons may serve as a signal for the quark-gluon plasma.

The dilepton production rate from the hadronic matter is usually calculated by assuming that the hadronic matter consists of only pions. Recently, it has been shown that, for temperatures $T>100$ $\mathrm{MeV}$, dilepton production from reactions involving higher-mass hadron resonances is important. ${ }^{2}$ Including both strange and non-strange pseudoscalar and vector mesons, it has been found that in the 
invariant mass region, $1.5 \mathrm{GeV}<M<3.0 \mathrm{GeV}$, the reactions involving vector mesons lead to significant dilepton production.

We have studied dilepton production from processes involving the $a_{l}$ meson in a thermalized hadronic matter using an effective chiral Lagrangian that includes not only pseudoscalar and vector mesons but also axial-vector mesons. ${ }^{3}$ In our effective Lagrangian, the pseudoscalar mesons are described by the non-linear $\sigma$ model while the vector and axial-vector mesons are included as massive Yang-Mills fields of the $S U(2) \times S U(2)$ chiral symmetry. The parameters of the effective Lagrangian have been determined from the experimental data on the decay widths and masses of $\rho$ and $a_{1}$ mesons. The electromagnetic interaction is introduced through imposing the $U(1)_{E M}$ gauge symmetry on the effective chiral Lagrangian.

The $a_{1}$ meson contributes to dilepton production mainly through the two processes, $\pi^{+}+a_{1}^{-}\left(\pi^{-}+a_{1}^{+}\right) \rightarrow e^{+}+e^{-}$and $a_{1}^{+}+a_{1}^{-} \rightarrow e^{+}+e^{-}$. We have calculated the dilepton production rate from hadronic matter at a temperature $200 \mathrm{MeV}$. The results are shown in Fig. 1. The dotted curve is the "usual" result from the reaction $\pi^{+} \pi^{-} \rightarrow e^{+} e^{-}$which dominates at low invariant masses. The contribution from the reaction $\rho^{+} \rho^{-} \rightarrow e^{+} e^{-}$is shown by the dashed curve. This process was found in Ref. 2 to be the dominant one for dileptons of invariant masses in the region $1.5 \mathrm{GeV}<M<3.0 \mathrm{GeV}$. The solid curves are from the reactions $\pi^{+} a_{1}^{-}\left(\pi^{-} a_{1}^{+}\right) \rightarrow e^{+} e^{-}$ and $a_{1}^{+} a_{1}^{-} \rightarrow e^{+} e^{-}$, both involving the $a_{l}$ meson. We note that in our calculations we have used the same form factors for $\pi^{+} \pi^{-} \rightarrow e^{+} e^{-}$and $\rho^{+} \rho^{-} \rightarrow e^{+} e^{-}$, as in Ref. 2. For the reactions $\pi^{+} a_{1}^{-}\left(\pi^{-} a_{1}^{+}\right) \rightarrow e^{+} e^{-}$ and $a_{1}^{+} a_{1}^{-} \rightarrow e^{+} e^{-}$, we assume that the form factors are also the same as for $\pi^{+} \pi^{-}$annihilation.

Our results show that dilepton production from $\pi^{+} a_{1}^{-}\left(\pi^{-} a_{1}^{+}\right) \rightarrow e^{+} e^{-}$is more important than that from the reaction $\rho^{+} \rho^{-} \rightarrow e^{+} e^{-}$and is most important in the invariant mass region $1.5 \mathrm{GeV}<M$ $<3.0 \mathrm{GeV}$. The reaction $a_{1}^{+} a_{1}^{-} \rightarrow e^{+} e^{-}$, on the other hand, becomes important only at higher
Dilepton Emission from Hadronic Matter

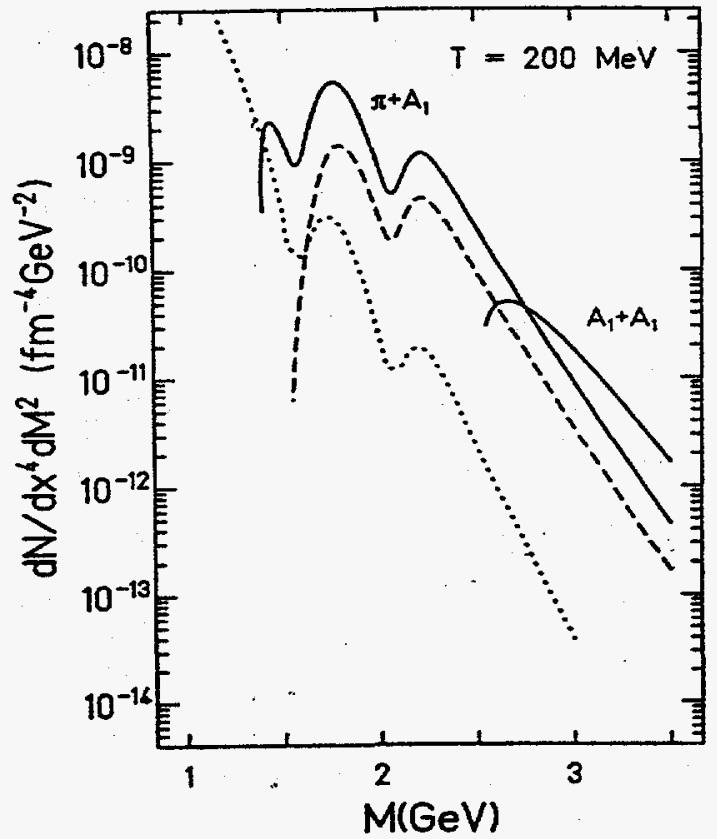

Figure 1. Dilepton production from the hot hadronic matter at $T$ $=200 \mathrm{MeV}$; the dotted and dashed curves are contributions from $\pi^{+} \pi^{-} \rightarrow e^{+} e^{-}$and $\rho^{+} \rho^{-} \rightarrow e^{+} e^{-}$, respectively, while the solid curves are from $\pi^{+} a_{1}\left(\pi^{-} a_{1}^{+}\right) \rightarrow e^{+} e^{-}$and $a_{1}^{+} a_{1}^{-} \rightarrow e^{+} e^{-}$.

invariant masses.

In using the dilepton as a signal for the quark-gluon plasma in future ultrarelativistic heavy-ion collisions, it is thus essential to understand the contribution to dileptons from the $a_{i}$ meson.

"Physics Department, McGill University, 3600 University St., Montréal, QC, H3A 2T8, Canada

\section{References}

1. P. V. Ruuskanen, Nucl. Phys. A544, 169c (1992), and references therein.

2. C. Gale and P. Lichard, Phys. Rev. D 49, 3338 (1994).

3. Chungsik Song, Phys. Rev. C 47, 2861 (1993); Phys. Rev. D 48, 1375 (1993); Phys. Rev. D 49, 1556 (1994). 


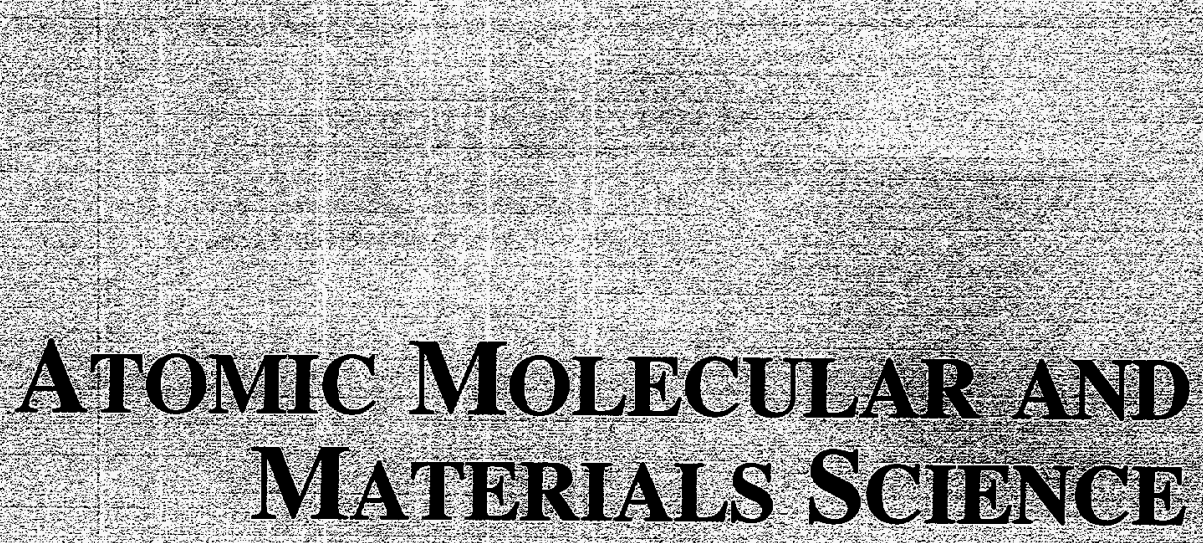




\title{
Electron Spectra from $\mathrm{Ag}$ and $\mathrm{NaCl}$ Targets Induced by Highly-Charged Ar Ions
}

\author{
C. Assad and R. E. Tribble
}

During this past year, we have obtained electron spectra from two different targets, $\mathrm{Ag}$ and $\mathrm{NaCl}$, that were produced by the interaction with $\mathrm{Ar}^{+q}$ ions at energies of $24 \mathrm{keV}$ with $\mathrm{q}=2,4,6,8$, and 9 , at 48 $\mathrm{keV}$ with $\mathrm{q}=4,6,8,9$, and 11 , and at $96 \mathrm{keV}$ with $q=8,9,11$, and 13 using Ar beams from the ECR ion source. The data were taken in the ultra high vacuum scattering chamber that is located on the beam line from the ECR source for atomic and surface science experiments. The experimental setup is shown in Fig. 1. The electron spectrometer is a model AC-902 from Comstock that was mounted inside a thin walled metal box that shielded the spectrometer and detector from stray electrons. A two stage micro-channel plate (MCP) detector was used at the end of the spectrometer to measure the electrons that were transported through the system. In front of the taget was a beam collimator which was followed by a ground plate, a wire mesh grid and a second ground plate. Beam currents were determined from the wire mesh grid which was calibrated against a $\mathrm{Cu}$ Faraday cup that could be inserted into the beam at the target location. An electron gun was mounted inside the vacuum system which was used to calibrate the spectrometer and to look at Auger spectra from the two targets.

The outside of the scattering chamber was lined with two layers of mu-metal shielding. With the cyclotron magnet off, this was sufficient to reduce the residual magnetic field in the central region of the chamber to levels of less than 0.1 Gauss. All electrical connections inside the chamber were isolated from each other with vacuum compatible insulation. In order to reduce static charge build up on these wires, they were wrapped with $\mathrm{Cu}$ wire that was then connected to the ground of the chamber. The spectrometer was operated in the constant transmission energy mode with the pass energy set at 50 electron volts. In this mode, the spectrometer efficiency varies as $1 / \mathrm{E}$ where $\mathrm{E}$ is the incident energy of the incoming electrons. Six high voltages on the spectrometer were adjusted by programmable power supplies that were connected to a personal computer (PC) through a CAMAC crate and a DSP Technology PC004 plug in interface card.

The $\mathrm{Ag}$ and $\mathrm{NaCl}$ targets and the $\mathrm{Cu}$ Faraday cup were mounted on a target ladder at the center of the scattering chamber. The $\mathrm{NaCl}$ target was similar to one that was used in our study of light emission. It was made by compressing $\mathrm{NaCl}$ powder around a $\mathrm{Cu}$ wire mesh grid at high pressure $(20,000 \mathrm{lbs}$.). The Ag target was $1 \mathrm{~cm} \times 1 \mathrm{~cm} \times 0.2 \mathrm{~cm}$ with a purity of $99.99 \%$.The target ladder was heated to about 390 degrees $\mathrm{C}$ and kept constant throughout the data acquisition. At this temperature, the $\mathrm{NaCl}$ target becomes slightly conducting so that static charge buildup from the beam is not a problem. Also heating the target increases the time required for surface contamination to build up on it. Even so, the targets were sputter cleaned by the beam prior to data acquisition with each new energy and charge state.

A spectroscopy preamplifier and amplifier were used to read out the MCP since high frequency noise (due to pick up from the ECR source power supplies) made it impossible to use fast electronics. Output signals from the amplifier were passed through a discrimator whose threshold was set above the noise. Data were collected in a PC via scalars that were read out in a CAMAC crate. The primary data that were collected were the number of discriminator outputs and the number of digitized current integrator outputs in a fixed time interval. After each counting time interval, the high voltages on the spectrometer were stepped to new values. Following a short delay to insure that the voltages were stable, the scalars were started and then data acquired for the next time interval.

Typical spectra showing electron yields as a 
function of electron energy for the two targets are shown in Fig. 2. The yields have been multiplied by E to correct for the spectrometer efficiency. Also the yields are normalized to the number of incoming particles. Several features are quite noticeble in the data. First, the $\mathrm{NaCl}$ target has a large yield in the region around $150-170 \mathrm{eV}$ for essentially all charge states and all energies. This is presumably due to Auger emission from the $\mathrm{Cl}$ in the target. It is interesting to note that the $\mathrm{Ar}$ and $\mathrm{Cl}$ targets are closely matched in $\mathrm{Z}$ and this appears to facilitate the electron transfer thus producing the peak, perhaps via a quasi-molecular mechanism. As the charge state of the incoming beam is increased, a peak emerges in both the $\mathrm{Ag}$ and $\mathrm{NaCl}$ spectra which appears to be due to Auger emission from the incoming $\mathrm{Ar}$ ion as it neutralizes. Also as the charge state and energy of the Ar ion increase, we see the Auger line in $\mathrm{Ag}$ at $350 \mathrm{eV}$ appear above the background. The yield to this line at $96 \mathrm{keV}$ and with charge states higher than $8^{+}$is quite large compared to its excitation both at lower Ar ion charge states and with light projectiles such as protons. Detailed analysis of the data is now in progress.

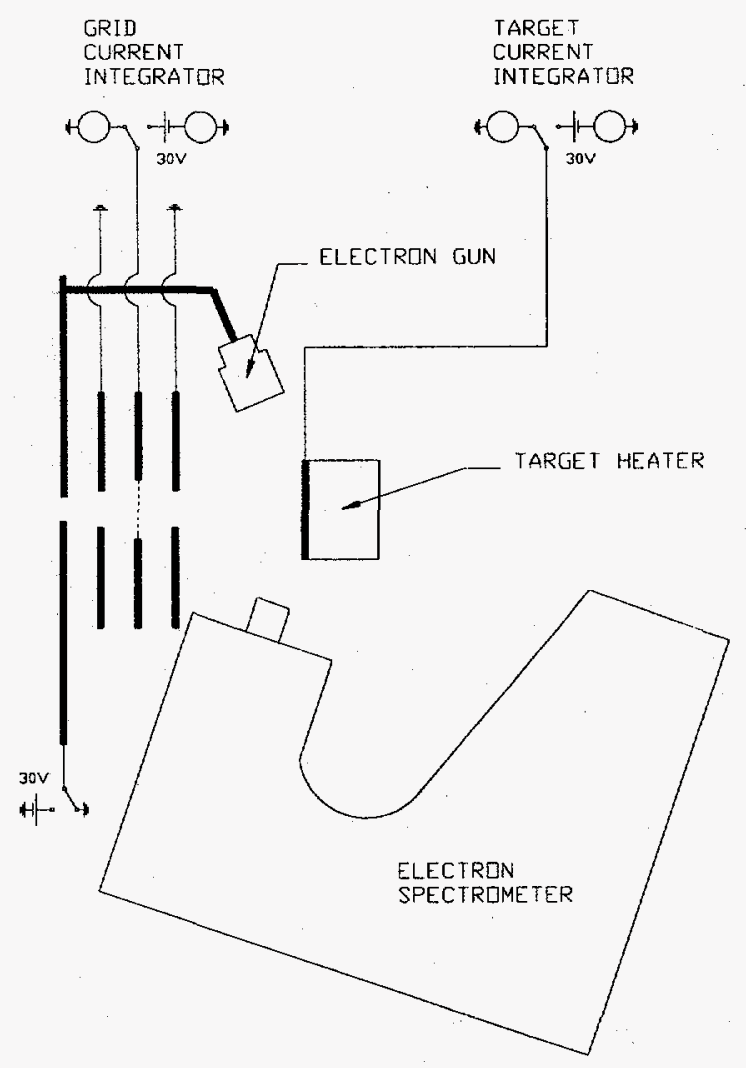

Figure 1. Schematic of the experimental setup in the UHV chamber. 

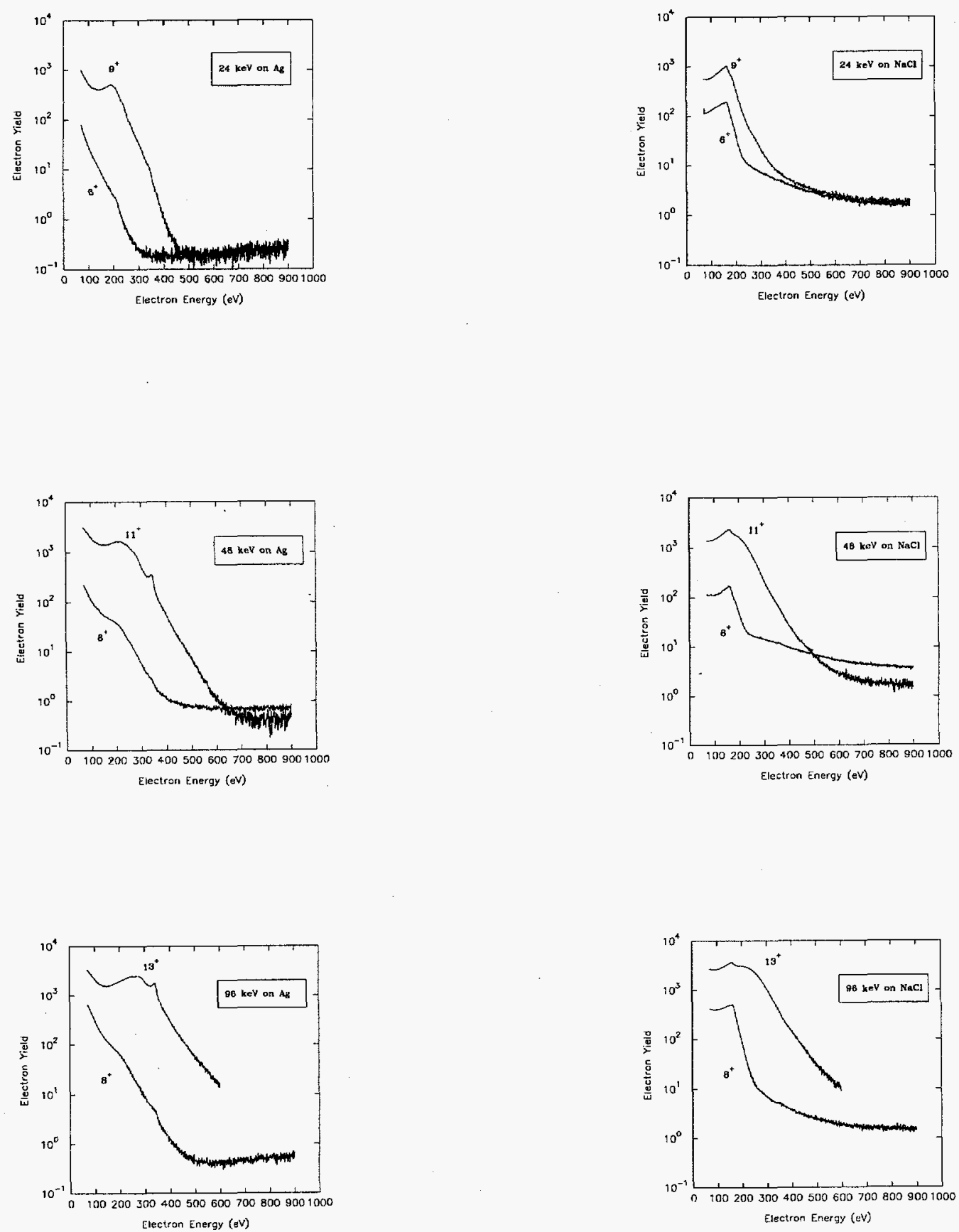

Figure 2. Spectra showing electron yield as a function of electron energy for the $\mathrm{Ag}$ and $\mathrm{NaCl}$ targets with incoming $\mathrm{Ar}^{+\mathrm{q}}$ ions at energies of $24 \mathrm{keV}$ with $q=6,9$, at $48 \mathrm{keV}$ with $q=8,11$, and at $96 \mathrm{keV}$ with $q=8,13$. 


\section{Spectra of L X-rays from Fast, Highly-charged Xe Ions Traveling in Solids}

\section{Horvat, R. L. Watson, and R. Parameswaran}

This report describes a study of the spectra of $\mathrm{L}$ $\mathrm{x}$-rays emitted by 6 and $8 \mathrm{MeV} / \mathrm{u}$ Xe projectiles traveling in thick and thin solid targets. At these velocities, the equilibrium charges of $\mathrm{Xe}$ (after emerging from a solid) are in the range of $43+$ to $45+$. In the ground state of $\mathrm{Xe}^{44+}$, electrons completely fill the $K$ and the $L$ shell, while the remaining shells are empty. However, the charge state equilibration process is a dynamic one in which there is a constant exchange of electrons between the ion and the medium. In the cases under consideration here, excitation/ionization of $\mathrm{K}$-shell electrons is extremely rare, as is electron capture to shells higher than the $\mathrm{M}$-shell. Therefore, the electronic configurations contributing to the equilibrium charge state distribution are expected to primarily consist of those formed from the ground state configuration by the removal of $\mathrm{L}$-shell electrons and the addition of $\mathrm{M}$-shell electrons. The object of the work described in this report was to examine the $\mathrm{L} x$-ray structure under moderately high resolution and identify the major $\mathrm{x}$-ray components. In a following report, this information is used to deduce the average $\mathrm{L}$ - and $\mathrm{M}$-shell electron population distributions of $\mathrm{Xe}$ ions inside the solid.

Beams of $6 \mathrm{MeV} / \mathrm{u} \mathrm{Xe}^{17+}$ and $8 \mathrm{MeV} / \mathrm{u} \mathrm{Xe} \mathrm{X}^{21+}$ were directed at thick targets of $\mathrm{Li}, \mathrm{Be}, \mathrm{B}, \mathrm{BN}, \mathrm{C}$, $\mathrm{NaF}, \mathrm{Al}, \mathrm{KCl}$, and the oxides of $\mathrm{Ca}, \mathrm{Sc}, \mathrm{Cr}$, and Mn. Thin foils of C (100 and $\left.200 \mu \mathrm{g} / \mathrm{cm}^{2}\right), \mathrm{Al}(150$ $\left.\mu \mathrm{g} / \mathrm{cm}^{2}\right)$, and $\mathrm{Ni}\left(180 \mu \mathrm{g} / \mathrm{cm}^{2}\right)$ were also used in some of the measurements. They were all oriented at $45^{\circ}$ with respect to the (horizontal) beam direction and at $45^{\circ}$ with respect to the (vertical) spectrometer axis such that the spectrometer viewed them from the front.

A $12.7 \mathrm{~cm}$ Johansson-type curved-crystal x-ray spectrometer with the focal circle oriented perpendicular to the beam axis was used to measure the $\mathrm{x}$-ray spectra. $\mathrm{X}$-rays diffracted from a $\mathrm{LiF}$ crystal were counted by a flow proportional counter (1 atm of argon (90\%)-methane (10\%)) having a 65 $\mu \mathrm{g} / \mathrm{cm}^{2}$ thick stretched polypropylene window. The spectrometer's view of the interaction region was defined by a $1.6 \mathrm{~mm}$ diam. aperture located at the top of the target cell. Energy calibration of the spectrometer was performed using the measured diffraction positions of the $K \alpha_{1,2}$ and $K \beta_{1}$ peaks of $K$, $\mathrm{Ca}, \mathrm{Sc}, \mathrm{Ti}, \mathrm{V}, \mathrm{Cr}$, and $\mathrm{Mn}$.

Data acquisition was accomplished by means of a Nucleus PC Analyzer card with the accompanying software, ${ }^{1}$ installed on an IBM XT computer: and operated in the multiscaler mode. The collected data were stored on a floppy disk. Data analysis was carried out off-line with a 486 PC-AT along with FORTRAN least-squares fitting programs written specifically for this purpose.

Spectra of L $\mathrm{x}$-rays emitted from $6 \mathrm{MeV} / \mathrm{u} \mathrm{Xe}$ ions traveling in a variety of thick solid targets having atomic numbers ranging from 25 down. to 3 are shown in Fig. 1. It is evident that the structure of the Xe $\mathrm{L} x$-ray spectrum exhibits a strong dependence on the target atomic number $\left(Z_{2}\right)$ and that partially resolved components emerge as $Z_{2}$ decreases below 10. Similar behavior was observed in the thin target measurements.

The range of 6 to $8 \mathrm{MeV} / \mathrm{u} \mathrm{Xe}$ ions in solids with atomic numbers between 3 and 25 is of the order of $20 \mathrm{mg} / \mathrm{cm}^{2}(0.1 \mathrm{~mm}) .{ }^{2}$ All the thick targets used in this experiment were much thicker than this, which means the beam particles were completely stopped. The charge changes very rapidly from its initial value reaching around $44+$ (the average equilibrium charge) within the first $150-200$ $\mu \mathrm{g} / \mathrm{cm}^{2} .^{3}$ Thereafter, the average charge decreases gradually as the ion slows down. By the time the projectile has lost $1 \mathrm{MeV} / \mathrm{u}$, the average charge has dropped approximately 1 unit. $^{3}$ The total distance traveled at this point is about $2 \mathrm{mg} / \mathrm{cm}^{2}(10 \mu \mathrm{m})$, 
which is $10 \%$ of the full range. Since the number of possible electronic configurations involving $\mathrm{L}$ vacancies is quite large for each contributing charge state, the resulting $L \mathrm{x}$-ray spectrum was expected to contain many closely spaced lines covering a wide energy range. Therefore, the structure displayed by the spectra for the low- $Z$ targets (see Fig. 1) is, at first sight, rather surprising.

The spectra indicate that the observed $x$-rays originate from $\mathrm{Xe}$ ions having a fairly restricted range of charges. This deduction is based on the following two spectral characteristics. (a) The observed L x-ray structure begins around $4.4 \mathrm{keV}$, whereas the most prominent $\mathrm{L} x$-ray line $\left(\mathrm{L} \alpha_{1}\right)$ from singly ionized Xe atoms has an energy of $4.1 \mathrm{keV} .{ }^{4}$ This large energy shift means (as will be demonstrated later) that only ions stripped of most of their outer electrons (down to the L-shell) contribute to the spectra. (b) The attenuation lengths of Xe L xrays vary widely over the range of target $Z$ examined and yet the overall positions and widths of the $L x$ ray distributions do not change very much (see Fig. 1). For example, in $\mathrm{KCl}$ the thickness required to attenuate $4.5 \mathrm{keV} \mathrm{x}$-rays by $90 \%$ is $4.6 \mathrm{mg} / \mathrm{cm}^{2} \mathrm{~s}$ while the range of $6 \mathrm{MeV} / \mathrm{u}$ Xe ions in $\mathrm{KCl}$ is about $14 \mathrm{mg} / \mathrm{cm}^{2}$. On the other hand, for $\mathrm{Li}$ the $90 \%$ attenuation thickness is $0.92 \mathrm{~g} / \mathrm{cm}^{2}$ and the range is $10 \mathrm{mg} / \mathrm{cm}^{2}$. Hence, if $\mathrm{L} \mathrm{x}$-ray emission from Xe ions having low charges (i.e. near the end of their range) was very probable, the spectrum obtained with the $\mathrm{Li}$ target would extend to much lower energies than the spectrum obtained with the $\mathrm{KCl}$ target. As may be discerned from Fig. 1, this is clearly not the case.

In order to predict the underlying $\mathrm{L} x$-ray structure contained in the measured spectra, the energies of transitions to the L-shell within the energy region viewed were calculated using the multiconfigurational Dirac-Fock (MCDF) program of Desclaux. ${ }^{6}$ These calculations were performed for all initial state configurations containing two K-shell electrons $+n_{L}$ L-shell electrons + one M-shell electron, where $n_{L}$ ranged from 7 to 0 .

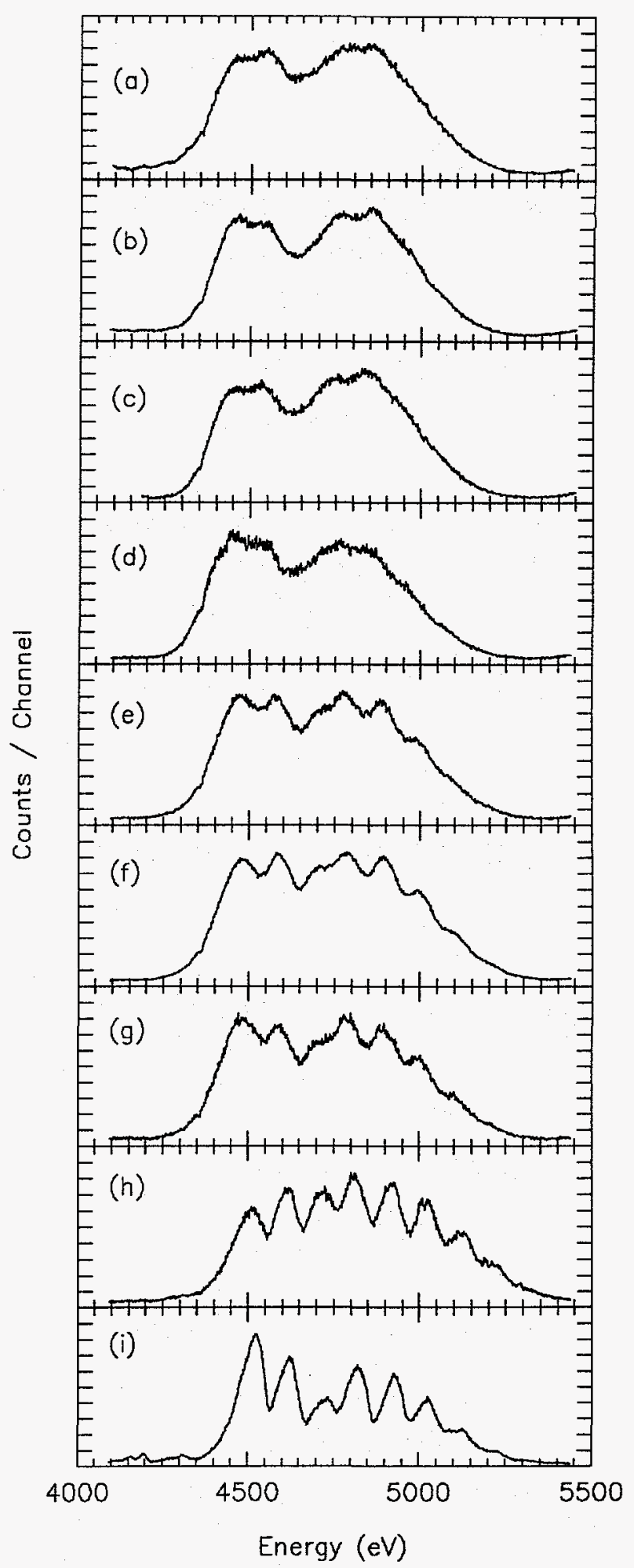

Figure 1. Spectra of Xe L x-rays emitted by $6 \mathrm{MeV} / \mathrm{u} \mathrm{Xe}$ ions traveling in thick targets of (a) $\mathrm{CaO}+\mathrm{MnO}$, (b) $\mathrm{KCl}$, (c) $\mathrm{Al}$, (d) $\mathrm{NaF}$, (e) BN, (f) C, (g) B, (h) Be, and (i) Li. 
Configurations for all possible combinations of the occupation numbers $n_{L 1}, n_{L 2}$, and $n_{L 3}$ of the $L_{1}, L_{2}$, and $L_{3}$ subshells were included. The specific transitions considered were $L \ell\left(M_{1} \rightarrow L_{3}\right), L \alpha_{1,2}\left(M_{4,5}\right.$ $\left.\rightarrow \mathrm{L}_{3}\right), \mathrm{L} \eta\left(\mathrm{M}_{1} \rightarrow \mathrm{L}_{2}\right), \mathrm{L} \beta_{1}\left(\mathrm{M}_{4} \rightarrow \mathrm{L}_{2}\right), \mathrm{L} \beta_{4}\left(\mathrm{M}_{2} \rightarrow \mathrm{L}_{1}\right)$, and $L \beta_{3}\left(M_{3} \rightarrow L_{1}\right)$.

A simulated spectrum was constructed for each value of $n_{L}$ by assuming a statistical population of initial states and using Scofield's ${ }^{7}$ calculated L-shell transition rates for single-vacancy atoms. The simulated spectrum for $n_{L}=7$ is shown together with a measured spectrum (Li target) in Fig. 2. From this comparison and those for other values of $n_{L}$, it was conciuded that the main components present in the measured spectra are $\mathrm{L} \alpha_{1}$ and $\mathrm{L} \beta_{1}$ pairs from Xe ions having $\mathrm{n}_{\mathrm{L}}$ from 7 to 4 . Additionally, the simulated spectra predicted that the $L \beta_{4}$ and $L \beta_{3}$ transitions should contribute to the structure at the high energy end. However, for $n_{L} \leq 5$, the calculations showed that the $\mathrm{L} \beta_{4}$ peak merges together with the $\mathrm{L} \beta_{1}$. The peaks in the simulated spectra were all shifted toward higher energies relative to the peaks in the measured spectra, as may be seen in Fig. 2. This indicates the $\mathrm{x}$-ray components in the experimental spectrum were emitted from atoms containing (on average) several additional M-shell (spectator) electrons. It was determined, for a selected number of representative cases, that adding spectator electrons to the M-shell causes all the calculated $\mathrm{L} \mathrm{x}$-ray energies to shift down in energy by an amount $\Delta \mathrm{E} \approx 24 \mathrm{eV}$ per spectator electron (to within a few $\mathrm{eV}$ ). Another characteristic revealed by the calculations was that the energy of the $L \beta_{1}$ transition in a Xe atom having $\mathrm{n}_{\mathrm{L}} \mathrm{L}$-shell electrons in the initial state is very close to the energy of the $\mathrm{L} \alpha_{1}$ transition in a Xe atom having $n_{L}-3$ L-shell electrons in the initial state when $n_{L} \geq$ 4. This helps explain why the peaks in the spectra for low-Z targets were better resolved than was first expected.

\section{References}

1. The Nucleus Inc., 761 Emory Valley Rd., Oak Ridge, TN 37830, USA.

2. F. Hubert, R. Bimbot and H. Gauvin, Atomic Data and Nucl. Data Tables 46, 1 (1990).

3. Y. Baudinet-Robinet, Nucl. Instrum. Methods 190, 197 (1981).

4. J. A. Bearden, Rev. Mod. Phys 39, 78 (1967).

5. H. McMaster, N. K. Del Grande, I. H. Mallet, and J. H. Hubell, Lawrence Livermore National Laboratory Report No. UCRL-50174, Sec. II, Rev. 1 (1969) (unpublished).

6. P. Desclaux, Comp. Phys. Commun. 9, 31 (1975).

7. H. Scofield, Phys. Rev. 179, 9 (1969).

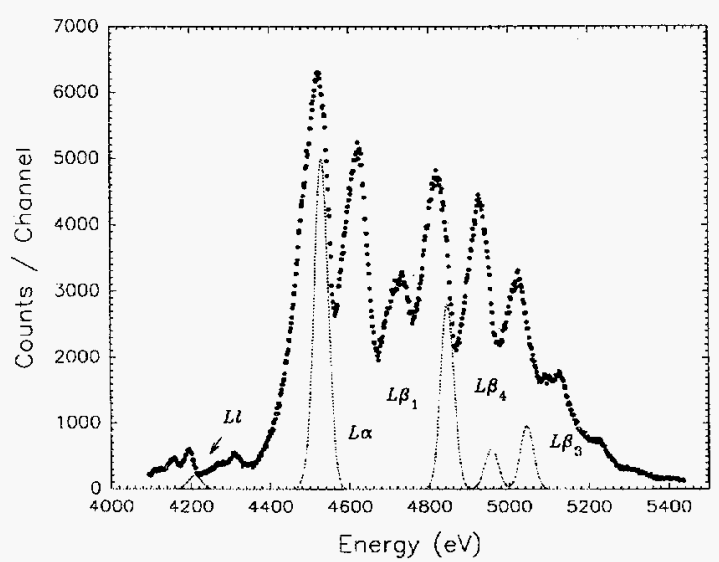

Figure 2. Comparison of the simulate spectrum for $n_{L}=7$ with the 6 $\mathrm{MeV} / \mathrm{u}$ Xe spectrum obtained with a thick $\mathrm{Li}$ target. 


\title{
Determination of Charge Distributions for 6 and $8 \mathrm{MeV} / \mathrm{u}$ Xe Ions Traveling in Solids from L X-ray Measurements
}

\author{
V. Horvat, R. L. Watson, and R. Parameswaran
}

In an effort to obtain information pertaining to the charge distributions of ions inside solid targets, detailed analyses of the spectra of $L \mathrm{x}$-rays emitted by 6 and $8 \mathrm{MeV} / \mathrm{u} \mathrm{Xe}$ ions traveling in thick, solid targets (described in the preceding report) were performed with a customized non-linear least squares fitting program. The analytical fitting function employed was based upon the following premises:

(a). During the $\mathrm{L} \mathrm{x}$-ray emission stage, the $\mathrm{K}$ shell remains filled while shells higher than the $\mathbf{M}$-shell are completely stripped of electrons.

(b). The population distribution of the L-shell is described by a Gaussian distribution function having a variable centroid and standard deviation.

(c). The L-electrons are statistically distributed among the L-subshells.

(d). The contributions of all $\mathrm{L} \mathrm{X}$-ray components other than $L \alpha_{1}$ and $L \beta_{1}$ are small enough to be neglected.

Premise (a) was tested by performing a spectral scan over the projectile $M \mathrm{x}$-ray region, whereupon it was discovered that the intensities of the $M x$-rays were negligible. Premise (b) is based on the statistical nature of the electron ionization and capture processes in high energy collisions and the fact that many collisions occur prior to $\mathrm{L} x$-ray emission. A statistical distribution of L-electrons (premise (c)) is reasonable in the absence of preferential population mechanisms. Premise (d) was adopted in order to keep the fitting function as simple as possible and because initial attempts to include the $L \beta_{4}$ and $L \beta_{3}$ components in the analysis indicated their contributions were negligible. These two transitions appear at the high energy end of the spectrum and have fairly small intensities. Evidently, the actual intensities are substantially less than those predicted by the calculated rates for singly-ionized atoms.

The fitting function $y(E)$ chosen for the spectral analysis was the following:

$$
\begin{aligned}
& y(E)=\sum_{n_{L_{1}}=0}^{2} \sum_{n_{L_{2}}=0}^{2} \sum_{n_{l_{3}}=0}^{4} A\left(n_{L}\right) P_{n_{l_{1}}}\left(n_{L_{1}}, n_{L_{2}}, n_{L_{3}}\right) \\
& \times\left\{\left(1-\delta_{n_{L_{3}}, 4}\right) G_{\alpha}\left(n_{L_{1}}, n_{L_{2}}, n_{L_{3}}, \bar{n}_{M}\right)\right. \\
& \left.+\kappa\left(1-\delta_{n_{L_{2}}, 2}\right) G_{\beta}\left(n_{L_{1}}, n_{L_{2}}, n_{L_{3}}, \bar{n}_{M}\right)\right\}+B,
\end{aligned}
$$

where

$$
A\left(n_{L}\right)=\frac{A_{0}}{\sqrt{2 \pi} \sigma_{L}} \exp \left[-\left(n_{L}-n_{L}^{c}\right)^{2} / 2 \sigma_{L}^{2}\right]
$$

$$
P_{n_{L}}\left(n_{L_{1}}, n_{L_{2}}, n_{L_{3}}\right)=\frac{\left(\begin{array}{c}
2 \\
n_{L_{1}}
\end{array}\right)\left(\begin{array}{c}
2 \\
n_{L_{2}}
\end{array}\right)\left(\begin{array}{c}
4 \\
n_{L_{3}}
\end{array}\right)}{\left(\begin{array}{c}
8 \\
n_{L}
\end{array}\right)}
$$

$$
\begin{aligned}
& G_{k}\left(n_{L_{1}}, n_{L_{2}}, n_{L_{3}}, \bar{n}_{M}\right)=\frac{1}{\sqrt{2 \pi} \sigma_{x}} \\
& \exp \left\{-\left[E-E_{k}\left(n_{L_{1}}, n_{L_{2}}, n_{L_{3}}, \bar{n}_{M}\right)\right]^{2} / 2 \sigma_{x}^{2}\right\},
\end{aligned}
$$

and

$$
\begin{aligned}
& E_{k}\left(n_{L_{1}}, n_{L_{2}}, n_{L_{3}}, \bar{n}_{M}\right) \\
& =E_{k}\left(n_{L_{1}}, n_{L_{2}}, n_{L_{3}}, 1\right)-24\left(\bar{n}_{M}-1\right) .
\end{aligned}
$$

The variable parameters of the fitting function are the overall amplitude $A_{0}$, the centroid $n_{L}{ }^{c}$ and standard deviation $\sigma_{\mathrm{L}}$ of the (initial state) L-electron population distribution, the average number of $\mathbf{M}$-shell electrons $\bar{n}_{M}$, the standard deviation of the individual $\mathrm{x}$-ray 
peaks $\sigma_{x}$, the $L \beta_{1} / L \alpha_{1}$ intensity ratio $\kappa$, and the background amplitude $B$. The $P_{n_{L}}$ factor gives the relative statistical probability that a projectile having $n_{L}$ electrons in the initial state assumes a particular configuration. The $\mathrm{L} \alpha_{1}$ and $\mathrm{L} \beta_{1}$ transition energies are represented by $E_{\alpha}$ and $E_{\beta}$, respectively. The Kronecker delta symbols $\delta$ in Eq. (1) ensure that the initial configurations having $n_{L_{3}}=4$ for $L \alpha_{1}$ transitions and $n_{L_{2}}=2$ for $L \beta_{1}$ transitions are excluded from the summations. Altogether, the fitting function contained 66 Gaussian peaks, but as was indicated above, it employed only 7 variable parameters.

It was discovered that the above fitting function was unable to account for the large tails on the low energy sides of the spectra for thick targets; nor was it was able to reproduce the structure near the centers of the spectra. Both problems caused the program to overestimate the $n_{L}=7$ and 6 contributions as it attempted to compensate for these deficiencies. The overall quality of the fits to the spectra for thin targets, on the other hand, were quite good. The reason for this is because the low energy sides of the spectra for thin targets do not display nearly as much tailing as is present in the spectra for thick targets. The differences between the fits for the spectra obtained with thick and thin carbon targets led to the conclusion that both the $\mathrm{L} \alpha_{1}$ and the $\mathrm{L} \beta_{1}$ peaks for $n_{L}=7$ in the spectra for thick targets contain asymmetric tails on their low energy sides. It is suspected that most of this tail arises from $x$-ray emission beyond the equilibration depth, after the projectile has slowed down significantly and consequently has picked up additional M-shell electrons.

As a means of accounting for the low energy tails, the function $G_{k}$ (Eq. 4) was modified for the specific cases where $n_{L_{1}}+n_{L_{2}}+n_{L_{3}}=7$ and $\mathrm{E} \leq \mathrm{E}_{\mathrm{k}}$ by replacing $\sigma_{\mathrm{x}}$ in the exponential with $\sigma_{\mathrm{x}}{ }^{\prime}$, a different and independently variable standard deviation, thus bringing the total number of fitting parameters to 8 . This procedure allowed the Gaussians representing the $n_{L}=7 \mathrm{~L} \alpha_{1}$ and $\mathrm{L} \beta_{1}$ components to have larger standard deviations on their left sides than on their right sides. Results of the fitting analysis are shown in Fig. 1 for the $6 \mathrm{MeV} / \mathrm{u}$ spectra obtained with thick

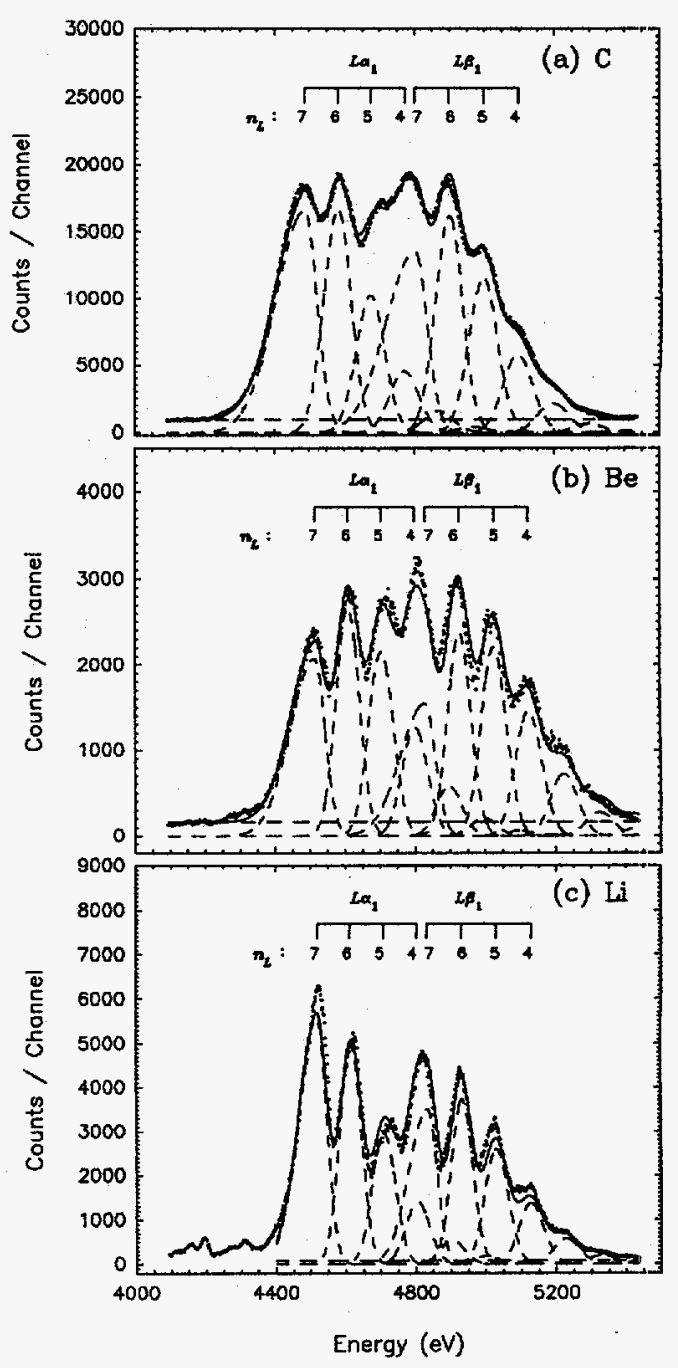

Figure 1. Least-squares fitting analysis of $6 \mathrm{MeV} / \mathrm{u}$ Xe spectra obtained with thick (a) C, (b) $\mathrm{Be}$, and (c) Li targets.

$\mathrm{Li}, \mathrm{Be}$, and $\mathrm{C}$ targets. For the sake of clarity, all $L \alpha_{1}$ components corresponding to the same value of $n_{L}$ are shown as single peaks, and likewise for the $\mathrm{L} \beta_{1}$ components. Overall, the fitting function reproduced the structure extremely well considering the fact that other low intensity $L \mathrm{X}$-ray lines (e.g. $L \beta_{4}$ and $L \beta_{3}$ ) probably contribute to the spectra.

An additional fitting analysis was performed on several of the spectra in which the $n_{L}$ distribution was 
assumed to be binomial instead of Gaussian. (The charge distributions resulting from multiple electron ionization in single collisions are generally well represented by binomial distribution functions). It was found that the best-fit binomial distribution widths were too small to account for the relatively broad overall shapes of the $\mathrm{x}$-ray spectra. This result is consistent with the conclusion that the $\mathrm{L}$ X-ray spectrum reflects the equilibrium distribution of excited states attained after a large number of collisions.

The average numbers of $L$-electrons associated with the L X-ray spectra were determined from the best-fit values of the amplitudes $A\left(n_{L}\right)$ using the relationship

$$
\bar{n}_{L}=\sum_{n_{L}=0}^{8} n_{L} A\left(n_{L}\right) / \sum_{n_{L}=0}^{8} A\left(n_{L}\right) .
$$

The average projectile charge was then obtained from

$$
<Q>=Z_{1}-n_{K}-\bar{n}_{L}-\bar{n}_{M},
$$

where $Z_{1}$ is the projectile atomic number (54) and $n_{K}$ is the number of $\mathrm{K}$-shell electrons (2). Corrections for fluorescence yield variation, absorption variation, and crystal reflectivity variation were judged to be small enough to neglect at the level of approximation being employed in this analysis. It should be noted that the tails on the $n_{L}=7$ peaks were not included in the determination of the values of $\bar{n}_{L}$ and $\bar{n}_{M}$ because these contributions were believed to arise from beyond the equilibration depth.

The numerical results of the spectral analyses are given in Table I. For $6 \mathrm{MeV} / \mathrm{u} \mathrm{Xe}$ ions interacting with thick targets, it is found that $\bar{n}_{L}$ is essentially independent of the target $Z$ except for the case of $B e$, which displays a significantly lower $\bar{n}_{L}$ than the rest. This latter observation is consistent with the distinctively different character of the spectrum obtained with the Be target, as may be verified by noting the relative contributions of the $n_{L}=7$ to 5
L $\alpha$ peaks in Fig. 1. The value of $\bar{n}_{M}$, on the other hand, rises steadily from $Z_{2}=3$ to $10(\mathrm{NaF})$ and remains approximately constant thereafter. As a consequence of this behavior, the average projectile charge decreases by about 3.4 units over the $Z_{2}$ range 3 to 10 and remains constant at approximately 40.7 thereafter. The same behavior is observed for $\langle Q\rangle$ at $8 \mathrm{MeV} / \mathrm{u}$ in that it also decreases by about 2 units as $Z_{2}$ increases from 6 to 28 , although it is caused, in this case, by an increase in both $\bar{n}_{L}$ and $\bar{n}_{M}$.

The $\bar{n}_{L}$ for the two thin $C$ target measurements at $6 \mathrm{MeV} / \mathrm{u}$ differ somewhat from each other with the result that the $\langle\mathrm{Q}\rangle$ for the $200 \mu \mathrm{g} / \mathrm{cm}^{2}$ target is substantially higher than that for the $100 \mu \mathrm{g} / \mathrm{cm}^{2}$ target. The predicted equilibrium charge of $6 \mathrm{MeV} / \mathrm{u}$ $\mathrm{Xe}$ ions after exiting a carbon foil is $42.7,{ }^{1}$ which agrees very well with the average projectile charges deduced from the thick and the $100 \mu \mathrm{g} / \mathrm{cm}^{2}$ carbon target spectra. The predicted value of the equilibrium charge at $8 \mathrm{MeV} / \mathrm{u} \mathrm{(44.8)}$ is also in excellent agreement with the $\langle Q\rangle$ deduced from the spectra obtained with $200 \mu \mathrm{g} / \mathrm{cm}^{2} \mathrm{C}$ targets. An estimate of the mean depth within a carbon target at which an Lvacancy is produced in the projectile may be obtained by assuming a geometrical L-shell ionization cross section $\left(\sim 5 \times 10^{-19} \mathrm{~cm}^{2}\right)$. This gives a mean depth $\sim$ $2 \times 10^{-5} \mathrm{~cm}$ and since the expected decay length is of the order of $7 \times 10^{-7} \mathrm{~cm}$, it must be concluded that most of the $L \mathrm{x}$-rays are emitted while the projectile is still inside a $100 \mu \mathrm{g} / \mathrm{cm}^{2}$ carbon target (which has a linear thickness of $4.4 \times 10^{-5} \mathrm{~cm}$ ). Therefore, the present results indicate that the average (equilibrium) charges for 6 and $8 \mathrm{MeV} / \mathrm{u} \mathrm{Xe}$ ions are the same inside and outside a carbon target. This conclusion is contrary to the expectation that the charge outside the target should be larger than the charge inside because of the occurrence of LMM Auger relaxation after the projectile exits from the foil. ${ }^{2,3}$ Each decay of this type would increase $Q$ by one unit, decrease $n_{M}$ by two units, and increase $n_{L}$ by one unit. However, in the systems under consideration, the Mshell is so devoid of electrons $\left(\bar{n}_{M} \approx 2\right.$ for the thin carbon targets) that $\mathrm{x}$-ray decay is probably able to 
win out over Auger decay, in which case there would not be a substantial difference between the average (equilibrium) charge inside and outside a solid.

The parameter $\sigma_{\mathrm{x}}$, listed in Table I, was assumed to consist of two components; $\sigma_{x}^{2}=\sigma_{y}^{2}+$ $\sigma_{\mathrm{m}}{ }^{2}$, where $\sigma_{\mathrm{I}}$ characterizes the contribution of the spectrometer resolution to the width of an X-ray peak for a single electron configuration and $\sigma_{\mathrm{m}}$ characterizes the broadening caused by the distribution of $M$-electrons. Using the average value of $\sigma_{1}(7.4 \mathrm{eV})$, the value of $\sigma_{\mathrm{m}}$ was obtained for each collision system and converted into the corresponding standard deviation of the M-electron distribution; $\sigma_{\mathrm{M}}$ $=\sigma_{\mathrm{m}} / 24$. A graph of $\sigma_{\mathrm{M}}$ versus $\overline{\mathrm{n}}_{\mathrm{M}}$ is shown in Fig. 2. The strong correlation between these two parameters supports the conclusion that the peak broadening is caused by the overlap of contributions from configurations having the same $n_{L}$, but different $n_{M}$.

Within the framework of the present model, the yield of each charge state $Y(Q)$ may be obtained by summing the yields $\mathrm{Y}\left(n_{L}, n_{M}\right)$ subject to the constraint that $n_{L}+n_{M}=52-\mathrm{Q}$ (see Eq (7)). Since it was assumed that the distributions of $n_{L}$ and $n_{M}$ are independent, then $\mathrm{Y}\left(n_{L}, n_{M}\right)=\mathrm{Y}\left(n_{L}\right) \cdot \mathrm{Y}\left(n_{M}\right)$. If it is further assumed that $Y\left(n_{M}\right)$ is also a Gaussian with centroid $\bar{n}_{M}$ and standard deviation $\sigma_{\mathrm{M}}$, then $\mathrm{Y}(\mathrm{Q})$ is merely a convolution of these two individual Gaussians. Therefore, the standard deviation of the charge distribution is $\sigma_{\mathrm{Q}}=\sqrt{\sigma_{\mathrm{L}}^{2}+\sigma_{\mathrm{M}}^{2}}$. The average $\sigma_{\mathrm{Q}}$ were $2.4 \pm 0.1$ and $2.2 \pm 0.1$ for the 6 and 8
$\mathrm{MeV} / \mathrm{u}$ charge distributions, respectively. These values are somewhat larger than the standard deviation of 1.8 predicted for the equilibrium charge distribution.' The broader charge distribution deduced from the $x$-ray spectra may reflect contributions from $x$-rays emitted by pre- and postequilibrium $X e$ ions.

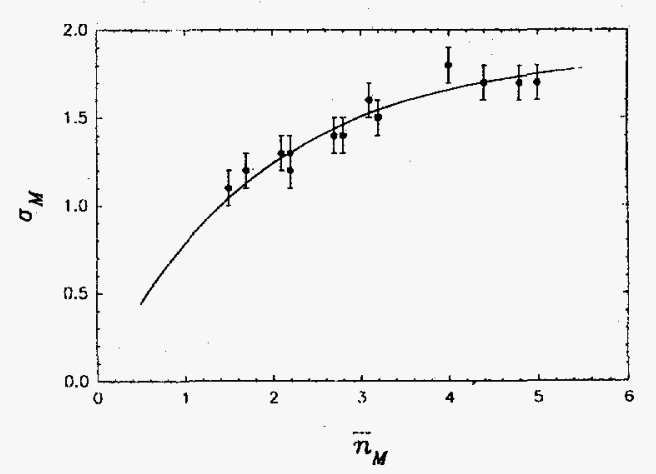

Figure 2. Standard deviation of the $M$-electron distribution as a function of the average number of $\mathrm{M}$-electrons.

\section{References}

1. R. O. Sayer, Rev. Physique Appl. 12, 1543 (1977).

2. H. D. Betz and L. Grodzins, Phys. Rev. Lett. 25, 211 (1970).

3. S. Della-Negra, Y. Le Beyec, B. Monart, and K. Standing, Phys. Rev. Lett. 58, 17 (1987). 
Table I. Results of the least-squares fitting analysis. (The estimated uncertainties are \pm 0.4 for $\bar{n}_{L}$ and $\bar{n}_{M}$, \pm 0.6 for $\langle\mathrm{Q}\rangle, \pm 0.4$ for $\sigma_{\mathrm{L}}, \pm 3 \mathrm{eV}$ for $\sigma_{\mathrm{x}}$, and \pm 0.2 for $\kappa$ ).

\begin{tabular}{|c|c|c|c|c|c|c|c|}
\hline $\begin{array}{c}\text { Energy } \\
(\mathrm{MeV} / \mathrm{u})\end{array}$ & Target $^{2}$ & $\overline{\mathrm{n}}_{\mathrm{L}}$ & $\overline{\mathbf{n}}_{\mathrm{M}}$ & $\langle Q\rangle$ & $\sigma_{\mathrm{L}}$ & $\begin{array}{c}\sigma_{\mathrm{x}} \\
(\mathrm{eV})\end{array}$ & $\kappa$ \\
\hline 6 & $\mathbf{L i}$ & 6.7 & 1.5 & 43.8 & 2.4 & 27 & 1.3 \\
\hline 6 & $\mathrm{Be}$ & 6.1 & 1.7 & 44.2 & 2.0 & 29 & 1.5 \\
\hline 6 & B & 6.8 & 2.7 & 42.5 & 1.7 & 35 & 1.6 \\
\hline 6 & $\mathrm{C}$ & 6.7 & 2.8 & 42.5 & 2.0 & 35. & 1.6 \\
\hline 6 & BN & 6.8 & 3.2 & 42.0 & 2.0 & 37 & 1.6 \\
\hline 6 & $\mathrm{NaF}$ & 6.8 & 4.8 & 40.4 & 1.9 & 41 & 1.6 \\
\hline 6 & $\mathrm{Al}$ & 6.6 & 5.0 & 40.4 & 1.8 & 42 & 1.8 \\
\hline 6 & $\mathrm{KCl}$ & 6.8 & 4.4 & 40.8 & 1.6 & 41 & 1.9 \\
\hline 6 & $\mathrm{Ca}+\mathrm{Mn}$ & 6.8 & 4.0 & 41.2 & 1.7 & 43 & 1.9 \\
\hline 6 & $C(100)$ & 6.6 & 2.2 & 43.2 & 1.9 & 33 & 1.3 \\
\hline 6 & $C(200)$ & 6.1 & 2.1 & 43.8 & 1.7 & 33 & 1.3 \\
\hline 8 & $C(200)$ & 5.1 & 2.2 & 44.7 & 1.9 & 29 & 1.1 \\
\hline 8 & $C(200)^{b}$ & 4.6 & 2.2 & 45.2 & 1.6 & 29 & 1.3 \\
\hline 8 & $\operatorname{Al}(150)$ & 5.8 & 3.1 & 43.1 & 1.7 & 40 & 1.3 \\
\hline 8 & $\mathrm{Ni}(180)$ & 6.2 & 3.1 & 42.7 & 1.8 & 39 & 1.6 \\
\hline
\end{tabular}

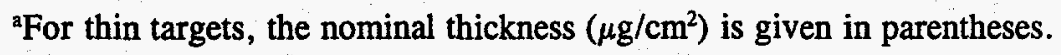

'This target was preceded by a $200 \mu \mathrm{g} / \mathrm{cm}^{2} \mathrm{C}$ stripper foil. 


\title{
Charge Distribution of $8 \mathrm{MeV} / \mathrm{u}$ Xe Ions Emerging from Carbon Foils
}

\author{
R. L. Watson, V. Horvat, R. Parameswaran, L. J. Cook, J. Knott, and A. Nash
}

The charge distributions of energetic ions passing through matter have been of continuing interest since the discovery of natural radioactivity. The close connection between the charge of an ion and the strength of its Coulomb interaction with atoms of a medium makes it one of the most important parameters in formulations of electronic stopping power. A large database of information on charge distributions has accumulated over the years (see for example Refs. 1,2) and it has provided a basis for the development of semi-empirical relationships describing equilibrium charge distributions. Some of these formulations, most notably those proposed by Nikolaev and Dimitriev, ${ }^{3}$ Baron and Delaunay, ${ }^{4}$ Sayer, and Shima et al., ${ }^{6}$ do a very credible job of predicting distribution widths and centroids (i.e. average equilibrium charges) over a wide range of projectile energy and atomic number. However, most of the data in existence is for energies up to a few $\mathrm{MeV} / \mathrm{u}$ and, as Martin et al. ${ }^{7}$ have pointed out, there is a need for charge distribution measurements with ions having $Z>36$ and energies above $1 \mathrm{MeV} / \mathrm{u}$. Moreover, very few investigations have provided detailed experimental information concerning the evolution of the charge state distribution as a function of target thickness.

In conjunction with our studies of recoil-ion and $\mathrm{L}$ x-ray production by $8 \mathrm{MeV} / \mathrm{u} \mathrm{Xe}$ ions, we have examined the dependence of the $\mathrm{Xe}$ charge distribution on the thickness of carbon foils. In these experiments, a beam of $8 \mathrm{MeV} / \mathrm{u} \mathrm{Xe}^{21+}$ was collimated to a diameter of $1 \mathrm{~mm}$ and passed through a carbon target. Targets ranging in thickness from 1 to 500 $\mu \mathrm{g} / \mathrm{cm}^{2}$ were placed in a 12 position wheel mounted perpendicular to the beam. The target wheel was remotely controlled by means of a small stepping motor. After exiting the target, the beam continued on through a charge state analyzing magnet and into a one-dimensional position sensitive microchannel plate detector (PSD) located approximately $10 \mathrm{~m}$ beyond. Since the radius of curvature of an ion in a magnetic field is inversely proportional to its charge, the position at which an ion was detected in the PSD identified its charge. Position spectra are shown in Fig. 1 for a very thin target $\left(1 \mu \mathrm{g} / \mathrm{cm}^{2}\right)$ and a relatively thick target $\left(400 \mu \mathrm{g} / \mathrm{cm}^{2}\right)$. The peaks corresponding to the different projectile charges are well resolved in the thin target spectrum, where the resolution is only limited by the incident beam diameter, however the peaks in the thick target spectrum are poorly resolved as a result of multiple scattering in the target. In order to extract accurate peak areas from the thicker target spectra, it was necessary to employ a least-squares peak fitting program.

The peak areas were corrected for the relative efficiency of the PSD to obtain the relative yields, which in turn were used to determine the average projectile charge as a function of target thickness. The results are shown in Fig. 2. As expected, the average charge at first increases rapidly with increasing target thickness, but soon the rate of increase slows and eventually goes to zero as the beam reaches charge equilibrium $\left(<\mathrm{Q}_{\mathrm{eq}}>=44.51 \pm\right.$ 0.11 ) at a thickness around $200 \mu \mathrm{g} / \mathrm{cm}^{2}$. The equilibrium charge distribution (obtained with the 400 $\mu \mathrm{g} / \mathrm{cm}^{2}$ target) is compared with the predictions of several semiempirical formulations in Fig. 3. All give good representations of the experimental data, especially the ones by Sayer ${ }^{6}$ and NikolaevDimitriev. ${ }^{3}$

Several of the targets were composed of two carbon foils sandwiched together, and it was noticed that the average charges obtained with these targets deviated slightly from the smooth curve defined by the data in Fig. 2. Further examination revealed that this effect depended on the relative ordering of the individual foils in the sandwich. For example, the 
charge distributions obtained with the same target sandwich, but in the two opposite orientations of $0^{\circ}$ and $180^{\circ}$ gave significantly different charge distiributions. This is illustrated in Fig. 4 for a target composed of $30 \mu \mathrm{g} / \mathrm{cm}^{2}+74 \mu \mathrm{g} / \mathrm{cm}^{2}$ foils. It is evident from Fig. 4 that the average charge of Xe ions after exiting the target in which the $30 \mu \mathrm{g} / \mathrm{cm}^{2}$ foil was encountered first was 0.6 units higher than the average charge of Xe ions after exiting the same target, but with the foils arranged in the opposite order. This same behavior, in which the average charge was higher when the thinner foil was first in line, was observed for the additional foil combinations of $22+64,60+90$, and $50+200 \mu \mathrm{g} / \mathrm{cm}^{2}$.

A simple model was developed in an effort to understand the effect described above. It was based upon the integrated rate equation expressing the thickness dependence of the ionic charge in terms of average ionization and capture cross sections with an additional term to represent the thickness dependence of charge multiplication by Auger decay after the ion exited the foil. The resulting equation for the average charge after exiting the foil $\left\langle\mathrm{Q}_{0}\right\rangle$ is given below:

$$
<Q_{0}>=q_{E}\left(1-e^{-a t}\right)+q_{I} e^{-a t}+n_{A}\left(1-e^{-b t}\right),
$$

where $q_{E}$ is the average equilibrium charge inside the foil, $\mathrm{q}_{\mathrm{I}}$ is the incident charge, and $\mathrm{n}_{\mathrm{A}}$ is the maximum number of Auger decays for ions with fully equilibrated inner-shells. In this preliminary analysis, the value of $n_{A}$ was fixed at 3 , while the values of $q_{E}$ and the cross section parameters $a$ and $b$ were determined by fitting Eq. (1) to the data presented in Fig. 2. The thickness dependence predicted by Eq. (1) for the case of the $30+74 \mu \mathrm{g} / \mathrm{cm}^{2}$ foil sandwich is shown in Fig. 5. It is apparent from this figure that the model reproduces the experimental average charges quite well and it accounts for the fact that the charge is higher when the thinner foil is first in line.

\section{References}

1. A. B. Wittkower and H. D. Betz, At. Data 5,
113 (1973).

2. K. Shima, T. Mikumo, and H. Tawara, At. Data and Nucl. Data Tables 34, 357 (1986).

3. V. S. Nikolaev and I. S. Dimitriev, Phys Lett. A28, 277 (1968).

4. E. Baron and B. Delaunay, Phys. Rev. A 12, 40 (1975).

5. R. O. Sayer, Rev. Phys. Appl. 12, 1543 (1977).

6. K Shima, T. Ishihara, and T. Mikumo, Nucl. Instrum. Methods 200, 605 (1982).

7. J. A. Martin, R. L. Auble, K. A. Erb, C. M. Jones, and D. K. Olsen, Nucl. Instrum. Methods A244, 187 (1986).

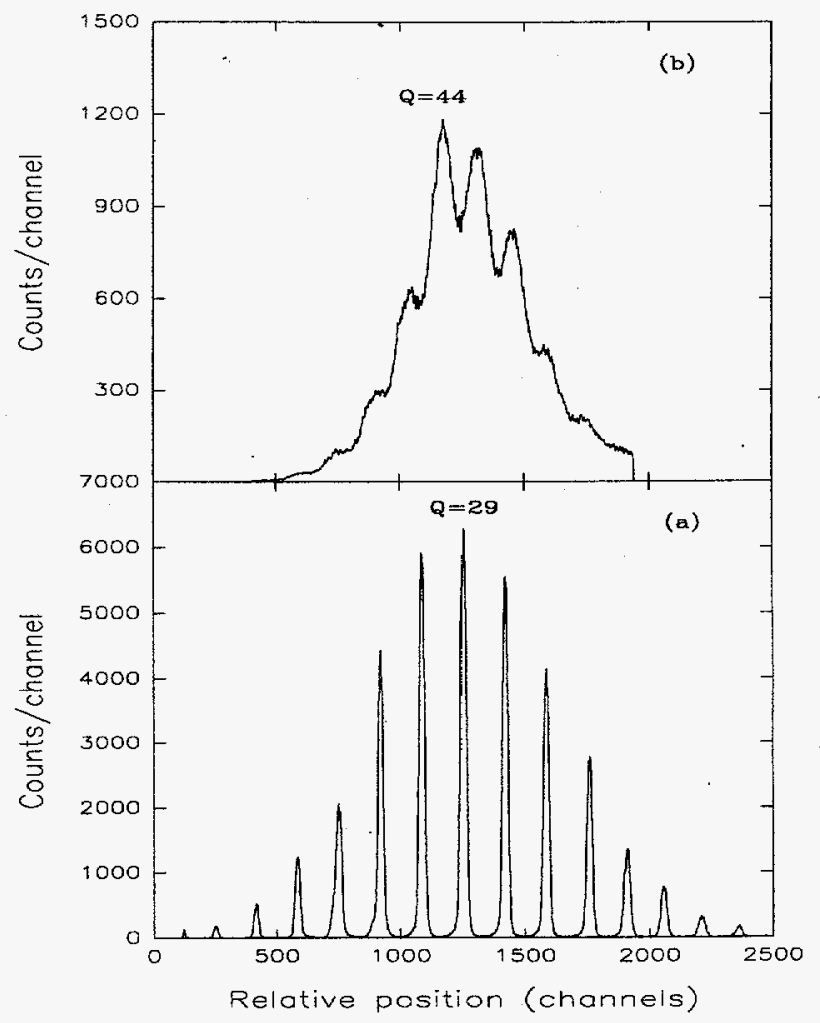

Figure 1. Position spectra of $8 \mathrm{MeV} / \mathrm{u} \mathrm{Xe}$ ions emerging from carbon foils of thickness (a) $1 \mu \mathrm{g} / \mathrm{cm}^{2}$, and (b) $400 \mu \mathrm{g} / \mathrm{cm}^{2}$ following magnetic dispersion. 


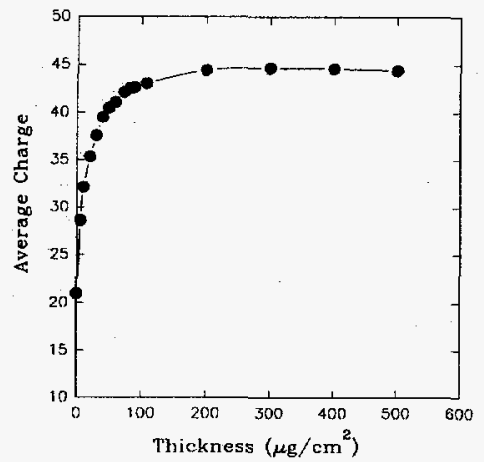

Figure 2. Dependence of the average charge of Xe ions on carbon foil thickness.

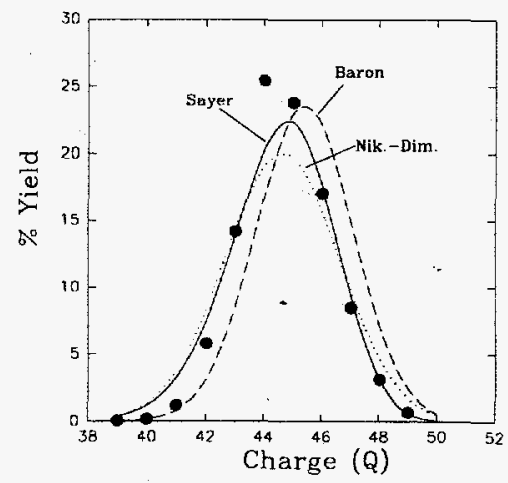

Figure 3. Comparison of the measured equilibrium charge distribution (solid circles) and those predicted by the semiempirical formulations of Nikolaev and Dimitriev, ${ }^{3}$ Baron and Delaunay, ${ }^{4}$ and Sayer. ${ }^{6}$

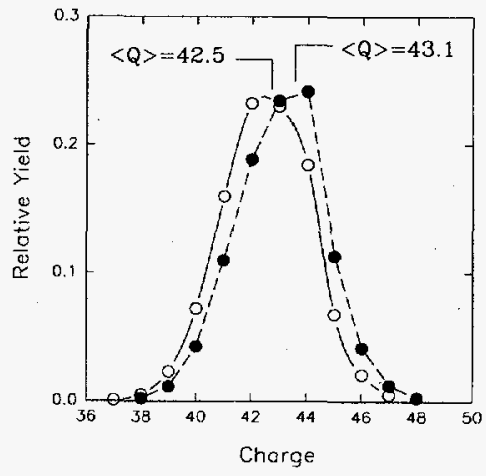

Figure 4. Charge distributions for Xe ions emerging from al $30+74$ $\mu \mathrm{g} / \mathrm{cm}^{2}$ carbon foil sandwich in opposite orientations; (open circles) the ions pass through the $74 \mu \mathrm{g} / \mathrm{cm}^{2}$ foil first; (solid circles) the ions pass through the $30 \mu \mathrm{g} / \mathrm{cm}^{2}$ foil first.

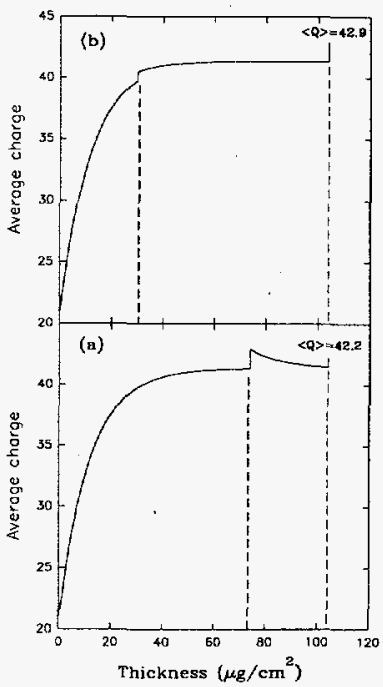

Figure 5. Results of the model calculations for the two orientations of the $30+74 \mu \mathrm{g} / \mathrm{cm}^{2}$ carbon foil sandwich; (a) $74 \mu \mathrm{g} / \mathrm{cm}^{2}$ foil first; (b) 30 $\mu \mathrm{g} / \mathrm{cm}^{2}$ foil first. 


\title{
Projectile and Target L X-ray Spectra Produced in Fast Kr Ion Collisions
}

\author{
V. Horvat, J. M. Blackadar, R. L. Watson, and R. Parameswaran
}

Spectra of L x-rays emitted by 6 and $12 \mathrm{MeV} / \mathrm{u}$ $\mathrm{Kr}$ ions traveling in a variety of thick, solid targets have been measured with a curved crystal spectrometer. A comparison of spectra obtained with a thick $\mathrm{Li}$ target at these two beam energies is shown in Fig. 1. The main structural features consist of three groups of lines produced by $M \rightarrow L, N \rightarrow L$, and $\mathrm{O} \rightarrow \mathrm{L}$ transitions. The peak structure within each of the transition groups is similar, indicating the relative subshell population distributions and fluorescence yields are about the same for the $M, N$, and $O$ shells. The relative intensities of the transitions within the groups and of the groups themselves are somewhat different at the two projectile energies. These differences primarily reflect changes in the charge state distribution and in the electron capture cross sections, respectively.

The predicted average equilibrium charges for $\mathrm{Kr}$ ions are $30.2(6 \mathrm{MeV} / \mathrm{u})$ and $32.9(12 \mathrm{MeV} / \mathrm{u}) .^{\prime}$ The $\mathrm{Kr}$-ion ground state configurations corresponding to charges of 30 and 33 are $1 \mathrm{~s}^{2} 2 \mathrm{~s}^{2} 2 \mathrm{p}^{2}$ and $1 \mathrm{~s}^{2} 2 \mathrm{~s}$, respectively. Therefore, it is to be expected that the most intense components in the spectra should be those associated with initial state configurations having L-shell occupation numbers $\left(n_{L}\right)$ of 0 to 4 . Using transition energies calculated with the DiracFock program of Desclaux ${ }^{2}$ and the theoretical singlevacancy transition rates of Scofield, ${ }^{3}$ simulated spectra of the $M \rightarrow L$ transitions were constructed for initial state configurations containing two $\mathrm{K}$-shell electrons $+n_{L}$ L-shell electrons + one M-shell electron. Shown in Fig. 2 is a comparison of the $6 \mathrm{MeV} / \mathrm{u} \mathrm{Kr} \mathrm{M} \rightarrow \mathrm{L}$ $\mathrm{X}$-ray spectrum ( $\mathrm{Li}$ target) with simulated spectra for $\mathrm{Kr}^{33+}, \mathrm{Kr}^{32+}$, and $\mathrm{Kr}^{31+}$ having initial state $\mathrm{n}_{\mathrm{L}}$ values of 0,1 , and 2 , respectively. It is evident from this figure that the four highest energy peaks in the measured spectrum primarily originate from $\mathrm{Kr}$ ions having a completely vacant L-shell. The average number of $\mathrm{M}$-shell electrons present was estimated by adding spectator M-electrons one at a time, until the calculated and measured peak energies matched. In a more detailed analysis of the measured spectra employing an expanded set of calculated transition energies and transition rates, it should be possible to extract information concerning the distributions of $\mathrm{L}$, $\mathrm{M}, \mathrm{N}$, and $\mathrm{O}$ electrons of $\mathrm{Kr}$ projectile ions inside solids.

The small peak around $2360 \mathrm{eV}$ in the $12 \mathrm{MeV} / \mathrm{u}$ spectrum (Fig. 1) may be due to $\mathrm{M} \rightarrow \mathrm{L}$ transitions in $\mathrm{Kr}$-ions having single $\mathrm{K}$-shell vacancies. Major contributions to the $\mathrm{L} x$-ray spectrum from ions with K-shell vacancies were not expected since, even at 12 $\mathrm{MeV} / \mathrm{u}$, the projectile velocity is well below the average $\mathrm{K}$-electron velocity.

Target L $x$-ray spectra produced by the interaction of $6 \mathrm{MeV} / \mathrm{u} \mathrm{Kr}$-ions with thick, solid targets of Rb, Mo, and La are shown in Fig. 3. These spectra are all characterized by several sharp lines adjacent to or superimposed upon one or more very broad peaks. Energy calibrations identified the sharp lines to be $L \alpha, L \beta_{1}$, and $L \beta_{2,15}$ transitions in target atoms having single $L$-vacancies. They are evidently excited as a result of photoionization by high fluxes of photons produced inside the targets with energies just above the $\mathrm{L}$ absorption edges. The broad peaks, on the other hand are apparently $L$ x-rays from target atoms having several $L$-vacancies and are produced by ion-atom collisions. In the Rb and Mo spectra, the broad collision-induced L $\alpha$ x-ray peaks are highly distorted by the $L_{3}$ absorption edge. The collisioninduced $L \alpha$ and $L \beta_{1}$ peaks in the La spectrum, on the other hand are almost intact as the $L_{3}$ absorption edge in this spectrum occurs above the $\mathrm{L} \beta_{3}$ line (at 5483 eV).

The spectra shown in Fig. 3 present an interesting puzzle. Unlike the projectile ions, the target atoms undergo single collisions. As a consequence of this, the cross sections for single ionization generally dominate over those for multiple ionization in the absence of impact parameter restrictions. For 
example, $\mathrm{K}$ x-ray emission from atoms with single $\mathrm{K}$ vacancies is always much more probable than $\mathrm{K} \mathrm{X}$-ray emission from atoms with double $\mathrm{K}$-vacancies in high velocity collisions. On the other hand, $\mathrm{K}$ x-ray emission from atoms having multiple $\mathrm{L}$-vacancies is frequently much more probable than $\mathrm{K} \mathrm{X}$-ray emission from atoms having zero L-vacancies, but this is because the $\mathrm{K} \mathrm{X}$-ray requirement selects a range of impact parameters well inside the $L$-shell where it is highly probable the atom will suffer multiple L-shell ionization. In the L X-ray spectra presented in Fig. 3, however, no such impact parameter restrictions are in force. Therefore it is quite interesting to find that essentially all of the collision induced $L \mathrm{x}$-rays arise from configurations involving multiple L-vacancies.

\section{References}

1. R. O. Sayer, Rev. Physique Appl. 12, 1543 (1977).

2. J. P. Desclaux, Comp. Phys. Commun. 9, 31 (1975).

3. J. H. Scofield, Phys. Rev. 179, 9 (1969).

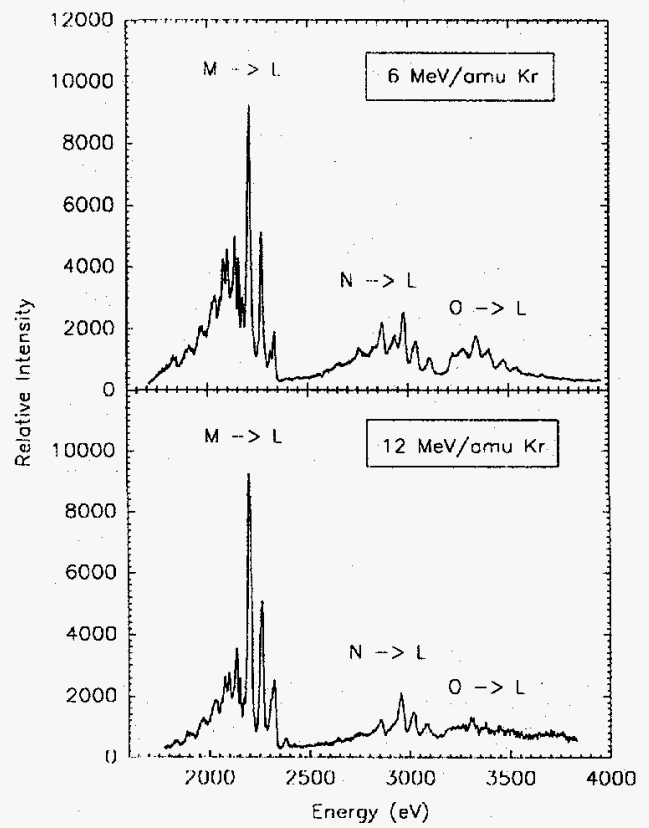

Figure 1. Spectra of Kr L x-rays emitted by 6 and $12 \mathrm{MeV} / \mathrm{u} \mathrm{Kr}$ ions traveling in a thick $\mathrm{Li}$ target:

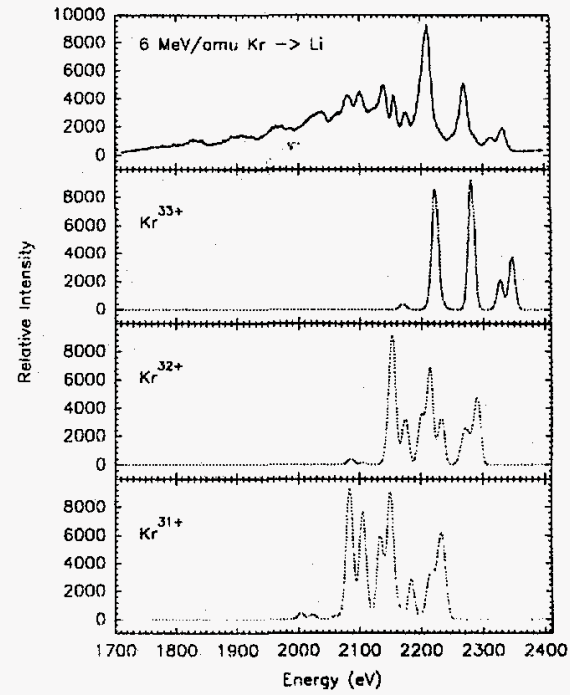

Figure 2. Comparison of $6 \mathrm{MeV} / \mathrm{u} \mathrm{Kr} \mathrm{M} \rightarrow \mathrm{L}$ x-ray spectrum $(\mathrm{Li}$ target) with simulated spectra showing the components expected for $\mathrm{Kr}$ ions having charges of $31+, 32+$ and $33+$.

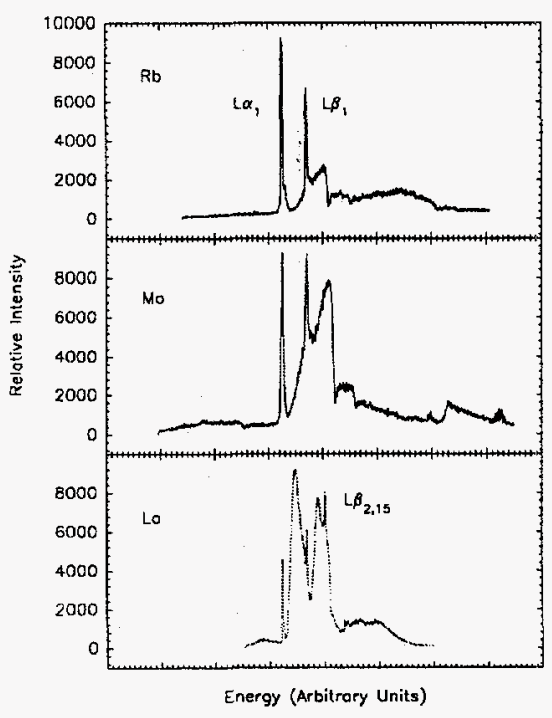

Figure 3. Spectra of $\mathrm{L} x$-rays emitted by target atoms of $\mathrm{Kb}, \mathrm{Mo}$, and La under bombardment by $6 \mathrm{MeV} / \mathrm{u} \mathrm{Kr}$ ions. The energy scales have been adjusted to align the $L \alpha$ and $L \beta_{1}$ lines. 


\title{
Charge Distributions of Noble Gas Recoil-Ions Produced in Fast Heavy Ion-Atom Collisions
}

\author{
R. Parameswaran, V. Horvat, and R. L. Watson
}

In fast ion-atom collisions, electron rearrangement occurs via the three principle processes of direct (or pure) ionization of the target (PI), ionization of the target accompanied by electron capture by the projectile $(\mathrm{CI})$, and ionization of the target accompanied by ionization (electron loss) by the projectile (LI). Many investigations of these processes have been conducted in the energy region around $1 \mathrm{MeV} / \mathrm{u}$, but very little information on recoil-ion charge distributions exists for the higher energy regime around $10 \mathrm{MeV} / \mathrm{u}$.

We have completed an investigation of recoil-ion production in $\mathrm{PI}, \mathrm{CI}$, and $\mathrm{LI}$ collisions at $8 \mathrm{MeV} / \mathrm{u}$ using projectiles of $\mathrm{Ar}^{7+}, \mathrm{Ar}^{17+}, \mathrm{Kr}^{13+}, \mathrm{Kr}^{32+}, \mathrm{Xe}^{21+}$, and $\mathrm{Xe}^{45+}$ incident on targets of $\mathrm{He}, \mathrm{Ne}, \mathrm{Ar}, \mathrm{Kr}$, and Xe. With the lower-charged projectile of each species, PI and LI were studied, while with the higher-charged projectile of each species, PI and CI were examined. The PI recoil-ion yields (which result from large impact parameter collisions) decreased monotonically with charge. This production mechanism accounted for about $99 \%$ of the total recoil-ion yield, with around $70-75 \%$ of this yield being attributed to singly-charged ions. Collisions involving CI and LI take place over a restricted range of relatively small impact parameters as dictated by the radii of the projectile shells involved. The recoil-ion charge distributions resulting from these types of collisions were characteristically bell-shaped.

The highest recoil-ion charges observed were produced in $\mathrm{CI}$ collisions. For example, the average charge of recoil ions produced in LI collisions of $\mathrm{Xe}^{21+}$ and $\mathrm{CI}$ collisions of $\mathrm{Xe}^{45+}$ on $\mathrm{Xe}$ were $14+$ and $25+$, respectively. This large difference in the average charge for the two types of collisions is attributable to several factors. Velocity matching arguments lead one to expect that electron capture will primarily involve the target atom K-shell in the cases of $\mathrm{Ne}$ and $\mathrm{Ar}$, or the L-shell in the cases of $\mathrm{Kr}$ and $\mathrm{Xe}$, whereas the projectile charges used for the LI studies were such that electron loss was most likely to occur from the projectile M-shell. Therefore, the generally smaller impact parameters required for $\mathrm{Cl}$ coupled with the higher projectile charges resulted in a greater ionizing power for the CI collisions. However, the most important factor was found to be charge multiplication resulting from Auger cascades which occur following the creation of inner-shell vacancies via the capture process. To illustrate the general characteristics of recoil-ion production in $\mathrm{CI}$ collisions at $8 \mathrm{MeV} / \mathrm{u}$, charge state distributions of Xe recoil-ions are shown in Fig. 1. The particular case of $\mathrm{Kr}^{32+} \rightarrow \mathrm{Xe}$ was the subject of a recent paper in which a simple model describing the limitations that are imposed on the post-collision Auger cascade was shown to account for both the charge change intervals and the width of the charge distribution as a function of the number of electrons captured.'

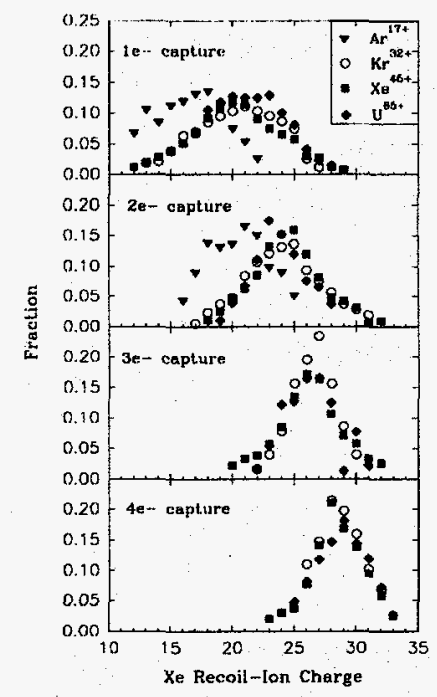

Figure 1. Charge distributions of Xe recoil-ions produced in $\mathrm{CI}$ collisions at $8 \mathrm{MeV} / \mathrm{u}$. The data for $9 \mathrm{MeV} / \mathrm{u} \mathrm{U} \mathrm{U}^{65+}$ is from Ref. 2. 
A noteworthy feature of the data shown in Fig. 1 is that the recoil-ion charge distributions for $\mathrm{Kr}^{32+}$ and $\mathrm{Xe}^{45+}$ projectiles are almost identical. Also shown are the charge distributions for $9.4 \mathrm{MeV} / \mathrm{u}$ $\mathrm{U}^{65+} \rightarrow \mathrm{Xe}$, obtained by Kelbch et al. ${ }^{2}$ The projectile charge independence exhibited by these $\mathrm{CI}$ recoil-ion charge distributions confirms and extends the previous observations of Müller et $a l^{3}$ Since CI collisions occur over a range of relatively small impact parameters where the outer-shell ionization probability is expected to remain almost constant, the present results imply that for some value of projectile charge between 17 and 32, the outer-shell ionization probabilities (at small impact parameters) begin to saturate.

Charge distributions of Xe recoil-ions produced in LI collisions at $8 \mathrm{MeV} / \mathrm{u}$ are shown in Fig. 2: The average impact parameter progressively decreases with the number of electrons lost. This

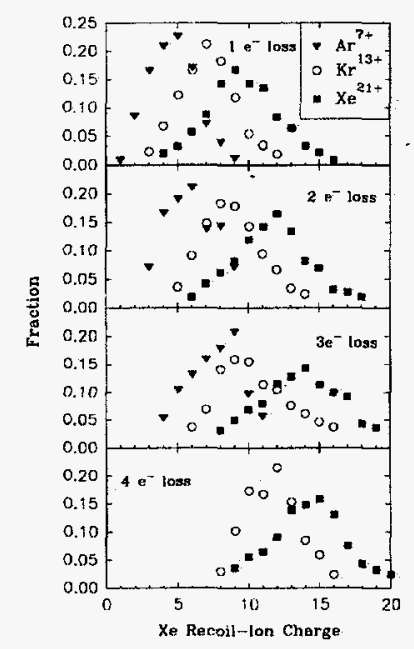

Figure 2. Charge distributions of Xe recoil-ions produced in LI collisions at $8 \mathrm{MeV} / \mathrm{u}$.

results in bell-shaped distributions of about the same width that shift to higher charges as the projectile electron loss increases. The average LI recoil-ion charge systematically increased with projectile charge and no evidence of an ionization probability saturation effect was observed for these relatively larger impact parameter collisions. Unlike the case of CI collisions, the creation of an inner-shell (K. or $L)$ vacancy is extremely rare in these LI collisions. Therefore, the LI recoil-ions do not undergo Auger cascade charge multiplication and have average charges that are considerably lower than those resulting from $\mathrm{CI}$ collisions.

As a means of investigating the idea of ionization probability saturation further, an analysis of the Ar recoil-ion charge distributions obtained with $8 \mathrm{MeV} / \mathrm{u}$ $\mathrm{Kr}^{32+}$ and $\mathrm{Xe}^{45+}$ projectiles was performed by employing the independent electron approximation model described by Heber et al. ${ }^{4}$ According to this model, the cross section for the production of a recoil-ion of charge $q$ in a CI collision in which $\mathrm{k}$. Kelectrons are captured by the projectile is given by

$$
\sigma_{k}^{q}=\sum_{l, m} 2 \pi \int_{0}^{\infty} P_{K k}(b) P_{L l}(b) P_{M m}(b) b d b
$$

where the summation is over all combinations of $l$ and $m$ which give charge $q$ under the assumption that Auger decay will occur whenever possible (i.e. Auger yield $=1$ ). The multiple ionization probabilities (or capture probabilities in the case of the K-shell) are given by the usual binomial factors;

$$
P_{l j}=\left(\begin{array}{l}
I \\
j
\end{array}\right) p_{I}(b)^{i}\left[1-p_{I}(b)\right]^{I-j},
$$

with $p_{I}(b)$ representing the impact parameter dependent single electron ionization or capture probability. Since capture from the K-shell dominates in these $\mathrm{CI}$ collisions with $\mathrm{Ar}$, the $\mathrm{L}$ - and $\mathrm{M}$-shell ionization probabilities were assumed to be constant over the range of relatively small impact parameters involved. The K-capture probability was represented by a step function and its value was determined using the measured total cross section; $\sigma_{\mathrm{C}}=\pi \mathrm{r}_{\mathrm{K}}{ }^{2} \overline{\mathrm{p}}_{\mathrm{k}}$. The best values of $\bar{p}_{L}, \bar{p}_{M}$, and $r_{K}$ 
were then obtained by fitting Eq. (1) to the 1- and 2electron capture charge distributions. The results are given in Table I, where it may be seen that the ionization probabilities appear to reach a limiting value of around 0.5. A similar analysis was also performed for PI by representing the impactparameter-dependent, single-electron ionization probabilities as $p_{I}(b)=p_{I} e^{-b / r_{I}}$. The fits to the PI and $\mathrm{CI}$ data are shown in Fig. 3.

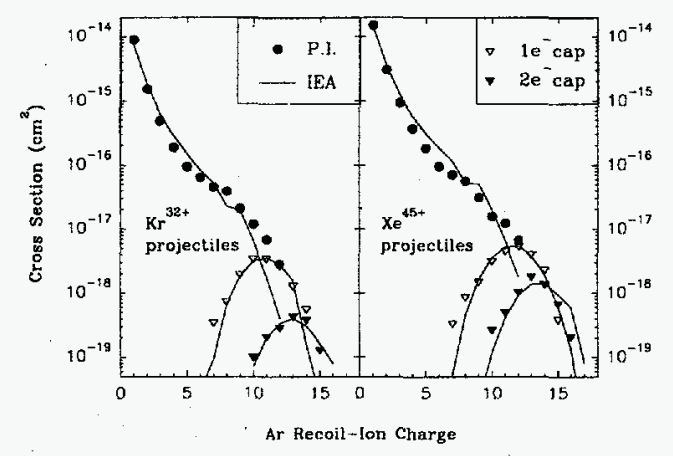

Figure 3. Results of the IEA analysis of Ar recoil-ion charge distributions produced in $\mathrm{PI}$ and $\mathrm{CI}$ collisions at $8 \mathrm{MeV} / \mathrm{u}$.
Table I. Results of the analysis of Ar recoil-ion charge distributions produced in 1- and 2- electron CI collisions at $8 \mathrm{MeV} / \mathrm{u}$.

\begin{tabular}{|l|l|l|l|l|}
\hline Projectile & $\bar{p}_{K}$ & $r_{K}(\AA)$ & $\bar{p}_{L}$ & $\bar{p}_{M}$ \\
\hline $\mathrm{Kr}^{32+}$ & 0.18 & 0.38 & 0.40 & 0.5 \\
\hline $\mathrm{Xe}^{45+}$ & 0.34 & 0.40 & 0.48 & 0.5 \\
\hline
\end{tabular}

\section{References}

1. R. Parameswaran, R. L. Watson, V. Horvat, G. Sampoll, and D. A. Church, Z. Phys. D (in press).

2. S. Kelbch, J. Ullrich, R. Mann, P. Richard, and H. Schmidt-Bocking, J. Phys. B 18, 323 (1985).

3. A. Müller, B. Schuch, W. Groh, E. Salzborn, H. F. Beyer, P. H. Mokler, and R. E. Olson, Phys. Rev. A 33, 3010 (1986).

4. O. Heber, R. L. Watson, G. Sampoll, and B. B. Bandong, Phys. Rev. A 42, 6466 (1990).

\title{
Lifetimes of Metastable Atomic Levels of Multicharged Ions
}

\author{
D. A. Church*
}

Certain atomic levels decay only by magnetic dipole, electric quadrupole, or other higher order transitions. These transitions are observed in certain plasmas, like Tokamaks or the solar corona, and are useful in determining electron densities. Lifetimes of these metastable atomic levels of multi-charged ions have been studied by capturing ions produced by the Electron Cyclotron Resonance Ion Source (ECRIS) into a Kingdon ion trap, and observing the spontaneous decays of the trapped ions. A Kingdon trap is an electrostatic device, similar to a Geiger tube, in which ions orbit a central wire in a lowcollision environment, and are confined through conservation of angular momentum, plus axial reflection from biased end electrodes. ${ }^{1}$

Low energy metastable multi-charged ions from ECRIS are selected in charge-to-momentum ratio using an electromagnet, and transported with electrostatic beam optics to the Kingdon trap. The ions are decelerated just as they enter the trap, which is floated at a potential near the value of the ECRIS extractor. The ions are captured into stable orbits in 
the Kingdon trap when the potential of the central wire is suddenly decreased to a low value. The ion beam is then deflected, and after a variable storage interval, the remaining ions are dumped by increasing the potential of the central wire. During the storage interval, photons in the infra-red, visible, or ultraviolet regions of the spectrum, emitted over time spans of a few ms to seconds in the metastable transitions, are detected using an optical system. This system consists of quartz lenses, an appropriate interference filter for wavelength selection, and a cooled photomultipliertube. Thousands of cycles are averaged, yielding a decreasing exponential optical decay. The time constant of this decay is determined by the unperturbed mean lifetime of the excited level, and collisional quenching, primarily due to electron capture. The quenching contribution was determined as a function of pressure (usually in the $10^{-8}$ Torr range). Extrapolation to zero pressure yielded the lifetime. The results for levels of Ar III, Ar IV, Ar $X$, and $\mathrm{Ar} \mathrm{Xi}$ appear in Table 1, together with theoretical calculations. Agreement with theory was adequate for these initial measurements.

"Physics Department, Texas A\&M University, College Station, Texas 77843

\section{References:}

1. L. Yang, D. A. Church, G. Weinberg, and Q. Wang; Nucl. Instrum. Methods B79, 37 (1993).

2. L. Yang and D. A. Church, Phys. Rev. Lett. 70, 3860 (1993).

3. L. Yang, D. A. Church, S. Tu, and J. Jin, Phys. Rev. A (in press). 
Table 1

\begin{tabular}{|c|c|c|c|c|c|}
\hline \multirow{2}{*}{$\begin{array}{l}A r^{2+} 3 s^{2} 3 p^{4} \\
\text { Transition }\end{array}$} & & \multicolumn{4}{|c|}{ Transition Rates $\left(\mathrm{s}^{-1}\right)$} \\
\hline & & $C \& K^{28}$ & $M \& K^{29}$ & $B \& H^{30}$ & $S \& K^{31}$ \\
\hline \multirow[t]{2}{*}{${ }^{3} P_{2}-1 S_{0}$} & M1 & - & - & - & - \\
\hline & E2 & $4.25(-2)$ & $4.17(-2)$ & $3.493(-2)$ & $3.018(-2)$ \\
\hline \multirow[t]{2}{*}{${ }^{3} P_{1}-{ }^{1} S_{0}$} & Ml & 4.02 & 3.91 & 3.972 & 4.166 \\
\hline & E2 & - & - & - & - \\
\hline \multirow[t]{2}{*}{${ }^{1} \mathrm{D}_{2}-{ }^{\prime} \mathrm{S}_{0}$} & M1 & - & - & - & - \\
\hline & $\mathrm{E} 2$ & 3.10 & 2.59 & 2.693 & 2.575 \\
\hline Net Transition Rates $\left(\mathrm{s}^{-1}\right)$ & & 7.1625 & 6.5417 & 6.6999 & 6.7712 \\
\hline Lifetime ${ }^{1} S_{0}(\mathrm{~ms})$ Exp. $159.7 \pm 9.7$ & & 139.6 & 152.9 & 149.3 & 147.7 \\
\hline$A r^{3}+3 s^{2} 3 p^{3}$ & & \multicolumn{4}{|c|}{ Transition Rates $\left(\mathbf{s}^{-1}\right)$} \\
\hline Transition & & $C \& K^{28}$ & $\mathrm{~B} \& \mathrm{H}^{32}$ & $\mathbf{H}^{33}$ & $M \& Z^{34}$ \\
\hline${ }^{4} S_{3 / 2}-{ }^{2} P_{3 / 2}$ & M1 & 2.55 & 2.336 & 2.582 & 2.11 \\
\hline & $\mathrm{E} 2$ & $1.56(-5)$ & $9.363(-5)$ & $2.694(-5)$ & $1.09(-6)$ \\
\hline \multirow[t]{2}{*}{${ }^{2} \mathrm{D}_{3 / 2}-{ }^{2} \mathrm{P}_{3 / 2}$} & M1 & 0.814 & 0.7635 & 0.7649 & 0.693 \\
\hline & $\mathrm{E} 2$ & $9.81(-2)$ & $9.643(-2)$ & 0.1063 & $9.63(-2)$ \\
\hline \multirow[t]{2}{*}{${ }^{2} \mathrm{D}_{5 / 2}-{ }^{2} \mathbf{P}_{3 / 2}$} & M1 & 0.444 & 0.4188 & 0.4251 & 0.379 \\
\hline & E2 & 0.226 & 0.2244 & 0.2449 & 0.219 \\
\hline Lifetime $\left({ }^{2} \mathrm{P}_{3 / 2}\right)(\mathrm{ms})$ & & 242 & 260.5 & 242.5 & 285.9 \\
\hline \multicolumn{6}{|l|}{ Exp. Lifetime $243 \pm 73 \mathrm{~ms}$} \\
\hline \multicolumn{2}{|l|}{$\mathrm{Ar}^{9+} 2 \mathrm{~s}^{2} 2 \mathrm{p}^{5}$} & \multicolumn{4}{|c|}{ Transition Rates $\left(\mathrm{s}^{-1}\right)$} \\
\hline \multicolumn{2}{|l|}{ Transition } & $\mathrm{E}, \mathrm{C}-\mathrm{M} \& \mathrm{Z}^{35}$ & $\mathrm{C}, \mathrm{K} \& \mathrm{D}^{36}$ & $K \& S^{28}$ & \\
\hline \multirow[t]{3}{*}{${ }^{2} \mathbf{P}_{3 / 2}-{ }^{2} \mathbf{P}_{1 / 2}$} & M1 & & $1.044(2)$ & & \\
\hline & $\mathbf{M} 1+\mathrm{E} 2$ & $1.05(2)$ & & $1.06(2)$ & \\
\hline & E2 & & $2.062(-3)$ & & \\
\hline Lifetime ${ }^{2} \mathrm{P}_{1 / 2}(\mathrm{~ms})$ & & 9.52 & 9.58 & 9.43 & \\
\hline \multicolumn{6}{|l|}{ Exp. Lifetime $8.53 \pm 0.24 \mathrm{~ms}$} \\
\hline \multicolumn{6}{|l|}{$A r^{10+} 2 s^{2} 2 p^{4}$} \\
\hline \multirow[t]{3}{*}{${ }^{3} P_{2}-{ }^{3} P_{1}$} & M1 & & $6.556(1)$ & & \\
\hline & $\mathrm{M} 1+\mathrm{E2}$ & 67 & & 66.3 & \\
\hline & E2 & & $4.382(-4)$ & & \\
\hline Lifetime ${ }^{3} \mathrm{P}_{1}$ (ms) & & 14.92 & 15.25 & 15.1 & \\
\hline
\end{tabular}

Exp. Lifetime $14.8 \pm 1.1 \mathrm{~ms}$ 


\section{SUPERCONDUCTING CYCLOTRON AND INSTRUMENTATION}




\title{
K500 Development and Operations
}

\author{
D. P. May, G. Mouchaty, G. Kim, and F. Abegglen
}

During the 1993-94 reporting period the K500 cyclotron logged the highest number of hours yet for beam on target, and the beams presented a much higher degree of difficulty than before. In addition, the cyclotron was shut down for extended periods in order to install the cryopanels, ${ }^{1}$ a beam-attenuator system in the injection line, and the new beam-line for the Single Event Effect (SEE) facility. ${ }^{2}$

\section{K500 Reliability of Operation}

The most striking feature of operations for the 1993-94 reporting period is the marked increase in the use of beams with higher total energy per charge (E/Q). The rf system and the deflectors must hold voltages which are directly proportional to $E / Q$ for any given beam. For best matching of the beam through the central inflector, the ECR extraction voltage and the inflector voltage are also directly proportional to $E / Q$, although these can be significantly relaxed with small degradation. Table 1 shows this increase in high $E / Q$ beams for 1993-94 reporting period over that of 1992-1993. In the 1992-93 reporting period beams below $\mathrm{E} / \mathrm{Q}=110$ composed $90 \%$ of the beam-on-target time, whereas in the 1993-94 period beams above E/Q $=110$ composed $48.7 \%$ of the beam-on-target time. In addition to the increase in time on target from 1992-93, the unscheduled maintenance time also decreased from $12.5 \%$ to $7.2 \%$ of the total time. For the period of April 1, 1993 to March 31, 1994 the operational time is summarized in Table 2 . The increase in scheduled maintenance probably accounts for part of the increase in reliability for higher E/Q beams. Half of the increase in scheduled maintenance arises from instituting an eight-hour maintenance shift scheduled on a two-week basis on a Monday day shift. Table 3 lists how the scheduled time was divided among experimenters.

\section{Beam List}

A list representative of the beams run so far by the K500 is presented in Table 4. Some of the high $E / Q$ beams were run in machine development (45 $\mathrm{AMeV}{ }^{12} \mathrm{C}^{4+}$ and 67.5 and $70 \mathrm{AMeV}$ deuterons, for example). In general, however, stable beams for experiments can be run for $E / Q$ less than or equal to 130. Table 5 gives a list of possible beams with either $E / Q=130$ (voltage limit for stable operation) or $\mathrm{K}=520$ (magnetic field limit) with an expected beam current taken from the highest for that particular ion. Beams of ${ }^{20} \mathrm{Ne}^{8+}$ and ${ }^{40} \mathrm{Ar}^{14+}$ have already been run for experiments close to these energies, leading to the possibility that reliable beams will approach the flutter limit of the cyclotron at $E / Q$ $=160$. Intensities at the lower energy limits are difficult to predict for the different ion beams. Extraction becomes more and more difficult as the main field drops to 3 Tesla $(K=200)$, and the total extracted current from the source drops with voltages lowered to match lower E/Q.

\section{References}

1. P. Smelser et al., Progress in Research, 19931994, Cyclotron Institute, TAMU, p. 131.

2. R. C. Rogers et al., Progress in Research, 19931994, Cyclotron Institute, TAMU, p. 138. 
Table 1. Beam on Target Time vs. E/Q of Beams

\begin{tabular}{rrrrrr} 
& \multicolumn{2}{c}{$92-93$} & & \multicolumn{2}{c}{$93-94$} \\
$\mathrm{E} / \mathrm{Q} \leq 90$ & 1598.25 & 70.0 & & 729.34 & 28.1 \\
\cline { 2 - 4 } \cline { 5 - 6 } $90<\mathrm{E} / \mathrm{Q} \leq 100$ & 289.75 & 12.7 & & 406.50 & 15.7 \\
$100<\mathrm{E} / \mathrm{Q} \leq 110$ & 166.50 & 7.3 & & 195.25 & 7.5 \\
$110<\mathrm{E} / \mathrm{Q} \leq 120$ & 228.25 & 10.0 & & 864.50 & 33.3 \\
\cline { 5 - 6 } $120<\mathrm{E} / \mathrm{Q} \leq 130$ & 0.00 & 0.0 & & 399.00 & 15.4 \\
\cline { 2 - 3 } Total & 2282.75 & 100.0 & & 2594.59 & 100.00
\end{tabular}

Table 2. 1993-94 Operational Time

\begin{tabular}{|c|c|c|}
\hline & Hours & $\%$ Time \\
\hline Beam on target & 2594.59 & 41.7 \\
\hline $\begin{array}{l}\text { Tuning cyc. \& opt., exp. } \\
\text { setup }\end{array}$ & 2137.00 & 34.3 \\
\hline Beam Development & 441.50 & 7.1 \\
\hline Scheduled maintenance & 496.00 & 8.0 \\
\hline Unscheduled maintenance & 446.08 & 7.2 \\
\hline Idle time & 85.25 & 1.4 \\
\hline Cool down, transfer & 20.75 & 0.3 \\
\hline Total & 6221.17 & 100.0 \\
\hline
\end{tabular}

Table 3. Scheduled Beam Time

\begin{tabular}{lrc} 
& Hours & \% Time \\
\cline { 2 - 3 } Nuclear Physics & 2708.25 & 47.5 \\
Nuclear Chemistry & 1844.25 & 32.3 \\
Atomic Physics & 590.50 & 10.4 \\
Outisde Collaboration & 0.00 & 0.0 \\
Outside Users & 120.00 & 2.1 \\
Beam Development & 441.50 & 7.7 \\
\cline { 2 - 3 } \multicolumn{1}{c}{ Total } & 5704.50 & 100.0
\end{tabular}


Table 4. Representative K500 Beams. E/Q is proportional to dee-voltage and $\mathrm{K}$ is proportional to the square of the beam rigidity, with $\mathrm{K}=520$ being maximum for the $\mathrm{K} 500$.

\begin{tabular}{|c|c|c|c|c|c|c|c|}
\hline Ion & $\mathrm{E} / \mathrm{A}$ & $\begin{array}{l}\text { I Extracted } \\
\text { (ena) }\end{array}$ & & Ion & $\mathrm{E} / \mathrm{A}$ & $\begin{array}{l}\text { I Extracted } \\
\text { (ena) }\end{array}$ & \\
\hline d & 52 & 5 & & ${ }^{20} \mathrm{Ne}^{8+}$ & 50 & 20 & $E / Q=125$ \\
\hline d & 65 & 15 & $E / Q=130$ & & & & \\
\hline d & 67.5 & 3 & & ${ }^{27} \mathrm{~A} 9^{9+}$ & 30 & 160 & \\
\hline$d$ & 70 & 5 & $E / Q=140$ & & & & \\
\hline $\mathrm{H}-\mathrm{d}^{+}$ & 30 & 250 & & ${ }^{32} \mathrm{~S}^{11+}$ & 30 & 6 & \\
\hline \multirow[t]{2}{*}{${ }^{3} \mathrm{He}^{+}$} & 21.5 & 18 & $K=193$ & ${ }^{40} \mathrm{Ar}^{3+}$ & 2 & 14 & \\
\hline & & & & ${ }^{40} \mathrm{Ar}^{11+}$ & 25 & 110 & \\
\hline${ }^{4} \mathrm{He}^{+}$ & 15 & 600 & & ${ }^{40} \mathrm{Ar}^{12+}$ & 35 & 25 & \\
\hline \multirow[t]{2}{*}{${ }^{4} \mathrm{He}^{+}$} & 30 & 3 & $K=480$ & ${ }^{40} \mathrm{Ar}^{14+}$ & 40 & 3.5 & \\
\hline & & & & ${ }^{40} \mathrm{Ar}^{14+}$ & 45 & 0.80 & $E / Q=128.6$ \\
\hline $\mathrm{H}^{-4} \mathrm{He}^{+}$ & 9 & 3 & $K=180$ & ${ }^{40} \mathrm{Ar}^{16+}$ & 50 & 0.030 & $E / Q=125$ \\
\hline${ }^{12} \mathrm{C}^{4+}$ & 45 & 3 & $E / Q=135$ & ${ }^{58} \mathrm{Ni}^{17+}$ & 31 & 50 & \\
\hline \multirow[t]{2}{*}{${ }^{12} C^{5+}$} & 50 & 3 & & & & & \\
\hline & & & & ${ }^{63} \mathrm{Cu}^{19+}$ & 35 & 4 & \\
\hline${ }^{14} \mathrm{~N}^{5+}$ & 45 & 4 & $\mathrm{E} / \mathrm{Q}=126$ & ${ }^{63} \mathrm{Cu}^{21+}$ & 40 & 0.15 & \\
\hline \multirow[t]{2}{*}{${ }^{14} \mathbf{N}^{6+}$} & 50 & 210 & & & & & \\
\hline & & & & ${ }^{129} \mathrm{Xe}^{17+}$ & 6 & 59 & \\
\hline${ }^{16} \mathrm{O}^{3+}$ & 7.5 & 330 & & ${ }^{129} \mathrm{Xe}^{23+}$ & 12.5 & 9 & \\
\hline${ }^{16} \mathrm{O}^{5+}$ & 30 & 700 & & ${ }^{129} \mathrm{Xe}^{25+}$ & 15 & 25 & $K=400$ \\
\hline${ }^{16} \mathrm{O}^{5+}$ & 35 & 300 & & & & & \\
\hline${ }^{16} \mathrm{O}^{6+}$ & 45 & 175 & & ${ }^{181} \mathrm{Ta}^{16+}$ & 2 & 0.45 & \\
\hline${ }^{16} \mathrm{O}^{7+}$ & 50 & 100 & & ${ }^{181} \mathrm{Ta}^{24 t}$ & 6 & 21 & \\
\hline${ }^{16} \mathrm{O}^{8+}$ & 62.5 & 12 & $E / Q=125$ & & & & \\
\hline
\end{tabular}

Table 5. Possible K500 Beams

\begin{tabular}{rrc}
\hline \hline Ion & E/A (MeV/n) & Intensity (ena) \\
\hline${ }^{4} \mathrm{He}^{+}$ & 32.5 & 600 \\
${ }^{14} \mathrm{~N}^{6+}$ & 55.7 & 210 \\
${ }^{16} \mathrm{O}^{6+}$ & 48.7 & 700 \\
${ }^{16} \mathrm{O}^{7+}$ & 56.9 & 100 \\
${ }^{20} \mathrm{Ne}^{8+}$ & 52 & 20 \\
& & \\
\hline
\end{tabular}

\begin{tabular}{ccc}
\hline Ion & E/A $(\mathrm{MeV} / \mathrm{n})$ & Intensity \\
\hline${ }^{40} \mathrm{Ar}^{12+}$ & 39 & 25 \\
${ }^{40} \mathrm{Ar}^{14+}$ & 45.5 & 3.5 \\
${ }^{58} \mathrm{Ni}^{17+}$ & 38.1 & 50 \\
${ }^{63} \mathrm{Cu}^{19+}$ & 39.2 & 4 \\
${ }^{129} \mathrm{Xe}^{25+}$ & 19.5 & 25 \\
${ }^{181} \mathrm{Ta}^{24+}$ & 9.14 & 21 \\
\hline \hline
\end{tabular}




\section{Cryopanel Installation and Operation}

P. Smelser, G. J. Derrig, F. P. Abegglen, S. M. Bielarnowicz, W. L. Dewees, D. P. May, W. H. Peeler, R. D. Russell, and W. J. Walterscheid

During the month of January, three cryogenically cooled copper panels were installed in the lower dee shells of the K500 Cyclotron. The design of the cryopanels closely follows the design of the Michigan State University cryopanels that are used in their K500 Cyclotron. The system for delivering the cryogens to the panels; developed uniquely for use with the Texas A\&M Cyclotron, consists of a cryostat that houses separate booster pumps for the liquid helium and for the liquid nitrogen. The booster pumps circulate the cryogens from the pit area under the cyclotron to the panels that are $6 \mathrm{~m}$ above the pit near the cyclotron median plane. The three cryopanels are connected in series and in between each cryopanel the cryogen passes through a coil of copper tubing in the cryogen reservoir of the cryostat. The cryopanels consist of an inner liquid helium cooled copper plate surrounded by a liquid nitrogen cooled shell. The nitrogen cooled surface that faces the center region of the cyclotron is fabricated from thin copper sheet into a layer of chevrons that block line of sight to minimize any radiant heat transfer to the cold head.

When the nitrogen panels are cooled the cyclotron vacuum typically improves from $8.9 \times 10^{-7}$ Torr to $6.0 \times 10^{-8}$ Torr. When the helium surface is also cooled the vacuum typically improves from 6.0 $\times 10^{-8}$ Torr to $2.6 \times 10^{-8}$ Torr. Installation of the complete cryopanel system was finished in the second week of February. The nitrogen cooled panels have been almost continuously in use. The helium cooled panels and associated pump system have only been used intermittently when the moderate vacuum improvement warrants the increased wear on the system.

In order to install the cryopanel supply system, major modifications to the existing magnet cryogen plumbing system were required. A 50 liter liquid nitrogen reservoir was installed approximately $4 \mathrm{~m}$ above the $\mathrm{K} 500$ cyclotron.

Liquid nitrogen was plumbed out of the dewar to the upper radiation shield of the main magnet and to another line that fed to a common chamber $2 \mathrm{~m}$ above the cyclotron at the north wall of the cyclotron vault. This common chamber was the gathering point for the nitrogen supply and return lines and the helium supply and return lines, from here these four lines were routed into a single 4 in. diameter vacuum jacketed line that transported the fluids to and from the pump cryostat in the sub-basement below the K500 cyclotron. An intermediate chamber was needed $2 \mathrm{~m}$ below the $\mathrm{K} 500$ median plane to allow liquid nitrogen to be fed to the lower radiation shield of the cyclotron.

Figure 1 shows the internal plumbing of the nearly completed pump cryostat with the nitrogen cooled radiation shielded liquid helium reservoir on the left of the picture and the liquid nitrogen reservoir on the right of the picture.

The nitrogen and helium pumps are indentical in construction and consist of a removable pump assembly that is inserted into the cryogen reservoir. The pump assembly is shown in Fig. 2. The pumps are electric motor driven, reciprocating piston pumps with ball type check valves for the pump inlet and outlet. The 4-3/8 in. long, 1 in. dia. piston has a 1 in. stroke. A series of grooves on the outside diameter of the piston form a labryinth type seal. Each piston is guided in its cylinder by a set of radially spring loaded carbon filled TFE inserts. The pistons are 304 S.S. material and after being coated with Xylan they are polished to fit the cylinders with minimal clearance.

From the pump station in the sub-basement three independent lines were routed to each of the three cryopanels. The lines feeding the cryopanels are 
routed through the inner conductors of the three lower R.F. dee stems. To allow replacement of dee stem insulators, it was necessary to design three multiple bayonet assemblies. Figure 3 shows one of these assemblies that contains all four of the cryogen lines and allows the joints to be separated without resorting to saw cutting any welds.

The use of the cryopanels has greatly increased the time that it takes to be able to raise the polecap of the cyclotron in order to gain access to the inside of the machine. The routine procedure for entering the cyclotron after the panels have been in use is to vent the cyclotron vacuum chamber to nitrogen gas at atmospheric pressure and then wait for a minimum of $6 \mathrm{hrs}$. This allows time for the cold surfaces to warm to room temperature in the gaseous nitrogen atmosphere in order to prevent condensation onto the cold surfaces of any moisture that may be present.

The cryopanels have required regenerating once since they were installed. The symptom that indicated the need for regenerating was fluctuations in the pressure of the liquid nitrogen reservoir. We theorize that an increased heat load on the panels due to the buildup of condensation caused local boiling to occur in the nitrogen feed lines and this in turn caused the fluctuations that were noted in the reservoir pressure. Once the panels were warmed up, pumped and recooled the situation corrected itself.

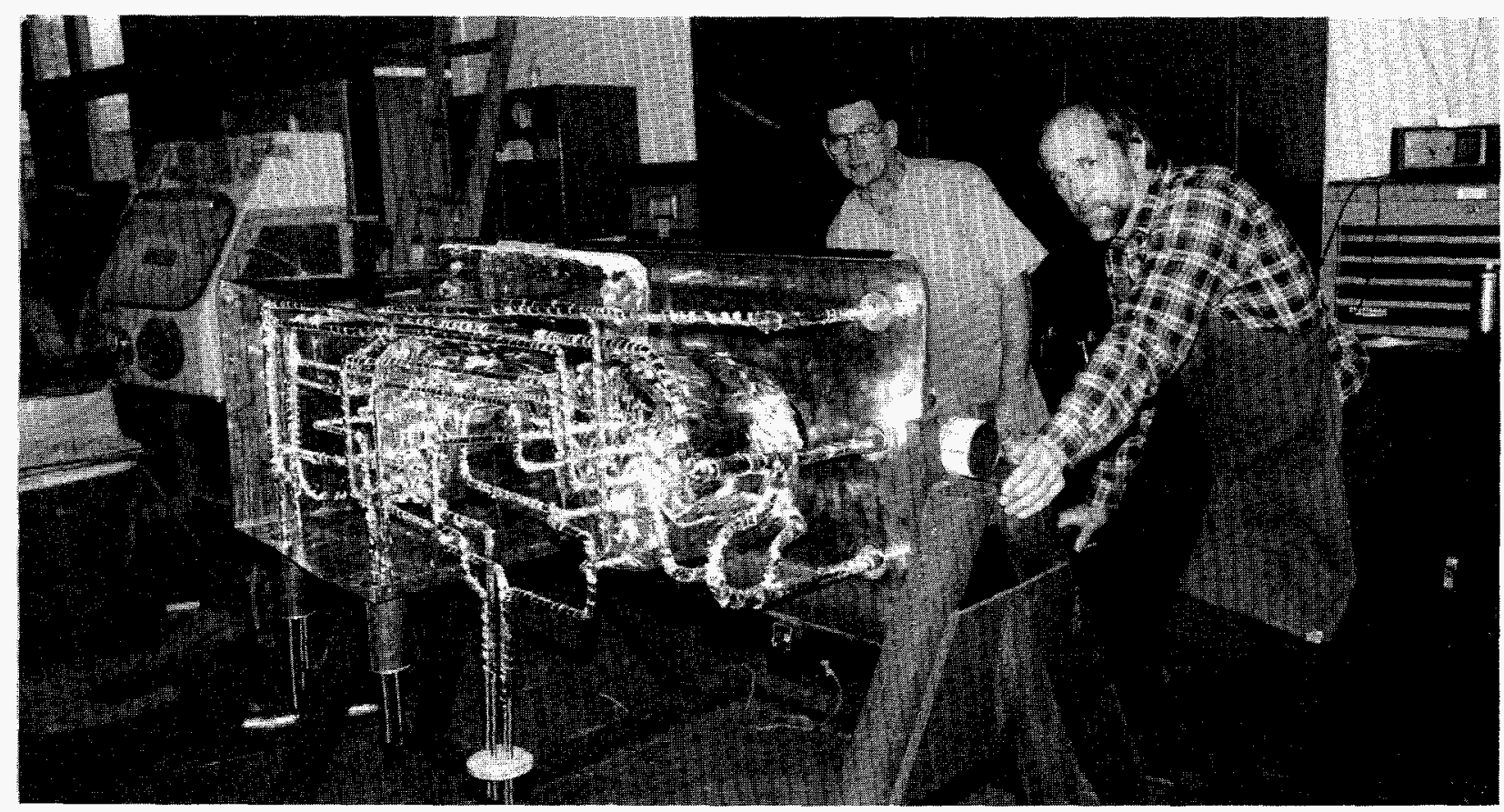

Figure 1. Internal plumbing of pump cryostat. 


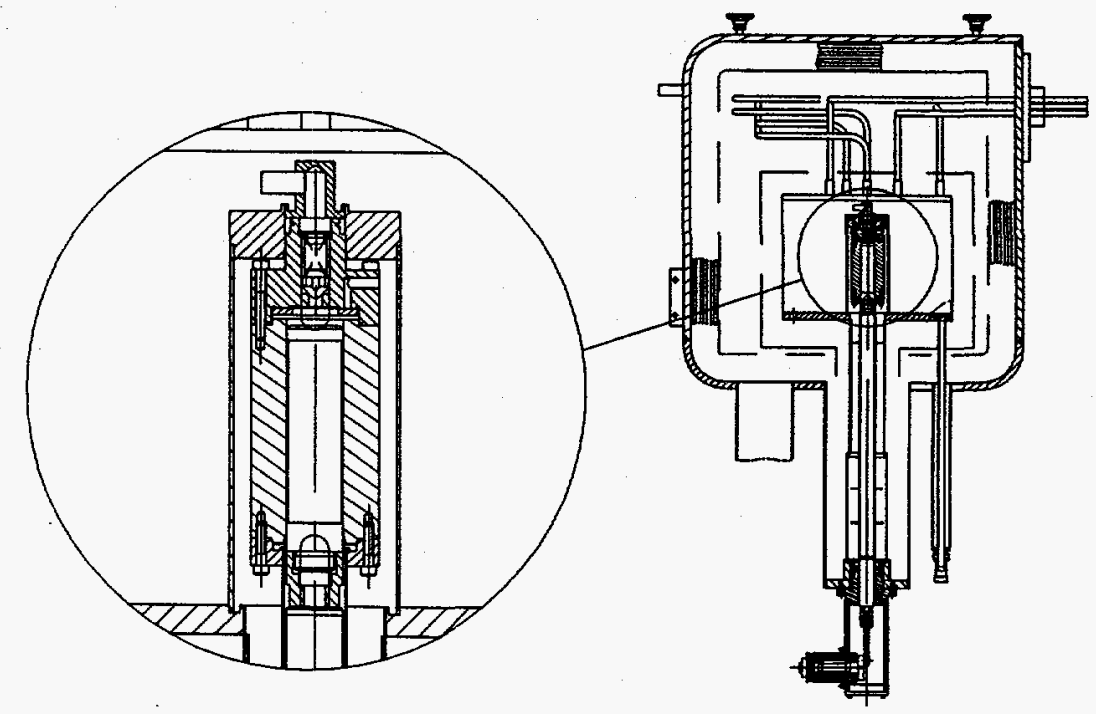

Figure 2. Sectional view of pump cryostat assembly with detail of piston-cylinder assembly.

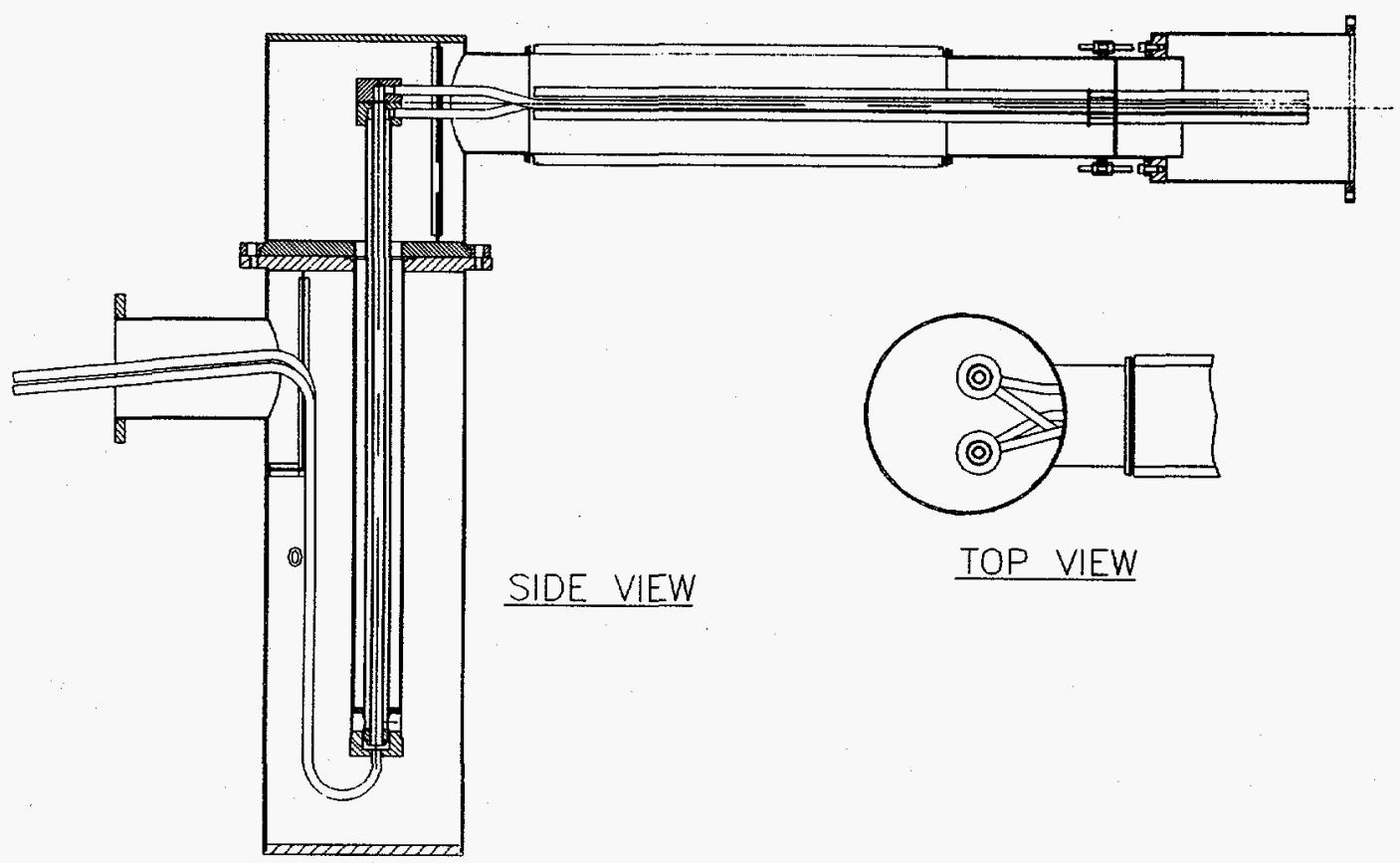

Figure 3. Typical Multiple Bayonet Assembly with helium supply and return and nitrogen supply and return lines in a common chamber. 


\title{
Computing at the Cyclotron Institute
}

\author{
H. Dejbakhsh and K. Hagel
}

We have reported the past two years on the upgrade to the computational facility at the Cyclotron Institute. In two years the computational power was increased from two or three MIPS (unit of computing power of the computer) to at least 300 MIPS. Many of the individual groups now have many tens of times the computing power of the institute as a whole before the upgrade. Almost all the post doctoral personnel and graduate students either have a workstation on their desk or have easy access to one. We reported on the upgrade last year ${ }^{1}$ and because there have not been many changes, we will not discuss that any further.

In the past year we have continued working on the network system at the cyclotron. The changes in the network system are as follows: 1 . We have eliminated the thinwire repeater shown in our network last year. This elimination required a major change in the LANs at the Cyclotron; 2. Re-wiring of the LANs was an essential task after the elimination of the thin wire repeater. We have established four different LANs at the Cyclotron instead of one large segment which included almost all the nodes associated with the repeater. We have provided a drop in each office regardless of the usage. This policy provides a more manageable network environment as the network need increases almost on a daily basis; 3- As the network industry rapidly moves toward twisted pair so do we. We will not eliminate the thinwire Ethernet, but in the future more twisted pair wiring will be added at the cyclotron. During last year we purchased and installed a 10BT HUB and placed two devices on the 8 port HUB. We are planning to provide twisted pair wiring to some newly remodel office space in the near future.

The major upgrade was in the area of the data acquisition system last year. We evaluated three data acquisition systems at three institutes which have similar needs in data acquisition. The first system we investigated was at the Michigan State University National Superconducting Cyclotron Laboratory (NSCL) the second system belonged to a group at the Oak Ridge National Laboratory (ORNL) which also carries out experiment at the TAMU Cyclotron and the third system was the data acquisition at the Indiana University Cyclotron Facility (IUCF). All these system use a VME based front end and all use a network link and protocol to move the data around. We decided to adapt the IUCF system for the following reasons: 1- We dismissed the ORNL system due to the slow read out of their Kinetic System CAMAC controller. The $7 \mu \mathrm{sec} /$ word CAMAC read out was unacceptable to many of the experimentalists at the Cyclotron. The future plan for this system was to move to FAST bus with a VME front end, discarding the CAMAC, which does not fit the Cyclotron Institute future plan; 2- The NSCL system was a mature and well established ${ }^{2}$ system based on the Motorola 68020 VME CPU. This system is well documented and has been in operation since the 1980s. There is a plan to upgrade the CPU to a 68030 the next more powerful CPU in Motorola line. However there is no provision to upgrade past that to say, the 68040 since that would require a major software revision. Due to the slow $68020 \mathrm{CPU}$ at the time NSCL was developing the system, early 1980 s, the developers of the system decided to provide their own protocol instead of using the commercial TCP/IP protocol in order to improve the throughput of the experiment; 3- The system at IUCF was the last system we evaluated. The system is based on the NSCL system with enough changes to make it attractive for us. At the time we investigated the system, the IUCF data acquisition was only used in one experiment. This system is not yet as established as NSCL system but is using the latest technology available. The system is not complete yet 
and is short in documentation. Since the system is based on the $6804033 \mathrm{MHZ} \mathrm{CPU}$ and uses the commercial TCP/IP protocol the Cyclotron decided to adopt this system.

Figure 1 shows the layout of the system with all the necessary hardware. We have purchased all the hardware needed to set up three data acquisitions. We only purchased one CAMAC crate and one CAMAC controller because we will use our existing systems in the future as we make the transition from the LAMF $Q$ system data acquisition to the new system. All the hardware components are in house and have been tested at this time. The first data acquisition system is already assembled and the front end and the link between the VME CPU and workstation have been tested. We have decided to purchase a VAXstation 4000/90 for the first system, and for the others we will use our existing data acquisition computers (two VAXstation 3200's). We have also decided to try installing the data acquisition on the ALPHA AXP open VMS system. This has not been done before and might require some modification. We will report in the future on the outcome of this project.

The layout of the software design and data flow for the data acquisition is shown in Fig. 2. This figure shows the final plan but not the one currently in use. The existing system only uses one CPU instead of two and tapping on the VME side is not available. The one CPU at present commuricates both with CAMAC and the workstation. The data throughput rate is given in Fig. 1 is based on one CPU and it will increase by $50 \%$ with the addition of the second CPU. In order to get the system running at the TAMU Cyclotron we have to make a few modifications. At IUCF the TCP/IP package (UCX) from Digital is used for communication but, we will use our existing TCP/IP software (multinet) by TGV. The TGV software allows us to use TFTP protocol for easy load of the front end program therefore eliminating user intervention for loading users front end applications. We have also purchased a C/Assembler Linker which should work better with our front end than the one at IUCF. This should diminish any problem associated with the compiler.

We expect to start taking data with this new system in the next few weeks and to make a complete transition within a year.

\section{References}

1. K. Hagel and H. Dejbakhsh, Progress in Research, 1992-1993, Cyclotron Institute, TAMU, p. 128.

2. R. Fox, R. Au, and A. Vander Molen, IEEE Trans. Nucl. Sci. 32, 1562 (1989), and references therein. 


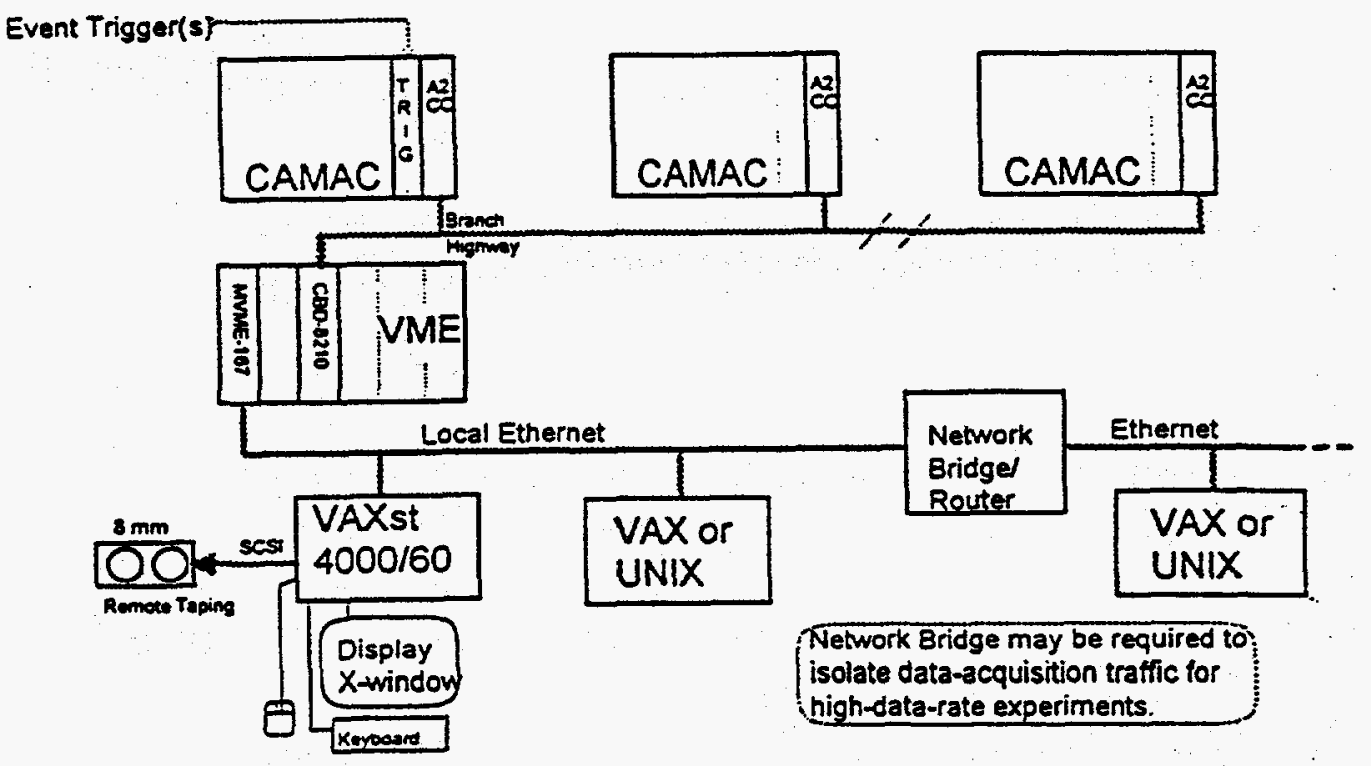

VME Components:

- One or Two Motorola MVME167 (68040) CPU modules

- CES CBD-8210 CAMAC Branch Highway Driver module

- pSOS+ Real-time kernel with TCP/IP network support

- Optional: Fastbus, VICbus, $8 \mathrm{~mm}$ tape

\section{Performance}

- CAMAC readout rate is $900 \mathrm{kB} / \mathrm{s}$ for 16-bit data words

- Minimum event busy time is $25 \mu \mathrm{s}$

- Ethernet transmission rate is $1 \mathrm{MB} / \mathrm{s}$ to a VAXstation $4000 / 60$

- Throughput using only one cpu for continuous beam is $315 \mathrm{kB} / \mathrm{s}$

- Throughput using only one cpu for pulsed operation is $420 \mathrm{kB} / \mathrm{s}$

- Estimated throughput using a second cpu for readout is $50 \%$ higher

\section{Status}

- One-cpu version is fully operational with remote taping

- Multiple data consumer version in development

- Host may be any VMS or UNIX computer

- Suppors XSYS event buffer format

\section{Applications}

- Earlier version based on MSU design was used for CE25 experiment

- IUCF version using TCPITP is currently in use for the ISiS detector experiment at the Satume accelerator, Saclay

Figure 1. A general view of the data acquisition system. 


\section{VME Data-Acquisition: Software Block Diagram}

Process: ROOT

Starts all other essential processes at boot time.

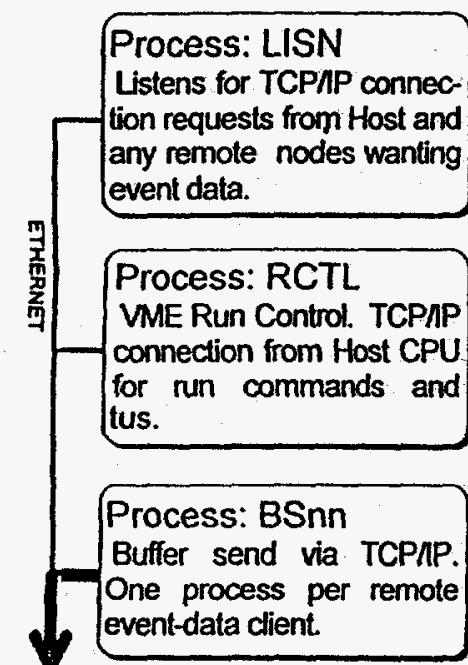

Process: BFMT

Gets event data from event

queue and formats output buffers for router.

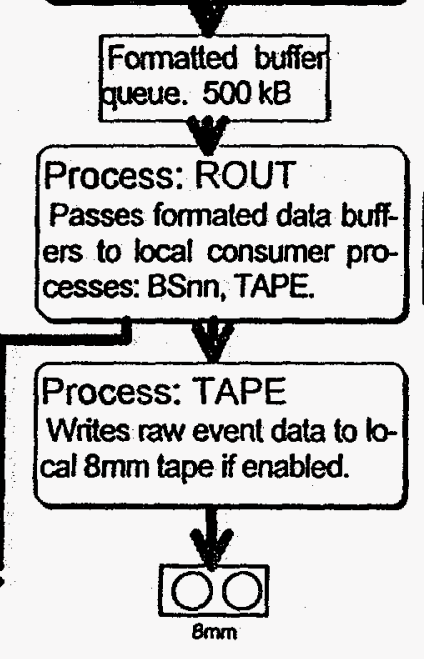

Process: ROOT

Starts all other essential processes at boot time.

Process: CMND

Slave Commands. VMEbus connection with master.

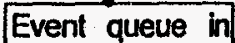

Event queve in

$500 \mathrm{kB}$

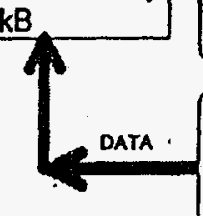

Process: CLOK

Generates clocked treggers

for scaler reads, etc.

\section{Process: EVTS}

Reads CAMAC data and puts event data records into event queue.

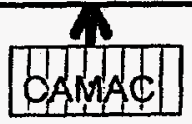

o HOST Processes in Master CPU

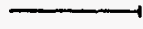

Figure 2. The software design of the future data acquisition. 


\section{Single Event Effect Facility}

\section{R. C. Rogers, R. A. Gutierrez, D. P. May, D. L. Utley and R. Wada}

The plans for the Single Event Effect (SEE) Facility were described in last year's report. ${ }^{1}$ The beamline and modified target chamber have been installed along with additional beam diagnostic and monitoring equipment. Figure 1 shows the present arrangement of the facility including the experimental area. Initial testing of the facility began on March 15 and continued on March 18.

The positioning mechanism as finally installed has one additional degree of motion beyond those previously described. The target mounting frame can be moved in the $\mathrm{X}$-axis (horizontal) and $\mathrm{Y}$-axis (vertical) directions that are normal to the direction of the beam travel. Rotation about the Y-axis is also possible. The additional motion is a $\mathrm{Z}$-axis in the direction of beam travel. This was added to accommodate integrated circuits with different mounting heights above the plane of the mounting frame. It also allows positioning of the phosphor target for beam imaging and alignment.

It can be seen in Fig. 1 that several safety features have been incorporated into the design of the SEE facility. The item noted as "Shield Wall" is a sandwich of two 3/4" thick plywood sheets laminated with a $3 / 16$ " thick lead sheet between them. The two interlocked gates insure that personnel cannot enter the area around the irradiation chamber while beam is being brought into the chamber. Opening either gate causes the main shield wall plug into the area to close and the RF system on the cyclotron to shut down. The experimental area has been surveyed for radiation level and verified as safe for all experimental beams up to intensity levels of one electrical nanoampere. This intensity level is two or

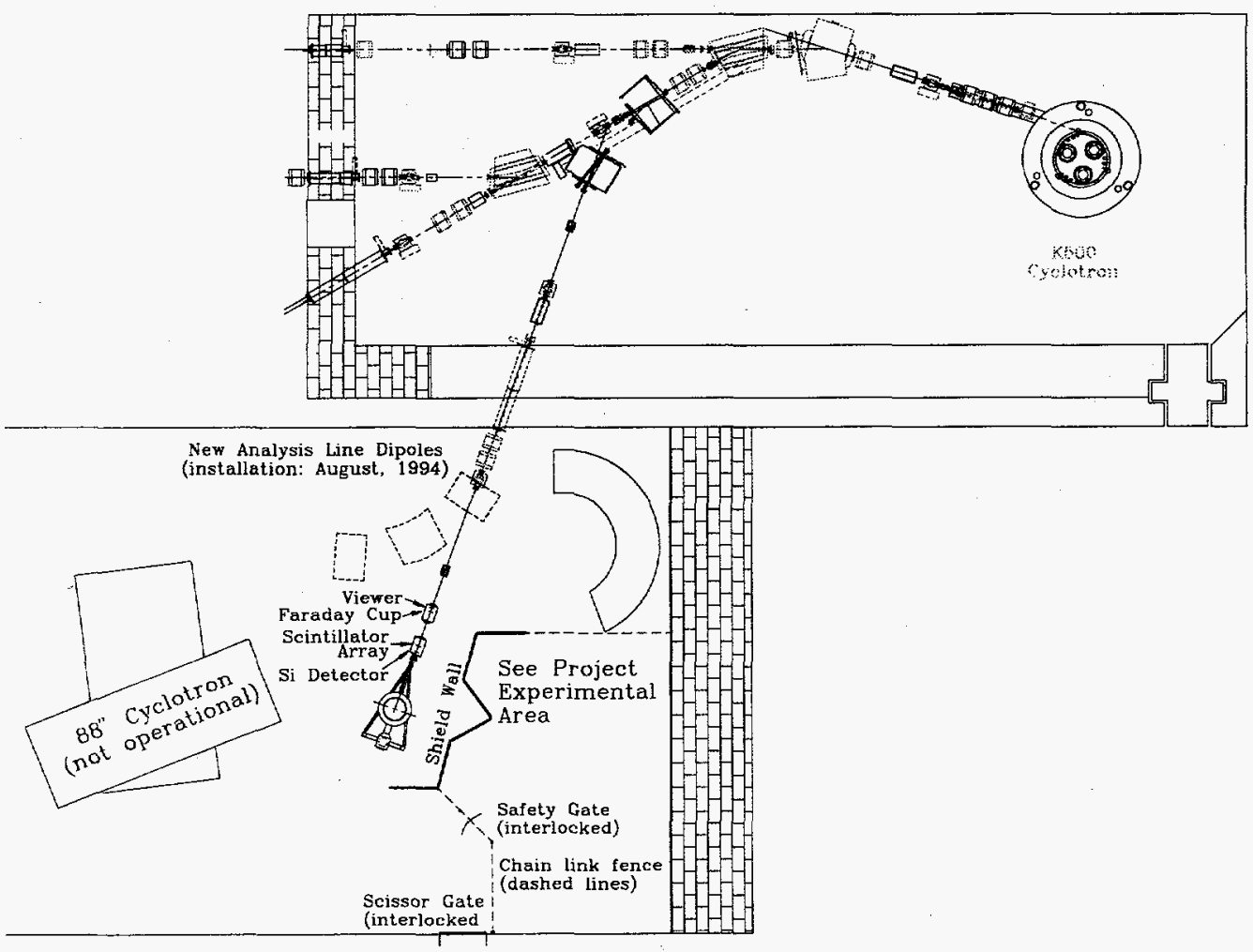

Figure 1. Location of Single Event Effect Facility relative to the K500 with some features noted. 
more orders of magnitude greater than any needed for the SEE experiments which require $<10^{4}$ part. $/ \mathrm{cm}^{2}{ }^{2}$ sec. The maximum charge state ion being used in these experiments is Xe 26+ which represents a total electrical current of only 0.15 picoamperes.

The first Viewer provides for initial focussing of the beam before it is introduced into the chamber where a more detailed inspection of the beam uniformity can be done.

The Faraday Cup located downstream from the viewer provides a convenient beam stop and preliminary current measurement location before the beam is allowed into the chamber. Excessive current striking the delicate integrated circuits that are to be tested can easily destroy them.

Following the Faraday Cup is an array of five $3 / 8$ " diameter by $1 / 2$ " long plastic scintillator and photomultiplier tube (Hamamatsu type R1635) assemblies. The array consists of four fixed PMT assemblies mounted on a five and one-half inch diameter ring and a single PMT assembly mounted on a movable arm that can be placed in the center of the beam path. This allows for both exact particle rate measurement with the central PMT assembly and continuous monitoring with the four ring assemblies.

As a final check on the beam, a $\mathrm{Si}$ detector mounted on a movable arm can be placed on the beam axis to measure total energy. This aids in particle identification and functions as a check for possible beam impurities.

A new technique for imaging the beam using a fluorescent material and a CCD camera was mentioned briefly in last year's report. That effort has matured in the past year and is now in a stage that it can be used as a tool for analysis of the uniformity of the beam.
The intent last year was to continue to use the Zinc-Cadmium Sulfide Fluorescent Indicator that had been used in the past for preparation of viewers in general use throughout the laboratory. This material is no longer as readily available as in the past because of concerns about the cadmium content. A different phosphor, $\mathrm{Y}_{3}\left(\mathrm{Al}_{1} \mathrm{Ga}\right)_{5} \mathrm{O}_{12}(\mathrm{Ce})$, was chosen from ones available for cathode ray tube use. This phosphor, Sylvania Type 1576, was chosen because of its short persistence and assumed higher light output (it is ordinarily used in flying-spot scanners).

This new phosphor was first deposited on a standard 3" diameter alumina viewer and marked with 1" and 2" reference circles and crosshairs. Figure 2, shows the phosphor target and Fig. 3 shows the superposition of a Xe beam image on the phosphor.

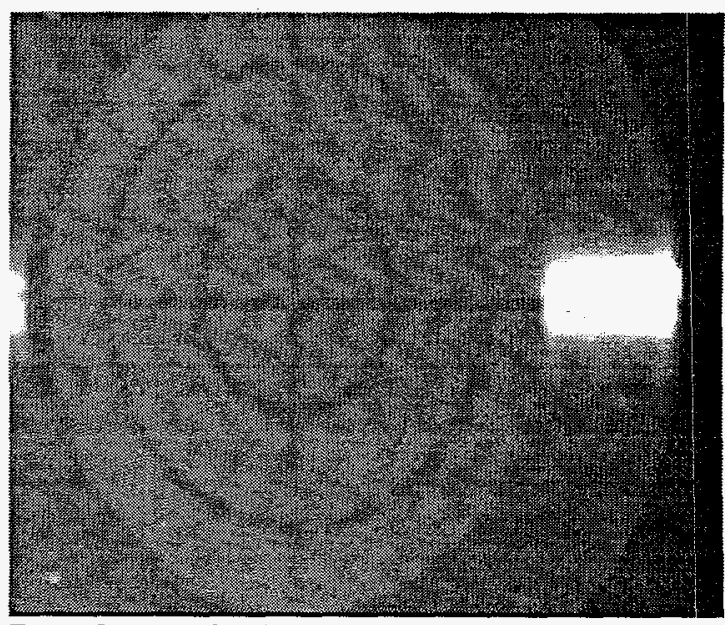

Figure 2. $\mathrm{Y}_{3}\left(\mathrm{Al}_{1} \mathrm{Ga}\right)_{5} \mathrm{O}_{12}(\mathrm{Ce})$ fluorescent target with reference markings.

The application of this imaging technique is to insure that beam uniformity is good across the area of the large scale integrated circuit being tested. Because of this a new imaging target was produced but with the reference markings omitted so that they would not interfere with the uniformity measurements. Figure 4 shows this target.

This target was tested for uniformity by stepping a small diameter beam image across the target and 


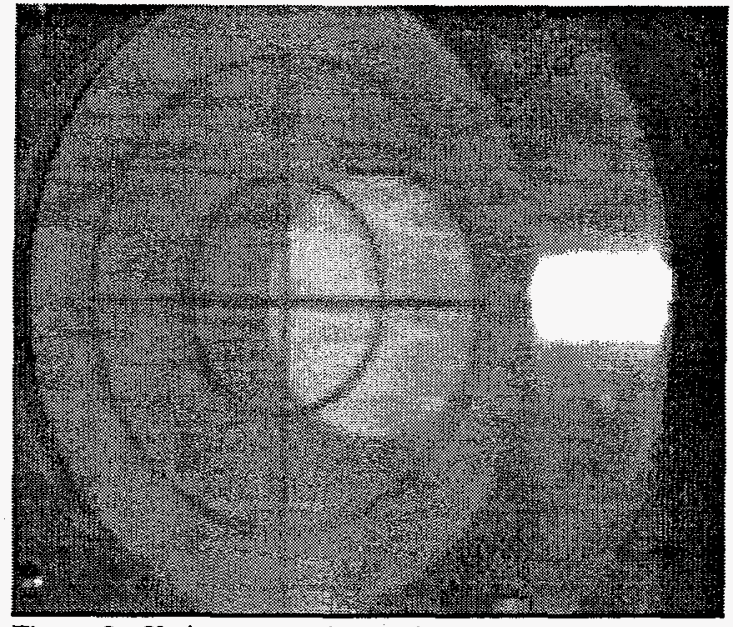

Figure 3. Xe image superimposed on target.

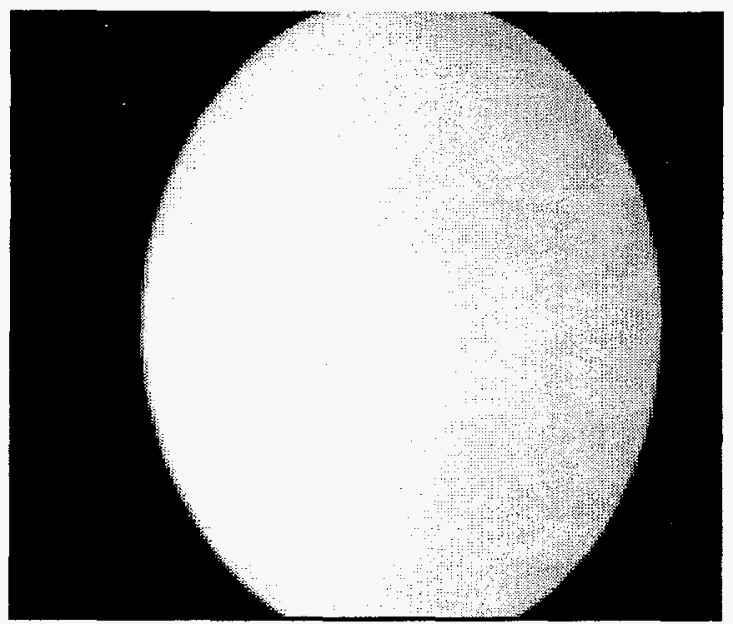

Figure 4. Target for beam uniformity measurements.

measuring the light output from each position. Figure 5 shows the step pattern while Fig. 6 shows the image of the $\mathrm{Xe}$ beam used for the test superimposed on the target at position 17 .

The target showed good uniformity across the entire surface with a variation in intensity of less than $5 \%$ when fluctuations of beam current were taken into account.

Future plans for improvements in the facility include: reducing the diameter of the PMT assemblies to a three inch diameter ring to improve count rate, collimation of all detectors to a well defined sensitive area, and further work on phosphor types to improve sensitivity in imaging.

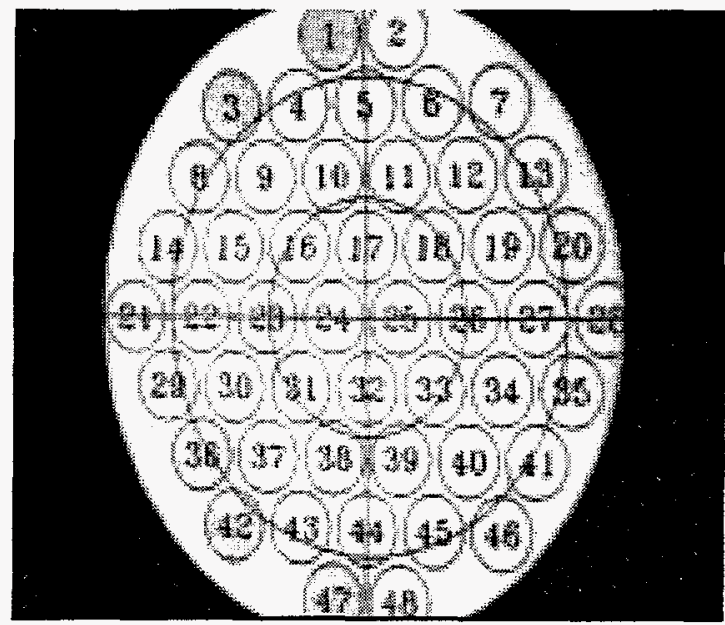

Figure 5. Step pattern for measurement of target uniformity.

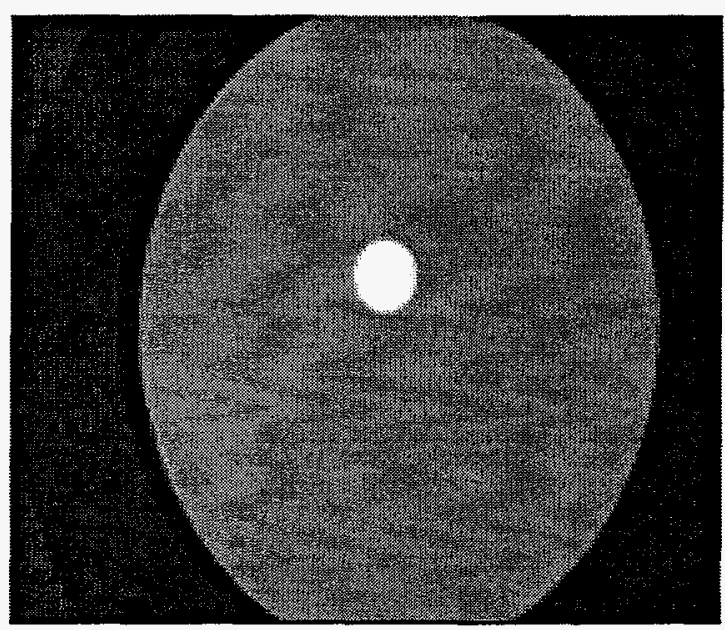

Figure 6. Superposition of Xe beam test spot on uniform target at location 17.

An experimental run for NASA to test a Motorola MC68HC000 microprocessor is already scheduled in the coming months. Experimental runs with the Laboratory for Radiation Studies at Prairie View A\&M University will be conducted in the fall months of 1994 to compare results with experiments done at the Brookhaven National Laboratory.

\section{Reference}

1. Progress in Research, 1992-1993, Cyclotron Institute, TAMU, p. 126. 


\section{J. Bronson, D. H. Youngblood, Y.-W. Lui, G. Derrig, and F. Abegglen}

The beam analysis system ${ }^{1}$ was discussed in detail in last year's progress report. Here we describe its current stage of development.

The facility layout, including the analysis system, is shown in Fig. 1. Dipoles D1 and D2 were part of the K500 cyclotron beam line. In June 1993, the beam line from the K500 cyclotron to the MDM Spectrometer was redone [quadruples, slits, viewers and Faraday cups moved and added] to provide a low resolution analysis system for the MDM Spectrometer (and two other beam lines). At this time all elements from the cyclotron through $\mathrm{Q} 4$ were placed in the position appropriate for the beam analysis system. Subsequently, we have a year of experience putting beams through the initial section of the system and find that the Transport/Raytrace calculations provide a reasonable description of the beam behavior in this portion of the BAS. A collimator and viewer had to be added immediately following the quadrupole triplet approximately $2 \mathrm{~m}$ upstream from the entrance slit to the analysis system as a guide for tuning the cyclotron extraction system and to limit the beam phase space.

In September 1993 dipoles D3 and D4 and quadrupoles Q5 and Q6 were added to the system. Subsequently beam has been tuned through the entire first half of the analysis system, and used for experiments in the ORTEC Chamber. Dipoles D3 and D4, each producing a bend of $20^{\circ}$, were constructed in house. After installation, preliminary field maps were made. However, during the installation of the rest of the system, detailed mapping of all the dipoles is planned.

The two dipoles D5 and D7 were originally to be designed and built here; however two surplus dipoles from the University of California Berkeley Bevelac Laboratory have been obtained that require only minor modification to perform these functions. These will require modification of the pole gap from eight inches to three inches and the addition of Rogowski tips. Dipole D6 will be a surplus magnet obtained from the "Histrap Project" at Oak Ridge National Laboratory. The quadrupole lens pair Q7 and Q8 have been refurbished and are ready for installation.

Slits are currently being constructed for $\mathrm{S} 02$ and S03. The boxes for the slits will also contain Faraday cups and viewers. The bids for construction of the boxes have been sent out. The Faraday cups and viewers have been designed and construction has begun.

The floor in the area from Q5 through Q8 is

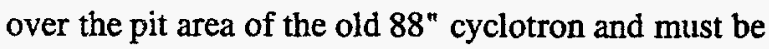
strengthened to support the weight of the magnets. This construction is scheduled for August, 1994. The floor strengthening was originally scheduled for May 1994, but it had to be redesigned because the Berkeley magnets each weigh about 12,000 pounds more than the magnets we were going to build.

Vacuum chambers for dipoles D5, D6 and D7 have been designed and will be built this summer. During the design and layout of this beam line, special attention is being given to precision alignment. Viewing ports and alignment pins are being placed in critical locations.

All power supplies for the dipoles and quadrupoles have been ordered and should be on site by the end of June, 1994. Installation and interfacing these power supplies to the control system will take approximately two months.

While use of the Berkeley magnets has saved money, the project has been delayed about 4 months for redesign of the floor modifications. The present schedule calls for floor modification in late August or early September with magnet installation beginning about 2 weeks after completion of the floor work. First tests with beam should occur in November. 


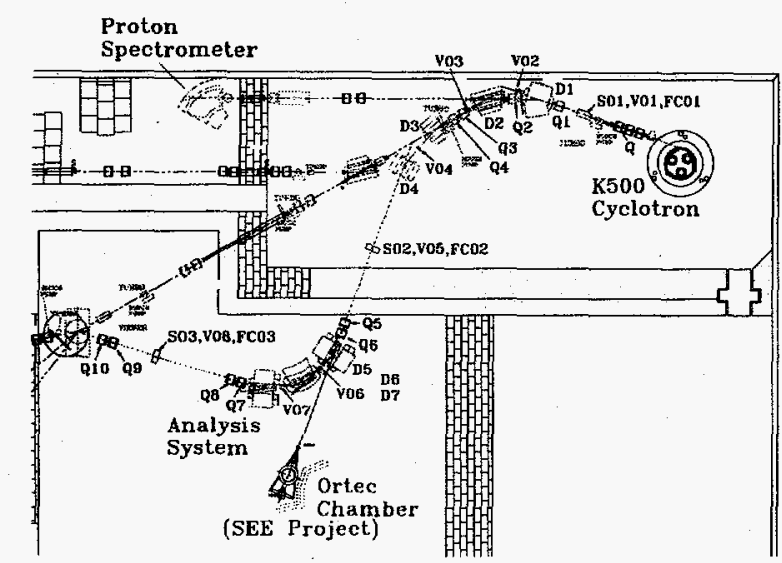

Reference

1. Progress in Research, 1992-1993, Cyclotron Institute, TAMU, p. 119.

Figure 1. Part of beam layout showing the beam analysis system (BAS).

\title{
MARS Modifications and Upgrades
}

\author{
R. Tribble, H. Dejbakhsh, R. Eiserer, C. Gagliardi, S. Hale, R. Rogers, D. Semon, L. Trache, \\ H. Xu and X. Zhou
}

During the past year, MARS has been used to carry out several different measurements of the beta decay of ${ }^{57} \mathrm{Cu}$. The results of these measurements are discussed in a different section of this year's report. In addition, test runs have been carried out to determine how well MARS will operate for particle-gamma coincidence measurements. A new, compact, scattering chamber along with a support system to hold seven Compton-suppressed $\mathrm{Ge}$ detectors is now being built for the gamma decay studies. The design of the chamber and support system can be found in a different section of this year's report. While setting up for the gamma test runs, we found that the pole tips of the first MARS quadrupole had been activated (the quadrupole was a part of the SREL synchrotron in the '60's) which results in about 100 counts/sec due to ${ }^{60} \mathrm{Co}$ gamma rays in the Ge detectors. As part of the new target chamber setup, the plumbing for the water lines on this quadrupole are being rerouted so that $\mathrm{Pb}$ shielding can be placed around the pole tips to reduce this background rate by about a factor of 100 .

In last year's annual report, ${ }^{1}$ we noted that there were several problems with the MARS high voltage system. For periods of up to several hours, a particle beam would be stable at the MARS focal plane but then it would move with what appeared to be a discrete jump in voltage. By a thorough system check-out, we were able to discover a diagnostic in the HV system that allowed us to pin down and ultimately repair the problem. The electronics that controls the power for the HV stack uses the signal from the resistor chain in the HV generator in a feedback loop to stabilize the voltage. In addition to this resistor chain, there is a second, higher impedance, resistor chain in parallel that is used as a 
monitor for the over-voltage protection circuit. By reading out the voltage from the two resistor chains simultaneously, we found that the two outputs from the negative high voltage system did not agree. In particular, the output from the resistance chain that was used in the feedback loop would remain constant while the auxiliary output would jump up by the equivalent of nearly $20 \mathrm{kV}$. This new value was not stable for long periods of time but would tend to drop, often back to the original value. After discovering this, we took the HV generator apart and found that two of the resistors in the main resistor chain had been damaged, presumably from sparking at some point. They had not been damaged to the point of being open circuits but rather their resistance values were not constant and would change depending upon the current through the resistors and their temperature. After the two resistors were replaced and the system was reassembled, the output voltages from both the primary chain and the over-voltage protection chain were quite stable.

Since this problem has been found and corrected, we have conditioned the high voltage plates up to just over $360 \mathrm{kV}$. At this voltage ( $180 \mathrm{kV}$ on each plate), we find that the positive side of the system draws a few micro-amps of current that does not go away with long conditioning times. We have not attempted to determined the source of this leakage (it could be in the HV stack or inside the vacuum system) because the system is operating in a very stable mode at voltages up to $360 \mathrm{kV}$ which is sufficient to carry out much of the physics program that was outlined for MARS. To increase the voltage beyond this will require a more thorough check-out of the system to find where the breakdown is occurring.

In last year's report, ${ }^{1}$ we noted that we had nearly completed the construction of a cryogenic gas target for MARS. The target was completed early last summer and tested in beam in July. We found a special cryogenic epoxy that we used to mount Havar windows onto the entrance and exit flanges of the gas cell and we have now cycled windows of $0.0002^{\text {" }}$ thickness several times to $\mathrm{LN}_{2}$ temperature without a problem. The major problem that we have had is a persistent (small) gas leak around a gas inlet which we have been unable to fix. Also we have noted that it takes about 6 hours for the cell to equilibrate during the initial cool down. In order to shorten this equilibration time and to eliminate the gas leak, we are making a new cell with somewhat simpler plumbing for the gas inlet. The body of the new cell is being made out of $\mathrm{Cu}$ instead of the stainless steel that we had used in the first version. With the simpler design, the gas will not be pre-cooled as it enters the cell. If we choose to flow gas in the cell at some point, an external system must be added to pre-cool the gas. The original gas cell was used as the production target for the measurements on the beta decay of ${ }^{57} \mathrm{Cu}$ which are discussed elsewhere in this report. Other than the small leak, it has worked quite well. We plan to use the new cell extensively in the future for the production of secondary beams through MARS.

\section{Reference}

1. Progress in Research, 1992-1993, Cyclotron Institute, TAMU, p. 136. 


\title{
Status Report of the $\boldsymbol{\gamma}$-Ray Spectroscopy
}

\author{
H. Dejbakhsh, C. A. Gagliardi, J. Jiang, D. R. Semon, L. Trache, R. E. Tribble, H. M. Xu, \\ X. G. Zhou
}

The domain of nuclides accessible for spectroscopic study has increased drastically during the last decade due to new accelerators and more sensitive detection systems. MARS (Momentum Achromat Recoil Spectrometer) will permit access to the region of isotopes lying far from the valley of stability. We will use this feature of MARS to investigate neutron-rich nuclei far from the valley of the stability.

The key to studying such nuclei is a good detection system. Early in 1993, we started negotiations with the nuclear structure group at Berkeley to acquire eight detectors from LBL's high energy-resolution array (HERA). ${ }^{1}$ The 30 detectors from the Gammasphere project came on-line for experiments at LBL in January, 1993 and freed the HERA detectors for such a request. This freed up the HERA detectors but not the electronics and data acquisition system which would not be available for use before January, 1994. A loan agreement was made between the two institutes and at the end of July, 1993 we borrowed 8 detector units. The HERA consists of 21 compton suppressed Ge detectors, with each detector consisting of a $5 \times 5 \mathrm{~cm}$ Ge detector, $20 \%$ efficiency, surrounded by six longitudinal pieces of BGO shield with a NaI cap.

Upon receiving the detectors we had to anneal all of them before their use. Six of the detectors were annealed in house after we purchased the necessary valve and heater. We used one of the in-house cryo pumps in an annealing station set up at the Cyclotron. All the units had a resolution of 2.0 to $2.2 \mathrm{keV}$ after annealing. We have borrowed the automatic cooling system for $\mathrm{Ge}$ detectors and electronic modules for eight Ge and eight BGO shields from Oak Ridge National Laboratory.

The eight detectors coupled to MARS provide a powerful tool for nuclear structure studies because of the unique design of MARS which allows the use of inverse kinematics with sufficient energy for the recoil masses to be separated at the focal plane according to their $\mathrm{m} / \mathrm{q}$ ratio. This feature will allow us to explore neutron-rich nuclei to higher angular momenta which enable the investigation of high spin states, band termination, and super deformation of these nuclei.

Since the MARS target chamber is made of thick stainless steel which is not designed for $\gamma$ ray spectroscopy, we must design a special target chamber and detector stand for the $\gamma$ ray detection system. The design of the spectroscopy station is shown in Fig. 1. Seven of the eight detectors are placed in two rings, three of the detectors are placed at $60^{\circ}$ relative to the beam in one ring, another three are at $25^{\circ}$ and one is at $45^{\circ}$ relative to the beam. We have placed a lead collimator in front of each detector. In our design, the distance from the target to the detector and the detector opening can be adjusted to limit Doppler broadening. The $\gamma$-ray resolution can be held to about 6 to $7 \mathrm{keV}$, Doppler broadened, for a $1 \mathrm{MeV} \gamma$ ray in the energy regime we are studying (inverse kinematics). We will use position sensitive focal plane detectors for mass measurements and any ambiguity regarding the $\mathrm{m} / \mathrm{q}$ ratio will be resolved by a $\Delta E-E$ telescope which we plan to use in future experiments.

We expect to carry out a $\boldsymbol{\gamma}$-ray spectroscopy experiment with proper mass gate at the focal plane of the MARS beam line soon. We will investigate the neutron-rich ${ }^{99-101} \mathrm{Tc}$ nuclei using a ${ }^{84} \mathrm{Kr}$ beam at 5-6 MeV/u. We expect the higher angular momentum transfer to excite these nuclei beyond the band crossing. The new data will complete the blocking information for the neighboring nuclei. The experiment will be carried out using coincidences between the specific group with the same $\mathrm{m} / \mathrm{q}$ ratio in the focal plane with the $\gamma$ rays in target area.

In the area of the $\mathrm{N}=\mathrm{Z}$ nuclei we will examine 
high spin states of ${ }^{24} \mathrm{Mg}$. The shell model description of nuclei in the sd shell predict a loss of collectivity near $J=8^{+}$, well below $J=12^{+}$, the highest spin in the model space. $^{2}$ This picture seems to be confirmed in the case of the ${ }^{24} \mathrm{Mg} \mathrm{K}=2$ band where the experimental $B(E 2)$ drops by a factor of two between spin $7^{+}$and $8^{+}$. There is evidence that the $\mathrm{K}=0$ (yrast) band may extend beyond $J=8^{+}$for ${ }^{24} \mathrm{Mg}$. This can modify the expectation of the spherical shell model in regard to the continuation of collectivity beyond $J=8^{+}$in the yrast band. We will examine the ${ }^{24} \mathrm{Mg}$ high spin states using $56 \mathrm{MeV}$ ${ }^{16} \mathrm{O}$ above $J=8^{+}$where superdeformation is expected to set in. ${ }^{3}$

Another study envisaged will heavily rely upon the design qualities of MARS (large angular and momentum acceptance, efficient beam suppression, isochronism) in order to efficiently select exotic reaction channels. They will focus on the isospin degree of freedom rather than on going higher in excitation energy or spin. Detailed spectroscopy studies of neutron deficient nuclei will be possible using inverse kinematic reactions at energies where fusion-evaporation is the dominant reaction mechanism. Very weak channels will be selected using coincidences between recoil nuclei separated by MARS and the $\gamma$-ray measured at the target position. Nuclei very far from the stability valley will be accessible and the channel enhancement factor will be 1000 or larger. So, in reactions like:

$$
{ }^{63} \mathrm{Cu}(5-6 \mathrm{MeV} / \mathrm{n})+{ }^{54,56} \mathrm{Fe}
$$

nuclei close to the proton-drip line can be populated, between the region of ${ }^{114} \mathrm{Xe}$ where octupole deformation in the ground state was recently predicted and that of the double magic ${ }^{100} \mathrm{Sn}$.

While the fusion-evaporation reactions induced by heavy ions studied in the past three decades allowed the study of neutron deficient nuclei in broad ranges of excitation energies, angular momenta and $\mathrm{N} / \mathrm{Z}$ ratios for neutron-rich nuclei current data are scarce and restricted to low energies and angular momenta available from the study of the fission fragments. A unique possibility to extend these studies at higher excitation energies, higher angular momenta and high $N / Z$ ratios is the use of the massive nucleon transfer that takes place in Deep Inelastic Reactions. The large number of open channels in such reactions makes difficult or impossible their study with $\gamma$-ray detector arrays alone. The study will only be possible if an appropriate selective device is used. Channel selection can be made with particle- $\gamma$ coincidences, using MARS for the detection of Projectile-Like Fragments in its focal plane.

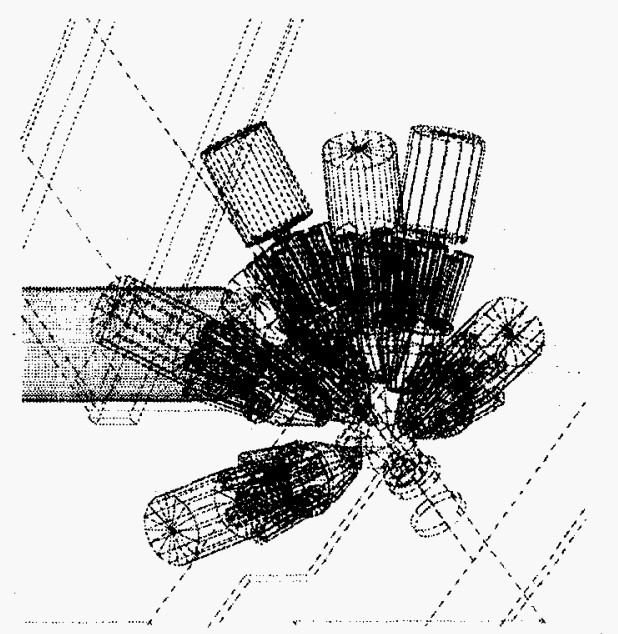

Figure 1. Layout of the seven detectors at the $\gamma$-ray station in the MARS beam-line at the Cyclotron.

\section{References}

1. R. M. Diamond, Instrumentation for Heavy Ion Nuclear Research, ed. by D. Shapira, p. 259 (New York: Harwood Acad. Pub. 1985).

2. A. Watt, D. Kelvin, and R. R. Whitehead, J. Phys. G6, 35 (1980).

3. H. Chandra and U. Mosel, Nucl. Phys. A298, 151 (1978). 


\title{
A High Resolution Large Dynamic Range Charged-Particle Detector for Nuclear Fragmentation Studies
}

\author{
T. C. White, J. A. Winger," H. Johnston, and S. J. Yennello
}

Six axial-field gas-ionization chamber telescopes were built for the collection of intermediate mass fragments (IMF) and light-charged particles. The telescope described here (Fig. 1) is based on $\mathrm{K}$. Kwiatkowski's ${ }^{1}$ design to measure light charged particles up to $\approx 70 \mathrm{MeV} /$ nucleon and intermediatemass fragments (IMF: $3 \leq \mathrm{Z} \leq 15$ ). This detector, with an active area of $25 \mathrm{~cm}^{2}$ consists of the following elements: (1) a gas-ionization chamber, (2) two passivated silicon detectors, and (3) either a single CsI(Tl) scintillator crystal or two CsI(Tl) crystals side-by-side read out by photodiodes.

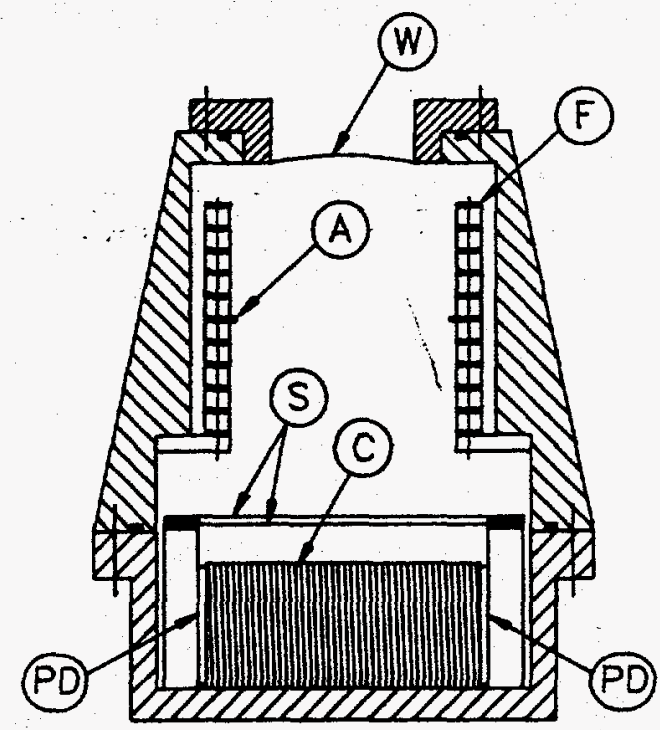

Figure 1. Schematic diagram of a large dynamic range charged particle detector for the collection of intermediate mass fragments (IMF) and light-charged particles. Legend: A - anode; W $0.0021 \mathrm{~mm}$ thick aluminized Mylar window; $\mathbf{F}$ - field shaping electrodes; S - silicon detectors; C - CsI(TI) scintillator, and PD photodiodes.

The gas-ionization chamber (GIC) element is of the axial-field design and is operated at 50.0 torr of $\mathrm{CF}_{4}$. The anode plane is positioned in the middle of the active length of the ion chamber in order to minimize the electron collection time. The grid of field shaping electrodes consists of brass frames with thicknesses of 0.031 " that are separated by $0.15^{\prime \prime}$ insulating spacers. On the center frame, the anode, five $0.005^{\prime \prime}$ diameter copper wires are strung across the frame. They are spaced $0.325 "$ apart. The front window is $0.0021 \mathrm{~mm}$ thick aluminized mylar and shows an energy loss of $0.17 \mathrm{eV}$ for alpha particles from an ${ }^{241} \mathrm{Am}$ source.

The pair of $5 \mathrm{~cm} \times 5 \mathrm{~cm}$ silicon detectors with thicknesses of $\approx 140 \mu \mathrm{m}$ and $\approx 1 \mathrm{~mm}$, serve several functions. The low energy or massive fragments that pass through the GIC but stop in the $\approx 140 \mu \mathrm{m}$ silicon detector are identified by a plot of energy deposited in the gas-ionization chamber versus energy deposited in the $140 \mu \mathrm{m}$ silicon detector. A typical plot is shown in Fig. 2. Fragments that pass through the first silicon but stop in the second $1 \mathrm{~mm}$ silicon are also identified by a $\Delta \mathrm{E}-\mathrm{E}$ plot. The $140 \mu \mathrm{m}$ silicon provides the $\Delta \mathrm{E}$ signal while the $1 \mathrm{~mm}$ silicon gives the $\mathrm{E}$ signal. The charge and mass identification of fragments up to oxygen which stop in the $1 \mathrm{~mm}$ silicon detector is shown in the plot of $\Delta \mathrm{E}$ versus $\mathrm{E}$ as shown in Fig. 3.

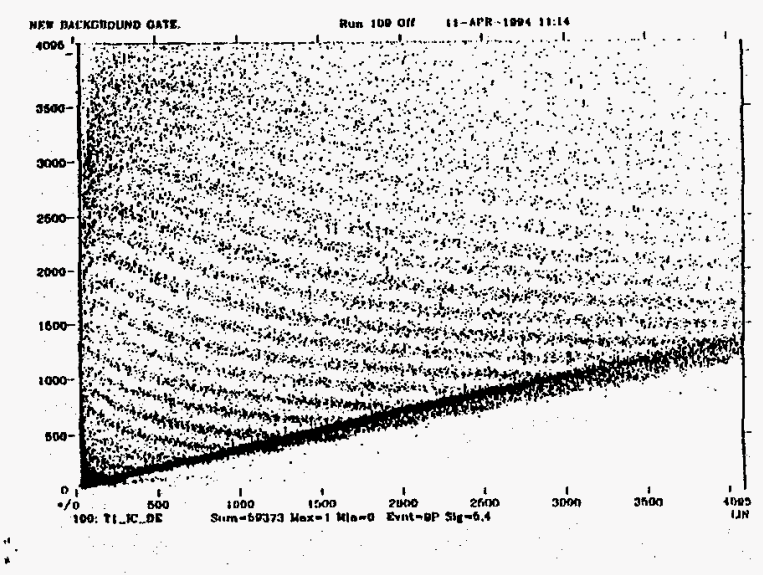

Figure 2. The $\mathrm{Z}$ resolution from energy deposited in the gasionization chamber versus energy deposited in the $140 \mu \mathrm{m}$ silicon detector for fragments up through $Z=18$. 


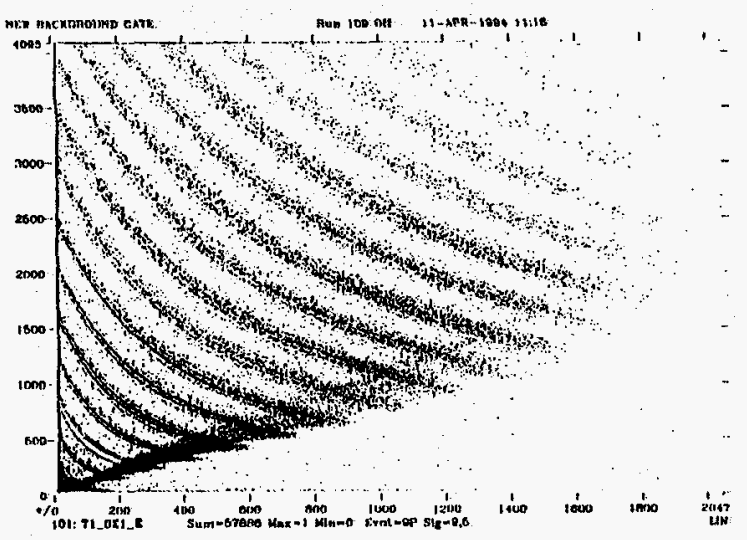

Figure 3. The A, $\mathrm{Z}$ resolution from energy deposited in the $140 \mu \mathrm{m}$ silicon detector versus the energy deposited in the $1 \mathrm{~mm}$ silicon detector for intermediate mass fragments (MF).

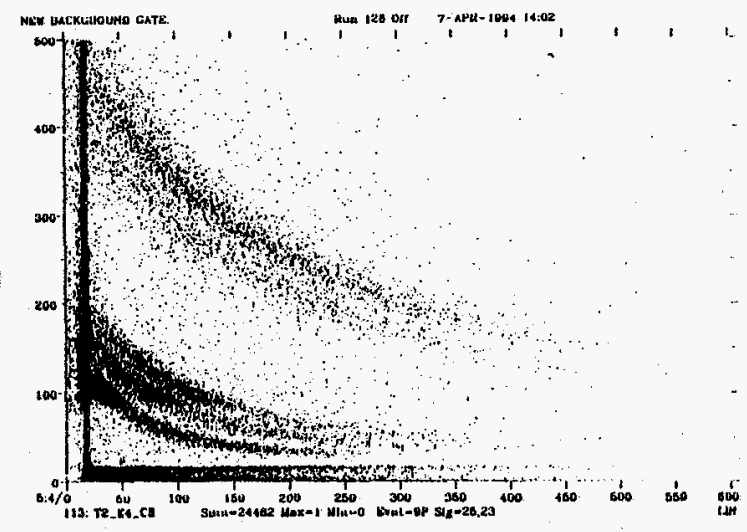

Figure 4. The $A, Z$ resolution from the energy deposited in the $1 \mathrm{~mm}$ silicon detector versus the energy deposited in the $\mathrm{Cs}$ (TI) scintillator crystal for light-charged particles.
The scintillation detector consists of either a single CsI(Tl) crystal with the dimensions of $2.165^{\prime \prime}$ $\times 2.165^{\prime \prime} \times 1.000^{n}$ or two crystals side-by-side measuring 0.886" $\times 2.165^{\prime \prime} \times 1.000^{\prime \prime}$ each. Photodiodes were attached with a silicon adhesive to both ends of each crystal. The photodiodes measure $10 \mathrm{~mm} \times 10 \mathrm{~mm}$ for the smaller crystals and $10 \mathrm{~mm} \times$ $20 \mathrm{~mm}$ for the larger ones. Each crystal was wrapped in Teflon tape to increase the reflectivity of the crystal. Plotting the energy deposited in the $1 \mathrm{~mm}$ silicon detector versus the energy in the scintillation crystal provides charge and mass resolution of light charge particles as shown in Fig. 4.

"Present address: Mississippi State University.

\section{References}

1. K. Kwiatkowski, et al., Nucl. Instrum. Methods A299, 166 (1990).

\title{
Monte Carlo Code Simulations of the Neutron Ball Response
}

\author{
B. Hurst and R. P. Schmitt
}

In order to simulate the response of the Texas A\&M Neutron Ball to various experimental conditions, a Monte Carlo simulation has been developed during the last few years. This code is based on the program DENIS, developed by Poitou and Signarbieux ${ }^{1}$ to simulate the neutron's behavior in a liquid scintillator. Like the original code, the current program traces the trajectory of a neutron with a given energy through the liquid and determines the types of interactions that occur while keeping track of the neutron's energy and position in the detector. These interactions are randomly selected 
from a weighted set of neutron cross section data. The history of the neutron is then recorded and another neutron is tracked. A more detailed description of the calculation procedure can be found elsewhere. ${ }^{1}$

The original code analytically handled the geometry of a spherical tank with a diametrical hole (cylindrical through tube), Because of the lower symmetry of our detector, an analytical approach is not efficient from a computational point of view. In our version of DENIS, the geometry of the neutron ball is treated using a cubical grid of $101^{3}$ elements with the neutron ball inscribed in the center. The grid points are assigned different values according to their position in the detector. This divides the array into 14 sections: the top hemisphere, the bottom hemisphere, and a ten-wedge median plane (the two most forward wedges subtend $\pm 20^{\circ}$ each, the remaining eight wedges subtend $40^{\circ}$ each), vacuum sections (i.e., target chamber and beam pipe) and outside the detector. With the graduation noted above, the spacing between grid elements is about 1.5 $\mathrm{cm}$. The number of grid points in each tank section reflects the volume of scintillator contained (or lacking in the case of the vacuum) in each section. The grid points in the vacuum and outside the detector are assigned the value of minus one and zero, respectively, while points in the various tank sections are given positive values that indicate the particular wedge or hemisphere. This makes it easy to determine path length corrections and to locate the neutron in the detector at any point during program execution. By zeroing elements in specific regions of the grid, one can easily simulate situations in which one or more wedges are removed.

The original code assumes that monoenergetic neutrons are emitted isotropically from a stationary source. In order to facilitate comparisons with reaction studies, the code was modified to incorporate a Maxwellian neutron kinetic energy distribution emitted from a moving source. For rapidly moving emitters, the neutron energies can be kinematically boosted well beyond the energy range assumed in
DENIS.

Neutron cross section data for the processes $\mathrm{Gd}(\mathrm{n}, \mathrm{n} \gamma) \mathrm{Gd}, \mathrm{C}(\mathrm{n}, \mathrm{n}) \mathrm{C}, \mathrm{p}(\mathrm{n}, \mathrm{n}) \mathrm{p}, \mathrm{p}(\mathrm{n}, \boldsymbol{\gamma}) \mathrm{d}$ are valid from $.01 \mathrm{eV}$ to about $15 \mathrm{MeV}$ in the original version. However, the carbon inelastic data is incomplete over this energy region. The reaction channel $\mathbf{C}(\mathbf{n}, \mathbf{n} \gamma) \mathbf{C}$ which opens at $4.43 \mathrm{MeV}$, is included, but this process is joined quickly by $\mathrm{C}(\mathrm{n}, \alpha) \mathrm{Be}$ at $7.28 \mathrm{MeV}$ and then by $\mathrm{C}(\mathrm{n}, \mathrm{n}) 3 \alpha$ at $12 \mathrm{MeV}$. These last two reactions are not accounted for in the original cross section data.

Modifications were made to compensate for these omissions and to extend the $p(n, n) p, C(n, n) C$, and inelastic carbon data sets to higher energies. Figure 1 shows the extended cross sections used in the new code. These were taken from compilations of neutron cross section data found in the literature. The cross sections for $\mathrm{p}(\mathrm{n}, \mathrm{n}) \mathrm{p}, \mathrm{C}(\mathrm{n}, \mathrm{n} \gamma) \mathrm{C}, \mathrm{C}(\mathrm{n}, \alpha) \mathrm{Be}$, $\mathrm{C}(\mathrm{n}, \mathrm{p}) \mathrm{B}, \mathrm{C}(\mathrm{n}, \mathrm{n}) 3 \alpha$, and $\mathrm{C}(\mathrm{n}, \mathrm{np} \alpha) \mathrm{Li}$ covering the energy region from $15 \mathrm{MeV}$ to $200 \mathrm{MeV}$ were taken from Fig. 12 of Strong et al. ${ }^{2}$ In the latter two reaction channels, a fraction of the maximum kinetic energy in the center of mass system was given to the neutron following the prescription of Strong. ${ }^{2.3}$ The elastic carbon $\mathrm{C}(\mathrm{n}, \mathrm{n}) \mathrm{C}$ data from $15 \mathrm{MeV}$ to 200 $\mathrm{MeV}$ were taken to be the difference between a tenth order polynomial fit to the $\sigma_{\mathrm{nC}}$ data found in Table 2 of Del Guerra et al. ${ }^{4}$ and the sum of the inelastic carbon channels mentioned above. The original elastic carbon cross sections between 5 and $15 \mathrm{MeV}$ were used since they account well for the resonances that occur in this region.

With these modifications it is possible to investigate the efficiency for a broad range of conditions. Figure 2 is a plot of the total calculated efficiency, $\epsilon$, as a function of the kinetic energy, $E_{n}$, for monoenergetic neutrons. The circles are calculations including wedge $1 \& 2$ while the triangles assume the two wedges are absent. This plot demonstrates that there is an average loss in efficiency of about $3.4 \%$, which is in line with analytically calculated laboratory solid angles.

As discussed by Bowman et al..$^{5}$ the angular 


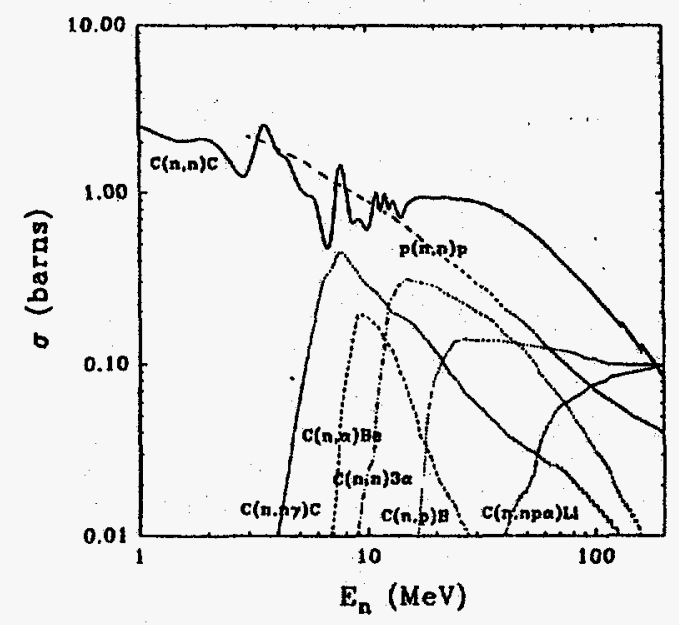

Figure 1. Neutron cross section data used to extend the original DENIS cross section data set.

distribution and energy spectra of ${ }^{252} \mathrm{Cf}$ neutrons are well described by emission from three sources: the two fragments and a scission component. Using their parameters for the source temperatures, velocities and multiplicities, our modified version of DENIS predicts a total detection efficiency of $84.9 \%$ with $800 \mathrm{keV} \gamma$ ray threshold settings. This is in excellent agreement with the measured efficiencies for ${ }^{252} \mathrm{Cf}$ of $83.5 \%$

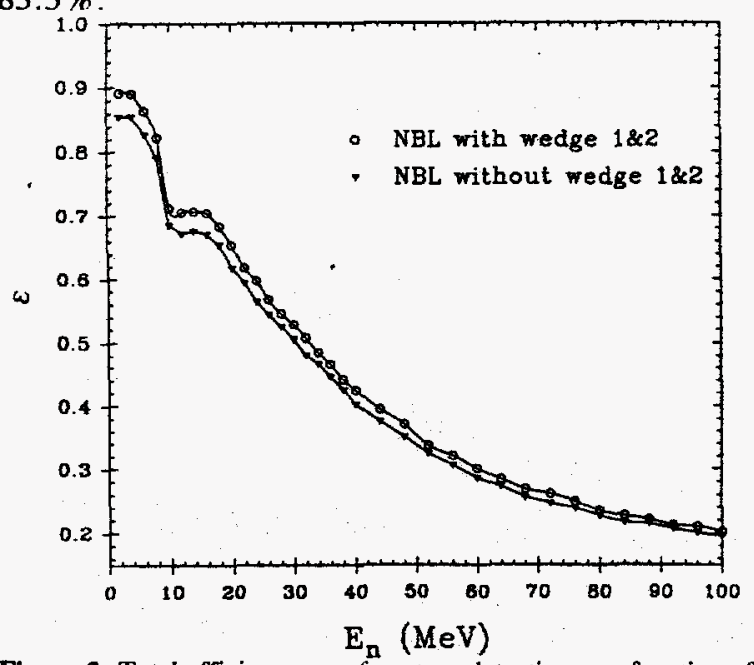

Figure 2. Total efficiency, $\epsilon$, of neutron detection as a function of laboratory kinetic energy, $E_{n}$, for monoenergetic neutrons emitted isotropically.

The above results refer to a geometry in which the ${ }^{252} \mathrm{Cf}$ fission fragments are emitted at the center of the ball in a direction perpendicular to the median plane. In this arrangement, the neutrons are expected to be isotropically distributed in the median plane.
The top of Fig. 3 shows the measured apparent neutron multiplicity distributions as a function of angle in the wedges on one side of the detector. These were determined using separate counting and background gates for each wedge. These are called apparent multiplicities because the same neutron capture can fire more than one detector due to inter-detector scattering of the capture $\gamma$ rays. The error bars given are statistical. The dashed curve is the calculated position of the neutron captures in the wedges. As expected both distributions are isotropic. However, the calculated values fall about $25 \%$ below the measured values due to neglect of $\gamma$-ray cross talk.

The $\gamma$-ray cross talk was incorporated into the calculations by keeping track of the total $\gamma$-ray energy deposited in each wedge. The detector was assumed to fire if the sum energy exceeded the experimental threshold requirement $(800 \mathrm{keV})$. The calculations predict more cross talk than actually observed. The solid curve shows the predicted distributions in the wedges after normalization by 0.64 . This discrepancy presumably arises because the simulation neglects $\gamma$-ray absorption in the walls of the scattering chamber, wedges, etc. Also, in a large detector the pulse height response is not completely uniform throughout the volume of the wedges. Thus, the total pulse height observed in a wedge is not strictly the sum of the contributions from individual photons. The middle and bottom panels of Fig. 3 show similar results for ${ }^{252} \mathrm{Cf}$ fission fragments which are emitted in the median plane either parallel or perpendicular to the beam axis, respectively. The solid curve gives the calculated multiplicities with cross talk normalized by the same factor mentioned above. The angular distributions in the wedges are attenuated compared to the primary neutron angular distribution due to the large angular acceptance of the wedges and inter-detector scattering. Nevertheless, there are still measurable differences between the wedge counters, implying that the neutron ball can be used to gather some neutron angular distribution information. 


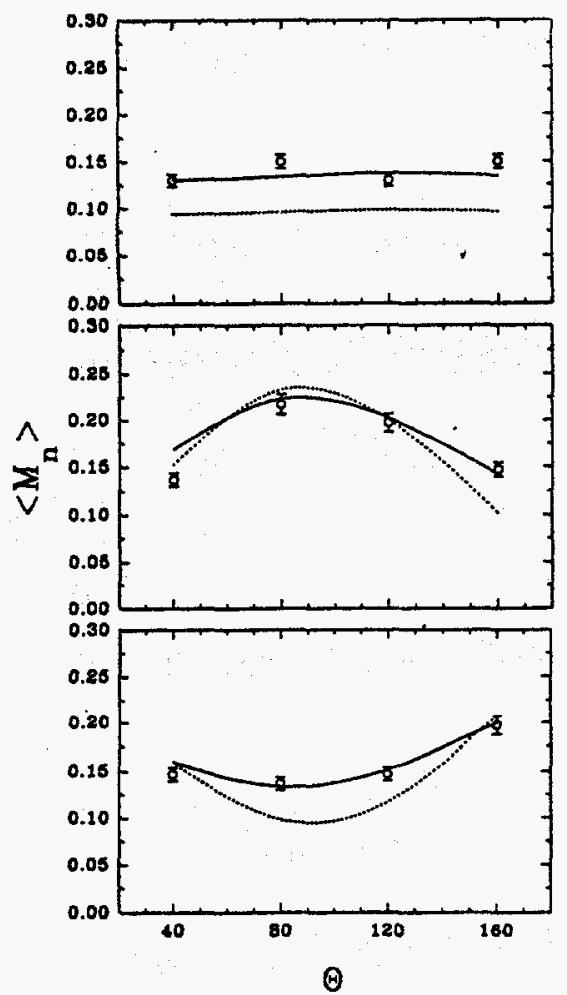

Figure 3. Comparison between measured and calculated neutron angular distributions in the median plane wedge for three different ${ }^{252} \mathrm{Cf}$ source orientations.

It is also interesting to note that the total efficiency for ${ }^{252} \mathrm{Cf}$ neutrons is weakly influenced by the orientation of the source. The total efficiency was measured and calculated for the three orientations mentioned above. The results are summarized in Table 1. As expected, the total efficiency decreases as the source is pointed parallel to the beam axis due to the loss of geometric efficiency with the forward most wedges removed.

In the case of heavy ion collisions, high temperatures and velocities can be realized by the recoiling system. This in turn gives rise to the production of more energetic neutrons than those produced during the spontaneous decay of ${ }^{252} \mathrm{Cf}$. Under these conditions the neutron ball can exhibit quite different detection efficiencies for the neutrons emitted from such sources. ${ }^{6}$ Figure 4 is a plot of the calculated neutron detection efficiency as a function of both source velocity and temperature. The neutron source is assumed to be traveling down the beam pipe again with the neutron ball's forward most wedges
Table 1. Measured and Calculated Total Detection Efficiency for Neutrons Emitted from ${ }^{252} \mathrm{Cf}$ for Three Source Orientations

\begin{tabular}{||l|c|c|}
\hline \multicolumn{1}{|c|}{$\begin{array}{c}\text { Source } \\
\text { orientation }\end{array}$} & $\begin{array}{c}\text { Measured } \\
\text { efficiency }\end{array}$ & $\begin{array}{c}\text { Calculated } \\
\text { efficiency }\end{array}$ \\
\hline $\begin{array}{l}\text { Perpendicular to } \\
\text { median plane }\end{array}$ & $83.5 \%$ & $84.9 \%$ \\
\hline $\begin{array}{l}\text { Parallel to beam } \\
\text { axis }\end{array}$ & $80.0 \%$ & $81.6 \%$ \\
\hline $\begin{array}{l}\text { Perpendicular to } \\
\text { beam axis }\end{array}$ & $82.7 \%$ & $85.3 \%$ \\
\hline
\end{tabular}

removed. From Fig. 4, it is evident that for a fixed temperature, the efficiency decreases with increasing source velocity. This is due mainly to the decreasing probability for thermalizing neutrons with larger kinetic energies. The dependence on temperature is more complex. The lowest efficiencies are seen for larger source velocities due to kinematic focusing. Neutrons emitted with low kinetic energies cannot overcome the forward velocity of the moving source and thus tends to be focused down the beam pipe. The focusing becomes less severe as the source temperature, and thus neutron kinetic energy, increases. This leads to an increase in the efficiency with temperature. For the lowest source velocities, the opposite trend is seen. In this case, there is little focusing, and one sees a decrease in efficiency with increasing temperature.

These simulations of the neutron ball response are being used to guide the analysis of a number of experiments. As described elsewhere, ${ }^{6}$ the estimated efficiencies seem to be fairly successful in explaining the behavior of the mean neutron multiplicities for a wide range of systems. In the future, the simulation could be coupled directly to reaction simulation programs.

\section{References}

1. J. Poitou and C. Signarbieux, Nucl. Instrum. Methods 114, 113 (1974). 
2. J. Strong et al., Nucl. Instrum. Methods 156, 411 (1978).

3. J. Galin, private communication (1993).

4. A. Del Guerra et al., Nucl. Instrum. Methods 135, 337 (1976).

5. H. R. Bowman, S. G. Thompson, J. C. D. Milton and W. J. Swiatecki, Phys. Rev. 126, 2120 (1962).

6. J. Boger et al., to be published.

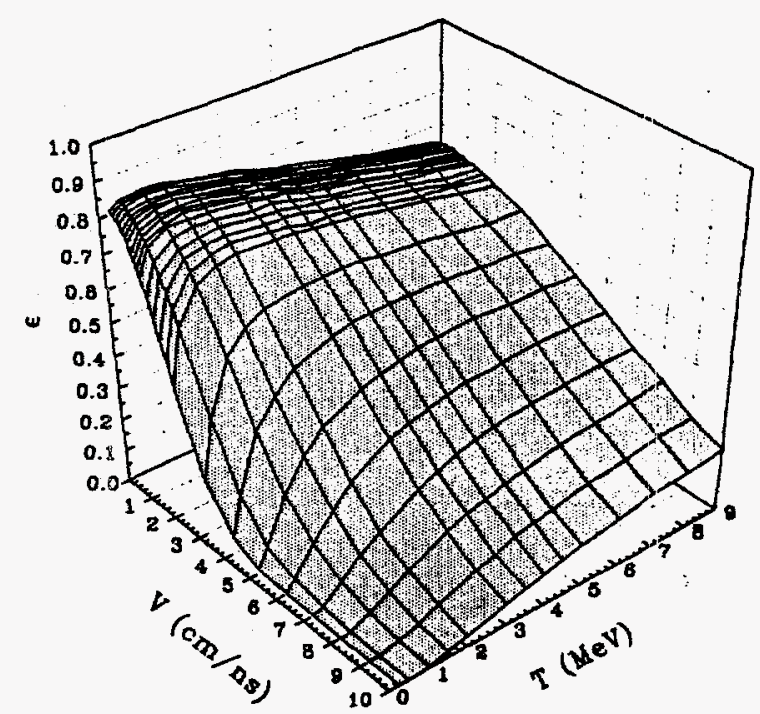

Figure 4. Calculated total efficiency, $\epsilon$, as a function of the source velocity, $\mathrm{V}$, and temperature, $\mathrm{T}$.

\title{
Running with the Neutron Ball $4 \pi$ Detector in a Fast Mode
}

\author{
F. Haddad, K. Hagel, J. Li, N. Mdeiwayeh, J. B. Natowitz, R. Tezkratt, D. Utley, R. Wada, \\ B. Xiao
}

The stability of the hot nucleus formed during heavy ion collisions remains one of the most important questions in nuclear physics. In particular, the limiting excitation energy that such nuclei can sustain is still an open problem.

Several studies have shown that neutron emission, due to the lack of the Coulomb barrier, is strongly related to the excitation energy reached by the compound system in a reaction. ${ }^{1}$ Several $4 \pi$ neutron detectors have been constructed, including the Neutron Ball (NBL) at the Cyclotron Institute. This detector allows a good determination of the emitted neutron multiplicities (the total efficiency is close to $85 \%$ for a ${ }^{252} \mathrm{Cf}$ source) and represents a powerful tool for energy deposition studies.
Unfortunately, the NBL is a slow detector $(\simeq 100$ $\mu \mathrm{s}$ ). This is mainly due to the neutron capture process (see Fig. 1).

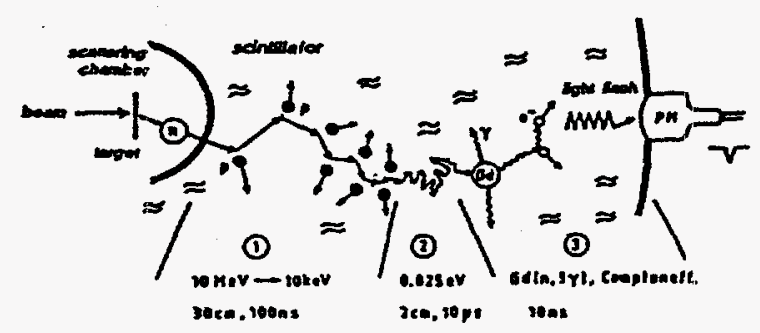

Figure 1. Principle of the Neutron Ball operation. Characteristic energies, lengths and times are given for the three steps in the process. ${ }^{1}$

The capture of a neutron can be split into three different stages. The first one takes place in about a 
hundred nanoseconds (ns). This thermalization stage consists of slowing down the neutron from about ten $\mathrm{MeV}$ to several $\mathrm{keV}$ by elastic scattering with the Hydrogen atoms present in the tank. This results in a fast light signal from the scintillator. The amplitude of this signal depends on the neutron kinetic energy loss. The fast signal also contains contributions from fast $\gamma$ decay processes and is often referred to as the $\gamma$ flash.

At low energy, the mean free path of the neutron increases. It takes $=10 \mu$ s before the capture of the neutron by Gadolinium. This constitutes the second part of the capture process.

In the last step, the Gadolinium nucleus emits three $\gamma$ rays. The observation of those $\gamma$ decays allows a precise determination of the neutron multiplicity.

In the regular use of the NBL, we often use the $\boldsymbol{\gamma}$-flash as a reaction trigger. When a large number of neutrons is emitted, i.e. in the most central collisions, we can expect the $\gamma$-flash to be correlated with the total neutron multiplicity. ${ }^{2}$

The study of this correlation between the $\gamma$-flash and the number of emitted neutrons was one of the goals of an experiment done the last week of March 1994. We studied the reaction of $15 \mathrm{MeV} / \mathrm{u}{ }^{129} \mathrm{Xe}$ with ${ }^{232} \mathrm{Th}$ and ${ }^{197} \mathrm{Au}$ targets. Our set up used the NBL $4 \pi$ detector, supplemented in the scattering chamber by different charged particle detectors (Ionization chambers, telescopes, hodoscopes and residue detectors).

In Fig. 2, the detected neutron number as a function of the $\gamma$-flash energy has been plotted for the ${ }^{197} \mathrm{Au}$ target. This spectrum has been recorded on line and no correction for the neutron background has been done. All the events have been triggered by one of two Ionization chambers located at $33^{\circ}$ and $90^{\circ}$ respectively. A quite linear correlation exists between the $\gamma$-flash and the detected neutron number.

It is then possible, after such a calibration of the $\gamma$-flash, to pick up the most central collisions by putting a high threshold on the $\gamma$-flash energy. This would allow us to reduce the neutron detection time from a few hundred $\mu$ s to a few hundred ns and, in

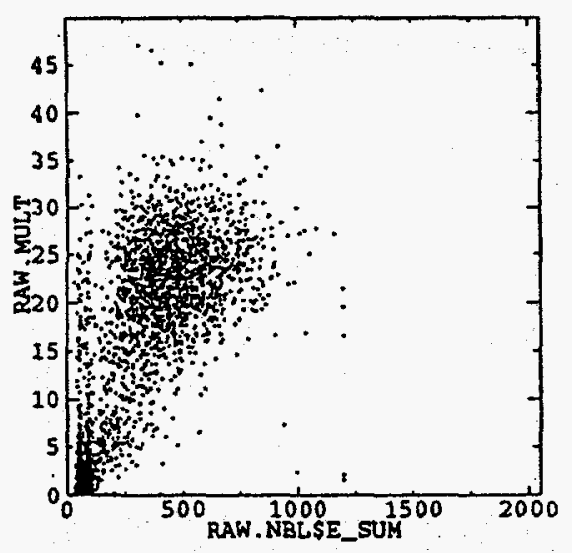

Figure 2. Detected neutron number as a function of the $\gamma$-flash energy for $15 \mathrm{MeV} / \mathrm{u}^{129} \mathrm{Xe}$ with ${ }^{197} \mathrm{Au}$ target.

combination with the beam sweeper, to work with much higher beam intensity. This will be useful for studying phenomena having small cross sections, allowing us a much more rapid data accumulation rate. The near future will be devoted to the precise analysis of these data in order to better understand the behavior of this $\gamma$-flash component of the NBL.

\section{References}

1. J. Galin et al., Proceedings of the Symposium on Hot Nuclei, Dallas, World Scientific, 241 (1988).

2. J. Galin, XXIst Summer School on Nuclear Physics MIKOLAJKI (Poland) August 26 September 5, 1990. 


\section{A New Method to Produce Plastic Scintillator Foils}

B. Xiao, S. Lee, K. Hagel, F. Haddad, J. Li, R. Tezkratt, R. Wada, D. Utley, and J. B. Natowitz

In recent years, a number of experiments performed on $4 \pi$ CsI-Plastic detector systems have shown that these relatively inexpensive detector systems are excellent tools to explore reaction mechanisms. The plastic scintillator foils used in these systems play a crucial role in identifying higher atomic number products. A good uniformity of the foils is the key to good charge resolution. In addition, to reduce the energy thresholds of the detectors, plastic foils, which are as thin as possible, should be used as long as they have reasonable light output.

The companies we have contacted during the construction of our CsI ball, could not provide products that are both very thin $\left(3 \mathrm{mg} / \mathrm{cm}^{2}-5\right.$ $\mathrm{mg} / \mathrm{cm}^{2}, 1 \mathrm{mg} / \mathrm{cm}^{2} \approx 10 \mu \mathrm{m}$ ) and have very good uniformity (less than 5 percent variation of the thickness in an area of $5 \mathrm{~cm} \times 5 \mathrm{~cm}$ ). We have successfully developed a new method of making and testing the plastic scintillator foils that meet our requirements.

We have adopted a traditional casting method of making thin film plastic scintillators. ${ }^{1}$ The basic idea of this method is that one can set up a leveling table with a glass plate. After the system is precisely leveled, one can simply pour the scintillator-solvent mixture on the surface of the glass. The gravity will cause the liquid to spread and it will stop at the point that the gravity and viscous forces are balanced.

In our work we have used a $45 \mathrm{~cm} \mathrm{x} 45 \mathrm{~cm} \mathrm{x}$ $0.5 \mathrm{~cm}$ normal glass plate resting on a $60 \mathrm{~cm} \times 60$ $\mathrm{cm} \times 2 \mathrm{~cm}$ square aluminum plate with a very uniform surface. The aluminum plate has height adjustment screws on the four corners. A precision level ( \pm 0.005 inch accuracy) is used to level the system. We used Bicron Organic Scintillator (BC498x Beta Paint) as the liquid material to make the plastic foils. The BC-498x is quite thick. To make thin foils, we added p-Xylene (from Fisher Scientific) as a solvent to the original $\mathrm{BC}-498 \mathrm{x}$ Beta Paint to reduce the viscosity. The ratio of $\mathrm{p}$-Xylene to $\mathrm{BC}$ $498 x$ depends on thickness of the foil desired. Table 1 shows several different ratios which we have used and the corresponding thicknesses of the foils.

Table 1. Different ratios of p-Xylene to BC-498x and corresponding foil thicknesses

\begin{tabular}{|c|c|c|c|c|c|}
\hline $\begin{array}{c}\text { Volume:p-Xylene/ } \\
\text { BC-498x }\end{array}$ & 1.0 & 1.5 & 3.0 & 4.5 & 6.0 \\
\hline Thickness $\left(\mathrm{mg} / \mathrm{cm}^{2}\right)$ & 123 & 73 & 40 & 28 & 7 \\
\hline
\end{tabular}

In early tests, we found it was very difficult to separate the plastic foils from the glass when the liquid is dry. Therefore we placed a mylar foil $(0.5$ $\mathrm{mg} / \mathrm{cm}^{2}$ ) on the surface of the glass. After the system is precisely leveled, we poured the well mixed liquid onto the mylar surface. Drying times of 2-4 days were required, depending on the viscosity of the mixture. By using this method, it was very easy to make large plastic foils and separate them from the mylar substrate.

Fourier Transform Infra-red Spectroscopy (FTIR) has been used to establish a thickness calibration and to measure the uniformity of the plastic foils. The FTIR instrument we used is the Galaxy 2000. Figure 1 shows a spectrum of absorbance vs wavenumber for a $1.4 \mathrm{mg} / \mathrm{cm}^{2}$ thick foil. We have made foils with thicknesses ranging from $0.8 \mathrm{mg} / \mathrm{cm}^{2}$ to $12 \mathrm{mg} / \mathrm{cm}^{2}$ and have used them to obtain a calibration curve with the FTIR. Figure 2 shows this calibration for 5 different wave numbers corresponding to the five sharpest peaks in Fig. 1. The thicknesses of the foils were obtained by weighing the samples of a known area with a microbalance ( $\pm 1 \mu \mathrm{g}$ ).

We chose a $40 \mathrm{~cm}$ diameter, $4 \mathrm{mg} / \mathrm{cm}^{2}$ foil to perform uniformity tests. Figure 3 shows the distribution of test points on the foil. Figure 4(a) shows the absolute absorbance for each test point 


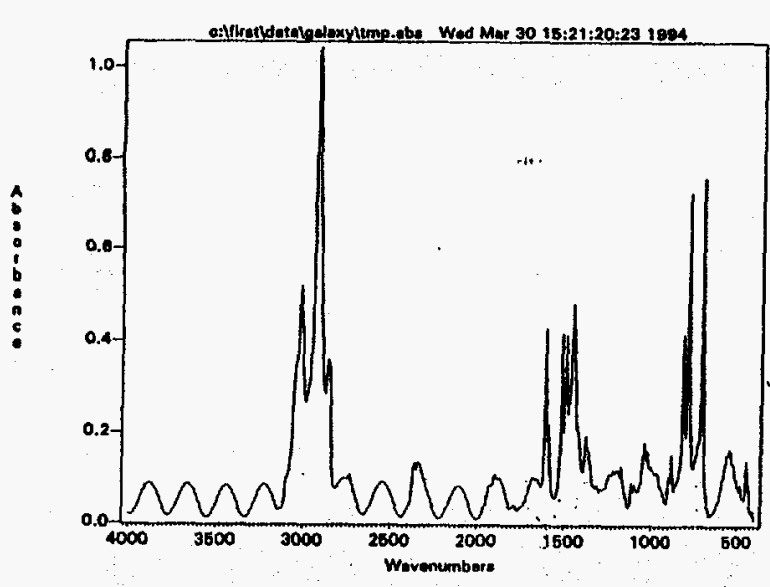

Figure 1. FTIR spectrum of absorbance vs wavenumber.

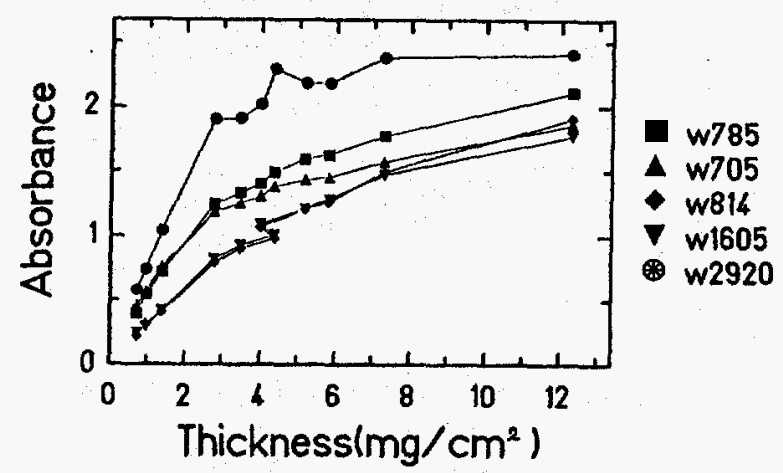

Figure 2. Thickness calibration curves for different wavenumbers.

along the $x$-direction and Fig. 4(b) shows relative uniformity derived from the absolute absorbance. One can see that the uniformity is quite good. The thickness variation is less than 3 percent in a range of $10 \mathrm{~cm}$ around any point. The thickness gradually becomes greater from one side to the other, apparently because the system was not perfectly level. In this case, it appears that the left side of the board was slightly higher than the right side. Measurements for the y-direction are shown in Figs. 5(a) and 5(b). The uniformity in the y-direction is as good as in the $x$-direction. From the trend of the thickness variation, one can see that there was a slight tilt such that the side labeled A in Fig. 3 was higher than that labeled $B$ when we leveled the system.

From these results, we conclude that we can successfully make large plastic foils which are very thin $\left(4 \mathrm{mg} / \mathrm{cm}^{2}\right)$ and have good uniformity.

\section{References}

1. Glatzhofer, Daniel T., Polymer-Chemist, University of Oklahoma, Private communication.

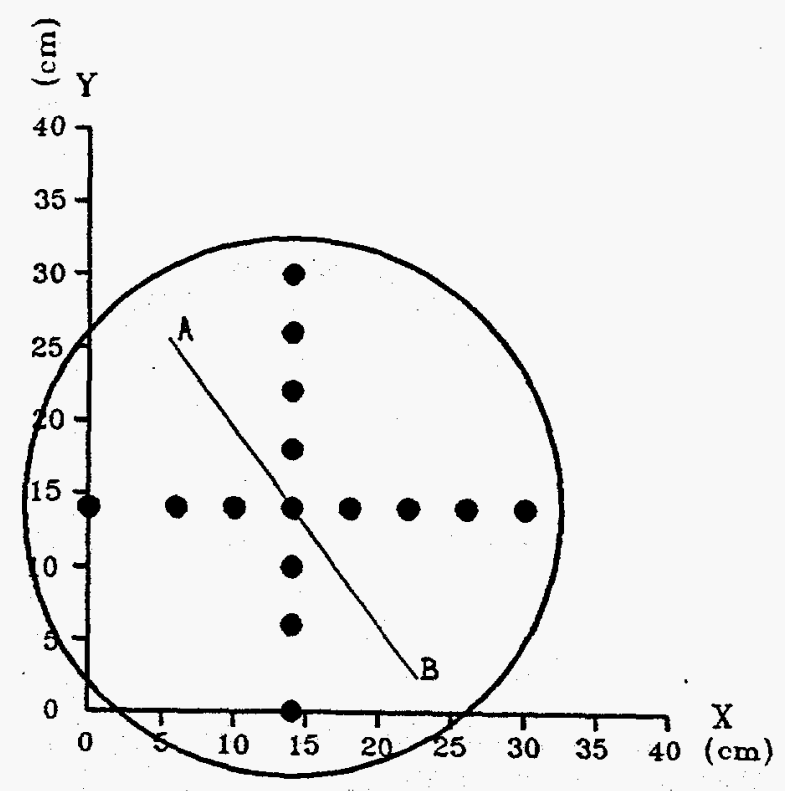

Figure 3. Distribution of test points on the foil. 


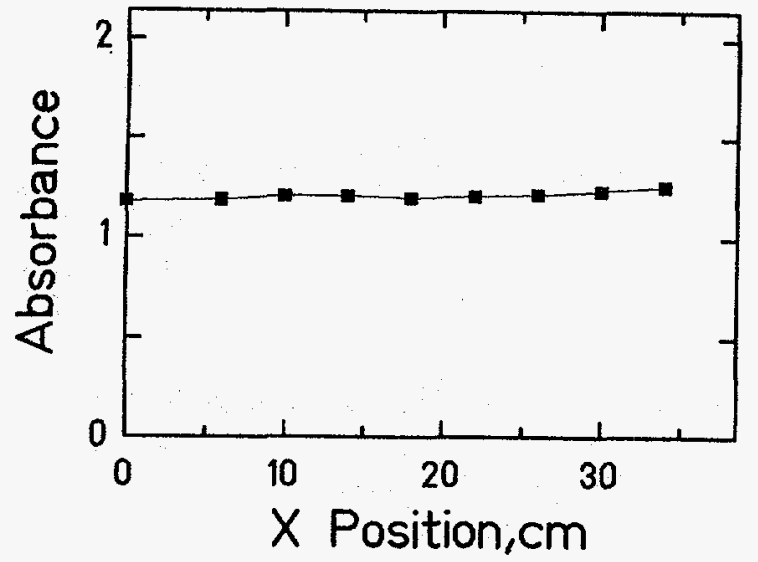

Figure 4(a). Absolute value of absorbance at $705 \mathrm{~cm}^{-1}$ for each test point along the $x$-direction.

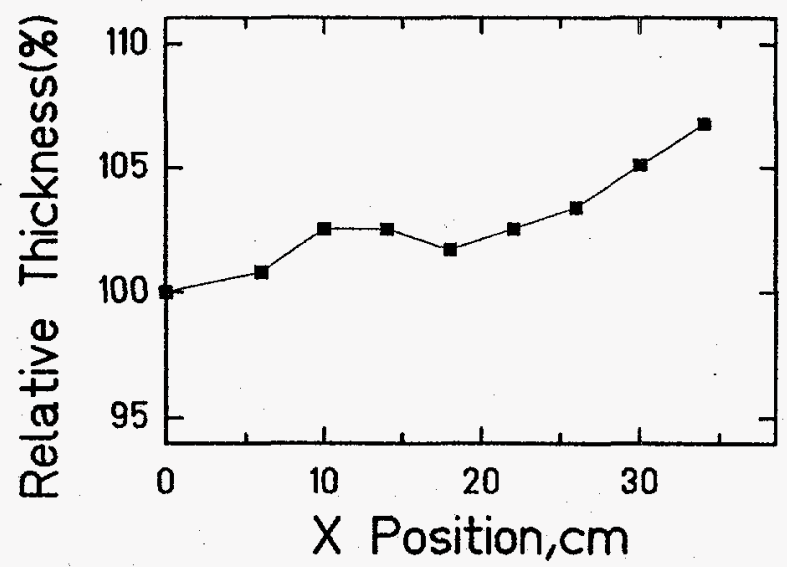

Figure $4(b)$. Relative uniformity in the $x$-direction.

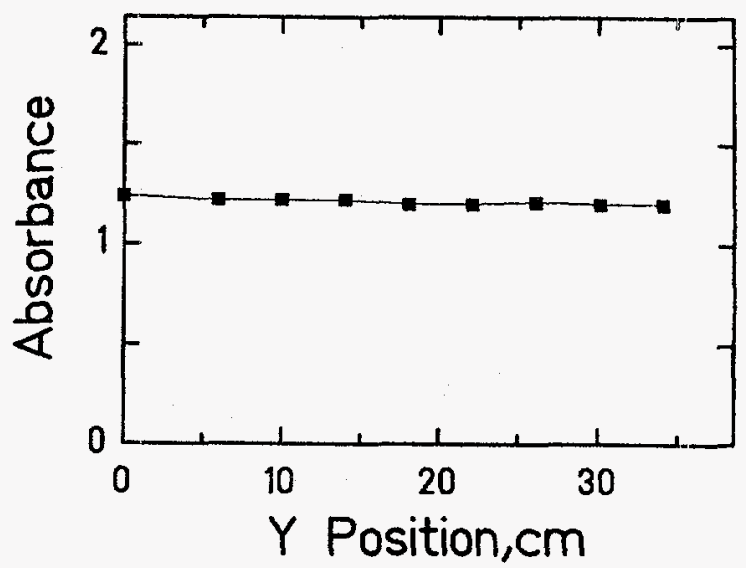

Figure 5(a). Absolute value of absorbance at $705 \mathrm{~cm}^{-1}$ for each test point along the $y$-direction.

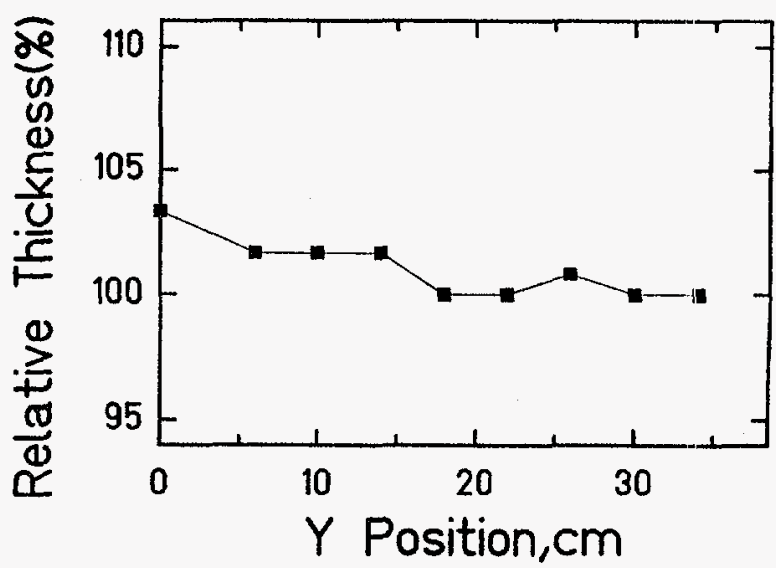

Figure 5(b). Relative uniformity in the $y$-direction. 


\section{Status of the CsI Ball}

B. Xiao, G. Derrig, K. Hagel, F. Haddad, S. Lee, J. Li, Y. Lou, N. Mdeiwayeh, R. Tezkratt, R. Wada, D. Utley, and J. B. Natowitz

Since last year's annual report, ${ }^{1}$ we have made progress on several aspects of our continued construction of the CsI ball. We briefly report this progress and the current status of the CsI ball.

To reduce the threshold of our detectors, we want to use plastic scintillator foils which are as thin as possible, as long as the foils have reasonable light output and good uniformity, which is the key to good charge resolution. The companies we have contacted, Bicron and Nuclear Enterprise, could not provide products that satisfied both of the above requirements at the same time, i.e., very thin (in the range between $30 \mu \mathrm{m}$ and $50 \mu \mathrm{m}$ ) and very good uniformity (less than 5 percent variation of the thickness in an area of $5 \mathrm{~cm} \times 5 \mathrm{~cm}$ ). We have successfully developed a method to make plastic foils that meet our requirements. The thickness of the foils we have made are from $10 \mu \mathrm{m}$ to $200 \mu \mathrm{m}$, and the thickness varies by less than 5 percent over a circular area with $40 \mathrm{~cm}$ diameter. More details about our method of making and testing these foils are presented elsewhere in this annual report.

Considerable effort has been made to construct the detectors. The light guides machined by an outside shop were not polished. To use thin foils to reduce energy thresholds, we need a high light transmission efficiency of the light guides. Our tests show that finely polished light guides can give 30-40 percent more transmission than the nonpolished ones. We hand polished all light guides for use in CsI ball.

Given the unusual shapes of our light guides and CsI crystals, we designed a special aluminum board to serve as a support frame to glue them together. GE RTV615 silicone rubber has been used as the glue. We also tried different wrapping methods to test the reflection efficiency. Our tests show that a combination of teflon tape, aluminum coated mylar and black tape around the CsI and light guide had no significant light collection difference compared to a teflon tape, black tape combination. The plastic foils were covered with aluminum coated mylar and attached to the CsI crystals to make plastic-CsI detector combinations.

Figure 1 shows the CsI ball, which consists of 95 detector elements (one detector of the $90^{\circ}$ ring is taken out to leave a space to insert the target holder), mounted in the vacuum chamber. The chamber also has 30 connectors for the silicon detectors, which will be used in the chamber as special trigger detectors or to provide easier calibration of the scintillator response.

Part of the electronics (including the fast bus), all the cable material and most of the connectors have already arrived in the Cyclotron Institute. We are presently fabricating some 2000 cables. The rest of the electronics and connectors are ordered and will arrive in the next three months. The target holder and the CsI ball support frame are nearly completed. We should be able to conduct experiments on this device in the fall.

\section{References}

1. B. Xiao et. al., Progress in Research, 19921993, Cyclotron Institute, TAMU, p. 144. 

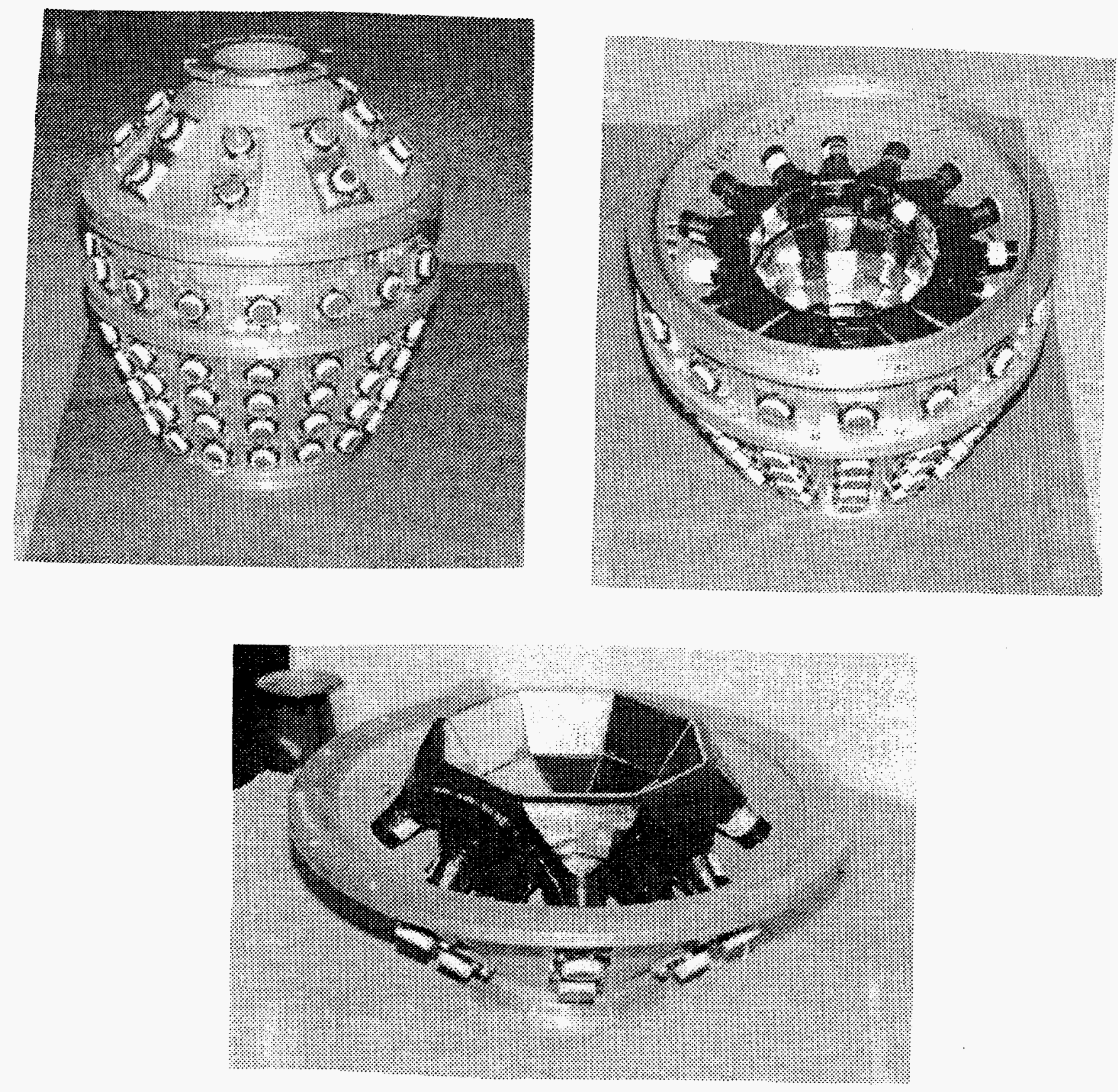

Figure 1. The $4 \pi$ CsI Ball. 


\title{
A Nearly Complete Detector System for Studying Heavy Ion Reactions
}

\author{
B. Xiao, G. Derrig, K. Hagel, F. Haddad, S. Lee, J. Li, Y. Lou, R. Tezkratt, R. Wada, D. Utley, \\ and J. B. Natowitz
}

To make more comprehensive studies of hot nuclei, we need a detector systcm which is capable of providing as complete information as possible on the decay of those hot nuclei. With the existing Texas A\&M $4 \pi$ Neutron Ball and the nearly completed CsI Ball, ${ }^{1}$ construction of a detection system which will allow nearly complete characterization of the intermediate system formed in individual nuclear collisions becomes possible.

Figure 1 shows a schematic design for such a system. To put the CsI ball inside the neutron ball, we need only to construct a set of new median plane wedges, and enlarge the gap between the upper and lower hemispheres. This will still allow the reconstructed neutron ball to provide nearly $4 \pi$ detection efficiency. Since the CsI ball only covers angles from $20^{\circ}$ to $170^{\circ}$ we propose to build a $\sim 120$ element charged particle detector forward wall which will provide high granularity in the forward $20^{\circ}$ cone. The forward wall will be placed inside a vacuum chamber which is connected to the CsI ball chamber. This system will provide us a excellent tool to explore the reaction mechanisms of complete and incomplete fusion reactions induced by heavy ion projectiles. More detailed design and tests of detectors for the forward array are now in progress.

\section{References}

1. B. Xiao et al., Progress in Research, 1991-1992, Cyclotron Institute, TAMU, p. 126; Progress in Research, 1992-1993, Cyclotron Institute, TAMU, p. 144.

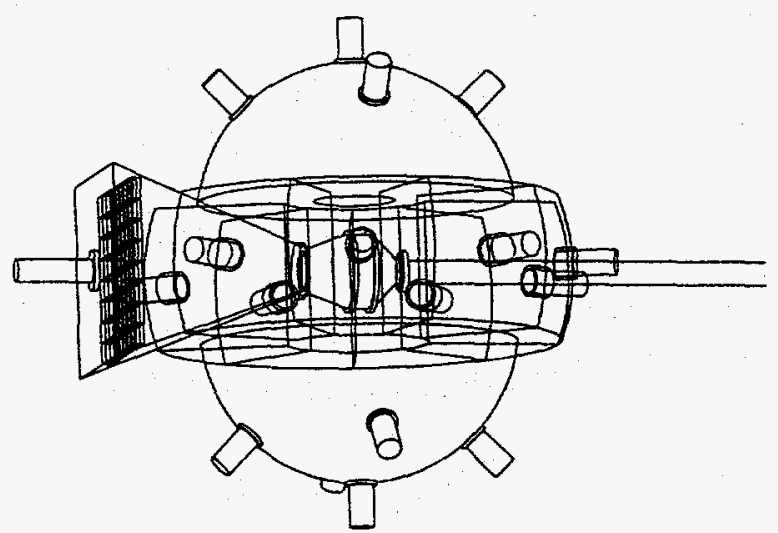

Figure 1. Design study for detector system with $4 \pi$ neutron ball, CsI hall and forward wall.

\section{An Angle Calibration Technique for Large Aperture Spectra}

\section{H. L. Clark, Y.-W. Lui, and D. H. Youngblood}

When using a large aperture spectrometer with ray tracing to determine scattering angle, a multi-slit collimator placed at the spectrometer aperture is normally used to obtain an angle calibration. Unlike energy spectra where discrete peaks provide a continuous check on the energy calibration, there are no well defined peaks in angle spectra to provide checks on angle calibration during normal data acquisition. Moreover some methods of angle measurement are susceptible to drift in absolute calibration, such as a vertical drift chamber (VDC) ${ }^{1}$ where the apparent angle of the ray is a function of the electron drift velocity.

Thus we have developed a technique which can reliably determine the effective position in each angle spectrum corresponding to the lowest angle defined by the solid angle determining collimator. This procedure can be used independently for each data 
run once the angle resolution function of the detector has been measured with a standard multi-slit collimator and an "idealized" distribution of the angle spectrum has been determined. The technique described below has proven to yield the effective slit edge reliably even for large spectrum shifts (Fig. 1).

Briefly, the technique is as follows: Both the angle resolution function of the detector and the spectrum calibration (in degrees per channel number) are determined by fitting the spectrum obtained with a multi-slit collimator, as illustrated in Fig. 2. The rest of the process uses an actual data spectrum from a run, such as the histogram in Fig. 3. A pseudo angular distribution is obtained by fitting the spectrum in the region well away from the actual slit edge (solid line in Fig. 3). This distribution is extrapolated to the assumed slit edges to produce an idealized distribution (dashed line in Fig. 4). The resolution function is then convoluted with the idealized distribution and the low angle aperture edge position iterated to produce the best fit to the actual data (solid line in Fig 4). By this procedure, the low angle aperture edge can be located to $\pm 0.04^{\circ}$.

The detector resolution and the angle spectrum calibration are determined by gaussian fits to the multi-slit collimator data (solid line in Fig. 2). For the MDM spectrometer, the angular magnification varies from approximately 2 to 3 over the $4^{\circ}$ horizontal aperture, therefore the peaks obtained with the multi-slit collimator have different widths and different position spacing (for a collimator with even angle spacing and slit openings). As a result, the angle calibration is obtained by a polynomial fit to the centroid channel numbers for the peaks. The top horizontal scale in Fig. 2 was obtained in this manner. The resolution function is determined by a polynomial fit to the corrected peak widths, as shown in Fig. 5. The corrected peak widths are obtained by subtracting the actual slit openings from the fullwidth-half-maximums determined by the gaussian fits $(2.354 * \sigma)$.

In order to model the angular distribution, a smoothed representation of the actual distribution was determined by fitting a second-order exponential to the angle spectrum. To reduce edge resolution effects in the pseudo distribution, only the region well away from the edges was included in the fit, as shown in Fig.3.

By extrapolating the pseudo distribulion to assumed slit positions, an idealized representation of the distribution, cut off by the collimator edges, is created as shown by the dashed line in Fig. 4. The validity of this position is tested by convoluting the idealized distribution with the detector resolution and then comparing the resultant shape to the actual data points through reduced- $\chi^{2}$. Before convoluting, the resolution function (expressed as a function of channel number) is appropriately shifted so that the assumed position corresponds to the actual angle defined by the multi-slitcollimator. This is necessary since the detector resolution is a function of angle, as illustrated by Fig. 5. Since the effective position is at first unknown, it is determined through an iterative process which steps over the range of possible positions. The routine begins at the low angle peak position and continues stepping positions until reduced $-\chi^{2}$ is minimized. Upon locating the correct position, the offset is removed and the spectrum is calibrated.

This technique was used to analyze large aperture data acquired with two different ray tracing detectors for a variety of reactions. The shifts were from different sources in the two detectors. In general the method was found to determine the aperture edges to within $\pm 0.04^{\circ}$ for both detectors.

\section{References}

1. W. Bertozzi, et al., Nucl. Instrum. Methods 141, 457 (1977). 


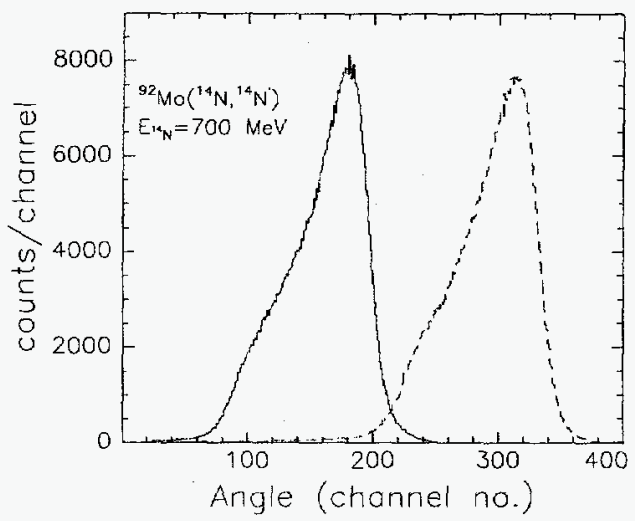

Figure 1. Plot of counts per channel versus angle in channel number for two successive data runs. The figure illustrates an extreme offset drift which could be corrected with the technique described. This data was taken with the multipole-dipole-multipole (MDM) spectrometer with an aperture having $\Delta \theta_{\mathrm{lab}}, \Delta \phi_{\mathrm{lah}}=2^{\circ}$ with central angles of $\theta_{\mathrm{lab}}=4^{\circ}$ and $\phi_{\mathrm{lab}}=0^{\circ}$.

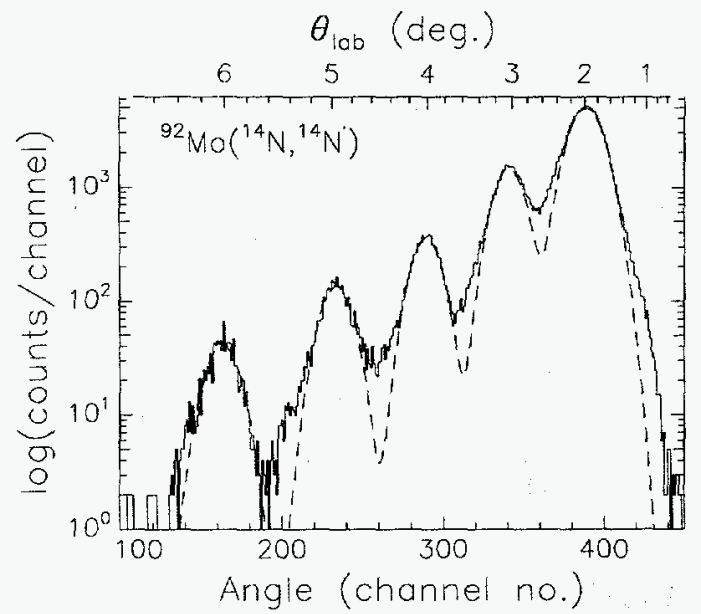

Figure 2. Semi-log plot of counts per channel versus angle for a 5 slit collimator taken in the MDM Spectrometer. The slit openings were spaced $1.02^{\circ}$ apart and were $0.12^{\circ}$ wide. The spectrometer was positioned such that the center slit was at $\theta_{1 \mathrm{ab}}=4^{\circ}$. The dashed line is a multi-gaussian fit as described in the text.

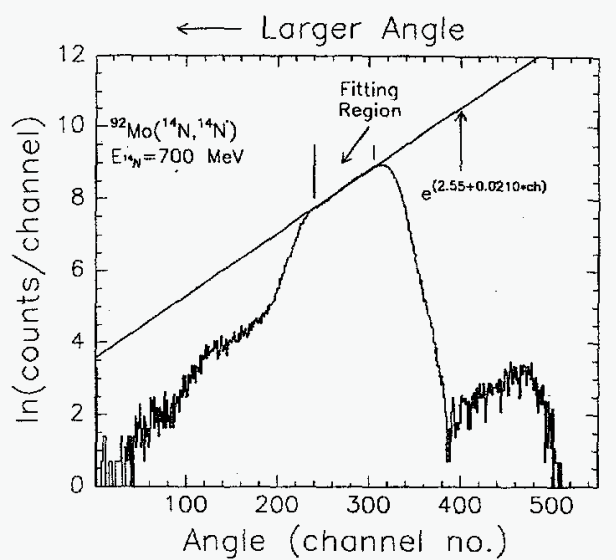

Figure 3. Semi-log plot of counts per channel versus angle. The line is a plot of the function obtained by a fit to the region indicated. The fit procedure is described in the text. This data was taken with the MDM spectrometer with an aperture having $\Delta \theta_{\mathrm{lab}}, \Delta \phi_{\mathrm{lab}}=2^{\circ}$ and with central angles of $\theta_{\text {lab }}=4^{\circ}$ and $\phi_{\text {tab }}=0^{\circ}$.

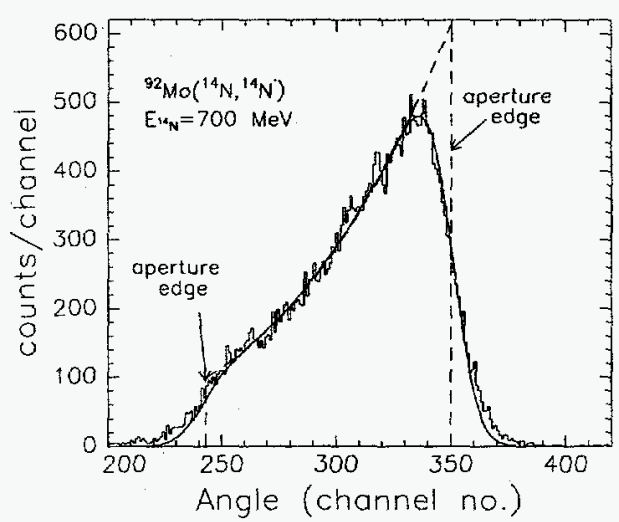

Figure 4. Plot of counts per channel versus angle. The dashed line represents the idealized distribution and the solid line is the result of the idealized distribution convoluted by the detector resolution. This data was taken with the MDM spectrometer with an aperture having $\Delta \theta_{\text {labs }}$, $\Delta \phi_{\mathrm{tab}}=2^{\circ}$ with central angles at $\theta_{\mathrm{lab}}=4^{\circ}$ and $\phi_{\mathrm{lab}}=0^{\circ}$. 


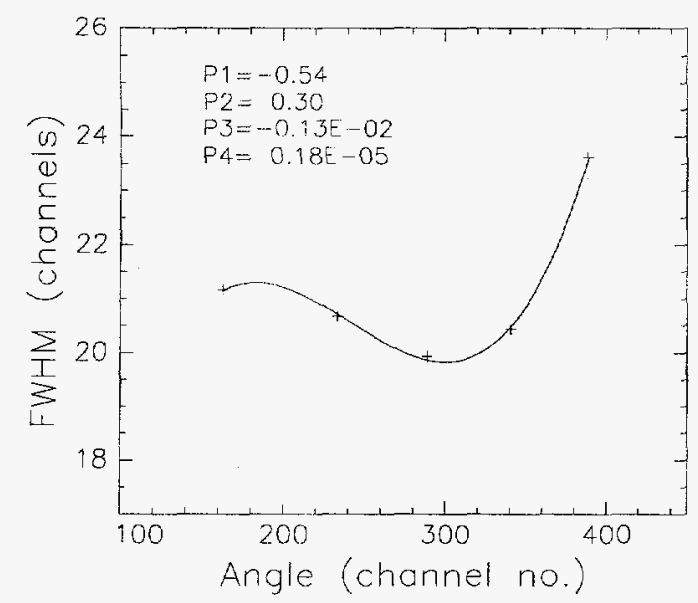

Figure 5. Plot of detector resolution as a function of angle. The widths $(+)$ were determined by the multi-gaussian fit to the multi-slit collimator spectra. The line is a polynomial fit to the widths defined by the equation $\mathrm{P} 1+\mathrm{P} 2{ }^{*} \mathrm{ch}+\mathrm{P}^{*} \mathrm{ch}^{2}+\mathrm{P}^{*} \mathrm{ch}^{3}$.

\section{Design of a Position Sensitive PPAC}

\section{R. Parameswaran and R. Gutierrez}

A position sensitive Parallel Plate Avalanche Counter (PPAC) has been developed as a particle detector for heavy ions. The present work was motivated by the need to have an efficient particle detector that can accommodate high counting rates of heavy ions. Furthermore it was necessary to have a detector that yielded particle timing signals and position information. Finally ease of use, ruggedness and reasonable cost of the detector were also important considerations in our effort in developing the PPAC described below. The PPAC was tested with fast $\mathrm{Xe}$ ions and its preliminary performance is compared with a position sensitive detector consisting of a microchannel plate with resistive anode (referred to as MCP henceforth).

The design of the PPAC detector closely follows the design of Gaulker et al. ${ }^{1}$ and is shown in Fig. 1. The heavy ions enter the PPAC through an aluminized mylar foil $200 \mu \mathrm{g} / \mathrm{cm}^{2}$ thick. This is the vacuum foil which separates the PPAC from the high vacuum of the experimental chamber. Next to this foil is a grid. A strong uniform electric field is maintained between the grid which is held at ground potential and the anode. A field of about $4000 \mathrm{~V} / \mathrm{cm}$ was applied during the test of the PPAC. A positive high voltage was applied to the anode through a load resistor of $1 \mathrm{M} \Omega$. A gas pressure of about 5 Torr of isobutane gas inside the PPAC was found to be optimal. Ions that enter the PPAC produce secondary electrons which are accelerated in the strong field between the grid and the anode thereby causing Townsend avalanches and a resulting fast potential change at the anode. This signal is fed into a fast preamplifier via a coupling capacitor of $10 \mathrm{pF}$.

The anode consists of two interleaving $\mathrm{Cu}$-wedge patterns (referred to as the top and bottom anodes here) etched on a printed circuit board $(5 \mathrm{~cm}$ long by $2 \mathrm{~cm}$ wide) and is the main element of the PPAC (Fig. 1). The wedges are $1 \mathrm{~mm}$ wide at one end and narrow down over a distance of $4.6 \mathrm{~cm}$. 


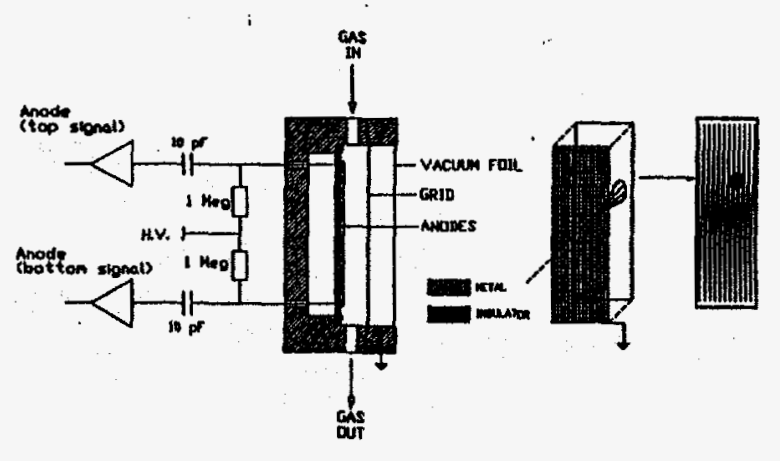

Figure 1. PPAC schematic.

The separation between any two parallel wedges is $0.2 \mathrm{~mm}$. This corresponds to an active area of about $80 \%$ of the anode area for the electron avalanche resulting from the passage of an ion. The split-anode was designed using the ACAD 12.0 software package. The drawing was used to obtain a photo negative to be used in the etching process on a printed circuit board. The design of the anode results in a position-dependent splitting of the electron avalanche signal along the length of the PPAC detector. The signals extracted from the top and bottom anodes were fed to a position sensitive detector analyzer (PSDA) after suitable pulse shaping. The particle position signals from the PSDA were then sent to a PC-based pulse height analyzer (PHA) to generate the particle position spectrum.

The experiment was performed using a beam of $8 \mathrm{MeV} / \mathrm{amu} \mathrm{Xe}^{21+}$ obtained from the $\mathrm{K} 500$ superconducting Cyclotron at Texas A\&M University and charge state distributions of the ions after passing through Carbon foils of different thickness were studied. The different charge states were dispersed using a magnet prior to detection. A comparison of the distribution obtained with the MCP detector and the PPAC is shown in Fig. 2 for Carbon foils of

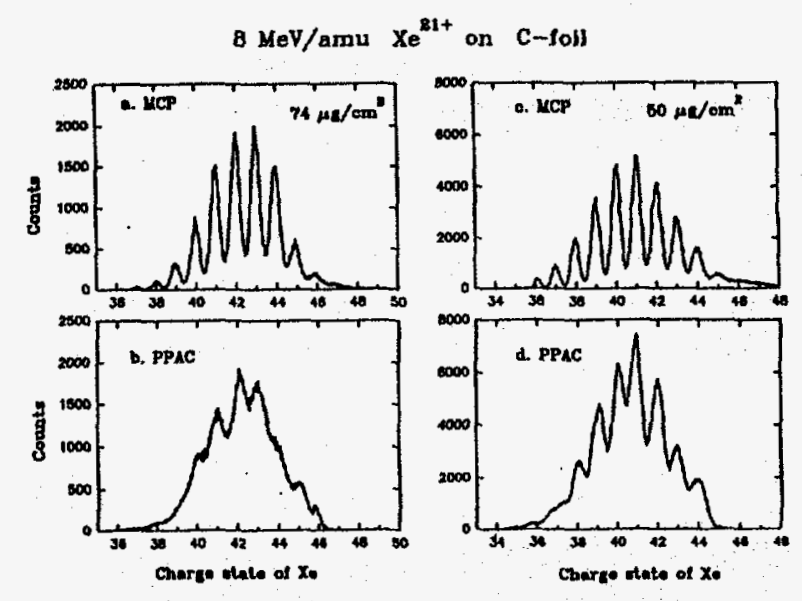

Figure 2. Position spectrum: Distribution comparison of MCP and PPAC for charge state range $\mathrm{Xe}^{36+}$ to $\mathrm{Xe}^{48+}$.

thicknesses 50 and $74 \mu \mathrm{g} / \mathrm{cm}^{2}$. At these thicknesses charge state equilibrium has still not been reached, and the various charge states of Xe exiting the foils were well resolved from each other. As seen in Fig. 2, the charge states range from $\mathrm{Xe}^{36+}$ to $\mathrm{Xe}^{48+}$ and are dispersed over a distance of about $4 \mathrm{~cm}$ along the length of the detector. With the $50 \mu \mathrm{g} / \mathrm{cm}^{2}$ there is a slightly better separation of the charge states. As seen for both cases, position resolution of the PPAC is poorer than that of the MCP detector under identical conditions.

The main reason for this might be due to the way the electron avalanche signal is split between the two anodes in the case of the PPAC and in the way the electron shower coming through the channels of the MCP is split in the resistive anode in the case of the MCP detector. With the PPAC, for the same physical separation of the $\mathrm{Xe}$ ion charge states the actual spectrum obtained on the pulse height analyzer gets contracted and spread over fewer PHA channels which results in poorer resolution. Either a longer PPAC or a modified design of the anode would help in improving the PPAC resolution. The timing signals from the PPAC after amplification in a fast amplifier were observed to have a risetime of about 20 nsec. 
In summary, we have developed an inexpensive detector that can be used as an efficient detector for fast heavy ions. An anode consisting of two interleaving $\mathrm{Cu}$-wedge split-anodes can be used for extracting position signals. The PPAC detector is also capable of generating timing signals and can be used as an effective projectile detector in coincidence experiments. With a simple modification of the anode design it is also possible to obtain two-dimensional projectile position information which can be useful in experiments where an impact parameter dependent study of the collision is desired, for example.
The complete detector was fabricated locally and the authors want to thank the mechanical shop at the Cyclotron Institute for the excellent workmanship. RG would like to thank the Electrical Engineering Department, Texas A\&M University for help with the etching of the anode pattern on the PC-board.

\section{References}

1. G. Gaulker, H. Schmidt-Bocking, R. Schuch, R. Schule, H.J. Specht and I. Tserruya, Nucl. Instrum. Methods 141, 115 (1977). 


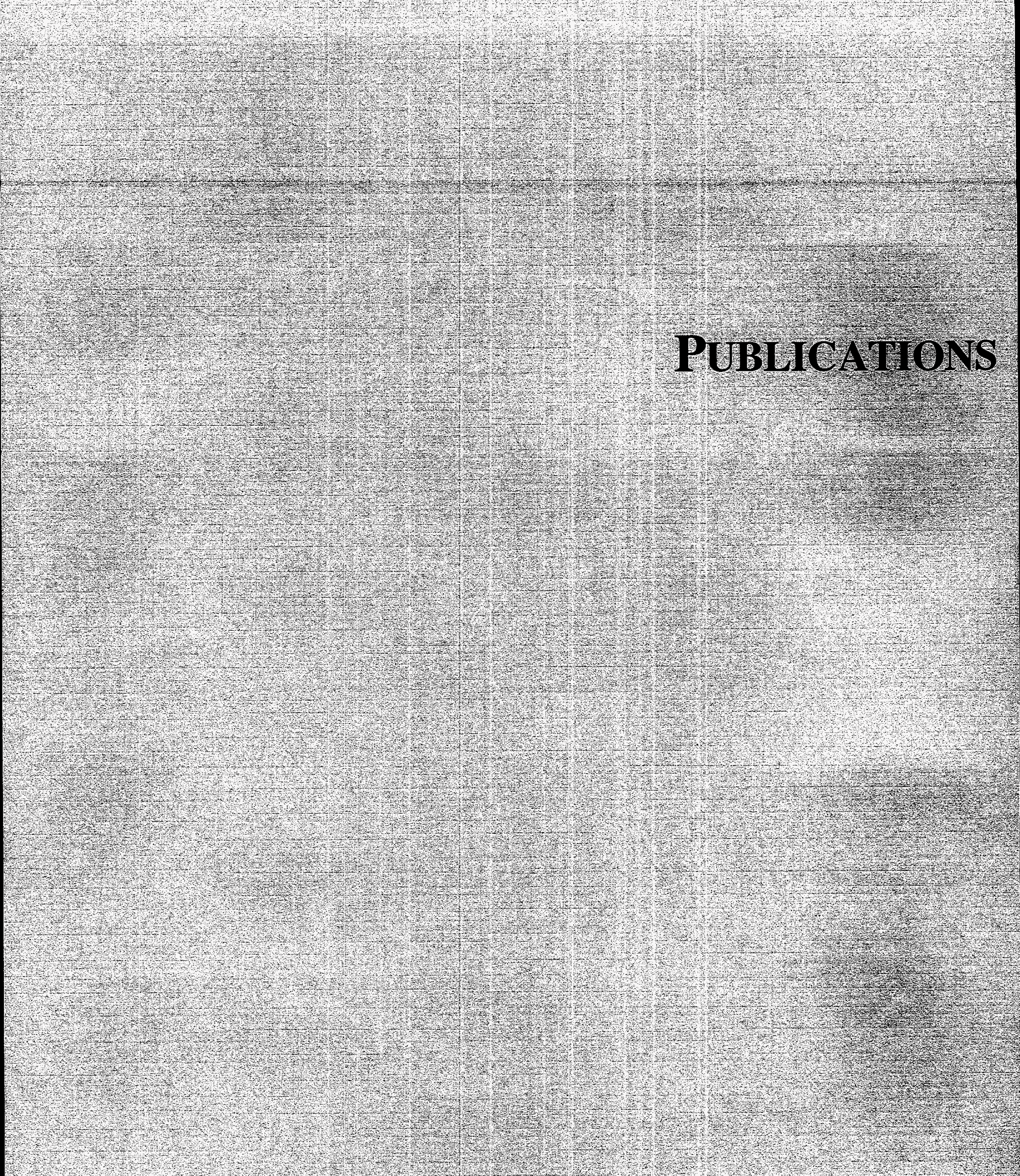




\title{
ABSTRACTS OF PAPERS PUBLISHED
}

\author{
April 1, 1993 - March 31, 1994
}

\section{SEEING THE QCD PHASE TRANSITION WITH PHI MESONS}

\author{
M. Asakawa and C. M. Ko \\ Phys. Lett. 322B, 33 (February, 1994)
}

A double phi peak structure in the dilepton invariant mass spectrum from ultrarelativistic heavy ion collisions is proposed as a signal for the phase transition from the quark-gluon plasma to the hadronic matter. The low mass phi peak results from the decay of phi mesons with reduced in-medium mass during the transition. Furthermore, the measurement of the transverse momentum distribution of these low mass phi mesons offers a viable means for determining the temperature of the phase transition.

\section{WHAT WE CAN LEARN FROM A SECOND PHI MESON PEAK IN ULTRARELATIVISTIC NUCLEAR COLLISIONS?}

C. M. Ko and D. Seibert

Phys. Rev. C 49, 2198 (April, 1994)

The decay width of a phi meson is reduced from its vacuum value as its mass decreases in hot hadronic matter as a result of the partial restoration of chiral symmetry. This reduction is, however, cancelled by collisional broadening through the reactions $\phi \pi \rightarrow K K^{*}, \phi K \rightarrow \phi K, \phi \rho \rightarrow K K$ and $\phi \phi \rightarrow K K$. The resulting phi mesons width in hot hadronic matter is found to be less than about 10 $\mathrm{MeV}$ for temperatures below $200 \mathrm{MeV}$. If hadronic matter has a strong first-order phase transition, this narrow phi meson with reduced mass will appear as a second peak in the dilepton spectrum in ultrarelativistic heavy-ion collisions. We discuss use of this second phi peak to determine the transition temperature and the lifetime of the two-phase coexistence region in the case of a strong first-order phase transition. We also discuss using the peak to determine the range of temperatures over which the transition occurs in the case of a smooth but fast change in entropy density.

\section{SUBTHRESHOLD ANTIPROTON PRODUCTION IN NUCLEUS-NUCLEUS COLLISIONS}

$$
\begin{aligned}
& \text { G. Q. Li, C. M. Ko, X. S. Fang, and Y. M. } \\
& \text { Zheng }
\end{aligned}
$$

Phys. Rev. C 49, 1139 (February, 1994)

Antiproton production in nucleus-nucleus collisions at energies below the threshold for its production from the nucleon-nucleon interaction in free space is studied in the Vlasov-Uehling-Uhlenbeck model. The antiproton self-energies in the medium are obtained in the mean-field approximation from the G-parity transformation of the nucleon self-energies. Due to the decrease of antiproton mass in the medium as a result of the attractive scalar mean field, there is an enhanced production of primordial antiprotons which are, however, mostly annihilated by nucleons as they propagate through the matter. The calculated final antiproton momentum spectrum is seen to agree reasonably with the experimental data.

\section{MEDIUM EFFECTS ON SUBTHRESHOLD KAON PRODUCTION FROM HEAVY-ION COLLISIONS}

\author{
X. S. Fang, C. M. Ko, G. Q. Li, and Y. M. \\ Zheng \\ Phys. Rev. C 49, R608 (February, 1994)
}

The relativistic transport model is extended to include the kaon degree of freedom. We also take into account the density dependence of the kaon effective mass in nuclear matter and the rescattering of kaons by nucleons. We find that the inclusion of kaon self-energy due to the attractive scalar mean field leads to an enhanced kaon yield in heavy-ion collisions at subthreshold energies. Also, kaon rescatterings are found to affect significantly the final kaon momentum spectra. We compare the theoretical results with recent experimental data from the HeavyIon Synchrontron (SIS) at GSI.

\section{DEUTERON ELASTIC SCATTERING AT 110 AND $120 \mathrm{MeV}$}
A. C. Betker, C. A. Gagliardi, D. R. Semon, R. E. Tribble, H. M. Xu, and A. F. Zaruba Phys. Rev. C 48, 2085 (October, 1993) 
Deuteron elastic scattering cross sections have been measured at 110 and $120 \mathrm{MeV}$ on $\mathrm{C}$, ${ }^{58} \mathrm{Ni}$, and ${ }^{208} \mathrm{~Pb}$. Optical model potentials have been extracted and compared to deuteron global optical model potentials.

\section{INTERCALIBRATION OF ${ }^{4} \mathrm{He}$ AND ${ }^{5} \mathrm{Li}$ NUCLEAR THERMOMETERS FOR HOT NUCLEI}

\section{J. B. Natowitz, J. C. Hagel, R. Wada, X. Bin, J. $\mathrm{Li}$, Y. Lou, and D. Utley}

Phys. Rev. C 48, 2074 (October, 1993)

For hot nuclei with $A \simeq 120$, a comparison is made between nuclear temperatures derived from spectral slope parameters for well-characterized equilibrated systems and those derived from state population ratios. When suitable account is taken of the deexcitation cascade, the two techniques lead to similar results, confirming the utility of the state ratio method. The general relationship between the measured quantities and the initial temperature for medium to heavy mass hot nuclei is discussed and a calibration of these is presented for measurements based on ${ }^{4} \mathrm{He}$ and ${ }^{5} \mathrm{Li}$ detection.

\section{NUCLEAR STRUCTURE STUDY OF THE ODD-A TC ISOTOPES WITHIN THE NEUTRON- PROTON INTERACTING BOSON-FERMION MODEL}

H. Dejbakhsh and S. Shlomo

Phys. Rev. C 48, 1695 (October, 1993)

We have investigated the odd-A Tc isotopes in the mass 100 region within the neutron-proton interacting boson-fermion model (IBFM-2). The calculation is based on the validity of the $\mathrm{Z}=38$ subshell closure. The results for the energy spectra and electromagnetic transitions are compared with experimental data and with results from other calculations which considered $Z=50$ as a valid closed shell for Tc isotopes.

MASS ASYMMETRY DEPENDENCE OF SCISSION TIMES IN THE REACTIONS OF 18.5 A MeV ${ }^{136} \mathrm{Xe}+{ }^{48} \mathrm{Ti}$

M. Gui, K. Hagel, R. Wada, Y. Lou, D. Utley, B. Xiao, J. Li, J. B. Natowitz, G. Enders, W. Kühn,
V. Metag, R. Novotny, O. Schwalb, R. J. Charity, R. Freifelder, A. Gobbi, W. Henning, K. D. Hildenbrand, R. Mayer, R. S. Simon, J. P. Wessels, G. Casini, A. Olmi, and A. A. Stefanini Phys. Rev. C 48, 1791 (October, 1993)

The multiplicities of $p$ and $a$ particles detected in coincidence with fragments emitted in fully relaxed collisions in the reactions of $18.5 \mathrm{~A} \mathrm{MeV}{ }^{136} \mathrm{Xe}+$ ${ }^{48} \mathrm{Ti}$ have been measured for different exit channel mass asymmetries. A kinematic source analysis of the spectra and angular distributions of the light particles has been used to separate the total multiplicities into prescission and postscission contributions. From these results, the excitation energies at scission are determined using an empirical technique based upon previous measurements of light charged particle multiplicities observed in coincidence with evaporation residues. These excitation energies are found to decrease from -400 $\mathrm{MeV}$ to $110 \mathrm{MeV}$ as the fragment mass asymmetry, $\mathrm{A}_{\mathrm{H}} / \mathrm{A}_{\mathrm{L}}$, varies from 4.8 to 1.0 . A corresponding increase of the mean lifetime of the scissioning nucleus from $\sim 5 \times 10^{-22} \mathrm{~s}$ to $\sim 1 \times 10^{-20} \mathrm{~s}$ is derived using calculated statistical model decay widths. The extent to which this variation of lifetime with mass asymmetry may be attributed to completely damped deep inelastic collisions or to dynamic delays in the decay of a compound nucleus is discussed as is the need for inclusion of dynamics in the deexcitation calculations for hot nuclei. Observed three fragment events are also discussed.

\section{EMISSION TEMPERATURES FROM WIDELY SEPARATED STATES IN ${ }^{14} \mathrm{~N}$ - AND ${ }^{129} \mathrm{Xe}$ - INDUCED REACTIONS}

C. Schwarz, W. G. Gong, N. Carlin, C. K. Gelbke, Y. D. Kim, W. G. Lynch, T. Murakarni, G. Poggi, R. T. de Souza, M. B. Tsang, H. M. Xu, D. E. Fields, K. Kwiatkowski, V. E. Viola, Jr., and S. J. Yennello Phys. Rev. C 48, 676 (August, 1993)

Charged-particle correlations were measured for the reactions ${ }^{14} \mathrm{~N}+{ }^{197} \mathrm{Au}$ and ${ }^{14} \mathrm{~N}+{ }^{27} \mathrm{Al}$ at $\mathrm{E} / \mathrm{A}=$ $75 \mathrm{MeV}$ and for ${ }^{129} \mathrm{Xe}+{ }^{27} \mathrm{Al}$ and ${ }^{129} \mathrm{Xe}+{ }^{122} \mathrm{Sn}$ at $\mathrm{E} / \mathrm{A}=31 \mathrm{MeV}$. Relative populations of widely separated states in ${ }^{4} \mathrm{He},{ }^{5} \mathrm{Li}$, and ${ }^{8} \mathrm{Be}$, only weakly affected by side feeding, indicate emission temperatures of $\mathrm{T}=4-5 \mathrm{MeV}$ for the ${ }^{14} \mathrm{~N}$-induced reactions and values about $1 \mathrm{MeV}$ lower for the ${ }^{129} \mathrm{Xe}$-induced reactions. 


\section{FORMATION AND DECAY OF TOROIDAL AND BUBBLE NUCLEI AND THE NUCLEAR EQUATION OF STATE}

H. M. Xu, J. B. Natowitz, C. A. Gagliardi, R. E. Tribble, C. Y. Wong, and W. G. Lynch Phys. Rev. C 48, 933 (August, 1993)

Multifragmentation, following the formation of toroidal and bubble nuclei is observed with an improved BUU model for central ${ }^{92} \mathrm{Mo}+{ }^{92} \mathrm{Mo}$ collisions. With a stiff equation of state, simultaneous explosion into several intermediate mass fragments (IMF) in a ring-like manner occurs due to the formation of metastable toroidal nuclei. In contrast, with a soft equation of state, simultaneous explosion into several IMF's in a volume-like manner occurs due to the formation of metastable bubble nuclei. Experimental signatures for the formation of these exotic shapes are discussed.

\section{MEASUREMENT OF TRANSVERSE ENERGY PRODUCTION WITH Si AND Au BEAMS AT RELATIVISTIC ENERGY: TOWARDS HOT AND DENSE HADRONIC MATTER}

J. Barrette, R. Bellwied, S. Bennett, P. BraunMunzinger, W. E. Cleland, T. M. Cormier, G. David, J. Dee, G. E. Diebold, O. Dietzsch, D. Fox, J. V. Germani, S. Gilbert, S. V. Greene, J. R. Hall, T. K. Hemmick, N. Herrmann, B. Hong, K. Jayananda, D. Kraus, B. Shiva Kumar, R. Lacasse, Q. Li, D. Lissauer, W. J. Llope, T. W. Ludlam, S. McCorkle, R. Majka, S. K. Mark, R. Matheus, J. T. Mitchell, M. Muthuswamy, E. O'Brien, S. Panitkin, C. Pruneau, M. N. Rao, M. Rosati, F. Rotondo, N. C. daSilva, J. Simon-Gillo, U. Sonnadara, J. Stachel, J. Sullivan, H. Takai, E. M. Takagui, T. G. Throwe, C. Winter, G. Wang, K. L. Wolf, D. Wolfe, C. L. Woody, N. Xu, Y. Zhang, Z. Zhang, and C. Zou Phys. Rev. Lett. 70, 2996 (May, 1993)

We present a systematic study of transverse energy $\left(\mathrm{E}_{\mathrm{T}}\right)$ production in collisions of $11.4 \mathrm{~A} \mathrm{GeV} / \mathrm{c} \mathrm{Au}$ and 14.6A GeV/c Si ions with targets of $\mathrm{Al}, \mathrm{Au}$, and $\mathrm{Pb}$. Comparison of data for $\mathrm{Au}+\mathrm{Au}$ and $\mathrm{Si}+\mathrm{Al}$ indicates that, for the heavier system, there is an increase in the amount of stopping which is accompanied by a decrease in the width of the $\mathrm{dE}_{\mathrm{T}} / \mathrm{d} \eta$ distribution. The ratio of the maximum $\mathrm{E}_{T}$ observed for the two systems is significantly greater than the ratio of the total energy available in the center of mass frame.

\section{THE USE OF RADIOACTIVE NUCLEAR BEAMS TO STUDY THE EQUILIBRATION OF THE N/Z DEGREE OF FREEDOM IN INTERMEDIATE-ENERGY HEAVY-ION REACTIONS}

S. J. Yennello, B. Young, J. Yee, J. A. Winger, J. S. Winfield, G. D. Westfall, A. Vander Molen, B. M. Sherrill, J. Shea, E. Norbeck, D. J. Morrissey, T. Li, E. Gualtieri, D. Craig, W. Benenson, and D. Bazin

Phys. Lett. 321B, 15 (January, 1994)

The effect of the neutron to proton ratio of the colliding system on the isotope ratio of the emitted fragments $(1 \leq Z \leq 5)$ was studied for a variety of constant mass beam-target combinations. To extend the $N / Z$ ratio as far as possible, one of the projectiles employed, ${ }^{40} \mathrm{Cl}$, was a radioactive secondary beam. The isotopic ratios depend on the $N / Z$ of the target and beam in a way which is not consistent with $N / Z$ equilibration on the timescale of the emission of intermediate-mass fragments.

\section{MEDIUM EFFECTS ON THE RHO MESON}

M. Asakawa and C. M. Ko

Phys. Rev. C 48, R526 (August, 1993)

The property of a tho meson in dense matter is studied using the QCD sum rule. The spectral function appearing on the hadronic side of the sum rule is evaluated in the vector dominance model that takes into account the interaction between the rho meson and the pion. Including pion modification by the delta-hole polarization in the nuclear medium, we find that as the nuclear density increases the rho meson peak in the spectral function shifts to smaller invariant masses and its width becomes smaller. We discuss the possibility of studying the rho meson property in dense matter via the dilepton invariant mass spectrum from heavy-ion collisions.

\section{MASS AND CHARGE DISTRIBUTIONS OF Cl- INDUCED HEAVY-ION REACTIONS}

A. A. Marchetti, A. C. Mignerey, H. Madani, A. Gökmen, W. L. Kehoe, B. Libby, K. Morley, H. Breuer, K. Wolf, and F. Obenshain Phys. Rev. C 48, 266 (July, 1993) 
Projectilelike fragments were detected and characterized in terms of $A, Z$, and energy for the reactions ${ }^{37} \mathrm{Cl}$ on ${ }^{40} \mathrm{Ca}$ and ${ }^{209} \mathrm{Bi}$ at $\mathrm{E}=7.3$ $\mathrm{MeV} /$ nucleon, and ${ }^{35} \mathrm{Cl}$ on ${ }^{209} \mathrm{Bi}$ at $\mathrm{E}=15$ $\mathrm{MeV} /$ nucleon, at angles close to the grazing angle. Mass and charge distributions were generated in the $\mathrm{N}-\mathrm{Z}$ plane as a function of energy loss, and parameterized in terms of their centroids, variances, and coefficients of correlation. The results are compared to the predictions of two current models based on a stochastic nucleon exchange mechanism. The drifts of the charge and mass centroids for the system ${ }^{37} \mathrm{Cl}$ on ${ }^{40} \mathrm{Ca}$ are consistent with a process of mass and charge equilibration mediated by nucleon exchange between the two partners, followed by evaporation. The asymmetric systems show a strong drift toward larger asymmetry, with the production of neutron-rich nuclei. It is concluded that this is the result of a net transfer of protons from the light to the heavy partner, and a net flow of neutrons in the opposite direction. Model predictions fail to reproduce in detail the evolution of the centroids for asymmetric systems. The variances for all systems increase with energy loss, as would be expected from a nucleon exchange mechanism. However, the variances for the reaction ${ }^{37} \mathrm{Cl}$ on ${ }^{40} \mathrm{Ca}$ are higher than those expected from that mechanism, and the variances for the reaction ${ }^{35} \mathrm{Cl}$ on ${ }^{209} \mathrm{Bi}$ start decreasing after about $100 \mathrm{MeV}$ of energy loss. The coefficients of correlation indicate that the transfer of nucleons between projectile and target is correlated, as expected from $Q$-value constraints to the valley of $\beta$ stability.

\section{np-ELASTIC ANALYZING POWER A $\mathrm{NO}_{\mathrm{NO}}$ AND SPIN TRANSFER $\mathrm{K}_{\mathrm{NN}}$}

M. W. McNaughton, K. Johnston, D. R. Swenson, D. Tupa, R. L. York, D. A. Ambrose, P. Coffey, K. H. McNaughton, P. J. Riley, G. Glass, J. C. Hiebert, R. H. Jeppesen, H. Spinka, I. Supek, G. E. Tripard, and $\mathrm{H}$. Woolverton

Phys. Rev. C 48, 256 (July, 1993)

We have measured the analyzing power $A_{N O}$ and the spin transfer $\mathrm{K}_{\mathrm{NN}}$ for np-elastic scattering from about $60^{\circ}$ to $170^{\circ} \mathrm{c} . \mathrm{m}$. at 485,635 , and $788 \mathrm{MeV}$. The new data clarify previous discrepancies and complete the first-order determination of nucleonnucleon elastic scattering at these energies.

\section{FORWARD ANGLE ANALYZING POWER IN p- n AND p-p QUASIFREE SCATTERING AT 643 AND $797 \mathrm{MeV}$}

\author{
G. Glass, T. S. Bhatia, J. C. Hiebert, R. A. \\ Kenefick, S. Nath, L. C. Northcliffe, W. B. \\ Tippens, and J. E. Simmons \\ Phys. Rev. C 47, 1369 (April, 1993)
}

Polarized proton beams and a liquid-deuterium target were used to measure the analyzing power for quasifree $\vec{p}-n$ and $\vec{p}-\mathrm{p}$ scattering in the forward direction at 643 and $797 \mathrm{MeV}$. Alternate use of a liquid-hydrogen target provided a comparison of free and quasifree $\bar{p}-\mathrm{p}$ scattering. The precision of the data is in general \pm 0.01 to \pm 0.02 , although some of the p-p data for $797 \mathrm{MeV}$ are at the precision level of \pm 0.005 . For c.m. angles $\theta^{*} \geq 22^{\circ}$ there is no difference between quasifree and free results at either energy. The data are compared with the predictions of several phase-shift analyses. In a search for more pronounced quasifree scattering effects, a small amount of $\vec{p}$-"p" data were taken with a carbon target.

\section{MEDIUM EFFECTS ON KAON AND ANTIKAON SPECTRA IN HEAVY-ION COLLISIONS}

\author{
X. S. Fang, C. M. Ko, G. E. Brown, and V. Koch \\ Phys. Rev. C 47, 1678 (April, 1993)
}

In the linear chiral perturbation theory, both kaon and antikaon masses decrease in dense matter. There is also a repulsive vector potential for the kaon and an attractive one for the antikaon. With these effects included in the relativistictransport model, it is found that the slope parameter of the kaon kinetic energy distribution is larger than that of the antikaon. This is consistent with the experimental data from heavyion collisions in the Alternating Gradient Synchrontron experiments at Brookhaven.

\section{TWO-PION CORRELATIONS AND MULTIPLICITY EFFECTS IN La ON La COLLISIONS}

H. Bossy, J. A. Bistirlich, R. R. Bossingham, A. D. Chacon, K. M. Crowe, Y. Dardenne, $M$. Justice, J. O. Rasmussen, A. A. Shilab-Eldin, M. 
A. Stoyer, K. D. Wyatt, J. P. Sullivan, and K. L. Wolf

Phys. Rev. C 47, 1659 (April, 1993)

Bose-Einstein correlations of negative pions in heavy ion collisions have been investigated for the reaction ${ }^{139} \mathrm{La}+{ }^{1{ }^{13}} \mathrm{La} \rightarrow 2 \pi^{-}+\mathrm{X}$ at 1.26 $\mathrm{GeV} /$ nucleon at two acceptances, centered at laboratory observation angles of approximately $0^{\circ}$ and $45^{\circ}$ with respect to the beam axis. A scintillation counter array downstream of the target was used to sample the charged particle multiplicity of each event and hence give some information on the impact parameter of the collision. Including results from previous experiments, space-time dimensions of the pion source are now available for mass-symmetric collisions in the mass range of $A=40$ to 139 . The sources are oblate for nearly all systems, except for $\mathrm{La}+\mathrm{La}$ central collisions viewed near $45^{\circ}$ in the laboratory $\left(90^{\circ}\right.$ in the center of mass) where the source is spherical. The perpendicular radius $R_{\perp}$ is never less than $4 \mathrm{fm}$, regardless of the centrality of the $\mathrm{La}+\mathrm{La}$ collision. Furthermore, $\mathrm{R}_{\perp}$ seems independent of the mass of the collision system.

GIANT DIPOLE RESONANCE GAMMA DECAY OF ${ }^{142}$ Nd NUCLEUS PRODUCED BY ${ }^{16} \mathrm{O}+{ }^{126} \mathrm{Te}$ AND ${ }^{22} \mathrm{Ne}+{ }^{120} \mathrm{Sn}$ REACTIONS AT $E^{*}=150 \mathrm{MeV}$

R. K. Choudhury, K. L. Wolf, H. Jabs, J. Shoemaker, J. B. Natowitz, K. Hagel, Y. Lou, R. Wada, R. P. Schmitt, W. Turmel, and H. Xu Nucl. Phys. A569, 93c (March, 1994)

The particle and gamma decay from ${ }^{142} \mathrm{Nd}$ nucleus produced at $\mathrm{E}^{*}=150 \mathrm{MeV}$ by ${ }^{16} \mathrm{O}+{ }^{126} \mathrm{Te}$ and ${ }^{22} \mathrm{Ne}$ $+{ }^{120} \mathrm{Sn}$ reactions were studied. The measured gamma ray spectra were analyzed to derive the GDR centroid and width parameters, which were found to be the same for both systems. No entrance channel effects were seen in the particle and gamma emission spectra in these systems.

\section{NUCLEAR MATTER INCOMPRESSIBILITY COEFFICIENT AND GIANT MONOPOLE RESONANCE}

S. Shlomo and D. H. Youngblood Nucl. Phys. A569, 303c (March, 1994)
We examine the current status of nuclear matter incompressibility coefficient, $\mathrm{K}_{\mathrm{nm}}$, deduced from experimental data on the strength function of the isoscalar giant monopole resonance (ISGMR) in nuclei. We review the results obtained by using a microscopic (continuum) random phase approximation (RPA) calculation and by using a semi-empirical expansion of the nucleus incompressibility coefficient $K_{A}$ in $A^{-1 / 3}$. We discuss the accuracy of the semiempirical expansion and present arguments indicating that all the coefficients in this expansion must be determined by a fit to the data. Contrary to recent claims, the present complete data set is not adequate to limit the range of $K_{n m}$ to better than 200-350 MeV when employing the semi-empirical approach.

\section{LIMITING TEMPERATURES AND DISAPPEARANCE OF FUSIONLIKE RESIDUES}

\author{
H. M. Xu \\ Nucl. Phys. A568, 365 (February, 1994)
}

With an improved Boltzmann-Uehling-Uhlenbeck (BUU) model, we have performed a comparative study of the reaction dynamics which limits the formation of fusionlike residues in ${ }^{40} \mathrm{Ar}+{ }^{27} \mathrm{Al}$ collisions at energies near the Fermi energy. By adjusting separately the values of the in-medium nucleon-nucleon cross section $\sigma_{\mathrm{NN}}$, we have obtained similar residue cross sections for both the stiff and the soft equations of state (EOS). Using these two exclusive parameters, we have calculated the thermal excitation energies, the angular momenta, and the temperatures for the fusionlike residues survived in the collisions. These calculations suggest that the reaction dynamics, not the Coulomb or thermal instabilities, plays the most important role in limiting the production of fusionlike residues at energies less than the Fermi energy. Furthermore, the calculations indicate that although the same residue cross sections can be obtained for different equations of state by adjusting separately the values of $\sigma_{\mathrm{NN}}$, one would still see significant differences in the residues temperatures. This sensitivity to the temperature arises from the sole sensitivity of the temperature to the EOS and the insensitivity of the temperature to the $\sigma_{\mathrm{NN}}$. Within the uncertainties of both the experimental data and the model calculations, the calculated cross sections and the residue temperatures are comparable to experimental observations. More systematical experimental studies; including the impact-parameter dependencies, are called for, in 
particular, to address the questions concerning the equation of state.

\section{MULTIFRAGMENTATION OF TOROIDAL AND BUBBLE NUCLEI WITHIN THE BUU APPROACH}

H. M. Xu, C. A. Gagliardi, R. E. Tribble, and C. Y. Wong

Nucl. Phys. A569, 575 (February, 1994)

Multifragmentation, following the formation of toroidal and bubble nuclei, is observed with an improved Boltzmann-Uehling-Uhlenbeck (BUU) model for central ${ }^{92} \mathrm{Mo}+{ }^{92} \mathrm{Mo}$ collisions. With a stiff equation of state, simultaneous explosion into several nearly-equal fragments (NEFs) in a ring-like manner occurs due to the formation of metastable toroidal nuclei. In contrast, with a soft equation of state, simultaneous explosion into several NEFs in a volume-like manner occurs due to the formation of metastable bubble nuclei. Guided by our BUU model, we propose two signatures: (1) because of the geometries of bubbles and toroids and because of the cold breakup at low temperatures, we predict enhanced cross sections for NEFs at small center-ofmass energies. (2) the coplanarity of these NEFs could carry important information concerning the geometry of the sources. This, in turn, could provide information about the stiffness of the equation of state. Finally, the dependence of these two predictions on the number of test particles, impact parameters, and the initial random seeds are assessed.

\section{SYMMETRIC MULTI-FRAGMENTATION BARRIERS AND THEIR TEMPERATURE- DEPENDENCE IN MEDIUM AND HEAVY MASS NUCLEI}

\section{G. X. Dai, J. B. Natowitz, R. Wada, Y. N. Lou, K. Hagel, and B. Xiao \\ Nucl. Phys. A568, 601 (February, 1994)}

Shapes consisting of a series of pear-like bodies surrounding a inner joined sphere are assumed to be those associated with symmetric fission or fragmentation into two or more fragments. The shapes evolve smoothly from that of an initial spherical nucleus to the scission point. The shapes are calculated using a generalization of the Cassinian ovaloid shape parameterization, which has been demonstrated to be a good approximation for binary fission. Using this parameterization fission barriers have been calculated for 2-, 3-, 4-, 6- and 8-fold multi-fragment final states. Proximity effects are included. The temperature dependence of these fission barriers is also calculated.

\section{SINGLE PARTICLE SPECTRA FROM NA44}

\author{
M. Murray
}

Nucl. Phys. A566, 515 (January, 1994)

We have measured $\pi^{ \pm}, \mathrm{K}^{ \pm}, \mathrm{P}$ and $\mathrm{P}$ spectra from proton and sulfur collisions with nuclear targets at the at beam energies of $450 \mathrm{GeV}$ and $200 \mathrm{GeV}$ per nucleon respectively. For central SA collisions we see that kaons and (anti)protons have large transverse energies. Protons are moved into central rapidity by rescattering where they annihilate antiprotons. The RQMD event generator qualitatively reproduces these trends while Fritjof does not.

\section{DOUBLE PHI PEAKS AS A SIGNATURE FOR THE QCD PHASE TRANSITION \\ C. M. Ko and M. Asakawa \\ Nucl. Phys. A566, 447 (December, 1993)}

Heavy-ion experiments offer the possibility to create in the laboratory the deconfined quark-gluon plasma. Many experimental observables have been proposed as possible signatures for its existence. We have shown previously that $\mathrm{M}_{\mathrm{T}}$ scaling in the dilepton spectrum between the $\phi$ and $\mathrm{J} / \psi$ peak is a plausible signature for the quark-gluon plasma expected to be formed in future experiments at the Relativistic Heavy Ion Collider (RHIC) and the Large Hadron Collider (LHC).

\section{QCD SUM RULES FOR A RHO MESON IN DENSE MATTER}

\author{
M. Asakawa and C. M. Ko
}

Nucl. Phys. A560, 399 (July, 1993) 
Using the QCD sum rules, we study the property of a rho meson in dense hadronic matter. Instead of the simple rho meson pole approximation for the rho meson spectral function at low momenta, we evaluate it using the vector dominance model that takes into account the interaction between the rho meson and the pion. Including pion modification by the deltahole polarization in the nuclear medium, we find that as the nuclear density increases the rho meson peak in the spectral function shifts to smaller invariant masses. We discuss the possibility of studying the rho meson property in dense matter via the dilepton invariant mass spectrum from heavy-ion collisions.

\section{RESCATTERING EFFECTS ON KAON ENERGY SPECTRA IN HEAVY-ION COLLISIONS}

\author{
X. S. Fang, C. M. Ko, and Y. M. Zheng \\ Nucl. Phys. A556, 499 (May, 1993)
}

Kaon production from heavy ion collisions at energies that are below the threshold for its production in free space is studied in the relativistic Vlasov-Uehling-Uhlenbeck model. Because of the small production probability, kaon production in baryon-baryon reactions is treated perturbatively. The rescattering of the kaon by nucleons is, however, included in the calculation. It is found that kaons undergo substantial rescatterings as they are mostly produced in high density region. This leads to significant effects on kaon final kinetic energy spectra.

\section{MODEL CALCULATIONS OF MULTIELECTRON IONIZATION OF $\mathrm{O}_{2}$ MOLECULES BY FAST-HEAVY-ION IMPACT}

\author{
K. Wohrer and R. L. Watson
}

Phys. Rev. A 48, 4784 (December, 1993)

A simple model employing the independentelectron approximation was developed to investigate orientation effects on the cross sections for multielectron ionization of diatomic molecules in fastheavy-ion collisions. The model was used to compare $\mathrm{K}$ - and L-shell ionization cross sections for $\mathrm{O}_{2}$ molecules with internuclear axes oriented parallel and perpendicular to the beam.
INCLUSIVE FRAGMENT PRODUCTION IN ${ }^{84} \mathrm{Kr}$ $+{ }^{197} \mathrm{Au} \mathrm{AT} \mathrm{E} / \mathrm{A}=35 \mathrm{MeV}$

U. Milkau, M. L. Begemann-Blaich, E.-M. Eckert, G. Imme, P. Kreutz, A. Kühmichel, M. Lattuada, U. Lynen, C. Mazur, W. F. J. Müller, J. B. Natowitz, C. Ngô, J. Pochodzalla, G. Raciti, M. Ribrag, H. Sann, W. Trautmann, and R. Trockel Z. Phys. A346, 227 (May, 1993)

Inclusive cross sections of intermediate mass fragments from the reaction ${ }^{84} \mathrm{Kr}+{ }^{197} \mathrm{Au}$ at $\mathrm{E} / \mathrm{A}=$ $35 \mathrm{MeV}$ were measured over the range $8^{\circ} \leq \theta_{\text {lab }} \leq$ $70^{\circ}$ with a low detection threshold. A moving-source parameterization was used to fit the doubledifferential cross sections. The integrated cross section for fragment production exceeds the total reaction cross section this indicating a large probability for multi-fragment processes. The deduced large temperature parameters can be explained by assuming emission from a rotating source. From the comparison to reactions with ${ }^{12} \mathrm{C}$ and ${ }^{40} \mathrm{Ar}$ projectiles at $\mathrm{E} / \mathrm{A}=30 \mathrm{MeV}$ a systematics of inclusive fragment production as a function of the projectile mass is obtained.

\section{IONIZATION OF NOBLE GAS ATOMS BY ALPHA PARTICLES AND FISSION FRAGMENTS FROM THE DECAY OF ${ }^{252} \mathrm{Cf}$}

\section{B. M. Hill, R. L. Watson, K. Wohrer, B. B. Bandong, G. Sampoll, and V. Horvat \\ J. Mass Spect. and Ion. Proc. 126, 37}

$$
\text { (April, 1993) }
$$

Charge state distributions of $\mathrm{He}, \mathrm{Ne}, \mathrm{Ar}, \mathrm{Kr}$, and $\mathrm{Xe}$ ions produced in single collisions with alpha particles and fission fragments from the decay of ${ }^{252} \mathrm{Cf}$ have been measured using time-of-flight spectroscopy. The measurements reveal that the maximum number of electrons removed in a fission fragment collision ranges from 8 in the case of $\mathrm{Ne}$ to 20 in the case of Xe. Recoil-ion production cross sections have been determined for the resolvable ionic charge states and compared with the predictions of a model based upon the independent electron approximation.

\section{MULTIFRAGMENTATION IN ${ }^{40} \mathrm{Ca}+{ }^{40} \mathrm{Ca}$ REACTIONS}

\author{
J. B. Natowitz
}

Proceedings of the Hirschegg Workshop on 
Multifragmentation, Hirschegg, Austria (January, 1994)

The multifragment emission of "completely characterized" events in the ${ }^{40} \mathrm{Ca}+{ }^{40} \mathrm{Ca}$ system at 35 $\mathrm{MeV} /$ nucleon has been compared to the predictions of several models. The observed multifragment emission is not in agreement with models based on conventional statistical binary decay, but is in agreement with both a simultaneous multifragmentation model and a sequential emission model in which expansion is treated.

\section{MEDIUM EFFECTS ON HEAVY-ION DYNAMICS}

C. M. Ko

Proceedings of the Workshop on Pre-Equilibrium Parton Dynamics, Berkeley, California (August, 1993)

The property of hadrons in hot dense matter is reviewed. Incorporating the medium effects in the relativistic transport model, which consistently treats the change of hadron masses and energies in dense matter via the scalar and vector fields, the expansion stage of heavy-ion collisions at the AGS and CERN energies is studied. With the in-medium particle production cross sections, this model provides a plausible explanation for the observed enhancement of strange particle production and differences in the slope parameters of the kinetic energy spectra for particles and antiparticles.

\section{SIGNATURES FOR QGP IN DILEPTON SPECTRUM}

\section{Asakawa}

Proceedings of the Workshop on Pre-Equilibrium Parton Dynamics, Berkeley, California (August, 1993)

We discuss two ideas to verify the formation of the quark-gluon plasma in ultrarelativistic heavy ion collisions using dileptons.

\section{FISSION TIME SCALES AND SHAPES FROM GDR $\gamma$ RAYS}

R. P. Schmitt, W. Turmel, L. Cooke, J. Boger, T.
Botting, B. Hurst, and D. O'Kelly

Proceedings of the Second International Conference on Dynamical Aspects of Nuclear Fission, Smolenice, CSFR (June, 1993)

Fission time scales have been investigated by studying GDR $\gamma$-ray emission in the reaction 120 $\mathrm{MeV}{ }^{16} \mathrm{O}+{ }^{208} \mathrm{~Pb}$. The gamma-ray spectra are well reproduced by statistical model calculations which hinder fission during the early stages of the deexcitation process. The analysis implies fission times in the range of 7.2-12.0 $\times 10^{-20} \mathrm{~s}$ over a fairly broad range of fragment masses. However, a drop off in yield of GDR $\gamma$ rays is observed at extreme asymmetries which suggests that the fission time scale may decrease by approximately a factor of two for very asymmetric fission.

\section{UPGRADE OF THE ECR ION SOURCE CAPABILITY AT TEXAS A\&M UNIVERSITY}

D. P. May and G. M. Mouchaty

Proceedings of the 11th International Workshop on Electron Cyclotron Resonance Ion Sources, Groningen, The Netherlands (May, 1993)

The operation of the ECR ion source at the Texas A\&M Cyclotron Institute has changed little since its commissioning and first use for injection into the K500 cyclotron. There has been some further development, specifically changing the analysis optics and adding a radial feed for rod material and crucibles. Operation of the source matches that of other 2-stage sources with $6.4 \mathrm{GHz}$ second stages. A proposal to upgrade the ion source capability of the Cyclotron Institute has been favorably received for funding in 1994. We are currently exploring options for a $14.5 \mathrm{GHz}$ ECR ion source. This could be an upgrade of the existing source or the construction of a new $14.5 \mathrm{GHz}$ ion source with dimensions approximating the LBL AECR. The latter option will be described along with our present capability.

\section{PROJECTILE BREAKUP REACTIONS INDUCED WITH $30 \mathrm{MeV} / \mathrm{u}{ }^{16} \mathrm{O}$ AND ${ }^{20} \mathrm{Ne}$}

R. P. Schmitt, H. Utsunomiya, D. O'Kelly, J. Boger, T. Botting, L. Cooke, B. Hurst, Y.-W. Lui, and W. Turmel

Proceedings of the International School-Seminar on Heavy Ion Physics, Dubna, Russia (May, 1993)

Breakup reactions of $30 \mathrm{MeV} / \mathrm{u}{ }^{16} \mathrm{O}$ and ${ }^{20} \mathrm{Ne}$ have 
been studied with high resolution techniques. Using a magnetic spectrograph, the dissociation of these projectiles was investigated for a variety of targets ranging from ${ }^{12} \mathrm{C}$ to ${ }^{208} \mathrm{~Pb}$. For all the systems, sequential breakup is the dominant reaction mechanism at least at small relative energies. A preliminary analysis of the yields of the various states indicates a statistical distribution is closely approached for total kinetic energy losses larger than about $30 \mathrm{MeV}$.

\section{HEAVY-ION REACTION MECHANISM STUDIES AND THE ROLE OF RADIOACTIVE BEAMS}

S. J. Yennello, B. Young, J. Yee, J. A. Winger, J. S. Winfield, G. D. Westfall, A. Vandermolen, B. M. Sherrill, D. J. Morrissey, T. Li, E. Gualtieri, W. Benenson, and D. Bazin

Proceedings of the Third International Conference on Radioactive Beams, East Lansing, Michigan (May, 1993)

The production of secondary beams of radioactive ions has begun to play an important role in the investigations of heavy-ion reaction mechanisms. The first experiment studying reaction mechanism by a radioactive beam was carried out at Michigan State University by combining the K1200 cyclotron, the

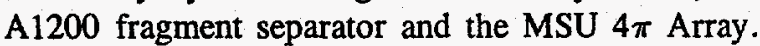
The reactions of ${ }^{40} \mathrm{Ca},{ }^{40} \mathrm{Ar}$ and ${ }^{40} \mathrm{Cl}$ beams with ${ }^{58} \mathrm{Ni}$ and ${ }^{58} \mathrm{Fe}$ targets were used to investigate the effects of changing the $N / Z$ of both the beam and the target within a constant mass system. Isotopically resolved intermediate-mass fragments (IMF) and light charged particles were detected by silicon detector arrays mounted in the $4 \pi$ Array, while complete event characterization was obtained by detection of nearly all IMF's and LCP's in the $4 \pi$ bragg curve and phoswich counters.

\section{DILEPTON PRODUCTION IN HEAVY ION COLLISIONS}

\author{
C. M. Ko \\ Proceedings of the 9th High Energy Heavy-Ion \\ Study, Berkeley, California \\ (October, 1993) \\ No abstract available

\section{SUBTHRESHOLD, KAON, ANTIKAON, AND ANTIPROTON PRODUCTION AT BEVELAC/SIS ENERGIES} \\ G. Q. Li and C. M. Ko \\ Proceedings of the 9th High Energy Heavy-Ion \\ Study, Berkeley, California \\ (October, 1993) \\ No abstract available
}

\section{MEDIUM EFFECTS ON HADRON STRUCTURE}

M. Asakawa, C. M. Ko, and P. Lévai

Proceedings of the International Workshop on QCD

Vacuum, Paris, France

(June, 1993)

No abstract available

\title{
ABSTRACTS OF PAPERS SUBMITTED
}

\author{
April 1, 1993 to March 31, 1994
}

\section{EFFECTS OF $N^{*}(1440)$ RESONANCE ON \\ SUBTHRESHOLD KAON, ANTIKAON, AND ANTIPROTON PRODUCTION}

\author{
B. A. Li, C. M. Ko, and G. Q. Li \\ Phys. Lett. B (Submitted, 1994)
}

We study the effects of $N^{*}(1440)$ resonance on the production of kaons, antikaons and antiprotons in heavy-ion collisions at subthreshold energies. Using free-space widths for the $\Delta(1232)$ and the $N^{*}(1440)$ resonance and free-space thresholds for particle production, it is found that the $\mathrm{N}^{*}$-baryon interactions contribute about $10 \%, 25 \%$ and $80 \%$ to the yield of kaon, antikaon and antiproton, respectively. Doubling the widths of resonances due to possible medium effects, both the total and the $\mathrm{N}^{*}$ induced antiproton yield decrease by about a factor of 3 . Using a reduced antiproton production threshold in a nuclear medium as a result of dropping in-medium nucleon and antiproton masses, the total and the $\mathbf{N}^{*}$ induced antiproton yield increase by a factor of 35 
and 27 , respectively, and the contribution of the $\mathrm{N}^{*}$ induced collisions is reduced to about $50 \%$ of the total.

\section{FIXED EXCITION NUMBER LEVEL DENSITY FOR A FINITE POTENTIAL WELL}

S. Shlomo, Ye. A. Bogila, V. M. Kolomietz, and

A. I. Sanzhur

Phys. Lett. B (Submitted, 1994)

We present a calculation of the nuclear level density $\mathrm{W}_{\mathrm{ph}}(\mathrm{E})$ for a fixed number of particles $p$ and holes $h$, taking into account the energy dependence of the single-particle level density $g(\epsilon)$. We demonstrate the significant effects of the finite depth of the potential well (continuum effect) and the finite surface thickness of the nucleus on the value of $\mathrm{W}_{\mathrm{ph}}(\mathrm{E})$.

\section{SUBTHRESHOLD ANTIKAON PRODUCTION IN NUCLEUS-NUCLEUS COLLISIONS}

\author{
G. Q. Li, C. M. Ko, and X. S. Fang \\ Phys. Lett. B (in press)
}

Antikaon production in nucleus-nucleus collisions at energies below the threshold for its production from the nucleon-nucleon interaction in free space is studied in the relativistic transport model. We include the in-medium self-energies of kaon and antikaon determined from either the mean-field approximation to the chiral Lagrangian or the empirical effective scattering lengths for the kaonnucleon and the antikaon-nucleon interaction. Also, the final-state interactions of antikaons with nucleons through both elastic scattering and absorption are explicitly treated in the study. Our results indicate that an attractive scalar potential for kaon and antikaon is needed to explain quantitatively the experimental data from the $\mathrm{Ni}+\mathrm{Ni}$ collision at 1.85 $\mathrm{GeV} /$ nucleon. to see more clearly the effect of the attractive scalar potential, we suggest that experiments at lower incident energies be carried out.

\footnotetext{
A COMPARISON OF BUU AND QMD CALCULATIONS FOR ${ }^{40} \mathrm{Ca}+{ }^{40} \mathrm{Ca}$ COLLISIONS NEAR THE FERMI ENERGY
}

J. Cibor, J. Lukasik, Z. Majka, and H. M. Xu Phys. Lett. B (in press)

The reaction dynamics for ${ }^{40} \mathrm{Ca}+{ }^{40} \mathrm{Ca}$ collisions near Fermi energy is investigated with a one-body BUU model and an n-body quantum molecular dynamics (QMD) model. Both models give similar predictions for quantities characterizing the early stages of reactions, i.e., the time scales for preequilibrium and thermalization, excitation energies and cross sections for residues surviving the collisions. The QMD model further predicts that evaporation residues can be produced in central collisions at energies much higher than those indicated by the BUU calculations.

\section{HADRON WIDTHS IN MIXED-PHASE MATTER \\ D. Seibert and C. M. Ko \\ Phys. Rev. Lett. (Submitted, 1994)}

We derive classically an expression for a hadron width in a two-phase region of hadron gas and quarkgluon plasma (QGP). The presence of QGP gives hadrons larger widths than they would have in a pure hadron gas. We find that the $\phi$ width observed in a central Au + Au collision at $\sqrt{s}=200 \mathrm{GeV} /$ nucleon is a few $\mathrm{MeV}$ greater than the width in a pure hadron gas. The part of observed hadron widths due to QGP is approximately proportional to $(d N / d y)^{-1 / 3}$.

\section{ANTIPROTON PRODUCTION IN Ni + $\mathrm{Ni}$ COLLISIONS AT $1.85 \mathrm{GeV} /$ nucleon}

\author{
G. Q. Li and C. M. Ko
}

Phys. Rev. C (Submitted, 1994)

Antiproton production in $\mathrm{Ni}+\mathrm{Ni}$ collisions at $1.85 \mathrm{GeV} /$ nucleon is studied in the relativistic Vlasov-Uehling-Uhlenbeck model. The self-energies of the antiproton are determined from the nucleon self-energies by the G-parity transformation. Also, the final-state interactions of the antiproton including both rescattering and annihilation are explicitly treated. With a soft nuclear equation of state the calculated antiproton momentum spectrum is in good agreement with recent experimental data from the heavy-ion synchrotron at GSI. The effect due to reduced nucleon and antinucleon masses in a medium is found to be more appreciable than in earlier Bevalac experiments with lighter systems and at 
higher energies.

\section{LARGE FRAGMENTATION OF THE PAIRING- VIBRATION-PARTICLE STRENGTH IN ${ }^{145} \mathrm{Sm}$}

L. Trache, A. M. Oros, Gh. Cata-Danil, K. O. Zell, P. von Brentano, G. Graw, D. Hofer and E. Müller-Zanotti Phys. Rev. C (in press)

The two-neutron transfer reaction ${ }^{147} \mathrm{Sm}(\mathrm{p}, \mathrm{t})^{145} \mathrm{Sm}$ was studied at $E_{p}=26 \mathrm{MeV}$. Angular distributions were measured for $\theta_{\text {lab }}=5^{\circ}-50^{\circ}$. The strength of the two neutron monopole pairing phonon-particle configuration ( $\left.\mathrm{n}_{-}=1, \mathrm{n}_{+}=1\right) \quad \nu 2 f_{72}$ is identified in old mass ${ }^{145} \mathrm{Sm}$ and is found to be quasi-uniformly spread in two narrow groups of as many as $8+5=$ 13 states. This unexpected large fragmentation is compared with the one in the core nucleus ${ }^{144} \mathrm{Sm}$ and with that from its isotone ${ }^{143} \mathrm{Nd}$ where MPV is less fragmented. The couplings with other excitation modes are extracted.

\section{EXCITATION ENERGY DEPOSITION IN CENTRAL COLLISIONS OF $40 \mathrm{AMeV}{ }^{40} \mathrm{Ar}$ WITH ${ }^{232} \mathrm{Th}$}

D. Utley, R. Wada, K. Hagel, J. Li, X. Bin, M. Gui, Y. Lou, R. Tezkratt, M. Gonin, and J. B. Natowitz Phys. Rev. C (in press)

Excitation energy depositions in the reactions of 40 $\mathrm{AMeV}{ }^{40} \mathrm{Ar}$ with ${ }^{232} \mathrm{Th}$ have been determined from measurements of neutron multiplicitiesin coincidence with mass identified heavy reaction products. For the most central collisions the derived excitation energies of $880 \mathrm{MeV}$ are 200 to $300 \mathrm{MeV}$ above previous estimates and in excellent agreement with momentum transfer systematics. Heavy evaporation residues are observed for these collisions indicating apparent dynamic delays in the fission channel of $1-5 \times 10^{-20}$ seconds. While a massive-transfer simulation incorporating pre-equilibrium emission is in generally good agreement with the experimental results evidence is also found for strongly damped collisions.

FRAGMENTATION BARRIERS OF TOROIDAL AND BUBBLE NUCLEI

H. M. Xu, C. A. Gagliardi, R. E. Tribble, and C.

\author{
Y. Wong \\ Phys. Rev. C (in press)
}

With an improved Boltzmann-Uehling-Uhlenbeck (BUU) model, the deformation energy surfaces in reactions leading to the formation of toroidal and bubble nuclei are examined for central ${ }^{92} \mathrm{Mo}+{ }^{92} \mathrm{Mo}$ collisions. We found that, the potential maximum, or the fragmentation barrier, occurs at times close to the times when these exotic shapes are formed. However, due to the dynamics of large amplitude compression and expansion, the fragmentation barriers are significantly higher than those estimated from the liquid-drop models.

\section{ROLE OF THE $a_{1}$ MESON IN DILEPTON PRODUCTION FROM HOT HADRONIC MATTER}

C. Song, C. M. Ko, and C. Gale Phys. Rev. D (Submitted, 1993)

Dilepton production from hot hadronic matter is studied in an effective chiral Lagrangian with pions, $\rho$-mesons, and $a_{1}$ mesons. We find that the production rates from reactions that involve axialvector mesons dominate over contributions from all other reactions when the dilepton invariant mass is above $1.5 \mathrm{GeV}$.

\section{RHO MESON SPECTRAL FUNCTION IN DENSE MATTER}

\section{M. Ko}

Phys. Rep. (in press)

The spectral function of a rho meson at rest in dense hadronic matter is studied in the vector dominance model by including both the effect of the delta-hole polarization on pion and the scaling inmedium hadron masses of Brown and Rho. It is found that as the nuclear density increases the rho meson peak moves to smaller invariant masses with diminishing strength. Also, a low mass peak around three times the pion mass appears and shifts down with increasing density. The change of the rho meson property in dense matter can be investigated via the dilepton invariant mass spectrum from heavyion collisions.

THE RELATIVISTIC TRANSPORT MODEL DESCRIPTION OF SUBTHRESHOLD KAON PRODUCTION IN HEAVY-ION COLLISIONS 
X. S. Fang, C. M. Ko, G. Q. Li, and Y. M. Zheng

Nucl. Phys. A (in press)

The relativistic transport model, in which the nucleon effective mass is connected to the scalar field while its energy is shifted by the vector potential, is extended to include the kaon degree of freedom. We further take into account the medium modification of the kaon mass due to explicit chiral symmetry breaking. Both the propagation of kaons in the mean-field potential and the kaon-baryon elastic scattering are explicitly treated in our study. We find that the attractive kaon scalar mean-field potential in the dense matter leads to an enhanced kaon yield in heavy-ion collisions at energies of about 1 $\mathrm{GeV} /$ nucleon that are below the threshold for kaon production from the nucleon-nucleon interaction in free space. The final-state kaon-baryon scattering is seen to affect significantly the kaon momentum spectra, leading to an enhanced yield of kaons with large momenta or at large laboratory angles. With a soft nuclear equation of state and including the attractive kaon scalar potential, the calculated kaon energy spectra agree with the preliminary data on subthreshold kaon production from the heavy-ion synchrotron at GSI.

\section{PHI MESON MASS IN HOT AND DENSE MATTER}

M. Asakawa and C. M. Ko

Nucl. Phys. A (Submitted, 1993)

The phi meson mass in hot and dense nuclear matter is studied in the QCD sum rules method. We find that it decreases substantially as a result of the abundant number of strange particles in the matter.

\section{ENERGY DEPENDENT MEASUREMENTS OF THE p-p ELASTIC ANALYZING POWER AND NARROW DIBARYON RESONANCES}

Y. Kobayashi, K. Kobayashi, T. Nakagawa, H. Shimizu, H. Y. Yoshida, H. Ohnuma, J. A. Holt, G. Glass, J. C. Hiebert, R. A. Kenefick, S. Nath, L. C. Northcliffe, A. J. Simon, S. Hiramatsu, Y. Mori, H. Sato, A. Takagi, T. Toyama, A. Ueno, and $\mathrm{K}$. Imai

Nucl. Phys. A (in press)
The energy dependence of the p-p elastic anallyzing power has been measured using an internal target during polarized beam acceleration. The data were obtained in incident-energy steps varying from 4 to $17 \mathrm{MeV}$ over an energy range from 0.5 to $2.0 \mathrm{GeV}$. The statistical uncertainty of the analyzing power is typically less than 0.01 . A narrow structure is observed around $2.17 \mathrm{GeV}$ in two proton invariant mass distribution. A possible explanation for the structure with narrow resonances is discussed.

\section{LIGHT EMISSION FROM A NaCl TARGET BOMBARDED BY MULTIPLY-CHARGED Ar IONS}

\section{Assad and R. E. Tribble}

Nucl. Instrum. and Methods (Submitted, 1994)

Light emitted by $\mathrm{Na}$ atoms following the bombardment of a $\mathrm{NaCl}$ target with $\mathrm{Ar}$ ions has been studied. Light yields that include contributions from both displaced surface and sputtered atoms have been obtained as a function of incident ion beam kinetic energy and with different Ar ion charge states. At a bombarding kinetic energy of $48 \mathrm{keV}$, we find that the light yield increases as the incident ion charge state increases. The light yield was found to increase and then slowly decrease as the ion beam kinetic energy changed from $8 \mathrm{keV}$ to $99 \mathrm{keV}$. Sputtering calculations have been carried out for both $\mathrm{NaCl}$ and $\mathrm{Ag}$ targets to investigate possible mechanisms for explaining the observed light yield in these two systems as a function of the incident beam kinetic energy.

\section{THE NUCLEAR SPECTRAL FUNCTION}

G. M. Vagradov and S. Shlomo

J. Phys. G (Submitted, 1994)

We consider some properties of the nuclear spectral function within the framework of the manybody field theory. We provide a proof for the existence of a lower energy limit for one particle spectrum. The general properties of the spectrum and the spectral function are demonstrated within a simplified model. We also consider the implication of the space-time translation invariance on the spectral function. 


\section{ON THE DECAY OF MANY-BODY SYSTEM}

\author{
G. M. Vagradov and S. Shlomo \\ Phys. Scripta (in press, 1994)
}

The existence of particle outflow in the decay of many body system leads to the special description with two sets of state eigenvectors and correspondent complex eigenvalues. Using such eigenvectors the general time behavior of the decaying system is obtained. We also consider the case of particle scattering by an optical potential and show that the existence of outflow also leads to two sets of state eigenvectors, but with real energy eigenvalues.

\section{STOCHASTIC ASPECTS OF NUCLEAR LARGE AMPLITUDE MOTION}

\section{M. Kolomietz}

Z. Phys. (Submitted, 1994)

A consistent description of the macroscopic large amplitude dynamics and processes of internal excitation of a nucleus is suggested. The cranking model approach is used for the calculation of the response function of the nucleus in a moving frame.
Using the spectral statistics smearing, the collective mass, friction and diffusion coefficients are derived. The relation of the response function in a moving frame to the correlation function in a classical chaotic system is established. The rate of dissipation due the Landau-Zener transitions and through the Kubo mechanism is considered.

\section{CONSTRUCTION OF THE MEGA PHOTON ARM DETECTOR}

M. Barakat, Y. Chen, S. Conrad, M. Cooper, M. Dzemidzic, J. A. Flick, C. Gagliardi, E. V. Hungerford, K. Johnston, G. J. Kim, K. Lan, F. Liu, Y. C. Lu, B. W. Mayes, R. Mischke, R. Phelps, L. S. Pinsky, D. Semon, R. E. Tribble, L. G. Tang, X. L. Tu, L. Van Ausdeln, W. Von Witsch, H. Z. Wang, X. Yao, and W. Yi Nucl. Instrum. Methods (in press, 1994)

The construction techniques used in the development of a set of large pair spectrometers for the MEGA detector are discussed. These spectrometers consist of carbon-fiber-composite cylinders on which the conversion foils are mounted, and appropriately spaced wire chambers in each layer which track the conversion leptons. Close tolerances on all dimensions are maintained. The detector has been operating well in the stopped muon beam line at LAMPF. 

TALKS PRESENTED

April 1993 - March 1994

Muon Decay Parameters, C. A. Gagliardi, Invited Talk, International Workshop on Low Energy Muon Science, Santa Fe, New Mexico (April, 1993).

Heavy Ion Reaction Mechanism Studies and the Role of Radioactive Beams, S. J. Yennello, B. M. Young, J. Yee, J. A. Winger, J. S. Winfield, G. D. Westfall, A. Vander Molen, B. M. Sherrill, J. Shea, E. Norbeck, D. J. Morrissey, T. Li, E. Gualtieri, D. Craig, W. Benenson, and D. Bazin, Invited Talk, Third International Conference on Radioactive Beams, East Lansing, Michigan (May, 1993).

In-Medium Strangeness Production, C. M. Ko, Invited Talk Topical Workshop on Meson Production in Nuclear Collisions, Darmstadt, Germany (May, 1993).

The Texas A\&M Cyclotron: Commissioning and Approaching the Limits, D. P. May, Invited Talk, Seminar at INFN, Catania, Sicily (May, 1993).

Upgrade of the ECR Ion Source Capability at Texas A\&M University, D. P. May, G. M. Mouchaty, 11th International Workshop on Electron Cyclotron Resonance Ion Sources, Groningen, The Netherlands (May, 1993).

Projectile Breakup Reactions Induced with $30 \mathrm{MeV} / \mathrm{u}^{16} \mathrm{O}$ and ${ }^{20} \mathrm{Ne}$, R. P. Schmitt, Invited Talk, International School-Seminar on Heavy Ion Physics, Dubna, Russia (May, 1993).

Fission Timescales and Shapes from GDR $\gamma$ Rays, R. P. Schmitt, Invited Talk, Second International Conference on Dynamical Aspects of Nuclear Fission, Smolenice, CSFR (June, 1993).

Dilepton as a Signature for the Quark-Gluon Plasma, C. M. Ko, Invited Talk, Nordic Workshop on Relativistic Heavy Ion Reaction Theory, Bergen, Norway (June, 1993).

Double Phi Peaks as a Signature for the QCD Phase Transition, C. M. Ko, Tenth International Conference on Ultra-Relativistic Nucleus-Nucleus Collisions, Borlange, Sweden (June, 1993).

De-Excitation Dynamics of Hot Nuclei, J. B. Natowitz, Invited Talk, Colloque GANIL, Toulon, France (June, 1993).

Medium Effects on Hadron Structure, M. Asakawa, C. M. Ko, and P. Lévai, Invited Talk, International Workshop on QCD Vacuum, Paris, France (June, 1993).

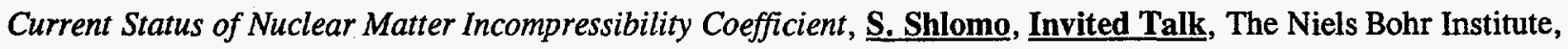
Copenhagen, Denmark (June, 1993).

Present Status of Proton, Neutron and Matter Density Distribution in Nuclei: Experiment and Theory, $\underline{\mathbf{S} \text {. Shlomo, }}$ Invited Talk, Summer Institute for Nuclear Astrophysics, The European Center for Theoretical Studies in Nuclear Physics and Related Areas (ECT*), Trento, Italy (July, 1993).

What We Know About Nuclear Compressibility, S. Shlomo, Invited Talk, The European Center for Nuclear Theory, Trento, Italy (July, 1993).

Nuclear Matter Incompressibility Coefficients and the Isoscalar Monopole Giant Resonance, S. Shlomo, Invited Talk, Summer Institute for Nuclear Astrophysics, The European Center for Theoretical Studies in Nuclear Physics and Related Areas (ECT*), Trento, Italy (July, 1993). 
Nuclear Level Density In Hot Nuclei, S. Shlomo, Invited Talk, University of Trento, Department of Physics, Povo, Italy (July, 1993).

Transport Theory of Heavy-Ion Collisions, C. M. Ko, Invited Talk, Workshop on Meson-Nucleus Dynamics at Intermediate and High Energies, Argonne, Illinois (August, 1993).

In-Medium Nucleon-Nucleon Cross Sections and Mean Field, G. O. Li, Invited Talk, Workshop on Meson-Nucleus Dynamics at Intermediate and High Energies, Argonne, Illinois (August, 1993).

Signals for the QCD Phase Transition, C. M. Ko, Invited Talk, Conference on Strong Interactions at Finite Temperature, Santa Barbara, California (August, 1993).

Medium Effects on Heavy-Ion Dynamics, C.M. Ko, Invited Talk, Workshop on Pre-Equilibrium Parton Dynamics, Berkeley, California (August, 1993).

Signatures of Quark-Gluon Plasma in Dilepton Spectrum, M. Asakawa, Invited Talk, Workshop on PreEquilibrium Parton Dynamics, Berkeley, California (August, 1993).

Nuclear Matter Compressibility Coefficient and Giant Monopole Resonance, S. Shlomo and D. H. Youngblood, Invited Talk, Gull Lake Conference on Giant Resonances, Gull Lake, Michigan (August, 1993).

Giant Dipole Resonance Gamma Decay of ${ }^{142} \mathrm{Nd}$ Nucleus Produced by ${ }^{16} \mathrm{O}+{ }^{126} \mathrm{Te}$ and ${ }^{22} \mathrm{Ne}+{ }^{120} \mathrm{Sn}$ Reactions at $E^{*}=150 \mathrm{MeV}$, R. K. Choudhury, K. L. Wolf, H. Jabs, J. Shoemaker, J. B. Natowitz, K. Hagel, Y. Lou, R. Wada, R. P. Schmitt, W. Turmel, and D. Utley, Invited Talk, Gull Lake Conference on Giant Resonances, Gull Lake, Michigan (August, 1993).

Equilibration Studies of Heavy Ion Collisions and the Role of Radioactive Beams, S. J. Yennello, Invited Talk, Fall Meeting of the American Chemical Society, Chicago, Illinois (August, 1993).

Initial Operation of MARS, R. E. Tribble, Invited Talk, Fall Meeting of the American Chemical Society, Chicago, Illinois (August, 1993).

Equilibration Studies of Heavy-Ion Collisions and the Role of Radioactive Beams, S. J. Yennello, Invited Talk, American Chemical Society, Chicago, Illinois (August, 1993).

Reaction Dynamics in the Formation and Decay of Hot Nuclei in the Reaction of ${ }^{63} \mathrm{Cu}+\mathrm{Au}$ at $35 \mathrm{AMeV}$, R. Wada, K. Hagel, R. Tezkratt, J. B. Natowitz, Y. Lou, B. Xiao, J. Li, D. Utley, N. Mdeiwayeh, J. Blackadar, and S. Lee, Invited Talk, Fall Meeting of the American Chemical Society, Chicago, Illinois (August, 1993).

Molecular Fission, R. L. Watson, Invited Talk, Fall Meeting of the American Chemical Society, Chicago, Illinois (August, 1993).

Isotopic Equilibration in the Reaction of $53 \mathrm{MeV} / \mathrm{n}^{40} \mathrm{Ca},{ }^{40} \mathrm{Cl},{ }^{40} \mathrm{Ar}$ with ${ }^{58} \mathrm{Fe}, \mathrm{Ni}, \mathrm{\mathbf {S }}$. J. Yennello, NSCL Seminar, East Lansing, Michigan (September, 1993).

Performance of the Texas A\&M K500, D. P. May, Seminar at National Superconducting Cyclotron Lab, Michigan State University (September 1993).

Dilepton Production in Heavy Ion Collisions, C. M. Ko, Invited Talk, 9th High Energy Heavy-Ion Study, Berkeley, California (October, 1993).

Subthreshold Kaon, Antikaon, and Antiproton Production at BEVALAC/SIS Energies, G. Q. Li and C. M. Ko, Invited Talk, 9th High-Energy Heavy-Ion Study, Berkeley, California (October, 1993). 
Multi-Fragment Emission in Intermediate Energy Heavy-Ion Collisions, $\underline{\mathbf{K}}$. Hagel, Invited Talk, Workshop on Multi-Fragment Emission, Asilomar, California (October, 1993).

Comparisons of Model Calculations to Experimental Data in the $35 \mathrm{MeV} / \mathrm{u}^{40} \mathrm{Ca}+{ }^{40} \mathrm{Ca}$ System, $\mathrm{K}$. Hagel, Invited Talk, Meeting of the American Physical Society, Asilomar, California (October, 1993).

Reaction Mechanism Studies with Radioactive Beams, S. J. Yennello, Invited Talk, NSCL Accelerator Upgrade Workshop, East Lansing, Michigan (November, 1993).

Multifragmentation in ${ }^{40} \mathrm{Ca}+{ }^{40} \mathrm{Ca}$ Reactions, J. B. Natowitz, Invited Talk, Hirschegg Workshop on Multifragmentation, Hirschegg, Austria (January, 1994).

Vector Mesons in Dense Matter, C. M. Ko, Invited Talk, Workshop on Mesons and Baryons in Hadronic Matter, Trento, Italy (February, 1994).

In-Medium Effects and their Consequences in Relativistic Transport Model Calculations, C. M. Ko, Invited Talk, Fourth Workshop on Dilepton Program at SIS, Darmstadt, Germany (March, 1994).

Isoscalar Monopole Giant Resonance and Nuclear Matter Incompressibility, S. Shlomo, Invited Talk, American Chemical Society, San Diego, California (March, 1994). 
Carl A. Gagliardi, Associate Professor of Physics Che Ming Ko, Professor of Physics

Joseph B. Natowitz, Professor of Chemistry, Director Lee Northcliffe, Professor of Physics

Richard P. Schmitt, Professor of Chemistry

Robert E. Tribble, Professor of Physics

Rand L. Watson, Professor of Chemistry

Kevin L. Wolf, Professor of Chemistry

Sherry J. Yennello, Assistant Professor of Chemistry

Dave H. Youngblood, Professor of Physics

\section{VISITING SCIENTISTS}

V. Kolomietz, ${ }^{1}$ Institute for Nuclear Research, Kiev, Ukraine

G. M. Vagradov, ${ }^{2}$ Institute for Nuclear Research, Moscow, Russia

\section{RESEARCH STAFF}

Jeff Bronson, Senior Scientist

Haydeh Dejbakhsh, ${ }^{3}$ Associate Research Scientist

John C. Hagel, Assistant Research Scientist

Vladimir Horvat, Assistant Research Scientist

George Kim, Accelerator Physicist

Yiu-Wing Lui, Research Scientist

Don May, Accelerator Physicist

George Mouchaty, Accelerator Physicist

A. Mukhamedzhanov, ${ }^{4}$ Assistant Research Scientist Shalom Shlomo, Senior Scientist

Livius Trache, ${ }^{5}$ Associate Research Scientist

Ryoichi Wada, Associate Research Scientist

\section{ENGINEERING STAFF}

Walter Chapman, Mechanical Engineer

Greg Derrig, ${ }^{6}$ Senior Mechanical Engineer

Robert Gutierrez, Electrical Engineer

Robert C. Rogers, Chief Engineer

Peter Smelser, ${ }^{7}$ Cryogenics Engineer

\author{
Masayuki Asakawa ${ }^{8}$ \\ John Boger ${ }^{9}$ \\ Aaron Chacon \\ Henry Clark ${ }^{10}$ \\ Guangxi Dai ${ }^{11}$ \\ Ferid Haddad ${ }^{12}$ \\ Guoqiang $\mathrm{Li}^{13}$
}

Wenzhong $\mathrm{Liu}^{14}$ Michael Murray

R. Parameswaran Chungsik Song ${ }^{15}$

Xiao-Lin Tu.

Jeff Winger ${ }^{16}$

Hongming $X \mathrm{U}$

Xiao-Gang Zhou

GRADUATE STUDENTS

John Blackadar ${ }^{17}$

Tye Botting

Bradley Hurst

Harry Jabs
Chemistry

Heather Johnston ${ }^{18}$

Shaleen Lee ${ }^{19}$

Donna O'Kelly

William Turmel ${ }^{20}$

Dennis Utley ${ }^{21}$

Physics

Gulnara Ajupova

Chahriar Assad

Angela Betker ${ }^{22}$

Xiao Bin

Svia Bouttchenko ${ }^{23}$

Xushan Fang

Jincai Jiang

Eric Hawker ${ }^{24}$

Ben Kokenge

Alexei Kolomiets ${ }^{25}$
Jiangtao Li

Fan Liu

Yunian Lou ${ }^{26}$ N. Mdeiwayeh ${ }^{27}$ Patrick Oliver ${ }^{28}$ David Semon George Simler ${ }^{29}$ Anthony Simon ${ }^{30}$ Leo Van Ausdeln ${ }^{31}$ Peiwen $\mathrm{Xia}^{32}$

\begin{tabular}{ll}
\hline${ }^{17}$ From 6-1-93 & ${ }^{25}$ From 6-1-93 \\
${ }^{18}$ From 6-1-93 & ${ }^{26}$ Through 1-31-94 \\
${ }^{19}$ Through 6-1-93 & ${ }^{27}$ From 6-1-93 \\
${ }^{20}$ Through 8-31-93 & ${ }^{28}$ From 6-1-93 \\
${ }^{21}$ Through 12-31-93 & ${ }^{29}$ From 6-1-93 \\
${ }^{22}$ Through 8-6-93 & ${ }^{30}$ Through 8-6-93 \\
${ }^{23}$ From 1-1-94 & ${ }^{31}$ Through 10-31-93 \\
${ }^{24}$ From 6-1-93 & ${ }^{32}$ From 6-1-93
\end{tabular}




\begin{tabular}{lll}
\multicolumn{1}{c}{ Name } & Year & \multicolumn{1}{c}{ Thesis Title } \\
\hline Angela Betker & 1993 & $\begin{array}{l}\text { Design \& Operation of a Facility to } \\
\text { Study Gamow-Teller Interactions with } \\
\text { the }\left(\mathrm{d},{ }^{2} \mathrm{He}\right) \text { Reaction }\end{array}$ \\
Yunian Lou & $1993 \begin{array}{l}\text { Excitation Energy Deposition and the } \\
\text { Fission Process in the Reactions }{ }^{63} \mathrm{Cu}+ \\
\end{array}$ \\
& $\begin{array}{l}{ }^{20,100} \mathrm{Mo} \text { at } 10,17,25 \text { and } 35 \mathrm{AMeV} \text { and } \\
{ }^{20}+{ }^{144,148,154} \mathrm{Sm} \text { at } 20 \mathrm{AMeV}\end{array}$
\end{tabular}

Anthony J. Simon

1993 High Precision Absolute Differential Cross-Section Measurements for ProtonProton Elastic Scattering @ 491.9, $575.5,641.6,728.2, \& 793.0 \mathrm{MeV}$

William Turmel

1993 Mass Asymmetry Dependence of GDR in Fission

Dennis Utley

Leo Van Ausdeln

1993 Excitation Energy and Fission Dynamics in $40 \mathrm{AMeV}{ }^{40} \mathrm{Ar}$ Induced Reactions

1993 A Photon Detector System for the
Advisor

Gagliardi

First Position

Post-doc - Indiana Univ.

Cyclotron Facility

Natowitz

Post-Doc - Indiana Univ.

Cyclotron Facility

Hiebert

Post-doc - Indiana University Medical School

Indianapolis, IN Search for the Rare Muon Decay $\mu \rightarrow \mathrm{e} \gamma$
Schmitt

Researcher

TAMU-Corpus Christi

Corpus Christi, TX

Natowitz

Lecturer - Texas A\&M

University - Chemistry

Gagliardi
Post-doc - TAMU - LAMPF MEGA Project, Los Alamos
Present Position

SAME

SAME

SAME

SAME

Instructor in Physics - Angelo State University

Researcher EG\&G, Idaho Falls 


\section{INSTITUTE COLLOQUIA AND SEMINARS}

$\underline{1993}$

April 2 J. Kolata, University of Notre Dame

April 20 Helio Dias, University of Sao Paulo, Brazil

April $27 \quad$ Jeff Winger, Texas A\&M University

May 4 Brian Gries, McDonnell-Douglas Corporation, Houston

May 4 Walter Henning, Argonne National Laboratory

May $7 \quad$ Peter Paul, State University of New York at Stony Brook

May 10

S. Freedman, University of Berkeley

May 13

May 17

May 21

June 8

June 14

June 17

June 29

July 7

August 27

August 31

September 2

September 3
B. Blank, University of Bordeaux, France

J. M. Alexander, SUNY at Stony Brook

M. Hussein, University of Sao Paulo, Brazil

K. M. Subotic, Boris Kidrich Institute, Belgrade

Felix Liang, SUNY at Stony Brook

Jim Brown, University of Michigan

Henry Clark, Ohio University

Brian Young, Michigan State University

M. Urin, Moscow, Russia

J. Parikh, Physical Research Laboratory, Ahmedabad, India

H. Schuessler, Physics Department, TAMU

E. Jacobson, Princeton University
Elastic Scattering and Fragmentation of Halo Nuclei

Study of Decay Modes of Giant Multipole Resonances

Search for the Termination Point of the rpProcess

The McDonnell-Douglas Single Event Effect (SEE) Project

Compressed Nuclear Matter: Neutron Stars and Heavy Ion Collisions

Hot Nuclear Matter, Giant Resonances and Superheavy Nuclei

The Demise of the $17 \mathrm{keV}$ Neutrino

Reaction and Decay Studies at the Proton Drip Line

Collective Motions in Central Collisions: ${ }^{40} \mathrm{Ar}+$ $\mathrm{Ag}(\mathrm{E} / \mathrm{A}=7-34 \mathrm{MeV})$

Deformed Gaussian Ensemble Approach to Symmetry Breaking in Nuclei

How to Measure the Density and Temperature of Nuclear Matter Using Isomer Beams

Quasi Elastic Transfer at Energies Near the Coulomb Barrier in ${ }^{32} \mathrm{Si}+{ }^{92,98,100} \mathrm{Mo}{ }^{93} \mathrm{Nb}$ Systems

Production and Scattering of Isomeric Beams

Studies with $500 \mathrm{GeV}$ Protons

Mass Measurements of ${ }^{10} \mathrm{Li}$ and ${ }^{11} \mathrm{Li}$

Partial Nucleon Escape Widths of Giant Resonances

Pre-equilibrium Collective Phenomena in QuarkGluon Plasma

On-Line Ion Transfer and Storing of Reaction Products

Exclusive Pion Production from $\mathrm{p}+{ }^{12} \mathrm{C}$ at the IUCF Cooler Ring 
September 7

Fred Moore, University of Texas at Austin

September 21 Gregory Ryjukh, Institute for Nuclear Research, Moscow, Russia

September 28 Fan Liu, Texas A\&M University

October 5 Livius Trache, Institute of Atomic Physics, Bucharest, Romania

October 14 Kris Hagel, Texas A\&M University

October 26 G. Viesti, University of Padova, Italy

October 28 William Turmel, Texas A\&M University

November 2 S. S. Kapoor, Bhabha Atomic Research Centre, Bombay, India

November 3 Bao-An Li, Hahn Meitner Institute, Berlin, Germany

November 16 Thomas Doessing, The Niels Bohr Institute, Copenhagen, Denmark

November 23 Nadine Barlow, Lunar and Planetary Institute, Houston

November 29 S. Lee, Yonsei University, Seoul, Korea

November 30 Gary Westfall, Michigan State University Cyclotron Lab

December 6 David Semon, Texas A\&M University

December 7 Jolie Cizewski, Rutgers University

December 10 Y. Z. Zhuo, Institute of Atomic Energy, Beijing, China

December 14 S. Moszkowski, University of California at Los Angeles

December 17 Mihai Horoi, Institute of Atomic Physics and Nuclear Engineering, Bucharest, Romania

$\underline{1994}$

January 10 Ramona Vogt, Lawrence Berkeley Laboratory
Double Giant Resonances

Dynamical Approaches for the Description of Light Nuclei

Measurement of the Michel Parameter Rho with the MEGA Positron Spectrometer

Exotic Multiphonon States in Odd Nuclei

Multifragment Emission in Heavy Ion Reactions

Recent Results on Gamma-Light Particle

Coincidences Using the Spectrometer GASP

Probing Nuclear Dynamics with GDR GammaRays

Nuclear Dynamics in Heavy Ion Induced FusionFission Reactions

Dynamical and Statistical Aspects of Nuclear Multifragmentation

Rotational Motion of Warm Atomic Nuclei

Physics Studies in the Martian Environment

Signature of Chiral Restoration in Low Temperature Pion Gas

The Disappearance of Flow and the Onset of Multifragmentation

Beta Decay of ${ }^{57} \mathrm{Cu}$ and the Weak Interaction

Dynamical Symmetries in Superdeformed Nuclei

Nuclear Fission Based on a Diffusion Model

The Triple Alpha Process in Nuclear Astrophysics: A Possible Application of Quark Chemistry

Recent Results in Parity Nonconserving Phenomena in Light Nuclei: A Large Shell Model Study

Rapidity Distributions of Dileptons from a Hadronizing Quark-Gluon Plasma 
January 17 Hongming $\mathrm{Xu}$, Texas A\&M University

January 19 Peter Levai, KFKI, Budapest, Hungary

January 25

V. Kolomietz, Institute for Nuclear Research, Kiev, Ukraine

February 8 A. Mukhamedzhanov, INR, Tashkent, Uzbekistan

February 15 A. Mukhamedzhanov, INR, Tashkent, Uzbekistan

March 1 A. Mukhamedzhanov, INR, Tashkent, Uzbekistan

March 8

Shirley A. Fry, Oak Ridge National Laboratory

March 22 Y. Oganessian, Joint Institute for Nuclear Research, Dubna, Russia (d, $\left.{ }^{2} \mathrm{He}\right)$ Reactions with Texas A\&M Di-Proton Spectrometer

Exotic Charmed Particle Production in Heavy Ion Collisions

Microscopic Properties of Nuclear Fermi Liquid

From Classical Nuclear Reactions to Nuclear Astrophysics

Experimental Proposals for Cyclotron Reactions to get Astrophysical Information

Anomalous Asymptotic Behavior of ManyParticle Bound State Wave Functions in TwoBody Channels and New Mechanism of Light Elements Nucleosynthesis at Very Low Energies. Possible Impact on Nuclear Astrophysics, Electron Screening, Muon-Catalyzed Fusion. Scientific Approach to Fusion at Very Low Energies ("Cold" Fusion)

Living with Radiation: How Risky Is It?

Discovery of Enhanced Nuclear Stability Near the Deformed Shells $N=162$ and $Z=108$ 